\title{
Emotionen und Erlebnisse beim Public Viewing
}

Explorative interdisziplinäre Analyse eines gesellschaftlichen

Phänomens

\section{Dissertation}

zur Erlangung des sozialwissenschaftlichen Doktorgrades an der Sozialwissenschaftlichen Fakultät der Georg-August-Universität Göttingen

\author{
vorgelegt von: \\ Britta Ufer \\ aus Lüneburg
}

Göttingen 2010 
1. Gutachter: Prof. Dr. Arnd Krüger

2. Gutachter: Prof. Dr. Axel Dreyer

Tag der mündlichen Prüfung: 26.06.2009 


\section{Danksagung}

Bevor auf der nächsten Seite die thematisch-wissenschaftlichen Ausführungen beginnen, möchte ich den Menschen danken, ohne die ich diese Dissertation nicht hätte verfassen können. An erster Stelle danke ich meinem Doktorvater, Herrn Prof. Dr. Arnd Krüger, der mich nicht nur zur Promotion motiviert, sondern der auch den wissenschaftlichen Entstehungsprozess permanent begleitet und stets neue Impulse geliefert hat. Ebenso möchte ich meinem zweiten Gutachter, Herrn Prof. Dr. Axel Dreyer, meinen Dank für seine Denkanstöße während des Doktorandenkolloquiums aussprechen und auch Herrn Prof. Dr. Heinz-Georg Marten für seine sofortige Prüfungsbereitschaft.

Wichtige Anlaufstellen waren mir während dieser Zeit, neben den anderen Kolloquiumsteilnehmern, so manche hilfsbereite Seele am IfS und das sozialwissenschaftliche Methodenzentrum. Einen besonderen Dank möchte ich der Sozialwissenschaftlichen Fakultät für die dreimonatige Auslauffinanzierung ausdrücken.

Des Weiteren gilt mein Dank meinem Vater, Armin Ufer, der nicht nur das tagelange Korrekturlesen nahezu klaglos übernommen hat, sondern mich auch immer wieder motiviert und mir in vielerlei Form diese akademische Ausbildung ermöglicht hat. Meiner Schwester, Annika Ufer, bin ich zu besonderem Dank verpflichtet, hat sie mir doch vor allem in langen Diskussionen immer wieder strukturelle, inhaltliche und mentale Unterstützung gegeben. Ohne sie hätte ich diese Promotion schlicht nicht schreiben können. Gerade letztere beide haben oftmals die Schattenseiten eines solchen 'Projektes' zu spüren bekommen und dennoch kühlen Kopf bewahrt.

Darüber hinaus sind auch Elfriede Blisse, mein Schwager Tobias Herzig-Ufer und mein Cousin Christian Schulz als schnelle Helfer hinsichtlich PC-Problemen zu nennen. Weiterhin möchte ich meiner Familie, insbesondere meiner Tante, Frau Edith Simon-Just, und meinem Patenonkel, Herrn Johannes Just, für ihr fortwährendes Interesse und ihre, auch finanzielle, Unterstützung danken.

Nicht zuletzt liegt es mir sehr am Herzen, meinen tollen Freunden, ich brauche sie nicht im Einzelnen zu nennen, denn ein jeder von ihnen weiß, wie wichtig er für mich ist, zu danken. Sie haben mir über diese nicht leichte Zeit in aller Form 
beigestanden, zu mir gehalten und mir den so nötigen Rückhalt gegeben. Nur wenige Dinge sind wirklich wichtig im Leben, eine Familie und gute Freunde gehören defi nitiv dazu! 


\section{Inhaltsverzeichnis}

Seite

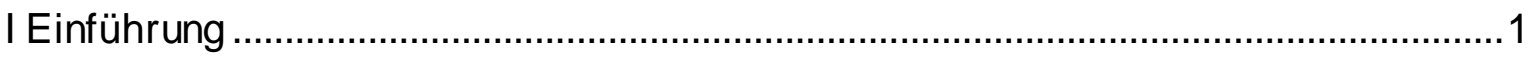

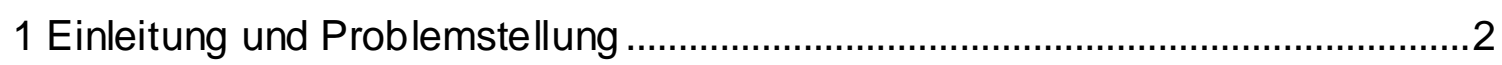

2 Perspektiven der Betrachtung ..........................................................................

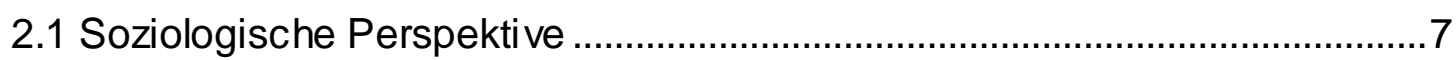

2.2 (Sozial-)Psychologische Perspektive ........................................................

2.3 Medienwissenschaftliche Perspektive .......................................................

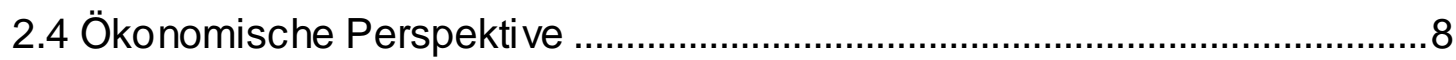

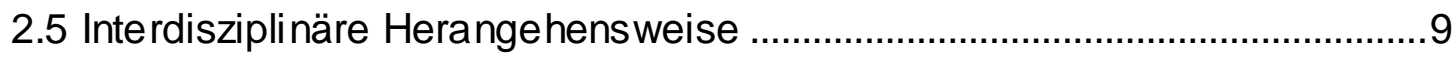

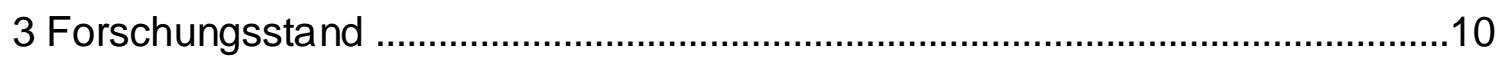

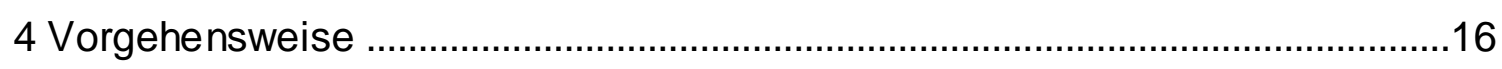

II Public Viewing als soziales Phänomen: Entwicklung und Grundlagen ...............21

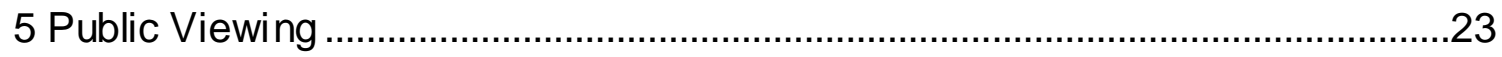

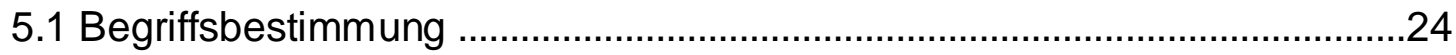

5.2 Wachsende Beliebtheit des Public Viewing ………..................................26

5.2.1 Fußball-Welt- und -Europameisterschaften als Quotengaranten........27

5.2.2 Schlüsselereignis: WM 2002 in Japan und Südkorea ..........................33

5.2.3 WM 2006: Die neue Qualität des Public Viewing .................................36

5.3 Zusammenfassung ................................................................................ 42

6 Modernisierungsprozesse in Gesellschaft, (Fußball-)Sport und Fernsehen....43

6.1 Postmoderne Gesellschaft: Wertewandel, Individualisierungs- und

Pluralisierungstendenzen .........................................................................4

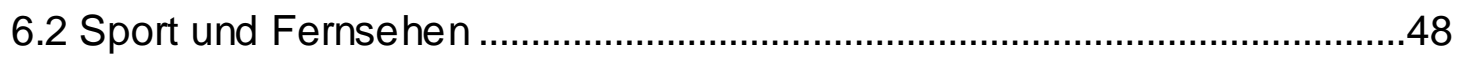

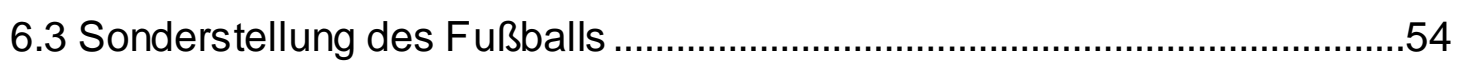

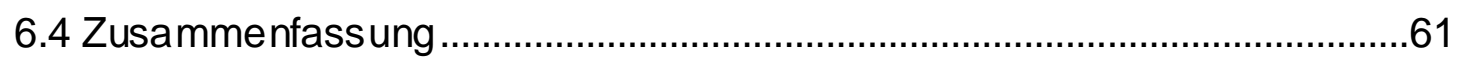

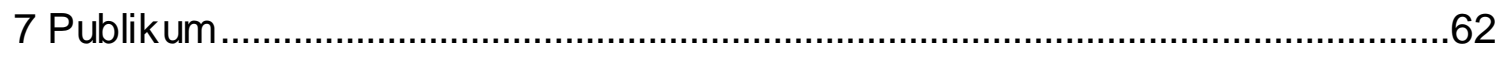

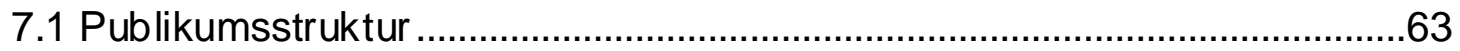

7.2 Determinanten der (Fernseh-)P ublikumsbildung .......................................66

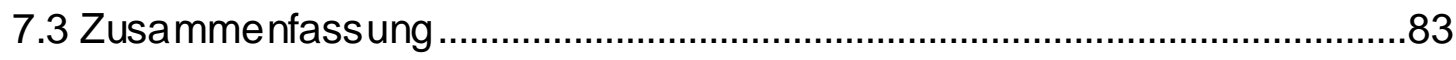

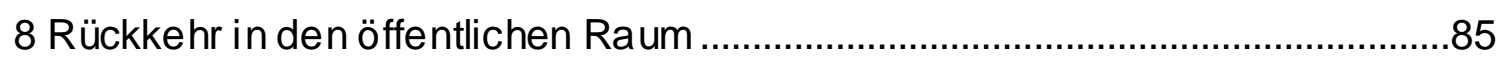

8.1 Determinanten des Public Viewing ..........................................................86

8.2 Technologische Entwicklung .................................................................... 


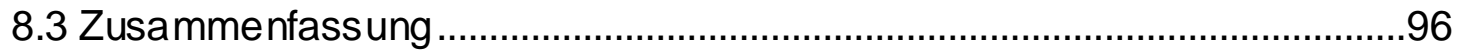

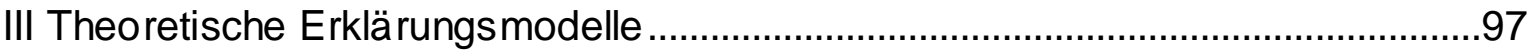

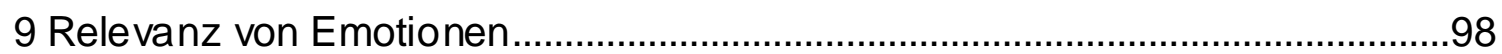

9.1 Emotionen: Begriffsabgrenzung und Definition ......................................... 100

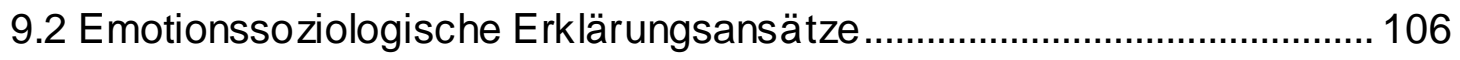

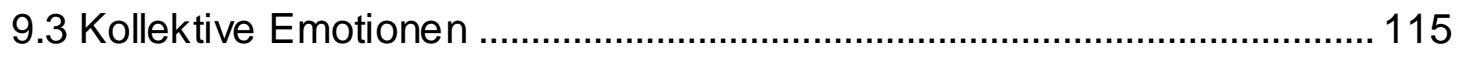

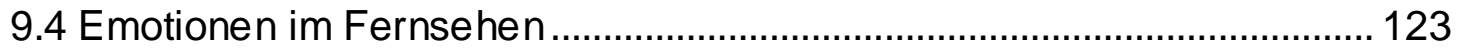

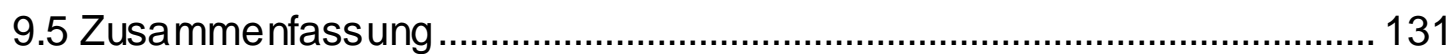

10 Suche nach und Inszenierung von Erlebnissen .......................................... 132

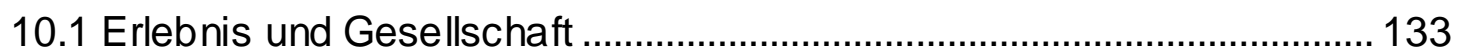

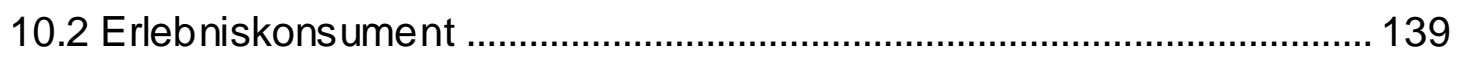

10.3 Public Viewing als Erlebnis-Setting? ................................................... 144

10.4 Inszenierung als Instrument des Erlebnis-Setting .................................. 148

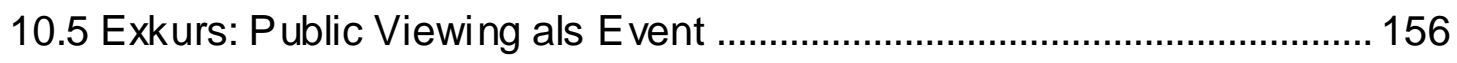

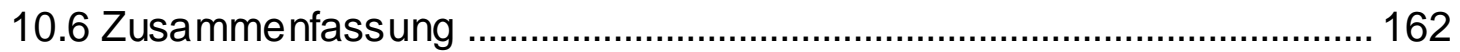

IV Methodik und Forschungsdesign.................................................................. 164

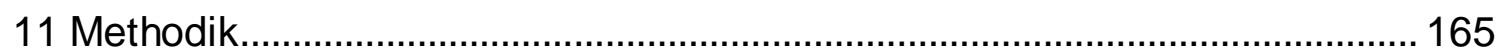

11.1 Triangulation: Qualitative und quantitative Verfahren im Methodenmix166

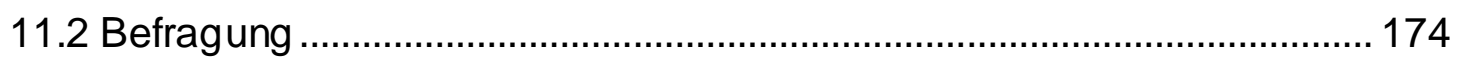

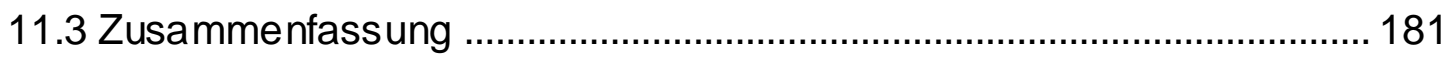

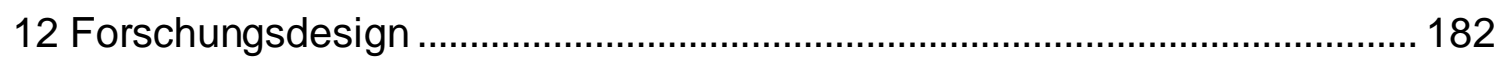

12.1 Design der Experteninterviews ........................................................... 183

12.1.1 Aufbau des Gesprächleitfadens .................................................... 184

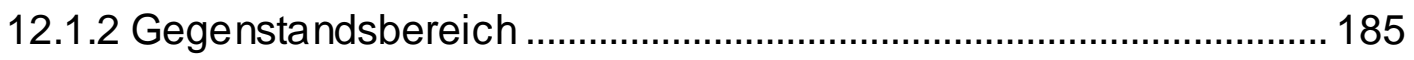

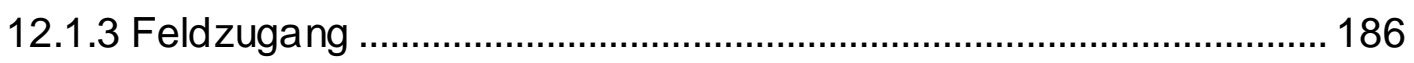

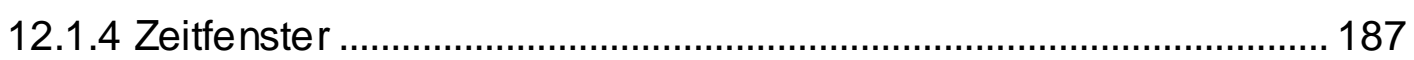

12.2 Design des Onlinefragebogens ........................................................... 187

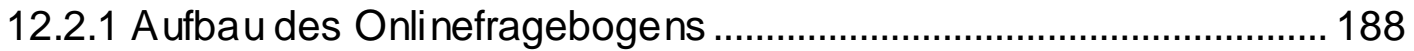

12.2.2 Gegenstandsbereich ...................................................................... 189

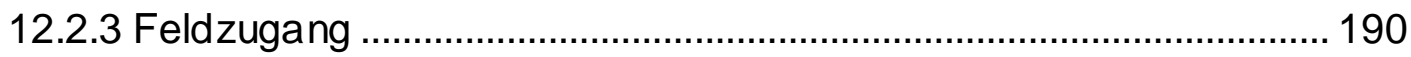

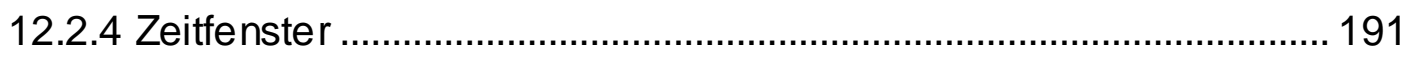

V Analyse der empirischen Daten ................................................................... 192

13 Diskussion der Untersuchungse rgebnisse .................................................. 193

13.1 Allgemeine Daten zu Experteninterviews und Onlinebefragung ........... 194 


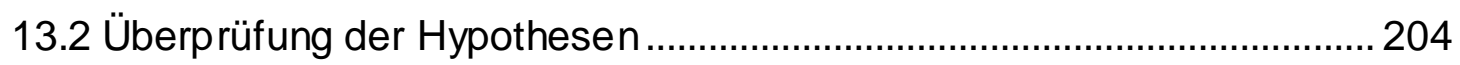

13.2.1 Entwicklungsfaktoren des Public Viewing ...................................... 205

13.2.2 Publikumsbildung beim Public Viewing ………............................... 216

13.2.3 Steuerbarkeit positiver Emotionen beim Public Viewing ................. 232

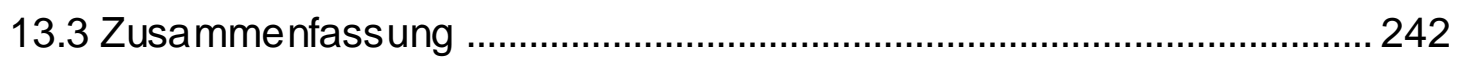

VI Schlussbemerkung und Ausblick.................................................................. 244

Literatur und Quellen ..........................................................................................

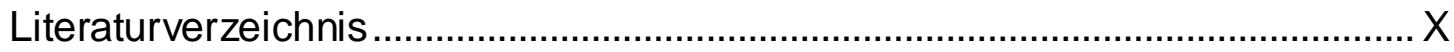

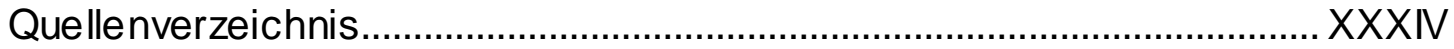

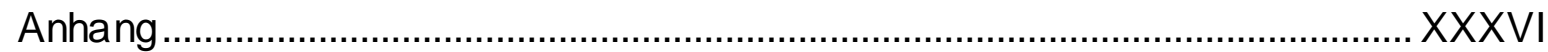

A. Anschreiben an die Public Viewing-Experten der FIFA Fußball-WM $2006^{\mathrm{TM}}$ $X X X V I$

B. Gesprächsleitfaden für die qualitativen Interviews mit den Public ViewingExperten der FIFA Fußball-WM $2006^{\mathrm{TM}}$ XXXVII

C. Onlinefragebogen für die Besucher von Public Viewing-Veranstaltungen ...XLI

D. Interviewtranskripte. .LI

E. Wissenschaftlicher Bildungsgang LII 


\section{Abkürzungsverzeichnis}

ADM

Arbeitskreis Deutscher Markt- und Sozialforschungsinstitute e.V.

ARD

Arbeitsgemeinschaft der öffentlich-rechtlichen Rundfunkanstalten der Bundesrepublik Deutschland

DFB

Deutscher Fußballbund

DOSB

Deutscher Olympischer Sportbund

DSF

Deutsches Sportfernsehen

FIFA

Fédération Internationale de Football Association

GEMA

Gesellschaft für musikalische Aufführungs- und mechanische Vervielfältigungsrechte

HBS Host Broadcast Service

HDTV

High Definition Television

Infront

Infront Sports \& Media AG

LED

Light-Emitting Diode

UEFA

Union des Associations Européennes de Football

$\mathrm{oFb}$

Online Fragebogen

RTL

Radiotélévision de Luxembourg

ZDF

Zweites Deutsches Fernsehen 


\section{Abbildungsverzeichnis}

Abb. 1: Zuschauerpotential (Zuschauer ab 3 Jahren) der Fußball-EM- und -WMSpiele 1992 bis 2008 in Deutschland (AGF/GfK; pc\#tv; Fernsehpanel D+EU nach Geese \& Gerhard, 2008, S. 443)

Abb. 2: Zuschauerstruktur (Zuschauer ab 14 Jahren) ausgewählter Spiele der Fußball-EM 2008 nach Geschlecht (AGF/GfK-Fernsehforschung, pc\#tv, Fernsehpanel D+EU nach Geese \& Gerhard, 2008, S. 445) 30

Abb. 3: Public Viewing bei der FIFA WM $2002^{\mathrm{TM}}$ in den Innenstädten Südkoreas (FIFA, o.J.c/Soo-min, 2002, p. 33)

Abb. 4: Orte der Außer-Haus-Nutzung (Personen, die mindestens ein Spiel außerhalb der eigenen Wohnung gesehen haben) im Verlauf verschiedener Fußballturniere "Wo haben Sie die Übertragungen der WM/EM gesehen?" (Mehrfachantworten möglich) (Telefonische Begleitumfragen anlässlich der Fußball-WM 2002 (MMA Frankfurt), Fußball-EM 2004 (forsa Berlin), und der Fußball-WM 2006 (IFAK Taunusstein) nach Geese et al., 2006, S. 459) .......40 Abb. 5: Traditionelle Arbeitswerte und neue Freizeitwerte (in Anlehnung an Becker, 2000, S. 12)

Abb. 6: Faktorengruppen für die Publikumsbildung (modifiziert nach Lucerna, 1997, S. 83).

Abb. 7: Contemporary football and the diffused audience (nach Abercrombie \& Longhurst, 1998, p. 178)

Abb. 8: Strukturelle Emotionen nach Kemper (nach Gerhards, 1988, S. 130) .... 108

Abb. 9: Kollektive Emotionen in einem emotionssoziologischen Modell (modifiziert nach Riedl, 2008, S. 245)

Abb. 10: Die wirtschaftliche Differenzierung (nach Pine \& Gilmore, 1999, S. 22) 140

Abb. 11: Fan Fest Frankfurt am Main (Stadt Frankfurt am Main, o.J.) 142

Abb. 12: Das Konzept des Erlebnis-Settings (nach Scheurer, 2003, S. 16) 145

Abb. 13: Einfluss von Umweltfaktoren und Wahrnehmung auf das Erlebnis (modifiziert nach Scheurer, 2003, S. 134) . 146

Abb. 14: Atmosphäre (modifiziert nach Scheurer, 2003, S. 139) 147

Abb. 15: Die Inszenierungspyramide (modifiziert nach Müller \& Scheurer, 2004, S. 14) 152 
Abb. 16: Inszenierungselemente Konzept des Erlebnis-Setting ( nach Scheurer, 2003, S. 214) 153

Abb. 17: Theoretische Grundannahmen qualitativer Forschung (nach Flick et al., 2005a, S. 22)

Abb. 18: Triangulationsmodell (nach Mayring, 2001, Abs. 21) ….......................... 168

Abb. 19: Typen der Befragung (nach Atteslander, 2003, S. 145) ........................ 178

Abb. 20: Drop-Out-Quote der Befragungsteilnehmer aufgeführt nach der letzten beantworteten Seite des Fragebogens $(n=595)$............................................ 199

Abb. 21: Altersstruktur der Teilnehmer der Onlinebefragung $(n=367)$................. 200

Abb. 22: Geschlechterverteilung der Teilnehmer der Onlinebefragung ( $n=364)$. 202

Abb. 23: Bildungsniveau der Teilnehmer der Onlinebefragung ( $n=367$ ) 202

Abb. 24: Herkunft der Teilnehmer der Onlinebefragung nach Bundesländern $(\mathrm{n}=356)$ 203

Abb. 25: Anlass, zu dem eine Public Viewing-Veranstaltung besucht worden ist

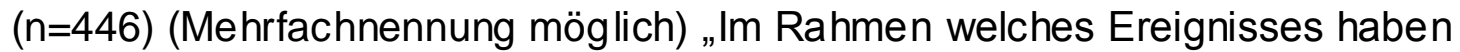
Sie eine Public Viewing-Veranstaltung besucht?" 209

Abb. 26: Vorhaben erneut ein Public Viewing zu besuchen ( $n=360)$ „Werden Sie wieder zum Public Viewing gehen?".

Abb. 27: Ereignis, zu dem eine Public Viewing besucht werden soll $(n=380)$ (Mehrfachnennung möglich) „Die nächste Public Viewing-Veranstaltung, die ich besuchen werde, wird zu folgendem Ereignis sein:..."...

Abb. 28: Sport als Freizeitinhalt (Mehrfachnennung möglich) „In meiner Freizeit..."

Abb. 29: In welchem Rahmen wurde über Public Viewing kommuniziert ( $n=384)$ (Mehrfachnennung möglich) „Außerhalb des Events war Public Viewing Gesprächsthema..."..... 213

Abb. 30: Besuchsmotivation ( $n=426$ ) (Mehrfachnennung möglich) „Ich habe eine Public Viewing-Veranstaltung besucht, weil..."..... 218

Abb. 31: Lokation der besuchten Public Viewing-Veranstaltung $(n=446)$ (Mehrfachnennung möglich) „Wo fand die von Ihnen besuchte Public ViewingVeranstaltung statt?" 219

Abb. 32: Begleitung für den Public Viewing-Besuch $(n=426)$ (Mehrfachnennung möglich) „Ich bin..." 220 
Abb. 33: Emotionserleben ( $n=396)$ (Mehrfachnennung möglich) „Ich habe positive Emotionen erlebt, weil..." 223

Abb. 34: Verhaltensunsicherheit ( $\mathrm{n}=359)$ (Mehrfachnennung möglich) „Vor meinem ersten Besuch bei einem Public Viewing..."

Abb. 35: Non-verbale Kommunikation I ( $\mathrm{n}=384)$ (Mehrfachnennung möglich) „Während des Public Viewing habe ich..." 225

Abb. 36: Non-verbale Kommunikation II ( $n=384)$ (Mehrfachnennung möglich) „Welche Handlungen haben die Personen um Sie herum während der Public Viewing-Veranstaltung gezeigt?" 226

Abb. 37: Qualität der Emotionen ( $n=396)$ (Mehrfachnennung möglich) „Wenn die von mir favorisierte Mannschaft ein Tor geschossen hat, weiß ich, welche Emotionen von mir erwartet werden“

Abb. 38: Intensität der Emotionen ( $n=396)$ (Mehrfachnennung möglich) „Wenn ein Spieler der von mir favorisierten Mannschaft gefoult wird, weiß ich welche Reaktion angemessen ist..." 228

Abb. 39: Dauer der Emotionen ( $n=396)$ (Mehrfachnennung möglich) „Die von Ihnen favorisierte Mannschaft hat gewonnen. Wovon machen Sie es abhängig, wie lange Sie sich über den Sieg freuen?“

Abb. 40: Public Viewing als Massenveranstaltung ( $n=368)$ (Mehrfachnennung möglich) „Public Viewing ist eine Massenveranstaltung“ 230

Abb. 41: Flow ( $n=366$ ) (Mehrfachnennung möglich) „Während des Public Viewing habe ich..." 233 


\section{Tabellenverzeichnis}

Tab. 1: Rangreihe der meistgesehenen Einzelsendungen des Jahres 2006 (Zuschauer ab 3 Jahre; Sendungslänge mind. 10 Min.) (AGF/GfK, pc\#tv, Fernsehpanel D+EU nach Zubayr \& Gerhard, 2007, S. 193) 32 (Telemetrische Daten-AGF/GFK, Fernsehpanel D+EU; telefonisch erhobene Daten forsa CATI- Studie zur Außer-Haus-Nutzung, fünf Wellen à 1.000 repräsentativ ausgewählte Personen nach Geese et al., 2006, S. 457 37

Tab. 3: Anteil der Personen mit Außer-Haus-Nutzung im Verlauf verschiedener Fußballturniere 39

"Wo haben Sie die Übertragung der WM/EM gesehen?" (Angaben in \%) (Telefonische Begleitumfrage anlässlich der Fußball-WM 2002 (MMA Frankfurt), Fußball-EM 2004 (forsa Berlin), und der Fußball-WM 2006 (IFAK Taunusstein) nach Geese et al., 2006, S. 458) 39

Tab. 4: Klassifizierung von Zuschauermotivationen im Sport (nach Gabler, 1998, S. 122) .70

Tab. 5: Motive der Sportbeobachtung (modifiziert nach Riedl, 2006, S. 84) .72

Tab. 6: Aufschlüsselung der Expertise der befragten Experten in die Kategorien Fan Fest, nicht-kommerzielles Public Viewing und kommerzielles Public Viewing (absolute Zahlen) ( $\mathrm{N}=21)$ 194

Tab. 7: Aufschlüsselung der Altersstruktur der befragten Experten in die Kategorien 20-30 Jahre, 30-40 Jahre, 40-50Jahre und über 50 Jahre (absolute Zahlen) ( $\mathrm{N}=21$ ) 195

Tab. 8: Aufschlüsselung der Positionen der befragten Experten zur WM 2006 in die Kategorien Pressesprecher, Leiter Stabstelle Presse- $u$. Öffentlichkeitsarbeit und Chefredaktuer; Geschäftsführer, Vorsitzender, Prokurist und Manager; leitender Angestellter, WM-Beauftragter der Stadt und WM-Projektleiter der Stadt; Finanzreferent und Schatzmeister; Sozialarbeiter (absolute Zahlen) ( $\mathrm{N}=21)$ 196 
Tab. 9: Aufschlüsselung des Bildungshintergrundes der befragten Experten in die Kategorien Rechtswissenschaften, Informatik; Wirtschaftsgeographie, ingenieurwesen und -wissenschaften; Verwaltungswissenschaften; Politikwissenschaften, Journalismus, Lehramt und Sozialpädagogik; Zweiter Bildungsweg, Eventmanagement und Messe-, Kongress- $u$. Tagungswirtschaft (absolute Zahlen) ( $\mathrm{N}=21)$.................................................. 197

Tab. 10: Aufschlüsselung der beruflichen Anstellung der befragten Experten in die Kategorien Stadt, Gesellschaft und Verein (absolute Zahlen) (N=21) .......... 198 


\section{Einführung}

Dieser Abschnitt befasst sich nach der Einleitung in das Thema Public Viewing und der Darlegung der bearbeiteten Problemstellung (1) mit einer kurzen Vorstellung des mehrperspektivischen Untersuchungsansatzes (2). Es wird ein Überblick über die in der vorliegenden Ausarbeitung eingenommenen Betrachtungsperspektiven gegeben. Der Schwerpunkt liegt auf der soziologischen Perspektive (2.1), es werden aber auch (sozial-)psychologische (2.2), medienwissenschaftliche (2.3) und ökonomische (2.4) Blickwinkel eingenommen, um die interdisziplinäre Herangehensweise (2.5) zu fundieren. Anschließend werden der bisherige Forschungsstand erläutert (3) und die Vorgehensweise der vorliegenden Arbeit erörtert (4). 


\section{Einleitung und Problemstellung}

Tausende von Menschen stehen dicht gedrängt zusammen. Es ist ein buntgemischter Haufen: Kinder und ihre Eltern, ein paar ältere Semester und viele junge Menschen. Es ist eine fröhliche und friedliche Gemeinschaft, die da steht in ihren oftmals schwarz-rot-goldenen Trikots, mit ihren Schals und witzigen Mützen oder Hüten. Sie schwenken Fahnen und singen, klatschen. Alle schauen nach vorne auf das Spielgeschehen: Fußball, Weltmeisterschaft 2006. Die deutsche Nationalelf spielt schnell nach vorne, die Stimmung ist gut: Tor!!! Frenetischer Jubel bricht los! Eine Lautsprecherdurchsage: „Es sind technische Probleme aufgetreten, die Datenkabel sind überlastet. Haben Sie einen Augenblick Geduld. Es geht gleich weiter." Die Zuschauer ${ }^{1}$ murren. Vom Spiel sehen sie einige Minuten lang nichts, sie befinden sich nämlich nicht im Stadion. Sie stehen auf einer Straße, der Straße des 17. Juni, mitten in Berlin. Diese ist abgesperrt und zur offiziellen Fanmeile erklärt. Das Publikum sieht auf eine Leinwand: Public Viewing. Public Viewing bei der der Fußball-Weltmeisterschaft 2006 in Deutschland.

"We can 'be there' without being there" (Croteau \& Hoynes, 2003, p. 304).

Wie diese kleine einleitende Szene beschreibt, sind die Zuschauer offenbar dabei, bei einem internationalen Großereignis wie der Fußball-Weltmeisterschaft 2006: Live und 'dabei', aber nicht direkt im Stadion. Sie können vermutlich besser sehen, das Spiel kompletter verfolgen als die Zuschauer vor Ort und sind doch nur mediale Rezipienten. Das heißt, der Einzelne sieht eben nicht das Spiel live vor Ort, sondern steht vor einem Großbildschirm und feiert die elektronische TV-Übertragung eines sportlichen Großereignisses.

Die Fragestellungen des Public Viewing sind äußerst vielfältig. Warum stellen sich Menschen vor eine überdimensionale Videowand, um ein Fußballspiel zu verfolgen? Public Viewing ist eine Erscheinung, ein soziales Phänomen, welches die Paradoxien unserer heutigen Gesellschaft widerspiegelt. Die mediale Omnipräsenz des Begriffs Public Viewing in Kombination mit der Diskussion über ver-

\footnotetext{
1 Auf die gleichzeitige Verwendung der weiblichen und männlichen Schreibform wird im Sinne der sprachlichen Einfachheit und besseren Lesbarkeit verzichtet. Die me hrheitlich verwendete männliche Form schließt im Folgenden selbstverständlich immer beide Geschlechter ein, es sei denn, es wird explizit eine Ausnahme gemacht.
} 
schiedenste Aspekte weckte das Interesse der Autorin. Gerade im Vorfeld der Fußball-Weltmeisterschaft 2006 in Deutschland überschlugen sich die Massenmedien mit Berichten über das 'Wie' der Übertragung auf Großbildschirme. Welche organisatorischen Möglichkeiten gibt es? Wie können sicherheitsrelevante Probleme geklärt werden? Wie verhält es sich mit der rechtlichen Situation? Und schließlich, wie können die verschiedenen Veranstaltungen finanziert werden? Dabei ist es gar nicht neu, dass sich Menschen vor große Leinwände stellen, um die Übertragung eines wichtigen gesellschaftlichen Ereignisses mitzuerleben. Im Rahmen von Musikveranstaltungen ist das Aufstellen von zusätzlichen Großbildschirmen gängige Praxis, der Besuch des Papstes oder Kirchentage werden auf diese Weise einem größeren Publikum nahe gebracht. Auch die Veranstalter im Sport arbeiten bereits seit mehreren Jahren mit dieser Methode, z.B. bei der Formel 1 oder den vergangenen Welt- und Europameisterschaften im Fußball. Eine neue Dimension in medialer Präsenz, organisatorischer Professio-nalität, in technologischer Qualität und in der Größe des Publikums-zulaufs erfuhr Public Viewing aber erst zur WM 2006, seit diesem Ereignis hat das Phänomen eine eigene Bezeichnung. Es darf also nicht verwundern, wenn im Folgenden viele Beispiele aus dem Umfeld dieses Ereignisses stammen. Die Mehrzahl der genannten Zahlen und Beispiele stellt sich aus dem Bereich der Sportart Fußball und der Weltmeisterschaftsturniere der letzten Jahre, vor allem aber des Jahres 2006, zusammen. Die Autorin hat ihre Ausführungen aus diesem Bereich abgeleitet, da Public Viewing bei der Fußball-WM 2006 eine große Dynamik entwickelt hat. Die Studie ist eine Momentaufnahme, sie sich auf die Vorgänge in Deutschland konzentriert. Für die Erörterung der Entwicklung wird aber auch z.B. auf die Fußball-Weltmeisterschaft 2002 in Japan und Südkorea geschaut. Darüber hinaus werden Seitenblicke vor allem auf das europäische Ausland und in die USA geworfen.

Mit Public Viewing als Forschungsgegenstand ergibt sich für diese Arbeit ein vielschichtiges und komplexes Themenfeld. Es bilden sich verschiedene Fragenblöcke; z.B. sind Fragen nach den Gründen für den enormen Zulauf während der Fußball-WM 2006, über Finanzierungsprobleme und Rechtsgrundlagen bis hin zu der Sicherheitsdiskussion denkbar. Es wäre also die Entwicklung mehrerer Problemstellungen vorstellbar. Das Hauptinteresse der vorliegenden Ausarbeitung liegt aber in der Frage nach dem 'Warum'. Das Ergründen einer Ursache bzw. 
mehrerer möglicher Aspekte für die Entstehung des 'gemeinsamen Sehens' ist eines der Ziele der Arbeit. Die Forschung zu diesem Gebiet steht ganz am Anfang. Lediglich Einzelpersonen wie Schulke (2006, 2006a, 2007) haben sich bisher mit verschiedenen Aspekten beschäftigt. Eine systematische interdisziplinäre Forschungsleistung fehlt allerdings noch. Aus diesem Grund handelt es sich bei der vorliegenden Dissertation um eine explorative Studie, die sich mit einem Aspekt der sich verändernden Stellung des Sports in der Gesellschaft beschäftigt.

\begin{abstract}
"Was in den neusten Tendenzen „Sport" heißt, ist nicht mehr unser alter, klassischer Sport, sondern ist gekennzeichnet durch eine Integration sportlicher Elemente in neuartige kulturelle Formen, in denen sich Elemente vieler verschiedener Bereiche verbinden - Mode, Popmusik, Film, Video, Tanz - und die sich von allem entfernen, was wir bisher kannten" (Gebauer, 2002, S. 3).
\end{abstract}

Aus der Natur des Sports und der Sportwissenschaft als wissenschaftliche Disziplin abgeleitet und insbesondere durch die Multidimensionalität des Phänomens begründet, liegt es nahe, theoretische Bausteine aus den sozialwissenschaftlichen Mutterwissenschaften zur Klärung dieses Diskurses einzusetzen, von denen erfolgversprechende Ergebnisse erhofft werden. Im Sinne des Theorienpluralismus werden schwerpunktmäßig Ansätze aus der Soziologie, der (Sozial-) Psychologie und der Medienwissenschaft herangezogen. Einen ergänzenden Blickwinkel liefert die ökonomische Perspektive.

Das Ziel dieser Arbeit ist es, aus bestehenden wissenschaftlichen Ansätzen Denkanstöße und theoretische Ableitungen für das Phänomen zu schaffen sowie aus diesen Erkenntnissen praktische Handlungsanstöße abzuleiten. Diese Dissertation eröffnet vor allem durch den interdisziplinären Zugang Perspektiven, die zum Verständnis des Public Viewing entscheidend beitragen können. Sie vermag den interdependenten Zusammenhang ein Stück weit zu entschlüsseln und ist als eine erste Basis eines zukünftigen wissenschaftlichen Diskurses zu sehen. Der Nachteil des sehr aktuellen Themas ist, dass nur in geringem Maße auf Vorwissen und akzeptierte Wissensbestände aufgebaut werden kann. So vermag diese Arbeit keine endgültigen Ergebnisse zu erbringen, liefert aber erste systematische Ideen und Erklärungsansätze.

Gegenstand der empirischen Untersuchung sind zum einen die Aussagen von Public Viewing-Experten während der WM 2006 und zum anderen die der 
Besucher von öffentlichen TV-Vorführungen. Aufgrund des bislang kaum vorhandenen Wissens in diesem Bereich bot es sich an, in einem ersten Schritt eine breit angelegte Befragung durchzuführen, um nicht von vornherein gewisse Aspekte auszuschließen. In diesem Sinne wurden explorative qualitative Interviews durchgeführt, um den Gegenstandsbereich überblicken zu können und Relevanzen und Handlungsorientierungen herauszufiltern. In der folgenden klassischen Literaturarbeit wurden interessante Themengebiete vertieft, die Fragestellungen spezifiziert und Hypothesen entwickelt. In einem letzten Schritt ist eine zweite Untersuchung in Form einer quantiativen Online-Umfrage durchgeführt worden, um spezifische Aufschlüsse über die ausgewählten Aspekte geben zu können. Anhand des Konglomerats aus theoretischem Wissen, qualitativen Expertenmeinungen und quantitativen Werten der Besucher von TV-Vorführungen werden die aufgestellten Hypothesen auf ihre Aussagekraft hin überprüft. Es lassen sich folgende forschungsleitende Fragestellungen formulieren:

1. Welche Faktoren führen zur Entwicklung des Public Viewing?

2. Warum besuchen Zuschauer Public Viewing-Veranstaltungen?

3. Ist das Entstehen von positiven Emotionen bei einer Public ViewingVeranstaltung steuerbar?

Diese Arbeit beinhaltet die Herausforderung der sehr aktuellen Thematik. Sie behandelt ein Phänomen, welches in den letzten Jahren eine rasante Entwicklung genommen hat, über das es aber noch kaum wissenschaftliche Abhandlungen gibt. Gleichzeitig macht diese Herausforderung aber auch die Stärke der Arbeit aus, nämlich ihre gesellschaftliche Aktualität und Relevanz. Dem Erhebungszeitraum der Datenbasis nach ist die Arbeit eine Momentaufnahme. Der Geltungsanspruch der Arbeit weist aber über diesen Zeithorizont hinaus, da Tendenzen und Entwicklungen für kommende sportliche Großereignisse benannt werden. 


\section{Perspektiven der Betrachtung}

Eine kurze Darlegung der genutzten Blickwinkel zur Verdeutlichung des methodischen und inhaltlichen Vorgehens dieser Arbeit ist unerlässlich. Jedoch erscheint es sinnvoll, eine Gewichtung der Perspektiven vorzunehmen. So liegt der Schwerpunkt auf der soziologischen Betrachtungsweise (2.1). Die Nähe zwischen (Sozial-) Psychologie und Soziologie lässt erstere aber ebenso einen wichtigen Blickwinkel darstellen (2.2). Simpel betrachtet handelt es sich beim Public Viewing um eine Fernsehübertragung, weshalb die medienwissenschaftliche Perspektive nicht fehlen darf (2.3). Als Ergänzung wird auch die ökonomische Sichtweise (2.4) ihre Beachtung finden. Abschließend erfolgt eine Diskussion der gewählten interdisziplinären Betrachtungsweise (2.5). 


\subsection{Soziologische Perspektive}

Die soziologische Perspektive wird im Rahmen dieser Arbeit vornehmlich eingenommen. In dieser Ausarbeitung wird der Blick sowohl in den Bereich der Makro- als auch der Mikrosoziologie geworfen. Das Feld der Makrosoziologie beschäftigt sich mit Entwicklungsgängen in großen sozialen Einheiten und umfassenden sozialen Prozessen. Es geht also von gesellschaftlichen Strukturzusammenhängen aus (vgl. Henecka, 2000, S. 29ff.). Diese Teilwissenschaft scheint geeignet, sich den gesellschaftlichen Bedingungen, die das Entstehen von Public Viewing beeinflusst haben, zu nähern. Die Mikroebene der Soziologie befasst sich mit den Grundbedingungen und -formen sozialen Handelns und Verhaltens. Genau genommen geht es also um Prozesse der Aneignung und Auseinandersetzung des Individuums mit der es umgebenden Kultur, z.B. mit gesellschaftlichen Rollen und Normen. Da die Mikroebene der Soziologie eng mit der Sozialpsychologie verwandt ist, liegt das Heranziehen von Konzepten und Theorien aus dieser Fachwissenschaft auf der Hand (s. Kap. 2.2). Eine soziologische Herangehensweise ist sowohl für die Erläuterung der Entwicklung und der Grundlagen, z.B. bei der Erörterung der postmodernen Gesellschaft und ihrer Wirkungen auf den Sport und das Fernsehen (s. Kap. 5 \& 6), als auch im Theoriekapitel über Emotionen (s. Kap. 9) und z.B. im Exkurs über Events (s. Kap. 10.5) erforderlich.

\section{2 (Sozial-)Psychologische Perspektive}

Mit dem Einbeziehen sozialpsychologischer Theorien und Konstrukte eröffnen sich wichtige Merkmale zur Analyse von Teilbereichen des Public Viewing. Insbesondere für die Ausführungen zum Publikum bieten sie geeignete Grundlagen. Theorien zur Identifikation und zum Gemeinschaftserleben gewähren Einblicke in die Sozietät des Sportzuschauers mit seinen vielfältigen ambivalenten Strukturen und wechselseitigen Bezügen (s. Kap. 7). Darüber hinaus spielen psychologische Prozesse auch bei der Entstehung von Emotionen eine wichtige Rolle. In diesem Rahmen wird neben den sozialpsychologischen Konstrukten auch auf theoretische Bausteine der Gruppen- und Massenpsychologie zurück-gegriffen. Diese können einen wichtigen Beitrag zur Diskussion von kollektiven Identitäten und ihren 
gemeinsamen Emotionen leisten (s. Kap. 9).

\subsection{Medienwissenschaftliche Perspektive}

Die Medienwissenschaft stellt ein wichtiges Glied für tiefergehende Einblicke in das mediale Ereignis Public Viewing dar. So ist die Evolution des Massenmediums Fernsehen stark mit der Entwicklung des Sports und insbesondere mit der des Fußballs in einer symbiotischen Form verbunden (s. Kap. 5 \& 6). Auch für die Beantwortung der Frage nach dem in dieser Arbeit zugrunde liegenden Verständnis des Wirkungszusammenhangs zwischen Publikum und Fernsehen geben die Ansätze der Medienwirkungsforschung Aufschluss. Mit diesen Wirkungsverbindungen einhergehen die Determinanten der medialen Publikumsbildung; hier bietet die Medienforschung wichtige Hinweise für die Besonderheiten der TV-Rezeption (s. Kap. 7). Darüber hinaus verändern sich die Orte der Fernsehnutzung, u.a. durch neue technologische Entwicklungen (s. Kap. 8). Des Weiteren liefern medienwissenschaftliche Theoriebausteine Erkenntnisse über die Entstehung von Emotionen und 'imaginierten Gemeinschaften' u.a. durch ihre Rolle in der gesellschaftlichen Kommunikation und ihre Multiplikatorfunktion (s. Kap. 9).

\section{4 Ökonomische Perspektive}

Die Aufnahme der ökonomischen Betrachtungsebene ist als ein ergänzendes Moment gedacht. Ansätze der Konsumenten- und Zuschauerforschung werden z.B. für die Diskussion der Publikumsbildung (s. Kap. 7) und für die Erörterung über die Möglichkeiten der Inszenierung von Public Viewing als Erlebnis und Event (s. Kap. 10) herangezogen. Im Allgemeinen soll es in dieser Ausarbeitung aber nicht um eine Analyse im wirtschaftlichen Sinne gehen. Es wäre allerdings falsch z.B. die wirtschaftlich bedingten Motivlagen der Zuschauer, was Eintrittspreise u.ä. angeht, ganz zu ignorieren. Darüberhinaus soll gerade ein Ziel dieser Arbeit sein, nicht ausschließlich im wissenschaftlichen 'Elfenbeinturm' zu verbleiben, sondern auch Ideen für die praktische Anwendung zu liefern. 


\subsection{Interdisziplinäre Herangehensweise}

Zur Analyse und Erörterung der genannten Fragestellungen wäre die Nutzung nur eines einzigen Blickwinkels von Vorteil. Nur so ist es möglich, ein Thema zu diskutieren ohne terminologische Schwierigkeiten zu provozieren. Denn kaum ein theoretisches Konstrukt arbeitet mit den gleichen Begriffen, noch lässt es sich inhaltlich konkret von anderen Theorien abgrenzen. Allerdings ist die Sportwissenschaft eine Wissenschaft, die für viele Disziplinen offen ist. In der vorliegenden sportwissenschaftlichen interdisziplinär angelegten Arbeit ist es daher unumgänglich, sich mit verschiedenen Perspektiven zu befassen. Denn nur so kann der Versuch unternommen werden, das vielschichtige komplexe Phänomen Public Viewing zu greifen. Folglich wird Public Viewing als eine gesellschaftliche Erscheinung betrachtet, die einer soziologischen Erklärungsbasis bedarf. Dennoch spielen gerade im Bereich der Motivkonstruktionen und der Emotionen auch (sozial-) psychologische Ansätze eine Rolle. Darüber hinaus ist Public Viewing eine Fernsehübertragung, weshalb nicht auf geeignete Konstrukte der Medienwissenschaft verzichtet werden darf. Letztlich hat diese Arbeit den Anspruch an die praktische Anwendbarkeit ihrer Schlussfolgerungen, so dass ein Blick für den wirtschaftlich geprägten Alltag nicht fehlen sollte. Allerdings ist die fehlende Tiefe in einigen Theoriebereichen eine Schwäche dieser, wie vieler anderer ähnlich angelegter Ausarbeitungen. Dieser 'Tiefgang' wäre ohne Frage wünschenswert, kann aber im Rahmen der vorliegenden Arbeit nicht geleistet werden. Hier ergeben sich allerdings Ansatzpunkte für zukünftige Studien. 


\section{Forschungsstand}

Über das Thema Public Viewing gibt es bislang wenige wissenschaftliche Abhandlungen. Die Forschung steht ganz am Anfang, und es kann folglich lediglich auf die wenigen zur Verfügung stehenden Texte zurückgegriffen werden. Einzelne Arbeiten beschäftigen sich vor allem mit den rechtlichen Aspekten (Arpagaus, 2008; Goes \& Wiedenfels, 2006; Reuter 2008) und ökonomischen Effekten (Habbel, 2005; Maennig, 2007; Schwark 2007 u.a.).

Bei Behn, Kuhlmann, Pilz und Wölki (2007) sowie Pilz (2007) findet sich die Evaluierung des Fan- und Besucherbetreuungsprogramms zur FIFA WM 2006 ${ }^{\mathrm{TM}}$ in Deutschland. In diesen Arbeiten bildet Public Viewing als ein Teil der offiziellen Fan Feste nur einen Aspekt der aber mit empirischen Daten unterlegt ist und somit zumindest in einem Bereich Vergleichsmöglichkeiten bietet. Hervorzuheben sind an dieser Stelle die theoretischen Ausführungen von Schulke $(2006,2007)$, die zunächst einen organisatorischen, rechtlichen und politischen Überblick über die Gegebenheiten anlässlich der Fußball-WM $2006^{3}$ in Deutschland liefern. Des Weiteren gewinnt man mit den größtenteils kultursoziologischen Begründungen wichtige Anhaltspunkte für die Erfassung des Phänomens, z.B. zur Faszination Fußball als Massenerlebnis (vgl. auch Roost \& Schöning, 2006) und Kommunikationsanlass. Er erwähnt aber auch die Bedeutung des technologischen Fortschritts in den Übertragungstechniken für die Entwicklung des Public Viewing. Leider liegen diesen Ausführungen, wie auch dem Werk von Cybulska (2007), keine empirischen Untersuchungen bei. Letzterer beschäftigt sich mit Public Viewing aus medienwissenschaftlicher Perspektive und ermöglicht einen Einblick in die Welt der Medienereignisse. Aus diesem Forschungsstand ergibt sich fast automatisch die grundsätzliche Ausrichtung der vorliegenden Arbeit. Zunächst erfolgt eine hauptsächlich explorative Diskussion des Themas mit dem Ziel des Zusammenführens eines datengestützten Theoriekonstrukts.

Es wäre sicherlich wünschenswert gewesen, explizit zum Thema Public Viewing auf eine größere Menge geeigneter wissenschaftlicher Literatur zurückgreifen zu können. Um aber dennoch einen Ertrag zu erreichen, ist es unumgänglich, ver-

\footnotetext{
3 Zur Fußball-Weltmeisterschaft 2006 gibt es vor allem Abhandlugen zu ökonomischen Aspekten (vgl. Brehm, Sygusch, Ballmert \& Reuter, 2007; Kurscheidt, 2002, 2005; Ohmann, Jones \& Wilkes, 2006).
} 
stärkt auf Arbeiten und Konzepte aus unterschiedlichen wissenschaftlichen Gebieten, vornehmlich jedoch auf Bereiche aus den sozialwissenschaftlichen Mutterdisziplinen, zurückzugreifen. Manche der verwandten Themengebiete sind recht zahlreich und ausführlich in der entsprechenden Literatur behandelt worden. Um die Vollständigkeit dieses Abschnitts nicht zu gefährden, werden selbst-verständlich alle relevanten Thematiken angeführt. Damit im Folgenden aber keine Aufzählung oder gar Wiederholung der in der vorhandenen wissenschaftlichen Literatur anzutreffenden Berichte über die Quellenlage entsprechender Bereiche entsteht, wird bei aktuellen Ausführungen auf diese verwiesen.

Neben den Arbeiten zum Public Viewing finden sich an der Medienforschung orientierte Aufsätze, die in ihren jüngsten Ausgaben auch Daten der Außer-HausSeher bei Fußball-Welt- und -Europameisterschaften darstellen (vgl. Geese \& Gerhard, 2008; Gerhard, 2006; Zubayr \& Gerhard, 2007 [u.a.]). Diese können durch Berichte der FIFA bzw. der UEFA und des Sportrechtevermarkters Infront Sports \& Media $\mathrm{AG}^{4}$ unterstützt werden. Nach diesen Erhebungen ist ein erhöhtes Interesse der Zuschauer festzustellen, wichtige internationale Ereignisse, vornehmlich des Fußballs, gemeinsam mit anderen zu verfolgen. Die damals noch namenlose Praxis des Public Viewing findet zur Fußball-WM 2002 in Südkorea und Japan mehrfach Erwähnung. Allerdings lassen sich fast ausschließlich bei Horne und Manzenreiter (2004) Versuche, einen Begründungszusammenhang zu erkennen, feststellen. Dieser wird vor allem in der Einflussnahme der öffentlichen Hand und der Medien gesehen. Dennoch kann nur ein hohes Interesse der Bevölkerung der Ausrichterländer den enormen Zulauf erklären. Public Viewing wird als ein Phänomen angesehen, welches sich u.a. aus den Entwicklungen der postmodernen Gesellschaft heraus entwickelt hat. Zu diesen Gegebenheiten zählt der sich verändernde Sport gleichermaßen wie andere gesamtgesellschaftliche Trends, z.B. die Individualisierungstendenzen oder das symbiotische Verhältnis zwischen Massenmedien -das Fernsehen sei hier hervorgehoben- und dem Spitzensport. Die postmoderne Gesellschaft ist stark mit einem Wandel der Werte verbunden. Grundlegende Literatur zum Wertewandel bieten z.B. die Klassiker von Klages (1984) und Inglehardt (1977), sowie Opaschowski $(2006,2008)$ und Horx (2007). Der Sport, insbesondere der Spitzensport, liefert für das Individuum in der

\footnotetext{
4 Im Folgenden zur Vereinfachung mit Infront abgekürzt.
} 
Postmoderne wichtige sozialstrukturierende Orientierung. Bezug auf die Individualisierungsthese und ihre Bedeutung für den Sport nehmen z.B. Gebauer (2002), Göring (2006) oder Krüger (1990). Beschäftigt man sich mit der hervorgehobenen Position, die der Fußball einnimmt, finden sich Untersuchungen bei Bausenwein (1995), Schiffer (2004) oder Schmidt (2002) u.v.m.. Von der Wechselwirkung zwischen Sport und Medien profitiert ersterer in Form von Ressourcen und zweitere durch die Darstellung des Sports vor einem breitgefächerten Publikum. Diese Spiralbewegung führt zu einem gesteigerten Interesse der Forschung an der Symbiose von Sport und Medien. Das Bildmedium Fernsehen nimmt hier eine Sonderrolle ein (vgl. Loosen, 2004; Riedl, 2006; Schwier, 2002 [u.a.]).

Die erörterten Wissensbereiche vermögen jedoch noch nicht vollständig zu klären, wieso es den Einzelnen vom heimischen Fernseher zu den öffentlichen Großbildschirmen zieht. Diesem Ansatz kommt man näher, wenn man sich der Literatur zur Mediatisierung, die die Veränderung der räumlichen Mediennutzung (vgl. Whannel, 2005) und die damit einhergehenden verschiedenen Publika beschreiben, zuwendet. Abercrombie und Longhurst (1998) bspw. sehen in der Interaktion des Publikums vor Ort mit dem massenmedialen und dem dispersen Publikum eine Neuordnung des Lokalen und des Globalen. In diesen Zusammenhang sind auch die Arbeiten von McCarthy (2001), McLuhan (2001) und Sydnor (2000) einzuordnen, die dem Fernsehen die Fähigkeit zuschreiben, die Grenzen zwischen Ort, Zeit und Realitäten verschwimmen zu lassen. TV-Übertragungen werden so immer mehr zu einem eigenständigen Ereignis, zu dem der technologische Fortschritt in den Übertragungs medien einen wichtigen Beitrag leistet.

Das Publikum spielt beim Public Viewing eine entscheidende Rolle. So muss das Interesse, ein Ereignis zu verfolgen, zunächst so hoch sein, dass es den Bedarf im Stadion übersteigt. Deshalb liegt es nahe, in dieser Arbeit nach den Beweggründen der verschiedenen Zuschauergruppierungen zu fragen, um diese anschließend auf das Public Viewing zu übertragen. Eine Übersicht über die Forschungshistorie zum Thema Sportzuschauer für die deutsch- und englischsprachige Literatur findet sich z.B. bei Stollenwerk (1996). Darüber hinaus gibt Lucerna (1997) einen sehr detaillierten Blick auf die Zuschauerforschung, indem er bestehende Ansätze aus sportpsychologischer Sicht bewertet. Riedl (2006) bietet eine strukturierte Übersicht über die wichtigsten Herangehensweisen und Arbeiten. Aus der Fülle an Beiträgen, die sich mit dem Sportpublikum beschäf- 
tigen, seien hier die Arbeiten von Bette und Schimank (1995), Gabler (1998), Schlicht und Strauß (2003) sowie Wann (1995) zu nennen. Mit den Motiven des Sportschauens im Fernsehen setzen sich bspw. Döhle, Klimmt und Schramm (2006), Wenner und Gatz (1998) oder Schramm, Döhle und Klimmt (2004) auseinander.

Es ergeben sich u.a. aus der Literaturarbeit zur Motivforschung von Sportzuschauern zwei grundlegende Gebiete, die in dieser Ausarbeitung vertieft behandelt werden sollen: Zum einen Emotionen und zum anderen Erlebnisse. Der Forschungsgegenstand Emotionen ist intensiv in verschiedenen Fachrichtungen bearbeitet worden. Der Schwerpunktlegung folgend, hat sich die Autorin in dieser Arbeit auf die in der Soziologie diskutierten Ansätze und Modelle konzentriert. Dennoch sind, um einen höheren Erkenntnisgewinn zu erzielen, auch (sozial-) psychologische Gedankengänge in die Analyse übernommen worden, weil die Auffassung vertreten wird, dass Emotionen nicht monokausal erklärbar. Um die Stringenz in der Darlegung des Forschungsstandes zum Thema Public Viewing nicht zu gefährden, sei an dieser Stelle für einen Überblick über die europäischen Klassiker der Soziologie auf die Bände von Flam (2002), Shilling (2002), Schmidt (2005) u.a. verwiesen, in denen detaillierte Ausführungen zur soziologischen Emotionsdiskussion zu finden sind. Für einen Einstieg in die Emotionspsychologie seien hier Ciompi (1997), Meyer, Schützwohl und Reisenzein (1993, 1997) oder Scherer (2001) zu nennen. Grundlage für die vorliegende Analyse ist die Arbeit von Gerhards (1988), die eine frühe fundierte Auseinandersetzung mit der soziologischen Theorie der Emotionen darstellt und die bis heute wenig an ihrer grundsätzlichen Bedeutung verloren hat. Gerhards beruft sich in seinen Ausführungen auf Kemper (1978), dessen Modell der 'Social Interactional Theory of Emotions' für die grundlegende soziale Funktionsweise von Emotionen als Argumentationsbasis dient. Allerdings besitzt dieses viel diskutierte Konstrukt kleine Schwachstellen, die in den Arbeiten von Kemper $(2002,2007)$ selbst, aber auch von Gerhards (1988), Flam (2002) oder im Werk von Turner und Stets (2007) erörtert werden. Die Arbeiten zur soziologischen Emotionstheorie von Ciompi (1997), Tritt (1992), Vester $(1991,1995)$ werden als Ergänzungen herangezogen. Erwähnt werden muss an dieser Stelle besonders Hochschild (1990, 1998), die die sozialkonstruktivistische Herangehensweise mit dem Begriff der Gefühlsregeln prägt. 
Bedauerlicherweise gibt es bislang nur eine auf soziologischen Theorien basierende Forschungsarbeit, die die Emotionen des Sportpublikums in den Blick nimmt (Riedl, 2006, 2008). Wichtige Gedankengänge sind aber auch z.B. bei Cordsen und Deilmann (2005) zu finden. So gilt es ebenso, an dieser Stelle auf weiterreichende Arbeiten zurückzugreifen und diese anschließend auf das Public Viewing zu übertragen. Da das Publikum in dieser Arbeit nicht nur als aus Individuen bestehendes Konglomerat, sondern als eigenständiges System angesehen wird, erscheinen sozialpsychologische und auch soziologische Analysen, die sich mit Gruppen- und Massentheorien auseinandersetzen, geeignet. Eine erste Grundlage bildet der frühe Klassiker von LeBon (1911/1982), der sinnvolle Ansätze liefert, der Masse aber eher negative Eigenschaften zuschreibt. Das Sammelwerk von Klein und Nullmeier (1999) oder Reicher (2001) widmen sich der Soziologie von Massen, wobei besonders Roth (1999) hervorzuheben ist, da er versucht, den Diskurs zu strukturieren. Neben Riedl (2006) bietet besonders Vester (1991, 1995) gute Gedankengänge, die sich auf das Sport- bzw. Public Viewing-Publikum übertragen lassen. Da Public Viewing simpel betrachtet eine Fernsehübertragung ist, bedarf die Bedeutung, die das Massenmedium auf Emotionen hat, ebenfalls einer Auseinandersetzung. Bezüglich des Forschungsstandes über die Rolle von Medien und Emotionen sei hier auf das Werk von Bösch und Borutta (2006) verwiesen. Dayan und Katz $(1994,2001)$ als gedankliche Väter der 'Medienereignisse' bieten wichtige Ansatzpunkte, und auch Rötzer $(1997,2006)$ ist an dieser Stelle zu erwähnen, der recht plakativ argumentiert, aber dennoch wichtige Denkanstöße liefert.

Von den Gedanken des gesellschaftlichen Wertewandels in der Postmoderne ausgehend, wird in dieser Arbeit die Möglichkeit eruriert die Entstehung von positiven Emotionen beim Public Viewing durch die Inszenierung von Erlebnissen zu unterstützen. Schulze prägt mit seinen Arbeiten $(1997,2000,2007)$ den Begriff und den Gedankengang der Erlebnisgesellschaft. Das Werk von Kagelmann, Bachleitner und Rieder (2004) zur Bedeutung des Erlebnisses in der Postmoderne ist hervorzuheben, da die Autoren Erlebnisse als emotionale Antwort auf die gesellschaftlichen Prozesse sehen. Pine und Gilmore (1999) geben wichtige Impulse aus der Erlebnisökonomie, in dem sie das Erlebnis als letzte Kette im Produktionsprozess ansehen. Für den Sport sind neben der soziologischen Ausarbeitung von Riedl (2006) die Arbeiten von Pfaff $(2002,2002 a, 2007)$ zu nennen, 
die einige wichtige Ansatzpunkte für diesen Bereich liefern. Das Buch des Erlebnismarketings für die Besucher von Sportveranstaltungen liefert aufgrund seines Schwerpunkts eine ökonomische Sicht auf die Vermarktung von Erlebnissen. Pfaff beschäftigt sich mit einem neuen Marketingansatz speziell für Erlebnisse im Sport. Die Thematik der Angebotsinszenierung von Tourismusdestinationen zur Steigerung der Erlebnisqualität der Gäste ist, wie die Tourismuswissenschaft, ein sehr interdisziplinäres Feld. Es mischen sich ebenfalls ökonomische mit psychologischen und soziologischen Sichtweisen auf die sich verändernden Kundenbedürfnisse und deren Auswirkungen auf die Angebotsgestaltung. Hervorzuheben sind hier besonders die Arbeiten von Dreyer (2002), Scheurer (2003) sowie der Sammelband von Weiermair und Brunner-Sperdin (2006).

Als eine der höchsten Stufen in der Gestaltung des Erlebnispotentials sind Events zu sehen. In der vorliegenden Ausarbeitung widmet sich ein kurzer Exkurs dieser Thematik mit dem Hintergedanken der Inszenierungsmöglichkeiten von Public Viewing als Event. Mit der generellen Eventthematik beschäftigen sich die Werke von Gebhardt, Hitzler und Pfadenhauer (2007), Nickel (2007) und Trosien und Dinkel (2000). Wobei sich erstgenannte mit soziologischen Aspekten und Nickel sowie Trosien und Dinkel mit eher ökonomischer Ausrichtung nähern. Für die Bedeutung von Events für die Destinationsvermarktung sei hier zunächst auf Dreyer (2002) und das Werk von Freyer und Groß (2002) verwiesen. Letzteres beschäftigt sich mit der Bedeutung von Sportevents, wie auch Pfaff (v.a. 2007, aber auch 2002, 2002a). Horne und Manzenreiter $(2004,2006)$ thematisieren u.a. die Fußball-WM 2002 als Mega-Event (vgl. auch Roche, 2000).

Diese Erläuterungen zeigen die Breite der Untersuchungs- und Erklärungsansätze und gleichzeitig auch die Grenzen, welche dadurch gegeben sind: Mit dieser Arbeit sollen Erklärungsansätze für das Phänomen Public Viewing erarbeitet werden, welche die veränderten gesellschaftlichen Rahmenbedingungen berücksichtigen. Gleichzeitig muss aber angemerkt werden, dass die einzelnen Teilbereiche im Rahmen dieser Arbeit nicht weiter erforscht werden können. Vielmehr müssen bestehende Resultate und Erkenntnisse der einzelnen Wissenschaftsbereiche als Ausgangspunkt aufgenommen werden, um durch deren sinnvolle Verbindung neue Erkenntnisse zu erhalten. 


\section{Vorgehensweise}

Der Untersuchungsansatz dieser Arbeit baut im theoretischen Bereich auf einer explorativen Erkundung des Forschungsgegenstandes Public Viewing auf. Als thematischer Einstieg werden die Entwicklung und die Grundlagen erörtert (II). In diesem Sinne wird zunächst das Thema Public Viewing eingegrenzt und in seiner Entstehung eruiert (5). Begonnen wird mit der Abgrenzung und der Bestimmung des Begriffs Public Viewing (5.1). Daran anschließend werden die Rahmenbedingungen für die wachsende Beliebtheit des Phänomens erörtert (5.2). Zu diesem Zweck werden die jüngsten Fußball-Welt- und -Europameisterschaften als Quotengaranten in den Blick genommen (5.2.1). Danach wird die WM 2002 als Schlüsselereignis für die Entwicklung von Public Viewing-Veranstaltungen nach heutigem Maßstab ausgemacht (5.2.2). Vor allem in Südkorea kam es während der Endrunde des Turniers der Fußball-Weltmeisterschaft im Jahr 2002 zur Versammlung von vielen tausend Menschen vor den meist wahllos aufgestellten Videowänden. Eine andere Dimension erreichte der Prozess in 2006. Deshalb wird anschließend nach der neuen Qualität des Public Viewing gefragt (5.2.3). Zusammengefasst werden diese Ergebnisse im resümierenden Kapitel (5.3).

Im Anschluss an dieses Kapitel thematisiert der nächste Abschnitt die gesellschaftlichen Modernisierungsprozesse, die ihren Teil zur Entstehung des Phänomens beitragen (6). Zu diesem Zweck wird ein Blick auf die postmoderne Gesellschaft geworfen, und die Prozesse des Wertewandels sowie Individualisierungs- und Pluralisierungstendenzen werden diskutiert (6.1). Diese sozialen Trends und Entwicklungen werden im Bereich der Medien und des (Fußball-)Sports weiter ausdifferenziert betrachtet. Mit der Symbiose zwischen dem Massenmedium Fernsehen und dem modernen Sport, die sich vor dem geschilderten gesellschaftlichen Hintergrund entwickelt hat, befasst sich das folgende Kapitel (6.2). Dabei wird deutlich, dass der Fußball eine besondere Rolle eingenommen hat. Aus diesem Grund wird explizit nach der Sonderstellung des Fußballs in der heutigen Sportwelt gefragt (6.3). Abschließend werden diese Ansätze zusammengefasst (6.4).

Des Weiteren scheint das Publikum beim Public Viewing eine gewichtige Rolle zu spielen. Somit gilt es, das Publikum als eine weitere Komponente näher zu bestimmen (7). Von Interesse erscheinen seine Struktur und die Faktoren, welche 
zu seiner Bildung führen. In diesem Sinn wird die Publikumsstruktur typisiert und klassifiziert (7.1), und die Determinanten der (Fernseh-)Publikumsbildung werden hinterfragt (7.2). Resümierend werden die theoretischen Bausteine an dieser Stelle gebündelt (7.3).

Natürlich ist der enorme Zulauf zu den TV-Vorführungen nicht allein aus einer sich verändernden Gesellschaft heraus möglich. Es scheint aber der Fall zu sein, dass das soziale Leben, und als ein Teil davon das Public Viewing, wieder in den öffentlichen Raum zurückkehrt (8). Um den Zusammenhang mit den Komponenten des Public Viewing soll es im folgenden Abschnitt gehen (8.1). Durch die symbiotische Beziehung des Sports zum Medium Fernsehen sind die technologische Entwicklung des Übertragungsmediums und sein Einfluß auf das Phänomen nicht zu unterschätzen (8.2). Eine Einordnung dieser Ergebnisse in das Gesamtgefüge Public Viewing erfolgt abschließend (8.3).

Zu Beginn der Arbeit sind Expertengespräche mit Public Viewing-Verantwortlichen während der Fußball-WM 2006 durchgeführt worden. Diese sind weit und offen angelegt, so dass sich aus dieser umfassenden Vorgehensweise Teilbereiche herauskristallisierten, die besonders interessant und wichtig erscheinen. Die in dieser Form gesammelten Erkenntnisse werden sowohl im folgenden Theoriekapitel (III) als auch in der zweiten empirischen Befragung spezifiziert. Im kommenden Kapitel werden zunächst theoretische Erklärungsmodelle eruiert. Im ersten Schritt wird die Relevanz von Emotionen für Public Viewing erörtert (9). Anschließend wird der Begriff der Emotionen abgegrenzt und klassifiziert (9.1). In einem zweiten Schritt erfolgt die Diskussion von emotionssoziologischen Erklärungsansätzen (9.2). In dem Zusammenhang Public Viewing erscheint die sozialstrukturell bedingte Herstellung kollektiver Emotionen interessant. Aus diesem Grund schließt sich die Auseinandersetzung mit kollektiven Emotionen an (9.3). Danach wird die Rolle der Emotionen im Fernsehen gewichtet (9.4). Im Anschluss werden die theoretischen Erkenntnisse dieses Kapitels in ihrer Gesamtheit eruiert und in den Kontext eingeordnet (9.5).

Der nächste Abschnitt durchleuchtet die Suche nach und die Inszenierung von Erlebnissen (10). Die Suche der Menschen nach Erlebnissen eröffnet einen weiterführenden Ansatz zur Näherung an das Phänomen Public Viewing. Ausgehend von den hedonistischen Erwartungen der Individuen an ihre Freizeit 
wird dann auf ein aus der Tourismuswissenschaft entlehntes Konzept zurückgegriffen. Dieses begreift den Besucher mehr als Konsumenten, was aber in der Arbeit nicht stört, sondern lediglich einen weiteren Blickwinkel öffnet. So hat doch die Übertragung dieser Annahmen auf Public Viewing-Veranstaltungen einen innovativen Charakter, der die Weitung des Blickes auf eine Varianz an theoretischen Konzepten notwendig macht. Als erstes wird auf die Rolle des Erlebnisses in der Gesellschaft eingegangen (10.1). Fortführend wird das Augenmerk auf den Erlebniskonsumenten erweitert (10.2). Auf diesen Ausführungen aufbauend, wird auf die Möglichkeit der Inszenierung von Erlebnissen eingegangen. Die Theorie der Erlebnisinszenierung als ein Konzept zur touristischen Angebotsgestaltung zu Hilfe nehmend, wird Public Viewing als Erlebnis-Setting diskutiert (10.3). Dieses für die Analyse von touristischen Angeboten entwickelte Konzept lehnt sich an den Klassiker von Pine und Gilmore (1999) zur Erlebnisökonomie an. Die Autoren gehen davon aus, dass Angebote einer Ökonomie des Erlebens immer mit dem Zusatznutzen 'Erlebnis' versehen sein sollten. Das Instrument der Angebotsgestaltung ist die Inszenierung. Diesen Gedanken weiter verfolgend, wird auf die Inszenierung als Instrument des Erlebnis-Setting für Public Viewing-Veranstaltungen eingegangen (10.4). Das Event stellt eine Extremform der Inszenierung dar. Den Optionen, die sich für das Public Viewing in dieser Gestaltungsweise bieten, widmet sich ein Exkurs (10.5). Abschließend erfolgt die Zusammenfassung der Erkenntnisse aus dem zweiten theoretischen Kapitel (10.6).

Der darauffolgende Abschnitt behandelt die Methodik und das Forschungsdesign der Untersuchung (IV). Zunächst wird auf die verwendete Methodik eingegangen (11). In dieser Arbeit erscheint es sinnvoll, die empirischen Erhebungen im Methodenmix aus einem qualitativen und einem quantitativen Verfahren durchzuführen. Die Bewertungs- und Gütekriterien einer solchen methodologischen Triangulation werden erörtert (11.1). Sowohl die qualitative als auch die quantitative Methode wurden als Befragung durchgeführt. In diesem Kapitel werden die Vor- und Nachteile dieser Vorgehensweise diskutiert (11.2). Das Kapitel endet mit der Zusammenfassung der Erkenntnisse (11.3).

Im nächsten Abschnitt erfolgt die Erörterung des Forschungsdesigns (12). Der erste Teil der empirischen Untersuchungen besteht aus qualitativen Experteninterviews. Es wurde ein halbstandardisiertes Befragungsdesign gewählt, 
um dem explorativen Charakter der Gespräche Platz zu geben (12.1). Der Aufbau des Gesprächleitfadens hat sich aus einer ersten Sichtung der Literatur ergeben. Dabei wurde das Ziel verfolgt, das Phänomen zunächst möglichst erschöpfend und objektiv zu erfassen. Zugleich sollte aber der Subjektivität der Empfindungen der Verantwortlichen Rechnung getragen werden. Da zu der behandelten Thematik wenige bis keine praktischen Erkenntnisse vorliegen, bot sich eine anfangs breite Anlegung an (12.1.1). Der Gegenstandsbereich der Untersuchung ergibt sich aus den im Umfeld der Fußball-Weltmeisterschaft 2006 durchgeführten Interviews. Spezialisten für Public Viewing-Veranstaltungen haben einen Fragenkatalog mit mehreren thematischen Bereichen beantwortet (12.1.2). Der Feldzugang ist durch einen Kontakt per E-Mail erfolgt (12.1.3); und das Zeitfenster der Befragung ist auf die Wochen nach Ende des Endturniers der WM 2006 gelegt worden (12.1.4).

Es kristallisierten sich besonders wichtige Aspekte heraus, die sich mit einer zweiten empirischen Untersuchung noch weiter spezifizieren lassen. Hierbei richtete sich das Design des standardisierten Fragebogens explizit nach den aus den Experteninterviews hervorgegangenen und theoretisch vertieften Interessenfeldern (12.2). Als erstes wird der Aufbau des Fragebogens diskutiert (12.2.1). Gegen-standsbereich dieser Befragung sind Public Viewing-Besucher, die bezüglich Fernsehübertragungen von sportlichen Ereignissen auf Großleinwänden befragt worden sind (12.2.2). Mit Hilfe des 'SchneeballVerfahrens' und dem Posten des Links in Internetforen sind interessierte Personen erreicht worden (12.2.3). Schließlich wird das Zeitfenster der quantitativen Onlineumfrage im Mai und Juni 2008 diskutiert (12.2.4).

Die Analyse der empririschen Daten erfolgt im Anschluss (V). Zu diesem Zweck werden zuerst die Forschungserkenntnisse eruiert (13). Anfangs werden allgemeine Daten zu den Experteninterviews und der Onlinebefragung erörtert. Es erfolgt die Aufschlüsselung des Untersuchungsgegenstandes mit Blick auf die Gütekriterien (13.1). Die Theoriekapitel, die qualitativen Expertenaussagen und die quantitativen Werte der Befragung der Besucher von TV-Vorführungen dienen als Diskussionsbasis für die im Anschluss folgende Überprüfung der Hypothesen (13.2). Im Fortlauf der in Kap. 1 vorgestellten Fragestellungen werden drei Hypothesen formuliert. Die erste Hypothese $(\mathrm{H} 1)$ fragt nach den Entwicklungs- 
faktoren des Public Viewing (13.2.1), die zweite (H2) nach der Publikumsbildung der TV-Vorführungen (13.2.2) und die dritte (H3) nach der Steuerbarkeit positiver Emotionen beim Public Viewing (13.2.3). Abschließend werden die Erkenntnisse dieses Kapitels zusammengefasst (13.3).

Letzlich gilt es im Rahmen der Schlussbemerkung die theoretischen und empirischen Ergebnisse in der gebotenen Kürze zu evaluieren. Zu diesem Zweck wird zum einen ein Rückblick auf das methodische und inhaltliche Vorgehen dieser Ausarbeitung erfolgen und zum anderen werden die forschungsleitenden Fragestellungen mit den Erkenntnissen aus der Diskussion der Hypothesen verglichen. Abschließend wird ein Blick auf Public Viewing bei zukünftigen sportlichen Großereignissen gewagt (VI). 


\section{Public Viewing als soziales Phänomen: Entwicklung und Grundlagen}

Im folgenden Teil der Arbeit wird das Fundament für die Entwicklungen und die Grundlagen des Public Viewing gelegt. Dazu erfolgt zunächst die Abgrenzung und Definition des Begriffs (5.1). Daran anschließend wird die wachsende Beliebtheit des gemeinsamen Fernsehens von Sportereignissen aufgezeigt (5.2). Beispielhaft wird anhand großer internationaler Fußballturniere der letzten Jahre das enorme Interesse vieler Zuschauer, Ereignisse dieser Art vor dem Bildschirm zu verfolgen, erörtert (5.2.1). Im Anschluss wird die Fußball-Weltmeisterschaft 2002 in Japan und Südkorea als der Schlüsselpunkt für die Zunahme und das wachsende Interesse der Öffentlichkeit für Public Viewing diskutiert (5.2.2). Schließlich kommt es während der WM 2006 in Deutschland zu einem nie da gewesenen Zulauf zu den Plätzen mit Großleinwänden; das Phänomen erhält eine neue Dimension (5.2.3). Es wird also in diesem Kapitel der Frage nachgegangen, inwiefern internationale Fußballgroßereignisse die Entstehung von Public Viewing beeinflussen. Resümierend erfolgt die Einordnung der gewonnenen Erkenntnisse in den Kontext (5.3).

Auf dieser Basis aufbauend, gilt es die Rolle von gesellschaftlichen Modernisierungsprozessen und ihren Einfluss auf die Entwicklung des gemeinsamen Fernsehens zu analysieren (6). In diesem Sinne wird die postmoderne Gesellschaft mit ihren Trends und Prozessen erfasst (6.1) und die Beziehung zwischen Sport und Fernsehen hergestellt (6.2). Der Sonderstellung des Fußballs wird ein weiteres Kapitel gewidmet (6.3), und die Ergebnisse werden abschließend gebündelt (6.4).

Das Publikum muss eine gewichtige Rolle beim Public Viewing spielen, so sind die Zuschauer im Gegensatz zum Stadionbesuch hier die Hauptakteure (7). Zunächst erfolgt die Strukturierung der Begrifflichkeit (7.1), während in einem weiteren Schritt auf die Determinanten der (Fernseh-)Publikumsbildung eingegangen wird (7.2). Abschließend wird das Kapitel zusammengefasst (7.3).

Schließlich wird dieser Abschnitt durch den Brückenschlag zwischen Gesellschaft, Sport und Fernsehen zum Abschluss gebracht. Die Rückkehr des sozialen Lebens in den öffentlichen Raum und damit auch das gemeinsame Erleben von 
Fernsehübertragungen wird im Anschluss eruiert (8). Dies kann nur geschehen, wenn die das Public Viewing determinierenden Faktoren wie bspw. die die Zahl der Stadiontickets übersteigende Nachfrage nach Karten, beachtet werden (8.1). Schließlich wären die Menschentrauben vor Großbildschirmen nicht ohne neue technologische Entwicklungen möglich gewesen (8.2). Letztlich werden die Erkenntnisse dieses Kapitels zusammengeführt (8.3). 


\section{Public Viewing}

Im folgenden Abschnitt wird das Ziel verfolgt, eine erste Basis für die folgende Analyse aufzubauen. Zu diesem Zweck wird erst einmal der Begriff des Public Viewing eingegrenzt und eine Arbeitsdefinition herausgestellt (5.1). Daran anschließend wird die wachsende Beliebtheit des gemeinsamen Fernsehens aufgezeigt (5.2). Es wird die Auffassung vertreten, dass sich anhand der Entwicklung der Zuschauerzahlen bei Fußball-Welt- und -Europameisterschaften bereits erkennen lässt, dass sich das Interesse der Zuschauer nicht nur auf die bloße sportliche Aktion beschränkt. Beispielhaft wird anhand großer internationaler Fußballturniere der letzten Jahre das enorme Interesse vieler Zuschauer, Ereignisse dieser Art vor dem Bildschirm zu verfolgen, erörtert (5.2.1). Im Anschluss wird die Fußball-Weltmeisterschaft 2002 in Japan und Südkorea als der Schlüsselpunkt für die Zunahme und das wachsende Interesse der Öffentlichkeit für Public Viewing diskutiert (5.2.2). Schließlich kommt es während der WM 2006 in Deutschland zu einem nie da gewesenen Zulauf zu den Plätzen mit Großleinwänden; das Phänomen erhält eine neue Dimension (5.2.3). Resümierend erfolgt die Einordnung der gewonnenen Erkenntnisse in den Kontext (5.3). 


\subsection{Begriffsbestimmung}

„Public Viewing ist 'en vogue'“ (Gelis, 2005).

Kaum einem anderen sportbezogenen Thema wurde im Jahr 2006 in den hiesigen Medien mehr Aufmerksamkeit geschenkt. Nie zuvor wurde so viel über die öffentlichen TV-Übertragungen diskutiert, verhandelt und lamentiert wie im Vorfeld der Fußball-Weltmeisterschaft 2006. Nun ist das Phänomen, welches sich hinter dem Begriff 'Public Viewing' verbirgt, nicht ganz neu. Wichtige Ereignisse des Sports, aber auch des öffentlichen Lebens und des Musikbusiness' wurden in den letzten Jahren verstärkt auf Großleinwänden gezeigt und von vielen Menschen davor verfolgt. Was aber genau verbirgt sich hinter dem Scheinang lizismus?

Es gestaltet sich schwierig, Public Viewing trennscharf zu definieren. Aufgrund der kaum vorhandenen wissenschaftlichen Quellen (s. Kap. 3) gibt es fast ausschließlich Definitionsversuche aus medialen Berichten, denen es meist an Genauigkeit und Ernsthaftigkeit mangelt. Deshalb werden an dieser Stelle vorhandene Begriffsbestimmungen eruiert und eine für den hiesigen Zusammenhang geeignete Arbeitsdefinition bestimmt.

Public Viewing übersetzt aus dem Englischen für "öffentlich“ und „zusehen/ansehen“" wurde im Vorfeld der WM 2006 von der FIFA eingeführt. Demzufolge beschränkt der Weltverband des Fußballs Public Viewing als Begriff für ",̈ffentliche Vorführungen von Spielen der FIFA WM $2006^{\text {TMk، }}$ (Infront Sports \& Media AG, o.J.a \& b). Bezüglich der WM 2006 ist mit Public Viewing eine Zusammenfassung von verschiedenen Veranstaltungstypen gemeint, bei denen die Spiele medial übertragen werden und die durch einen öffentlichen Charakter bestimmt sind (vgl. Cybulska, 2007, S. 73). Das Süddeutsche Zeitung Magazin (2006, S. 26) definiert Public Viewing als „Fernsehübertragung von Sportveranstaltungen auf öffentlichen Plätzen“. Nach der FIFA bzw. Infront und dem Süddeutsche Zeitung Magazin sind Public Viewing Events öffentlich, d.h. für die Allgemeinheit zugänglich. Doch während die FIFA den Begriff ausschließlich auf die Vorführung von Spielen der Fußball-WM 2006 bezieht, spricht das Süddeutsche Zeitung Magazin bereits von einem allgemeinen Terminus, nämlich von Sportveranstaltungen. Womit sich die Begrifflichkeit eindeutig einem erweiterten Bedeutungshorizont öffnet. Weg von der Übertragung eines Events in einer bestimmten Sportart in einer definierten Zeitspanne, hin zu der Öffnung des 
Begriffs für die Gesamtheit aller Sportarten. Es kann angenommen werden, dass dieses divergierende Verständnis von Public Viewing mit der Veränderung des Begriffs im Laufe des Endrundenturniers 2006 zu erklären ist. Ursprünglich nur für die Übertragungen auf den Fan Festen gedacht, öffnete sich das Begriffsverständnis für alle Arten von öffentlichen Übertragungen, für Vorführungen in Kneipen gleichermaßen wie in Universitätshörsälen oder Parks (vgl. Süddeutsche Zeitung Magazin, 2006, S. 26).

Meyers Lexikonverlag (2007) definiert das Phänomen Public Viewing als „öffentliche Liveübertragung von publikumswirksamen Großereignissen“. Hier wird eindeutig der ursprüngliche Bedeutungszusammenhang noch weiter geöffnet. $\mathrm{Er}$ wird aus dem Bereich des Sports herausgenommen und auf publikumswirksame Großereignisse übertragen. Demnach gilt Public Viewing auch für bisher namenlose Praxen, wie die Aufstellung von Leinwänden im Londoner Hyde Park zur Begräbniszeremonie von Lady Diana und das Mitverfolgen der Feierlichkeiten von tausenden Menschen jetzt als Namensgeber.

Der Weltverband des Fußballs und Infront haben den Begriff des Public Viewing für ihre Belange in drei Einheiten unterteilt. Diese richten sich allerdings hauptsächlich nach rechtlichen und vermarktungsorientierten Gesichtspunkten und sind für einen allgemeinen Definitionsversuch nicht geeignet. Jedoch stellen sie für die späteren Befragungen (s. Kap. IV \& V) eine nützliche Abgrenzung dar und werden in genannten Bereichen ihre Anwendung finden. Aus diesem Grunde sind sie hier kurz aufgeführt:

- $\quad$ Das gemeinsame Fernsehen auf den Fan Festen der FIFA WM $2006^{\mathrm{TM}}$,

- $\quad$ das nicht-kommerzielle Public Viewing, bei dem der Veranstalter keinerlei kommerziellen Nutzen verfolgen darf, sowie

das kommerzielle Public Viewing. Hier handelt es sich um Veranstaltungen mit gewerblichem Charakter. ${ }^{5}$

Die Public Viewing-Veranstaltungen neben den Fan Festen können ganz unterschiedlicher Natur sein; kleine halb-öffentliche Firmenfeste sowie große öffentliche Fernsehvorführungen auf Marktplätzen oder Werbeevents von

\footnotetext{
5 Die Einteilung in offizielle FanFeste, kommerzielles und nicht-kommerzielles Public Viewing mit den jeweiligen rechtlichen Auflagen ist von der UEFA für die EURO 2008 übernommen worden (vgl. UEFA, 2008). Es kann angenommen werden, dass sich diese Abgrenzung im praktischen Sektor bewährt hat.
} 
Unternehmen sind denkbar. Diese können einer sportlichen Ausrichtung mit Kleinfeldturnieren oder Torwandschießen folgen, einen Partycharakter haben, als eine Attraktion in eine Art Stadt(teil)fest eingebaut oder ein Präsentations-Event einer Firma für ihre Hauptkunden sein.

Eine Verfeinerung der Begriffsbestimmung ist zur Europameisterschaft 2008 erfolgt. Die UEFA (2008) definiert die öffentlichen Vorführungen von Spielen der UEFA EURO $2008^{\mathrm{TM}}$ als

„jede Vorführung [...], die ausserhalb der Privatsphäre bestehend aus Familie und privaten Gästen auf einem Bildschirm oder einer Grossleinwand mit einer Diagonale von mehr als 3 Metern stattfindet".

In dieser Ausarbeitung wird sich dieser präzisierten Begriffsbestimmung angeschlossen, und die erforderliche Begrenzung der Definition auf öffentliche Live-Übertragungen von sportbezogenen Großereignissen wird vollzogen. Dennoch wird, sofern dem Erkenntnisgewinn zuträglich, auf Übertragungen außerhalb des Sports zurückgegriffen, um nicht von vornherein wichtige Ansätze auszuschließen.

\subsection{Wachsende Beliebtheit des Public Viewing}

Bei vielen Ereignissen von besonderem öffentlichem Interesse gehören Großleinwände, die das Ereignis möglichst vielen Menschen zugänglich machen, inzwischen zum gewohnten Bild. Dies ist nicht nur bei großen Sportveranstaltungen der Fall, wie bspw. in der Formel 1, bei Olympischen Spielen oder bei der Handball-WM im Jahr 2007, bei denen sich viele Fans vor den großen Bildschirmen versammeln. Als 1997 weltweit 2,5 Milliarden Menschen die Trauerfeier zu Ehren von Lady Diana verfolgten, waren die zwei in London aufgestellten Großbildschirme allerdings zu klein, so dass viele Tausende die Übertragung nur hören konnten (vgl. Krüger, 1999, S. 1226; Reng, 1997). Neben Ereignissen des öffentlichen Lebens, wie der Beerdigung Lady Dianas, ziehen Musikkonzerte eine große Zahl an Zuschauern an. Die Karten für das einzige Konzert von Robbie Williams im Jahr 2005 in Berlin waren sofort ausverkauft. Daraufhin organisierte das Marketing-Unternehmen des Musikers LiveÜbertragungen des Konzerts in 27 Großkinos in elf europäischen Ländern. Auch diese Konzert-Vorführungen waren alle ausverkauft (vgl. Buhr, 2005). 
Wie kommt es aber, dass zur Fußball-Weltmeisterschaft 2006 in Deutschland das Public Viewing solche Ausmaße annimmt, so dass erstmals ein eigener Begriff kreiert wird? Dieser Frage widmet sich der kommende Abschnitt mit einem Blick auf die enorme Präsenz von internationalen Fußballturnieren im Fernsehen (5.2.1) und dem Schlüsselereignis für die Entwicklung des Public Viewing, der WM 2002 in Japan und Südkorea (5.2.2). Letzten Endes bietet die WM 2006 mit ihren Besonderheiten die Grundlage für die Entstehung des Phänomens in seiner uns bekannten Form (5.2.3).

\subsubsection{Fußball-Welt- und -Europameisterschaften als Quotengaranten}

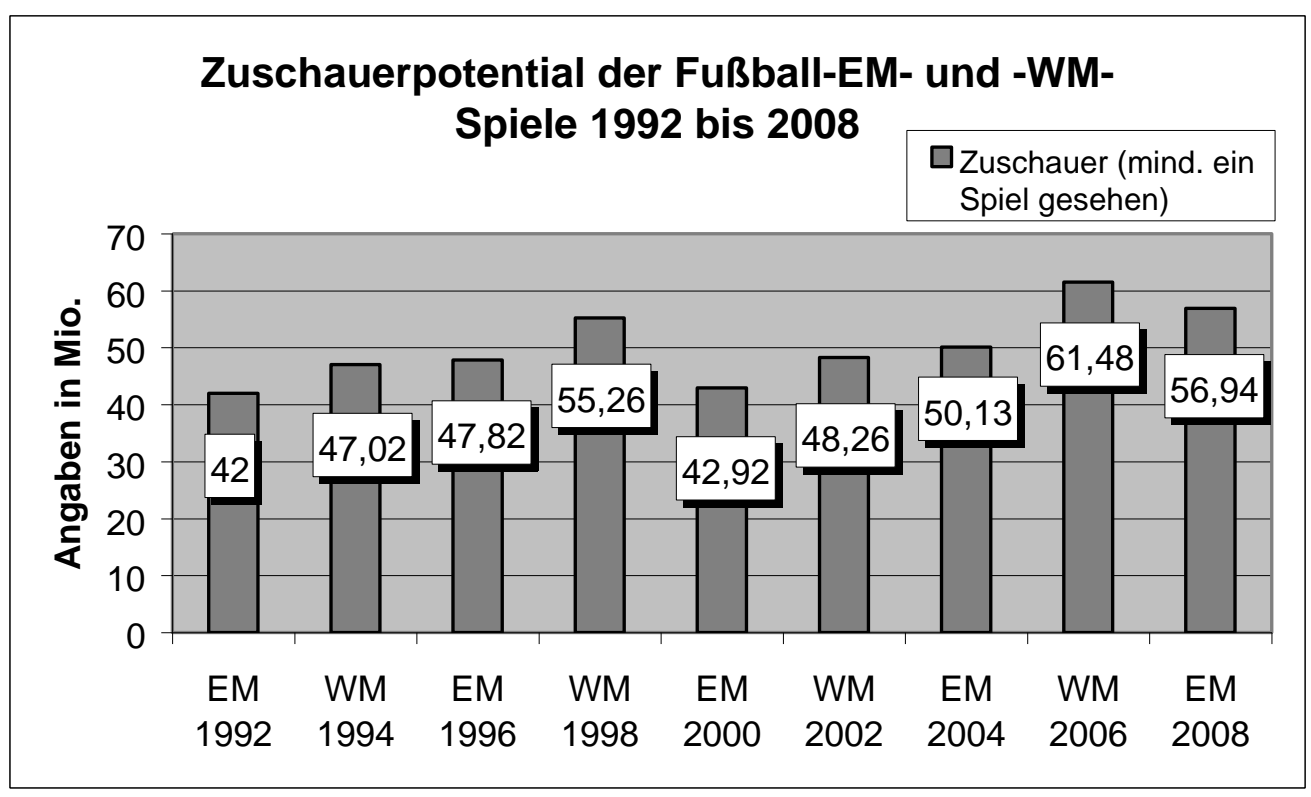

Abb. 1: Zuschauerpotential (Zuschauer ab 3 Jahren) der Fußball-EM- und -WM-Spiele 1992 bis 2008 in Deutschland (AGF/GfK; pc\#tv; Fernsehpanel D+EU nach Geese \& Gerhard, 2008, S. 443)

Wie in Abb. 1 ersichtlich, steigt das Interesse des deutschen Fernsehpublikums an Fußball-Europa- und Weltmeisterschaftsspielen seit 1992 sukzessive an. Dabei ist zunächst nicht verwunderlich, dass das Interesse der deutschen Bevölkerung, die WM im eigenen Land zu verfolgen, in 2006 höher war als in 1998 und 2002. Waren es 1998 mit insgesamt 55,26 Mio. Zuschauern etwa 77\% der Bevölkerung und in $2002^{6}$ mit 48,26 Mio. Zuschauern 68\% der Einwohner, so haben 2006 83\% aller Deutschen mindestens ein Spiel live bei den öffentlich-rechtlichen Sendern

6 Bei den Zahlen für 2002 muss allerdings beachtet werden, dass die Spiele mit neun Stunden Zeitverschiebung zu eher unattraktiven Fernsehzeiten ausgestrahlt wurden (vgl. Geese et al., 2006, S. 454). 
oder RTL verfolgt, was 61,48 Mio. Zuschauern entspricht (vgl. Geese et al., 2006, S. 454). Die EM in Österreich und der Schweiz im Jahr 2008 verfolgten insgesamt 56,94 Mio. Rezipienten, wobei aber die durchschnittliche Sehbeteiligung mit einem Wert von 15,84 Mio. Zuschauern über dem Wert der WM 2006 mit 12,06 Mio. liegt, was nach Geese und Gerhard (2008, S. 442) an den späteren Spielzeiten gelegen haben kann. ${ }^{7}$ In besonderem Maße ist das Turnier in Japan und Südkorea 2002 hervorzuheben (s. Kap. 5.2.2). Es kann ohne Zweifel als

"24 hours a day, seven days a week viewing phenomenon" (FIFA Marketing AG M edia Services, o.J.)

bezeichnet werden. Trotz der, durch die Zeitverschiebung zwischen den Austragungsorten in Asien und dem Hauptteil der Rezipienten in Europa und Amerika bedingten, ungünstigen TV-Übertragungszeiten sahen sich weltweit viele Menschen die Spiele an. So wurden zahlreiche 'non-prime-time' LiveÜbertragungsrekorde gebrochen. Zum Beispiel verfolgten 18,1 Mio. Deutsche das Spiel ihrer Nationalmannschaft gegen Paraguay, was der ARD, die das Spiel übertrug, einen Marktanteil von $88 \%$ bescherte (vgl. ebd., o.J.). Im Fußballland Brasilien sahen sich kumulierte 54,8 Mio. Rezipienten diese WM an, 20 Mio. mehr als beim vorherigen Turnier 1998 (vgl. Infront Sports \& Media AG, O.J.c). Der brasilianische Fernsehsender TV Globo erreichte mit der Übertragung des Viertelfinalspiels zwischen England und Brasilien einen sehr hohen Marktanteil von $94,2 \%$. Bei einer gemessenen Einschaltquote von $30,2 \%$ bedeutet das hochgerechnet, dass sich 46 Mio. Brasilianer die Partie ansahen, obwohl sie um drei Uhr dreißig in der Nacht ausgestrahlt wurde (vgl. FIFA, o.J.b). In Asien, wo die

\footnotetext{
7 Während bei der WM 2006 etwa ein Drittel der Spiele am Nachmittag stattfand, wurden die EM Spiele alle um 18 bzw. um 20.45 Uhr ausgetragen (vgl. ebd., S. 442).

8 Die rückläufigen TV-Quoten der WM 2002 führt die FIFA (o.j.b) bei den vom Weltfußballverband ermittelten Werten auf, die ersten zuverlässig ermittelten und geprüften Erhebungen in China zurück. Die chinesischen Angaben für die Turniere von 1990 bis 1998 wären bei weitem zu hoch, da sie auf den Qutoten von Shanghai City basierten, die auf das ganze Land hochgerechnet worden sind. Diese unbefriedigenden Ergebnisse führten zu dem Entschluss der FIFA, für die Weltmeisterschaft 2002 gründlichere und genauer geprüfte Zuschauerzahlen zu ermitteln. So betrug die kumulative Einschaltquote aller Tage und Länder der Weltmeisterschaft im Jahr 2002 insgesamt 28,8 Mrd.. Was bedeutet, dass die Zuschauerzahl im Vergleich zum vorherigen Wettbewerb in Frankreich um 4,6 Mrd. gesunken ist, denn der für dieses Turnier von der FIFA ermittelte Wert lag bei kumulierten 33,4 Mrd. TV-Zuschauern (vgl. FIFA, o.J.a). Würden jedoch bei beiden Veranstaltungen die Daten aus China nicht berücksichtigt, sei für die Weltmeisterschaft in Südkorea und Japan 2002 ein Ansteigen der Einschaltquoten um 431,7 Mio. Rezipienten, was eine Steigerung von $2 \%$ ausmache, festzustellen, so der Weltfuß ballverband. Kumulative Angaben, die in dieser Arbeit gemacht werden, beziehen sich auf „that in many instances, the football fans not only watch one but rather several matches on TV, hence the incredible totals that are registered“ (ebd.).
} 
Spiele zur Hauptsendezeit übertragen wurden, lagen die Zuschauerzahlen im Vergleich noch höher. So sahen auf dem japanischen Sender CX beachtliche 62,3 Mio. Menschen das Spiel Japan gegen Russland (vgl. Dentsu/Video Research zit. n. FIFA Marketing AG Media Services, o.J.). Sicherlich waren Sport und insbesondere Fußball- Weltmeisterschaften schon immer ein Garant für gute TVQuoten, aber diese Zuschauermassen wurden zum ersten Mal erreicht;

"[I]n terms of television viewing, the FIFA World Cup is the absolute no. 1 sports event" (FIFA, o.J.a).

Die Entwicklung der letzten Jahre zeigt also, dass viele Menschen ein enormes Interesse haben, wichtige nationale wie internationale Ereignisse, insbesondere des Sports, intensiv vor dem Fernseher zu verfolgen.

Was können nun aber die Gründe dafür sein? Kruck und Stuke (2003, S. 36) sehen die Besonderheit der Fußball-Weltmeisterschaft im Vergleich zu den Olympischen Spielen in ihrem Aufbau. Während bei letzteren die Spannung auf viele Entscheidungen verteilt wird, baut sich die Spannung bei der WM sukzessive auf, und am Ende verbleibt nur ein einziger Gewinner. Dieses einzelne Ergebnis bestimme auch die kommunikative Struktur des Turniers bei den Medien wie bei den Zuschauern. Ein anderes Argument ist das im Vergleich auffällig hohe Interesse der weiblichen Bevölkerung an den Begegnungen. Der ARD-Trend: Programm und Marketing (2002 zit.n. Rühle, 2003, S. 217) gibt an, dass das Sportinteresse von weiblichen und männlichen TV-Sehern unterschiedlich ist. Während 66\% der Männer im Alter von 14-49 Jahren und 69\% der Männer ab 50 Jahren dem Sport eine „wichtige“ oder sogar „besonders wichtige“ Position einräumen, sind es bei den Frauen (14-49 Jahre) gerade einmal 23\% und bei den Frauen ab 50 Jahren mit 29\% ebenfalls nur geringfügig mehr. Die WM-Spiele 2006 erreichen aber bei allen soziodemographischen Bevölkerungsgruppen hohe und relativ gleichmäßige Marktanteilswerte. Das heißt, dass die WM-Livespiele auf hohem Akzeptanzniveau gleichmäßig alle Zuschauergruppen relativ unabhängig von Alter, Geschlecht, Bildung und Berufsstatus erreichen (vgl. AGF/GfKFernsehforschung, pc\#tv, Fernsehpanel D+EU zit. n. Gerhard, 2006, S. 473). Nach Geese et al. (2006, S. 456) war während des Endturniers der Fußball-WM im eigenen Land das Interesse der weiblichen Zuschauer erstaunlich hoch. Im 
Vergleich mit der vorangegangenen Bundesligasaison gesehen ${ }^{9}$, interessierten sich weit mehr Teile der weiblichen Bevölkerung für die Live-Begegnungen der WM. Während sich in der Bundesliga nur ein Drittel der weiblichen Zuschauer wirklich für Fußball begeisterten, waren es bei den Livespielen der Weltmeisterschaft 2006 beachtliche 43\%. Bei den Begegnungen, an denen die deutsche Mannschaft beteiligt war, machten die Frauen sogar die Hälfte der Gesamtseher aus, und bei den letzten drei WM-Spielen der deutschen Männer saßen erstmals mehr Frauen vor den Fernsehgeräten als Männer. Dieser Trend setzt sich weiter fort.

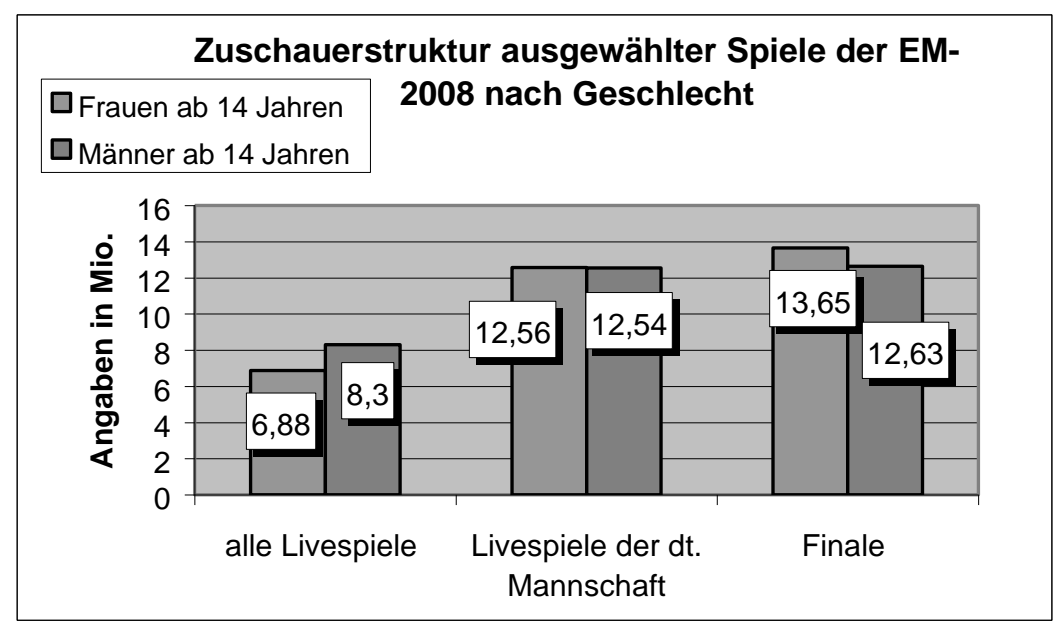

Abb. 2: Zuschauerstruktur (Zuschauer ab 14 Jahren) ausgewählter Spiele der Fußball-EM 2008 nach Geschlecht (AGF/GfK-Fernsehforschung, pc\#tv, Fernsehpanel D+EU nach Geese \& Gerhard, 2008, S. 445)

Wie Abb. 2 verdeutlicht, sahen bei der Europameisterschaft 2008 durchschnittlich ebenso viele Frauen wie Männer die Begegnungen der deutschen Nationalelf. Ab dem Viertelfinale überstieg die Zahl der weiblichen Rezipienten die der männlichen erstmals, und dem Finale sahen 13,65 Mio. Frauen und 'nur' 12,63 Mio. Männer zu (vgl. auch Kap. 13.2). Woran kann dieser enorme Publikumszustrom bei Fußball-Europa- und -Weltmeisterschaften liegen? Diese Frage wird, da sie in unmittelbarem Zusammenhang mit der Entstehung und Entwicklung des Public Viewing steht, in den folgenden Kapiteln immer wieder von unterschiedlichen Blickwinkeln beleuchtet. An dieser Stelle bietet Gerhard (2006, S. 470f.) zunächst folgende Merkmale zur Beschreibung der Beliebtheit von Fußball-Europa- und -Weltmeisterschaften an:

- die Marktanteile,

\footnotetext{
9 Gemeint ist hier die Saison 2005/06.
} 
- $\quad$ die erzielten Reichweiten einzelner Begegnungen,

- $\quad$ die durchschnittlich erzielten absoluten Zuschauerzahlen und

- $\quad$ den weitesten Seherkreis des Events ${ }^{10}$.

Bei der WM 2006 haben mehr als 61 Mio. Zuschauer, das sind 83\% der Bevölkerung, mindestens eine der Übertragungen im Free-TV verfolgt (vgl. Geese et al., 2006, S. 454; ebd., S. 465). Nach Rühle (2003, S. 216) gilt Sport als Zuschauermagnet, was ihm eine besondere Position für die Ausgestaltung von Senderprofilen verleiht. Er wird neben Information und Unterhaltung als dritter wichtiger Programmkomplex gesehen. Der positive Aspekt des Sports ist, dass er in den verschiedenen Alters-, Bildungs- und Einkommensgruppen gleichermaßen beliebt zu sein scheint. Diese breite Zielgruppenansprache macht den Sport zu einem besonderen Programmbestandteil, was insbesondere für internationale Fußballturniere, allen voran Weltmeisterschaften, gilt. Die durchschnittlich erzielten absoluten Zuschauerzahlen von ARD und ZDF für die ersten vier deutschen Spiele lagen bei der Weltmeisterschaft 1998 bei kumulierten 85,5 Mio. Zuschauern. In 2006 waren es mit durchschnittlich 87,6 Mio., also jeweils ca. eine halbe Mio. mehr Zuschauer pro Begegnung (vgl. Infront Sports \& Media AG, 2006a). Die Reichweiten einzelner Begegnungen betrachtend, muss festgestellt werden, dass 31 der 56 Spiele von mehr als zehn Mio. Zuschauern gesehen wurden. Acht Spiele fesselten sogar mehr als 20 Mio. Menschen und erstmals erreichte mehr als die Hälfte der übertragenen Spiele ein zweistelliges Millionenpublikum (vgl. AGF/GfK-Fernsehforschung, pc\#tv, Fernsehpanel D+EU zit.n. Geese et al., S. 454). Die größte Reichweite überhaupt erzielte das Halbfinalspiel Italien gegen Deutschland. 29,66 Mio. Zuschauer verfolgten den Einzug der Italiener ins Finale. Dies bedeutet einen Marktanteil von $84,1 \%$, was der höchsten in Deutschland je gemessenen TV-Reichweite entspricht (vgl. ebd., S. 454). Diese Zahlen decken sich mit den zehn meistgesehenen TV-Sendungen des Jahres 2006 in den Öffentlich-Rechtlichen, die ausnahmslos Übertragungen der Fußball-WM sind.

10 Diese Determinante gibt an, wie viele Zuschauer mindestens eines der Live-Spiele gesehen haben (vgl. ebd., S. 470f.). 
Tab. 1: Rangreihe der me istgesehenen Ein zelsendungen des Jahres 2006 (Zuschauer ab 3 Jahre; Sendungslänge mind. 10 Min.) (A GF/GfK, pc\#tv, Fernsehpanel D+EU nach Zubayr \& Gerhard, 2007, S. 193)

\begin{tabular}{||l||l|l||l|l||l|}
\hline $\begin{array}{l}\text { Rang- } \\
\text { platz }\end{array}$ & Sender & & Datum & $\begin{array}{l}\text { Zuschauer } \\
\text { in Mio. }\end{array}$ & $\begin{array}{l}\text { Marktanteil } \\
\text { in } \%\end{array}$ \\
\hline 1. & ZDF & Fußball-WM 2006: Deutschland - Italien & 04.07. & 29,66 & 84,1 \\
\hline 2. & ARD & Fußball-WM 2006: Italien - Frankreich & 09.07. & 25,88 & 72,3 \\
\hline 3. & ARD & Fußball-WM 2006: Deutschland - Argentinien & 30.06. & 24,74 & 86,1 \\
\hline 4. & ZDF & Fußball-WM 2006: Deutschland - Portugal & 08.07. & 23,97 & 76,1 \\
\hline 5. & ARD & Fußball-WM 2006: Deutschland - Polen & 14.06. & 23,88 & 72,5 \\
\hline 6. & ZDF & Fußball-WM 2006: Deutschland - Schweden & 24.06. & 22,34 & 86,3 \\
\hline 7. & ARD & Fußball-WM 2006: Ecuador - Deutschland & 20.06. & 21,3 & 82,1 \\
\hline 8. & ZDF & Fußball-WM 2006: Deutschland - Costa Rica & 09.06. & 20,06 & 75,7 \\
\hline 9. & ARD & Fußball-WM 2006: Portugal - Frankreich & 05.07. & 15,3 & 53,2 \\
\hline 10. & ARD & Fußball-WM 2006: Brasilien - Kroatien & 13.06. & 15,16 & 52,3 \\
\hline \hline
\end{tabular}

Diese überwältigende Akzeptanz der WM 2006 bestätigt in zugespitzter Form die außerordentliche Rolle von Fußballübertragungen für Weltmeisterschaftsturniere im Fernsehen. Keine andere Sportart scheint so für das Fernsehen geeignet wie der Fußball, nicht nur bei einer Fußball-WM (vgl. Gerhard, 2006, S.465). ${ }^{1}$ Allerdings hat diese Aufwärtsspirale offenbar ihre Grenzen. Bei der WM 2002 verkaufte die FIFA in den meisten nationalen Märkten die Ausstrahlungsrechte an Pay-TV-Sender, die die meisten Spiele live zeigten. ${ }^{12}$ Dies hatte rückläufige Reichweiten der Endrunde in 2002 zur Folge, so dass u.a. aus diesem Grund in 2006 deutlich mehr Übertragungen im Free-TV stattfanden (vgl. ebd., S. 468). ${ }^{13}$

Zusammenfassend kann an dieser Stelle zunächst gesagt werden, dass besonders die Endrunden der Fußball-Welt- und -Europameisterschaften große Aufmerksamkeitswerte in breiten Bevölkerungskreisen, auch bei der weiblichen Bevölkerung, erreichen. Das Interesse steigt sukzessive an, es scheint jedoch im Falle eines kostenpflichtigen Zugangs Grenzen zu geben.

11 Bei den erwähnten Reichweiten muss man berücksichtigen, dass die Messungen der GfK sich auf Fernsehnutzung in Privathaushalten beschränkt (vgl. Zubayr \& Gerhard, 2007, S. 192f.).

12 Für die freien Stationen blieb nur ein begrenztes Kontingent von wichtigen Spielen für die jeweiligen heimischen Märkte übrig. Das Vorgehen des Weltverbandes ist vor allem zur Steigerung der Abonnentenzahlen der Pay-TV-Sender gedacht gewesen.

13 Für Deutschland erwarb RTL die Rechte für die acht Sonntagsspiele bis zum Viertelfinale von den öffentlich-rechtlichen Sendern. Diese strahlten insgesamt 48 Spiele aus. Acht Partien übertrug der Bezahlsender Premiere exklusiv. Somit wurden erstmals 56 der 64 Spiele im Free-TV ausgestrahlt, bei der WM 2002 erwarben ARD und ZDF nur Rechte für 26 Spiele (vgl. Geese et al., 2006, S.454). Dieses waren die Spiele des letzten Vorrundentages, die in den Gruppen zeitgleich stattfanden. 


\subsubsection{Schlüsselereignis: WM 2002 in Japan und Südkorea}

"During the 1990s the culture of collective viewing in public spaces, long common in continental Europe, became a more significant phenomenon around the world" (Whannel, 2005, p. 414).

Bereits bei Ereignissen wie der FIFA WM 1998 in Frankreich oder bei den Olympischen Sommer-Spielen in Sydney 2000 wollten mehr Menschen an dem Ereignis teilhaben, als die Stadienkapazitäten zuließen. Die damaligen Übertragungen auf Großleinwände sind vielerortens schon ein großer Erfolg gewesen (vgl. FIFA, o.J.d; Neue Zürcher Zeitung, 2001). Unter anderem dank neuer Übertragungstechniken (s. Kap. 8.2) ist das Public Viewing im Rahmen großer Veranstaltungen nun mehr und mehr in den Fokus gerückt (vgl. Habbel, 2005, S. 7). Aber erst bei der WM 2002 in Südkorea und Japan ist das öffentliche Schauen von Fußballspielen zu einem wirklich interressanten Faktor geworden.

Es kann von einem Wandel des Zuschauer- und Fanverhaltens gesprochen werden, der sich dadurch auszeichnet, dass außergewöhnlich viele Menschen wichtige Spiele außer Haus sehen (vgl. Chi-dong, 2002, p. 28; FIFA Marketing AG Media Services, O.J.). Darüber hinaus ändert sich die Art und Weise, wie Menschen außerhalb der Stadien die Spiele verfolgen (vgl. FIFA Marketing AG Media Services, o.J.). Eine auffällig hohe Zahl sieht sich die Spiele nicht vor dem heimischen Fernseher an, sondern gemeinsam mit Freunden oder Kollegen in Bars oder auf großen Plätzen. Das Miterleben von Spielen auf Videowänden ist zu einem wichtigen Faktor geworden (vgl. FIFA, o.J.b). Nach Angaben von Sponsoring Insights (zit.n. FIFA Marketing AG Media Services, o.J.) sollen bis zu 43\% der Fernsehzuschauer im Vereinigten Königreich die Begegnung zwischen Argentinien und England in Pubs oder Bars gesehen haben. Für die wichtigen europäischen Fußballmärkte Italien und Spanien gehen andere Quellen von 20 bis 25\% aushäusiger Zuschauer aus (vgl. SponsorMetrix zit. n. ebd.).

Offenbar scheint der Einzelne zu besonders wichtigen Sportereignissen den gemütlichen heimischen Fernsehsessel gerne gegen einen Barhocker einzutauschen. Der Fußball rückt so ein Stück weiter in die Öffentlichkeit, in die Gesellschaft hinein. Auch in Deutschland gab es Public Viewing zur WM 2002. Es wurden an zentralen Plätzen in Großstädten die wichtigen Spiele der eigenen Nationalmannschaft übertragen. Viele tausende Menschen versammelten sich vor den Leinwänden (vgl. Schulke, 2006b, S. 36). Nach Röger (2006) hat die WM in 
Südkorea und Japan den Fußball noch ein wenig mehr in die Gesellschaft gebracht. Durch die Zeitverschiebung fielen die meisten Spiele in Mitteleuropa auf den Vormittag. Somit ruhte in so manchem deutschen Büro die Arbeit, wenn die Nationalelf spielte, es wurden Tippspiele veranstaltet und die Mittagspause nach dem Spielplan gelegt. Dadurch haben auch nicht fußball-affine Gruppen der Gesellschaft am Ereignis teilgenommen, einfach, weil es Spaß machte und kaum zu umgehen war.

Ein mancher wird sich an das erste große Fußballereignis im deutschen Fernsehen erinnern: Die Fußball-WM 1954. Die ARD übertrug neun der 26 Spiele live. Zu dieser Zeit muss nach Gerhard (2006, S. 465f.) davon ausgegangen werden, dass sich weniger als 100.000 TV-Geräte in Benutzung befanden. Dennoch scheinen Hunderttausende, wenn nicht Millionen von Zuschauern das Endspiel im Fernsehen -vor allem in Gaststätten- gesehen zu haben (vgl. auch Breitmeier, 2004). Der Fußball(-Sport) ist also in die Mitte der Gesellschaft zurückgekehrt.

In Südkorea, einem der Aus richterländer, warben Regierung und Ausrichterstädte rege für das 'Gemeinschaftsgucken' vor Großleinwänden. Es wurden insgesamt 2.021 sogenannte Big-Screens an 1.868 Plätzen im ganzen Land aufgestellt, um den Menschen das Zusehen mit Freunden zu ermöglichen. Hinzu kamen private, meist für Werbezwecke genutzte Leinwände, die auf Trucks befestigt für eine noch größere Anzahl an zusätzlichen Public Viewing-Möglichkeiten sorgten (vgl. SoonHee, 2004, p. 149ff.). Meist Jugendliche und junge Erwachsene feuerten ihre Mannschaft vor TV-Wänden in den Innenstädten der Ballungsräume an. Hierbei handelte es sich meist um kurzfristig und punktuell iniziierte Maßnahmen (vgl. Schulke, 2006b, S. 30). Nach Angaben des Korea Pool ${ }^{14}$ (zit.n. FIFA Marketing AG Media Services, o.J.) gingen 2,8 Mio. Menschen in Korea auf die Straßen, um sich bei 223 offiziellen Veranstaltungen das Spiel ihrer Mannschaft gegen Portugal anzusehen. Beim Spiel gegen Italien sollen es sogar 4,2 Mio. gewesen sein.

\footnotetext{
14 Der Korea Pool ist eine Gemeinschaft von Fernsehstationen. Er besteht aus der KBS (Korea Broadcasting System), der MBD (Munwha Broadcasting Corporation) sowie der SBS (Seoul Broadcasting System) (vgl. Pank, o.J.).
} 

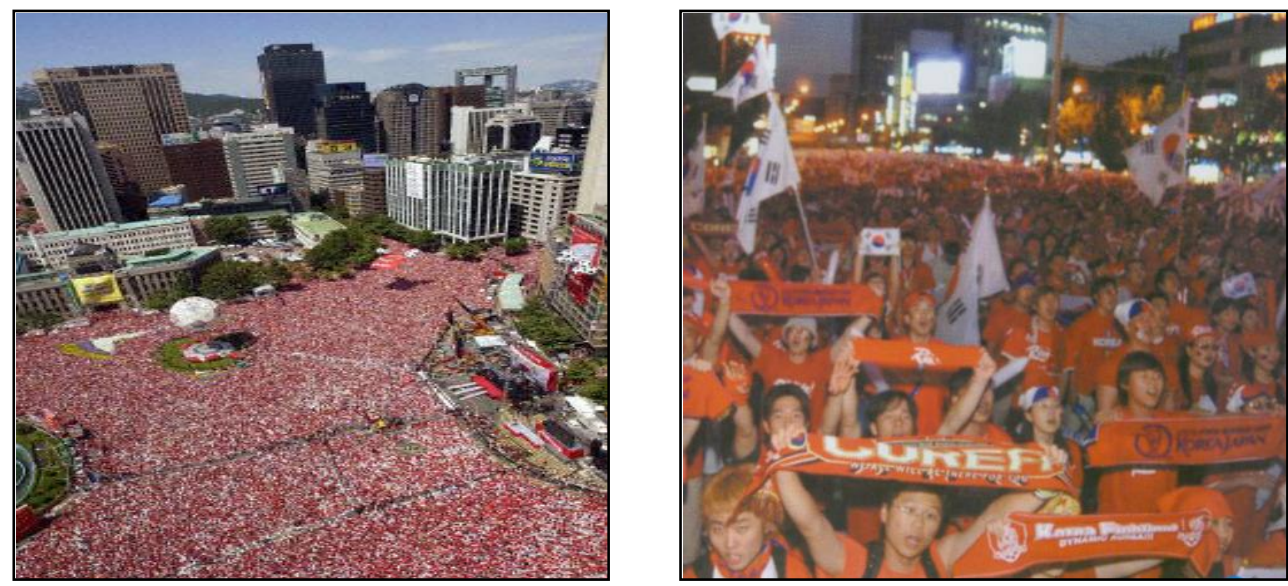

Abb. 3: Public Viewing bei der FIFA WM $2002^{\mathrm{TM}}$ in den Innenstädten Südkoreas (FIFA, o.J.c/Soo-min, 2002, p. 33)

Wieso ist es gerade dort zu diesen Zusammenkünften von tausenden Personen gekommen? Nach Horne und Manzenreiter (2004, p. 195) hätten besonders die koreanischen Medien einen großen Einfluss auf das Phänomen geno mmen:

"Korean Media had instructed their nation how to cheer for national success. Oh, pilseung Korea is an adapted version of a football cheer sung by K-League team Bucheon SK supporters."

Durch einen Fernseh-Werbespot mit einem koreaweit bekannten Filmschauspieler und hunderten Fans der 'Red Devils' wurde die Bevölkerung angeleitet „Oh, pilseung, Korea" und einen anderen Fangesang zu singen (vgl. Soo-min, 2002, p. 32f.). Während die Bilder der WM-Partys mit bis zu 100.000 in Nationalfarben gekleideten, friedlich feiernden Koreanern um die Welt gingen, verbot die japanische Regierung das öffentliche Spektakel. Sie hatte Bedenken, dass junge gewaltbereite Fans mit der Polizei aneinander geraten könnten (vgl. Hamburger Abendblatt, 2005a; Soon-Hee, 2004, p. 151). Horne und Manzenreiter (2004, p. 195) postulieren, dass Public Viewing in Japan nur in eingegrenzten erlaubten Zonen möglich war. Die anwesende Polizei soll verdutzt die jungen Japaner mit ihren „Nippon! Nippon!“-Rufen und typischen Verhaltensweisen siegreicher Fußballanhänger wie dem Fahneschwenkenden-auf-die-Straße-Laufen beobachtet haben. Horne und Manzenreiter (2007, p. 574) sehen in diesem Verhalten, „a symbolic battleground for a 'new Japan'“. Ähnlich wie in Deutschland 195415 und 2006 könnten die Fans eine seltene Gelegenheit genutzt haben, um das seit 1945 andauernde seltsame Gefühl beim Zeigen von nationaler Identität zu

15 Siehe hierzu Kap. 6.3 
entschärfen und zu überwinden. So habe der Fußball sogar die Macht im Scheinwerferlicht der Globalisierung als „indigenization, re-invention of tradition and creaolisation" weiterreichende soziale Funktionen zu erfüllen.

Die Beispiele der Ausricherländer Südkorea und Japan verdeutlichen sehr gut die vielschichtigen Beweggründe, die sich hinter dem Zusammenfinden der Zuschauer auf den Straßen verbergen können. Immer mehr Menschen möchten ihre Emotionen augenscheinlich unmittelbar (mit-)teilen und derartige Turniere nicht alleine zu Hause verfolgen (vgl. Habbel, 2005, S. 89), und das überall auf der Welt. Ein neuartiges Zuschauerverhalten bei internationalen Fußballspielen ist erkennbar. Wann genau allerdings die Abwanderung der Menschenmassen vom heimischen Fernsehsessel auf den Barhocker oder vor die Großbildleinwand auf dem Marktplatz begonnen hat, kann nicht eindeutig bestimmt werden. Denn erst während der WM 2002 wurden zum ersten Mal die Zahlen der 'öffentlichen Seher' ermittelt (vgl. FIFA, o.J.b; Infront Sports \& Media AG, 2002), die teilweise die Zuschauerzahlen im Stadion übertreffen (vgl. Schulke, 2006, S. 17).

\subsubsection{WM 2006: Die neue Qualität des Public Viewing}

"Out-of-home viewing for the FIFA World $\mathrm{Cup}^{\mathrm{TM}}$ has become a huge phenomenon" (Infront Sports \& Media AG, 2006).

Durch die große Kartennachfrage im Vorverkauf haben sowohl die Städte als auch Infront und FIFA rechtzeitig vor der Weltmeisterschaft in Deutschland das enorme Potential von TV-Übertragungen der Spiele erkannt (vgl. Schulke, 2006a, S. 19) (s. auch Kap. 8.1). So wurde Public Viewing erstmals offiziell vom Verband und den zwölf Ausrichterstädten organisiert ${ }^{16}$, und die Rechte für weitere Veranstaltungen wurden von Infront vermarktet. Erstmals wurde neben den üblichen Panel-Untersuchungen zur Akzeptanz der WM-Partien bei Fernsehzuschauern per Telefoninterview eine demoskopische Befragung durchgeführt. Das Institut Forsa wurde vom ZDF beauftragt, in fünf Wellen telefonisch zu ermitteln, wo das Publikum welche Spiele der deutschen Mannschaft an welchen Orten außer Haus verfolgt hat (vgl. Geese et al., 2006, S. 456). ${ }^{17}$

\footnotetext{
16 Die FIFA plant die Fan Feste für zukünttige Turniere als festen Veranstaltungsteil zu etablieren (vgl. Schulke, 2007, S. 27, 60-63).

17 Es wurden in jeder Befragungswelle mit je 1.000 repräsentativ ausgesuchten B efragten ab 14 Jahren computergestützte Telefoninterviews durchg eführt (vgl. ebd., S. 464).
} 
Tab. 2: Nutzung der Fernsehübertragung der Spiele der deutschen Mannschaft zur WM-2006 innerhalb und außerhalb der eigenen Wohnung. Verg leich der tele metrisch gemessenen Zuschauerzahl (durchschnittl. Sehbeteiligung) mit tele fonisch erhobenen Außer-Haus-Reichweiten (Tele metrische Daten-AGF/GfK, Fernsehpanel D+EU; tele fonisch erhobene Daten forsa CATI- Studie zur Außer-Haus-Nutzung, fünf Wellen à 1.000 repräsentativ ausgewählte Personen nach Geese et al., 2006, S. $457^{18}$

\begin{tabular}{||l|c|c|}
\hline & $\begin{array}{l}\text { Zuschauer ab 3 Jahren } \\
\text { im eigenen Haushalt } \\
\text { durchschnitt. } \\
\text { Sehbeteiligung in Mio. }\end{array}$ & $\begin{array}{l}\text { Erwachsene ab 14 } \\
\text { Jahren außerhalb des } \\
\text { eigenen Haushalts } \\
\text { Personen in Mio. }\end{array}$ \\
\hline $\begin{array}{l}\text { Deutschland - Costa Rica } \\
\text { Fr, 09.06.2006, 18 Uhr, ZDF }\end{array}$ & 20,06 & 12,09 \\
\hline $\begin{array}{l}\text { Deutschland - Polen } \\
\text { Mi, 14.06.2006, 21 Uhr, ARD }\end{array}$ & 23,88 & 10,71 \\
\hline $\begin{array}{l}\text { Deutschland - Ecuador } \\
\text { Di, 20.06.2006, 16 Uhr, ARD }\end{array}$ & 21,3 & 10,82 \\
\hline $\begin{array}{l}\text { Deutschland - Schweden } \\
\text { Sa, 24.06.2006, 17 Uhr, ZDF }\end{array}$ & 22,34 & 15,79 \\
\hline $\begin{array}{l}\text { Deutschland - Argentinien } \\
\text { Fr, 30.06.2006, 17 Uhr, ARD }\end{array}$ & 24,74 & 16,15 \\
\hline $\begin{array}{l}\text { Deutschland - Italien } \\
\text { Di, 04.07.2006, 21 Uhr, ZDF }\end{array}$ & 29,66 & 16,38 \\
\hline $\begin{array}{l}\text { Deutschland - Portugal } \\
\text { Sa, 08.07.2006, 20 Uhr, ARD }\end{array}$ & 23,97 & 16,93 \\
\hline
\end{tabular}

Die Spiele der deutschen Nationalelf gehören auch außer Haus zu den beliebtesten Partien. Es ist anzunehmen, dass die Reihenfolge dieser Begegnungen in der Beliebtheitsskala von der Attraktivität der Partien abhängt. Weitere Anhaltspunkte sind die Anstoßzeit der Begegnung sowie der Wochentag. Während bei den Spielen mit einer Anstoßzeit um 21 Uhr grundsätzlich höhere Zuschauerzahlen in den eigenen Wänden gemessen wurden als bei den früher angepfiffenen Begegnungen, geht die Außer-Haus-Nutzung für die späten Spiele tendenziell zurück. Des Weiteren scheint die Zeit und die Motivation, am Wochenende die Spiele außer Haus zu sehen, größer. In diesem Fall kann die Uhrzeit eine zweitrangige Rolle spielen. Denn für die Partie um den dritten Platz Deutschland gegen Portugal am Samstag, 8. Juli 2006, wurden trotz der Anpfiffzeit um 21 Uhr die meisten Besucher registriert. Die Erhebung ergab für dieses Spiel eine Außer-Haus-Nutzung von 16,93 Mio. Zuschauer ${ }^{19}$ (vgl. ebd., S. 457). Nach wie vor findet aber ein wesentlicher Teil der externen Fernsehnutzung

18 Wobei an dieser Stelle darauf hinzuweisen ist, dass sich die im telefonischen Interview erhobenen Daten nicht direkt mit denen der qualitativen Erhebung zum Fernsehkonsum vergleichen lassen. So sind wohl die meisten, die in der telefonischen Befragung angeben, zu einer Public Viewing Veranstaltung zu gehen, in der kontinuierlichen Fernsehforschung als 'Seher' einzurechnen. Die Anzahl dieser 'Seher' liegt meist deutlich über dem Wert der Zuschauerzahl, die für die 'durchschnittliche Sehbeteiliung' in der Paneluntersuchung genommen wird (vgl. ebd., S. 456).

19 Gewertet wurden Zuschauer ab 14 Jahren. 
nicht öffentlich statt, sondern bei Nachbarn, Freunden oder Verwandten. Von den knapp 17 Mio. Bürgern, die das 'kleine Finale' nicht am eigenen Gerät verfolgten, war ungefähr die Hälfte, nämlich etwa acht Mio., bei Anderen zu Besuch. ${ }^{20}$ Auf öffentliche Großbildleinwände entfielen ca. 4,3 Mio., auf gastronomische Einrichtungen ungefähr 3,2 Mio. Zuschauer. Demnach lässt sich sagen, dass das gemeinsame Sehen im privaten Rahmen auch bei der WM 2006 jenes im öffentlichen Raum noch immer mindestens aufwog (vgl. ebd., S. 457).

Verlässliche Angaben über die Höhe der Zunahme von Außer-Haus-Sehern können nicht gemacht werden, da für die vergangenen Europa- und Weltmeisterschaften keine genauen Daten vorliegen (s. Kap. 5.2.2). Geese et al. (2006, S. 458) wagen aber den Vergleich mit Blick auf das Gesamtereignis. Im Verlauf der WM 2006 haben danach 49\% des Publikums, das sind annähernd 30 Mio. Menschen, auch mal ein Spiel woanders, das heißt nicht im eigenen Haushalt, gesehen, während bei den vorherigen Turnieren, der Fußball-WM 2002 und der Europameisterschaft 2004, jeweils nur Werte von etwa 30\% erreicht wurden (vgl. auch Zubayr \& Gerhard, 2002, S. 309; Zubayr \& Gerhard, 2004a, S. 424).

20 Die Kategorie „Schrebergarten, Wochenendgrundstück“ (ca. 0,4 Mio.) wird hier hinzugezählt, wodurch die 50\%-Marke fast erreicht wird (vgl. ebd., S. 464). 
Tab. 3: Anteil der Personen mit Außer-Haus-Nutzung im Verlauf verschiedener Fußballturnie re „Wo haben Sie die Übertragung der WM/EM gesehen?“" (Angaben in \%) (Tele fonische Begleitu mfrage anlässlich der Fußball-WM 2002 (MMA Fran kfurt), Fußball-EM 2004 (forsa Berlin), und der Fußball-WM 2006 (IFA K Taunusstein) nach Geese et al., 2006, S. 458)

\begin{tabular}{|c|c|c|c|c|c|c|}
\hline $\begin{array}{l}\text { Basis: Personen die mind. ein } \\
\text { Spiel des Turniers gesehen } \\
\text { haben }\end{array}$ & & Geschlecht & & Alter & & \\
\hline Turnier & $\begin{array}{c}\text { BRD } \\
\text { gesamt }\end{array}$ & Frauen & Mănner & $14-29 \mathrm{~J}$. & $30-49 \mathrm{~J}$ & ab $50 \mathrm{~J}$. \\
\hline \multicolumn{7}{|l|}{ Fußball-WM 2002} \\
\hline Außer-Haus-Nutzung gesamt & 32 & 24 & 39 & 58 & 32 & 19 \\
\hline $\begin{array}{l}\text { Nutzung im eigenen Haushalt } \\
\text { und Außer-Haus-Nutzung }\end{array}$ & 29 & 22 & 35 & 52 & 29 & 17 \\
\hline exklusive Außer-Haus-Nutzung & 3 & 2 & 4 & 6 & 3 & 2 \\
\hline \multicolumn{7}{|l|}{ Fußball-EM 2004} \\
\hline Außer-Haus-Nutzung gesamt & 30 & 22 & 36 & 60 & 31 & 12 \\
\hline $\begin{array}{l}\text { Nutzung im eigenen Haushalt } \\
\text { und Außer-Haus-Nutzung }\end{array}$ & 27 & 19 & 33 & 54 & 27 & 11 \\
\hline exklusive Außer-Haus-Nutzung & 3 & 3 & 3 & 6 & 4 & 1 \\
\hline \multicolumn{7}{|l|}{ Fußball-WM 2006} \\
\hline Außer-Haus-Nutzung gesamt & 49 & 45 & 55 & 78 & 53 & 33 \\
\hline $\begin{array}{l}\text { Nutzung im eigenen Haushalt } \\
\text { und Außer-Haus-Nutzung }\end{array}$ & 44 & 41 & 49 & 67 & 47 & 32 \\
\hline exklusive Außer-Haus-Nutzung & 5 & 4 & 6 & 11 & 6 & 1 \\
\hline
\end{tabular}

Gut ersichtlich ist zunächst, dass der Wunsch, die Weltmeisterschaftspartien mit anderen zu erleben, besonders bei den 14 bis 29-jährigen ausgeprägt ist. Im Jahr 2006 bescheinigten fast $80 \%$ der jungen WM-Zuschauer, schon einmal ein Spiel extern verfolgt zu haben. Für $11 \%$ fand das Zuschauen sogar ausschließlich Außer-Haus statt (vgl. Geese et al., 2006, S. 458).

Bis jetzt konnten wir sehen, dass die Zahl der Menschen, die sich gemeinsam mit Freunden und Fremden vor Großleinwänden versammeln, um wichtige Fußballspiele zu verfolgen, generell zugenommen hat. Doch wo findet das 'gemeinsame Sehen' der Partien statt? 


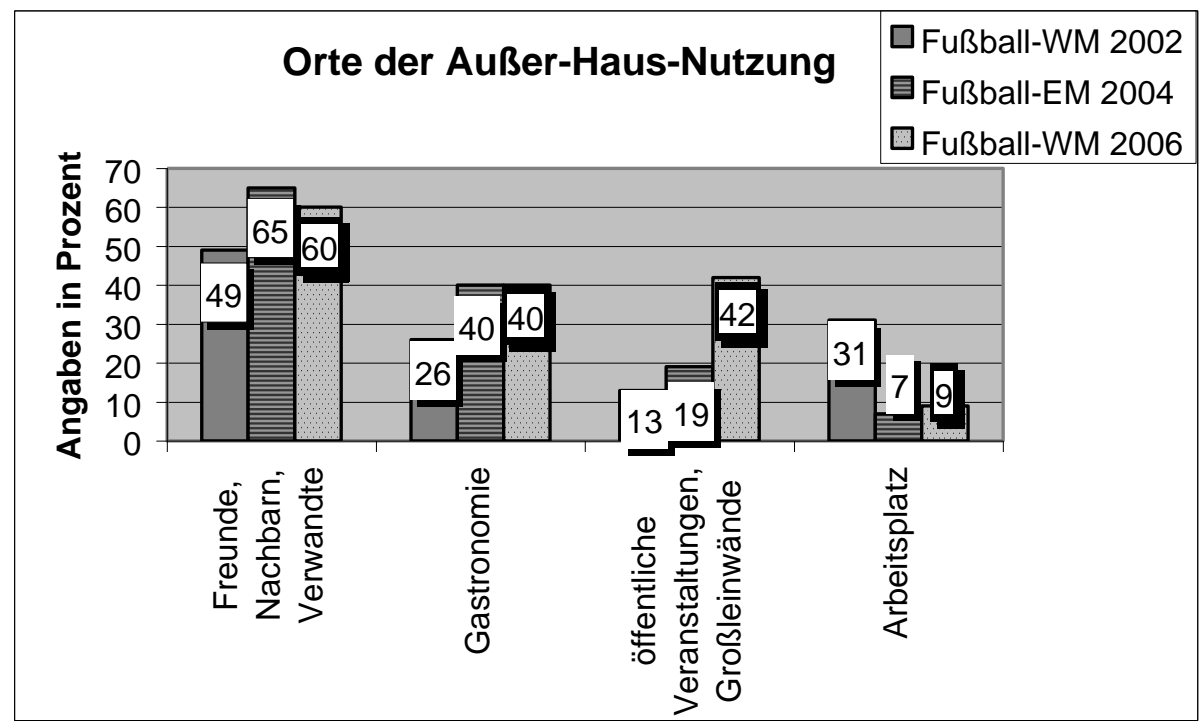

Abb. 4: Orte der Außer-Haus-Nutzung (Personen, die mindestens ein Spiel außerhalb der eigenen Wohnung gesehen haben) im Verlauf verschiedener Fußballturniere „Wo haben Sie die Übertragungen der WM/EM gesehen?" (Mehrfachantworten möglich) (Telefonis che Begle itu mfragen anlässlich der Fußball-WM 2002 (MMA Frankfurt), Fußball-EM 2004 (forsa Berlin), und der Fußball-WM 2006 (IFAK Taunusstein) nach

Geese et al., 2006, S. 459)

Es ist ersichtlich, dass die Orte des gemeinsamen Sehens einem Wechsel unterworfen sind. Während die Prozentwerte für Übertragungen in gastronomischen Einrichtungen von 2004 bis 2006 keine erkennbaren Änderungen zeigen, verzeichnet das "öffentliche Sehen"21 auffallend steigende Werte. Bei der WM in Japan und Korea im Jahr 2002 nutzten nur 13\% die Möglichkeiten des Public Viewing. Allerdings sahen $31 \%$ die Spiele am Arbeitsplatz, was eindeutig mit den durch die Zeitverschiebung bedingten frühen Anstoßzeiten zusammenhängt (vgl. ebd., S. 459). Diese Zahlen decken sich in der Tendenz mit einer Umfrage der Mediaedge: CIA (2002). ${ }^{22}$ In dieser Untersuchung gaben $78,5 \%$ an, die Spiele allein oder mit der Familie zu Hause anzusehen. Die Werte für das Zuschauen im Lokal lagen bei 9,5\% und die Zahlen für das gemeinsame Gucken an öffentlichen Orten sogar nur bei 1,9\%. Die ZDFMedienforschung (zit.n. Geese \& Gerhard, 2008, S.447) fand für die EM 2008 heraus, dass bei allen Spielen der Anteil der Außer-Haus-Seher, die das Spiel bei Freunden, Verwandten oder Bekannten sehen, am größten ist. Der nächst geringere Anteil sah sich zumeist die Begegnung in einer gastronomischen

21 Mit 'öffentlichem Sehen' sind all jene Veranstaltungen gemeint, bei denen sich das Publikum im öffentlichen Raum zusammenfindet. Geese et al. (2006, S. 459) stellen aber die begründete Vermutung an, dass die Befragten vermutlich Biergärten zu dieser Kategorie gezählt haben, obwohl diese eigentlich in den Bereich Gastronomie gehören würden.

22 Die Agentur befragte 1.672 Personen zu ihrem Fernsehverhalten hinsichtlich der WM 2002. 
Einrichtung an, auch bei den Spielen der deutschen Mannschaft. Bei den K.o.Rundenspielen der deutschen Mannschaft allerdings lag das gemeinsame Verfolgen der Spiele auf öffentlichen Plätzen an zweiter Stelle. Allgemein nach der beliebtesten Form der Außer-Haus-Nutzung gefragt, geben 56,3\% an, dass sie Spiele bei Freunden, Bekannten oder Verwandten sehen, dann folgen die gastronomischen Einrichtungen mit 39,8\%. Das Verfolgen der Spiele auf öffentlichen Plätzen geben nur $36,2 \%$ an. ${ }^{23}$

Aber nicht nur in Deutschland sahen viele Menschen die Spiele außer Haus. Das Finale ihrer Mannschaft gegen Frankreich sahen 200.000 Italiener im antiken Circus Maximus in Rom auf drei großen Leinwänden. Auch in Mailand sahen 150.000 Fans den Sieg der Squadra Azzurra gegen die Equipe Tricolore auf dem Piazza del Duomo (vgl. Infront Sports \& Media AG, 2006).

Resümierend kann festgehalten werden, dass der Besuch von Public ViewingVeranstaltungen von verschiedenen Faktoren wie bspw. den Mannschaften, der Uhrzeit und von den gegebenen Möglichkeiten abhängt. Public Viewing hat zu der WM 2006 in Deutschland definitiv eine neue Qualität entwickelt. Trotz des großen Zulaufs zum 'öffentlichen Sehen' der Übertragungen der Spiele, ist die Rezeption im Verhältnis zum Sehen im privaten Raum als ausgeglichen anzusehen. Das Publikum zwischen 14- und 29 Jahren scheint das öffentliche Schauen am attraktivsten zu finden.

23 Geese et al. (2006, S. 459) geben zu bedenken, dass es zunächst geeignete Lokalitäten geben muss, in denen die Spiele außerhalb des privaten Rahmens zu verfolgen sind. Es hätte, so argumentieren sie, vielleicht bei den Turnieren vor 2006 mehr Außer-Haus-Seher geben, wenn diese mehr Möglichkeiten gehabt hätten. 


\subsection{Zusammenfassung}

Das Ziel dieses Kapitels war es, eine erste Grundlage für die weiteren Ausführungen zu schaffen. Der Begriff Public Viewing ist für diese Ausarbeitung auf öffentliche TV-Vorführungen von sportlichen Großereignissen außerhalb der Privatsphäre von Familie und privaten Gästen mit einer Bildschirmdiagonale größer als drei Meter definiert worden. Das Interesse, Fußball Welt- und Europameisterschaften im Fernsehen zu verfolgen, steigt in vielen Staaten, so auch in der Bundesrepublik sukzessive an. Dabei tritt ein sich veränderndes Sehverhalten zutage; die WM 2002 in Südkorea und Japan kann als Schlüsselereignis gelten. Viele Personen ziehen das gemeinsame Schauen der Spiele auf einer Videowand dem heimischen Fernsehsessel vor, vor allem in den Ausrichterländern, aber auch im Rest der Welt. Öffentliche Stellen und Medien in Südkorea haben auf dieses Verhalten unterstützend eingewirkt. Zur Weltmeisterschaft im Jahr 2006 ist eine neue Qualität des Public Viewing festzustellen. Erstmals wurden im Ausrichterland Deutschland offiziell organisierte Public Viewing-Veranstaltungen eingerichtet und das Zuschauerverhalten diesbezüglich evaluiert. Der Zulauf zu den öffentlichen Fernsehübertragungen der WM-Spiele scheint demnach bspw. von demographischen Faktoren wie dem Alter, der Attraktivität der Begegnung oder alternativen Veranstaltungen abhängig zu sein. 


\section{Modernisierungsprozesse in Gesellschaft, (Fußball-)Sport und}

\section{Fernsehen}

In diesem Teil der Arbeit wird der Frage nachgegangen, inwiefern soziale Prozesse in der postmodernen Gesellschaft die Entwicklung des Sports mitgetragen haben, inwieweit sie Motor sind für neue Formen der sportlichen Unterhaltung, und was es mit der Sonderstellung des Fußballs auf sich hat. An dieser Stelle wird ein weiterer Grundstein für die Analyse des Public Viewing gelegt. Aus dem vorhergehenden fünften Kapitel ist ersichtlich, dass das Interesse an der Übertragung von internationalen Fußballturnieren stetig wächst und sich das Sehverhalten in den öffentlichen Raum verlagert. Es wird hier die Annahme verfolgt, dass gesellschaftliche Rahmenbedingungen einen großen Anteil an der Entstehung des Public Viewing tragen. Individualisierungs- und Pluralisierungstendenzen sowie die Hinwendung zu hedonistischen Werten in der postmodernen Gesellschaft machen den Sport zu einem geeigneten sozialen Projektionsfeld (6.1). Dies führt in einem weiteren Schritt zur Veränderung des Sports und seines Umfeldes. Zu letzterem gehören in zunehmendem Maße die Massenmedien und insbesondere das Fernsehen. Sport und TV stehen in einem wechselseitigen Verhältnis (6.2). Das Sportspiel Fußball nimmt in diesem Konglomerat eine hervorgehobene Position ein (6.3). Abschließend erfolgen ein Resümee der Erkenntnisse und die Einordnung dieser in den Kontext der Arbeit (6.4). 


\subsection{Postmoderne Gesellschaft: Wertewandel, Individualisierungs- und Pluralisierungstendenzen}

Public Viewing wird in dieser Ausarbeitung als eine Erscheinung betrachtet, die ihren Ursprung z.T. in der sich wandelnden Gesellschaft hat. Ziel dieses Abschnittes ist es, die sozialen Voraussetzungen für die Entwicklung des Phänomens zu diskutieren. In diesem Sinne werden im Folgenden die Diskurse des Wertewandels, der Individualisierung und Pluralisierung eruiert und in ihrer Bedeutung für die Beliebheit des gemeinsamen Fernsehens erörtert.

Bei einem Blick in die wissenschaftliche Literatur erhält man den Eindruck, dass Individuen in westlichen hoch entwickelten Gesellschaften in einer Varianz von Sozialsystemen zu leben scheinen. Bezeichnungen wie Leistungsgesellschaft, Wohlstandsgesellschaft, Risikogesellschaft, Mediengesellschaft, Erlebnisgesellschaft oder eben postmoderne Gesellschaft sind nur einige der anzutreffenden Schlüsselbegriffe. Sie beziehen sich jeweils auf verschiedene Schwerpunkte und Blickwinkel, haben aber den gleichen Aussagekern (vgl. Göring, 2006, S. 168; Heinemann, 2007, S. 317f.). Diese „Gesellschaftsdiagnosen" (Göring, 2006, S. 156) versuchen die Wandlungsprozesse unserer Gesellschaft zu beschreiben. ${ }^{24}$ Die Vertreter der Postmoderne versuchen diesen Entwicklungen gerecht zu werden und gehen z.B. davon aus, dass es nicht eine rationale Theorie zur Erklärung der Verhältnisse gibt, sondern dass eine Vielfalt nebeneinander existierender Perspektiven besteht. Das heißt, dass die Wirklichkeit nur annäherungsweise erfasst und interpretiert werden kann (vgl. Ritzer, 1997, p. 3). So bieten postmoderne Theorien keine rationalen Lösungen für gesellschaftliche Probleme an, wie es bspw. Theoretiker der Moderne versuchen (vgl. ebd., p. 6). Sie gehen vielmehr davon aus, dass durch die Individualisierungstendenzen häufiger Emergenz auftritt. Sawyer (2005, p. 193) schlägt deshalb vor, dass Erforschen von sozialer Emergenz immer auf auf drei gleichberechtigten Ebenen stattfinden sollte: Individuen, die Kommunikation zwischen diesen und makrosoziologischen Gruppendynamiken.

\footnotetext{
24 Grundlegende Literatur zum Wertewandel, seinen Bedingungen und der Entwicklung find et sich neben den Klassikern von Inglehart (1977) und Klages (1984) u.a. bei Horx und Opaschowski (2006; 2008). Der Umschwung der US-amerikanischen Gesellschaft wird z.B. von Godbey (1993) erörtert. Weiterführende Informationen zu soziologischen Gegenwartsmodellen finden sich u.a. in Beck (1997), Göring (2006) und Schulze (1997).
} 
Zentrales Element und gemeinsamer Nenner der zahlreichen gesellschaftichen Modernisierungsmodelle ist der schrittweise Umschwung von traditionellen Handlungsweisen, Mustern und Strukturen hin zu teilweise völlig neuen Sinnzusammenhängen. Die sozialen Werte wandeln sich. Traditionell vorhandene Orientierungswerte wie Klassen, Schichten und soziale Gruppen mit ihren vorgegebenen Rollenstrukturen und Verhaltensnormen verblassen. Ein jedes Individuum steht für sich allein, es muß sich seine eigenen, individuellen Strukturen schaffen. Pluralisierungstendenzen beschreiben die Auflockerung der historisch gewachsenen Glaubens- und Sozialstrukturzwänge und die damit einhergehende Auflockerung des Zusammenlebens und des GemeinschaftshandeIns (vgl. Vester, Oertzen, Geiling, Hermann und Müller, 1993, S. $38 f$.). ${ }^{25}$ Das heißt allerdings nicht, dass die alteingebürgerten Gesetzmäßigkeiten gänzlich ihren Wert verlieren, es erfolgt vielmehr eine Bedeutungsübertragung. Vielfach, z.B. von Gebauer (2002, S. 166), Heinemann (2007, S. 321), Keupp (1995, S. 50) oder Schulze (1997, S. 17) wird postuliert, dass das soziale Miteinander keine verbindenden Werte mehr hat und keine besonderen Körpereigenschaften. Die Orientierungsmöglichkeiten an sozialen Gruppen wie Alter, Geschlecht oder die Zugehörigkeit zu einem bestimmten Beruf schwinden, und Instanzen wie Tradition, Familie und Beruf verlieren allmählich ihre sinnstiftende Funktion.

Der Einzelne ist also, als Individuum gezwungen, sich eigene Strukturen zu suchen bzw. zu schaffen:

„Die Individualisierungstheorie beschreibt und analysiert den tiefgreifenden Strukturwandel moderner Gesellschaften als einen, der sich in der Auflösung traditioneller Klassen- und Sozialbindungen zeigt und dabei dem Individuum weit mehr Freiheiten und Aufgaben als früher zumutet“" Beck, 1997, S. 261).

Es wird vielerorts die Auffassung vertreten, dass der Einzelne im Rahmen der Individualisierung die Möglichkeit verliert, sich selbst auf der Grundlage von allgemein gültigen Modellen sinnstiftender Wirklichkeitskonstruktionen zu definieren (vgl. Bette, 1993, S. 36). Verhaltensweisen, ebenso wie Werte und Einstellungen von Einzelpersonen scheinen in zunehmendem Ausmaß auf

25 Eine interessante Weiterentwicklung wäre an dieser Stelle der Diskurs der Pluralisierung von Lebensstilen, der u.a. von Bordieu und Vester geprägt wurde (vgl. Spellerberg, 1996, S. 54). Um eine stringete Argumentation nicht zu gefährden, soll es an dieser Stelle aber lediglich bei dieser Anmerkung bleiben. 
autonomen Entscheidungen der Individuen zu basieren (vgl. Friedrichs, 1998, S. 13). Diese Entwicklung führt dazu, dass das Individuum in starkem Maße selbst für seine eigene Identitätsstiftung sorgen muss. Für diesen Zweck können nun entweder alte Sinnzusammenhänge, wie die Religiosität (vgl. Opaschowski, 2008, S. 572) eine neue Wertigkeit erfahren oder sich neue Wertvorstellungen als identitätsstiftend erweisen, wie z.B. die eigene Selbstverwirklichung (vgl. Keupp, 1995, S. 50).

Da liegt es nahe, Orientierung in einem Sozialsystem zu suchen, in dem es noch wahrnehmbare Kategorien und Strukturen gibt (vgl. Guttmann, 1986, S. 177; Beck, 1997, S. 140; Digel \& Burk, 2001, S. 23). Der Sport, als eine „Eigenwelt“ (Güldenpfennig, 1996, S. 39), aber auch wiederum als ein Spiegel der Gesellschaft, ist bereits im antiken Griechenland immer auch soziale Darstellung gewesen. Es wird vielfach betont, dass bereits damals sportliche Wettkämpfe ebenso wie Theateraufführungen soziale Darstellungen, in denen Gemeinsamkeit gebildet und in regelmäßiger Wiederholung bekräftigt wurde, waren. Nach Gebauer (2002, S. 158-166) sind die Olympischen Spiele somit nicht nur ein Teil der griechischen Kultur, sondern auch ein wichtige Quelle der sozialen und nationalen Repräsentation. ${ }^{26}$ Die sich regelmäßig wiederholenden sportlichen Wettkämpfe sind ein wichtiges Mittel, um Gemeinschaft fühlbar und sichtbar zu machen.

Marsh und Morris (1989, p. 113) sprechen dem Sport, insbesondere dem sportlichen Spektakel, auch heute noch einen rituellen Status zu. In diesem Sinne kann Sport sogar als religiöser Ersatz gesehen werden. Sie berufen sich auf die Anthropologin Cheska, die in den modernen sportlichen Ritualen ähnliche Komponenten wie in alten Stammeskulturen erkennt. Genannt werden können hier die regelmäßige Wiederkehr, die Regelförmigkeit, die Dramatik und Symbolik der sportlichen Rituale. Sie strukturieren, sie stereotypisieren, und sie wiederholen. Der moderne Sport selbst ist ein großes Ritual, dem der Einzelne in sozial unsicheren Situationen gerne folgt (vgl. Krüger, 1990, S. 141).

"The dream lived within the rituals and ceremonies of football fandom cannot be anything other than an illusion, a self-collusive infatuation, of disassociated individuals living in late modern society" (Manzenreiter \& Horne, 2007, p. 575).

Erinnern wir uns an das schwarz-rot-goldene Farbenmeer während des WM-

26 Zumindest für all jene, die ein minimales Interesse am Sport besitzen (vgl. ebd., S. 162). 
Sommers 2006. Haben wir nicht alle durch das Tragen eines Trikots, durch das Schwenken einer Fahne oder Ähnlichem einen kleinen Beitrag zum postulierten 'gesunden Patriotismus' geleistet? Oder darf dieses 'Nach-außen-Tragen' der Gefühle gar nicht ausschließlich in diesen rituellen Rahmen gepresst werden? Zeigt sich nicht vielmehr die in der Postmoderne so typische Multikausalität? Vielleicht präsentiert sich hier an dieser Stelle vielmehr der anfangs bereits angedeutete Wertewandel: Die Fußball-Weltmeisterschaft 2006 als riesiges Straßenfest, ein Spaß für jedermann?

\begin{tabular}{|c|c|}
\hline Traditionelle Arbeitstugenden & Neue Freizeitwerte \\
\hline Zielsetzungen & Zielsetzungen \\
\hline $\begin{array}{l}\text { `Leistung, Erfolg, Ehrgeiz } \\
\text { `Besitz, Eigentum, Vermorgen }\end{array}$ & 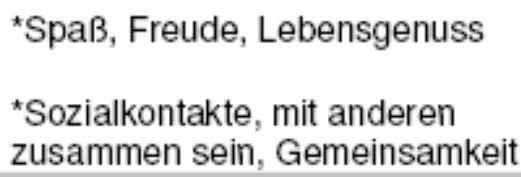 \\
\hline Făhigkeiten & Făhigkeiten \\
\hline${ }^{\star}$ Fleiß, Ehrgeiz & ${ }^{*}$ selbst machen, selbst aktiv sein \\
\hline *Disziplin, Gehorsam & `Spontanităt, Selbstentfaltung \\
\hline${ }^{\text {*Pflichterfällung, Ordnung }}$ & ${ }^{*}$ sich entspannen, wohlfühlen \\
\hline
\end{tabular}

Abb. 5: Traditionelle Arbeitswerte und neue Freizeitwerte (in Anlehnung an Becker, 2000, S. 12)

Die grundlegende Aussage, der eher plastisch gehaltenen Abb. 5 ist kaum von der Hand zu weisen: Der Stellenwert der Arbeit mit den dazugehörigen Werten geht zugunsten der Freizeit zurück. Allgemein gesagt: Die Person, das Individuum an sich rückt mehr in den Vordergrund, während die Gemeinschaft in den Hintergrund gerät (vgl. Schlicht \& Strauss, 2003, S. 122). Godbey (1993, p. 52) sieht in diesem Prozess die Grundlage für die Identitätssuche, welche sich im Streben nach Erlebnissen und Erfahrungen ausdrückt. Beck (1997, S. 18) fasst diese Begriffe zusammen in „immaterielle Gesichtspunkte der Lebensqualität”, zu denen auch der Spaß und das Zusammensein-mit-Freunden gehört. 27

Den Gedanken der verschiedenen Gesellschaftsmodelle wieder aufnehmend, kann der Blick auf den Sport und seine Phänomene nur aus der jeweiligen

27 Es sei an dieser Stelle darauf hingewiesen, dass Abb.8 bewusst eine Vereinfachung der gesellschaftlichen Tendenzen darstellt. Der Eventisierung konträre Entwicklungen, wie sie bspw. Opaschowski (2008, S. 566-615; 640-650) beschreibt und grafisch zusammenfasst (2008, S. 589) sind durchaus auch geläufig. In diesen Sozialentwürfen bilden gerade die verlorengeglaubten und -gegangenen Strukturen wie Familie und Tradition, genauso wie die Werte von Zufriedenheit, Glück, Ehrlichkeit, Zuverlässigkeit etc. das Ziel. 
Perspektive heraus erfolgen (vgl. Digel, 1995, S. 20; Heinemann, 2007, S. 317f.). Für unseren Zusammenhang ist es wichtig, Public Viewing mit seinen Akteuren in den gesellschaftlichen Kontext einzuordnen. So lässt sich festhalten, dass das Individuum in der Postmoderne einem Mangel an Sozialstruktur ausgesetzt ist, welches es in Eigenleistung auszugleichen versucht. Der Sport mit seinen gesetzmäßigen Ritualen kann einen Beitrag dazu leisten.

\subsection{Sport und Fernsehen}

Die gesamtgesellschaftlichen Entwicklungen haben Einfluss auf den Sport und die Medien, insbesondere auf das Fernsehen. Nach Bette (1993, S. 34-39) und Digel (1995, S. 25) erzeugt insbesondere der Individualisierungsprozess die sozialstrukturellen und individualpsychologischen Voraussetzungen für einen Boom des Sportmarktes. Der soziale Wandel sorgt für den Bedeutungszuwachs und die gestiegene gesellschaftliche Wertschätzung des Sports und des sportiven Körpers. Selber aktiv Sport zu treiben, gehört für viele Menschen in den Alltag. ${ }^{28}$ Es soll an dieser Stelle darum gehen, den Zusammenhang zwischen gesellschaftlicher Modernisierung, dem Sport und dem Massenmedium Fernsehen zu durchleuchten, um aus diesen Erkenntnissen Rückschlüsse auf die Entstehung des Public Viewing zu ziehen.

Ein Sozialsystem, welches Schichten, Geschlechter und Altersgruppen erfasst, kann als Verbindung für Menschen unterschiedlicher Gruppierungen funktionieren und den stärkeren Bedürfnissen nach einer bewussten Freizeit- und Erlebnisgestaltung Rechnung tragen (vgl. Rittner \& Breuer, 2002, S. xiv). Die massive Hinwendung $\mathrm{zu}$ einem körperbetonten Handlungsfeld, welches unmittelbare Wirksamkeitserfahrungen und Ich-Erlebnisse verspricht, ist eine

\footnotetext{
28 Die Bestandserhebung des Deutschen Olympischen Sportbundes (DOSB) von 2008 (vgl. Deutscher Olympischer Sportbund, 2008, S. 3, 14f.) verzeichnet insgesamt über 27 Mio. Mitglieder in den einzelnen Mitgliedsorganisationen. Im Alter zwischen 19 und 26 Jahren sind bspw. 42,3\% der männlichen und $24,1 \%$ der weiblichen Bevölkerung in den alten Bundesländern in einem der Mitglieds vereine des DOSB organisiert Zudem kann davon ausgegangen werden, das die Zahl der nicht im Verein organisierten Sportler noch um ein Vielfaches höher liegt. Allerdings gilt es hier nach Opaschowski (2001, S. 82f.) kritisch zu hinterfragen, ob der Mitgliederstatus wirklich Rückschlüsse über die Sportlichkeit der deutschen Bevölkerung zulässt. So vermutet er, dass das Sporttreiben der Deutschen bei weitem überschätzt wird. Diese Diskussion soll aber an dieser Stelle nicht vertieft werden.
} 
mögliche Folge ${ }^{29}$ Dabei kann nur in bedingtem Maße von der direkten eigenen Sportausübung gesprochen werden. Vielmehr ist damit eben auch der indirekte Einfluss, vom Tragen von Sportkleidung im Alltag hin zu sportiven Aussagen in Werbespots gemeint, die der Sport in der postmodernen Gesellschaft hat. Individuen, die in angestammten Rollen und Traditionen keinen Halt und keine Orientierung mehr finden, lenken die Aufmerksamkeit auf den Körper und das 'Sich-Erfahren', als Zeichen einer aktiven Selbstgestaltung und -inszenierung auf der Suche nach der eigenen Identiät. Wer auf sich selbst zurückgeworfen ist, nutzt folgerichtig auch verstärkt seinen Körper für eine Stabilisierung des eigenen Ichs. Im Sport lässt sich Identität 'erarbeiten'. Wer an seiner Physis ${ }^{30}$ und seinem Äußeren arbeitet, der macht sich und seinen Körper zu einem Symbol des Sports. Die Personen machen sich den Gesundheits- und Leistungscharakter des Sports zunutze, um ihre Identität darzustellen. Das Feld des Sports eigne sich eben in besonderer Weise zur Darstellung von Identität und Distinktion ${ }^{31}$ (vgl. Bette, 1993, S. 39f.; Gebauer, 2002, S. 34).

Aber nicht nur derjenige, der selber aktiv ist benutzt den Sport zur Identitätsstiftung (vgl. Schulke, 2006a, S. 20). Auch die Zuschauer von Sportveranstaltungen benutzen den Sport und die aktiven Sportler zur Orientierung in der Gesellschaft. Hierzu gibt es in der wissenschaftlichen Literatur eine Reihe von divergierenden Theorien. Im Folgenden werden exemplarisch zweierlei Gedankengänge erörtert. Gebauer (2002, S. 2, 157) oder auch Schulke (2006, S. 21) sehen den Sport als Abbild der Gesellschaft. Das Theater als Vergleich zu Hilfe nehmend, wird der Sport als ein soziales Feld beschrieben, welches die zentralen Themen moderner Gesellschaften behandelt. Im Gegensatz zum Theater oder Film-Genres, denen ebenfalls eine gesellschaftliche Spiegelfunktion unterstellt werden kann- besitzt der Sport kein Skript. Er ist nicht vorhersehbar. Sport ist nicht intellektuell abgehoben, sondern er ist für alle verständlich. Man ist nicht bloß Zuschauer, der in den dunklen Rängen sitzt, sondern man befindet sich mitten im Jetzt und Hier, im Diesseits und im Alltag.

\footnotetext{
29 Diese Sehnsucht wird besonders im Zusammenhang mit dem erlebnisorientierten Menschenund Gesellschaftsbild beschrieben (s. Kap.10).

30 Die Physis ist das zentrale Thema des Sports. Sie ist ein gesellschaftlich gelernter und akzeptierter Ort des Affekt- und Emotionsauslebens (s. Kap. 9).

31 Distinktion deshalb, weil auch die Abgrenzung, die Differenzierung von anderen einen gewichtigen Teil der eigenen Identitätsfindung ausmacht.
} 
Auch die bereits erwähnte Ritualfunktion des Sports kann hier angeführt werden. Gleich (2001, S. 178) weist darauf hin, dass sportliche Großereignisse, die von den Medien entsprechend inszeniert werden, die Qualität moderner Rituale oder Zeremonien annehmen. Die modernen Massenmedien scheinen folglich eine wichtige Funktion zu haben. Diese sind, wie der Sport, Mittler, die die Beziehungen zwischen den Mitgliedern einer Gesellschaft erzeugen. Sie verbinden nicht nur als Partner einer Mannschaft oder eines Teams, sie trennen sie auch als Kontrahenten und ermöglichen eine Teilnahme für alle als Publikum (vgl. Gebauer, 2002, S. 161).

In einem weiteren Ansatz sieht Gebauer (2002, S. 163ff.) in der sozialen Motorik einen möglichen Grund für die unbestreitbar stattfindende Identifikation mit Spitzensportlern. Demnach stellt die Bewegungslehre die Teilhabe des Einzelnen an der Gesellschaft dar. So übernimmt beispielsweise das Neugeborene die Motorik seiner Eltern, wie der Nachwuchsathlet die bevorzugte Technik seines Trainers nachahmt. Daher führen junge Menschen durch Vorbilder erlernte Bewegungen als Gemeinsamkeiten im Sinne der sozialen Motorik fort. Für den Sportzuschauer verbindet sich die eigene Motorik mit den Bildern der Athleten. Sportler können also als ein exemplarischer Teil einer Kultur angesehen werden. Die eigene Gesellschaft wird durch sie widergespiegelt durch die Gemeinsamkeit der Bewegungen. Spitzenathleten repräsentieren demnach auf der einen Seite jeden Einzelnen, aber auch die Kultur in ihrer Gesamtheit.

Letztere ist das Gemeinsame an diesen Ansätzen, und dies bildet auch die Grundlage der Diskussion: Sport leistet, so ist betont worden, durch die Möglichkeiten der Identifikation und Repräsentation sowie seiner Ritualfunktion einen wichtigen Beitrag für die Identitätsbildung in hoch entwickelten, postmodernen Gesellschaften. Besonders im Rahmen von internationalen Sportgroßveranstaltungen fällt dies auf; Einzelne, die sich vorher nicht besonders für Sport, eine bestimmte Disziplin und schon gar nicht für einen einzelnen Sportler interessiert haben, identifizieren sich und ihre Nation plötzlich mit dem Topathleten des Turniers (vgl. ebd., S. 156-167). Demnach kann durchaus angenommen werden, dass sich „eine Gesellschaft, die sich bei ihrer symbolischen Selbstkonstitution zusieht und dieses Spektakel genießt, (...) sich ihrer selbst [vergewissert]“ (ebd., S. 167). Der Sport scheint folglich auf mehreren Ebenen mit dem sich vollziehenden sozialen Wandel in Beziehung zu stehen. 
Bette (1993, S. 49) oder auch Digel und Burk (2001) sehen in der steigenden Individualität sogar einen Motor, mit dem der Sport einen großen Teil seiner sachlichen, sozialen und zeitlichen Variationen produziert. Digel (1995, S. 14) und Heinemann (2007, S. 327) gehen zunächst davon aus, dass sich die Entwicklungen des Sports anhand der genannten Prozesse mindestens beschreiben lassen.

Jedoch sind es nicht nur die sozialen Prozesse und gesamtgesellschaftlichen Entwicklungen, die Einfluss auf die Entwicklung des Sports haben. Prozesse der Mediatisierung $^{32}$ stehen ebenso in Wechselwirkung mit diesen Abläufen. Eine nahezu symbiotische Verbindung besteht zwischen den Medien und dem Sport.

"Sport begleitet die Medienevolution: Mediengeschichte ist immer auch SportMediengeschichte" (Loosen, 2004, S. 16).

Der Sport ist unabdingbar mit der Evolution der Medien verbunden. Seit der Einführung des dualen Rundfunksystems in Deutschland im Jahr 1984 stieg die Zahl der dem Sport gewidmeten Stunden der Berichterstattung in den öffentlichrechtlichen und in den privatwirtschaftlichen Fernsehanstalten kontinuierlich an (vgl. Digel \& Burk, 1999, S. 22). Besonders der Spitzensport „erweist sich (...) [dabei] als Dauerproduzent von berichtenswerten Ereignissen für die Massenmedien" (Riedl, 2006, S. 64).

Sportgroßveranstaltungen wie die Fußball-Weltmeisterschaften, Olympische Spiele, aber auch das Lokalderby in der Fußball Bundesliga können als Medienereignisse beschrieben werden, die eine große Zahl an Zuschauern anziehen. Nach Cybulska (2007, S. 23) sind Medienereignisse Ereignisse, bei denen es „zu einem Zusammenwirken zweier Ereignisse [kommt]. Auf der einen Seite gibt es ein Ereignis, das eintrifft; und auf einer weiteren Ebene ein Ereignis, das dieses zeigt". Rivenburgh (2003, p. 31) definiert Media Events als "a unique media genre that results when television's visual and narrative power taps into public fascination with a story that transcends daily experience" ${ }^{33}$ Es treffen an dieser Stelle folglich zwei wichtige Determinanten zusammen: ein großes Ereignis,

32 Eine gute Zusammenfassung über Begriff und Inhalt der Mediatisierung für den Sport findet sich z.B. bei Bernstein und Blain (2003), Dohle und Vowe (2006) oder Krüger (1993).

33 Die weiterreichendere Begriffsbestimmung der 'Väter' der Media Events, Dayan und Katz (1994), werden an dieser Stelle noch nicht genannt, um die Stringenz der Argumentation nicht zu gefährden. In Kap. 9.4 wird darauf zurückgekommen. 
welches an sich bereits Interesse weckt, und als zusätzlicher Faktor ein weiteres Ereignis, welches die Massenmedien -zumeist das Fernsehen- produzieren.

Kehren wir aber zunächst zu den Sportveranstaltungen zurück. Ihre große Reichweite ist es, welche die Massenmedien primär interessiert. Natürlich hat vor allem das Fernsehen vorrangig finanzielle Verwertungsinteressen im Blick, was die Auswahl der übertragenen Sportarten stark begrenzt. Nichtsdestotrotz beschaffen paradoxerweise auch die Massenmedien dem Sport das Publikum. Sie weiten den Zuschauerkreis auf die Fernsehrezipienten aus. Dieser Kreislauf ist es, der beiden Systemen in der Verbindung gute Entwicklungsmöglichkeiten bietet.

„Sportereignisse genießen als rituelle Medienereignisse in der Medienlandschaft eine Sonderstellung und gehören zu den quotenstärksten Formaten im deutschen Ferns ehen" (Cybulska, 2007, S. 34).

So werden TV-Stationen nicht müde, zu jeder wichtigen Spitzensportveranstaltung neue mediale Technologien anzubieten, um die Attraktivität des Fernsehsports weiter zu erhöhen und die Telegenisierung von Sportereignissen weiter voran zu treiben (vgl. Schauerte \& Schwier, 2004, S. 164).

\footnotetext{
"[T]echnical innovations are symbolically staged and popularized mainly in the context of sports events-whether they be new image technologies like slow motion replay or 'virtual replay', or broadcast and recording technologies such as cable and satellite TV after 1984, the introduction of DVD and PVR" (Keilbach \& Stauff, 2006, p. 165).
}

Die fortwährende Entwicklung von technischen Neuheiten wie der Vergrößerung der Bildschirme, Slow-Motion oder optische Unterstützungen der Torentfernung haben das Erlebnis, zu Hause Sportberichterstattungen zu verfolgen, auf eine neue Stufe gehoben (vgl. Schulke, 2006, S. 19) (vgl. auch Kap. 8).

So hat Schwier (2002, S. 2) mit Blick auf das Public Viewing Recht, wenn er postuliert:

"Der Sport und die Massenmedien gehören gegenwärtig sowohl zu den
ökonomischen Wachstumsbranchen als auch zu den einflussreichsten kulturellen
Kräften unserer Zeit und weisen darüber hinaus äußerst velfältige
Wechselbeziehungen auf, die eine Verschmelzung beider Phänomene zu einem
einheitlichen Produktionskomplex schon in näherer Zukunft möglich erscheinen lässt."

Die Medien haben ihren Anteil dazu beigetragen, dass sich der Fernsehsport und besonders der Fernsehfußball zu einer eigenen Unterhaltungssparte entwickelt haben. Der Prozess der funktionalen Ausdifferenzierung in der Medienlandschaft (vgl. Digel \& Burk, 2001, S. 20) lässt sich z.B. an der variableren Form der Berichterstattung oder der räumlichen Vielfalt (s. Kap. 8) beobachten. Mit Vor- und 
Nachberichten, Interviews usw. ebenso wie mit der Möglichkeit, strittige Szenen zu wiederholen und heranzuzoomen, sind die TV-Sender in der Lage, die Übertragung von Fußballspielen im Vergleich mit einem Besuch im Stadion nicht als zweitrangig dastehen zu lassen. Dem visuellen Medium Fernsehen ist es möglich, die bereits vorhandenen Inszenierungen des Sports aufzugreifen und zu verstärken (vgl. Cybulska, 2007, S. 38). Die Sportberichterstattung hat sich, wie bereits Krüger (1993, S. 28f.) anführte, vor allem im Fernsehen geändert. Loosen (2001, S. 137) bezeichnet diese als „Hybrid aus Information und Unterhaltung“, welches sich einer klaren Einreihung in die Facetten Unterhaltung und Information entzieht. Gleich (2000, S. 512; 2001, S. 170f.) beschreibt die Sportberichterattung als weit über das sportliche Ereignis hinausgehend. Scherer (2004, S. 218) betont, dass diese Tendenzen aber keinesfalls einseitig ausgelegt sind, ebenso unterstützen die Ausrichter von Sportveranstaltungen die Bemühungen der Medien um Dramatisierung, Emotionalisierung und Spannungssteigerung. Dayan und Katz (2001, S. 413) gehen sogar so weit, TV-Übertragungen als „funktionales Äquivalent“ im Vergleich mit dem Live-Ereignis anzusehen.

\begin{abstract}
„Das Fernsehen überlagert die performance der Organisatoren mit seiner eigenen performance, es präsentiert seine Stellungnahme den Stellungnahmen der Zuschauerinnen und Zuschauer und es bietet stellvertretende Formen der Teilnahme an, um die Zuschauerinnen [spectators] und Zuschauer für die ihnen vorenthaltene Teilnahme zu entschädigen. Fernsehen wird so zum Hauptdarsteller [primary performer] bei der Ausführung öffentlicher Zeremonien“ (Dayan \& Katz, 2001, S. 413f.).
\end{abstract}

Dayan und Katz (2001, S. 414) gehen bei ihren Ausführungen davon aus, dass die Performance der Ereignisse im Fernsehen im Sinne einer "qualitativen Umwandlung der Beschaffenheit öffentlicher Ereignisse“ gesehen werden kann. Es wird hervorgehoben, dass das Fernsehen mit seiner eigenen Rhetorik den Versuch unternimmt, einen Ersatz oder eine Alternative für das Live-Erlebnis zu sein.

Es gilt also festzuhalten: Bezug nehmend auf das Phänomen Public Viewing ist hier ein wichtiger Ansatz zu sehen. Vor dem Hintergrund der gesellschaftlichen Modernisierungsprozesse erscheinen der Sport und seine Berichterstattung im Fernsehen als ein geeignetes Projektionsfeld für Identifikations-, Repräsentationsund Distinktionsentwicklungen. Insbesondere internationale sportliche Großereignisse bieten aufgrund der fortgeschrittenen Übertragunstechnik und der dramaturgisch ausgerichteten Sportberichterstattung gute Vorraussetzungen für 
die Inszenierung als Medienereignisse. Neben dem eigentlichen Ereignis produziert das Fernsehen durch eigene Anreicherung, z.B. durch die Integration der Zuschauer, ein weiteres mediales Ereignis. In diesem Sinne stellt Public Viewing eine neue Form von Medienereignis und somit eine neue Stufe in der Sport-Medienentwicklung dar.

\subsection{Sonderstellung des Fußballs}

In dieser Arbeit werden viele Beispiele und Ansätze aus dem Bereich des Fußballs stammen. Dies hat den einfachen Grund, dass Public Viewing zum ersten Mal bei einer Fußball-Veranstaltung in großem Maße 'aufgetreten' ist, nämlich bei der WM 2002 in Südkorea und Japan. Darüber hinaus ist Public Viewing bei der FußballWeltmeisterschaft 2006 erstmalig in das organisatorische Gesamtkonzept aufgenommen worden (s. Kap. 5). Dieses soll keine Beschränkung auf den Bereich des Fußballs darstellen. Allerdings darf und kann nicht außer Acht gelassen werden, dass von dieser Ballsportart und seinen Akteuren die wichtigen Impulse zur Entstehung des Phänomens Public Viewing ausgingen, so dass der Fußball eine hervorgehobene Rolle spielen muss. Demnach ist die Sonderrolle des Fußballsports im Zusammenspiel zwischen gesellschaftlichen Modernisierungsprozessen und dem Massenmedium Fernsehen zu eruieren. Was hat es aber mit der besonderen Stellung des Fußballs auf sich? Warum konnte sich Public Viewing gerade in diesem Umfeld entwickeln? Das sind die Fragen, deren Beantwortung sich der folgende Abschnitt widmen wird. Unter den vielen Sportarten, die es auf der Welt gibt, spielt der Fußball einen ausne hmend auffälligen Part. Um es nach Feldenkirchen (2006) zu sagen: Fußball ist längst kein beliebiges Ballspiel mehr, er hat seinen gesellschaftlichen Stellenwert verändert. Er selbst -der Fußball- könne nichts dafür, er sei im Wesentlichen der alte geblieben, so Feldenkirchen, sondern die Menschen haben sich rund um das Spiel verändert. Dieser Ballsport ist der populärste Sport unserer Zeit. In Deutschland sind etwa 40 Mio. Menschen an Fußball interessiert, was einem Prozentsatz von 60 der über 14-Jährigen entspricht. Auch im übrigen Europa und in Südamerika, ebenso wie in Afrika und Asien, ist der Fußballsport das beliebteste Freizeitvergnügen (vgl. Bausenwein, 1995, S. 11; Kurscheidt, 2004, S. 2). 
Der genaue Ursprung des Spiels ist nicht bekannt. Ein wichtiger Aspekt aber sind die sich über den gesamten Globus verteilenden Vorläufer. Frühe Varianten des beliebten Sports wurden im dritten Jahrtausend vor Christi Geburt in China, im zweiten Jahrtausend vor Christus in Mittelamerika und später in Japan, Italien und Spanien praktiziert. ${ }^{34}$ Fußball ist in all seinen Formen bereits ein global verbreitetes Spiel gewesen, als es seine entscheidende Entwicklung in England und Schottland nahm (vgl. FIFA, o.J.e). So ist eine der frühesten Erwähnungen einer Handschrift von 1175 zu entnehmen (vgl. Marsh \& Morris, 1989, p. 118). Im 14. Jahrhundert erfreute sich nach Ellias und Dunning (1966 zit.n. Schmidt, 2002, S. 15; Schulke, 2006, S. 21) ein Spiel Namens Hurling ${ }^{35}$, eine Mischung aus Fußball, Rugby und Handball, großer Beliebtheit. Dieser von zwei Parteien auf freiem Feld organisierte Wettstreit half -vor dem sozialen Hintergrund des Mittelalters gesehen-, Konflikte zwischen niederen sozialen Gruppen und Ortschaften körperbetont auszutragen. Der Fußball fand hierzulande zunächst kaum Anklang. Die Obrigkeit und Vertreter der Kultus- und Schulbehörden bevorzugten das militärisch anmutende Turnen und verachteten den spielerischen Wettstreit. Während Reformer -hervorzuheben sei hier der Braunschweiger Pädagoge Konrad Koch- die englische Spielform einführten, warnten Konservative vor den negativen Einflüssen des Fußballs. Dieser stellte ihrer Meinung nach eine Gefahr für das Gemeinschafts- und Vaterlandsbewusstsein dar. Wenig später freilich trat der Fußballsport seinen Siegeszug an, der bis heute andauert (vgl. Schmidt, 2002, S. 16; Murray, 1996, p. 25f.). ${ }^{36}$

Bereits in der Weimarer Republik entwickelte sich der Fußball zu einem

\footnotetext{
34 Da die Darlegung und Diskussion der Erkenntnisse um die Entstehung des Sportspiels für den Verlauf dieser Arbeit nicht von herausragendem Interesse ist, wird im Folgenden nur kurz darauf eingegangen. Näheres über die historische Entwicklung des Fußballs kann z.B. in Bausenwein, (1995), Brinker (2004), König (2002), Marsh und Morris (1989) und Murray (1996) nachgelesen werden.

35 Hurling wird noch heute gespielt. Die schnelle Mannschaftssportart ist vor allem in Irland beliebt. 36 Einen nicht unwichtigen Part trägt der 1904 in Paris gegründete Weltfußballverband. In 100 Jahren ist aus der wagemutigen Idee einiger Fußballliebhaber, ein mächtiges Organ des internationalen Fußballs geworden (vgl. Eisenberg et al., 2004, S. 9, 57). Die FIFA achtet stets darauf, ihren Einfluss weiter auszubauen. Mit Rücksichtsnahme auf neue Märkte verschob die FIFA die Anzahl der Plätze von den angestammten Fußballgebieten Südamerika und Europa zu Gunsten der Verbände Asiens, Mittel- und Nordamerikas plus Karibik sowie Ozeaniens. Um den bislang noch wenig erschlossenen Fußballmärkten gerecht zu werden, hat sich auch die Vergabestrategie für das Endturnier geändert. Der traditionelle Wechsel der Ausrichterrolle zwischen Europa und Südamerika wurde erstmals 1994 mit der Festlegung auf die USA durchbrochen. Nach den Ländern Südkorea und Japan als Organisatoren für die Weltmeisterschaft im Jahr 2002, wird das Turnier 2010 in Südafrika stattfinden. Zukünftig erfolgt die Ausschreibung nach einem regionalen Rotationsprinzip (vgl. Kurscheidt, 2004, S. 3ff.).
} 
Massenereignis bei Aktiven und Zuschauern (vgl. Pyta, 2005, S. 7-30; Schulke, 2006, S. 22).

\begin{abstract}
"Massensport das heißt heute: Zweiundzwanzig spielen Fußball, Tausende und Zehntausende sehen zu. Sie stehen um das Spielfeld herum, kritisieren, johlen, pfeifen, geben ihr sachverständiges Urteil ab, feuern Spieler an, bejubeln ihre Lieblinge, beklatschen einzelne Leistungen, reißen den Schiedsrichter herunter, fanatisieren sich, spielen innerlich mit. Sie verfallen der Fußballpsychose, und sie benehmen sich auf dem Sportplatz, als hinge nicht nur ihr eigenes Wohl und Wehe, sonder das Wohl und Wehe der ganzen Welt von dem Ausgang dieses lumpigen Fußballspiels ab“ (Helmut Wagner im Jahr 1931 zit.n. Pilz, 2002, S. 59).
\end{abstract}

Aber warum ist gerade der Fußball das „erstaunlichste(n) Massenphänomen der Moderne“ (Bausenwein, 1995,S. 11)? ${ }^{37}$ Nun, es kann ohne Zweifel angenommen werden, dass der Erfolg des Fußballs auf mehr basiert als bloß auf dem Spiel an sich. Dieser Sport spiegelt mit seiner Dramatik unser Leben wider. Nach Weinhold (2002, S. 34f.) können wir uns an die Höhepunkte des Spiels erinnern, weil in ihnen die zentralen Inhalte des Wettkampfs, also Siegen und Verlieren, Anerkennung von Leistung, Teamgeist usw. sichtbar werden. Die Identifikation mit hervorragenden Athleten, das Mitfühlen ihrer Tränen nach einem verlorenen Spiel, genauso wie die Freude nach einem Sieg sind symbolhafte Handlungen unseres eigenen Lebens. Zudem sorgt nicht zuletzt die Unvorhersehbarkeit des Ausgangs für echte Emotionen und somit für mehr Realismus als bspw. im Theater oder Kino. Der moderne Fußball(-Sport) mit seinen Ritualen, Inszenierungen, Betrugsversuchen usw. ist jederzeit authentisch, öffentlich und unmittelbar (vgl. Schulke, 2006, S. 21). Diese Bilder machen die Begeisterung der Akteure und der Zuschauer aus. Besonders anonymer werdende Gesellschaften brauchen, so wird vielfach betont, Orte, an denen die Kunst, das Leben zu meistern, symbolhaft dargestellt wird, wie es eben im antiken Griechenland bereits der Fall war. Demnach kann im Spiel mit dem eigenen und dem gegnerischen Körper, das sich durch Dribbeln, Schießen und Täuschen darstellt, ein Rollenkampf gesehen werden. Die Angriffs- und Abwehrspieler symbolisieren ein Charakterspiel zwischen 'guten' und 'bösen' Spielern, welches für die Fans das Leben in vereinfachter bildhafter Form darstellt (vgl. Gebauer, 2002, S. 172). Marsh und Morris (1989, p. 112) heben zudem hervor, dass in den weltweit populären Massensportarten sich die Merkmale wieder finden, die einst für

37 Im Folgenden sollen beispielhaft einzelne in der wissenschaftlichen Literatur fort während wiederkehrende Argumente und Positionen weiterführend erörtert werden. Ein Anspruch auf Vollständigkeit kann nicht erhoben werden und erscheint auch nicht zweckmäßig. 
Jagdgesellschaften wichtig waren. Schnelligkeit und Wendigkeit, koordinative Fähigkeiten, Kraft, Zähigkeit und Treffsicherheit sind Eigenschaften, die hier in den Augenschein fallen.

Dem könnte entgegengehalten werden, dass dieses ja bei jeder Sportart möglich ist, dennoch scheint das Sportspiel Fußball besonders zu sein. Murray (1996, p. 174) streicht bspw. die Förderlichkeit des Teamsports gegenüber Individualdisziplinen bzgl. der Identifikation heraus. Mannschaften bestehen im Gegensatz zu Einzelathleten über Generationen hinweg. So besitzt eine Familie meist Jahrzehnte lang Dauerkarten für einen Verein, während sich die jüngere Generation nicht mehr mit dem inzwischen nicht mehr aktiven Lieblingsindividualsportler des Vaters identifizieren kann. 38

Welchen wechselseitigen Einfluss Sport und Fernsehen aufeinander haben, ist bereits erörtert worden. So ist es nicht verwunderlich, dass die Ausstrahlungsquantität sowie -qualität eines Sports im Fernsehen oftmals als Argument genannt wird. Schließlich trägt das TV einen nicht unerheblichen Anteil an der Beliebtheit des Fußballs. Die hohe Telegenität dieses Sportspiels sichert seine umfassende Bedeutung und weit reichende Verbreitung (vgl. Gebauer, 2002, S. 175). Ballsportarten, wie vor allem Fußball, aber auch Basketball oder Handball lassen sich ideal im Fernsehen zuschauergerecht umsetzen. Durch die physische Einheit von Ort, Handlung und Zeit lässt sich der Ablauf eines Fußballspiels ohne größere dramaturgische Eingriffe des Fernsehens auf eine spannende Art abbilden. Bei vielen anderen Sportarten ist dies nicht der Fall. Bei einer Reihe greift das Fernsehen ein und stelt einen linearen und somit zuschauerfreundlichen Ablauf eines sportlichen Wettbewerbs erst her, wie z.B. bei den Wintersportarten Biathlon und Skilanglauf. Oft muss der Moderator durch erklärende Eingriffe den Ablauf und die Entscheidungen einer Sportart aufbereiten, wie beim Fechten. Fußball versteht der Zuschauer auch direkt meist ohne Erklärungen (vgl. Gerhard, 2006, S. 472; 2006a, S. 45).

Als eine weitere Begründung für die herausragende Stellung des Fußballs wird das in Europa herrschende Überangebot an Fernsehfußball angeführt. Nach Eisenberg, Lanfranchi, Mason und Wahl (2004, S. 267) stehen den

\footnotetext{
38 Bezogen auf diese Thematik ist die Rolle der Vereine besonders im Ruhrgebiet mit Sicherheit eine eigene Arbeit wert. Weitergehende Ausführungen finden sich bei Pyta (2004) oder Breitmeier (2004).
} 
Endverbrauchern diesbezüglich mehr als 250 Kanäle zur Verfügung. Auf diese Weise werden sogar diejenigen zu Kennern des Spiels, die sich eigentlich überhaupt nicht dafür interessieren. Wann man auch immer durch die Kanäle schaltet, immer ist auf einem Sender eine Tabelle mit den Spielergebnissen des Wochenendes oder ein Interview mit einem Spieler oder Trainer zu sehen. Gerhard (2006a, S. 44) hält dagegen fest, dass selbst in den 'Fußballjahren' jeweils zu den Welt- und Europameisterschaften der Anteil bei der LiveÜbertragungen bei der ARD nur 0,9\% und selbst beim Deutschen Sportfernsehen (DSF) nur bei 2,4\% liegt.

Aus den genannten Gründen lässt sich schlussfolgern, dass es offenbar die Summe der Details ist, die die Sonderstellung des Fußballs ausmacht. Ein weiteres vielfach genanntes Argument für die große Bedeutung des Sportspiels ist die sich quer durch alle Bevölkerungsschichten und Landesteile ziehende Beliebtheit. Denn auch im Handball oder Eishockey wird die gleiche lebensechte Dramatik erzeugt, nur sind diese Sportspiele zumindest hierzulande ausschließlich regional bedeutsam. Für den Fußball dagegen begeistern sich neben den Profis eine Masse an Freizeitkickern und Millionen von Menschen, die passiv an diesem Sport teilhaben (vgl. Messing und Lames, 1996, S. 26; Rahmann, Weber, Groening, Kurscheidt, Napp \& Pauli, 1998, S. 63). Schiffer (2004, S. 15) geht sogar so weit, dass er den Fußball zu einem „das Leben prägenden gesamtkulturellen Phänomen“ emporhebt, welches als einziges Klassen und Nationen kulturell vereint. Gebauer (2002, S. 172) hingegen mahnt zur Vorsicht, weil Mädchen und Frauen im Normalfall zu viel geringeren Anteilen fußballinteressiert seien. Nach Rühle (2003, S. 218) kann differenziert werden in Sportarten, die polarisieren, und solche, die nicht polarisieren. Fußball gehört zu denjenigen, die weitgehend unabhängig von Alter und Geschlecht der Zuschauer ein breites Publikum ansprechen. Bertling und Eggers (2004, S. 202-205), Gebauer (2002, S. 177ff.) oder Murray (1996, p. 201) schreiben, dass der Fußball seinen Status als schichtübergreifendes kulturelles Phänomen jedoch erst durch seine Akzeptanz bei den Eliten in den 1970er Jahren erlangte. Diese äußert sich zum einen dadurch, dass es heute für viele Politiker, Manager und Schauspieler 'zum guten Ton' gehört, sich auf der Ehrentribüne oder in der VIP-Loge ihres 
Heimatklubs sehen zu lassen ${ }^{39}$ und zum anderen anhand der wissenschaftlichen Auseinandersetzung mit dem Thema.

Eine Besonderheit stellt der Gewinn des Weltmeistertitels 1954 durch die deutsche Nationalmannschaft dar. Der sportliche Sieg über die Ungarn im Finale von Bern wurde für die gesamte Bevölkerung zum politischen Symbol des Wiederaufbaus, sowohl des kriegszerstörten Landes als auch des deutschen Selbstbewusstseins. Das „Wunder von Bern“ sei nach Pyta (2004, S. 7, 29) als Stilisierung, als Ausdruck einer Symbolpolitik zu begreifen, aus der sich als letzte Konsequenz der Gründungsmythos der Bundesrepublik ergibt. Die deutsche Bevölkerung hätte neun Jahre nach Kriegsende nach neuen identitätsstiftenden Symbolen gesucht, die durch den Glauben an die starke D-Mark zum Einen und durch die Leistungsfähigkeit der sozialen Marktwirtschaft zum Anderen befriedigt wurden. Mit letzterem korrespondiere das „Wunder von Bern“, weil es durch die Verzahnung mit dem Wirtschaftswunder Platz in der kollektiven Erinnerung eines ganzen Volkes gefunden hat (vgl. Breitmeier, 2004, S. 142). Breitmeier (2004, S. 143-147) geht sogar so weit, dass er die Feierlichkeiten zum Erfolg der Weltmeisterschaft 1954 als Gründungsmythos der BRD ausbaut. Die damalige Regierung hätte die Gelegenheit genutzt, negativ besetzte nationale Symbole wie das Berliner Olympiastadion, die Nationalhymne oder auch die, allerdings neue, Nationalflagge mit dem positiven Sieg der Arbeiterelf im gesamten Volk zu etablieren. Die genannten Aspekte sorgen letztlich dafür, dass der Gewinn der Fußball Weltmeisterschaft 1954 sich bei fast allen Bevölkerungsteilen weit über die Zeit hinaus als symbolischer Erinnerungsort in das kollektive Gedächtnis gebrannt hat.

Feldenkirchen (2006) beschreibt diese Vorgänge aus heutiger Sicht wie folgt;

„Er [der Fußball] ist einer der letzten Kleber für eine auseinander fallende Gesellschaft, der letzte gemeinsame Nenner von Hartz-IV-Empfängern, Investmentbankern und Intellektuellen, vor allem während der Weltmeisterschaft. Er lässt die Grenzen sozialer Herkunft für die Dauer eines Turniers verschwinden, er sorgt für zeitlich befristeten Zusammenhalt."

\footnotetext{
39 Vor allem im südlichen Europa und in Lateinamerika gibt es eine andere Repräsentationskultur. Die Verbindung von sportlichen Begegnungen mit kirchlichen Riten und Handlungen lässt Feste entstehen, an denen sich die nationalen Eliten selbstverständlich beteiligen. In diesen Ländern wird Fußball längst als nationale Repräs entation angesehen (vgl. Gebauer, 2002, S. 178).
} 
Es ist deutlich geworden, dass es schwer ist, dem 'Mythos Fußball' 40 gerecht zu werden. So könnten die angeführten Argumente problemlos um die Erfolgsgeschichte des Fußballs im deutschen Fernsehen oder die Markenstärke des Fußballs erweitert werden. Eine möglichst vollständige Diskussion kann aber hier nicht zweckdienlich sein. Vielleicht lässt sich die Massenaffinität des modernen Fußballs schlicht mit dem folgenden Zitat zusammenfassen:

„Es ist eines der Paradoxien der Postmoderne, dass diese Individualisierung am besten in einem Massensport erfolgen kann" (Krüger, 1999, S. 1228).

In diesem Kapitel ist das Ziel verfolgt worden, einige wichtige Aspekte, die ihren Teil zum Verständnis beitragen können, zu erörtern. Gesagt werden kann zunächst, dass die Gefahren, die z.B. Gebauer (2002, S. 185) als Folge der Professionalisierung für die Identifikation und Repräsentation durch die Spieler sieht, für die Mehrzahl nicht zu greifen scheinen. Die Annahme, dass die Kommerzialisierung des Spitzensports und insbesondere des Fußballs die Identifikations- und Repräsentationsfunktion der Akteure mindert, bewahrheitet sich nicht. Wie Feldenkrichen (2006) betont, raube das moderne Fußballgeschäft den Anhängern die Möglichkeit sich zu identifizieren, aber dennoch scheine das Bedürfnis nach Fußball unaufhaltsam. An dieser Stelle haben allerdings die Medien eine wichtige Vermittlerrolle eingenommen. Sie verstehen es, fortwährende Spannung zwischen Nähe und Distanz sowie zwischen Sportler und Fan zu erzeugen, die den Starkult aufrechterhalten. Dieses veränderte Verhältnis macht den Showcharakter des modernen Fußballs aus (vgl. Pilz, 2002, S. 61). Eisenberg et al. (2004, S. 267ff.) gehen sogar davon aus, dass gerade dieses sportliche Entertainment, was u.a. durch begleitende Reportagen, Hintergrundinformationen oder Interviews unterstützt wird, zur Bildung oder Festigung des sozialen Netzes moderner Gesellschaften beitragen kann, indem nämlich das medial vermittelte Wissen, z.B. durch Computeranimation der Abseitsregel, in der sozialen Gemeinschaft thematisiert werden kann.

Resümierend lassen sich die anfangs aufgestellten Fragen wie folgt beantworten. Für die hervorgehobene Position des Fußballs muss das Zusammenspiel einer Vielzahl an Faktoren verantwortlich gemacht werden. Anführen lassen sich Determinanten von der bereits in der frühen Entwicklungshistorie angesiedelten

40 Weiteres zum Mythos Fußball ist z.B. nachzulesen in Breimeier (2004, S. 129f.) und Pyta (2004, S. 6). 
weltweiten Verbreitung bis zu den Repräsentations - und Identifikationskonstrukten oder der Telegenität der Sportart. Die Beantwortung der zweiten Frage, die da lautete, warum sich Public Viewing gerade in diesem Umfeld entwickeln konnte, kann nur angedeutet werden. So ist offensichtlich ein großes, möglichst in der Bevölkerung breit angelegtes Interesse nötig, um die notwendige Zuschaue rzahl zu generieren.

\subsection{Zusammenfassung}

Durch gesellschaftliche Modernisierungsprozesse ist der Einzelne im gesteigerten Maße gezwungen, sich individuelle Sozialstrukturen aufzubauen. Im Zusammenspiel mit dem Wertewandel und den durch diesen hervorgerufenen Bedeutungszuwachs an hedonistischen Freizeitwerten kann der körperorientierte Sport eine neue Bezugsquelle darstellen. In anonymer werdenden individualisierten Gesellschaften kann vor allem die Identifikation mit den und die Repräsentation durch die Athleten dem individuellen Sozialprofil Struktur geben. Besonders bei internationalen Sportgroßveranstaltungen repräsentieren die Top-Athleten jeden EinzeInen, aber auch die gesamte Nation und rufen ein Zusammengehörigkeitsgefühl hervor. Eine starke Identifikation kann demnach z.B. mit den Nationalmannschaften, weil diese im Vergleich zu Individualsportlern fortwährend eine Möglichkeit der Repräsentation bieten, erfolgen. Eine herausragende Bedeutung für die Befriedigung dieser Bedürfnisse kommt in Deutschland, wie in vielen anderen Ländern dem Nationalsport Fußball zu. Die hervorgehobene Position, die das Sportspiel innehat, ist nur aus einem multifaktoriellen Begründungszusammenhang zu erklären. Faktoren wie die weltweite Verbreitung oder die Telegenität des Fußballs haben diesen zu einem Massenphänomen gemacht. Mit den modernen Massenmedien und insbesondere mit dem Fernsehen steht der Sport in einer symbiotischen Beziehung. Diese hat u.a. zu fortwährendem technologischem Fortschritt bei den Übertragungsmedien und einer sich verändernden Sportberichterstattung geführt. Sportgroßveranstaltungen werden vom Fernsehen als eigene Ereignisse auf anderer Ebene produziert. Mit Hilfe von zusätzlichen narrativen Mitteln wird das Fernsehen zum Hauptakteur der Gattung des Medienereignisses. Public Viewing wird als eine neue Form von Medienereignis und somit eine neue Stufe in der Sport-Medienentwicklung verstanden. 


\section{Publikum}

In diesem Teil geht es darum, die Grundlagen um die Komponente des Publikums zu erweitern und damit das Fundament der Analyse des Phänomens Public Viewing zu festigen. Das Publikum spielt für das Phänomen Public Viewing eine unübersehbar wichtige Rolle. Denn es ist kein Zusatz wie bei anderen Sportveranstaltungen, die zum sportlichen Vergleich stattfinden. Beim Public Viewing spielt das Publikum die Hauptrolle; ohne Zuschauer würde es kein Public Viewing geben, und niemand würde auf die Idee kommen, Großleinwände aufzustellen. Um das Phänomen Public Viewing diskutieren zu können, ist es also erforderlich, die Verbindung zwischen dem Sport und seinem Publikum zu analysieren. Obwohl es bereits seit den ersten Sportereignissen immer Zuschauer oder Unterstützer der Athleten gab, scheinen bei besonderen Begebenheiten extrem viele Menschen zu Zuschauern oder besser, zu einem Teil des Publikums zu werden. Außerordentlich auffällig ist dieses Phänomen während der FußballWeltmeisterschaften 2002 und 2006 zu beobachten gewesen.

Ein erster Schritt zur Klärung dieser Beobachtung wird mit der Strukturierung des extrem heterogenen Sportpublikums unternommen. Dabei steht nicht die vollständige Klassifizierung im Fokus, sondern es geht vielmehr um die Verdeutlichung der Existenz von unterschiedlichen Zuschauerarten (7.1). Im zweiten Schritt wird erörtert, wie sich die Publikumsbildung und -bindung determiniert. Zu diesem Zweck muss zunächst die Bedeutung des Sports für das (Medien-)Publikum diskutiert werden. Für dieses Anliegen liefert die Arbeit von Riedl (2006) den nötigen strukturellen Hintergrund. Auch hier kann es nicht darum gehen, einen möglichst vollständigen Überblick über die Motive und Bedürfnisse von Individuen zu geben, sich Sport anzusehen. Stattdessen werden wesentliche Forschungslinien mit ihren zentralen Aussagen aufgegriffen und für die Erklärung des Phänomens Public Viewing genutzt (7.2). Abschließend erfolgt die Zusammenfassung der Erkenntnisse dieses Kapitels (7.3). 


\subsection{Publikumsstruktur}

„Wie schon bei den [Fußball-]Weltmeisterschaften 1998 und 2002 war die Zahl der weiblichen Zuschauer auch bei der WM im eigenen Land [gemeint ist Deutschland] überdurchschnittlich hoch. Interessierte sich während der letzten Bundesligasaison ein Drittel der weiblichen Zuschauer für Fußball, waren es bei den Livespielen der Fußball-Weltmeisterschaft 43 Prozent" (Geese, Zeughardt \& Gerhard, 2006, S. 454).

Dieses Zitat verdeutlicht die außergewöhnliche Zuschauerstruktur, die bei internationalen Großereignissen des Sports anzutreffen ist. Bei Veranstaltungen, wie Weltmeisterschaften beliebter Sportarten oder Olympischen Spielen, scheinen mehr Menschen die Athleten zu unterstützen, als es im normalen Ligabetrieb oder bei weniger wichtigen Wettkämpfen der Fall ist. Diese müssen andere Motive oder Beweggründe haben, sich eine Veranstaltung anzusehen, als diejenigen, die langjährige Fans einer Mannschaft oder eines Vereins sind. Gerade beim Public Viewing zu den Fußball-Weltmeisterschaften 2002 und 2006 fallen vor allem die vielen weiblichen Besucher auf, die sich den Fans angeschlossen haben.

An dieser Stelle ist es daher sinnvoll, sich mit dem Publikum zu beschäftigen. Denn augenscheinlich ist dieses ein wichtiger Bestandteil des Public Viewing. In der wissenschaftlichen Literatur herrscht diesbezüglich ein Begriffswirrwarr. Einen Zuschauer definieren Tedeschi, Madi und Lyakhovitzky (1998, S. 94) als

\footnotetext{
,jemanden, der eine bewusste (häufig mit einigen Kosten verbundene) Entscheidung trifft, ein Ereignis zu beobachten, das zu dem Zweck organisiert wird, ein Publikum entstehen zu lassen."
}

Wichtig ist in diesem Fall die Freiwilligkeit des Zuschauens. Der Einzelne trifft also eigenständig die Entscheidung für den Besuch der Sportveranstaltung. So lassen sich bereits am Anfang Zeugen eines Verbrechens oder Unfalls als Zuschauer ausschließen. Denn sie sind nicht aus eigener Motivation bei dem Ereignis anwesend, und sie haben keine bewusste Entscheidung getroffen (vgl. Tedeschi et al.., 1998, S. 94), sondern sind zufällig anwesend gewesen, als ein Ereignis eingetreten ist. Bei Strauß und Jürgensen (1998) findet sich ein interessanter Zuschaueransatz, der auf die Intendierung bzw. Nicht-Intendierung des Ereignisses abzielt. Sie differenzieren den Besucher nach der vorhandenen bzw. nicht-vorhandenen Absicht des Besuchs. Des Weiteren sehen sie Zuschauer als „nicht wesentliche[n] Bestandteil des eigentlichen Geschehens". Das heißt, der Besucher ist nur ein Beobachter von außen, er scheint hier nicht in das Geschehen inbegriffen und kann auch nicht einwirken. Wie bei obigem Zitat von 
Tedeschi et al. (1998, S. 94) deutlich wird, trifft der einzelne Zuschauer die bewusste Entscheidung, einem Ereignis beizuwohnen, welches u.a. dazu initiiert wurde, ein Publikum entstehen zu lassen. Der Begriff Publikum bestimmt also

\begin{abstract}
„Zuschauermengen, die sich zu einer bestimmten Zeit an einem bestimmten Ort befinden - zum anderen aber auch massenmediale Publika - d.h. viele Zuschauer, die sich zur gleichen Zeit an verschiedenen Orten aufhalten" (vgl. Strauß \& Jürgensen, 1998, S. 18).
\end{abstract}

An dieser Stelle werden folglich die einzelnen Zuschauer zu einem Publikum zusammengeführt. Dieses wird aber wiederum in das Publikum vor Ort und das disperse massenmediale Publikum unterschieden.

In der englischsprachigen Literatur wird oftmals auch die Bezeichnung 'audience' für die Zuschauer oder das Publikum verwendet. Da dessen Verwendung aber dem Verständnis des Publikumbegriffs in dieser Arbeit ähnelt, wird die deutsche Umschreibung vorgezogen. Entscheidend ist hier jedoch nicht das Heischen nach der vermeintlich richtigen Begrifflichkeit, wie es in einigen Werken verfolgt wird, sondern eine Festlegung der Auffassung, als was in dieser Ausarbeitung das Publikum verstanden werden wird. Zu diesem Zwecke lassen sich der 'Uses-andGratification-Approach' und der 'Selective-Exposure-Ansatz' aus der Medienforschung heranziehen. Der Kern des 'Uses-and-Gratification-Approach' ist die Vorstellung von einem aktiven Publikum. Der Zuschauer verfolgt mit dem Sehen einer Fernsehübertragung ein Ziel, er selektiert und bewertet die Nützlichkeit seines Tuns durch die Wahl, die er trifft, eben diese Sendung zu sehen oder nicht (vgl. Bösch \& Borutta, 2006a, S. 28; Zillmann \& Bryant, 1998, S. 197). Der Einzelne, so wird angenommen, ist kognitiv motiviert, verschiedene Medieninhalte zu bestimmten Zeiten zu sehen bzw. nicht zu sehen, um seine sozialen und psychischen Bedürfnisse zu befriedigen (vgl. Raney, 2004, p. 51). Die Auswahl für ein bestimmtes Programm fällt zwischen den von den Rezipienten gesuchten Gratifikationen und jenen Gratifikationen, die sich die Zuschauer von der ausgestrahlten Sendung versprechen. Die Entscheidung fällt für das Programm, bei dem diese Diskrepanz am geringsten ist (vgl. Döhle, Klimmt \& Schramm, 2006, S. 53).

Diesem kognitiven Ansatz steht das Modell der 'Selective-Exposure' gegenüber. Dieser geht davon aus, dass sich Zuschauer zwar explizit für das Sehen einer Sportübertragung entscheiden, aber sie das zu Sehende bewerten mit Mögen, Gleichgültigkeit oder Nicht-Mögen; dies geschieht aus „spontanen hedonistischen 
Launen“ (Zillmann \& Bryant, 1998, S. 199) heraus. Raney (2004, p. 50) fügt dem hinzu, dass Individuen "tend to selectively expose themselves to media content that is presumed to be (for the most part) consistent with their existing attitudes, beliefs, and thoughts".

Eine Verbindung dieser beiden Ansätze ist das dynamisch-transaktionale Model von Früh (1991). Dies ist weder kommunikator- noch rezipientenorientiert, stattdessen schreibt es dem Wirkungsprozess die entscheidende Rolle zu. Die zentralen Perspektiven des Wirkungsprozesses sind demnach gleichberechtigt, und als eigenständige Komponente tritt die Wechselbeziehung hinzu (vgl. Früh, 1991, S. 39). In diesem Sinne wird der einzelne Zuschauer als Teil des Publikums angesehen. Das Augenmerk liegt hier weniger in der Begriffsabgrenzung als auf dem Bedeutungs- und Wirkungszusammenhang mit dem Medium Fernsehen.

Ein ähnliches Begriffs-Wirrwarr herrscht in der Fachliteratur bezüglich der Zuordnung einzelner Gruppierungen. Eine der wenigen klaren Strukturierungsversuche schlagen Wann, Melnick, Russell \& Pease (2001, S. 1-4) vor. Sie unterscheiden zunächst grob in

„sport fans" und

„sport spectators" oder „sport consumers“.

Demnach sind 'sport fans' als Individuen zu bezeichnen, die am Sport interessiert sind und die Karriere eines Teams oder eines Athleten verfolgen. Der Begriff 'sport fan' spaltet sich in den 'lowly identified sport fan' und den 'highly identified sport fan' auf. Erstere sind Personen mit einem geringen Niveau an Identifikation. Die Rolle, die die Mannschaft oder der Sportler für ihr Selbstkonzept einnimmt, ist eher gering, und demzufolge reagieren 'lowly identified sport fans' nicht sonderlich stark auf das Auftreten der Sportler. Nach König (2002, S. 48f.) entscheidet sich dieser Typus eher nach rationalen Faktoren wie, 'wenn Zeit dazu da ist' oder dem Wetter, dem übrigen Freizeitangebot etc. für oder gegen den Veranstaltungsbesuch. Für die 'highly identified sport fans' auf der anderen Seite, ist die Rolle des Fans eine sehr wichtige in seiner Identitätskonzeption.

Die Kategorie der 'sport spectators' oder 'sport consumers' verfolgt aktiv das Sportereignis, sei es live oder mit Hilfe eines Mediums. Diese Einteilung differenzieren Wann et al. in 'direct sport consumers' und 'indirect sport consumers'. Die erste Bezeichnung bezieht sich auf Individuen, die bei einem 
Sportereignis anwesend sind, und die zweite auf Personen, die die Veranstaltung über eine Form der Massenmedien wahrnehmen. Kruck und Stuke (2003, S. 51) finden für diese Art Zuschauer den Begriff „temporärer Fußball-Fan“. Dieser interessiere sich für den sonstigen Profi-Fußball nicht sonderlich, würde sich aber zu Zeiten großer interationaler Turniere ein Expertentum aneignen. Als Gründe nennen sie hier den kaum zu vermeidenden Medienhype, das Mitreden-Können, das Dabeisein-Wollen. ${ }^{41}$ Selbstverständlich kann diese Einteilung nur beispielhaft sein und eine grobe Struktur darstellen. Denn das Sportpublikum ist in hohem Maße heterogen. Dennoch sind so die grundsätzlichen Strukturen des Publikums erkenn- und anwendbar. Eine Differenzierung der Zuschauertypen, die den medialen Konsum von Sport integriert, findet sich z.B. bei Pritchard und Funk (2006). Sie untersuchen die Beziehung zwischen der Häufigkeit des Live-Besuchs und des TV-Konsums von Fans der US-amerikanischen Major League Baseball. Dazu benutzen sie die Be-griffe "light consumption“, „heavy consumption“, und diese sind entweder „event dominant" oder „media dominant“. In diesem Kapitel konnte zunächst die Begrifflichkeit Publikum strukturiert werden. Darüber hinaus ist die in der vorliegenden Arbeit verfolgte Gleichberechtigung des Einflusses bzw. des Wirkungszusammenhangs zwischen dem Medium Fernsehen und dem Publikum erläutert worden.

\subsection{Determinanten der (Fernseh-)Publikumsbildung}

Ein großes Publikum ist auch im Spitzensport keine Selbstverständlichkeit, und sportliche Höchstleistungen scheinen kein Garant für ein hohes Publikumsaufkommen zu sein. Um es plakativ auszudrücken: Ein deutschlandweites Public Viewing bei einer Weltmeisterschaft im Abfahrtsski oder auch Basketball würde kein großes Publikumsaufkommen generieren können und somit nicht erfolgreich sein. Welche Gründe haben also Individuen Sport zu verfolgen? Gibt es Unterschiede in den Bedürfnissen nach Live- oder TVRezeption? Um die Klärung dieser Fragen geht es im folgenden Abschnitt.

\footnotetext{
41 Auf die Motive und Beweggründe des Publikums wird in Kap. 7.2 näher eingegangen.
} 


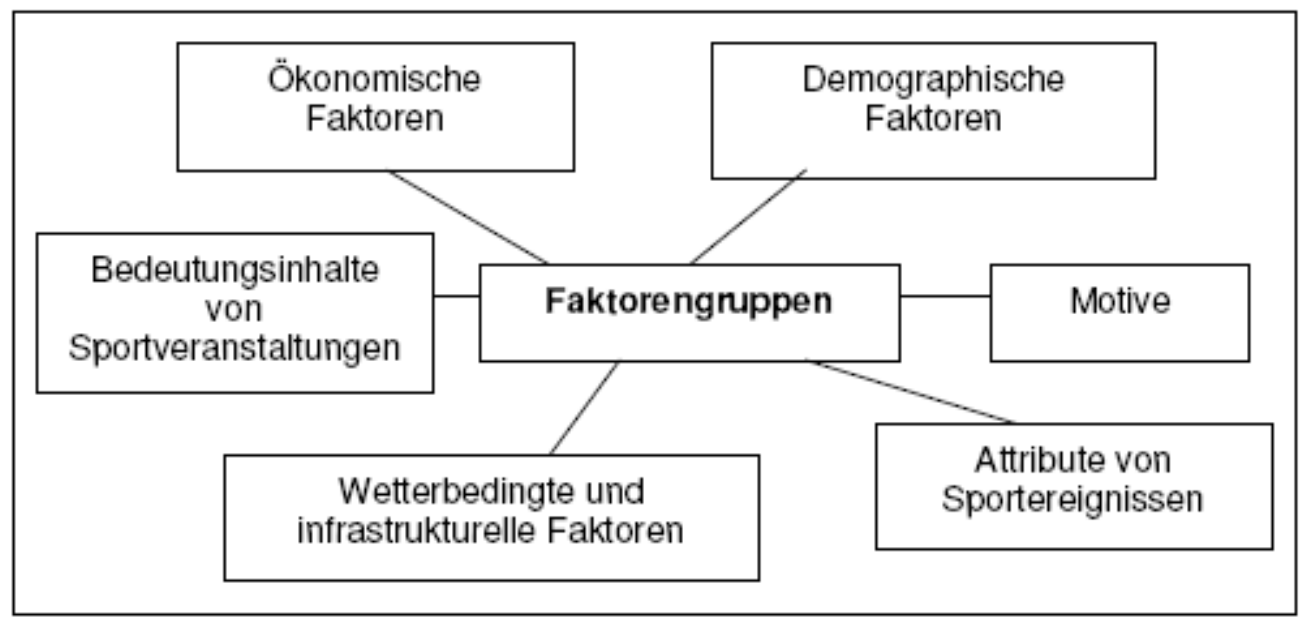

Abb. 6: Faktorengruppen für die Publiku msbildung (modifiziert nach Lucerna, 1997, S. 83)

Generell lassen sich verschiedene Faktorengruppen, die in der Forschung zur Erklärung der Beweggründe von Zuschauern benutzt werden, grob in 'Ökonomische Faktoren', 'Demographische Faktoren', 'Bedeutungsinhalte von Sportveranstaltungen', 'Motive', 'Attribute von Sportveranstaltungen' und 'Wetterbedingte und infrastrukturelle Faktoren' einteilen (s. Abb. 6). Zu dem Zwecke der vorliegenden Arbeit ist allerdings eine Schwerpunktsetzung auf den Bereich der Motive nötig. Es werden aber aus den anderen Faktorengruppen, wie ökonomischen und demographischen Merkmalen, den Bedeutungszuschreibungen der einzelnen Sportereignisse oder ihren Attributen, sowie wetterbedingte und infrastrukturelle Faktoren ergänzend herangezogen. Eine Vielzahl an Autoren setzt sich mit der Nachfrage, den Bedürfnissen oder den Motiven der Zuschauer auseinander, sich sportliche Wettkämpfe anzusehen. Dabei werden unterschiedliche Ebenen der Motivlage sich eine Sportveranstaltung live anzusehen herausgearbeitet. Eine geringere Anzahl von Arbeiten beschäftigt sich mit den Gründen der Sportrezeption im Fernsehen. Seit ca. 30 Jahren ist der Sportzuschauer Gegenstand der Forschung. Unter anderem haben sich Soziologen, Psychologen und Medienwissenschaftler, aber auch Ökonomen der Thematik angenommen. Sportsoziologen haben sich bspw. mit Gewalt unter Sportfans oder Fragen der nationalen Identität auseinandergesetzt. Viele dieser Arbeiten setzen sich explizit mit dem Fußballpublikum auseinander. In der psychologischen Literatur überwiegen Themen wie Verhaltens- oder Motivationsforschung. Medienwissenschaftler beschäftigen sich mit dem Anreiz von Sport- 
stars auf das Publikum oder den Akzeptanzwerten für Sportübertragungen (vgl. Schimmel, Harrington \& Bielby, 2007, p. 581f.). Hervorzuheben sind unter der Masse an Arbeiten zum Thema Sportzuschauer die motivationstheoretischen Forschungen von Wann und Branscombe (1993), Wann (1995) und Wann et al. (2001) mit der Entwicklung der 'Sport Fan Motivation Scale'. Wann (1995) erarbeitet anhand eines empirisch geprüften Fragebogens ein Klassifikationssystem. Es bilden sich acht Kategorien heraus: Gruppenanbindung, Familie, Ästhetik, Selbstwert, Ökonomie, Spannung, Flucht und Unterhaltung. Diese Dimensionen ähneln und überlappen sich bspw. mit denen von Sloan (1989), der in seiner "diversion theory" vom zentralen Motiv der Zuschauer nach der Flucht aus dem affektarmen Alltag ausgeht. Auch Opaschowski (2000, S. 242) schnürt Motivpakete, die sich aber vor allem auf das Erleben von etwas 'Nichtalltäglichem' beziehen. Schlicht und Strauß (2003, S. 140) kürzen diese Motivdimensionen zu vier Clustern, nämlich Identifikation, Selbstdarstellung, Stimmungsregulation und Kontrolle zusammen. In das erste Motivbündel integrieren sie sozialpsychologische Ansätze der Identitätsbildung und -festigung. Hierzu werden oft verschiedene Theorien, wie die zur sozialen Identität von Tajfel (1978) herangezogen. Tedeschi et al. (1998) oder Mummendey (1995) beschäftigen sich mit diesem thematischen Komplex. Wobei sich die Arbeiten von Tedeschi et al. u.a. mit dem Zuschauer im US-amerikanischen College-Basketball oder im professionellen Baseball befassen. Im deutschsprachigen Raum hat sich bspw. Mummendey (1995) in einer allgemeinen Herangehensweise ausführlich mit der Psychologie der Selbstdarstellung befasst, die sich auch auf das Sportpublikum anwenden lässt. Ein Klassiker der generellen Thematik ist Goffman (1969), der davon ausgeht, dass jedes Individuum eben nicht nur seine, um in die Begrifflichkeiten Tajfels einzusteigen, personale, sondern auch seine soziale Identität auslebt. Weiteres zu Selbst- und Gruppenkonzepten ist ebenso in der Konsumentenpsychologie, z.B. in Wiswede (2000) nachzulesen. An diesem Punkt stößt man fast automatisch auf eine weitere Dimension der komplexen Beweggründe von Zuschauern. Denn es scheint neben den individue llen Motiven auch immer soziale Hintergründe zu geben. Der Mensch in der Gruppe als Anhänger eines Teams oder eines Sportlers. Soziale Phänomene wie das BIRGing, also das 'Basking in reflected glory' (Cialdini, Borden, Thorne, Walker, Freeman \& Sloan, 1976), CORFing 'Cutting off reflected Failure' (Synder, Lassegard \& Ford, 1986) 
oder das Blasting (Cialdini \& Richardson, 1980), dass Abwerten der gegnerischen Mannschaft treten auf (vgl. Schlicht \& Strauß, 2003, S. 143ff., Stollenwerk, 1996, S. 21). In den erweiterten Sektor der Gruppenpsychologie gehört auch das frühe Werk der Massenpsychologie von Le Bon (1911/1982).

Zunächst wollen wir aber auf die von Schlicht und Strauß (2003) vorgeschlagenen Cluster zurückgreifen. Als einen dritten Punkt nach Identifikation und Selbstdarstellung nennen sie die Stimmungsregulation. Sie beziehen sich auf Zillmann und Bryant (1998), die sich im englischsprachigen Raum mit Motivforschung und Fernsehsport beschäftigt haben. Im Rahmen der MoodManagement-Theorie gehen sie davon aus, dass Menschen im Allgemeinen bestrebt sind, sich in einem Zustand positiver Stimmung zu erleben. Um diese Gemütsverfassung zu erreichen, würden sie eben auch zum Sportkonsum greifen, unerheblich ob live oder vor dem Fernseher. An dieser Stelle scheint es also zunächst keinen Unterschied zwischen den Beweggründen für das live- oder mediale Sehen zu geben.

Als vierten Faktor wird die 'Kontrolle' genannt. Schlicht und Strauß (2003, S. 148) berufen sich hier, unter Zuhilfenahme der vier Möglichkeiten der Kontrolle von Thompson (1981) darauf, dass Individuen bestrebt sind, Kontrolle über Ereignisse und Zustände zu besitzen. Da dieses Motiv in nur wenigen anderen Studien erwähnt wird und sich die vorliegende Arbeit in diesem Zusammenhang auf die wesentlichen Beweggründe konzentriert, erscheint es nicht sinnvoll, diesen Strang weiter zu verfolgen. Beckmann (1993), Pfaff (2002a) und Lucerna (1997) geben ebenfalls Versuche $a b$, das Konglomerat an Motiven zu strukturieren. Wobei Lucerna einen sehr ausführlichen Einblick in die Welt der sportsoziologischen, sportpsychologischen und auch wirtschaftlichen Ansätze zur Erklärung von Zuschauerverhalten bietet. Eine ergänzende Rolle können Arbeiten in Richtung der Konsumforschung, angefangen bei Gärtner und Pommerehne (1978), Frick (1997) oder auch Hafkemeyer (2003) einnehmen, die den Sportzuschauer von seiner wirtschaftlich denkenden Seite untersuchen. Im Rahmen dieser Arbeiten wird allerdings lediglich auf ökonomische Einflussgrößen, wie Eintrittskosten oder infrastrukturelle Vorgaben geachtet, um das Entscheidungsverhalten für oder gegen den Besuch einer Sportveranstaltung zu hinterfragen.

Für die Zielsetzung dieser Arbeit sind die Beiträge von Gerhard (2006), Zubayr und Gerhard (2005; 2007), Raney (2004), Hagenah (2004) und Gleich (1998) von 
Bedeutung, da sie sich mit der Akzeptanz des Fernsehsports auseinandersetzen. Gerhard (2006) diskutiert hauptsächlich die großen internationalen Fußballturniere der letzten Jahre, während Zubayr und Gerhard (2005; 2007) die generellen Tendenzen im Zuschauerverhalten interpretieren. Im englischsprachigen Raum ist Whannel (2000) ebenso wie Wenner und Gantz (2000) zu nennen, die sich ebenfalls mit den Motivclustern der TV-Zuschauer auseinandersetzen. Das Werk von Gabler (1998) ist letztlich noch zu erwähnen, da es einen anderen perspektivischen Blick auf das Zuschauerverhalten eröffnet.

Tab. 4: Klassifizie rung von Zuschauermotivationen im Sport (nach Gabler, 1998, S. 122)

\begin{tabular}{||l|l|l||}
\hline Zuschauen & Ich bezogen & Im sozialen Kontext \\
\hline Bezogen auf das Sporttreiben & - Freude an sportspezifischen & - Miterleben der Gesamt- \\
selbst & Bewegungsformen & Atmosphăre des \\
& - Neugier & Sportereignisses \\
& - Erlebnis von Risiko, Abenteuer, & - Prăsentation \\
& Spannung, Dramatik & \\
& - selbst aktiv sein & \\
\hline Bezogen auf das Ergebnis des & - sich identifizieren mit dem Erfolg und & - Prestige \\
Sporttreibens & mitleiden mit dem Misserfolg der & \\
& Akteure & \\
& - selbst zum Erfolg aktiv beitragen & \\
\hline Bezogen auf sportexterne & - Entspannung, Zerstreuung, & - Kontakt, Anschluss \\
Zwecke & Abwechslung & - Geselligkeit, Kameradschaft \\
& - Ausgleich (Kompensation) & - Aggression \\
& - Freizeitgestaltung & \\
\hline
\end{tabular}

Für diesen Kontext ist es von Interesse, dass er die Motive sowohl auf das Individuum als auch auf den Einzelnen im sozialen Umfeld bezieht. So scheinen Faktoren wie Entspannung und Ausgleich ihre Erweiterung in sozialen Annehmlichkeiten wie Geselligkeit zu finden. Diese Cluster finden sich übrigens wiederum in einer von Aimiller und Kretschmar (1995) für das DSF durchgeführten Studie wieder. Sie geben u.a. 'parasoziale Motive' und 'soziale Motive' als Beweggründecluster an.

Wie Gleich (1998, S. 144), Schlicht und Strauß (2003, S. 138) oder auch Stollenwerk (1996, S. 17) richtig feststellen, liegt es wohl an der empirisch wenig übereinstimmenden und aufeinander aufbauenden Forschung und ihrer Klassifikationssysteme, dass sich nur schwer differenzierte und fundierte Aussagen treffen lassen. Bedingt durch die erörterte Differentialität der vorhandenen Ansätze und vielleicht auch durch die große Vielzahl der Arbeiten zu diesem Thema, gibt es eine fast ebenso große Spanne an Ergebnissen bzw. Motivdimensionen. Es könnte an dieser Stelle eine noch um ein Vielfaches weiterreichende und breitere Diskussion der Konzepte geführt werden. Es ist 
jedoch nicht die Aufgabe der vorliegenden Ausarbeitung, neue Motivdimensionen hinsichtlich der Publikumsmotivation für den Besuch von Sportveranstaltungen zu bilden oder einen bis ins Kleinste differenzierten Vergleich der bisher erfolgten Forschungsliteratur anzustellen. Die Bedürfnisse des Publikums, die dem Besuch einer Sportveranstaltung oder dem Ansehen einer Sportsendung im Fernsehen zu Grunde liegen, sollen lediglich als eine Hinleitung dienen, um das Phänomen Public Viewing näher zu erläutern. So erscheint es sinnvoll, sich eine Arbeit exemplarisch herauszugreifen, die so viele Punkte wie möglich anspricht, aber dennoch klar strukturiert ist. Zudem beschränken sich die meisten Arbeiten auf die individuellen Bedürfnisse der Besucher. Diese können aber allein keine ausreichenden Aufschlüsse über die Beweggründe von Individuen liefern, sich mit tausenden anderen vor eine Großbildleinwand zu stellen. Deshalb ist es vonnöten, sich den „integrierenden Blick für das Soziale dieses Phänomens [gemeint ist hier das Phänomen Publikumsbindung generell] offen zu halten" (Riedl, 2006, S. 7).

So wird dann auch das Werk von Riedl (2006), in dem er Ansätze einer soziologischen Theorie der Publikumsbildung ${ }^{42}$ entwickelt hat, als Grundlage dienen. Er fasst die Fülle an Beweggründen in vier übergeordnete Motivdimensionen zusammen. Dazu vergleicht Riedl die Arbeiten von Gabler (1998), Bette und Schimank (1995) und Wann (1995) mit den Forschungsergebnissen des Projektes 'Global Player-Local Hero, ${ }^{43}$ zu den Motiven des Stadien- bzw. Hallenpublikums sechser deutscher Spitzenclubs in den Sportarten Basketball, Fußball und Handball (vgl. Riedl, 2006, S. 77). Es kristallisieren sich vier übergreifende Motivmuster ${ }^{44}$ heraus:

- Emotionserleben

- $\quad$ Sportliche Attraktion und Unterhaltung

- $\quad$ Identifikation und Gemeinschaft ${ }^{45}$

\footnotetext{
42 Riedl beschränkt sich allerdings auf das Live-Publikum.

43 Das Projekt 'Global Player-Local Hero' ist ein an der Universität Bielefeld durchgeführtes Projekt, das den Sportverein zwischen Spitzensport, Publikumsbindung und Vermarktung untersucht hat. Aus diesem Grund liefern Daten von sechs deutschen Vereinen aus drei Sportarten (Basketball: Alba Berlin, Opel Skyliners; Fußball: FC Bayern München, Bayer 04 Leverkusen; Handball: THW Kiel, TBV Lemgo) die Basis (vgl. Riedl, 2006, S. 8).

44 In der Literatur sind immer wieder ähnliche Bündelungs versuche zu finden. So findet Beckmann (1993) in seiner Untersuchung von Handballzuschauern die Motivcluster 'sportliches Interesse', 'Fan', 'Unterhaltung' und 'Freunde gehen hin' heraus, die sich auf die von Riedl (2006) genannten Bündel übertragen lassen.

45 Guttmann (1986, p. 180) benutzt in diesem Zusammenhang explizit den deutschen Begriff 'Geselligkeit'.
} 
- $\quad$ Integrität des Vereins ${ }^{46}$.

Diese setzen sich aus folgenden einzelnen Aspekten zusammen.

Tab. 5: Motive der Sportbeobachtung (modifiziert nach Riedl, 2006, S. 84)

\begin{tabular}{|c|c|c|c|c|}
\hline & $\begin{array}{l}\text { Global Player - } \\
\text { Local Hero }\end{array}$ & Gabler (1998) & Wann (1995) & $\begin{array}{c}\text { Bette \& Schimank } \\
\text { (1995) }\end{array}$ \\
\hline 1 & Emotionserleben & $\begin{array}{l}\text { Stress-Stimulation- } \\
\text { Motiv } \\
\text { Sensation-Seeking- } \\
\text { Motiv } \\
\end{array}$ & $\begin{array}{l}\text { Eustress } \\
\text { Flucht aus dem } \\
\text { Alltăglichen } \\
\end{array}$ & $\begin{array}{l}\text { Spannungserleben } \\
\text { Affektives Sich- } \\
\text { Ausleben }\end{array}$ \\
\hline 2 & $\begin{array}{l}\text { Identifikation und } \\
\text { Gemeinschaft }\end{array}$ & $\begin{array}{l}\text { Achievement-Motiv } \\
\text { Hero-Identification- } \\
\text { Motiv } \\
\text { Affiliation-Motiv }\end{array}$ & $\begin{array}{l}\text { Steigerung der } \\
\text { Selbstachtung } \\
\text { Bedarfnis nach } \\
\text { Gruppenzugehorrigkeit } \\
\text { Zeit mit der Familie }\end{array}$ & $\begin{array}{l}\text { Heldenverehrung } \\
\text { Biographische } \\
\text { Kontinuităt } \\
\text { Gemeinschaftserleben } \\
\text { Gesprăchsthema } \\
\text { Sportunspezifische } \\
\text { Sozialităt }\end{array}$ \\
\hline 3 & $\begin{array}{l}\text { Sportliche } \\
\text { Attraktion und } \\
\text { Unterhaltung }\end{array}$ & Entertainment-Motiv & $\begin{array}{l}\text { Ästhetischer Wert } \\
\text { Wunsch nach } \\
\text { Unterhaltung }\end{array}$ & $\begin{array}{l}\text { Ästhetische } \\
\text { Darbietung }\end{array}$ \\
\hline
\end{tabular}

\section{Emotionserleben}

Unter dem ersten Begriff fasst Riedl (2006, S. 83) sechs Motive zusammen, die alle das Erleben von Gefühlen in den Mittelpunkt stellen: Spannungserleben, Eustress, Flucht aus dem Alltäglichen, Affektives Sich-Ausleben, StressStimulation-Motiv und das Sensation-Seeking-Motiv. Alle diese Bedürnnisse sind positiv besetzt. Eustress ist bspw. der positive Stress eines Sprinters vor dem Start des Rennens. Er steigert die Anspannung des Körpers, bis eine maximale Aufmerksamkeit erreicht ist. Mit diesen Beweggründen, so wird ausgeführt, lässt sich der Wunsch der Besucher nach einem positiv spannungsgeladenen Ereignis ausdrücken. Dieses kann als Kontrast zum routinierten Alltag gewollt sein, aber auch als Ablenkung von Problemen gesehen werden.

"Sport Spectating is an ordered and regimented form of participated consumption in which pleasures are experienced in terms of drama uncertainty and intensity" (Whannel, 2005, p. 407).

Die Dimension Emotionserleben wird von den Besuchern sportlicher Großveranstaltungen oft als das bedeutendste Motiv hervorgehoben. So wollen viele Zuschauer etwas von dem Flair, der Internationalität von globalen

\footnotetext{
46 Wobei der Punkt 'Integrität des Vereins' für die Fragestellung dieser Arbeit unerheblich ist und im Folgenden nicht mehr beachtet wird.
} 
Ereignissen wie den Olympischen Spielen oder der Fußball- Weltmeisterschaft erleben. Von den Veranstaltungen geht eine starke Anziehungskraft aus. Uta Herbst, Co-Leiterin der Fußball-Langzeit-Studie der Universität Hohenheim ${ }^{47}$, sieht im Erleben der WM-Atmosphäre sogar das Hauptziel der Besucher (vgl. Doelfs, 2005). In diesem Wunsch kann eine Chance für Public Viewing gesehen werden. Die FIFA und das deutsche Organisationskomitee haben dies anlässlich der Fußball-WM 2006 als Anbieter des Gutes Fußball-Weltmeisterschaft mit dem

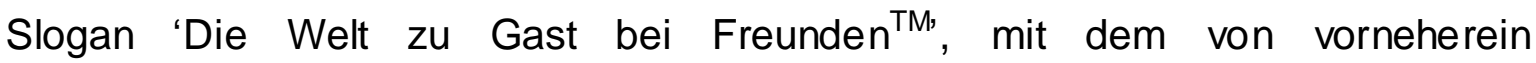
gastfreundliche internationale Fröhlichkeit vermittelt werden sollte, umgesetzt. Aber auch die Städte und Gemeinden hofften auf "den kollektiven Glückseligkeitsrausch“ (Hüttl, 2005). Sie haben erkannt, dass mit FernsehVorführungen nicht nur die Bedürfnisse der Fans befriedigt werden können, sondern auch eine gute Werbung im eigenen Interesse möglich ist. Im Rahmen der Fußball-Langzeit-Studie wurden 2.093 Personen aller Altersgruppen über 14 Jahren im Mai und Juni 2005 befragt, ob diese sich vorstellen können, das Event 2006 vor der Großleinwand mitzuerleben. Damals konnten sich lediglich 33\% der Befragten vorstellen, Spiele vor öffentlichen Großbildleinwänden zu verfolgen, und $26 \%$ schlossen dieses sogar aus (vgl. Klebs, 2005).

\section{Sportliche Attraktion und Unterhaltung}

Riedl (2006, S. 85) weist darauf hin, dass die zweite Bedürfnisgruppe Sportliche Attraktion und Unterhaltung das Entertainment-Motiv, den ästhetischen Wert der Darbietung und den Wunsch nach Unterhaltung mit einschließt. Es bezieht sich damit auf das Erzeugen eines in sich geschlossenen Ereignisses bzw. einer eigenständigen Welt durch Ästhetik der sportspezifischen Bewegungsformen und die Inszenierung des Spitzensportereignisses. Wie bereits in Kap. 5 erörtert und z.B. von Heinemann (1995, S. 185) angeführt, hat sich die Art der Nachfrage und folglich auch das Angebot des Zuschauersports deutlich verändert. Der eigentliche sportliche Wettkampf rückt mehr und mehr zugunsten des Erlebniswerts in den Hintergrund. Die Besucher eines Spitzensportevents wollen attraktiven Sport, eine

47 Die WM-Langzeit-Studie des Marketing-Lehrstuhls der Universität Hohenheim soll durch Befragungen der Bevölkerung einen Stimmungsindikator ermitteln. Zudem ist es das Ziel der Forscher durch im Jahresrhythmus wechselnde Themenschwerpunkte wie die Standortwahl der Stadien oder Merchandising ihren Beitrag zur erfolgreichen Organisation zu leisten (vgl. Klebs, 2005). 
angenehme Umgebung und zusätzlich Erlebnisangebote. Entsprechend kommen immer weitere Geselligkeits- und Unterhaltungselemente hinzu, die den Weg bereiten für ein vieldimensionales Zuschauersportangebot.

Im Projekt 'Global Player-Local Hero' (vgl. Riedl, 2006, S. 79) wurden die Dimensionen Sportliche Attraktivität und Unterhaltung zusammengelegt. Dem Besucher wird an dieser Stelle suggeriert, dass er kaum zwischen der sportlichen Darbietung und dem Rahmenprogramm unterscheidet, sondern dass er vielmehr beide Komponenten als ein Gesamtprodukt wahrnimmt. Der Zuschauer ist an hoher sportlicher Qualität, gepaart mit der Attraktivität und den Annehmlichkeiten des Rahmenprogramms, interessiert. Die Richtigkeit dieser Vermutung kann und soll an dieser Stelle nicht geklärt werden. Um Motivverästelungen zu vermeiden, wird diese Annahme im Folgenden übernommen. Es konnte festgestellt werden, dass weibliche Besucher nach anderen Arten von sportlicher Ästhetik suchen als Männer. Während Männer eher an rasanten Spielzügen oder technischen Tricks von Spitzenspielern interessiert scheinen, sehen Frauen sich gern elegante Bewegungen, wie sie oftmals beim Eiskunstlauf oder Turnen gezeigt werden, an. Mit Bestimmtheit kann aber gesagt werden, dass die Zuschauer die jeweilige spezifische Attraktivität der besuchten Sportart suchen (vgl. auch Olsson \& Viscovi, 2006, p. 44).

Von größerer Relevanz ist der Punkt der Unterhaltung. Nach Riedl (2006, S. 200) wird bei den im Projekt 'Local Heros-Global Players' untersuchten Clubs ein entsprechendes Rahmenprogramm als 'notwendiger Bestandteil' des Sportereignisses gesehen und auch von den Besuchern erwartet. Während es kaum Ansätze gibt, die Publikumsbindung über die ästhetische Komponente zu erhöhen, werden Aktivitäten der Inszenierung von allen untersuchten Vereinen verfolgt. Somit scheint die Inszenierung im Spitzensport zum Standardrepertoire der Vereine zu zählen und für das Publikum von großer Wichtigkeit zu sein. Der Inszenierung von Sportveranstaltungen wird in den hier angeführten Überlegungen eine zentrale Rolle in der Publikumsbindung zugesprochen. Denn, überspitzt dargestellt, ist eine jede Public Viewing-Übertragung eine Inszenierung des Live-Ereignisses in Form einer medialen Rezeption.

Identifikation und Gemeinschaft

Dieses Cluster beinhaltet nach Riedl (2006, S. 84f.) elf Motive: 
Gemeinschaftserleben, Zeit mit der Familie, Bedürfnis nach Gruppenzugehörigkeit, Steigerung der Selbstachtung, Affiliation-Motiv, HeroIdentifikation-Motiv, Achievement-Motiv, Biographische Kontinuität, Gesprächsthema, Heldenverehrung und sportunspezifische Sozialität. Beweggründe wie die Steigerung der Selbstachtung oder die Hero-Identifikation richten sich auf spezifische Identitätsprozesse einzelner Zuschauer, während Motive wie Zeit mit der Familie verbringen oder das Bedürfnis nach Gruppenzugehörigkeit auf das gewünschte Gemeinschaftserlebnis abzielen.

An dieser Stelle wird deutlich, dass die Motivmuster nicht von einander zu trennen sind, sondern oftmals ineinandergreifen. Denn es können natürlich kaum Emotionen erlebt werden, wenn sich der Besucher nicht mit dem Sportler oder der Sportlerin identifiziert. Die Ergebnisse des Wettstreits wären dann bedeutungslos und ließen uns kalt. Eine Ausnahme stellen internationale Spitzensportveranstaltungen dar. Denn wenn zwei Mannschaften aus zwei Ländern oder gar die Athleten der Nationen bei Olympischen Spielen gegeneinander antreten, braucht der Zuschauer nur die gemeinsame Nationalität als Grundlage für Identifikations- und Repräsentationsprozesse (vgl. ebd., S. 155). Für das Konstrukt Identifikation und Gemeinschaft gibt es eine Reihe an psychologischen Ansätzen, die sich mit der Rolle des Individuums in der Gesellschaft beschäftigen. Im Grunde geht es immer um die Bildung der Identität zwischen eigenen Ambitionen und gesellschaftlichen Erwartungen. So nähert man sich dem Phänomen Publikum über Identität und Identifikation, diese gehen, wie die Theorie der Sozialen Identität von Tajfel (1978), immer von der Identität von Einzelnen aus, die sich nach Gruppen ausrichten.

Riedl (2006, S. 158f.) nimmt die soziologische Systemtheorie zu Hilfe, um das Forschungsfeld der Identifikation zu erweitern. Er schlägt den Begriff der 'kollektiven Identitäten' ${ }^{48}$ für eine spezifische Art von sozialen Systemen vor, nämlich denen, die keine eigene Kategorie bilden können.

\footnotetext{
48 Bereits Le Bon (1911/1982) schlug in seinen Arbeiten zur Massenpsychologie den Begriff der kollektiven Persönlichkeit vor. Allerdings attestiert Le Bon der kollektiven Persönlichkeit und Massen generell eine verminderte Intellektualität. Diese negative Besetzung der Begrifflichkeiten wird in dieser Arbeit nicht verfolgt.
} 
Der Begriff der kollektiven Identität bietet die Möglichkeit einer Klammerfunktion, die systemübergreifend $\mathrm{bzw}$. für soziale Zusammenhänge, die kein eigenständiges System bilden, wie z.B. ethnische Gemeinschaften, Nationen und auch das Sportpublikum, verwendet werden kann. Kollektive Identitäten sind Resultate kommunikativer Prozesse (ebd., S. 159).

Das Publikum ist also ein „Personenkollektiv, das durch den gleichzeitigen Konsum eines bestimmten Erlebnisangebotes [und die Kommunikation darüber] konstituiert wird“" (Schulze, 1997, S. 743). Worauf aber kann ein solches Kollektiv aufbauen, wenn ihm die konstitutiven Merkmale eines sozialen Systems fehlen? Normalerweise gründen sich gesellschaftliche Einheiten auf spezifischen Eigenschaften oder charakteristischen Merkmalen. Diese Einordnung in soziale Systeme stellt für den Menschen eine Komplexitätsreduktion dar. Aufgrund der gesellschaftlichen Wandlungsprozesse (s. Kap. 6 \& 10) wird es aber zunehmend schwieriger, solche identitätsbildenden Maßnahmen zu verfolgen (vgl. Riedl, 2006, S. 160f.). An dieser Stelle ist es klar, dass sich der Großteil der Bundesbürger zur Weltmeisterschaft 2006 mit der deutschen Nationalmannschaft identifiziert hat. Die gemeinsame Nationalität ist zumeist Grundlage genug, ein kollektives Merkmal zu erstellen. Möchte man den Blick weiten und auch auf andere Ereignisse schauen, bei denen es großen Zulauf zu Großbildleinwänden gibt, sind die bisherigen Ausführungen nicht ausreichend. Es kann angenommen werden, dass sich kollektive Identitäten über Kommunikation manifestieren. Das Publikum beschreibt sich selbst, indem es über sich spricht, sei es während des Ereignisses oder am nächsten Morgen bei der Arbeit. Das Publikum ist aber auch Gegenstand von Kommunikation in Zeitungen oder im Fernsehen. Die Selbst- und Fremdinteraktion ist keinem festen Muster unterlegen, durch permanente Kommunikation erklärt sich die Identität des Publikums jedoch immer wieder neu. Diese Beschreibungen seien dann, so führt Riedl (2006, S. 161) weiter aus, die kommunizierte kollektive Identität.

Nachdem geklärt ist, was kollektive Identitäten sind, muss im nächsten Schritt erläutert werden, wie diese erzeugt werden können. Auch sie, genauso wie individuelle Identitäten, bilden sich, so Riedl (2006, S. 161f.), durch Ein- und Abgrenzung. Eine Reihe von psychologischen Konstrukten hat sich, wie bereits angemerkt, mit diesem Thema beschäftigt. Die Theorie der Sozialen Identität von Tajfel und das dazugehörige Teilkonzept der Theorie der Selbstkategorisierung (Turner et al., 1987) sind vielzitierte Klassiker. Sie gehen davon aus, dass wir uns 
selbst kategorisieren. Das Individuum ordnet sich selbst und andere in Gruppen ein. Als Folge entsteht ein Wir-Gefühl zu der Gruppe, zu der sich der Einzelne selbst zugeordnet hat. Dabei ist es unerheblich, ob wir einen Großteil der Gruppe kennen, oder ob es völlig Unbekannte sind (vgl. Lucerna, 1997, S. 103f.). Denn die Mitglieder identifizieren sich mit der Gruppe, nehmen sich als zugehörig wahr und werden von außerhalb der Einheit als ein Mitglied erkannt. Natürlich muss das Individuum die Teilhabe in der Gemeinschaft als positiv ansehen. Tajel schlussfolgert, dass sich das Selbst aus der Beziehung zwischen Gruppen ableitet (vgl. Mummendey, 1995, S. 106). Als Folge der Identifikation mit einer Gruppe, die sich beispielsweise nach außen durch das Tragen von Trikots und das Anstimmen von Fangesängen zeigt, entsteht ein typisches Intergruppenverhalten, in dem die eigene Gruppe aufgewertet und andere Gemeinschaften abgewertet werden. ${ }^{49}$ So ist nach Lucerna (1997, S. 104) die Theorie der Sozialen Identität geeignet, das Wir-Gefühl mit Publikum(-massen) zu erklären. Sie bringt aber keine direkten Aufschlüsse über die Entstehung von kollektiven Identitäten.

Wie aber kann Gemeinschaft entstehen, die sich nicht durch die Abgrenzung nach außen manifestiert? Wie entwickelt sich ein Kollektiv aus sich selbst, als von innen heraus begründet? Zur Beantwortung dieser Fragen soll zunächst wieder auf psychologische Konzeptionen zurückgegriffen werden. So zeigt der 'affiliation und power-Ansatz' (Tedeschi \& Felson, 1994), dass Individuen das grundlegende Motiv der Selbstdarstellung besitzen. Affiliation, ins Deutsche zu übersetzen mit Anbindung, assoziiert den Wunsch, von Anderen gemocht zu werden und einen gewissen Status (power) zu erzielen. Mit diesem Ansatz erklären Tedeschi und Felson sich veränderndes Verhalten, sobald Publikum in der Nähe ist. Ähnlich verhält es sich beim Prozess des BIRGing. Die Person, die BIRGing betreibt, versucht, sich selbst als erfolgreich darzustellen, indem sie ein gemeinsames Merkmal des Erfolgreichen sucht und sich entsprechend in der Öffentlichkeit präsentiert. Das Individuum sonnt sich also in dem Ruhme anderer; dies kann eine Einzelperson genauso wie eben eine Gruppe sein. Dieses Merkmal ist häufig flüchtig, wenn die Person sich bspw. damit rühmt, bei einem glorreichen Spiel live dabei gewesen zu sein, und kann auch nur ein Symbol darstellen, man denke hier z.B. an die in Vereinsfarben bemalten Fangesichter (vgl. Tedeschi et al., 1998, S.

49 Mehr zu sozialpsychologischen Intergruppenprozessen kann in Lucerna (1997), Mummendey (1995) oder Stroebe, Jonas \& Hewstone (2001) nachgelesen werden. 
103). Das BIRGing ist der Versuch, in einer Gemeinschaft akzeptiert und als Mitglied anerkannt zu werden. Ähnliches gilt für die Normkonformität (Jones, 1964) bzw. Depersonalisation ${ }^{50}$ in sozialen Konglomeraten. Einschmeichelnde Handlungen sollen dazu dienen, die Zuneigung anderer zu gewinnen. Diese Verhaltensweise zeigt sich wiederum auch nach außen in Form von gleicher Kleidung oder entsprechenden Kommentaren zu der Leistung einzelner Sportler (vgl. Tedeschi, Madi \& Lyakhovitzky, 1998, S. 105). Identifiziert sich der Einzelne in einer bestimmten Situation oder längerfristig mit einer Gruppe, führt Lucerna (1997, S. 153) aus, übernimmt er Normen, Werte, Einstellungen und Gefühle, aber auch prototypische Verhaltensweisen. Individuen neigen demnach dazu, in bestimmten Kontexten nicht als Einzelne zu handeln, sondern durch die Übernahme gruppenspezifischer Verhaltensweisen- und normen als Repräsentanten dieser Gemeinschaft. Stollenwerk (1996, S. 21) und Tedeschi et al. (1998, S. 104) weisen darauf hin, dass es wichtig ist zu betrachten, was die Besucher überhaupt in die Stadien verschlägt, wenn sie das Ereignis auch vor dem TV verfolgen können. Sie betonen auch in diesem Zusammenhang den BIRGing Effekt. Gewinnt die favorisierte Mannschaft, könne der Einzelne behaupten, dabei gewesen zu sein. Er war Teil des Geschehens, er hat ein besonderes Ereignis unmittelbar miterlebt, was wiederum den Selbstwert, aber auch Anerkennung und Prestige steigere. Einen kurzen Überblick über die Konstrukte der Identitäsbildung und -Erhaltung in Bezug auf den Fußballzuschauer liefert Haut (2006).

Es lässt sich festhalten, dass die Gemeinschaft von innen heraus, vor allem durch die Kommunikation von Selbstbeschreibungen, Werten, Normen und Einstellungen aber auch Solidarität, Nähe, Loyalität, Reziprozität erzeugt wird. Dadurch entstehen sich intern stabilisierende Erwartungsbündel und eine Grenze nach außen. In dieser Form kann die Gemeinschaft zu einem nicht hintergehbaren Wert werden, d.h. Gemeinschaft und die Erfüllung ihrer zentralen Normen kann und muss normativ erwartet werden. Kollektive Identitäten existieren ausschließlich durch Kommunikation, die sich permanent erneuern muss. Kollektive Identitäten sind eine soziale Größe, die sich aus Selbst- und

\footnotetext{
50 Der Begriff Depersonalisation ist zwar an den von Le Bon (1911/1982) genutzten Begriff der Deindividation bzw. Deindividualisierung angelehnt, wird hier aber wertneutral gesehen und nicht wie bei Le Bon negativ besetzt.
} 
Fremdbeschreibungen sowie aus der Abgrenzung nach innen und nach außen begründet. Darauf basiert auch die Funktionsweise von Gruppenkohäsion, und in analoger Form stabilisiert die Gemeinschaftssemantik die kollektive Ide ntität (vgl. Riedl, 2006, S. 163f.).

Die diskutierten theoretischen Konzeptionen beziehen sich in der Literatur zumeist auf das Live-Publikum. Public Viewing-Zuschauer verfolgen aber ein Ereignis zweiter Ordnung, ein Medienereignis. Zudem handelt es sich meist, wiederum die Fußball-WM 2006 zu Hilfe nehmend, um ein disperses Publikum. Es gab in den Weltmeisterschaftswochen in jeder Großstadt und in vielen Kleinstädten, Gemeinden, Vereinen (Clubs) usw. in Deutschland und der ganzen Welt öffentliche Fernseh-Vorführungen der Spiele. Wie nehmen sich bspw. die Fans der brasilianischen Mannschaft als Gemeinschaft wahr, wenn nicht alle im Stadion sind, sondern einige vor dem heimischen Fernseher oder vor der Großleinwand das Spiel verfolgen, und dieses noch quer über den Globus verstreut? Einen interessanten Aspekt liefert Riedl in Bezugnahme auf Brint (2001) und Anderson (1988): Den Begriff der „imagined community ${ }^{{ }^{4}{ }^{51}}$. Anderson (1988, p. 15) bezieht den Begriff ursprünglich auf die Annahme, dass sich Nationen als eine Gemeinschaft fühlen, obwohl sie die meisten anderen Mitglieder niemals kennen lernen werden. Allerdings sei in den Köpfen eines jeden Individuums die Vorstellung über diese Gemeinschaft vorhanden (vgl. Anderson, 1988, p. 15). Brint (2001, p. 10f.) entwickelt unterschiedliche Formen von 'communities'. Die'imagined community' entwickelt er weiter als eine auf der Annahme beruhenden Unterart der Gemeinschaft, bestehend aus dispersen Individuen, die ohne face-to-face Interkation auskommen. Seiner Meinung nach können die Fans einer Sportmannschaft eine solche 'imagined community' darstellen.

Muth (1999, S. 134) beschäftigt sich mit dem Einfluss von gesellschaftlichen Sinnstrukturen bzgl. Lady Dianas auf die Entstehung einer modernen Massengemeinschaft. Sie stellt heraus, dass die gemeinsam trauernde DianaGemeinschaft eine „kollektive Wertegemeinschaft gegen Ungerechtigkeiten dieser Welt" darstellt. Sie betont die Reflexibilität der Gemeinschaft, die sich darin

51 Brint (2001, p. 8) definiert "communities" als

"aggregates of people who share common activities and/or beliefs and who are bound together principally by relation of affect, loyalty, common values, and/or personal concern." 
ausdrückt, dass sich der Einzelne freiwillig anschließt und die Verbindungen der Mitglieder untereinander sowohl global als auch in zeitlich abgeschlossenen Phasen geschehen. Die Diana-Gemeinschaft würde in ihrem Charakter eine gemeinsame Bedeutung finden, die wiederum einen lebenspraktischen Sinn widerspiegelt:

„Im substantiell Guten der Lady Diana verbirgt sich eine Struktur, die das Massenphänomen des Trauerns erklärt.“

Rötzer (2007) bringt in seinem Essay die Rolle des Mediums bzw. des Fernsehens als unterstützendes Werkzeug für die Bildung einer imaginierten Gemeinschaft mit ins Spiel.

\begin{abstract}
„Das Massenereignis, das Ereignis der Masse, die sich um eine schöne Leiche kristallisierte, erzeugte auf einmal eine Gemeinschaft, eine wirkliche Öffentlichkeit, ein Beteiligtsein an der Geschichte, auch wenn sie wie in diesem Fall banal war. Plötzlich vermittelte das Medium eine Verbindung, über die die Aufmerksamkeit erhalten blieb und sich mit der der anderen über den medialen Anschluß verkettete“ (Rötzer, 1997).
\end{abstract}

Die Besucher von Public Viewing-Veranstaltungen befinden sich in einer Art Zwischenstellung, denn weder sind sie live bei dem Ereignis dabei noch sitzen sie zu Hause vor dem Fernseher. Vielfach wird davon ausgegangen, dass Individuen ihr Fernsehverhalten ihren Bedürfnissen, diese können natürlich je nach Stimmung, Tageszeit, Stress etc. variieren, anpassen (vgl. Raney, 2004, p. 52). Die Zuschauer zu Hause setzen sich also, wie die Live-Zuschauer vorsätzlich einer kontrollierten Situation mit körperlichen und kognitiven Stimuli aus, auf die sie in einer vorhersehbaren Art und Weise reagieren werden, u.a. auch durch den Ausdruck von Emotionen (vgl. Hediger, 2006, S. 49). Ist also die medial vermittelte Erlebnisqualität mit der 'live' erlebter Veranstaltungen zu vergleichen? Bislang hat sich seit dem von Gleich (1998, S. 144) beklagten Mangel an Studien zu dieser Fragestellung nicht viel geändert, und vielleicht kann es das auch nicht. Die Motive, die z.B. Döhle, Klimmt und Schramm (2006), Hagenah (2004), Olsson und Viscovi (2006), Schramm, Döhle und Klimmt (2004) oder Wenner und Gatz (2000) zur Sport- (bzw. Fußball-)rezeption im Fernsehen herausfanden, gehen konform mit den bereits für die Live-Rezeption genannten. Raney (2004, pp. 52-67) bspw. hebt generell emotionale, kognitive und „behavioral or social needs“ als Motive des TV-Sportkonsums hervor. Unter den emotionalen Gründen werden Entertainment, Eustress, self esteem und escape motivation genannt, und unter den kognitiven Motiven sind Lernmotivation und Ästhetik aufgeführt. Der letzte 
Aspekt der 'behavioral or social needs' spaltet sich in Erholung, Gemeinschaft, Gruppenmotive, Familie und schließlich ökonomische Gründe auf. Führer und Ross (2006, p. 4) sehen die Fernsehrezeption nicht nur als abhängig von einer Bandbreite an Faktoren, wie der Generation, dem Geschlecht, der Klasse, der Religion und der Bildung. Vielmehr gehen sie davon aus, dass die Medienrezeption nicht einfache Reflektionen dieser sozialen Unterschiede ist, sondern dass Medienrezeption „a highly variegated and 'creative' process" ist.

Eine andere Erklärungsmöglichkeit bieten Zillmann und Bryant (1998) mit ihrer Theorie des 'Mood Management' an. Diese baut auf den Theorien der Medienwirkungsforschung des 'selective-exposure' und des 'uses-andgratification-approaches' auf (vgl. Raney, 2004, p. 51). Sie beziehen ihren Ansatz hauptsächlich auf das Unterhaltungsmotiv von Sportzuschauern im Fernsehen und gehen davon aus, dass

\section{„Fernsehpräferenzen dazu dienen, schlechte Laune zu verbessern oder zu beenden, eine gute Stimmung zu schaffen und zu unterstützen sowie einen Übergang von schlechter zu guter Laune zu fördern“ (Zillmann \& Bryant, 1998, S. 203).}

Dabei gilt die Theorie primär zur Erklärung von Medienkonsum. All diese Aspekte sind in den von Riedl (2006) verwandten Motivbündeln inkludiert. Wobei es zu beachten gilt, dass angenommen werden kann, dass die Entscheidung für oder gegen die Nutzung von Medienangeboten selten von nur einem einzigen Motiv bestimmt wird. Ebenso können Rezipienten zu dem Schluss kommen, dass sie einmal zum Public Viewing gehen und einmal zu Hause sich ein Spiel ansehen müssen, um ihren Bedürfnisse gerecht zu werden (vgl. Döhle, Klimmt \& Schramm, 2006, S. 54).

Aus diesem Grund wird im Folgenden davon ausgegangen, dass sich die Motive und Bedürfnisse für die Sportrezeption zwischen Live-Besuch und medialem Zusehen nicht gravierend unterscheiden. Schlicht und Strauß (2003, S. 139) unterstützen die Aussage von Riedl, dass sich die verschiedenen Motivskalen im Großen nicht wesentlich unterscheiden. Die in einigen Bereichen fehlenden Motive führen sie auf die verschiedenen Methoden zurück. Zudem betonen sie aber, dass auch die Motivation zum Mediensportkonsum sich hier wieder findet. ${ }^{52}$ Es ist

52 Gerhard (2006, S. 465) stellt zehn Kriterien zusammen, von denen die Akzeptanz von Fernsehübertragungen seiner Meinung nach abhängt: Der Sportart und ihrer Tradition, den Erfolgschancen deutscher Sportler/Mannschaften, der Persönlichkeit der Akteure, dem übertragenden Fernsehsender, dem Eventcharakter der Übertragung, der fernsehgerechten 
immer zu bedenken, dass der Publikumszulauf eine variable Größe ist, die von vielen Ursachen beeinflusst wird. Für einen Besucher kann das Emotionserleben das einzige Motiv sein, für einen anderen kann das Konglomerat aller genannten Bedürfnisse den Reiz ausmachen. Darüber hinaus lassen sich die Motivcluster schwer unabhängig voneinander betrachten. So sind die erlebten Emotionen natürlich wesentlich von der Relevanz des Ergebnisses abhängig. Die Relevanz bestimmt wiederum die Identifikation mit den Sportlern.

Logischerweise dürften Faktoren wie die Informationsdichte durch die Medien, die Menge und das Niveau der (Sport-) Veranstaltungen im Umkreis oder auch demographische Daten Einfluss auf die Sportnachfrage haben. Als weitere Faktoren sind u.a. der Fortgang des Turniers und die Leistung der favorisierten Mannschaft zu nennen. Diese können jedoch in dieser Arbeit nicht ausführlich berücksichtigt werden, sind aber z.B. bei Gebauer (2006), Hagenah (2004) oder Stollenwerk (1996) nachzulesen. Schulke (2006b, S. 6) führt Faktoren, wie die Kosten für den Besuch, die gute Errreichbarkeit durch die zentrale Lage und Möglichkeit sich für spontanen Besuch zu entscheiden, das gute Wetter, Erfolg der deutschen Mannschaft ${ }^{53}$, die zentrale geographische Lage Deutschlands als Garanten für den Erfolg der Fan Feste 2006 an.

Das Fernsehverhalten muss immer rückführend auf die Verflochtenheit von Systemfaktoren wie Werten und Normen, Wirtschaft, Politik und Technologie der umgebenden Gesellschaft, die familiäre Umgebung und Fernsehindustrie analysiert und beurteilt werden. Diese Faktoren interagieren und beeinflussen das Fernsehverhalten in starkem Maße. Bei der Fußball-WM 2010 in Südafrika wird das Public Viewing in starkem Umfang von der Sicherheitslage im ausrichtenden Land abhängen. Will man die perspektivische Schere noch weiter öffnen, sind natürlich auch immer die regions- oder landestypischen Traditionen zu berücksichtigen. Zubayr und Gerhard (2004, S. 42) schreiben, dass Veranstaltungen der Leichtathletik oder des Radsports in den USA trotz des Erfolges der US-amerikanischen Athleten kaum nachgefragt werden.

Umsetzung, der Tageszeit der Ausstrahlung, dem eigenen Programmumfeld und der breiten Zielgruppenansprache. Da diese Punkte sich verstärkt auf den Fernsehsport beziehen, sollen sie hier nur am Rande erwähnt sein. Es würde sich mit Sicherheit aber lohnen, diese in späteren Studien deutlicher zu berücksichtigen.

53 Wobei Schulke (2007, S. 29) zu bedenken gibt, dass bei anderen internationalen Sportereignissen bspw. in der Leichtathletik, in einer Sportart also, in der die Deutschen in den letzten Jahren keine vergleichbaren Erfolge erzielt haben, dennoch die Massen begeistert sind. 
In diesem Kapitel wurden die Gründe von Zuschauern, Sport zu verfolgen, eruiert. Dies ist beispielhaft in Orientierung an die von Riedl (2006) angeführten vier bzw. drei Motivcluster: Emotionserleben, Sportliche Attraktion und Unterhaltung erfolgt. Dabei ist zunächst die individuelle Bedürfnislage diskutiert worden. In einem zweiten Schritt wurde der Blickwinkel auf das Soziale des Phänomens Publikumsbildung ausgeweitet. Hierbei stellten Konzepte wie das der 'imagined communities' wichtige Bausteine für den weiteren Aufbau des theoretischen Fundamentes dar. Kommunikation durch das Medium Fernsehen trägt zur Bildung bzw. Stabilisierung bei. Letztlich konnte die Frage nach divergierenden Beweggründen zwischen live- und medialer Sportrezeption verneinend beantwortet werden.

\subsection{Zusammenfassung}

Das Publikum ist eine wichtige Determinante des Public Viewing. Es stellt keinen bloßen 'Zusatz' wie bei anderen Sportveranstaltungen dar, sondern kann durchaus als der Hauptakteur bezeichnet werden. Denn ohne die Erwartung von Zuschauern hätte sicher niemand Großbildschirme z.B. anlässlich der WM 2006 aufgebaut. Nach der Klärung und Strukturierung der Begriffe Publikum und Zuschauer und der Erörterung der in der vorliegenden Ausarbeitung verfolgten Wirkungssichtweise sind die Determinanten der (Fernseh-)Publikumsbildung erfasst worden. Es konnte aufgezeigt werden, dass das Publikum sowohl in seiner Struktur als auch in seinen Beweggründen extrem heterogen dargestellt werden muss.

Mit der Frage nach den Motiven der Zuschauer, Sport zu verfolgen, konnten wichtige Hinweise auf die Publikumsbildung und -bindung gewonnen werden. Dabei stelte sich zum Einen heraus, dass sich die Gründe für die Fernsehrezeption nicht grundlegend von denen der live Rezeption unterscheiden, zum Anderen sind neben den individuellen Beweggründen auch soziale Motive ein wichtiger Faktor. So konnte mit Hilfe von Ansätzen der sozialen Systemtheorie gezeigt werden, dass das Publikum über das gemeinsame Erlebnis, in diesem Falle Public Viewing, zu einem Personenkollektiv zusammenwächst. Diese kollektiven Identitäten halten sich über Kommunikation zusammen, die über jeden EinzeInen, aber auch über die Medien abläuft. Innerhalb dieser werden 
fortwährend Selbst- und Fremdzuschreibungen manifestiert, die intern stabilisieren und nach außen abgrenzen. Eine Fortführung dieser Bausteine stellt das Konstrukt der „imagined community“ dar. Dieses geht davon aus, dass die dispers verteilten Individuen über die Vorstellung der Gemeinschaft ohne face-to-face Interaktion Gemeinschaft empfinden können. Hilfreich sind hier z.B. gemeinsame Wertevorstellungen und wiederum die Kommunikation über das Fernsehen. 


\section{Rückkehr in den öffentlichen Raum}

„Doch war Fernsehen lange Zeit verbannt in Räume. Und wegen der kleinen Bildschirme war zudem die Menge der Zuschauer beschränkt. Die Teilnahme an der Welt war so beschränkt auf mehr oder weniger zurückgezogene Höhlenbewohner" (Rötzer, 2006).

In diesem letzten Teil des einführenden Grundlagenkapitels geht es um die Rückkehr des Fernsehers und des Fernsehens in den öffentlichen Raum. Laut Digel und Burk (2001, S. 21) kann der Prozess der Mediatisierung in der Gesellschaft Rückschlüsse über die Veränderungen der räumlichen Mediennutzung zulassen. Sie beschreiben die Ausdehnung der Fernsehnutzung vom Wohnzimmer in immer weitere (Wohn-)Räume und begründen u.a. damit die gesteigerte Bedeutung des Mediums in der alltäglichen Kommunikation. Ergänzend zu den vorherigen Ausführungen wird in diesem Kapitel zunächst auf die determinierenden Faktoren des Public Viewing eingegangen. Die Erfahrungen der Fußball-WM 2006 zeigen, dass das Aufstellen von Videowänden auf zentralen öffentlichen Flächen nicht alleiniger Erfolgsgarant ist. Während die Fan Feste sehr häufig Menschenmassen anzogen, machte doch jeder Veranstalter seine eigenen Erfahrungen, und außerhalb der Austragungsorte kam es sogar gelegentlich zum Scheitern der Public Viewing-Veranstaltungen (8.1).

Obwohl das eigentliche Ereignis vielleicht tausende Kilometer weit entfernt liegt, bekommt man das Gefühl, live dabei zu sein. Zeit und Raum erscheinen irrelevant, vielleicht erfährt ein Fernsehzuschauer in Australien das Ergebnis eines Spieles schneller als ein Anwohner direkt neben dem Stadion, der nicht den Fernseher eingeschaltet hat. Wie McLuhan (2001, S. 138) ausführte: „Die Allgegenwart ist eine altägliche Dimension des menschlichen Lebens geworden." Technologischer Fortschritt scheint somit eine entscheidende Funktion in der Entwicklung des Public Viewing zu haben (8.2). Schließlich werden die Ergebnisse dieses Abschnitts zusammengefasst und in den Kontext der Arbeit eingeordnet (8.3). 


\subsection{Determinanten des Public Viewing}

„Die größte Fußball-Party aller Zeiten kann steigen. Wir haben in jeder Stadt jetzt zwei Austragungsstätten: das Stadion und das offizielle Fan-Fest. Das gab es noch nie“ (Franz Beckenbauer zit.n. Hamburger Abendblatt, 2005b).

Die Grundvorrausetzung für das Funktionieren von Public Viewing ist die Nachfrage für die ausgewählte Veranstaltung. Es muss ein außerordentliches Interesse an einem (Sport-)Ereignis existieren; mehr Menschen müssen die Veranstaltung besuchen wollen, als für sie Plätze in der Sportstätte selber vorhanden sind. Im Nationalsport Fußball ist das oftmals der Fall (s. Kap. 5 \& 6). Greifen wir beispielhaft wieder auf die Weltmeisterschaft 2006 zurück. Während der Endrunde im Sommer gab es für die zwölf Stadien insgesamt 3,2 Mio. Eintrittskarten. Ein gutes Drittel war für den Normalbürger käuflich. Die übrigen Tickets erhielten die internationalen Fußballverbände, Sponsoren usw. (vgl. Hamburger Abendblatt, 2005a). Natürlich konnten die frei verkäuflichen 1,12 Mio. Karten niemals der enormen Nachfrage gerecht werden. Im fußballbegeisterten Deutschland allein wollte ein Großteil der etwas über 82,5 Mio. Einwohner das Riesenspektakel miterleben, ein Teil der vielen tausend Fußballbegeisterten sein, die ihre Mannschaft live im Stadion anfeuern. ${ }^{54}$ Diejenigen, die keine Stadionkarte erwerben konnten, sehen sich nach alternativen Möglichkeiten um, die Spiele zu verfolgen. Ebenso jene, die am Turnierverlauf als Ganzes und nicht nur an einzelnen Spielen interessiert sind. ${ }^{55}$

\footnotetext{
"The sport spectacle, as it developed in the early 20th century, was a ritual of place and space, in which the enclosed space of the stadium and the mythologized significance of the great stadia - Yankee Stadium, Soldiers Field, Wembley, Bernabeu, Sydney Cricket Ground, Lords, the Maracana, the Aztec played an important role" (Whannel, 2005, p. 413).
}

Die zweite Determinante stellt die Lokalität des Veranstaltungsortes dar. Die klassischen Sportereignisse waren früher nur im Stadion oder auf dem Platz, jedenfalls nur an dem Originalschauplatz an sich, erlebbar. Röger (2006) verdeutlicht, dass lange Zeit hauptsächlich in geschlossenen Räumen ferngesehen wurde. Mit der Weiterentwicklung des Fernsehens kam für eine kurze

54 Darüber hinaus gibt es selbstverständlich einige spezifische Faktoren, wie die Höhe der Stadionticketpreise, die Lage der Lokalität und des Ausrichterlandes, die Einfluss auf die Nachfrage haben (s. auch Kap. 7.2).

55 Die Public Viewing-Veranstaltungen zu WM in Deutschland 2006 besuchten über 25 Mio. Zuschauer, 18 Mio. waren es allein bei den Fan Festen (vgl. FIFA nach Schulke, 2007, S. 60). 
Zeit die Kneipe um die Ecke dazu. Als weltweit erstes größeres Fernsehereignis gelten die Olympischen Spiele in Berlin 1936. In 27 Fernsehstuben konnten Zuschauer die Wettkämpfe verfolgen (vgl. Guttmann, 1986, p. 134). Bei der WM 1954 traf man sich in den wenigen mit TV-Geräten ausgerüsteten Wohnzimmern, Kneipen oder vor den Schaufenstern der Rundfunkgeschäfte, um die ersten LiveÜbertragungen zu sehen (vgl. Kap. 5.2.2). Whannel (2005, p. 413f.) betont, dass spätestens ab den 1960er Jahren mehr Zuschauer solche Ereignisse vor dem Bildschirm zu Hause verfolgten, als in einem Stadion untergebracht werden könnten. Zu diesem Zeitpunkt ist das Stadion bereits als gewachsener Ort des Zuschauens abgelöst, denn mehr Fans sahen das Ereignis zu Hause als vor Ort. Aber wo wird der moderne Sport heute erlebt? Vor dem Fernseher zu Hause, im Stadion, in der Kneipe, in der Sportsbar oder im Stadtzentrum oder einem anderen öffentlichen Raum? Es kann festgehalten werden, dass die Großereignisse des heutigen Sports dezentralisiert stattfinden. Das Publikum ist dispers, mit all seinen Verhaltensweisen und Ritualen. Die Zuschauer sind also über einen Staat, über einen Kontinent oder auch wie bei der Fußball-WM, über die ganze Welt, verteilt.

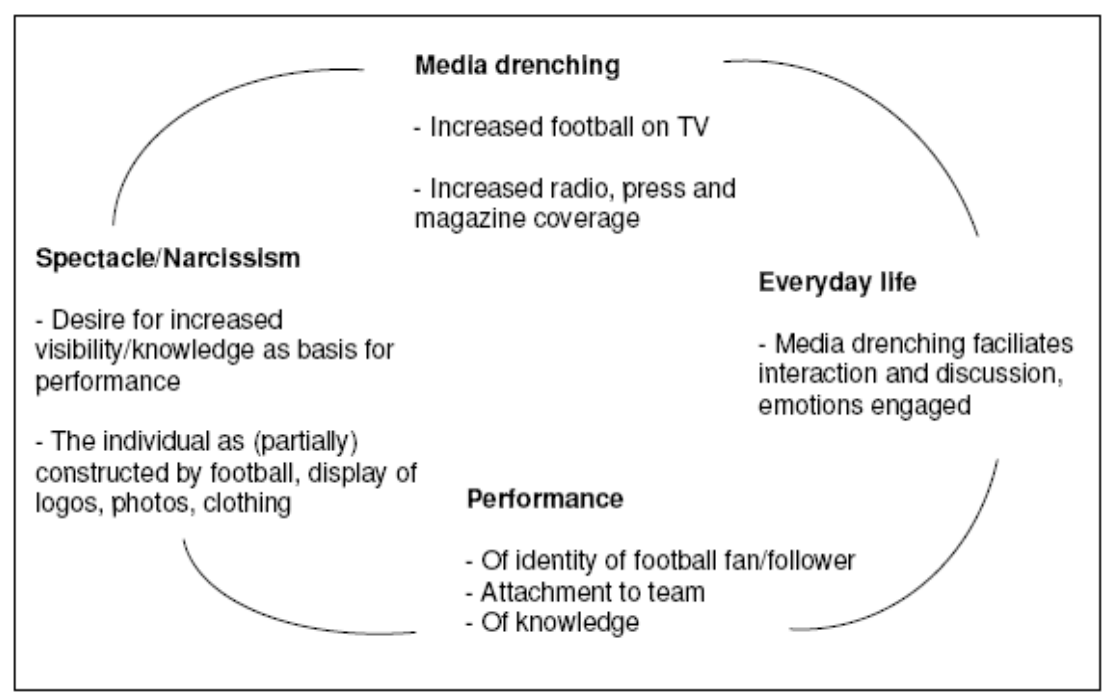

Abb. 7: Contemporary football and the diffused audience (nach Abercrombie \& Longhurst, 1998, p. 178)

In ihrem Werk von 1998 gehen Abercrombie und Longhurst davon aus, dass aufgrund des veränderten Platzes, den die Medien in heutigen fortgeschrittenen westlichen Gesellschaften einnehmen, sich das Medienpublikum in drei Typen unterteilt: „the simple, the mass and the diffused ${ }^{56}$. Im Grunde gehen sie davon

56 Wobei "diffused" im Folgenden mit dispers übersetzt wird. 
aus, dass ein jeder zu jeder Zeit ein Teil einer Zuschauerschaft ist (vgl. Couldry, 2005, p. 190). Am Beispiel des zeitgenössischen Fußballs postulieren Abercrombie und Longhurst, dass die 'simple audience' direkt bei dem Spiel anwesend ist und dazu tendiert, sich in einer bestimmten Weise anzuziehen, so dass die Zugehörigkeit zu einem Team erkennbar ist. Das Tragen von Trikots wird aber auch im Alltag verfolgt. Hier kommt die disperse Zuschauerschaft ins Spiel. Trikots werden z.B. im Urlaub im Ausland getragen, wobei sich ein jeder der Existenz von anderen Fans versichern kann. Das Massepublikum sieht Fußball im Fernsehen und interagiert mit dem dispersen Publikum und innerhalb dessen. So würden nach Abercrombie und Longhurst (1998, p. 159f.) das Lokale und das Globale neu geordnet und neue disperse Universialitäten gebildet. Couldry (2005, p. 192) betont, dass

"Performances for diffused audiences are public and private. Indeed, they erode the difference between the two."

Die bereits in Kap. 7.2 eingeführten Begriffe der 'imagined community' bzw. der imaginierten Gemeinschaft betonen, dass das Fernsehen über seine Möglichkeiten der Kommunikation zu ihrer Entstehung und Festigung beiträgt. Die Unterteilung der Zuschauerschaft in das Publikum vor Ort, das massenmediale Publikum und das disperse Publikum weitet das Konstrukt aus, indem es explizit davon ausgeht, dass durch die Zusammenarbeit der verschiedenen Publika die imaginierte Gemeischaft gefestigt wird. McCarthy (2001, p. 140) schreibt, dass der Bildschirm einen Raum in einem anderen Raum darstellt, d.h., der Zuschauer schaut, obwohl er sich in einem Raum befindet, auf eine andere Welt in einen anderen Raum durch den Fernseher hi nein.

In der Vergangenheit fanden Angebote für TV-Vorführungen vor allem in bestehenden Lokalitäten statt. In Kinos sind beispielsweise bereits große abgegrenzte Räume mit Sitzplätzen sowie den technischen Vorraussetzungen vorhanden. Zudem gibt es sanitäre Einrichtungen und Reinigungspersonal, Infrastruktur in Form von Parkplätzen oder Anbindung an öffentliche Verkehrssysteme. Schulke (2006b, S. 35) betont, dass Public Viewing außerhalb geschützter Räume zumindest in Mittel-und Nordeuropa extrem witterungsabhänig sei. Liegt die Zukunft des Public Viewing also in den Kinos? Während der WM 2006 erfreuten sich nicht nur die Großbildleinwände im Freien großer Beliebtheit, sondern auch Kinosäle waren gut gefült. Der Hamburger Kinovermarkter 
Werbeweischer hatte die Übertragungsrechte für die deutschen Kinos erworben und zeigte sich so zufrieden mit dem Ergebnis, dass das Konzept auch auf ausgewählte Bundesliga-Begegnungen, DFB-Pokalspiele oder die Champions League erweitert werden soll (vgl. Horizont Sportbusiness 2006). Bereits Ende der 1990er Jahre hatte allerdings der Chef der Münchner Kinowelt Medien AG Michael Kölmel versucht, mit der TV-Übertragung von Fußballspielen auf Big-Screens Gewinn zu erzielen. Kölmel investierte mit Borussia Mönchengladbach nur in einen Erstligisten, ansonsten förderte er Klubs der zweiten und dritten Liga. Als Gegenleistung haben ihm die Vereine einen Großteil ihrer Vermarktungsrechte übertragen. Leider konnten die großen Kinosäle nicht mit entsprechend vielen Menschen gefüllt werden, und die Klubs hatten das Geld schnell verbraucht. Die Geschäftsidee scheiterte u.a. an der trügerischen Hoffnung des Finanziers, dass die großzügige finanzielle Unterstützung den Vereinen mittelfristig zu einem Aufstieg in die erste Liga verhelfen und damit Kölmel beträchtlichen Gewinn bescheren würde (vgl. Tagesspiegel, 2001). Es scheint also kaum eine Regel für den Erfolg bzw. Misserfolg von Public Viewing zu geben. Schwark (2007, S. 105) weist für die von ihm untersuchte Veranstaltung in Duisburg auf eine Spannweite von 8.500 bis 300 Zuschauer zwischen den einzelnen Spieltagen hin. Er begründet dies mit der Attraktivität der Spieltage, die er aber nicht weiter definiert bzw. erforscht. 57

Schulke (2006b, S. 6) weist z.B. darauf hin, dass die Möglichkeit, sich spontan für einen Besuch zu entscheiden, eine Rolle spiele. Der Nachteil der Kinos, Mehrzweckhallen o.ä. ist die eingeschränkte Öffentlichkeit der Veranstaltung. Natürlich ist in geschlossenen Räumen allein aus Sicherheitsgründen eine Beschränkung der Zuschauerzahl nötig, was aber für den Rezipienten bedeutet, dass er den Besuch planen muss. Die Besonderheit des Public Viewing ist offensichtlich, dass sich die Übertragungen auf öffentlichen Plätzen „in einem architektonisch, zeitlich, sozial und emotional weitgehend offenen Raum [abspielen], in dem sich die Fußballbegeisterung anarchisch bzw. spontankollektiv entfalten kann" (Schulke, 2006, S. 24). Dem entgegen argumentieren

\footnotetext{
57 Allerdings gibt es hier eine $P$ arallele mit den dieser Arbeit zugrunde liegenden Untersuchungen (vgl. Kap. 13). Die Bedingungen für den Misserfolg von Public Viewing-Veranstaltungen können in diesem Zusammenhang nur rudimentär bleiben. Alllerdings bietet sich an dieser Stelle ein Anknüpfungspunkt, z.B. anhand einer umfangreichen Zeitungsanalyse weiter zu forschen.
} 
Roost und Schöning (2006, S. 38f.), dass es sich bei den Public Viewing-Arealen zumeinst um "temporär privatisierten Raum" handele, der vom jeweiligen Veranstalter gesteuert würde. Sie weisen darauf hin, dass nur für diejenigen Zuschauer, die augenscheinlich dem Bild des Veranstalters entsprechen, der Zugang überhaupt möglich gewesen sei und so spontan, überraschende Emotionen wohl eher weniger auftraten.

Unbeachtet dessen erobert sich nicht nur das Fernsehen die öffentlichen Plätze in den Innenstädten zurück, sondern auch der Sport, sei es im Rahmen eines Skilanglaufrennens durch Düsseldorf oder die BeachVolleyball Tour auf dem Rathausmarkt in Hamburg (vgl. Schulke, 2006b, S. 17). Der Sport rückt ins Zentrum der Städte und somit auch wieder ins Zetnrum des gesellschaftlichen Interesses und Diskurses. Einen weiteren wichtigen Hinweis zur Neugestaltung des Raums bietet Sydnor (2000, S. 226f.). Sie nennt das Konzept des „liminal space" als einen Ort, der von Zeitlosigkeit geprägt ist, der ohne eigentliche Bedeutung verbleibt und irgendwo zwischen den Realitäten angesiedelt ist. Ein Raum, in dem Individuen für ein paar Sekunden berühmt werden können, in dem die alten Regeln nicht mehr gelten, wo Identitäten verschwimmen. 'Liminal spaces' seien Orte, an denen "sport scenes on screens (...) provide opportunities for aesthetic experiences for watchers" (Sydnor, 2000, p. 226). Public ViewingLokalitäten als 'liminal spaces' bieten demnach hervorragende Bedingungen zur Festigung individueller Sozialstruktur und zur Entstehung von emotionalen Erlebnissen.

Ein weiterer Faktor, der für das Funktionieren von Public Viewing-Vorführungen immens wichtig ist, ist die Atmosphäre. Fraglich ist, ob diese im heute stark gesicherten Stadion zu finden ist. Schulke sieht in diesem Punkt die Chance für öffentliche TV-Vorführungen:

\footnotetext{
„Public Viewing wird den Fußballfans eine neue emotionale Erlebniswelt eröffnen, die wir aufgrund der zahlreichen Reglementierungen in den Stadien nicht vorfinden werden, es wird auf dem Heiligengeistfeld eine Stimmung wie einst beim legendären Musik-Festival Woodstock 1969 in den USA herrschen“ (Schulke, zit.n. Grünberg, 2005).
}

Sportveranstaltungen sollen heute Spaß machen und unterhaltsam sein. Public Viewing bietet dem Rezipienten neue emotionale Räume für den Genuss von Fußball. Ob eine Art Woodstock-Atmosphäre entsteht, sei dahingestellt. Generell 
kann gesagt werden, dass das wachsende Interesse, Fußball und andere beliebte Sportarten in Bars und öffentlicher Umgebung zu verfolgen, einen ganz neuen Kontext für die Konsumierung bzw. das Erleben von Sport erzeugt hat. Die Anbieter von Fernseh-Vorführungen befinden sich in einer Art Zwitterstellung zwischen dem Stadion und der TV-Rezeption zu Hause. Whannel (2005, p. 414) betont, dass Public Viewing-Veranstaltungen näher am Stadionerlebnis liegen, durch die Möglichkeit, Emotionen und Rituale direkt zu teilen. Sie haben die Chance, das Erlebnisbedürfnis der heutigen Sportzuschauer direkt und in vielerlei Hinsicht anzusprechen. Die heutigen Stadien, in denen Großereignisse stattfinden, sind normalerweise ausschließlich mit Sitzplätzen bestückt und von sicherheitsbedingten Restriktionen eingeengt. Auf einem öffentlichen Platz an dem man sich bewegen kann und auf dem Interaktionen uneingeschränkt stattfinden können, ist das Erlebnis Fußball näher an seiner ursprünglichen Form als in den stark reglementierten hochkomplexen Arenen. ${ }^{58}$ Diesen Effekt können Veranstalter z.B. durch eine geeignete bauliche Gestaltung des Public Viewing-Areals unterstützen (vgl. Kellermann, 2007, S. 155). Bereits im antiken Olympia ist erkannt worden, dass eine bestimmte Bauweise eine optimale Sicht auf die Athleten und eine geordnete Struktur der Zuschauerplätze verspricht, aber auch für eine stimmungsvolle Atmosphäre sorgen kann. Beispielsweise durch rund um den Ort des Geschehens nach oben ansteigend platzierte Zuschauerränge können sich Emotionen optimal verbreiten (vgl. Messing \& Lames, 1996, S. 24). Schulke (2006b, S. 16) weist zudem noch auf die körperliche Nähe zwischen Akteuren und Zuschauern wie auch der Zuschauer untereinander und die Möglichkeit der sich dadurch verdichtenden Emotionen hin (s. Kap. 9) ${ }^{59}$

Massenveranstaltungen stellen freilich immer ein Risiko dar. Es muss davon ausgegangen werden, dass es dort, wo Menschenmassen aufeinander treffen und Alkohol und Emotionen im Spiel sind, immer zu Auseinandersetzungen kommen kann. Die Determinante Sicherheit ist der letzte Aspekt für das Funktionieren von Public Viewing. Eine wichtige Aufgabe für die Veranstalter von öffentlichen TVVorführungen muss sein, den sicheren oder zumindest den 'gefühlt sicheren'

\footnotetext{
58 Kellermann (2007, S. 156) bezeichnet diese Situation des freien emotionalen Erlebens und der dafür notwendigen Sicherheitsmaßnahmen, die in dieser Form nur beim Public Viewing möglich sind als „Balance aus Sicherheit und Offenheit“.

$59 \operatorname{Er}(2007$, S. 64) bezeichnet dies als „Arenaatmos phäre“.
} 
Besuch zu gewährleisten. Kellermann (2007, S. 156ff.) bietet in diesem Zusammenhang erste Überlegungen bzgl. der Auswirkungen durch das beim Public Viewing enstehende Gemeinschaftsgefühl auf die Sicherheitsfrage. Hier bietet sich eine hervorragende Möglichkeit für weitere Untersuchungen, besonders im Vorfeld des bevorstehenden Turniers in Südafrika, an. Interessanterweise gaben in der Evaluierung der Besucherbetreuung während der Fußball WM 2006 (vgl. Behn et. al., 2007, S. 52; Pilz, 2007, S. 81) mehr deutsche (zusammen 93\%) als ausländische Besucher (zusammen 83,1\%) ihre Stimme für die Kategorien von sehr entspannt bis eher entspannt ab. So dass angenommen werden könnte, dass sich die heimischen Besucher insgesamt sicherer fühlten als die ausländischen Gäste. Da dieses subjektive Sicherheitsgefühl aber eine Grundlage für das Entstehen von positiver Atmosphäre ist, werden die FIFA und die Verantwortlichen in Südafrika alles Mögliche unternehmen müssen, um diese Differenz so gering wie möglich zu halten.

Letztlich kann gesagt werden, dass Public Viewing nur mit einer die Sportstättenkapazität übersteigenden Ticketnachfrage möglich ist. Sport findet heute in diversen Räumen -und Kontexten- wie dem Stadium, öffentlichen Plätzen weltweit, in Cafés, zu Hause usw. statt. Das Fernsehen besitzt eine Mittlerposition, indem es die dispersen Publika verbindet und in Form von 'liminal spaces' für ein Verschwimmen der Zeit-Ort-Relation sorgt. Besonders die öffentliche Umgebung öffnet neue emotionale Räume. Whannel (2005, S. 414f.) hebt hier hervor, dass es dadurch immer schwieriger zu bestimmen gelingt, wo nun eigentlich die Hauptaktion stattgefunden hat. So scheint es fast, als ob die Fan Feste als besondere Medienereignisse während der Fußball-WM 2006 den Stadien nahezu den Rang abgelaufen hätten. 


\subsection{Technologische Entwicklung}

"A toaster with pictures would really be much more interesting than a regular appliance" (McCarthy, 2001, p. 117).

McCarthy (2001, p. 118) geht davon aus, dass "things do, in some sense 'talk". Fernsehgeräte seien diskursive Objekte, die "as a distinctive kind of object" auf ihre Art und Weise in öffentlichen Kontexten kommunizieren. Die Möglichkeit des Fernsehens, Bilder und Töne live zu übertragen im gleichen Augenblick, in dem sie entstehen, ist der große Vorteil des Fernsehens. Durch vertonte Livebilder ist es also möglich, die Begrenzung von Zeit und Ort aufzuheben. Ein globales Ereignis wie die Fußball-WM erzeugt eine große Öffentlichkeit. Seit es den Rundfunk und insbesondere das Fernsehen gibt, können die Zuschauer nahezu überall auf der Welt dem Ereignis gleichzeitig oder zumindest zeitnah beiwohnen, obwohl es natürlich in den Anfängen technologische Schwierigkeiten gab. So verpassten die Kameras im November 1936 das erste Tor während der Begegnung Italien gegen Deutschland, und die Spieler und der Ball waren für die Zuschauer vor dem Bildschirm kaum auszumachen (vgl. Keilbach \& Stauff, 2006, S. 166). Das 'Live-Dabeisein' scheint in den letzten Jahren einen großen Imagezuwachs in der Gesellschaft bekommen zu haben. So wird betont (vgl. Levine, 2008, p. 398; Sydnor, 2000, p. 224), dass die Entwicklungen der neuen (Kommunikations-)Medien wie Mobiltelefone oder Internet auf die 'Liveheit' als zentrale Qualität abgerichtet sind. Siegel (2002, p. 49) schreibt, dass sich die Präsenz von Bildschirmen im öffentlichen Raum enorm erhöht hat. Im Sommer 1980 wurde im Dodger Stadium in Los Angeles, USA, das erste „large-screen color video display system" installiert.

"In many grounds, too, the spectactor can watch live (with slight delay) replays of the action on giant screens..." (Redhead, 2007, p. 234).

Dieses Zitat verdeutlicht, egal, ob der Zuschauer im Stadion oder am anderen Ende der Welt auf dem Sofa sitzt, ein jeder ist live dabei. Diese bipolaren Zuschauer-Positionen, auf der einen Seite bspw. gerade in der Loge einer Fußballarena zu sitzen, andererseits aber auf dem Bildschirm die Olympischen Spiele zu verfolgen, machen das Publikum zu einem „multimedia product of many different visual and spatial ideologies" (McCarthy, 2001, p. 119). Heutzutage sind neben den großflächigen Bildschirmen im Stadion Fernsehbildschirme allgegen- 
wärtig. Sei es an der S-Bahn-Haltestelle, im Supermarkt oder in der Bank, an all diesen Orten wird das Individuum mit Informationen in Bild und Text versorgt. Ein jeder kann internationale Medienereignisse live verfolgen, ob er auf dem Flug hafen in Dubai ist oder in einer australischen Stadt,

Eine Zeit lang war es nicht möglich, bei starkem Tageslicht eine Übertragung für viele Menschen zugänglich zu machen. So war das TV-Erlebnis noch meilenweit vom Live-Genuss eines Sportereignisses entfernt. ${ }^{60}$ Schulke (2006b, S. 3) weist darauf hin, dass neue Übertragungsformen stets Katalysatoren für sportliche, ökonomische, gesellschaftliche und technische Entwicklungen gewesen sind. Dass dies auch umgekehrt gilt, betont Krüger (1999, S. 1226). Nach Gerhard (2006, S. 472) hat die visuelle Aufbereitung der Liveübertragung bei der FußballWM 2006 einen qualitativen Sprung gemacht: 16:9 Format, mehr Kameras, mehr Totalen. Der ballführende Spieler ist mit der Kamera verfolgt worden, es gab grafische Zusatzelemente oder computeranimiertes Nachstellen von Spielszenen. Zwei weitere Nova sind für den Markt der Sportübertragung proklamiert worden, die für ein noch authentischeres Live-Erlebnis sorgen sollen. Zunächst ist die außergewöhnlich hohe Bildauflösung, das High Definition Television (HDTV) ${ }^{61}$, zu nennen. HDTV soll eine völlig neue Qualität hinsichtlich Schärfe, Detailgenauigkeit sowie Farbbrillanz ermöglichen und ein Live-Seherlebnis vermitteln. ${ }^{62} \mathrm{Im}$ akustischen Bereich wird ein Dolby-Surround-System für verbesserte Qualität sorgen. Der Ton wird von je 30 Mikrofonen pro Stadion zunächst in ein spezielles Studio geleitet, wo er von einem Toningenieur gemischt und dann übertragen wird. Dank der genannten Technologien müssen die Zuschauer nun laut FIFA (2005) nicht mehr direkt im Stadion sitzen, um Atmosphäre zu schnuppern. Die Neuheiten HDTV und Dolby-Surround-Sound machen die Ausstrahlung der

60 In der wissenschaftlichen Literatur zu dieser Thematik wird in der Regel näher auf die historische Entwicklung eingegangen (vgl. Cy bulska 2007; Loosen, 2004; Whannel, 2005, p. $407 \mathrm{ff}$. [u.a.]). Da diese im hier vorliegenden Zusammenhang keine unabdingbare Bedeutung hat, wird auf eine Darstellung verzichtet.

61 HDTV, ist ein Sammelbegriff für Fernsehnormen, die sich durch höhere vertikale, horizontale und bzw. oder temporale Auflösung auszeichnen. Durch die bessere Auflösung kommt es zu einer prägnanteren Bildqualität (vgl. Woldt, 2006, S. 235).

62 Der Host Broadcast Service (HBS) ist bei allen 64 Spielen der Fußball-WM 2006 mit mindestens 20 HDTV-Kameras vor Ort gewesen und hat versucht, die Stimmung in den Stadien einzufangen(vgl. Kaiser, 2005). Die Kameras des HBS werden das Turnier in „wide-screen“-Optik aufnehmen, welches die zweite Neuerung der FIFA darstellt. Dieses ist ein ursprünglich für Spielfilme entwickeltes 16:9 Format. Da jedoch nur spezifische Geräte dieses Format empfangen können, wird es auch weiterhin alle Übertragungen im Standardformat 4:3 geben (vgl. Infront Sports \& Media AG, o.J.). 
Begegnungen noch realer und könnten zu einer erstmals vollen Ausschöpfung des Public Viewing-Potentials führen. Allerdings ist Premiere der einzige Sender, der HDTV gezeigt hat. Während der Fan Feste musste sich der Zuschauer also mit der normalen Auflösung zufrieden geben, weil dort die Signale von ARD, ZDF und RTL gesendet wurden. Auf einigen anderen Veranstaltungen, die in Kooperation mit dem Pay-TV-Kanal ausgestrahlt wurden, ist es aber möglich gewesen, die Begegnungen in hochauflösenden Bildern zu verfolgen. Allerdings machten, so Schulke (2006, S. 20), erst die "epochalsten medientechnologischen Fortschritte“ das Phänomen Public Viewing möglich. Sie sorgten dafür, dass Übertragungen auf Großbildschirme immer mehr in den Mittelpunkt von Ereignissen rücken. Durch komplexe Steuerungsprozesse von Lichtsignalen in kleine Präsentationsmodule sind scharfe Bilder auf Flächen von bis zu 200 qm möglich. Diese machten laut Habbel (2005, S. 7) die Übertragung bei hellem Tageslicht erst möglich. Diese neuartigen Videowalls ermöglichen es auch für den in $150 \mathrm{~m}$ Entfernung stehenden Zuschauer, bei Wind und Wetter ein gestochen scharfes Bild zu sehen. Die mögliche Größe und die Leuchtkraft dieser Wände erlauben es großen Menschenmengen, direkt und nahezu live dabeizusein (vgl. Schulke, 2006b, S. 12; 2007, S. 36). Neue Technologien ermöglichen es also, dass das Fernsehen auf die öffentlichen Flächen und Plätze der Städte zurück wandert. Diese sind plötzlich wieder zentraler Punkt der Stadt, auf dem sich soziales Leben abspielt. Die vorangegangenen großen Sportereignisse, vor allem aber die internationalen Fußballturniere, haben gezeigt, dass solche Veranstaltungen, auch wenn sie für den Zuschauer nur auf dem Bildschirm stattinden, für inn attraktiv sind. Das Medium Fernsehen ist mit dem großen Erfolg des Public Viewing in 2006 ein Stück weit aus dem Konsum in den eigenen vier Wänden in die Öffentlichkeit gewandert.

Die symbiotische Beziehung des Fernsehens und des Spitzenfußballs treibt die Entwicklung in den Übertragungsmedien voran. Gerade zur WM 2006 wurden drei Neuheiten auf den Markt gebracht. Die LED (Light-Emitting Diode)-Technik ist für das Public Viewing am bedeutsamsten, weil sie TV-Vorführungen auch bei starkem Tageslicht ermöglicht. Aber auch die Einführung von HDTV und des 16:9Formats machen deutlich, dass die Fernsehübertragungen von internationalen Sportveranstaltungen immer mehr zu eigenständigen Medienereignissen werden. Wie Rötzer (2006) betont, scheint das eigentliche Ereignis immer mehr in den 
Hintergrund zu rücken. Dieses gilt für Public Viewing in besonderem Maße.

\subsection{Zusammenfassung}

Das Grundlagenkapitel abschließend ist in Kap. 8 ein weiterer Faktor des Public Viewing angesprochen worden. Durch die Mediatisierung der Gesellschaft hat sich nicht nur die Bedeutung des Fernsehens geändert und z.B. die Entwicklung des Sports vorangetragen, sondern es hat sich auch die räumliche Nutzung gewandelt. Dies ist zunächst durch einen Blick auf die das Public Viewing determinierenden Faktoren der Kartennachfrage, der Lokalität, der Atmosphäre und der Sicherheit erfolgt. Die die Kapazität der Sportstätte übersteigende Nachfrage nach Tickets, die Lokalität im Sinne des 'liminal spaces' als der Faktor für die Entstehung einer spontan-kollektiven, emotionalen Atmosphäre und die zu vermittelnde 'gefühlte' Sicherheit sind die bestimmenden Größen des Public Viewing. Sport und Sportübertragungen im Fernsehen finden heute in diversen Räumen und Kontexten weltweit statt. Eine Vielzahl an Menschen kann Großveranstaltungen gemeinsam an öffentlichen Orten verfolgen. Technologische Entwicklungen, nicht zuletzt hervorgerufen durch die Symbiose zwischen dem Fernsehen und dem Spitzenfußball in den Übertragungsmedien, hervorzuheben sind hier die LED-Videowände, sorgen immer mehr dafür, dass TV-Vorführungen und in besonderem Maße das Public Viewing zu vom eigentlichen Ereignis zu zunehmend unabhängigen Ereignissen werden. 


\section{Theoretische Erklärungsmodelle}

Im folgenden Kapitel werden zwei grundsätzliche theoretische Ansätze zur Aufschlüsselung des Phänomens Public Viewing eruiert. Sowohl Emotionen als auch Erlebnisse sind im Kapitel II und in der vorangehenden explorativen Expertenbefragung als wichtige Bestandteile von Sportübertragungen auf Videowände herausgefiltert worden. Das Ziel ist es, aufbauend auf den bislang erörterten Ergebnissen den Komplex der Emotionen und den der Erlebnisse zur weiteren Erkenntnisgewinnung theoretisch zu vertiefen.

Begonnen wird mit der Diskussion über einen auffälligen Bestandteil des Public Viewing, nämlich die Emotion. Zunächst wird ihre soziologische Relevanz erörtert (9) und anschließend der Begriff definiert und in seinem Bedeutungsrahmen eingegrenzt (9.1). Im nächsten Schritt werden emotionssoziologische Werkzeuge vorgestellt, die Erklärungsansätze und Interpretationsebenen bieten (9.2). Ein besonderes Augenmerk wird hier mit einem eigenen Kapitel auf kollektive Emotionen gelegt (9.3). Abschließend darf die Analyse von Emotionen und Fernsehen in diesem Kontext nicht fehlen (9.4). Resümierend wird letztlich die Rolle der Emotionen für das Phänomen Public Viewing zusammengefügt (9.5).

Danach erfolgt die Erörterung der Suche nach und Inszenierung von Erlebnissen (10). Zu diesem Zweck wird die Stellung des Erlebnisses in der Gesellschaft eruiert (10.1) und das Individuum als Konsument von Erlebnissen analysiert (10.2). Weiterführend wird der Ansatz des Erlebnis-Setting auf Public Viewing übertragen (10.3) und die Inszenierung als Instrument dessen erfasst (10.4). Der Vollständigkeit halber wird ein Exkurs zum Thema Event dieses Kapitel abrunden (10.5). Abschließen wird diesen Teil erneut eine Zusammenfassung der Ergebnisse und ihre Einordnung in den Gesamtzusammenhang (10.6). 


\section{Relevanz von Emotionen}

„Da schien eine ganze Nation, vor allem die jungen Menschen, in eine taumelnde Feierstimmung zu geraten, bei der es auch um das Fußballspiel, die eigene Mannschaft und eine Art nationaler Stimmung ging, aber in der die riesigen Leinwände den Kern bildeten, der die Massenerregung der Menschen in roter Kleidung massierte (...) Die Masse feierte sich selbst, die Spiele waren dafür nur der notwendige Katalysator, der für die Grunderregung, die Einigkeit und ausreichende Menge an Menschen draußen auf den Plätzen und Straßen sorgte" (Rötzer, 2006).

Emotionen spielen beim Public Viewing eine kaum übersehbare zentrale Rolle. Das Ausdrücken und Ausleben von Gefühlen besonders in der Freizeit scheint in der Gesellschaft nötig und erwünscht zu sein. Besonders auffällig ist dieses Phänomen bei Massenveranstaltungen, gerade im Sport. Wobei Public Viewing hier anscheinend nochmals eine Sonderstellung einnimmt. Es besteht an dieser Stelle also eindeutig ein Forschungsinteresse nach der Bedeutung von Emotionen für das Phänomen Public Viewing.

Für die Erörterung der Dimension Emotion ist es nahe liegend, anfangs zu klären, welche Bereiche diese Dimension beinhaltet. Befasst man sich mit Emotionen, wird schnell deutlich, dass der Begriff an sich und seine Bedeutungszuweisungen sehr vielseitig und vielschichtig sind. Zu Beginn des Kapitels steht die definitorische Begrenzung des Emotionsbegriffs für die folgende Verwendung im Vordergrund (9.1). Die Rolle der Emotionen für das Phänomen Public Viewing soll näher bestimmt und der Zusammenhang dieser beiden Komponenten verdeutlicht werden. Dazu wird im ersten Schritt eine Schwerpunktlegung auf soziologische Aspekte erfolgen, um eine Zerfaserung der Erkenntnisse zu vermeiden. Um Aufschluss über diese Frage zu gewinnen, wird diskutiert, wie Gefühle soziologisch entstehen und wie selbige bedingt sind. Für diesen Zweck werden theoretische Konzeptionen vorgestellt, diskutiert und für die Fragestellung der Ausarbeitung ausgewertet. Dennoch wird nach Brown (2000, p. 36) das Ziel verfolgt, die traditionelle Trennung der Psychologie, die sich mit dem Individuum beschäftigt, und der Soziologie, die sich mit der Gesellschaft auseinandersetzt, zu lockern, um einen Erkenntnisgewinn zu erzielen. Es wird die Frage nach dem sozialen Einfluss für Emotionen gestellt. Zu diesem Zweck werden hauptsächlich die Arbeiten von Kemper (1978, 2002, 2007), bzw. Gerhards (1988) sowie die Gedanken Riedls (2006) als Grundlage genutzt, um sowohl einen sozialkonstruktivistischen als auch einen systemtheoretischen Zugang zu erhalten 
(9.2).

Um der Rolle von Emotionen im Hinblick auf Public Viewing gerecht zu werden, wird im zweiten Schritt das Augenmerk auf kollektiv geteiltes (emotionales) Wissen und die Kommunikation dieses Wissens von Menschenmassen gelegt. Von sozialpsychologischen Theorien ausgehend, wird der Versuch unternommen, einen an der soziologischen Systemtheorie orientierten Aspekt für das Zusammenspiel von Emotionen, Massen und Public Viewing zu liefern (9.3). Daran anschließen wird sich ein Einblick in den Zusammenhang zwischen Gefühlen und dem 'Kern' des Public Viewing, nämlich dem Fernsehen und seiner Wechselwirkung mit Emotionen (9.4). Den Abschluss bilden erneut die Zusammenfassung der Erkenntnisse und die Eingliederung in den Horizont der Arbeit (9.5). 


\subsection{Emotionen: Begriffsabgrenzung und Definition}

“...Emotions lie at the juncture of a number of fundamental dualisms in western thought such as mind/body, nature/culture, public/private" (Bendelow \& Williams, 1998, p. xvi).

Auf der Suche nach im weitesten Sinne sozialwissenschaftlichem Material zum Thema Emotionen wird man zunächst auf psychologische Literatur stoßen. Das liegt nahe, denn Emotionen scheinen primär als personengebundene und -bezogene Gegebenheiten, wird doch von Individuen erlebt und gefühlt (vgl. Gerhards, 1988, S. 12). So sieht Compi (1997, S. 67) in seinem Werk über Affektlogik Emotionen -er bezeichnet sie allerdings als Affekte-als ein

„Von inneren oder äußeren Reizen ausgelöste, ganzheitliche psycho-physische Gestimmtheit von unterschiedlicher Qualität, Dauer und Bewußtseinsnähe“.

Zudem scheint ebenso sowohl ein gewisser Körperbezug vorhanden, der sich z.B. im umgangssprachlichen Gebrauch -das Herz schlägt mir bis zum Hals- wieder findet, als auch etwas im Inneren des Subjektes vorhanden zu sein, was nach außen gezeigt wird. Denn an der äußeren Reaktion eines Subjektes erke nnen wir schließlich als Erstes, dass Emotionen im Spiel sind (vgl. Riedl, 2006, S. 91). ${ }^{63}$

Der Zielstellung dieser Arbeit kann der eingeschränkte Horizont der Psychologie nicht reichen, so geht es denn um ein soziales Phänomen. Mit dem Einstellen des Fokus auf die Soziologie der Emotionen öffnet sich ein weites Feld. Es würde den Erkenntnisstand dieser Arbeit aber nicht fördern, unnötig detaillierte Ausführungen zum Forschungsgegenstand Emotionen in allen nützlichen soziologischen Fachbereichen und Wissensgebieten zu liefern. Ein Überblick über die europäischen Klassiker der Soziologie des letzten Jahrhunderts, die sich mit Emotionen beschäftigt haben, findet sich z.B. bei Flam (2002), Maier (1999), Schmidt (2005) oder Shilling (2002). Wie Williams und Bendelow (1998, p. xv) richtig feststellen:

"It is really only within the last decade or so that a distinct 'corpus' of work, mostly American in origin, has begun to emerge in the sociology of emotions."

Allerdings sind diese Arbeiten eher pluralistisch orientiert und gehen formell wenig konform (vgl. Flam, 2002, S. 165; Hochschild, 1998, S. 3). Es können jedoch aus

63 Ein Einstieg in die Emotionspsychologie ist u.a. bei Ciompi (1997), Meyer, Schützwohl \& Reisenzein $(1993,1997)$ oder Scherer (2001) zu finden. 
einzelnen Werken Ansätze und Gedankengänge genutzt werden, um dem Phänomen Public Viewing näher zu kommen. In diesem Sinne wird hier das Ziel verfolgt, die vorhandenen Ergebnisse zu selektieren und für den Erkenntnisgewinn dieser Ausarbeitung zu nutzen.

So soll an dieser Stelle die Arbeit von Hochschild (1990, 1998) genannt werden. Denn sie postuliert in einer konstruktivistischen Denkweise das Selbst als fähig, Gefühle zu erleben, zu reflektieren und zu managen (vgl. Flam, 2002, S. 127). Hochschild verfolgt hier den bereits von Goffman (1969) verfolgten Gedanken, dass sich Individuen in ihren Interaktionen normkonform verhalten möchten. Auf die von Hochschild erstmals erwähnten Begriffe 'Gefühlsarbeit' bzw. 'Gefühlsregeln/feeling rules' wird im weiteren Verlauf der Ausarbeitung noch eingegangen. Mit diesen Gefühlsregeln verbindet Hochschild Emotionen, die sie zunächst auf der Mikroebene ansiedelt und mit der Makroebene, indem sie Organisationen oder Eliten die Macht über diese zuschreibt (vgl. Flam, 2002, S. 133f.).

Das Werk von Gerhards (1988) ist eine frühe fundierte Auseinandersetzung der Soziologie mit der Thematik der Emotionen. Dabei geht es Gerhards (1988, S. 19) darum, das Phänomen Emotionen aus einem soziologischen Blickwinkel zu betrachten, indem er das soziale Element „im Sinne der wechselseitigen, sinnhaften Bezugnahme der Handelnden aufeinander" in den Fokus der Diskussion stellt. Er bezieht Stellung im Sinne einer postmodernden Sicht,
„in welcher gerade nicht die Kontrolle von Emotionen, sondern die Orientierung des Verhaltens an den emotionalen Befindlichkeiten selbst, mit dem hedonistischen Ziel des Vermeidens von Negativ-Emotionen und dem Erreichen von angenehmen Gefühlen zur gesellschaftlichen Norm werde“ (Ciompi, 1997, S. 239).

Nach Brown und Richards (2000, p. 31) wächst die Bedeutung von Emotionen als Forschungsfeld der Sozialwissenschaften zum Verständnis unserer Gesellschaft.

\footnotetext{
"Not only is it recognised that there has to a degree been an emotionalisation of public life, so that emotion in an explicit way intrudes into the sociological view, but the emotional dimensions of all social processes are now beeing seen as a proper and necessary object of study."
}

In diesem Diskurs gibt es zunächst zwei Blickwinkel. Gerhards (1988, S. 11f.) betont, dass Emotionen auf der einen Seite immer das Ergebnis von sozialen Konstellationen und auf die Reaktion auf sie sind (vgl. auch Riedl, 2008, S. 237). Der WM-Zuschauer erlebt Freude und Glücksgefühle, weil die deutsche Nationalmannschaft ein Spiel gewonnen hat. Die Emotionen beziehen sich also 
auf eine Situation, ein Geschehnis. So wird hier auch diese Perspektive, also die Frage nach den sozialen Bedingungen der Entstehung von Emotionen, in den Vordergrund gestellt. Gleichzeitig geben unsere Emotionen aber auch den sozialen Zusammenhängen des täglichen Lebens Struktur. Oftmals sind es scheinbar keine rationalen Entscheidungen, die wir in unserem Alltag treffen. Positive Emotionen bestimmen vielleicht letzlich, ob wir uns ein Fußballspiel ansehen oder lieber einer alternativen Freizeitbeschäftigung nachgehen. Darüber hinaus besteht noch eine weitere Ebene, nämlich die der kulturellen Kodierung von Emotionen. In unserer komplexen Umwelt verwenden wir Emotionsregeln, die die Informationsverarbeitung entlasten. Bei vielen alltäglichen Ereignissen verwenden Individuen bereits vermittelte Wissenselemente zur Bewältigung der Lebenswelt. Diese können bspw. in der Sozialisation erfahren werden und auf der Wertebildung früherer Generationen basieren oder auch durch sprachliche Medien vermittelt werden (vgl. Tritt, 1992, S. 168). Emotionen scheinen also wesentlicher Bestandteil der gesellschaftlichen Struktur zu sein. Folgerichtig sind die meisten soziologischen Ansätze zur Bestimmung der Emotionalität also durch den Wunsch geprägt, die Auswirkungen von Emotionen auf Interaktionen bzw. die Effekte von vorhandenen gesellschaftlichen Mustern auf die Emotionalität von Einzelpersonen zu untersuchen (vgl. Tritt, 1992, S. 46).

Dennoch ist dieses Feld nicht einfach zu bearbeiten und es darf nicht vergessen werden, dass auch andere Faktoren wie die Physiologie eine entscheidende Rolle spielen. In diesem Zusammenhang jedoch soll das Augenmerk hauptsächlich auf der soziologischen Perspektive liegen. Eine wichtige Anmerkung macht Schmidt (2005, S. 16) unter Berufung auf Bartsch und Hübner (2004, S. 109) zu diesem Thema. Diese sind der Meinung, dass es nicht wichtig ist, ob Emotionen primär biologische oder soziale Phänomene sind, sondern wie biologische und soziale Faktoren bei der Entstehung von Emotionen zusammenwirken. So bilden soziokulturelle mit biologischen Faktoren im Entwicklungsgang der Emotionsentstehung, der Bewertung und schließlich des Ausdrucks, des Auslebens von Emotionen einen Wirkungszusammenhang. Die Einflüsse beziehen sich wechselseitig aufeinander und stellen eine dynamische Gesamtstruktur dar. Diese Feststellung lässt sich allein mit dem Gedanken untermauern, dass der Einzelne im Bewusststeinsprozess des Emotionalen gar nicht unterscheiden kann, ob die erlebten Gefühle nun biologischen, kognitiven, 
körperlichen oder soziokulturellen Ursprungs sind (vgl. Schmidt, 2005, S. 16f.). Ebenso ist Brown (2000, p. 35) der Meinung, dass es im Sinne der psychoanalytischen Soziologie der Emotionen wichtig ist, „to avoid studying the individual in isolation from his or her social context".

Verschiedentlich wurde betont (z.B. von Gerhards, 1988, S. 15; Meyer et al., 1993 \& 1997; Schmidt, 2005, S. 11f.; Tritt, 1992, S. 30-41; Vester, 1991, S. 28), dass es zum Begriff Emotionen so viele Definitionen zu geben scheint wie Autoren, die sich im weitesten Sinne mit diesem Thema beschäftigen. Angesichts der geschilderten Situation emp fiehlt es sich für diese Arbeit nicht, eine eigene Suche nach der richtigen und allumfassenden Definition zu beginnen. Stattdessen bietet es sich an, den Emotionsdiskurs ein Stück weit zu systematisieren, um die Referenzbereiche, von denen im weiteren Verlauf der Arbeit gesprochen wird, erfassen zukönnen.

Riedl (2006, S. 93-97) stützt seine Überlegungen zu einem emotionssoziologischen Modell zur Steuerung des Emotionserlebens im Publikum auf die allgemein gehaltene Definition von Meyer et al. (1993, S. 22ff.). Emotionen sind demnach Erscheinungen mit vier Eigenschaften:

1. Emotionen sind aktuelle Zustände von Personen, wie Freude, Traurigkeit, Angst, Stolz etc. oder weitere Vorkommnisse, die diesen hinreichend ähn lich sind.

2. Emotionen lassen sich nach ihrer Qualität und ihrem Typus unterscheiden.

3. Emotionen richten sich no rmalerweise nach einem Objekt.

4. Personen, die Gemütsbewegungen fühlen, spüren häufig charakteristische physiologische Veränderungen, dazu haben sie üblicherweise ein typisches Erleben und Reaktionen.

Einen wichtigen Aspekt sprechen Meyer et al. im dritten Punkt an, dass nämlich Emotionen meist in irgendeiner Weise einen Objektbezug haben. Dabei muss dieser Objektbezug nicht der Realität entsprechen, nur die momentane Realität der Person ist für diesen Faktor von Bedeutung. Die vierte genannte Größe besitzt drei einzelne Komponenten. Die körperliche, physiologische Ebene bemerken wir, wenn wir vor Aufregung feuchte Hände bekommen. Dieser Körperbezug wird im Verlauf der Arbeit nicht völlig ausgeklammert, aber da er zum größten Teil nicht in den sozialwissenschaftlichen Forschungsraum fällt, nur eine Randrolle spielen. Mit dem Erlebnisaspekt von Emotionen ist die subjektive Komponente der Gefühle 
gemeint. Es gibt ein bestimmtes Gefühl, das dem Einzelnen das Vorhandensein von Emotionen anzeigt, und dieses Gefühl fühlt sich anders an, als wenn die Person sich in anderen bewussten Zuständen befindet. Mit der Reaktion auf ein bestimmtes Gefühl sind nachgeschaltete Verhaltensweisen gemeint, wie etwa Mimik, Gestik und Bewegungen des Körpers. Genauso können dies aber komplexere Verhaltensweisen wie Angriffsbemühungen bei Wut oder Flucht bei Angstgefühlen sein (vgl. ebd., S. 27-32).

Gerhards $(1988$, S. 16) gibt folgende Arbeitsdefinition für sein Werk aus:

\begin{abstract}
„Emotionen sind eine positive oder negative Erlebnisart des Subjektes, eine subjektive Gefühlslage, die als angenehm oder unangenehm empfunden wird. Emotionen entstehen als Antwort auf eine Bewertung von Stimuli und Situationen; sie können mit einer physiologischen Erregung einhergehen und können in Form von Emotionsexpressionen zum Ausdruck gebracht werden. Sie wirken selbst wieder strukturierend auf den sozialen Zusammenhang zurück."
\end{abstract}

Das Erleben von Emotionen wird demnach als subjektiv beschrieben. Diese Erlebnisart kann positiv oder negativ sein. Sie entsteht als Reaktion auf und Folge von Situationsinterpretation auf Zustände und Reize. Mit ihr einhergehen können körperliche Erregung und andere Gefühlsäußerungen. Physiologische Erregungen und Emotionsexpressionen können als Folge auftreten, müssen dies aber nicht zwingend. Rückwirkend strukturieren Emotionen wiederum selbst den sozialen Interaktionszusammenhang, aus dem sie hervorgegangen sind (vgl. Gerhards, 1988, 16f.).

Diese Beschreibung wird in der Definition von Cordsen und Deilmann (2005, S. 314) um weitere wichtige Komponenten ergänzt. So sprechen sie von der Entstehung von Emotionen als einem

„sukzessiven, kognitiven Verarbeitungsprozess in der Gegenwart wahrgenommener Reize (...), aus dem eine spezifische Reaktion resultiert - im Rückgriff auf in der Vergangenheit erworbenes Wissen und orientiert an in der Zukunft liegenden Wünschen und Handlungszielen."

So kommt in dieser Auslegung klar die zeitliche Komponente zur Geltung. Emotionen geschehen demnach zeitnah, können aber durch Erfahrungen in der Vergangenheit sowie in der Zukunft liegende Ziele kognitiv beeinflusst werden.

Diese drei Versuche, Emotionen zu begreifen, liefern zunächst ein ausreichendes Grundverständnis. Während Meyer et al. eine eher psychologische Perspektive wählen, greifen Gerhards sowie Cordsen und Deilmann bereits sozialkonstruktivistische Aspekte auf, die im Folgenden vertieft werden sollen. 
Denn das Phänomen Public Viewing wird in dieser Arbeit als ein in der Gesellschaft liegendes Geschehnis begriffen. Insofern wird selbige als Bezugsbereich interessant, da Aspekte der Sozialisation und Kommunikation von Emotionen, ihr Zusammenspiel in den Dimensionen von Macht und Status und die Verankerung ihrer Position in Augenschein genommen werden (vgl. Schmidt, 2005, S. 28).

Eine wichtige Hilfestellung zur Ordnung der strukturellen Komponenten bietet Schmidt (2005, S. 26f.) mit der von inm vertretenen Differenzierung der Bezugnahme auf das Emotionsgeschehen in fünf Ebenen:

1. Die neuronale Ebene im Hinblick auf körperliche Grundlagen und Prozesse,

2. die psychische Ebene im Bezug auf das bewusste Erleben von Gefühlen,

3. die kognitive Ebene hinsichtlich des Wissens von Emotionen,

4. die kommunikativ-mediale Stufe im Hinblick auf die Emotionsperformanz in allen ihren Aspekten und die

5. soziokulturelle Ebene, die sich auf den Stellenwert, die Interpretation und Bewertung von Gefühlen im individuellen wie im gesellschaftlichen Bereich bezieht.

Schließlich macht er zwei Hauptbezugsrichtungen aus. Zum einen die Richtung des Körper, des Gehirns, des Bewusstseins und Kognition und zum anderen die der Sprache, der Kommunikation, der Gesellschaft und der Kultur. Wobei hier der Standpunkt von Brown (2000, p. 46) vertreten wird: „This reveals a complex view of emotion which is tied to mind, body, transitional phenomena, unconscious communication and discourse". ${ }^{64}$

Auf der letztgenannten Richtung soll der Schwerpunkt dieser Ausarbeitung liegen, wobei hier wiederum die Gesellschaft, die Kultur und die Kommunikation im Vordergrund stehen werden. Kultur wird nach Lyon (1998, p. 39) als ein komplexes System von Symbolen, Bedeutungen, Kategorien, Modellen oder Schemata re-präsentiert, welches Erfahrungen und Aktionen steuert. In diesem Sinne liefert sie eine zusammenhängende, in unserem Kontext am passendsten erscheinende Definition von Emotionen.

64 Siehe hierzu auch Hochschild (1998) und Williams und Bendelow (1998). 
"Emotion -including feelings, sentiments, motivation, expression, and their representation- is seen from the persperctive to be the product of cultural construction through an individual's socialization and his or her continuing experience in a particular sociocultural context" (Lyon, 1998, p. 40).

Es läßt sich schließlich festhalten, dass aus der großen Menge an soziologischen Perspektiven zum Thema Emotionen ein Bereich extrahiert wurde. So geht es in diesem Zusammenhang im Sinne der sozialkonstruktivistischen Denkweise um Emotionen als Ergebnis und auch als Reaktion auf soziale Konstellationen. Das Erleben von Emotionen wird als subjektiv und als positiv oder negativ beschrieben. Emotionen geschehen zeitnah, können aber durch Erfahrungen in der Vergangenheit sowie in der Zukunft liegende Ziele kognitiv beeinflusst werden. Um dem dieser Arbeit zugrunde liegenden Ausgangspunkt, nämlich dem Verständnis des Public Viewing als gesellschaftliches Geschehnis gerecht zu werden, werden die Komponenten Kommunikation und Kultur im Folgenden schwerpunktmäßig erfasst. Wobei Emotionen generell als multikomplexes System verstanden werden, welches in seiner Ganzheit kaum zu erfassen ist.

\subsection{Emotionssoziologische Erklärungsansätze}

"...The sociologist of emotion does not usually focus on a person's childhood development per se, or on injury and repair, but instead on the sociocultural determinants of feeling, and the sociocultural bases for defining, appraising and managing human emotion and feeling" (Hochschild, 1998, p. 5).

In diesem Kapitel wird dem Gedanken gefolgt, sich auf wenige wichtige Konzeptionen der Emotionssoziologie zu beschränken, um eine Zerfaserung des Gesamtkontextes zu vermeiden. ${ }^{65}$ Zu diesem Zwecke wird im Folgenden zunächst ein Überblick über drei soziologische Emotionsmodelle gegeben. Als Ergebnis soll kein eigenes emotionssoziologisches Modell entstehen, sondern bestehende Ansätze sollen als Basis der Analyse dienen. Hierbei wird das Ziel verfolgt, geeignet erscheinende Arbeiten zu wählen und zur Erklärung des Zusammenhangs von Emotionen und Public Viewing zu nutzen. Die zu analysierende Grundlage bilden die sozialkonstruktivistischen Arbeiten von Kemper (1978) bzw. Gerhards (1988). Kempers Arbeit von 1978 stell einen

65 Gerade im Bezug auf weitere Konzeptionen zum Thema Emotionen aus dem Bereich Soziologie, aber sicherlich auch aus den Gebieten der Psychologie und der Konsumforschung lassen sich an dieser Stelle gute Anknüpfungspunkte für weitere Forschungsarbeit finden. 
Ausgangspunkt dar, der durch die Überlegungen Gerhards, aber auch durch spätere Aufsätze Kempers $(2002,2007)$ und andere Arbeiten um wichtige Komponenten erweitert wird. Riedls (2006) systemtheoretischer Zugang ergänzt die Beispiele um den Aspekt der Kommunikation als strukturelle Kopplung zwischen psychischen und sozialen Systemen.

Gerhards (1988) baut seine Überlegungen auf der 'Social Interactional Theory of Emotions' von Kemper (1978) auf. Dabei sind Emotionen nach Gerhards einerseits als Ergebnisse sozialer Beziehungsmuster zu sehen und andererseits als Konstrukteure von Wirklichkeit. Nach Flam (1999, S. 183) geht Kemper davon aus, mit seinem Ansatz die Lücke zwischen Makro- und Mikrosoziologie zu schließen, weil er Gefühle zum Produkt sozialer Verhältnisse, aber auch zur wichtigen Quelle dieser macht. Es wird z.B. von Flam (2002, S. 134ff.) und Gerhards (1988, S. 124) hervorgehoben, dass Kemper in drei intervenierende Interaktionsebenen Sozialstruktur, Emotionen und Physiologie unterscheidet. Seiner Meinung nach führen soziale Situationen zu spezifischen physiologische Zuständen, die spezifische Emotionen auslösen. Das Zusammenspiel dieser drei Ebenen versucht Kemper in seinen Arbeiten zu erläutern. Im Bereich des Sozialen bilden Macht und Status für Kemper (1978, pp. 26-42) die fundamentalen Dimensionen. Alle Interaktionen zwischen zwei Akteuren können zwischen diesen beiden Dimensionen lokalisiert werden. Aus diesen realen, imaginierten oder antizipierten Stellungen im sozialen Raum resultieren entsprechende Emotionen. Die Machtdimension definiert Kemper (1978, p. 29) als jede Chance, innerhalb einer sozialen Beziehung den eigenen Willen auch gegen Widerstände durchzusetzen, gleichgültig, worauf diese Chance beruht. Riedl (2006, S. 108) schreibt, die Machtdimension kann ein Verhalten des Gehorsams und der Unterordnung erzeugen, welches nicht auf Freiwilligkeit beruht. Auf der anderen Seite steht die Statusdimension für alle Verhaltensweisen, die als generell positiv gesehen werden können, also Haltungen, die als Form der Gewährung von Achtung, Akzeptanz oder Sympathie zu sehen sind. Darüberhinaus sind in der Statusdimension Aktionsweisen integriert, die als gebend, unterstützend, liebevoll usw. bezeichnet werden können, also alle positiv orientierten Verhaltensformen. Flam (2002, S. 150) weist jedoch darauf hin, dass Kemper immer davon ausgeht, dass die Mächtigen in einer Gesellschaft in der Lage sind, die Macht- und Statusdimensionen zu strukturieren. 
Auf dieser Grundlage entwickelt Kemper ein zunächst aus wenigen Komponenten bestehendes Modell. Dieses eignet sich damit für den Einstieg in die Welt der Emotionen. Kemper (2007, p. 96) beschreibt die Konzeption, geht vom idealen Zustand von nur zwei interagierenden Akteuren, als Alter und Ego bezeichnet, aus. Als Ergebnis der Positionen dieser im sozialen Raum und der Bewertung des eigenen und fremden Verhaltens in den Dimensionen exzessiv, adäquat oder insuffizient, resultieren die Emotionen Egos.

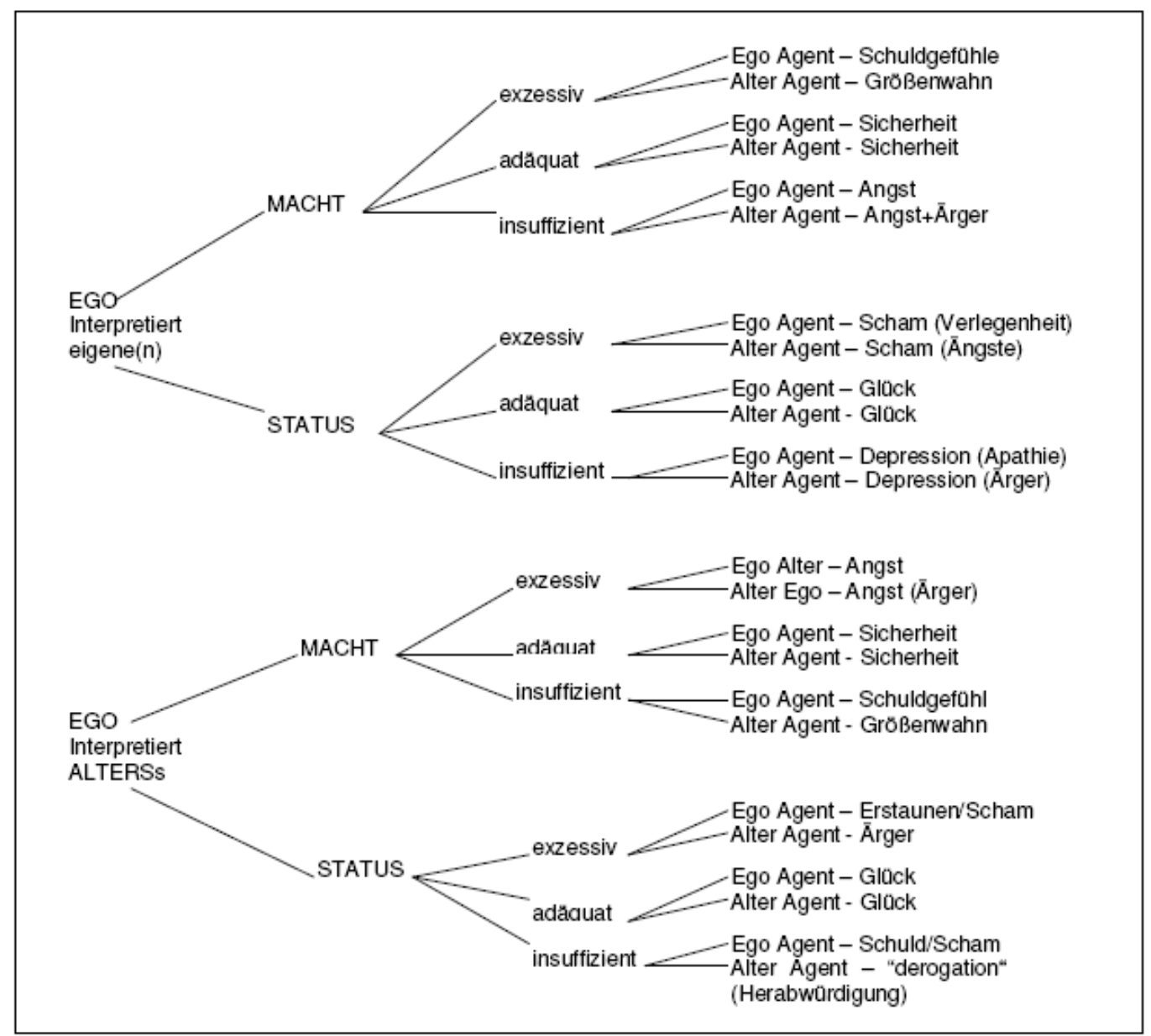

Abb. 8: Strukturelle Emotionen nach Kemper (nach Gerhards, 1988, S. 130)

Gerhards (1988, S. 128) führt aus, dass Kemper diese Grundstruktur des Modells zunächst um einen dritten verantwortlichen Akteur, den er mit 'agency' bezeichnet, erweitert. Dabei kann es sich auch um Ego oder Alter selber handeln, oder es kann sich um ein drittes Subjekt handeln. Wobei der Dritte sowohl ein konkreter Akteur oder auch ein imaginierter Handelnder sein kann. Nach Kemper (2007, p. 97) kann die 'agency' aber auch „the way things are“ sein.

Dieses Muster der Emotionsentstehung lässt sich auf spezifische Situationen im Sport anwenden. Nehmen wir eine Szene im Fußballstadion: Der Zuschauer Ego 
ist eher gelegentlicher Besucher der Spiele des Vereins. Alter dagegen geht regelmäßig zu den Spielen und ist sogar im Fanclub organisiert. Ego besucht nun mit Alter ein Spiel des Vereins in ihrer Stadt, wobei Ego gerne in den erweiterten Kreis von Alters Fanfreunden aufgenommen werden möchte. Alter zeigt sich aber genervt. Ego würde Alter die Agency zurechnen und das Verhalten von Alter als insuffizient interpretieren. Folglich wird sich Ego herabgewürdigt fühlen. Kemper spezifiziert sein Modell noch um weitere Variable, wobei das Konstrukt dadurch schnell an Komplexität gewinnt. Da diese eher der Verwirrung als dem Erkenntnisgewinn zuträglich sind, wird auf die Ausführung weiterer Spezifikationen des Konstrukts verzichtet. Für eine differenziertere Darstellung der 'Social Interactional Theory of Emotions' vgl. besonders Kemper (1978, aber auch 2002, 2007) und Gerhards (1988). Weitere Interpretationen sind bei Flam (2002) oder auch im Band von Turner und Stets (2007) nachzulesen. Peterson (2007, p. 121) schreibt, der Ansatz Kempers würde deutlich zeigen, wie Änderungen in Beziehungen emotionale Reaktionen zur Folge haben. Die in diesem Falle erwarteten emotionalen Reaktionen seien als Resultate der gesellschaftlichen Emotionskultur zu sehen.

Gerhards (1988, S. 139f.) erweitert die Grundstruktur Kempers, indem er zunächst Kritikpunkte anmerkt. Zum einen erläutert er, dass Handelnde nicht automatisch soziale Situationen in den Kategorien Status und Macht erfassen müssen. Emotionen entstünden nicht automatisch aufgrund der Sozialstruktur, sondern es komme darauf an, ob Ego die soziale Situation überhaupt in den vorgeschlagenen Kategorien Macht und Status interpretiert. Zum anderen fehle in der Konzeption Kempers der Gedanke, dass allein aus Gründen der Komplexitätsreduktion die Interpretation von Situationen nicht immer wieder neu geschähe, sondern das spezifische Deutungsmuster bereits existieren. Kemper versuche die sozialen Bedingungen zu rekonstruieren, die verschiedene Emotionen auslösen, übersieht dabei aber laut Gerhards (1988, S. 140ff.) den Bereich der kulturellen Kodierung von Emotionen. Gerhards verdeutlicht diese These am Beispiel einer Trauerfeier. Die Trauer der Gäste lässt sich zwar mit Hilfe der Kemperschen Kategorien aus einer Beziehungssituation heraus erklären, dennoch darf nicht vergessen werden, dass die Anwesenden einer Beerdigung auch Trauer fühlen, weil es kulturell so vorgeschrieben ist. Aus diesen Defiziten heraus erweitert Gerhards die Theorie 
um folgende Punkte;

1. Er misst der Interpretationsleistung eines jeden Akteurs eine eigenständige Größe zu, und

2. integriert er das kulturelle Deutungsschema in seiner Relevanz für Emotio nen in den theoretischen Rahmen.

Er versucht mithilfe der Systeme des Organismus, der Persönlichkeit, des Sozialsystems und der Kultur sich der Entstehung von Emotionen zu nähern. Emotionen werden nach Gerhards (1988, S. 205) definiert als das Ergebnis des Zusammenspiels der vier Ebenen Organismus, Persönlichkeit, Sozialstruktur und Kultur.

Subjekte bzw. psychische Systeme und deren Gefühlslagen und Gemütszustände, die durch wertende Prozesse von Stimuli und Situationen ausgelöst werden, stehen im Mittelpunkt der Diskussion. Gerhards (1988, S. 91) sieht Gefühle nicht eindimensional aus sozialstrukturellen Situationen, Impulsen des Organismus oder kulturellen Normen erklärbar. Diese spielen eine große Rolle, aber Emotionen entstehen erst aus der subjektiven Interpretation dieser Bedingungen durch die Akteure heraus. Weiter führt er aus, dass die Determinante Persönlichkeit aber eindeutig dem Analysezusammenhang der Psychologie zuzuschreiben ist. Deshalb wird sie im Folgenden nicht weiter aufgegriffen. Die zentrale Aussage der Beachtung der spezifischen Interpretationsleistung, die Gerhards dem Cluster Persönlichkeit zurechnet, muss allerdings festgehalten werden. Der Organismus gehört, genauso wie die Persönlichkeit nicht in den Bereich der Soziologie. Er soll hier als Parameter zwar kurz erklärt werden, im weiteren Verlauf der Ausführungen wird er aber nicht weiter betrachtet. So können physiologische Impulse z.B. bei der Entstehung von Emotionen eine Rolle spielen, sie müssen dies aber nicht. Des Weiteren ist es als unabdingbar anzusehen, dass die Impulse mittels einer sinnhaften Interpretation ins Bewusstsein transportiert werden, damit sie als Emotion ins Bewusstsein treten können (vgl. ebd., S. 195f.).

Im Fokus der soziologischen Analyse Gerhards stehen die Sinnzusammenhänge Sozialstruktur und Kultur, was sie von der Ausrichtung her als Grundlage für diese Arbeit geeignet macht. In der sozialstrukturellen Ebene beruft er sich auf die vorgestellte Theorie Kempers, die allerdings durch die genannten Kritikpunkte und die Anmerkungen zum System Persönlichkeit erweitert werden muss. Konkret 
heißt dies, dass nicht Status und Macht soziale Zusammenhänge strukturieren, sondern die Interpretation sozialer Situationen in den Dimensionen Macht und Status. An dieser Stelle muss die entscheidende Modifikation des sozialstrukturellen Modells nach Kemper gesehen werden: Erst die Deutung durch das Individuum entscheidet über die Entstehung der Emotionen.

\footnotetext{
"This powerful process of matching inner experience to a cultural dictionary becomes, for the sociologist of emotion, a mysterious, important part of the drama" (Hochschild, 1998, p. 6).
}

Gerhards (1988, S. 197) führt weiter aus, dass die interpretierte Sozialstruktur durch die genannten physiologischen Determinanten, die kulturelle Definition usw. gedanklich vervollständigt wird. Kultur und Sozialstruktur müssen in diesem Zusammenhang als Gegenbegriffe verstanden werden. In Gerhards (1988, S. 200) Sinn heißt Kultur „ein System kollektiver Sinnkonstruktionen, meint die Schemata der Weltinterpretation, mit denen Menschen Wirklichkeit definieren“. Bezieht man den Begriff der Kultur auf Emotionen, bedeutet es, dass die den Mitgliedern einer Gesellschaft oder Gruppe gemeinen Sinnkonstruktionen auch für die Deutung von Gefühlen gelten.

Hier ist der Begriff der 'feeling rules' 66 zentral.

„Gefühlsregeln sind Deutungsmuster, meist über die Sozialisation vermittelt und über Formen der sozialen Kontrolle stabilisiert, die festlegen, was und wie in welchen Situationen gefühlt und zum Ausdruck gebracht werden soll“ (ebd., S. 199).

Dabei beziehen sich die normativen Emotionscodes auf

1. die Qualität der Emotionen, also die Frage, welche Emotion in welcher Si tuation erwartet wird,

2. die Intensität der Gefühle, d.h., wie stark soll man sich ärgern, traurig sein etc., sowie

3. die Dauer der erwarteten Emotion, ob diese nur situativ oder an eine die Situation überspringende Rolle gebunden ist (vgl. ebd., S. 199).

Es kann folglich gesagt werden, dass das System Kultur auf zwei Ebenen in die Entstehung von Emotionen eingreift. Auf der einen Seite kann Kultur unmittelbar über die genannten Gefühlsregeln intervenieren, und auf der anderen Seite besteht die Möglichkeit eines mittelbaren Einflusses über die kulturelle Kodierung von Sozialstruktur (vgl. ebd., S. 201f.). Nach Gerhards (1988, S. 201f.) kann die 
Kultur und in Verbindung mit dieser auch die Sozialstruktur durch folgende Ansatzpunkte in Form von Gefühlsregeln auf die Emotionen einwirken:

1. Kultur wirkt unmittelbar durch kulturelle Gebote des richtigen und angemessenen Fühlens und des angemessenen Ausdrucks der Gefühle auf die Entstehung des Emotionalen ein. 67

2. Kulturelle Deutungen leiten die Interpretation von Sozialstrukturen an und wirken auf diese Weise mittelbar auf die Entstehung von Emotionen.

Zwischen Sozialstruktur und Kultur lassen sich drei Ansatzpunkte erkennen:

- $\quad$ Kulturelle Normen legen das Maß an Macht- und Statusgebrauch fest. Das heißt, dass sie involviert sind in die Interpretation von Verhalten in den Dimensionen exzessiv, adäquat oder insuffizient.

- $\quad$ Normierte Codes legen fest, ob wir uns die Macht- und Statuskonstellationen in einer bestimmten Situation als selbstverschuldet zuschreiben oder dem Gegenüber dieses zurechnen.

- Positionen und Handlungen können in verschiedenen Kulturen mit divergierenden Macht- bzw. Statusanteilen versehen sein. Somit können Handlungen und Positionen in zwei Gesellschaften das Gleiche bedeuten oder aber etwas anderes ausdrücken und demzufolge andere emotionale Reaktionen auslösen.

Der Einfluss der kulturellen Kodierung von Sozialstruktur lässt sich nach Gerhards (1988, S. 203) weiter u.a. an der kulturellen Definition von Persönlichkeitsentwürfen, dem spezifischen Entwurf von Identität und über die Stellung dieser Identitäten in der Gesellschaft erkennen. Resümiert man die vorgestellten Überlegungen, so stellt sich heraus, dass der Sinnzusammenhang Kultur in vielerlei Hinsicht, durch Gefühlsregeln, die Kodierung von Sozialstruktur sowie die kulturelle Definition von Identität, Einfluss auf die Entstehung von Gefühlen nimmt.

Turner und Stets (2007, p. 39) weisen richtig darauf hin, dass sich die vorgestellte Theorie von Kemper, wie die meisten anderen Theorien, die sich mit Macht und Status befassen, auf einem Mikrolevel bewegt. So ist es nötig, nach Ansätzen Ausschau zu halten, die diese Ansätze weiterführen, aber soziale Faktoren mehr

67 Peterson (2007, p. 115) führt das Beispiel an, dass die Kultur steuere, ob wir eine erhöhte Herz rate in einer bestimmten Situation mit Aufregung oder Angst gleichs etzen. 
integrieren. Gerhards' Erweiterung der Kemperschen Theorie ist hier bereits eine Hinleitung, weitere Anknüpfungspunkte lassen sich u.a. in der soziologischen Systemtheorie finden. So ist auch Riedl (2006, S. 130ff.) der Meinung, dass der Bereich des Sozialen für die Entstehung, Interpretation und Regulierung von Emotionen in psychischen Systemen unabdingbar ist. Es darf aber nicht vergessen werden, dass soziale Systeme für sich keine Emotionen empfinden können, dies können nur die Person selbst, also das psychische System. In der Systemtheorie gibt es jedoch den Terminus der 'Strukturellen Kopplung', und „das Medium der strukturellen Kopplung des psychischen Systems mit dem sozialen System ist Sprache" (ebd., S. 132).

Nach Riedl (2006, S. 133; 2008, S. 233f.) haben soziale Systeme also zwei Möglichkeiten im Bezug auf Emotionen:

1. Sie können über Emotionen kommunizieren. Gefühle sind Inhalt bzw. The ma von Kommunikation.

2. Soziale Systeme können aber auch auf emotionalisierter Kommunikationen basieren. Das meint, dass sich aus der Art und Weise, wie kommuniziert wird und Informationen mitgeteilt werden, Emotionen erschließen lassen.

Tritt (1992, S. 180) hebt hervor, dass Emotionszuschreibungen bei Mitmenschen in der Face-to-face-Interaktion maßgeblich an Prozessen der Sinngebung und bei der Wahl von Handlungsentwürfen beteiligt sein können. Gerhards (1988, S. 90) schreibt weiter, dass non-verbale Kommunikationsformen als die dominanten Kanäle durch die Emotionen ihren Ausdruck erhalten. Zudem könne z.B. zwischen dem Gefühl Freude und bestimmten Gesichtsausdrücken ein Zusammenhang bestehen. Diese sind teilweise kulturell universell.

Was Gerhards (1988) als den Einfluss der Kultur auf die Sozialstruktur und die Emotionen beschreibt, fasst Riedl (2006, S. 135-138; 2008, S. 234ff.) unter dem Oberbegriff der Emotionsregeln zusammen. Als Unterkategorien nennt er Emotionsnormen, Kodierungsregeln, Manifestationsregeln und Korrespondenzregeln, wobei letztere auf untergeordneter Ebene den Kodierungsund Manifestationsregeln zuzuschreiben sind. Unter Emotionsnormen versteht Riedl Regelungen, die festlegen, welche Gefühle für eine spezifische Situation von anderen sozial erwartet werden und aus Sicht des Subjekts angemessen erscheinen. Emotionsnormen erhöhen also die Wahrscheinlichkeit, dass auf eine gewisse Situation bestimmte Emotionen erfolgen und ausgedrückt werden. Riedl 
bietet an dieser Stelle ein Beispiel aus dem Bereich des Fußballs an. Ein rüdes Foul ist nicht nur durch die Spielregeln verboten und wird von den Unparteiischen geahndet, sondern bietet den Zuschauern auch die Möglichkeit, über das unnötig harte Einsteigen des Spielers wütend zu sein. Diese Normen der Emotionen geben also Verhaltensmuster vor, die zur Komplexitätsstrukturierung beitragen. Werden diese wiederholt und auf ähnliche Situationen übertragen, kommt es zur Festigung der Norm. Die Kodierungsregeln und die Manifestationsregeln setzten am Verhaltensaspekt der Emotionen und ihrer entsprechenden Darstellung an. Während erstere bestimmt, welche Verhaltensweisen wie Gestiken, Mimiken, Ausrufe usw. als Expression von Gefühlen gelten, legen die Manifestationsregeln fest, welcher Emotionsausdruck in welchem Maße in welcher Situation angemessen ist. Die Kodierungsregeln ermöglichen es umstehenden Personen, aus dem Verhalten des Subjektes etwas über die momentane Gefühlslage zu erfahren. So ist z.B. Nägelkauen ein verbreitetes Signal für Nervosität. Das Publikum im Sport kann sich also einerseits an sich selber orientieren, andererseits mit Blick auf das Verhalten der anderen Fans oder aber auch auf die Athleten entscheiden, welche Emotionen sich aus deren Verhalten ableiten lassen. Die Manifestationsregeln blicken auf die soziale Situation und kodieren so die zu zeigenden Emotionen sowie ihre Qualität. Das heißt, dass das Publikum anhand dieser Regelungen im Normalfall weiß, dass ein genormtes Ärgern in dem obig beschriebenen Fall toleriert, ja sogar erwartet wird, ein totales Ausrasten allerdings in der Regel als unangemessen erfahren wird.

\footnotetext{
"Each culture has it's unique emotional dicitionary, which defines what is and isn't, and it's emotional bible, which defines what one should and should not feel in a given context" (Hochschild, 1998, p. 7)
}

Unter dem Begriff der Korrespondenzregeln, die Riedl als Unterkategorie der Kodierungs- und Manifestationsregeln einordnet, ist die Steuerung der emotionalen Reaktion auf Emotionen zu verstehen. Für die Zuschauer beim Public Viewing heißt das, dass sie anhand der Korrespondenzregeln wissen, wie sie auf den Jubel ihrer Nationalmannschaft reagieren sollen, nämlich auch mit Jubel. Oder genauso heißt es für die Organisatoren, dass sie wissen müssen, welche Inszenierungsmethoden und -techniken geeignet sind, um die positive Stimmung zu erhalten oder zu verbessern (s. Kap. 10.4 \& 13.2). Natürlich kann es vorkommen, und es ist sogar wahrscheinlich, dass sich diese Normen und Regeln 
zeitweilig überschneiden oder widersprüchlich sind. Sie sind immer vom Kontext abhängig, fast nie eindeutig und direkt übertragbar. So wie hier dargestellt, stellen sie lediglich ein Abstraktum dar, was der Verdeutlichung zuträglich ist. „Emotionen limitieren die Möglichkeiten sozial anerkannter Formen des Emotionserlebens und emotionaler Reaktionen, aber sie determinieren Emotionen nicht“ (Riedl, 2006, S. 138).

In diesem Kapitel ist das Ziel verfolgt worden, aus bestehenden emotionssoziologischen Ansätzen wichtige Erkenntnisse für den Zusammenhang zwischen Emotionen und Public Viewing zu extrahieren. Als Ergebnisse der erörterten Theorien muss festgehalten werden, dass Emotionen ausschließlich multidimensional aufgefasst werden können. Sie entstehen letztlich aus der subjektiven Interpretation eines jeden Einzelnen auf der Basis von kulturellen und gesellschaftlichen Hintergründen. Diese Regeln und Normen stellen die soziale Basis der emotionalen Deutung dar, anhand derer verdeutlicht werden konnte, wie geteiltes Wissen Einfluss auf die Emotionsentstehung und -regulierung beim Sportpublikum nehmen kann.

\subsection{Kollektive Emotionen}

Im Verlauf des vorliegenden Kapitels gilt es das kollektiv geteilte, emotionale Wissen in seiner Bedeutung für Public Viewing zu erörtern. Ein Phänomen, welches gleichzeitig systematisch zur Emotionalisierung der Massen und zu emotionalen Ausbrüchen führen kann. Ausgehend von sozialpsychologischen Konstrukten wie der Theorie der Sozialen Identität oder der Normkonformität wird versucht, sich dem Phänomen Public Viewing in einem makrosoziologischen Rahmen zu nähern. Zusätzlich werden erneut Ansätze der soziologischen Systemtheorie zu Hilfe genommen, um Erklärungsmöglichkeiten für das Zusammenspiel zwischen Emotionen und Public Viewing zu liefern. In diesem Kapitel soll der Frage nachgegangen werden, ob und wie aus geteiltem Wissen kollektive Emotionen entstehen können. Von den Ansätzen der soziologischen Systemtheorie ausgehend, bedarf es einer gemeinsamen emotionalen Wissensbasis, wenn die Kommunikation über Emotionen und auch die emotionalisierte Kommunikation ve rstanden werden soll (vgl. Riedl, 2006, S.140). 
„Es war ein Ausdruck einer tiefsitzenden, gelegentlich wie beim Mauerfall an die Oberfläche kommenden und doch immer wieder gerade in Deutschland enttäuschten Sehnsucht des modernen Menschen nach persönlichem wie landesmannschaftlichem, letztlich kulturellem Halt und Nähe“" (Schulke, 2006b, S. 18).

In einer individualisierten Gesellschaft wird oft von einer großen oder kleinen Gemeinschaft geträumt.68 Dieser kann sich offenbar z.B. im Wunsch nach der zeitgleichen Betrachtung einer wichtigen Begegnung eines internationalen Fußballturniers äußern (vgl. Schulke, 2007, S. 38). Auch die Nachricht vom Tod Lady Dianas schien etwas ausgelöst zu haben: „Globalität oder zumindest eine große Kollektivität war plötzliche ein verbindendes Gefühl“ (Rötzer, 1997). Was ist aber zunächst kollektives emotionales Wissen? Das emotionale Wissen ist das Produkt von Erfahrungen. Dieses Wissensgut kann sowohl aus eigenen Erfahrungen resultieren wie auch aus subjektfremden Wissen. Letzteres basiert aus der Fremdbeobachtung von Mitmenschen und ist auch über ein sprachliches Medium, wie z.B. das Fernsehen, vermittelbar. Gerade das Fernsehen ermöglicht es dem Individuum, ohne persönliche Anwesenheit, dennoch am kulturellen Wissen, welches nicht selten bereits auf Erfahrungen früherer Generationen beruht, teilzuhaben (s. Kap 9.4). Die fremdvermittelten Normen vermischen sich mit den eigenen, bewährten durch die Sozialisation geprägten Einstellungen. Tritt (1992, S. 168) hebt hervor, dass Menschen in einer Generation eines Kulturkreises durch eine ähnliche Sozialisation geprägt und durch eine gemeinsam geteilte Lebenswelt bedingt simultane emotionale Einstellungen haben. Dabei gilt es nach Barbalet (2002, p. 5) zu beachten, dass diese Emotionen keinesfalls identisch sein müssen, denn sie bilden sich in Abhängigkeit der Dimensionen Status und Macht und der Position des Individuums in der Gruppe. Womit an dieser Stelle wieder an die ursprüngliche theoretische interpretative Basis angeknüpft wird, indem nämlich das soziale Entstehen von Emotionen auf die subjektive Interpretation der Situation in den Dimensionen Status und Macht erfolgt.

"Emotion is fundamentally and incontrovertibly an individual level phenomenon. Only individuals experience emotions. Thus, when we speak of group emotion, we can mean only that some aggregate of individuals is feeling something that is sufficiently alike to be identified as the common emotion of the aggregate" (Kemper, 2002, p. 62).

Dies scheint weltweit beim Sport der Fall zu sein. Denn verbindet man den

68 Offenbar (vgl. Kap. 5.2.2) kann dies nicht nur in individualisierten Gesellschaften, sondern in allen Gesellschaften denen es an nationalen Symbolen und Werten mangelt, der Fall sein. 
Gedanken der gemeinsamen emotionalen Wissensbasis mit dem in Kap. 7.2 bereits angeführten Konstrukt der kollektiven Identität bzw. der imaginierten Gemeinschaft, bedarf es einzig der gemeinsamen Nationalität oder des gemeinsamen Erlebens bzw. 'Dabeiseins' bei einem bedeutenden Großereignis, um ähnliche emotionale Einstellungen zu haben.

Wie kann man aber nun das Emotionserleben von kollektiven Identitäten erklären? In der wissenschaftlichen Literatur stößt man zunächst auf die sozialpsychologischen Massentheorien. Bereits Le Bon (1911/1982) ging davon aus, dass erst bestimmte Einflüsse aus Menschen, die sich auf einem Platz versammeln, eine 'psychologische Masse' machen würden. Die Personen müssten noch nicht einmal zur gleichen Zeit am gleichen Ort sein, um sich zu einer solchen Masse zusammenzuschließen. Reize, wie ein großes nationales Ereignis, reichten aus, so Le Bon (1911/1982, S. 10ff.), um aus einzelnen Personen eine kollektive Persönlichkeit 'zu formen'. Allerdings, wie auch verschiedentlich in der Literatur betont, z.B. von Reicher (2001, p. 186), beschäftigte er sich eher mit den negativen Auswirkungen der Masse auf den EinzeInen. So attestierte er der kollektiven Persönlichkeit, die sich aus der Masse herausbildet, negative Eigenschaften wie Beeinflussbarkeit oder Machtgefühle.

Ein weiteres frühes Konzept, welches den Einfluss der Masse ebenso als eher negativ beurteilt, ist die Theorie der Deindividuation (Zimbardo 1969). Es wird davon ausgegangen, dass die Anonymität in der Masse es dem Einzelnen erlaubt, sich anders zu verhalten. Normative Standards, das Eindrucksmanagement sowie die Konsequenzen des Verhaltens werden als weniger wichtig bewertet. Durch das 'Eintauchen' in die Masse verliert der Einzelne ein Stück weit seine eigene Identität. Es entsteht Anonymität und Verantwortungsdiffusion (vgl. Neal, 1993, pp. 94f.; Stroebe et al., 2001, S. 376f.). Es könnte demnach angenommen werden, dass auch das Tragen von Trikots als Uniformierung, als Eintauchen in die Masse verstanden werden kann. Nach Riedl (2006, S. 143ff.) ist dies aber nicht der Fall, denn die Zuschauer haben die Wahl, sich das Mannschaftsshirt überzustreifen. Sie müssen dies aber nicht, anders als es beim Militär der Fall ist. Vielmehr ist davon auszugehen, dass die Besucher dies tun wollen; sie wollen sich und ihr Fantum darstellen und dadurch auffallen. Harney und Jütting (2007, S. 12) betonen sogar, dass die Attraktivität des Zuschauens auf Identifikation beruhen würde und diese damit auf einer Leistung beruhen würde, die weitestgehend 
selbst erbringbar ist. Diese Selbstdarstellung ist nur vor Publikum sinnvoll, d.h. der einzelne Zuschauer sucht unter den anderen Besuchern die Möglichkeit nach Abgrenzung, Eintauchen und Darstellung gleichermaßen. Natürlich sind einige Veranstaltungen mehr für dieses Verhalten geeignet als andere. So ist in einem dunklen Kinosaal die Möglichkeit eher gering, während sich die Logenplätze in einem Stadion sehr gut eignen (vgl. Tedeschi et al., 1998, S. 93). Es macht aber auch einen qualitativen Unterschied, ob es sich um ein Pokalspiel oder eine Weltmeisterschaft handelt. Die Möglichkeit einer solch identitätsstiftenden Verhaltensweise gibt es aber nicht nur im Sport. So sind z.B. auch Opernbesucher auf die Gelegenheit aus, sich vor und nach der Vorstellung sowie in den Pausen darzustellen. Nicht zuletzt aus diesem Grund wird dort ein hoher Wert auf ein gepflegtes Äußeres gelegt. Mit einem Blick auf den Kontext und den weiteren Verlauf der Arbeit lässt sich folgende Aussage von Horne und Manzenreiter (2004, p. 189) bereits an dieser Stelle anbringen:

"Football and sports mega-events in general can present novel social contextes within which produce, display and explore the late modern identity."

Aber zunächst zurück zu den genannten Konzeptionen der Massenpsychologie: Sie beziehen sich zwar hauptsächlich auf die negativen Eigenschaften von Massen und sind eher für die Erörterung von gewalttätigen Ausschreitungen geeignet, dennoch zeigen sie aber, dass es kollektive Emotionen zu geben scheint. In der neueren Literatur, z.B. im Überblick von Reicher (2001) oder in der Zusammenstellung von Klein und Nullmeier (1999), werden die Komponenten Masse, Macht und Emotionen im Rahmen einer politischen Soziologie der Emotionen untersucht. Hervorzuheben ist hier besonders Roth (1999, S. 241f.), der zunächst versucht, den Massendiskurs zu strukturieren. So beklagt er zu Recht, dass heute geläufige Begriffe wie Massenkonsum oder Massenveranstaltung eigentlich nur Merkmale sind, denen eine große Zahl an Individuen folgt, diese würden aber ohne soziale Beziehung zueinander bleiben. Dass dies beim Sport- bzw. Public Viewing-Publikum nicht angenommen werden kann, ist bereits erörtert worden (vgl. Kap. 7.2).

Wenn man die Frage stellt, wie es aber nun dazu kommt, dass das Verhalten einiger weniger von vielen nachgeahmt und übernommen wird, kommt man zwangsläufig zu der Überlegung, dass eventuelle Ansteckungsmechanismen 
existieren. „Das Ganze (...) [ist] mehr als die Summe seiner Teile“; zu dieser Annahme kommt Vester (1991, S. 15). So kann, identifiziert sich der Einzelne in hohem Maße mit dem Ganzen (vgl. Riedl, 2008, S. 238) bei der Partizipation und Kommunikation gemeinsamer emotionaler Normen eine z.T. rasante Ausbreitung beobachtet werden. Dies ist z.B. bei kollektiven Reaktionen eines ganzen Volkes erkennbar. Ein jeder kann sich an die Eigendynamik erinnern, die schlie Blich die Berliner Mauer zum Fallen brachte (vgl. Schmidt, 2005, S. 20). Vester (1991, S. 15) betont, dass aber Protestbewegungen oder Massenaufstände durch spezifische Verzögerungen, aber auch Beschleunigungen gekennzeichnet sind. Wut, Angst oder Traurigkeit sind demnach keine rein privaten Erlebnisse; ihr individuelles Auftreten sowie die Art und Weise ihres Ausdrucks sind abhängig von kollektiven Situationen und Ereignissen sowie von kollektiven Zeichensystemen. Die sich z.B. in Form von Ritualen und Inszenierungen finden (vgl. Riedl, 2008, S. 240). Wie vielfach (z.B. von Neal, 1993, pp. 95f.; Reicher, 2001, p. 192f.; Stroebe et al. 2001, S. 377 [u.a.]) beschrieben wurde, geht die 'Emergente-NormenTheorie' von Turner und Killian (1972) davon aus, dass in Gruppen- bzw. Massensituationen neue Normen entstehen. Die Teilnahme an einer Massenveranstaltungen und die Situation, sich in einer Menschenmenge zu befinden, stellt für den Einzelnen normalerweise etwas Außergewöhnliches und Außeralltägliches dar. Nach Riedl (2006, S. 144) resultiert daraus oftmals eine große Unsicherheit hinsichtlich der zu wählenden Verhaltensweisen, weil die entsprechenden Normen nicht internalisiert oder zumi ndest nicht bekannt sind. So nimmt der Einzelne die neuen gruppeninternen Werte an. Bezug nehmend auf den obig bereits beschriebenen Wunsch nach identitätsstiftender Abgrenzung, Eintauchen und Selbstdarstellung schreibt Neal (1993, S. 109), dass gerade diese Verhaltenswiesen einzelner Zuschauer kollektives Verhalten bedingen könne. Reicher (2001, S. 193) fügt dem hinzu, dass Vorreiter unsicheren Besuchern als Vorbilder und Orientierungsgrößen dienen, deren Verhalten übernommen wird. Während bei normalen Sportveranstaltungen die Verhaltensunsicherheit relativ gering ist, denn Sportwettkämpfe folgen einem festen Rahmen, und die Reaktionen auf spezifische Ereignisse sind weitgehend festgelegt, routiniert und ritualisiert (vgl. Riedl, 2006, S. 144), kann mit Sicherheit angenommen werden, dass eine große Verhaltensunsicherheit hinsichtlich Public Viewing vorhanden war. Das wohl auffälligste Anzeichen dieser Unsicherheit waren die fortwährenden 
Diskussionen der Polizei und Städte mit Wissenschaftlern und Experten sowie Organisatoren über die Sicherheit der TV-Übertragungen. Diese Unsicherheit bzgl. des individuellen Emotionsmanagements verstärkt die Orientierung an Anderen und deren Verhaltensweisen (vgl. Peterson 2007, p. 116) und zuträglicher für die durch Inszenierung vorgegebenen kollektiven Emotionen (vgl. Riedl, 2008, S. 243). Riedl (2006, S. 146) bezeichnet den Prozess der Ansteckung, aus dem ein Gleichlauf des Erlebens und ein Nachahmen von Verhaltensweisen resultiert, im Rahmen der soziologischen Systemtheorie als eine „systeminterne Zustimmung hinsichtlich der eigenen Beobachtungen psychischer Systeme“. Der Prozess bezieht sich folglich auf die Wahrnehmung von Verhaltensweisen anderer und deren Annahme bzw. Ablehnung. Genau in dieser Differenz zwischen Annahme und Ablehnung ist dann die Ansteckung zu sehen. Es ist nach Vester (1993, S.195) davon auszugehen, dass das Überschreiten dieser Schwelle maßgeblich von der Identifikation des Anzusteckenden mit dem Ansteckenden anhängt. Insbesondere in den Situationen, in denen wenig Zeit zur Reflextion bleibt, kommt dem Zugehörigkeitsgefühl eine wichtige Funktion bei der Informationsverarbeitung zu, da es Kontingenz ausblendet. Soziale Nähe und Identifikation sind zentrale Größen für die Wahrscheinlichkeit der Ansteckung. Wie Müller und Scheurer (2004, S. 9) hervorheben, zeigen verschiedenste Untersuchungen, dass viele Erlebnisse nur im Kontakt mit Menschen möglich sind. Beziehungen und Begegnungen mit anderen Menschen sind demnach als wichtige Quelle von emotionalem Erleben anzusehen.

Die genannten Aspekte betrachtend, fällt ein zusätzlicher Faktor auf, der bisher noch nicht erwähnt wurde: Das 'Crowding'. Allein durch die räumliche Dichte der in der Masse eng beieinander stehenden Menschen können sich Stimmungen besser ausbreiten als auf Sitzplätzen im Stadion. Zum Teil sicherlich auch durch die räumliche Nähe bedingt, stecken sich die Zuschauer mit ihrer Performanz der emotionalen Ausdruckgestaltung 69 gegenseitig an und schaukeln sich hoch. Am intensivsten ist dies während eines Live-Wettkampfes oder eines an einen LiveWettkampf heranreichenden Ereignisses mit großer Zuschauerdichte möglich. Laut Cordsen und Deilmann (2005, S. 336f.) ist für einige Zuschauer der emotionale Kick des gemeinsamen Erlebens und der gegenseitigen Aufheizung

69 Gemeint sind hier Gestik, Mimik, Körperhaltung, Stimmverhalten und die Sinnrichtung des kommunikativen Verhaltens. 
und Ansteckung wichtiger als das Spiel selbst. Hier wird also neben den fachlich Interessierten noch ein ganz anderes Publikum angesprochen, nämlich eher die 'sport spectators' bzw. 'sport consumers' (s. Kap. 7.1).

Riedl (2006, S. 147) geht davon aus, dass durch Identifikation mit den Sportlern und den anderen Zuschauern ein kollektiver Denkstil eingenommen wird, aus dem als Reaktion eine gemeinsame Affektlogik entsteht. Diese wiederum steuert dann das synchrone Erleben und Ausdrücken von Emotionen. Denn wie wären sonst Phänomene wie Massenhysterien und Massenpaniken auf der negativen Seite zu verstehen und eben ein kollektiver Freudentaumel in Bezug auf das Public Viewing? Compi (1997, S. 247f.) betont, dass Emotionen nicht nur den sozialen Raum organisieren, also z.B. Teams und Fanclubs zusammen halten würden, in Gut und Böse differenzieren, sondern auch kollektive Gedächtnistüren öffnen und schließen. Da das Erfahren und Encodieren von Gefühlen hauptsächlich im episodischen Gedächtnis geschieht, prägen sich, so Halbwachs (1985, S. 53) und Vester (1993, S. 75), Emotionen besser ein, wenn sie einer bestimmten Situation zugeordnet werden können. An dieser Stelle ist erneut das „Wunder von Bern“ als moderner symbolischer Erinnerungsort zu erwähnen, ein Mythos der das kollektive Gedächtnis des deutschen Volkes geprägt hat (vgl. Kap. 5.2.2). Die Erinnerung an ein Gefühl lässt sich also nicht von der Situation trennen, in der wir es erlebt haben. Eine Möglichkeit, diesen Vorgang der Verbindung von Emotion und Situation zu festigen und wieder abrufbar zu machen, sind Rituale. Eine Zunahme von kollektiven Emotionen ist durch ein gesteigertes gefühlsmäßiges Aufladen von Ereignissen wie bei Zeremonien, Bräuchen oder eben Ritualen möglich (vgl. Riedl, 2006, S. 149). ${ }^{70}$ Riedl (2008, S. 245) bietet ein emotionssoziologisches Modell kollektiver Emotionen an, welches einen makrosoziologischen Theorierahmen für die Entstehung und Regulation von kollektiver Emotionalität wie sie beim Public Viewing anzutreffen ist, veranschaulicht.

\footnotetext{
70 Beim Public Viewing der Fußball-WM 2006 wurde Gebrauch von im Stadion üblichen Ritualen
} gemacht, wie das 'Vorname-Zuname-S piel' oder die 'La-Ola-Welle' (s. Kap. 13.2). 


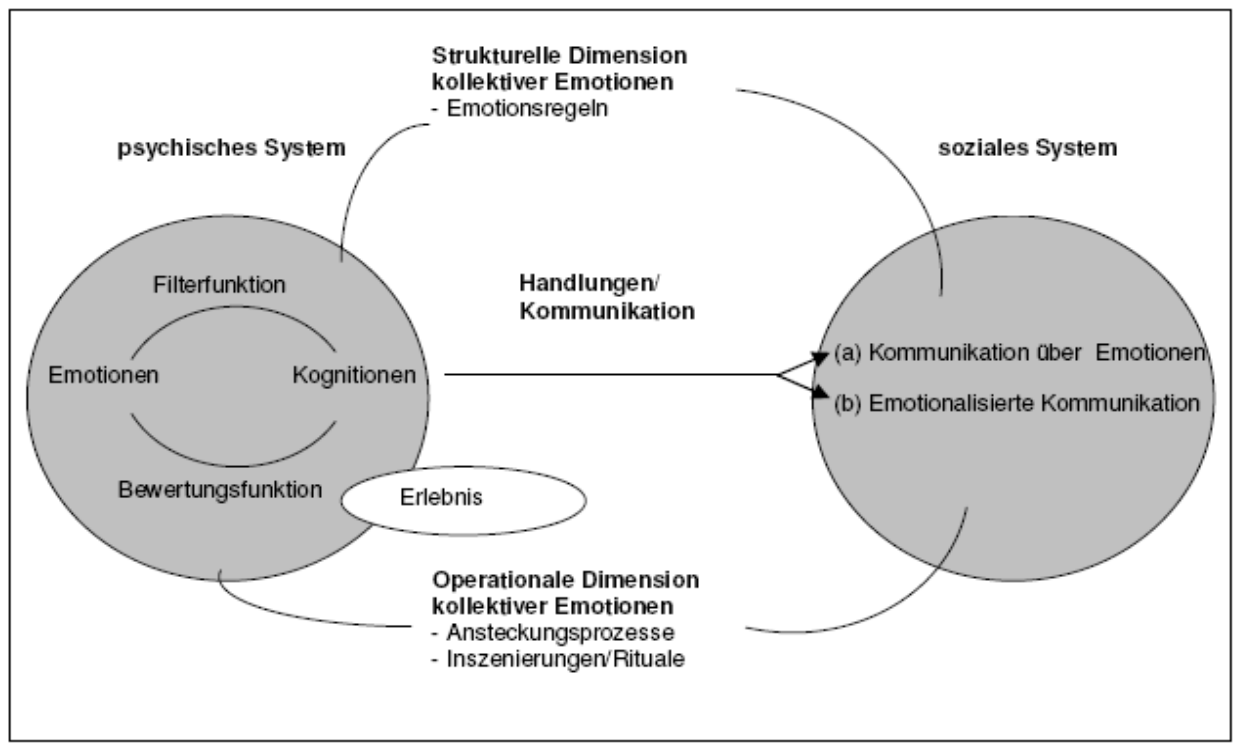

Abb. 9: Kollektive Emotionen in einem emotionssoziologischen Modell (mod if iziert nach Riedl, 2008, S. 245)

Abb. 9 hebt das Zusammenwirken von psychischem und sozialem System in der Entstehung und Regulierung von kollektiven Emotionen hervor. Auf der Seite des psychischen Systems werden Emotionen und Kognitionen bewertet und gefiltert. Als Ergebnis dieses Prozesses, folgen Handlungen bzw. Kommunikation mit dem sozialen System. In diesem wird entweder über Emotionen kommuniziert (a), oder es erfolgt eine emotionalisierte Kommunikation (b). Aus diesen beiden Abläufen ergeben sich zwei Dimensionen kollektiver Emotionalität: die strukturelle und die operationale Dimension. Unter Ersterer versteht Riedl im weiteren Sinne Emotionsregeln, -normen, -codes etc. und unter Zweiterem Rituale, Inszenierungen und Ansteckungsprozesse. Diese beiden Ebenen wirken wiederum auf das psychische System ein und generieren z.B. das subjektive Erleben eines Ereignisses als Erlebnis (vgl. Riedl, 2008, S. 244f.).

Allgemein kann festgehalten werden, dass Emotionen als eine Form der Sinngebung dienen können, da sie sich durch kein anderes soziales System regulieren lassen (vgl. Gerhards, 1988, S. 252). In einer Gesellschaft, der es an Strukturen und gemeinsamen Wertvorstellungen mangelt, können Emotionen diese ersetzen. Denn Gefühle sind das, was authentisch ist, was sich ein Jeder selber zuschreiben kann. Sie sind das Prinzip, das nicht versagt, wenn alle anderen versagen. Oder wie Schulke (2006b, S. 17) es beschreibt: 
„Es ist das suggestive, im individualisierten Alltag nicht mehr vorkommende Gefühl Teil einer unendlich erscheinenden großen Gemeinschaft zu sein - beseelte Empfindungen wie sie bei Kirchentagen, politischen Umbrüchen, bewegenden Turnfesten oder jugendbewegten Musikfestivals gelegentlich auftreten."

In den vorherigen Absätzen haben wir uns mit der Entstehung und Festigung von kollektivem Emotionswissen und seiner Funktionsweise sowie Bedeutung für Public Viewing auseinandergesetzt. Das Publikum als kollektive Identität und die Intention des gemeinsamen Teilhabens an einem Großereignis reichen beim Public Viewing aus, um kollektives Emotionswissen zu generieren. Es kann festgehalten werden, dass es sowohl einer gemeinsamen emotionalen Wissensbasis als auch einem Konglomerat aus verschiedenen psychologischen und soziologischen Prozessen bedarf, die Erzeugung von kollektiven Emotionen zu entwickeln. Zu nennen sind hier beispielhaft Ansätze wie die 'Emergente Normen Theorie' oder das 'Crowding', die über emotionale 'Ansteckungsmechanismen' kollektive Emotionen begünstigen. Besonders bei ungewohnten Ereignissen scheint die Orientierung an anderen Personen ausgeprägt zu sein. Diese determiniert sich durch verschiedengeartete Formen von Kommunikation. So soll im nächsten Kapitel der Frage nach der Rolle der massenmedialen Bindung des Fernsehens mit Emotionen nachgegangen werden.

\subsection{Emotionen im Fernsehen}

Der Fernsehsport ist für viele nicht die zweitbeste Lösung, denn er bietet viele Vorteile. Die Fans müssen sich nicht vor Hooligans in Acht nehmen, sie sind nicht dem schlechten Wetter ausgesetzt oder verpassen das entscheidende Tor, weil gerade jemand in der Reihe vor innen seinen Schirm aufspannt. Sie können strittige Szenen in der Wiederholung noch einmal genauer betrachten, und sie vermögen es, bei allen Spielen der Lieblingsmannschaft dabei zu sein, sowohl im Heimatstadion als auch beim Auswärts- oder Auslandsspiel. Zudem bietet die Vorund Nachberichterstattung über die reine Spielzeit hinaus wichtige Informationen zu den Athleten, Vereinen und zur Sportart. Es werden Experten befragt oder kritische Szenen analysiert. Diese Art der Berichterstattung wird stetig zu den großen Sportereignissen weiter ausgebaut und hat sich sogar zu einem eigenen Formatelement in der Übertragung entwickelt (vgl. Gerhard, 2006, S. 472). Zu diesem gehören eine lang angelegte Vorberichterstattung ebenso wie 
Spielanalysen und Magazinberichte (vgl. Schulke, 2006b, S. 9). Darüber hinaus, so führen u.a. Scherer (2004) und auch Röger (2006) aus, spielen Emotionen eine bedeutende Rolle in der Sportberichterstattung. Sie nennen eine Vielzahl an Faktoren, die dazu beitragen; bspw. die bereits im bisherigen Verlauf dieser Ausarbeitung angesprochene emotionalisierte Berichterstattung der Sportkommentatoren, die Regeländerungen zur Verbesserungen der Telegenität einzelner Disziplinen oder auch die Ambitionen der Ausrichter von Sportveranstaltungen. Diese Form der Übertragung wird inszwischen sogar erwartet. So weist Horak (1995, S. 137) auf eine Erhebung der Österreichischen Gesellschaft für Marketing hin. Sie zeigt, dass $60 \%$ der Besucher von Spielen in der ersten Liga im Raum Wien die Installation einer Großleinwand begrüßen würden. Aus dem einfachen Grund, dass auf dieser Wiederholungen spannender und entscheidener Spielszenen gezeigt werden können.

\footnotetext{
„Medien repräsentieren und erzeugen Emotionen, sie verändern die Ausdrucksform und Intensität von Gefühlen, und sie produzieren Diskurse über Emotionen" (Bösch \& Borutta, 2006b, S. 9).
}

Es ist demnach unerlässslich, in diesem Kapitel über die Soziologie der Emotionen auch das Massenmedium Fernsehen in seiner Bedeutung für Public Viewing miteinzubeziehen. Im Zusammenhang mit großen internationalen Ereignissen, auch des Sports, sind die Medien ein wichtiger Faktor. Bei dem Sieg der deutschen Nationalelf 1954 war das Radio noch das Medium für die Massen (vgl. Oswald, 2006), heute ist es das Fernsehen. Der Fernsehsport, und der Fernsehfußball ganz besonders, bieten dem Zuschauer also mehr Informationen an, als im Stadion gegeben werden. Diese vermehrten Informationen steigern als Konsequenz auch das gemeinsame kollektive Wissen und dadurch die Menge an emotionalem Kommunikationspotential bei den Besuchern. Riedl (2006, S. 62) betont, dass das Ereignis mit seinen strittigen Szenen, schönen Momenten und tollen Toren somit in der Face-to-face Kommunikation wie auch in den Massenmedien wieder und wieder Thema gesellschaftlicher Konversation wird. Damit wäre es in der öffentlichen Meinung präsent und somit auch in der Gesellschaft existent. Dayan und Katz (2001, S. 427) ordnen dem Fernsehen eine starke Position zu. Sie gehen davon aus, dass es die Aufmerksamkeit der Rezipienten von den expliziten Aussagen auf die visuellen Aspekte des Geschehnisses verschiebe. Das Fernsehen, so führen sie weiter aus, 
„naturalisiere“, es bezeuge die Echtheit der Botschaften, verwandele die Aspekte von Ritualen in visuelle Charakteristika, die von den Kameras aufgenommen würden. Alfes (1995, S. 169) fasst die Rolle der Medien wie folgt auf:

„[M]ithilfe der Medien werden einerseits Gefühls-Konventionen und -Schemata aktualisiert, thematisiert und gefestigt. Andererseits liegt in der Vielfalt an Darbietungsmöglichkeiten die Chance, Gefühls-Konventionen zu variieren, zu erweitern und in Frage zu stellen."

Nach den in Kap. 9.2 evaluierten Emotionsregeln erlernt jedes Individuum im Laufe der Sozialisation Regeln und Normen, die bestimmte soziale Situationen mit den erwarteten sozialen Emotionskategorien verbinden. Am Beispiel Friedhof wird das Beschriebene deutlich sichtbar, denn einen Friedhof besetzt man automatisch mit Trauer. Ebenso wird erlernt, welche Bandbreite an Performanzmodi ${ }^{71}$ sozial akzeptiert ist, d.h. wie sehr darf Freude über die Niederlage des verhassten Nachbarvereins gezeigt werden, und was würde hier unangemessen oder übertrieben wirken?

Schmidt (2005, S. 11, 29f.) weist darauf hin, dass emotionalisierte Performanzmodi uns täglich über die Medien angeboten werden. Die Medien böten uns eine Orientierung, ein emotionalisiertes Wirklichkeitsmodell an. Diese Leistung der Kodierung und Schematisierung von kollektivem emotionalem Wissen, welche früher in großem Maße Literatur und Theater geboten haben, finden wir heute in den Medien wieder. Sie bieten sowohl für die Selbst- als auch für die Fremdbeobachtung eine Orientierung, welche die Beobachtungsmöglichkeiten erhöht und sich sowohl auf die Emotionssozialisation als auch auf die Zeitgeist- bzw. Mentalitätsgestaltung auswirke. Nach Diederichsen (1999, S. 331) teilt das Massenereignis den Beteiligten etwas mit, was in bestimmten Phasen den weiteren Verlauf einer Szene oder Kultur mitgestaltet. Die Masse hilft den an ihr Beteiligten in erster Linie zu einem „sozialen, politischen, generationsspezifischen oder anderweitig überschriebenen kulturellen Selbstbild“. Rötzer (1997) könnte dem hinzufügen, dass das Objekt der Aufmerksamkeit von den Medien als kollektivem Aufmerksamkeitsorgan generiert wird. Dabei darf dieses, um als "Trojanisches Pferd“ zur Anfechtung von kollektiven Emotionen zu wirken, nicht zu sehr gegen mentale Abwehrkräfte

71 Mit Performanzmodi sind die „konkrete Realisierung von Ausdrücken in einer bestimmten Situation" (Schmidt, 2005, S. 24) gemeint. 
verstoßen und muss mehr oder weniger neutral sein. Dies trifft bei der Gutherzigkeit der Lady Diana ebenso zu wie bei einem Kirchentag, einem Popkonzert oder eben einer Fußball-Weltmeisterschaft. Würde es in dieser Arbeit um politische Phänomene gehen, müsste der Ansatz ein völlig anderer sein. Auch Dayan und Katz (2001, S. 227) betonen in der Tradition des 'DynamischTransaktionalen-Modells' (s. Kap. 7.1), dass das Fernsehen das Publikum zu aktiven Partnern in der Interpretation von Ereignissen mache. Es stachle das "hermeneutische Vergnügen bei der Entzifferung von Hinweisen und der Suche nach 'Symbolen' an“. Medien sind nach Gerhards (1988, S. 96) -und hier beruft er sich auf Niklas Luhmann- Zusatzeinrichtungen zur Sprache und damit der Kommunikation. Sie stellten Spezialsprachen dar, die die Übermittlung von komprimierten Überlegungen ermöglichten. Immer dann, wenn Akteure ihr Handeln an den symbolisch generalisierten Medien orientieren, können sie ein hochselektives Regelsystem benutzen, ohne dies selbst thematisieren zu müssen. Alfes (1995, S. 169f.) gibt an dieser Stelle allerdings zu bedenken, dass es in bestimmten Situationen vorkommen kann, dass wir persönliche Einschätzungen und Empfindungen vernachlässigen. Seiner Meinung nach bestünde in der Rezipientensituation ohnehin eine begrenzte Einwirkungsmöglichkeit. So kann tendenziell emotionale Individualität passiviert und emotionales Stereotypenverhalten akti viert werden. Die sozial schematisierten Performanzmodi ermöglichen und sichern die (Wieder-)Erkennbarkeit von Emotionen. Schmidt (2005, S. 24, 31) schreibt, dass Massenmedien mit Inszenierungsformen von Emotionen in diesen Prozess eingreifen. Allerdings ist Medium nicht gleich Medium. Medien eignen sich unterschiedlich stark für emotionale Kommunikation. Die spezifische Medientechnologie ist aus dem Grund bedeutend, weil sie den Gefühlen in gewissem Maße einen "wahrnehmbaren und erlebbaren Körper" gibt, sowie ihr Verhältnis in Raum und Zeit aufzeigt. Cordsen und Deilmann (2005, S. 337) betonen, dass es für emotionale Kommunikation in Performanzmodi unbedingt wichtig ist, dass bestimmte Körperhaltungen, symbolische Handlungen wie das Zeigen des 'Stinkefingers' oder stilisierte Jubelszenen von Sportlern und Zuschauern beobachtet und mit der eigenen Gefühlsregung verglichen und bewertet werden. Mimik, Gestik und Körperbewegungen sind die wichtigsten Projektionsflächen für Emotionen. Diese sind aber nur in Film und Fernsehen beobachtbar. Somit haben sich selbige zu einer wichtigen Instanz in der 
Sozialisation von kollektivem emotionalem Wissen etabliert. Das Publikum vor dem Fernseher sehe nicht nur mehr, weil der Blick auf das Geschehen frei sei, so Dayan und Katz (2001, S. 434), sondern auch weil das Fernsehen die von seinen Organisatoren für das Geschehen vorgeschlagene Definitionen unterstreiche und zusätzlich interpretiere. Der TV-Rezipient wisse „weit mehr von der symbolischen Bedeutung des Ereignisses als das primäre Publikum vor Ort". Schmidt (2005, S. 35) führt aus, dass körperlich ausdrucksstarke Gefühle wie Wut oder Freude am besten in audiovisuellen Medien darstellbar sind. Unterschiedliche Medientypen sind folglich in Bezug auf ihre emotionale Nutzbarkeit mit spezifischen Erwartungen verbunden, diese Annahmen resultieren aus den bisherigen Erfahrungen der Zuschauer mit entsprechenden Medienangeboten. Im Zuge der Aufmerksamkeitsökonomie schmematisieren und stereotypisieren Medien Emotionen, was sich z.B. in der Prägung von Prototypen wie Helden oder Stars äußert.

Im Bereich des Sports, so beschreiben Schauerte und Schwier (2004, S. 169), ist im Zusammenhang mit den Handlungen der Sportler von mitreißenden Szenen, Kabinettstückchen oder tragischen Helden die Rede. Darüber hinaus würden ganze Wettkämpfe als Krimis, Dramen oder Horrorszenarien beschrieben. Hackforth (2001, S. 37) betont, dass die emotionale Inszenierung oder Initiierung des Sports in den Medien im Moment Konjunktur hat. Auch in den großen Arenen, so Schulke (2006, S. 20), wird die Tendenz zu Videowänden sichtbar. Um auch den Zuschauern in den hinteren Rängen die Möglichkeit zu geben, das Spielgeschehen bis ins Detail mitzuverfolgen, setzen die Betreiber auf die Wiederholbarkeit von strittigen Szenen in Slowmotion o.ä., so können Zuschauer die flüchtige Wahrnehmung festhalten, wiederholen, vertiefen, interpretieren und diskutieren. So haben, äußert sich Henecka (2000, S. 67f.), die Medien die Wirkung von Emotionen erkannt und es mache den Anschein, dass nichts mehr ohne Gefühle zu gehen scheine. Sie würden in der Politik genauso instrumentalisiert wie eben auch im Sport. Henecka führt weiter aus, dass durch den Bedeutungsverlust ehemals allgemeingültige Institutionen ihre ursprünglichen Funktionen anderen Institutionen übertragen werden. Allerdings könne ihre normative Regulierungsfunktion auch aufgrund des gesellschaftlichen Wertewandels im Bewusstsein der Gesellschaftsmitg lieder an Gültigkeit einbüßen. Keilbach und Stauff (2006, p. 176) sind der Meinung, dass eben die 
Sportberichterstattung so eine 'Ersatzinstitution' bieten könne. Sie schreiben, dass die soziale Funktion des Fernsehsports nicht auf die Übertragung oder das Hervorbringen von Sportereignissen reduziert werden dürfe:

"Any consideration of the role of television in society or of the gerneral workings of a highly medialized public sphere find in televiced sport a central reference point. Central because it garantees both widespread comprehension and passion, and moreover because it (more than any other broadcast content) affects almost everyone in some form or another."

Aus diesem Grund, so wird vielfach betont, würden die Diskussionen um die Reichweite des Sports im Fernsehen oft repräsentativ über das Fernsehen und die Gesellschaft gehalten.

In der Postmoderne bleiben den Menschen nur zwei Möglichkeiten: Entweder sie ziehen ihre Emotionalität in den privaten Raum zurück, oder sie bringen diese in die Öffentlichkeit. Infront (2006b) sieht Public Viewing als „sozialen Trend“, der die Menschen zusammenbringt, um mehr Vergnügen aus der Erfahrung wichtiger Spiele über Großleinwände zu ziehen. Rötzer (2006) sieht Public Viewing als einen „kollektiven Rausch“ an. Es komme also nicht zu einer Vereinsamung der Menschen vor ihren Bildschirmen, denn die in den öffentlichen Raum gerückten Bildschirme erwiesen sich als „Katalysator eines Masse nrausches“.

Schmidt (2005, S. 30) beschreibt, dass soziale Wandlungsprozesse wie Individualisierung und Wertewandel das Menschen- und Gesellschaftsbild verändern. Dies wiederum hätte starke Effekte auf die Legitimierung, die Interpretation und Performanz von Emotionen, sowohl im privaten wie im öffentlichen Bereich. Welche Rolle aber nimmt das Fernsehen in diesem Zusammenhang ein? Dayan und Katz (2001, S. 417f.) gehen davon aus, dass das Fernsehen zunächst die Reaktion der bei dem Ereignis selbst anwesenden Zuschauer hervorhebt. Diese könnten von dem Publikum zu Hause als Verhaltenshinweise angenommen werden. Das Ereignis sollte demnach im Idealfall so dargestellt sein, dass es die gesamte Gemeinschaft angemessen repräsentiert. Wie Dayan und Katz weiter ausführen, würde allerdings das Fehlen der primären Zuschauer im Stadion das Fehlen an Verhaltensvorschlägen bedeuten und die Zuschauer zu Hause sich selbst überlassen. Diederichsen (1999, S. 330) schlussfolgert, dass die spektakuläre Massenhaftigkeit genau den technischen und sozialen Vorrichtungen geschuldet sein kann, die sie dann wieder als massenkulturelle Bilder in die Haushalte senden. Sei es, dass diese neuen 
Massen die natürlichen, altäglichen, zufälligen Massen im öffentlichen Raum kompensieren oder elektronische Bilder neue Massenhaftigkeit erst ins Bild und damit in die Wirklichkeit bringen.

Das Fernsehen ist somit nach Klein, Nullmeier und v. Wersch (1999, S. 10) in der Lage, die Emotionen, die mit neuen kulturellen Phänomenen einhergehen, zu vermassen. Emotionale Geschehnisse mit großer kollektiver Erregung wie die Love Parade oder der Tod von Lady Diana, würden öffentlich verwertet und unterliegen einer Vermassung - Emotionen würden demnach reproduzierbar gemacht. Rivenburg (2003, p. 32) dagegen sieht das Fernsehen zunächst nur in der Rolle eines Erzählers emotionaler Geschichten. Es schmückt die Geschehnisse aus, z.B. als historisch oder als ehrenhaft. So würden sie mit dominanten oder idealisierten Werten verbunden. Weiter führt auch er allerdings aus, dass Medienereignisse, wie eben auch Fußball Welt- und Europameisterschaften, über die Probleme und Alltägliches hinausgingen. Als ein Ergebnis könnten sie die Möglichkeit haben Menschen zu integrieren und zu vereinen. Sie können eine Stimmung hervorrufen, die neue 'Dinge' möglich mache. Ein solches Charakteristikum von Medienereignissen sehen Stiehler und Friedrich (2003, S. 55f.) in der Anpassung des Tagesablaufs. Die Zuschauer würden ihre Altagsroutinen dem Ereignis anpassen. Harney und Jütting (2007, p. 13) betonen, dass das Massenpublikum durch die Massenmedien sich selbst zusähe und dadurch selbst zum Bestandteil der Szenen und Narrationen würde. Es rege sich dadurch selbst, in Form einer Selbstreferentialität, an, so die Autoren. Nun hat die technologische Entwicklung der Medien dazu geführt, dass sich Einstellungen zu Raum und Zeit ändern. Zum Beispiel das Internet, aber auch die LED-Technik eröffnen neue Möglichkeiten, mit denen sich die Wahrnehmung, der Ausdruck und die Einschätzung von Emotionen in technisch definierter Kommunikation ändern (vgl. Schmidt, 2005, S. 30). Während Ciompi (1997, S. 245) noch davon sprach, dass eine Fernsehübertragung vom emotionalen Geschehen bei einem Ereignis höchstens einen „blassen Abklatsch“ zu übermitteln in der Lage sei, wird von Rötzer (2006) betont, dass die Medien die Massen "massieren“, sie in Schwingungen versetzen und Konformität erzeugen. Nach Alfes (1995, S. 169) unterstützen Medien also permanent emotionale Kommunikation, indem sie mit dem Wissen um prototypische Gefühle emotionale Bedürfnisse wecken. Sie bieten emotionalisierte Gefühlsklischees an und 
stereotypisieren Verhalten. Somit versorgt das Fernsehen seine Ereignisse mit Narration, mit einer Perspektive und mit einem Inhalt (vgl. Dayan \& Katz, 2001, S. 442).

Kann es denn aber eine kollektive Konformität in Form von Public Viewing geben, wenn das Publikum dispers ist? Dayan und Katz (2001, S. 451f.) führen in diesem Zusammenhang den Begriff der „Mikro-Ereignisse“ ein. Durch diese in den einzelnen Haushalten erfolgende Übersetzung eines großen Ereignisses würde dieses in seiner zeremoniellen Form bestätigt (vgl. auch Röger, 2006). Das kann nur be-stätigt werden, denn sonst würden in Deutschland am 24. Dezember in den Wohnzimmern nicht Weihnachtsbäume aufgestellt und Geschenke verteilt. Solche 'Mikro-Ereignisse' bauen auf dem Prinzip des gemeinsam geteilten emotionalen Wissens auf. Diese beruhen wie aber wieder auf der Erwartung, dass es andere Personen gibt, die dieses auch tun, also auch dieser imaginierten Gemeinschaft angehören, und dass ihre Erwartungen ihrerseits bestätigt werden. Dies geschieht, ohne dass sich die beiden Parteien direkt kennen müssen. Diese Ausführung lässt die Überlegung zu, ob sich nicht Public Viewing-Publika genau auf diesem Wege zusammenfinden. Denn der Fernsehzuschauer, wie der Stadionbesucher, ist sich sicher, dass zur gleichen Zeit tausende Anderer das gleiche Spiel verfolgen. Die permanente Fremdbeschreibung der Fan Feste durch die Medien könne diesen als „Symbol für die vorgestellte Gemeinschaft“ (Riedl, 2006, S. 170) dienen.

Folglich lässt sich festhalten, dass Medienereignisse, wie z.B. eine FußballWeltmeisterschaft, Gegenstand der täglichen individuellen und massenmedialen Kommunikation sind. Aus dieser Mehrinformation resultiert ein gesteigertes kollektives Wissen und somit die Menge an emotionalem Kommunikationspotential bei den Rezipienten. Insbesondere das Fernsehen ist in der Lage, Gefühlsregeln auch vor dem Hintergrund des durch soziale Modernisierungsprozesse geänderten Gesellschaftsbildes zu thematisieren, zu festigen und zu aktualisieren. Geschehnisse mit großer kollektiver Emotionalität „massieren die Massen“ (Rötzer, 2006) und sind in der Lage, durch Mikro-Ereignisse aus dispersen Publika imaginierte Gemeinschaften herzustellen. 


\subsection{Zusammenfassung}

Dieses Theoriekapitel hat sich mit der Bedeutung von Emotionen für das Phänomen Public Viewing beschäftigt. Dazu ist zunächst der Relevanzhorizont auf vornehmlich soziologische Theoriebausteine eingegrenzt worden. Hier wiederum wurde der Schwerpunkt auf sozialkonstruktivistische und systemtheoretische Denkansätze gelegt, wobei Emotionen generell als multikomplexes System verstanden werden, welches in seiner Ganzheit kaum zu erfassen ist. Sie werden hier als aus der subjektiven Interpretation und Sozialisation eines jeden Einzelnen auf der Basis von kulturellen und gesellschaftlichen Hintergründen heraus, aber auch von zukünftigen Zielen und Wünschen abhängig, verstanden. Dieses geteilte kollektive Wissen in Form von Emotionsregeln wird im Rahmen von außergewöhnlichen Ereignissen allein durch das Wissen um die imaginierte Gemeinschaft und die Intention gemeinsam an diesem Ereignis teilhaben zu wollen kommuniziert. Insbesondere das Fernsehen besitzt in diesem Zusammenhang zum einen eine Multiplikatorfunktion, indem es Medienereignisse zum Gegenstand emotionaler Kommunikation macht. Zum anderen kann es aber auch vor dem Hintergrund des durch soziale Modernisierungsprozesse geänderten Gesellschaftsbildes Emotionsnormen thematisieren, festigen und aktualisieren. Geschehnisse mit großer kollektiver Emotionalität wie Public Viewing werden durch das Fernsehen massiert und können so durch viele MikroEreignisse aus dispersen Publika imaginierte Gemeinschaften herstellen. 


\section{Suche nach und Inszenierung von Erlebnissen}

Im Rahmen des vorliegenden Kapitels wird diskutiert, inwiefern es möglich ist, die Entstehung von Emotionen durch die Inszenierung von Erlebnissen zu unterstützen. Für die Erörterung der Suche des Einzelnen nach Erlebnissen scheint es hilfreich, einen näheren Blick auf das Verhältnis von Gesellschaft und Erlebnis zu werfen. Die veränderten gesellschaftlichen Rahmenbedingungen führen zu dem Wunsch, hedonistische Bedürfnisse auszuleben. Das Subjekt sucht als Umkehrschluss auf die sozialen Wandlungsprozesse besonders in der Freizeit nach Befriedigung innenorientierter Wünsche und wählt seine Aktivitäten nach den eigenen Bedürfnissen aus (10.1). Individuen sind bestrebt, sich bei fehlenden äußeren Strukturen nach sich selbst also nach innen und den persönlichen Empfindungen bzw. Emotionen zu richten. Somit eröffnen sich vor allem für Anbieter von Freizeitangeboten neue Möglichkeiten, Gäste zu gewinnen und zu binden. Das Erlebnis wird in der Erlebnisökonomie als Endglied der Differenzierung von Produkten gesehen, denn die Besucher erwarten im Tausch gegen ihr Eintrittsgeld und ihre Anwesenheit ein Erlebnisangebot. Mit der Angebotsanreicherung durch Erlebnisse werden Chancen gesehen, sich auf dem Markt abzugrenzen (10.2). Vor dem Hintergrund dieser Ausarbeitung wird sich der Frage gewidmet, wie es machbar ist, die bestmöglichen Vorraussetzungen für die Wahrscheinlichkeit des Entstehens von positiven Emotionen bei einer Public Viewing-Veranstaltung zu schaffen. Zu diesem Zweck wird auf das ursprünglich für den Tourismus entwickelte Konzept des Erlebnis-Setting zurückgegriffen (10.3). Die in diesem Modell verankerten Inszenierungsinstrumente werden erörtert und auf ihre Tauglichkeit hinsichtlich der Realisierung bei sportlichen Fernseh-Vorführungen diskutiert. Anhand von verschiedenen Inszenierungsmöglichkeiten werden z.B. die Schaffung günstiger Rahmen-bedingungen und der Aufbau eines Spannungsbogens erörtert (10.4). In einem kurzen Exkurs wird abschließend eine der höchsten Stufen dieser Entwicklung, nämlich die Eventisierung von Sportveranstaltungen und die Rolle des Public Viewing in diesem theoretischen Rahmen, analysiert (10.5). Den Abschluss dieses Abschnittes bildet ein Resümee mit der Einordnung der gewonnenen Erkenntnisse in den Kontext der Arbeit (10.6). 


\subsection{Er lebnis und Gesellschaft}

Anfang der 1990er Jahre deklarierte Gerhard Schulze als eine Folge des Übergangs zur Überflussgesellschaft die Erlebnisgesellschaft. Es wird vielfach (vgl. u.a Bachleitner, 2004; Heinemann, 2007, S. 325; Köck, 2005, S. 6.) davon ausgegangen, dass in dieser Gesellschaftsform verschiedene und individualisierte Formen des psychischen und physischen Genusses das soziale Leben dominieren. Mögliche hedonistische Lebensweisen, in denen das Leben im Hier und Jetzt generell wichtig ist, nehmen in den Gedanken von Schulze zur Erlebnisgesellschaft einen wichtigen Platz ein. Dabei wird in der wissenschaftlichen Literatur (s. Kap. 6) die Meinung vertreten, dass der Sport mit seinen vielfältigen Möglichkeiten des Erlebens, der Selbstverwirklichung und damit der Vermittlung eines "unmittelbaren Lebensgefühls" eine strukturgebende Bedeutung haben kann. Im Folgenden wird die Schere der theoretischen Erklärungsansätze weiter geöffnet. Es gilt die Rolle der erlebnisorientierten postmodernen Gesellschaft für das Phänomen Public Viewing auszuloten.

Schulze (1997, S. 15) bezeichnet sein theoretisches Modell als „ein graduelles Prädikat, das die im historischen und interkulturellen Vergleich relativ große Bedeutung von Erlebnissen für den Aufbau der Sozialwelt" hervorhebt. Vielerorts wird darauf hingewiesen (vgl. Opaschowski, 2000, S. 11-31, 2001; Pfaff, 2002a, S. 28; Scheurer, 2003, S. 50-53 [u.a.]), dass die gesellschaftlichen Rahmenbedingungen der westlichen Industrienationen den Hang zur Erlebnisorientierung verstärken. Neben der gestiegenen Lebenserwartung wird bspw. die damit einhergehende prozentual geringere Lebenszeit genannt, die mit Arbeit verbracht wird. Deutlich ersichtlich ist auch die Verschiebung des Stellenwertes der Arbeit. Die ehemals hohe Wertschätzung der Arbeit scheint -so wird betont- zu Gunsten von Freizeit und Konsum in den Hintergrund zu rücken. Die traditionellen Milieus lösen sich auf, deshalb sucht man nach neuen Orientierungspunkten. Schulze (2007, S. 310) geht von der Leitthese aus, dass die Gesellschaft in „kollektiven Lernschritten auf einem Pfad der Subjektorientierung" vorangeht. Die Subjektorientierung rühre von der seit den fünfziger Jahren anhaltenden Steigerung der Möglichkeiten her, die sich einem jeden von uns bieten. Der Lebensmittelpunkt der Menschen sei weniger von äußeren (über-)lebenswichtigen Handlungen bestimmt. Dies lasse die Aufmerksamkeit immer weiter von außen 
nach innen wandern, so Schulze. Das Individuum orientiere sich also nach innen, ziehe die eigenen Wünsche und Bedürfnisse zur Befriedigung heran (vgl. auch Weiermair, 2006, S. 14).72 Bachleitner (2004, S. 16) sieht die Erlebnisorientierung als Folge des Individualisierungsprozesses der postmodernen Gesellschaft. Erlebnisse würden dem Einzelnen als emotionale Qualitäten in Aussicht gestellt, die letztlich eine Anwort auf das Massendasein der Postmoderne darste lle.

Können nun aber emotionale Erlebnisse dem Einzelnen einen Ersatz für ehemals äußere Wegweiser wie Schichtmitgliedschaft, Beruf, Familie oder regionale Zugehörigkeit bieten? Können emotionale Erlebnisse sozialstrukturelle Orientierungshilfen bieten?

Schulze (1997, S. 54) definiert die Erlebnisgesellschaft als

"Gesellschaft, die (im historischen und interkulturellen Vergleich) relativ stark durch
innenorientierte Lebensauffassungen geprägt ist. Die Untersuchung der
Erlebnisgesellschaft zielt auf Gemeinsamkeiten ab, die sich unter dem Einfluss
innenorientierter Lebensauffassung entwickeln."

„Erlebnisgemeinschaften“, so schreiben Müller und Scheurer (2004, S. 3), die sich etwa für den Besuch eines Public Viewing verabreden oder sich dort erst kennen lernen, fungieren als die neuen Orientierungspunkte (vgl. auch Grötsch, 2006, S. 61ff.). Soziale Bedürfnisse und Erlebnisse haben also eine ganz neue gesellschaftliche Bedeutung erhalten (vgl. ebd. S. 51; Weiermair, 2006, S. 16). Auch Wöhler (2004, S. 221) sieht die Bindung des Individuums an die gesellschaftlichen Strukturen mittels Erleben. Die heutige Gesellschaft lebt erlebnisorientiert. Schulze (1997, S. 41f.) betont, dass das Bedürfnis nach Erlebnissen der Oberbegriff für die umfassende Einstellung ist. Dieses impliziere kleine Alltagsfreuden genauso wie den Konsum expliziter Erlebnisangebote. Er könne aber auch weit gefasst wie eine Lebenseinstellung gemeint sein kann. Erlebnisse sieht er nicht bloß als Begleiterscheinung des Handelns, sondern als dessen hauptsächlichen Zweck an. Dies geschehe, so führt er weiter aus, als Gegenstand der Handlungsplanung, in voller Absicht des Individuums. Schulke (2007, S. 46) sieht den Public Viewing-Besucher als ein Art feierfreudigen, dem emotionalen Erleben zugewandten Weltbürger, der offen gegenüber neue Medien ist und den die ungezwungene, nicht bindende Atmosphäre eines offenen Marktplatzes anspricht.

72 Grötsch (2006, S. 51) kreiiert in diesem Zusammenhang den Begriff 'Selfbewusstsein'. 
Scheurer (2003, S. 86) gibt an, dass die verstärkte Erlebnisorientierung besonders im Freizeit- und Reiseverhalten zutage tritt, da diese Bereiche durch einen hohen Grad an Handlungs- und Entscheidungsfreiheit definiert sind. In der Freizeit steigen hedonistische, auf innere Bedürfnisse gerichtete Werte, wie Spaß mit anderen gemeinsam erleben, Genuss und die Freude an Sozialkontakten (s. auch Abb. 5). Zudem reicht der Erwerbslohn bei vielen immer weniger für große und lange Urlaubsreisen. Pfaff (2002a, S. 28) sieht in der Kombination der genannten Punkte die Möglichkeit, dass kurze Ausflüge bzw. Ausflüchte aus dem Alltag, die am Wochenende oder an einem Abend erlebt werden können, in der Beliebtheit steigen. In diesem Aspekt kann ein weiterer Ansatzpunkt für kommerziell gesteuerte (Sport-)Erlebnisse gesehen werden. So betont Opaschowski (2006, S. 241): "Sport dient altersher der Unterhaltung („disportare" = sich zerstreuen)“. Bachleitner (2004, S. 17) weist auf den abwechslungs- und aufregungsarmen sowie meist unkörperlichen Arbeitsalltag der Postmoderne hin. Demnach eignet sich gerade der Sport als Pendant.

Was verstehen wir aber als Erlebnis? In der wissenschaftlichen Literatur finden sich einige Definitionsversuche, deren Eindeutigkeit aber immer wieder an der nicht trennscharfen Schnittlinie zu dem Begriff Emotion scheitert. Trotz der Schwierigkeit, eine terminologische Trennung vornehmen zu können, muss an dieser Stelle eine Abgrenzung erfolgen. So ist eine Tendenz zu erkennen, nach welcher Emotionen in der Gegenwart gelagert sind (s. Kap. 9.1), und Erlebnisse oftmals um vorheriges Wissen angereichert erlebt werden (vgl. z.B. Grötsch, 2006, S. 50). Wie Emotionen, können auch Erlebnisse durch spezifische Veränderungen der externen Umwelt ausgelöst werden. Müller und Scheurer (2004, S. 8f.) nennen hier soziale Umweltreize genauso wie Farben, Töne oder Gerüche. Sie können aber auch eine Reaktion auf innere Reize wie Körperempfindungen oder bzw. und kognitive Prozesse wie Bewertungen darstellen. Grötsch (2006, S. 53) postuliert, dass im „Spannungsbogen zwischen Innensteuerung und Außensteuerung der Emotionen" das Potential für Erlebnisse läge. Es darf jedoch nicht vergessen werden, dass Erlebnisse als Folge von Emotionen Konstruktionen sind, die individuell und sozial emotional wahrgenommen werden. Resultierend aus den bisherigen Erkenntnissen muss in dieser Arbeit die Auffassung vertreten werden, dass Erlebnisse nicht per Dienstleistung herstellbar sind (vgl. auch RomeißStracke, 2006, S. 43). Nach Müller und Scheurer (2004, S. 1) können aber 
besonders geeignete äußere Bedingungen geschaffen werden, also z.B. ein Ereignis, welches die Entstehung von Erlebnissen als Konsequenz begünstigt. Diesen Vorgang versteht Schulze (2000, S. 20; 2007, S. 312) als „Erlebnisrationalität":

„Erlebnisrationalität bewährt sich manchmal gut, manchmal nicht, und die beglückendsten Momente im Leben sind diejenigen, die man überhaupt nicht beabsichtigt hat."

Einfache Alltagssituationen beschreiben diesen Ansatz. So lässt sich ein Besuch im Wellnesscenter mit vielen entspannenden Behandlungen buchen, aber ob sich der Einzelne danach wohler fühlt, kann er nur für sich selbst entscheiden. Im schlimmsten Fall kann sich die Massage so unangenehm anfühlen, dass der Besucher den Entspannungstag als Negativerlebnis einordnet. Natürlich ist das auch beim Public Viewing in dieser Form möglich. Ein Veranstalter kann noch so viele Voraussetzungen für einen angenehmen aufregenden Besuch getätigt haben, wenn ein Zuschauer Angst vor Menschenmassen hat, wird er sich auf einem großen Fan Fest nicht wohl fühlen. Etwas anderes wäre dann schon wieder ein Biergarten o.ä.. Müller und Scheurer (2003, S. 15) führen folglich weiter aus, dass ein Jeder maßgeblich selber daran beteiligt ist, ob ein Ereignis -oder eine Veranstaltung- zu einem positiven Erlebnis wird. Somit ist es nicht möglich, Erlebnisse zu produzieren, es können jedoch günstige Rahmenbedingungen geschaffen werden (vgl. auch Grötsch, 2006, S. 50f.). Sie (2003, S. 4) gehen davon aus, dass Erlebnisse Ereignisse voraussetzen, die aber erst durch Erkenntnisse zur persönlichen Erfahrung werden. Daraus ergeben sich die vier ' $E$ ' der Erlebnisgesellschaft:

- $\quad$ Ereignis

- $\quad$ Erlebnis

- $\quad$ Erkenntnis

- $\quad$ Erfahrung.

Demnach sind emotionale Erlebnisse nur über Ereignisse zu erreichen. Diese führen zur Erkenntnis, und über die Erkenntnis gelangt der Gast zur Erfahrung. Auch Schulze (1997, S. 35) ist der Meinung, dass Erlebnisse in singuläre subjektive Kontexte eingebunden sind, sich diese aber durch Reflexion verändern und verstärken lassen. Es gibt verschiedene Ansätze zum Modell der Eindrucksbildung. Schulze (1997, S. 43f.) verankert die Konstitution von 
Erlebnissen fast ausschließlich im Subjekt. Was von außen kommt, wird erst durch die Verarbeitung zum Erlebnis: „Schöne Erlebnisse verlangen Eigenbeteiligung: Erlebnisarbeit" (ebd., 2000, S. 21). Ebenso sieht Csikszentmi halyi (2003, S. 103106) in seinen Ausführungen über das Flow-Gefühl73, dass die Wahrscheinlichkeit des Eintretens von Flow aus einer strukturierten Gegebenheit heraus höher sei (vgl. auch Brunner-Sperdin, 2006, S. 26-33). Er beschreibt Sport und Rituale als Konstrukte, die den Menschen in einen geordneten und damit angenehmen Bewußtseinszustand versetzen würden. Dennoch gehöre eine Eigenaktivität dazu, die den Einzelnen in eine andere Realität, in einen anderen Bewußtseinszustand versetze. Günther (2006, S. 58) schreibt, dass der Mensch in der Lage ist, Ereignisse, die nicht in den Erlebnisrahmen zu passen scheinen, auszublenden, während andere Ereignisse wiederum vom Individuum in den Erlebnisrahmen eingebaut werden. Diese Vorgänge sind als 'Erlebnisarbeit' zu verstehen. Nach Schulze (1997, S. 35) wird sie als „die Absicht, die Umstände so zu manipulieren, dass man darauf in einer Weise reagiert, die man selbst als schön reflektiert" definiert. Wöhler (2004, S. 220) spezifiert:

„Im Empfinden, d.h. der emotionalen Widerspiegelung der Realität, erlebt das Individuum zugleich sein Ich und Sich mit der Welt bzw. der Gesellschaft. Der Bezug zur Gesellschaft stellt sich demzufolge durch Erleben her, wobei das subjektive Empfinden darüber entscheidet, welche Gesellschaft bzw. gesellschaftlich vorgehaltene Welt integriert wird."

Schulze (1997, S. 44ff.) erörtert drei Elemente der Erlebnistheorie der Verarbeitung, die hier am Beispiel Public Viewing illustriert werden sollen:

1. Subjektbestimmtheit

2. Reflexion

3. Unwillkürlichkeit.

Unter Ersterem ist zu verstehen, dass ein Ereignis erst durch die Verbindung mit einem subjektiven Zusammenhang zum Erlebnis werden kann. Wichtig ist, dass dieser Kontext, wie er für die Einzelperson in dem Augenblick ist, nicht wieder so sein wird. Darüber hinaus führt die Verarbeitung zu einzigartigen Erlebnissen. Dieses bedeutet also, dass ein jeder Besucher Public Viewing anders wahrnimmt,

\footnotetext{
73 Csikszentmihalyi (2003, S. 103) definiert Flow als „ein Gefühl, dass die eigenen Fähigkeiten ausreichen, eine gegebene Herausforderung in einem zielgerichteten, regelgebundenen Handlungssystem zu bewältigen, das deutliche Rückmeldung bietet, wie gut man dabei abschneidet. Die Konzentration ist dabei so intensiv, dass keine Aufmerksamkeit übrig bleibt, um an andere unwichtige Dinge zu denken oder sich um Probleme zu sorgen“.
} 
ein jeder das Ereignis in seinen eigenen momentanen subjektiven Horizont einordnet. Dennoch muss in Anbetracht der Menschenmassen auf den großen Plätzen der Städte im WM-Sommer 2006 eine gewisse emotionale ErlebnisIntersubjektivität vorhanden sein.

Mit Reflexion ist die Verarbeitungsphase des Subjektes gemeint. Durch eigenes Erinnern an die Geschehnisse, das Kommunizieren der Empfindungen und die Interpretation sowie Bewertung dieser, nimmt das Ursprungsereignis an Stabilität zu. Die dritte Stufe ist die der Unwillkürlichkeit. Die hier vertretene Hypothese konstatiert, dass durch die zweite Stufe der Reflexion aus dem erlebten Ereignis etwas Neues, nämlich das Erlebnis entsteht.

Kommt man aber auf die Fragen nach der Möglichkeit von Intersubjektivität von Ereignissen bzw. Erlebnissen zurück, leuchtet es ein, dass in der Phase der Reflexion eine Menge kognitive Erlebnisarbeit auf das Individuum zukommt. Im Sinne der Reizreduktion ist der Mensch darauf ausgerichtet, sich bekannter gesellschaftlich gelernter Formen der Selbstanschauung zu bedienen. Schulze (1997, S. 53) weist darauf hin, dass in der Erlebnisgesellschaft kollektive Schemata von Einzelpersonen genutzt werden. Es werden intersubjektive Modelle gebraucht, um die eigenen kognitiven Prozesse möglichst gering zu halten. Seiner Meinung nach lässt die Frage nach gemeinsamen Erlebnissen ein Für und Wider zu. Auf der einen Seite beschreiben Personen, die eine Situation oder ein Ereignis erlebt haben, dieses im Nachhinein oft ähnlich. Andererseits sind die Begründungen, wieso sie das Ereignis so und nicht anders beschreiben, oft unterschiedlich. Wenn eine Person wiederkehrend mit Abwehr auf den Besuch eines Public Viewing reagiert, hat diese eine stabile Verbindung zwischen Public Viewing und Abwehr aufgebaut. Tritt nun diese Verknüpfung ebenso bei den Bekannten und Freunden der Person auf, ist die dem Public Viewing abgewandte Haltung in diesem Freundeskreis allen gemein. Intersubjektivität basiere zum einen auf Wiederholung und zum anderen auf der Verbindung von Zeichen und ihrer Bedeutung.

Abschließend muss gesagt werden, dass nach den Modellen der Erlebnisgesellschaft der individualisierte Einzelne geneigt ist, sich über eine innenorientierte Lebenssauffassung soziale Struktur zu verschaffen. In sogenannten Erlebnisgemeinschaften, die sich über das gemeinsame Miterleben von emotionalen Ereignissen definieren, die auch im Rahmen von Public Viewing- 
Veranstaltungen denkbar sind, wird gesellschaftliche Orientierung gesucht. Soziale gemeinschaftliche Ereignisse erhalten so einen neuen Stellenwert. Erlebnisse sind jedoch nicht per se herstellbar, sondern nur durch individuelle Erlebnisarbeit erlebbar. Trotz dieser Eigenartigkeit scheint es eine gewisse Erlebnis-Intersubjektivität z.B. beim Public Viewing zu geben.

\title{
10.2 Er lebniskonsument
}

\begin{abstract}
"The stress in accounts of our 'postmodern' world on subjectivities and identities inevitably points to the affective grounding of identity, while the acknowledged centrality of consumption to the life of the contemporary subject leads to consideration of the powerfully affective nature of the consumer's relationship to goods and to the social contexts of consumption" (Brown \& Richards, 2000, p. 32).
\end{abstract}

Das Individuum in der postmodernen Gesellschaft handelt oftmals erlebnisorientiert und kann sich zu diesem Zweck zu Erlebnisgemeinschaften zusammenschließen, das konnte bis zu dieser Stelle festgestellt werden. Was bedeutet es jedoch für das Public Viewing und die verantwortlichen Experten, wenn der Zuschauer als Konsument von emotionalen Erlebnissen zu begreifen ist? Dieser Frage widmet sich das folgende Kapitel.

Vielfach wird betont (vgl. z.B. Opaschowski, 2001, S. 87; Romeiß-Stracke, 2006, S. 35ff.; Scheurer, 2003, S. 25), dass sich die erlebnisorientierte Lebenseinstellung auch in veränderten Konsumwünschen und -bedürfnissen widerspiegelt. Weiermair und Brunner-Sperdin (2006) führen aus, dass die verstärkte Erlebnisorientierung und das Bedürfnis, diese Erlebnisse in der freien Zeit zu erleben, eine Anpassung der Freizeit-Anbieter an die gegebene Situation bewirkt hat (vgl. auch Müller und Scheurer, 2004, S. 4f.;Opaschwoski, 2001, S. 87). Die Tendenzen der Erlebnisgesellschaft führen dazu, dass der Erlebniswert einer Dienstleistung, eines Angebotes oder auch eines Produktes stärker in das Zentrum des Interesses rückt. Diesen Sehnsüchten versuchen viele Anbieter zu entsprechen. Auch Bachleitner (2004, S. 18) betont den heutigen „Erlebnisfetischismus“ im Verständnis der Etablierung von Gegenwelten und Gegenentwürfen zu den empfundenen Bedingungen der Postmoderne. Als logische Konsequenz hat sich ein Markt für Erlebnisse gebildet. 


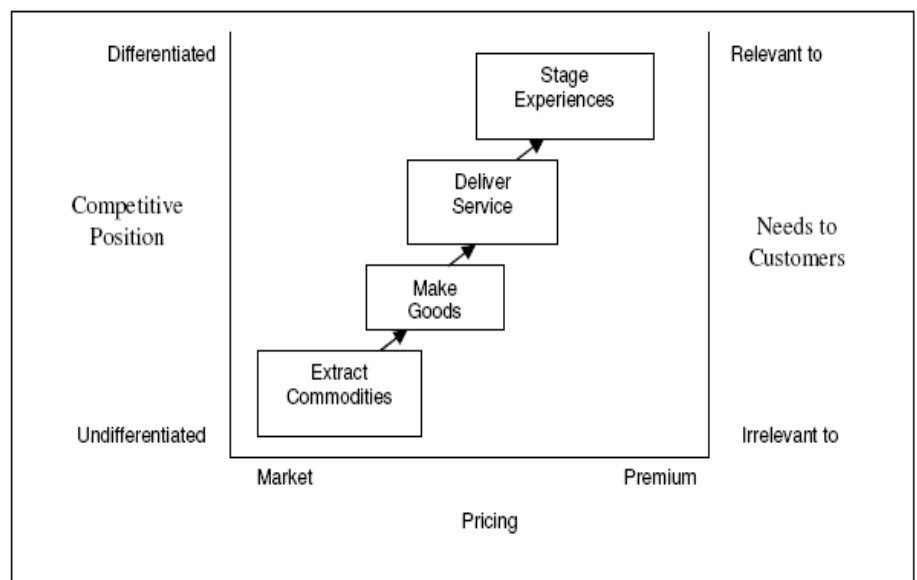

Abb. 10: Die wirtschaftliche Differenzierung (nach Pine \& Gilmore, 1999, p. 22)

Der Prozess der wirtschaftlichen Differenzierung, den Pine und Gilmore (1999, S. 1) in ihrem Standardwerk zur Erlebnisökonomie am Beispiel Kaffee erklären, kann auch auf Public Viewing übertragen werden. Der Kaffee wird auf seinem Entstehungsweg von der rohen Bohne, über die Verarbeitung, hin zu der Aufbereitung und Präsentation sukzessive aufgewertet. Mit seiner Verarbeitung erhält der Kaffee eine Wertsteigerung. Dieser Prozess ist eine Wertschöpfungskette, wie sie bei vielen Produkten anzutreffen ist. Der entscheidende Aspekt wird jedoch im vierten Kettenglied gesehen. Es macht einen großen Unterschied aus, ob Kaffee in einem gewöhnlichen Restaurant oder in einem szenigen Coffeeshop ausgeschenkt wird. In ersterem wird man einen Preis von 1,50 € bezahlen, während im zweiten Fall eine Kaffeekreation durchaus $4 €$ kosten kann. Die Differenz zwischen dem Restaurant und dem Coffeeshop besteht in dem Erlebnis. In letzterem gibt es eine Auswahl an Kaffeesorten und arten, es ertönt entspannende Musik, und der Kunde kann sich in gemütlichen Sesseln erholen. Somit wird aus dem Kaffeegenuss eine kleine Flucht aus dem Alltag, für die viele Besucher tiefer in die Tasche greifen. Das Erlebnis, den oft durch Sirup, Sahne oder andere Geschmackstoffe angereicherten und dekorierten Kaffee an einem schönen Ort zu genießen, stellt das Endglied in der Produktdifferenzierung dar. Der Gast erhält im Austausch gegen sein Geld, seine Anwesenheit und Aufmerksamkeit, die nicht zuletzt für Werbende interessant ist, ein Erlebnisangebot.

Das kann der eben beschriebene Kaffeegenuss genauso sein wie das Erleben einer TV-Vorführung in einem stadionähnlichen, entsprechend geschmückten 
Areal mit zum Setting passender Musik. Gerhard (2006a, S. 46) hebt hervor, dass der Fußball,

„mehr als andere Sportarten (...) im Fernsehen Erlebnisqualitäten [eröffnet], die sowohl kognitiver wie auch emotionaler Art sind: einfache überschaubare Regeln, der stetige Wechsel von antizipierbaren und nicht antizipierbaren Abläufen, die Ästhetik der Bewegungsabläufe, der affektiv verankerte Wunsch nach einem Sieg der $<$ eigenen> Mannschaft in einer Wettbewerbssituation, die Spannung des unsicheren Ausgangs und letztlich die Möglichkeit des stetigen Wechsels dieser emotionalen und kognitiven Reaktionsmöglichkeiten der Fernsehzuschauer selbst."

Einen wichtigen Aspekt im Hinblick auf den Kontext Public Viewing hebt hier Pfaff (2002a, S. 18) hervor: Inszenierte Sportveranstaltungen müssen sich mit dem Live-Erlebnis messen können. Dies gilt insbesondere für nur über Leinwand oder Fernsehen dargestellte Ereignisse, die oftmals genau aus diesem Grund zu Medienereignissen angereichert werden. Public Viewing muss in diesem Fall an das mediale Ereignis vor dem heimischen Fernseher heranreichen, gleichzeitig aber den Vergleich mit dem Spiel im Stadion nicht scheuen müssen. Es muss also konkurrenzfähig zu dem sicheren Sitzplatz, der guten Sicht und dem freien Weg zu den sanitären Anlagen zu Hause bzw. zum Live-Erlebnis im Stadion sein. Internationale (Fußball-)Sportturniere sind oftmals an sich bereits ein Ereignis, dennoch bietet es sich geradezu an, das Erlebnispotential für die Besucher zu steigern. Maennig (2007, S. 37) weist auf den erheblichen Erlebnisnutzen für die deutsche Bevölkerung hin, den er mit der gestiegenen Zahlungsbereitschaft der Deutschen die WM im eigenen Land zu haben, begründet. Er konstatiert, dass vor dem Turnier nur jeder fünfte Bewohner Deutschlands bereit war zu zahlen: 4,26€ im Durschnitt. Während nach der Weltmeisterschaft 2006 42,6\% der Bevölkerung eine positive Zahlungsbereitschaft aufwiesen, die im Mittel bei 10,07€ lag. Maennig impliziert den Erlebnisnutzen der Bevölkerung als den größten Effekt der WM aus ökonomischer Sicht.

Den Begriff des Erlebnisangebots definieren Müller und Scheurer (2004, S. 5) wie folgt:

„Eine Leistung kann als Erlebnisangebot bezeichnet werden, wenn es vorrangig dem Erlebnisnutzen dient und mit atmosphärischen Bezeichnungen wie schön, spannend, interessant, gemütlich oder gar geil beschrieben wird. ${ }^{74}$

Ist es folglich sinnvoll, Public Viewing mit Erlebnisangeboten zu offerieren? Kann

74 Pfaff (2002a) bietet für diese Aktion den Begriff 'verstärkte Erlebnisse' an. Im Rahmen dieser Arbeit soll aber die von Scheurer und Müller (2004, S. 4) proklamierte Trennung von Ereignis und Erlebnis weitergeführt werden. 
damit das Erlebnisbedürfnis befriedigt sowie dem Wunsch nach Atmosphäre und emotionalem Erleben Genüge getan werden? Wie im Rahmen dieser Arbeit bereits deutlich wurde, ist das Entstehen von Emotionen und Erlebnissen einzig multikausal erfassbar. Bereits bei den meisten offiziellen Fan Festen zur WM 2006 in Deutschland wurde Wert auf den Erlebnisnutzen gelegt (vgl. auch Kap. 13.2).

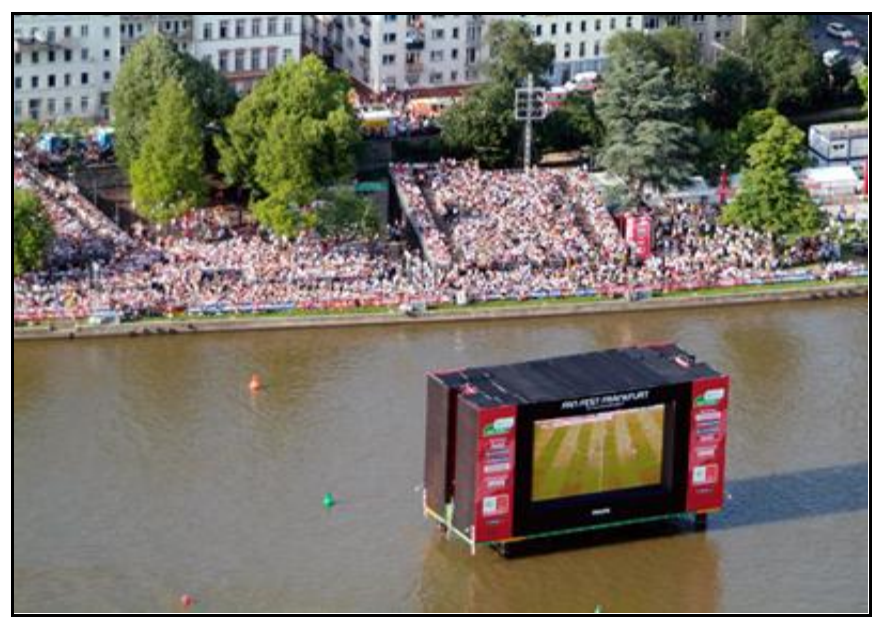

Abb. 11: Fan Fest Frankfurt am Main (Stadt Frankfurt am Main, o.J.)

Die Ausrichter der Fan Feste während der Fußball-Weltmeisterschaft 2006 haben z.T. ein enormes Repertoire um das eigentliche Public Viewing herum angerichtet. Das Fan Fest der Stadt Frankfurt fand bspw. direkt am Main statt: Die rund 15.000 Fans, die an den aufsteigenden Ufern des Flusses Platz finden, schauen auf schwimmende Leinwände. Für dieses Szenario werden anstatt der ansonsten 60 bis 80 qm großen Screens zwei 144 qm große benötigt, damit die Spielszenen für die Zuschauer auch genau zu verfolgen sind. Ein 'Hingucker' ist die Sky Arena, die Verwandlung der typischen Hochhauskulisse in eine überdimensionale LichtKlang-Skulptur. Für die Zuschauer ist fast alles wie im Stadion; es gibt Sitztribünen, Stehplätze, Zugangskontrollen und einen VIP-Bereich (vgl. Paperlein, 2005; Zils, 2005).

Es wird bereits hier deutlich, dass die Fan Fest-Verantwortlichen in Erlebnisangeboten einen Zusatznutzen gesehen haben. Vielleicht zog genau das die vielen Zuschauer zu diesen Veranstaltungen. Denn gerade die Fan Feste wurden von reichlich 'Gelegenheitsfans' besucht. Godbey (1993, p. 53), der das Freizeitverhalten im postmodernen Nordamerika beschreibt, offeriert den Begriff „time deepening“. Er nimmt an, dass die Menschen in ihre Freizeit am liebsten 'zwei Fliegen mit einer Klappe' schlagen. Das soll heißen, dass eine Person es als 
erstrebenswert ansieht, mehr als eine Aktivität zur gleichen Zeit zu tun. Es ist denkbar, dass die Besucher die Verbindung von Public Viewing auf dem Großbildschirm und das Zusammensein und Feiern mit Freunden gesucht haben. Ebenso sehen Harney und Jütting (2007, p. 15) im Public Viewing eine „mittelbare Unmittelbarkeit", dem Publikum würde das 'Dabeisein' suggeriert und verbünde sich mit mit dem Gemeinschaftserlebnis. Diese Kombination ließe die Abwesenheit des Spiels unwichtig werden.

In der Erlebnisgesellschaft kommt es vielen weniger auf das Konsumgut selber an, als auf die innere Wirkung, die Besagtes in innen auslöst. Dass es das tut, wird oft von der Werbeindustrie und den Medien suggeriert. Der Gast ist deshalb versucht zu glauben, es könne das Erlebnis, die innere Wirkung gekauft werden (vgl. Schulze, 2007, S. 312). Köck (2005, S. 14) beschreibt diesen Vorgang anhand des Beispiels eines beliebten Urlaubsortes der deutschen Bevölkerung:

\section{„Erst die Beschreibung und Etikettierung durch die kommerzielle Erlebnisindustrie und durch die mediale und wissenschaftliche Tourismuskritik haben das sommerlich karnevaleske Leben an diesem Strandabschnitt [gemeint ist El Arenal auf Mallorca] zur außergewöhnlichen Erfahrung gemacht."}

Demnach können Massenmedien eine wichtige Funktion für Erlebnisangebote erfüllen. So fiel während der Fußball-Weltmeisterschaft 2006 die vorrangig positive Berichterstattung über die Public Viewing-Areale auffällig ins Gewicht (vgl. Kap. 13.2).

Schulze (2007, S. 312) gibt andererseits zu bedenken, dass viele Konsumenten die Botschaft gerne glauben, weil es sie von der eigenen Verantwortung erlöst. Was aber vergessen würde, ist die psychische Aktivität, die man selbst leisten müsse, um ein Erlebnis zu haben. Dennoch scheint das Fortschreiten der Erlebnisorientierung unumgänglich zu sein (vgl. Opaschowski, 2000, S. 166ff.).

Es gilt festzuhalten, dass das Bedürfnis von Individuen, emotionale Erlebnisse in der Freizeit zu erfahren, gerade in der postmodernen Gesellschaft ausgeprägt ist. Der Erlebniswert eines Freizeitangebots, wie z.B. Public Viewing, rückt weiter in das Zentrum des Interesses der Besucher. Diesen Ansprüchen versuchen viele Anbieter mit ihrer Angebotsgestaltung bereits zu entsprechen. Gerade für das Public Viewing, welches letztlich eine mediale Rezeption zweiter Ordnung darstellt, scheint die Verstärkung des Erlebnispotentials geeignet, sich in der Zwitterstellung zwischen der Bequemlichkeit des heimischen TV-Genusses und dem Live-Erlebnis im Stadion etablieren zu können. Die Medien können auch hier 
durch Beschreibung und Etikettierung eine Multiplikatorfunktion einnehmen, die die Assoziationen bzgl. des Public Viewing positiv unterstützt.

\title{
10.3 Public Viewing als Erlebnis-Setting?
}

\begin{abstract}
„Wenn ein nicht unbeträchtlicher Teil der gesellschaftlichen Produktion in der Hervorbringung von Spektakeln besteht und die Schauspiele immer mehr zu körperlichen performances-Spiel, Aufführung, Ästhetik- werden, dann nähern sich die Handlungsbereiche von Unterhaltung, Business, Kultur und Sport einander immer mehr an" (Gebauer, 2002, S. 3).
\end{abstract}

Opaschowski führte in seinen Berichten und Trendaussagen zur Zukunft des Sports (1996, S. 57; 2000a, S. 166) aus, dass sich der Sport im Erlebniszeitalter nur als „vermarktete Freizeitwelt zwischen Körperkultur und Erlebnismarketing behaupten“ könne. Ob diese Aussage absolut zu sehen ist, bleibe dahingestellt. Dennoch stellt z.B. Beckmann (1993, S. 153) fest, dass ein Heimspiel des von ihm untersuchten Handballvereins VfL Fredenbeck ein „Fest“ sei, ein „Handballspiel, aber rundherum, vorher und nacher [böten] sich ausgezeichnete Möglichkeiten, den Unterhaltungswert zu steigern“. In den vorangehenden Kapiteln ist es jedoch deutlich geworden, dass das emotionale Erleben innerhalb von Freizeitangeboten in den Vordergrund rückt. Public Viewing-Veranstaltungen als mediale Rezeption können von der Verstärkung des Erlebnispotentials profitieren. Aufgabe ist es nun, Möglichkeiten für Public Viewing-Verantwortliche am Beispiel des Erlebnis-Setting aufzuzeigen.

Erlebnis-Setting ist als ein „Erklärungsmodell zur touristischen Angebotsgestaltung in der Erlebnisökonomie“ (Müller \& Scheurer, 2004, S. 10) entwickelt worden. "Setting“, aus dem Englischen von „to set“, ist zu übersetzen mit Umgebung oder Schauplatz. Unter Setting ist in diesem Zusammenhang nach Scheurer (2003, S. 13) die „Gesamtheit der Umgebungsmerkmale, in deren Rahmen bestimmte Erlebnisse [bzw. zunächst Ereignisse, um bei der in dieser Arbeit verfolgten Differenzierung der Begriffe Ereignis und Erlebnis zu bleiben] stattfinden“, gemeint. Dabei kann es sich um kommerzielle und nicht-kommerzielle Schauplätze handeln. Für den ursprünglich touristischen Hintergrund bedeutet dies, dass eine möglichst optimale Angebotsgestaltung von Urlaubszielen angestrebt wird. Denn, obwohl Erlebnisse schon immer Bestandteil des touristischen Angebots waren, hat sich auch im Tourismus die Angebotslage 
verändert: Romeiß-Stracke (2006, S. 35f.), Schober (1995, S. 12ff.) und Scheurer (2003, S. 13) heben hervor, dass Erlebnisse nicht mehr nur erwünschte Zusatzleistungen sind, sondern operatives Ziel der Angebotsgestaltungen. Zu diesem Zweck sollte dem Besucher zunächst einmal ein Ereignis in einem spezifischen Setting offeriert werden. Dieses wird nach Scheurer (2003, S. 133) mit 'Settingprogramm' bezeichnet. Erlebnis-Settings zeichnen sich durch drei Eigenschaften aus:

1. Ein einziges Subjekt oder mehrere handelnde Subjekte,

2. eine Umgebung, die die typischen Inhalte im Erlebnis-Setting unterlegt, und

3. eine spezifische, für den jeweiligen Schauplatz charakteristische Reihenfolge.

Für eine Public Viewing-Veranstaltung könnte das folgendes bedeuten:

1. Die Besucher und die Mitarbeiter sind die handeInden Personen.

2. Das Settingprogramm ist die Fernseh-Vorführung mit den begleitenden Attraktionen, wie Moderation, Musikeinspielungen, Imbissbuden usw., und

3. die Umgebung ist automatisch durch die begrenzte Sicht auf die Leinwände eingeschränkt. Wobei ein Marktplatz genauso die Umgebung darstellen kann wie ein eingegrenztes Fan Fest-Areal, etwa den Münchner Olympiapark oder die Glückauf-Kampfbahn in Gelsenkirchen wie bei der WM 2006.

Daraus leitet Scheurer (2003, S. 133) folgende Minimalkriterien für einen ErlebnisSchauplatz ab:

1. Es muss mindestens ein "relativ gleich bleibendes" Settingprogramm vorhanden sein,

2. eine temporale sowie physische Abgrenzung des Settings existieren, und

3. das Programm des Settings sollte mit der Umgebung übereinstimmen.

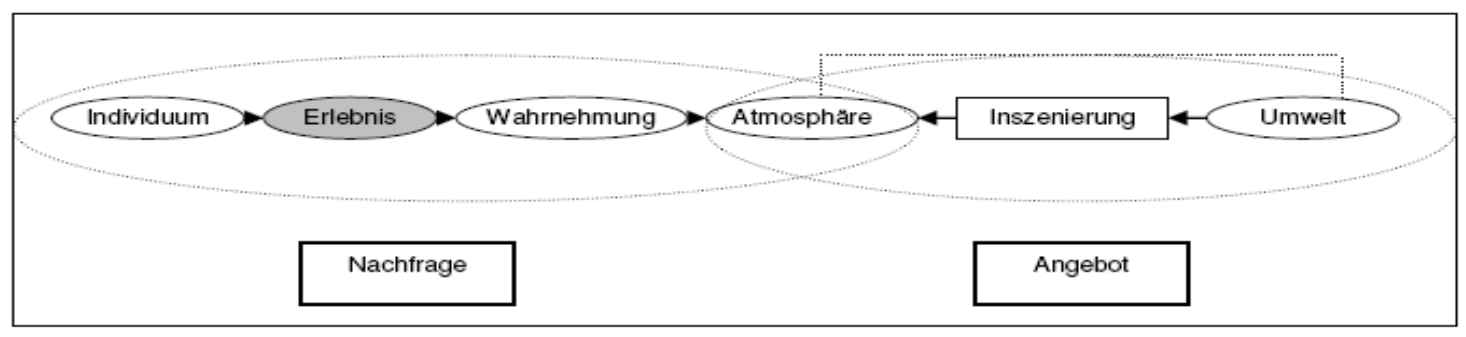

Abb. 12: Das Konzept des Erlebnis-Settings (nach Scheurer, 2003, S. 16) 
In jede Situation, in die wir uns absichtlich oder auch unabsichtlich begeben, wirken Reize aus der Umwelt auf uns ein. Diese können natürlich sowohl positiver als auch negativer Natur sein. Scheurer (2003, S. 133f.) bezieht sich hier auf das Modell zur 'Environmental Psychology' von Russel und Mehrabian (1974), wenn er sagt, dass Umweltreize und Personenvariable in einem bestimmten Setting Erlebnisse auslösen können.

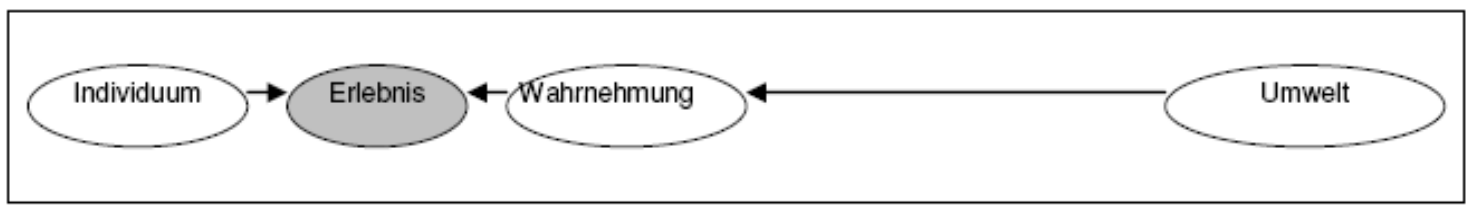

Abb. 13: Einfluss von Umweltfaktoren und Wahrnehmung auf das Erlebnis (modifiziert nach Scheurer, 2003, S. 134)

Müller und Scheurer (2004, S. 10) betonen, dass die Wahrnehmung durch das Individuum eine unabdingbare Vorraussetzung für den Einfluss von Umweltreizen sei. Wenn wir etwas wahrnehmen, verarbeiten wir Informationen aus der Umwelt über die Sinnesorgane. Der Mensch ist in der Lage, Stimulatoren über den Geruchs-, Tast-, und Hörsinn wie auch über Muskeln und den Gleichgewichtssinn wahrzunehmen. Erstaunlicherweise jedoch nimmt das Individuum 80\% der Reize über die Augen auf. Ein Faktum, das für Fernseh-Vorführungen nicht zuletzt eine wichtige Rolle spielt. Alle diese Sinneseindrücke sind jedoch immer subjektiv, d.h., was wahrgenommen wird und was sich im besten Fall als Erlebnis (bzw. Erfahrung) heraus stellt, ist bei jedem unterschiedlich. Das Subjekt wird einerseits durch das Wahrnehmungsvermögen und andererseits durch Erwartungen und Erfahrungen beeinflusst (vgl. Kap. 9). ${ }^{75}$

Scheurer (2003, S. 134) stellt heraus, dass die Gesamtheit der Einzelreize und Reizcluster vom Individuum im Wahrnehmungsprozess gefiltert wird. Die wahrgenommene Reizessenz bildet die Umweltwirkung.

Nach der Theorie der Atmosphäre von Schober (1995, S. 26) wird in folgende Atmosphärearten unterschieden:

Anregende Atmosphäre (anziehend-erregend):

Als themenbezogenes Beispiel kann hier das maritim gestaltete Fan Fest in

75 Diese kurze Erörterung über die Wahrnehmung soll hier genügen. Weiteres zum Wahrnehmungsprozess ist nachzulesen in Mehrabian und Russel (1974), Scheurer (2003), Pfaff (2002) u.a.. 
Hamburg zur WM 2006 genannt werden.

- $\quad$ Ruhige, entspannende Atmosphäre (anziehend-beruhigend):

Ein Wellness bereich eines Hotels oder ein Ruheraum

Bedrückende, deprimierende Atmosphäre (abweisend-beruhigend):

Alles was eintönig und kahl ist, z.B. graue Hochhausfronten oder

aggressive Atmosphäre (abweisend-erregend):

Eine Reizüberflutung in dem Public Viewing-Areal, etwa in Form von zu vielen Besuchern, zu vielen Unterhaltungsangeboten oder zu lauter Musik.

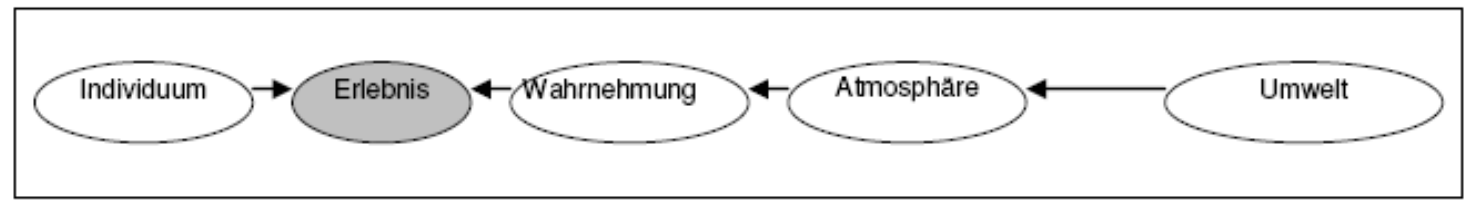

Abb. 14: Atmosphäre (modifiziert nach Scheurer, 2003, S. 139)

Innerhalb eines Settings sind meist einige der Reize dominanter als andere. Die Dominanten kann man nach Schober (1995, S. 24ff.) als 'Atmosphäreträger' benennen. Diese werden in Atmosphärestifter und Atmosphärekiller unterteilt. Erstere wirken anregend, also emotional positiv und abhängig von der Gesamtatmosphäre erregend/aktivierend oder beruhigend. Negative Atmosphäreträger, die Atmosphärekiller, beeinflussen demgegenüber negativ. Zusätzlich existieren so genannte 'Leerfaktoren' mit einer in den meisten Fällen neutralen emotionalen Wirkung. Die Wirkfaktoren lassen sich in konstante und variable Faktoren differenzieren. Konstante Faktoren sind z.B. das Landschaftsbild oder die Aufbauten beim Public Viewing. Der deutlichste variable Faktor ist wohl das Wetter. Des Weiteren kann in direkte Wirkfaktoren, wie Farbe, Licht, Töne, Gerüche etc. unterschieden werden, diese Eindrücke sind über die Sinnesorgane wahrnehmbar. Indirekte Wirkfaktoren dagegen bestehen schon vor dem Ereignis oder dem Urlaub. So nehmen Motive oder Erwartungen direkten Einfluss auf die Wahrnehmung und spiegeln sich in Vorstellungen, Assoziationen oder Images wider. Zur Prüfung und letztendlich zum gelungenen Aufbau von Atmosphäreträgern ist demnach eine Reihe von Aspekten von wahrnehmungsphysiologischen bis zur psychologischen Wirkung von Reizclustern zu beachten (vgl. Müller \& Scheurer, 2004, S. 11; Scheurer, 2003, S. 140f.). Diese drückt die emotionale Wirkung eines Standortes aus. Müller und Scheurer (2004, 
S. 10) betonen, dass nur, wenn die Atmosphäre als positiv bewertet würde, sich die gewünschten Erlebnisse einstellen könnten. Folglich ist es nicht ausreichend, Ereignisse zu schaffen oder sich auf gegebene Anlässe zu verlassen, sondern die passende Gesamtatmosphäre ist entscheidend. Daraus kann geschlossen werden, dass sich eine Vielzahl der auf den Besucher einwirkenden Reize nicht beeinflussen läßt. Es gibt jedoch auch Reize, die der Veranstalter mittels der Angebotsgestaltung beeinflussen oder moderieren kann (vgl. Müller \& Scheurer, 2004, S. 12). Es sollte das Ziel eines jeden Public Viewing-Veranstalters sein, Angebote so zu gestalten, dass sie für die Gäste eine angenehme Atmosphäre darstellen. Denn nur so können Ereignisse letztlich als positive Erlebnisse interpretiert werden.

Resümierend kann gesagt werden, dass die optimale Angebotsgestaltung des Public Viewing als Erlebnis-Setting sinnvoll ist. Durch das Settingprogramm sollten die Voraussetzungen für eine anziehend-erregende Atmosphäre geschaffen werden, die von den Besuchern als positiv bewertet wird. Nur wenn die Gesamtatmospähre positiv gesehen wird, können sich gewünschte Erlebnisse einstellen.

\subsection{Inszenierung als Instrument des Erlebnis-Setting}

Wie kann nun das Konzept des Erlebnis-Setting in die Public Viewing Praxis umgesetzt werden? Worum muss es den Veranstaltern von Public Viewing-Events gehen? Aus dem vorangehenden Kapitel kann entnommen werden, dass sie versuchen sollten, ihr Angebot durch die Schaffung einer zielgruppenorientierten Atmosphäre zu attraktivieren. Im Folgenden werden anhand des Leitfadens zur Angebots-Inszenierung im Tourismus von Müller und Scheurer (2004) Gestaltungsmöglichkeiten für Public Viewing vorgestellt und eruiert.

Auf Inszenierungsstrategien wird vornehmlich im Eventmanagement bzw marketing $^{76}$ (vgl. z.B. Pfaff, 2002, S. 161-181, Dreyer, 2002, S. 353-368) und im Tourismus (vgl. u.a Grötsch, 2006, S. 49-80; Waiermair \& Brunner-Sperdin, 2006)

\footnotetext{
76 Unter Eventmarketing wird im Allgemeinen die Vermarktung von Veranstaltungen bzw. Events verstanden. Ziel des Eventmarketing ist es, ein Event möglichst gut zu verkaufen (vgl. Dreyer, 2002, S. 353). Dabei wird nach Zanger (2007, S. 3) vermehrt Wert auf die Prozessorientierung des Marketing gelegt. Diese beinhaltet die „zielorientierte, systematische Planung, konzeptionelle und organisatorische Vorbereitung, Realisierung sowie Nachbereitung von erlebnisorientierten Veranstaltungen".
} 
zurückgegriffen. Vielfach wird im Marketing aber von einer weitergreifenden Einflussmöglichkeit ausgegangen, als sie in dieser Arbeit vertreten wird (s. Kap. 9). Für den weiteren Verlauf soll die Definition von Weinberg und Nickel (2007, S. 38) zum erlebnisorientierten Marketing als Orientierung dienen. Sie sehen dieses als „konsequent nach den emotionalen Bedürfnissen, die sich im Lebensgefühl und Lebensstil der Zielgruppe“ wiederfinden, ausgerichtet.

Mit dem Begriff der Inszenierung ist laut Hickethier und Bleicher (1998, S. 369) zunächst ganz allgemein „etwas 'in Szene' setzen“ gemeint, „einem Geschehen Gestalt und Form geben, eine Geschichte in einen Rahmen fügen, sie in einem Raum in eine Aktion von Figuren zu überführen“.

Seel (2001, S. 49) definiert Inszenierungen als Handlung mit drei Merkmalen:

„1. Absichtsvoll eingeleitete oder ausgeführte sinnliche Prozesse, die 2. vor einem Publikum dargeboten werden und zwar 3. so, dass sich eine auffällige spatiale und temporale Anordnung von Elementen ergibt, die auch ganz anders hätte ausfallen können."

Scheurer (2003, S. 11) bezieht den Begriff direkt auf das Konzept des ErlebnisSetting. Inszenierung ist demnach das

\footnotetext{
„Instrument des Erlebnis-Settings zur gezielten Schaffung und Verstärkung einer zielgruppenorientierten Atmosphäre mittels verschiedener Teilinstrumente unter Berücksichtigung des Umfeldes und der Rahmenbedingungen."
}

Wie bereits erörtert wurde, sind Sportveranstaltungen an sich schon emotionsund spannungsgeladen, mehr als eine Theatervorführung oder eine touristische Destination. Die Voraussetzung zur Erzeugung von emotionalen Erlebnissen ist bei den Besuchern gut. Demnach dürfte die adressatengerechte Inszenierung bei einem sportlichen Ereignis leichter fallen als z.B. bei einer Ausstellung. Zudem ist die aktive Einbindung des Zuschauers, welche die kognitive Gefühlsarbeit erleichtert, von den Gegebenheiten her schon einmal besser als z.B. im traditionellen Theater, wo der Besucher nur passiver Zuseher des Geschehens auf der Bühne ist, und er sich kaum in den Ablauf involvieren lässt. Aber auch im Bereich des Theaters werden zunehmend erlebnisorientierte Neuformen, wie z.B. das Improvisationstheater oder das Kriminaltheater angeboten. Es stellt sich die Frage, ob erfolgreiche Inszenierungsstrategien von Public Viewing-Vorführungen in der Angebotsgestaltung umgesetzt werden können. Damit würden sie den veränderten Besucherbedürfnissen entsprechen und könnten im Wettbewerb konkurrenzfähig werden. Nach Scheurer (2003, S. 82f.) und Weiermair und 
Brunner-Sperdin (2006) ist der Begriff der Inszenierung im Tourismus zu einem Leitthema geworden. Inszenierung wird als Instrument des Erlebnis-Setting verwandt, um Angebote zu profilieren und sie aus der Masse hervortreten zu lassen. Dabei wird sich, so Scheurer, an bekannte Formen der Inszenierung aus dem Bereich des Schauspiels angelehnt (vgl. auch Romeiß-Stracke, 2006, S. 37f.). Angenommen, das Public Viewing-Areal ist das Theater, dann ist der Bereich der TV-Vorführungen die Bühne, und die einzelnen Spiele sind die Szenen. Der Organisator ist der Regisseur, die Mitarbeiter vom DJ zu den Gastronomen sind die Schauspieler, und der Besucher ist je nach Perspektive entweder beobachtender Gast oder selbst Darsteller, indem er sich mit Fanartikeln einkleidet und Gesänge anstimmt. Riedl (2006, S. 148) betont, dass

\footnotetext{
„der Spitzensport durch Inszenierung und Rituale gekennzeichnet ist, die darauf abzielen, kollektive Emotionen beim Publikum zu erzeugen und in den Stadien und Hallen entsprechende Stimmungen und Atmosphäre herzustellen."
}

Nach Opaschowski (2001, S. 92) ist die Inszenierung die logische Konsequenz der Kommerzialisierung des (Fußball-)Sports, denn wer mit dem Sportinteresse der Gesellschaft Geld verdienen will, darf das Zuschauen nicht einfach dem Zufall überlassen. Aus diesem Grund verlässt sich wohl kaum ein Sportveranstalter mehr darauf, ausschließlich über die sportliche Darbietung Emotionen hervorzurufen. Harney und Jütting (2007, p. 12) betonen jedoch, dass es der permanenten Gegenwart des „Sportmythos“ bedürfe, in den sich die Vorstellungen und Projektionen der Zuschauer hineinstellen könnten. Ein perfekt inszeniertes Produkt sehen sie nicht als zielführend an.

Müller und Scheurer (2004, S. 12ff.) haben in ihrem Leitfaden zur AngebotsInszenierung im Tourismus anhand von sieben Mitteln ein Rüstzeug zusammengestell.t. ${ }^{77}$ Die Inszenierungselemente, das Thema, das Inszenierungskonzept, die Attraktion und Aktivitäten, die Gestaltung der Szenerie, die Vorkehrungen zur Sicherheit des Wohlbefindens, die Besucherlenkung und der Gast selber sind miteinander verknüpft. Die Touristiker gehen vielfach davon aus, dass nur die Beachtung aller Instrumente einen Erlebnis-Schauplatz

\footnotetext{
77 Diese Ausführung soll hier als klar strukturierte Orientierungshilfe genügen. Sicherlich kann sie nicht als allumfassend und allgemeingültig gelten, dies wird aber, um die Stringenz der Ausführungen und damit letztlich auch den Erkenntnisgewinn nicht zu gefährden, in Kauf genommen. Weitere Anregungen finden sich z.B. bei Gundlach (2007) oder Inden-Lohmar (2007).
} 
vervollständigt. Die Mittel sollen nun im Folgenden erörtert werden:

1. Thema - das Leitinstrument

Um die Einheitlichkeit der Veranstaltung zu gewährleisten, ist ein Thema unerlässlich. Das Thema sollte veranstaltungs- und zielgruppenaffin sein.

Die anderen, im Folgenden aufgezeigten Mittel orientieren sich am Thema.

2. Inszenierungskonzept - das Planungs- und Koordinations-Instrument

Das Inszenierungskonzept zielt darauf ab, die einzelnen Bereiche der Veranstaltung in Einklang zu bringen. Mögliche Zielgruppen können festgelegt und ihr Verhalten und ihre Erwartungen bestmöglich bestimmt werden. Für ein gastadäquates Programm werden die Inszenierungsinstrumente geplant und koordiniert.78

3. Attraktion und Aktivität - das Auslöser-Instrument

Attraktionen und Aktivitäten sorgen für die Schaffung oder Unterstützung von Ereignissen, die als Folge Erlebnisse ermöglichen. Diese sollten durch die anderen Inszenierungsmittel getragen werden, den Besuchern genügen und natürlich zum Leit-Instrument, also zum Thema konform sein.

4. Szenerie - das Ästhetik-Instrument

Das Umfeld des Events mit seiner natürlichen Szenerie, der Landschaft und den Häusern hat großen Einfluss auf die Wahrnehmung der Gäste. Natürlich fallen Faktoren wie Licht und Wetter als erstes auf, aber auch die Bauweise und Möblierung sind wichtig. Das Ästhetik-Instrument hinterlegt den dramaturgischen Ablauf des Events und unterstützt die Besucherlenkung. Zudem stützt sie die Attraktionen und Aktivitäten durch ihre Authentizität.

5. Besucherlenkung - das Lenk-Instrument

Hier wir der Versuch beschrieben, die Besucher unter Zuhilfenahme von gestalterischen Elementen zu leiten. Aussichtspunkte, Ruhezonen können diese Punkte sein, aber auch die Aktivitäten und Attraktionen können die Besucherlenkung dramaturgisch aufladen.

6. Wohlbefinden - das Unterstützungs-Instrument

Dieses Inszenierungs-Tool meint die Sicherung der physiologischen

\footnotetext{
78 Romeiß-Stracke (2006, S. 39) weist zu Recht darauf hin, dass der Leitfaden genau an der Stelle der Inszenierung relativ vage bleibe und auch in Scheurer (2003) wie in den folgenden Erläuterungen deutlich wird, nicht viel deutlicher wird. Mit dem Hinweis auf die Abhängigkeit der Inszenierungselemente zu dem zu Inszenierende, z.B. eine touristische Destination oder ein Event, liefert er knappe aber einleuchtende Hilfestellungen (s. Kap. 10.5).
} 
Grundbedürfnisse der Besucher, so dass diese sich wohl fühlen können. Genügend Toilettenhäuschen sind damit genauso gemeint wie die Wahrung der Sicherheit. Das unterstützende Element des 'Wohlfühl-Managements' ist eng mit der Besucherlenkung verbunden.

7. Besucher/Gäste - das Bewertungs-Instrument

Zufriedene Gäste, die die Veranstaltung oder den Urlaub in guter Erinnerung behalten, sind das, worauf es ankommt. Deshalb sind Beurteilungsverfahren sehr sinnvoll. Allerdings darf nicht vergessen werden, dass Besucher gleichzeitig auch immer selber ein Teil der Attraktion und der Veranstaltung sind. Aus diesem Grund solllte den Gästen immer das Bedürfnis, sich selbst in Szene zu setzen, zugestanden werden. Bekanntermaßen ist eine Achterbahn ohne Gekreische genauso wenig vorstellbar wie ein Sportereignis ohne Zuschauer oder ein Public Viewing-Event ohne entsprechend gekleidete Fans und ihre Gesänge.

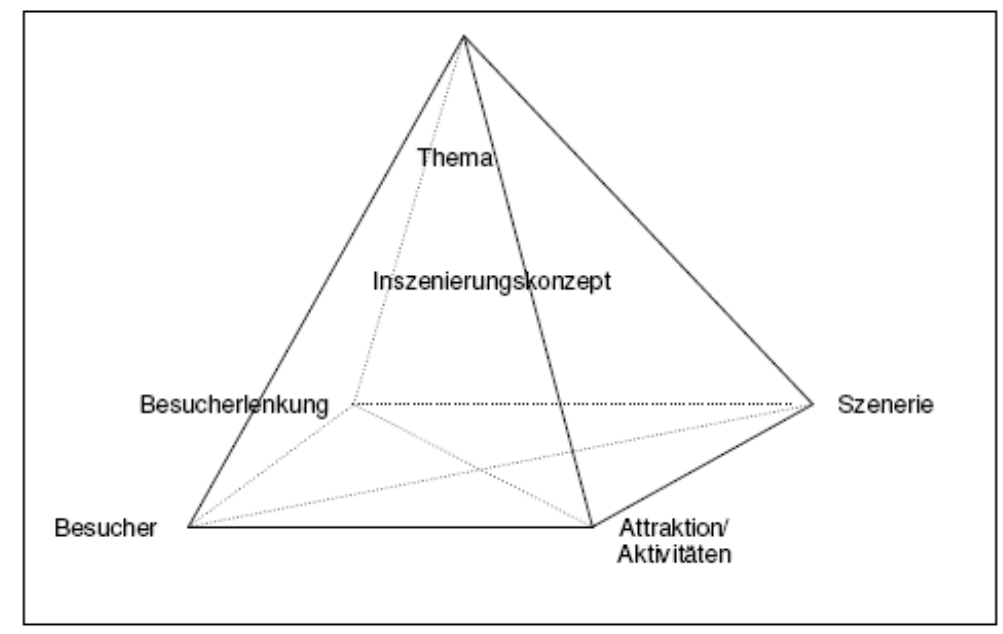

Abb. 15: Die Inszenierungspyramide (modifiziert nach Müller \& Scheurer, 2004, S. 14)

Müller und Scheurer (2004, S. 14) weisen darauf hin, dass das Thema nicht immer der Ausgangspunkt einer Inszenierung sein muss. Es kann auch eine spezielle Attraktion -wie beim PV- oder eine Aktivität sein, von der aus die übrigen Inszenierungs-Instrumente bewusst und systematisch eingesetzt werden, um die Wahrscheinlichkeit von positiven Erlebnissen zu erhöhen. 


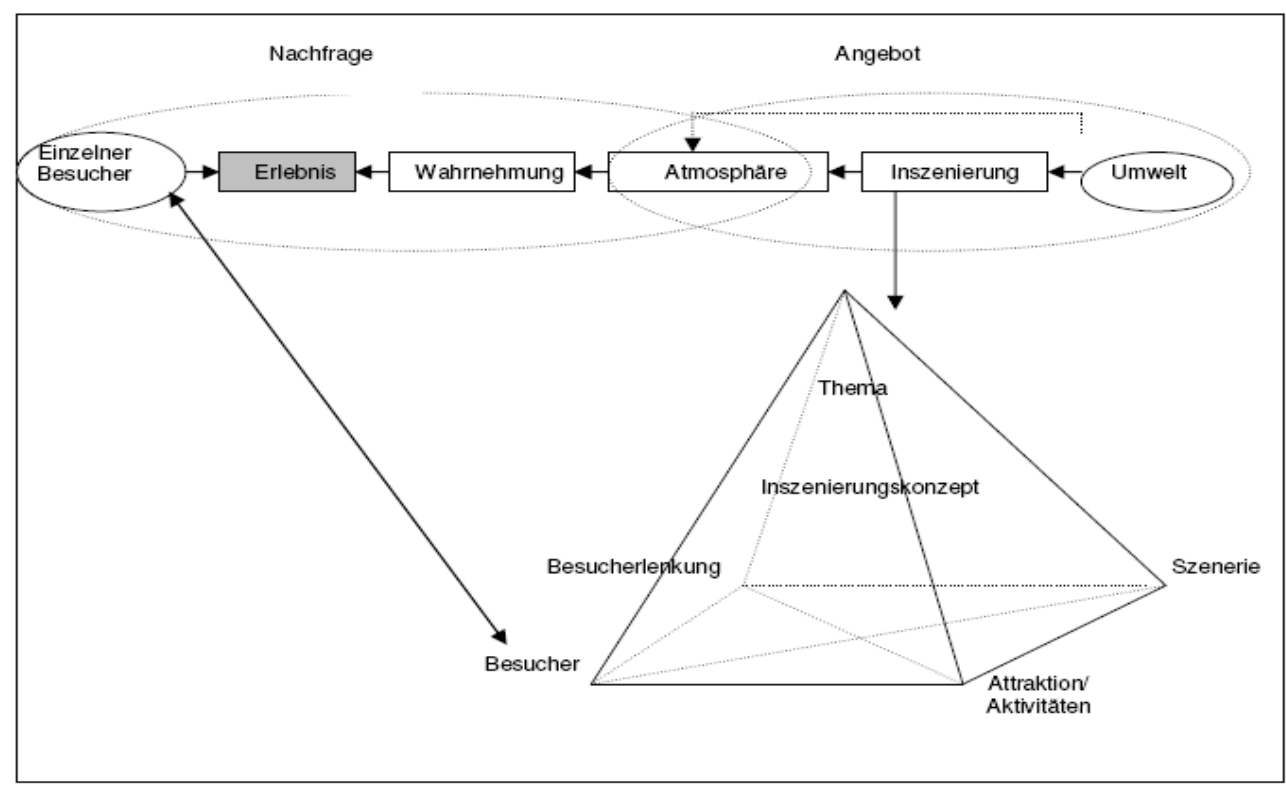

Abb. 16: Inszen ierungselemente im Konzept des Erlebnis-Setting (nach Scheurer, 2003, S. 214)

Im Tourismus wie im Sport steht das Miterleben von etwas Besonderem -eines Ereignisses oder das Besuchen einer antiken Stätte- als Erlebnis immer im Mittelpunkt. Was sich aber, wie Müller und Scheurer (2004, S. 7) hervorheben, verändert hat, ist die Ausrichtung des Schauplatzes. Anfangs wurde im Tourismus nur wenig inszeniert, wohingegen heute jede Destination mit Erlebnispotential angereichert oder gar hauptsächlich zur Vermittlung von Erlebnissen errichtet wird. Als Extrembeispiel sind hier Freizeitparks, Erlebnisgastronomie u.ä. zu nennen.

Die Inszenierung scheint auch bei vielen Public Viewing-Feiern anlässlich der WM 2006 in Deutschland bereits eine Rolle gespielt zu haben. In Hamburg versuchte man das maritime Flair der Stadt auf das Fan Fest zu übertragen. Dort wurden für die Region typische Elemente in die Inszenierung mit aufgenommen. In der Hansestadt war das Heiligengeistfeld neben dem Fußball-Stadion Zentrum des Geschehens. Hier gab es von mittags bis Mitternacht ein buntes Rahmenprogramm, welches die Besucher unterhalten hat ( $\mathrm{vgl}$. Meyer-Odewald und Grünberg, 2005). Auf der Bühne haben Live-Auftritte stattgefunden, davon rankten sich einige um das Thema Fußball, während andere Musikacts gewesen sind. Es gab eine internationale Meile am Hafen und Wassersportvergnügen auf der Binnenalster. Für weitere Unterhaltung wurden eine Tribüne und der 'Soccer Dome' aufgebaut. Letzterer ist eine etwa zehn Mio.€ teure Halbkugel, in deren 
Innern sich 3.500 qm reine Ausstellungsfläche befinden. Sie sollte das Publikum in eine erlebnis- und eventorientierte Fußballwelt entführen (vgl. Hanauer, 2005). Für die Besucher sollte durch Torwandschießen, einen XXL-Kicker und einen künstlichen See eine Fußballjahrmarktsatmosphäre entstehen, die genau auf die Zielgruppe feierfreudiger Fans zugeschnitten war (vgl. Goy, 2005). Riedl (2006, S. 211) betont, dass das Gelingen von Erlebnisangeboten bzw. Inszenierung zu einem Großteil vom genauen Wissen über die spezifische Zusammensetzung des Publikums und dessen Wünschen, Präferenzen und Bedürfnisse abhängig ist.

Als gemeinsames Planungs- und Koordinationsinstrument kann bei der Weltmeisterschaft 2006 das gemeinsame Inszenierungskonzept aller Austragungsstädte gesehen werden. Jede Stadt gestaltete ihr WMRahmenprogramm zwar individuell, weshalb auch die Organisationsformen des 'Fernsehguckens' unterschiedlich sind. Einige der Ausrichterstädte organisieren und vermarkten das offizielle Public Viewing ${ }^{79}$ selbst, während andere die Veranstaltung für Event-Dienstleister ausgeschrieben haben (vgl. Städele, 2005). Die lokal unterschiedlich ausgerichteten Feste vereint aber neben dem Design das gemeinsame Rahmenkonzept, was u.a. den kostenfreien Eintritt zur LiveÜbertragung mit Rahmenprogramm beinhaltet (vgl. Rheinland-Pfalz WM-Portal, o.J.).

Wie wichtig bereits die Szenerie genommen wurde, kann am Beispiel Berlins verdeutlicht werden. In der Hauptstadt gibt es viele prestigeträchtige Bauten und Orte, vor denen die Stadtväter gerne das medienwirksame Fan Fest aufgebaut hätten. So kam es zu monatelangen Debatten, bis man sich schließlich auf den neuen Spreebogenpark mit der Ausweitung des Areals auf die Straße des 17.Juni geeinigt hat.

Bezüglich der Besucherlenkung können Maßnahmen, wie der Versuch, den Fokus der emotionalen Aufmerksamkeit mit dem Näherrücken des Spielbeginns auf den sportlichen Wettstreit zu leiten, erwähnt werden. Riedl (2006, S. 203) nennt hier Maßnahmen, wie das Beleuchten der Spieler mit Spotlicht beim Vorstellen, wie es

\footnotetext{
79 Die elf Fan Feste FIFA WM 2006 wurden in Dortmund auf dem Friedensplatz, in Frankfurt am Main am Mainufer, in Gelsenkirchen auf der Glückauf-Kampfbahn und in Hamburg auf dem Heiligengeistfeld ausgerichtet. In Hannover trafen sich die Fußballbegeisterten auf dem Waterlooplatz, in Kaiserslautern auf dem Stiftsplatz, in Köln auf dem Roncalliplatz und in Leipzig auf dem Augustusplatz. Darüber hinaus fanden die WM-Partys im Münchner Olympiapark, auf dem Nürnberger Volks festplatz und auf dem Stuttgarter Schlos splatz statt.
} 
z.B. beim Basketball gängig ist. An dieser Stelle muss wiederum auf den hohen Stellenwert der Kommunikation hingewiesen werden. Die Vor- und Nachberichterstattung soll und kann Erwartungen an ein zukünftiges Ereignis schüren und auf das mediale Sportereignis hinfiebern lassen (vgl. Cordsen \& Deilmann, 2005, S. 340). Es lässt sich festhalten, dass die Möglichkeiten der Inszenierung bereits bei der WM 2006 teilweise zur Anwendung gekommen sind. Jedoch scheinen die Mittel vorrangig bei den offiziellen Fan Festen angewandt worden zu sein (s. Kap. 13.2).

Vielfach wird, z.B. von Hickethier und Bleicher (1998) und Schwier und Schauerte (2006), bzgl. der Inszenierung im Fernsehen ${ }^{80}$ unterstrichen, dass diese mediale Gestaltung eine doppelte sei. Die erste würde von den Akteuren, den Spielern selbst, vor der Kamera getätigt, und die andere würden die technischen Medien durch das Auswählen der Spielszenen, die Schnitttechnik usw. bereiten. Das heißt für unser Beispiel Public Viewing, dass bereits die Fernseh-Übertragung ein Stück Inszenierung ist, einen Teil der Gestaltung des Public Viewing darstellt. Darüberhinaus schreiben Hickethier und Bleicher (1998, S. 382), würde die Inszenierung eine ordnende Funktion besitzen. Sie würde uns zeigen, dass wir Zuschauer seien und das Geschehen in den Zusammenhang einordnen. Faktoren, die in der postmodernen Gesellschaft zur Orientierung genutzt werden können.

Es kann also gesagt werden, dass die Public Viewing-Veranwortlichen mit der Inszenierung einer zielgruppenorientierten Atmosphäre unter Berücksichtigung des Umfeldes und der Rahmenbedingungen die Möglichkeit haben, Public Viewing als Erlebnis-Setting zu platzieren. Die vorgestellten sieben Werkzeuge können als Leitfaden dienen, um eine adäquate Inszenierung im Public Viewing zu gestalten.

80 Bei Horky (2006) findet sich z.B. eine detaillierte Aufschlüsselung über die Inszenierungsformen die RTL beim Beachvolleyball unternommen hat. 


\subsection{Exkurs: Public Viewing als Event}

„Aus der Authentizität des eigenen Sporterlebens wird zunehmend ein Sport der Inszenierung, der neue Erlebnis-Welten und Mega-Ereignisse jenseits des wirklichen Lebens erfindet und produziert" (Opaschowski, 1996, S. 58).

In einem kurzen Exkurs wird abschließend eine der höchsten Stufen der aufgezeigten Entwicklung, nämlich die Eventisierung von Sportveranstaltungen und ihre gesellschaftliche Begründung, analysiert. Auf der Suche nach Literatur zum Thema Event öffnet sich ein sehr weites Feld, in dem der Begriff 'Event' in vielen Variationen bestimmt wird und seine Anwendung findet. Zuächst muss hier also geklärt werden, was in dieser Arbeit als Event verstanden wird.

Was ist nun überhaupt ein Event? Ist es eine Veranstaltung, oder ist es mehr als das? Welche Arten von Events gibt es, und wie unterscheiden sich diese? Diesen Fragen widmen sich ausführlich z.B. der Sammelbände von Gebhardt, Hitzler und Liebl (2000), Nickel (2007) und Trosien und Dinkel (2000). Dreyer (2002, S. 345) der sich mit der Bedeutung von Events für die Destinationsvermarktung beschäftigt, sieht den Unterschied zwischen dem Begriff Veranstaltung und Event in dem Mitschwingen des Erlebnis- und Einmaligkeitswerts bei letzterem. Auch Freyer und Groß (2002a, S. 1) verfolgen diese Abgrenzung vom Alltäglichen, indem ihrer Meinung nach Events „als Veranstaltung oder Ereignis zu sehen [sind], die über eine 'Besonderheit' verfügen und die sich anhand dieser Besonderheit von 'normalen' Ereignissen oder Veranstaltungen (...) abgrenzen" (vgl. auch Gebhardt, 2000, S. 18f.). Auch Trosien und Dinkel (2000a, S. 16) betonen die gegenwärtige Offenheit des Begriffs und dessen vielfältige Einbeziehung in den Sport. Zum einen erscheinen im vorliegenden Zusammenhang Events als eine extreme oder ganzheitliche Form der Inszenierung. Sie dienen dem Zweck, die vom Einzelnen zu leistende Erlebnisarbeit so gering wie möglich zu halten, bei maximaler Erlebnissicherheit (vgl. Schulze, 2007, S. 313). Zum anderen interessiert die soziale Relevanz des Events. Gebhardt, Hitzler und Pfadenhauer (2000, S. 12) begreifen Events aus Sicht der individualisierten Gesellschaft als „die typischen außeralltäglichen Vergemeinschaftungsformen grenzenloser, sich zunehmend individualisierender und pluralisierender Gesellschaften". An dieser Stelle soll allerdings auf eine weitere Ausdifferenzierung der verschiedenen Eventarten, die vornehmlich nach 
Größe und Reichweite vorgenommen wird, verzichtet und auf die obig erwähnten Sammelbände verwiesen werden. Einzig der Mega-Event soll hier Erwähnung finden, da Fußball-Welt- und -Europameisterschaften als solche bezeichnet werden, und sie aus diesem Grund besondere Bedeutung für den Kontext dieser Arbeit besitzen.

Roche (2000, p. 1) begreift Mega-Events als

\begin{abstract}
"large scale cultural events of mass popular appeal and international importance which are typically stagemanaged by a combination of national governement and international none-governemental actors."
\end{abstract}

Horne und Manzenreiter (2006, p. 2) fügen hier zwei Merkmale von Mega-Events an; sie müssen zum einen signifikante Konsequenzen für die Ausrichterstädte habe ${ }^{81}$ und zum anderen eine beachtliche Medienabdeckung erreichen.

"Mega-Events such as the Football World Cup Finals provide multiple meanings for different groups of agents - as they happen, when they have taken place and, perhaps especially, as they are being bid for" (Horne \& Manzenreiter, 2004, p. 199).

Weiter weisen sie darauf hin (2004, p. 200), dass diese Art von Events einen großen Einfluss auf viele kulturelle Bereiche, wie Sportstars und ihre Rolle als Teile der Popkultur, die Modeindustrie und ihre spezifischen Produkte sowie die multinationale Produktion dieser Dinge haben. Dies ist nur durch die modernen medialen Technologien möglich, die dafür sorgen, dass die ganze Welt an einem solchen Ereignis teilhaben kann.

Was macht aber nun ein Event aus? Schulze (2000, S. 22-25; 2007, S. 313f.) nennt vier Faktoren, die ein Event wirkungsvoll machen:

- $\quad$ Einzigartigkeit,

- $\quad$ Episodenhaftigkeit,

- $\quad$ Gemeinschaftlichkeit und

- $\quad$ Beteiligung.

Mit der Einzigartigkeit des Events ist gemeint, dass es sich um ein Ereignis handeln muss, das an einem spezifischen Ort zu einem bestimmten Zeitpunkt stattfindet. Zudem sollte es mit der Möglichkeit, besondere Erfahrungen zu sammeln, angereichert sein. Das Kriterium der Einzigartigkeit ist bei sportlichen

81 Kurscheidt (2002; 2005) erfasst z.B. mit einer sozioökonomischen Kosten-Nutzen-Analyse die Bedeutung der Fußball-Weltmeisterschaft 2006. Ohmann, Jones und Wilkes (2006) bieten z.B. eine Studie über die soziale Wirkung der Fußball-WM 2006 auf die Bewohner Münchens an. 
Wettkämpfen immer erfüllt, da kein Turnier oder Spiel gleich sein oder wiederholt werden kann. Allerdings sei die Einzigartigkeit lediglich bei Live-Events gegeben, eine Fernsehübertragung wäre demnach kein Event. Dem kann anhand der bisherigen Erkenntnisse der vorliegenden Arbeit nur wiedersprochen werden. Denn gerade weil Public Viewing-Veranstaltungen über das Medium Fernsehen an vielen Orten gleichzeitig zu sehen sind, wachsen sie zu einem gemeinsamen Event zusammen.

Weiter führt Schulze aus, dass die Episodenhaftigkeit auf die Dramaturgie des Ereignisses anspielt. Der Veranstaltungsablauf muss einen gewissen emotionalen Spannungsbogen $^{82}$ aufweisen. Der Faktor Gemeinschaftlichkeit steht für das Entstehen der kollektiven Zuschaueridentität, welche sich auch im Kriterium Beteiligung wieder findet. Denn zu einem erfolgreichen Event gehört immer das Mitmachen und Sich-in-Szene-setzen des Publikums (vgl. auch Gebhardt, 2000, S. 21). Bette und Schimank (2000, S. 308) erweitern die Gemeinschaft um das Attribut 'emotional'. So sehen sie gerade Sportereignisse

„in dem Sinne event-tauglich, dass viele Gesellschaftsmitglieder sie als Gelegenheiten nutzen, ein ästhetisches Spektakel in einer emotionalen Gemeinschaft zu erleben. "

Dreyer (2002, S. 365f.) ergänzt die Ausführungen von Schulze um die Faktoren der
Inszenierung und
Thematisierung,
Perfekte Organisation,
Multisensualität,
Wetterunabhängigkeit und das
angemessene Preis-Leistungsverhältnis.

Unter dem Aspekt der Inszenierung und Thematisierung wird das Aufeinanderabstimmen des kompletten 'Besucherpfades' ${ }^{83}$ verstanden, d.h., dass der Gast von der Information über die Veranstaltung bis zur Heimfahrt geleitet wird. Die Perfekte Organisation meint selbsterschließend den reibungslosen Ablauf des Events. Gebhardt (2000, S. 21f.) weist darauf hin, dass zumeist eine

\footnotetext{
82 Weiterführende Ausführungen zur emotionalen Dramturgie finden sich u.a. in Gundlach (2007), Pfaff (2007) und Schober (1995).

83 Nach Pfaff (2007, S. 61) ist der 'Besucherpfad' der Weg, auf dem sich der Gast während seines Aufenthalts bewegt. Alle kundenrelevanten Prozesse werden auf diesem Weg hinsichtlich ihrer Erlebnisfunktion kontrolliert und gegebenenfalls modifiziert.
} 
monothematische Fokussierung des Events verfolgt wird. Die Olympischen Spiele würden demnach eine Ausnahme darstellen. Mit Multisensualität ist das Ansprechen aller Sinne gemeint. Also bspw. der Einsatz von taktilen Elementen im Rahmenprogramm in Kombination mit den üblichen visuellen Medien. ${ }^{84}$ Mit Wetterunabhängigkeit sind Vorkehrmaßnahmen gemeint, die möglichen wetterbedingten Unanehmlichkeiten vorbeugen. Nicht zuletzt ist ein angemessenes Preis-Leistungs-Verhältnis wichtig, um Zuschauer zu einem Event zu 'locken'. Gebhardt (2000, S. 19) weist darauf hin, dass Events planmäßig erzeugt sind. Schulze (2000, S. 24) merkt dazu an, dass sich im Eventmarketing allmählich die Meinung durchzusetzen scheine, dass sich der verbreitete Denkfehler, die Erlebnisarbeit des Individuums zu unterschätzen, relativiert habe (vgl. auch Günther, 2006, S. 57ff.). Als Erfolgsgeheimnis von Events schlägt er vor, den Konsumenten von vornherein mehr Verantwortung für das eigene Erleben zu ermöglichen und auch abzuverlangen sowie gleichzeitig massive Unterstützung zukommen zu lassen. Denn wie Bette und Schimank (2000, S. 307) betonen, sind auch Events nur in Maßen inszenierbar. Romeiß-Stracke (2006, S. 44) gibt aber den Hinweis, dass der Besucher bei einem Event kurzweilig in eine „emotional geladene Ausnahmesituation" eintauchen möchte und da könne man „schon einmal dicker auftragen“.

Nachdem geklärt wurde, als was Events in unserem Zusammenhang zu begreifen sind und was diese ausmacht, wird nun auf die Frage nach dem Enstehen von Events eingegangen.

„Die Verbindung von sportlichen Wettkämpfen mit Entertainment-Elementen hat hierbei letztlich die Funktion, zwischen Sport und eventisierter Kultur vermittelnd einzugreifen und damit auch eine Konkurrenzfähigkeit herzustellen“ (Pfaff, 2007, S. $59)$.

Wie Pfaff betont auch Riedl (2006, S. 201ff.) die Chancen von eventisierten Sportveranstaltungen. Er schreibt, dass in Großstädten meist ein Überangebot an Freizeitangeboten und Sportveranstaltungen herrsche. Die Konkurrenz der Anbieter sei entsprechend groß, und demnach sei es besonders in Ballungsräumen wichtig, größtmög liche Attraktivität zu bieten. In kleineren Städten

84 Mehr zur Multisensualität findet sich bei z.B. bei Gebhardt (2002), Pfaff (2002, 2002a) oder Weinberg und Nickel (2007). Ersterer benutzt allerdings die Bezeichnung des 'kulturellen und ästhetischen Synkretismus' und meint damit die Vermischung unterschiedlichster ästhetischer Ausdrucksfomen, wie Tanz oder Musik, um die Sinne anzusprechen. 
bestünde dieses Problem nicht in dem Maße, da sportliche Ereignisse viel mehr im örtlichen Geschehen verankert seien und ein Besuch für viele zum sozialen Leben gehöre (vgl. auch Kap. 13.2). Aus dieser Konkurrenzsituation und dem veränderten Freizeitkonsum erkläre sich die Eventisierung von Sportveranstaltungen. Opaschwoski (2001, S. 79) sieht die Events als neue Marktund Rummelplätze des vorherigen Jahrhunderts. Schulze (2000, S. 26ff.; 2007, S. 317) sieht in dem Eventphänomen eine Radikalisierung der Erlebnisgesellschaft. Events werden sich zukünftig immer mehr von den eigentlichen Produkten lösen, an die sie zunächst gekoppelt waren, etwa Sportartikel oder Autos. Events werden zu einer neuen Kulturform und zu einem eigenständigen Produkt. Demnach können zumindest die Fan Feste FIFA WM $2006^{\mathrm{TM}}$ als Events bezeichnet werden. Denn sie wurden von den Besuchern als eigenständiges Ereignis wahrgenommen. Sie sind von dem eigentlichen Produkt, der WM 2006 und ihren Spielen, zwar geprägt, und diese stellen auch die Basis dar, aber die Fan-Meilen waren doch eigenständig.

Riedl (2006, S. 201) weist darüber hinaus darauf hin, dass neben der Abgrenzung zu anderen Freizeitangeboten die Inszenierung als Event einen nicht unbedingt sofort erkennbaren Vorteil bietet. Mit dem zusätzlichen Angebot kann der Erwartungsdruck auf ein positives Sportgeschehen deutlich gemildert werden. So waren beim Spiel um den 3. Platz dennoch viele Besucher beim Public Viewing anzutreffen, denn sie wussten, dass sie ein gesichertes Maß an Unterhaltung erwarten konnten. Also können viele Zuschauer das Event trotz Niederlage oder schlechter sportlicher Leistung positiv bilanzieren, aus dem einfachen Grund heraus, dass sie sich trotzdem gut unterhalten und aufgehoben fühlen: Stimmungssteuerung.

Mehr aus der soziologischen Perspektive betrachtet, sind nach Bachleitner (2004, S. 19) gerade Erlebniszentren eine Antwort auf die mit der postmodernen Erlebnisorientierung einhergehende ökonomische Orientierung. Nach Roche (2000, p. 225) haben große Events die Möglichkeit, Ressourcen für „a meaningful social life in relation to a changing societal environment that has the potential to destabilize and threaten these things" zu bieten. Auch Horne und Manzenreiter (2006, p. 1) betonen, dass sportliche Mega-Events wichtige Elemente in der 
Orientierung der nationalen und internationalen oder globalen Gesellschaft sind. ${ }^{85}$ Sie (ebd., p. 17) weisen weiter darauf hin, dass z.B. Gruppen entstehen, die sich im kollektiven Zelebrieren spiegeln können. Welche Bedeutung diese Erlebnisgemeinschaft hat, darauf wurde bereits eingegangen. Gebhardt, Hitzler und Pfadenhauer (2000, S. 11) nennen als weitere gesellschaftliche Funktion von Events die „Wiederverzauberung“. Knoblauch (2000, S. 36-43) thematisiert in seinem Artikel das Event als „soziale Situation“, die vornehmlich der „fokussierten Interaktion" diene (vgl. Kap. 13.2). Sie sei quasi die personifizierbare Kommunikation und demnach im Sinne der Luhmannschen Systemtheorie die ideale Strukturelle Kopplung. Bette und Schimank (2000, S. 316-322) unterstellen Events eine kompensatorische Funktion zur Behebung der Defizite der Postmodernde, wie Affektdämpfung, Gemeinschaftsverlust oder Entzauberung z.B. durch Säkularisierung-. ${ }^{86}$

Somit sind Events und gerade Mega-Events als Antwort auf die postmoderne Gesellschaft zu sehen (vgl. Gebhardt, Hitzler und Pfadenhauer, 2000, S. 11; Gebhardt, 2000, S. 28; Roche, 2000, pp. 217-235). Jedoch handle es sich, so Gebhardt (2006, S. 23), letztlich nicht um eine bahnbrechende Neuschöpfung, sondern um eine „von einer professionellen Organisationselite perfekt in Szene gesetze Veranstaltung mit ausgearbeitetem Programm“. Womit der Bogen zur ursprünglichen Intention dieses Kapitels geschlagen wäre. Public Viewing als Event ist also letztlich genau das, was vor allem im Rahmen der offiziellen Fan Feste während der Fußball-WM 2006 in Deutschland bereits umgesetzt worden ist. Nämlich eine perfekt in Gesellschaft, Medien, Sport und Politik in Szene gesetze sehr gut organisierte Veranstaltung mit einem unterhaltsamen Rahmenprogramm.

Es konnte in diesem Kapitel gezeigt werden, dass Events als Extremform der Inszenierung, als Antwort auf die postmoderne Gesellschaft gesehen werden können. Gerade Mega-Events wie die Endrunde der Fußball-WM besitzen multifaktorielle Wirkungen und Funktionen, die von den Medien als Mutliplikatoren und Kommunikatoren unterstützt werden können. Events können als ideale Strukturelle Kopplung angesehen werden, weil sie sich vermehrt von dem

\footnotetext{
85 Ein Beispiel über die Bedeutung der Olympischen Spiele 2008 für China gibt Xu (2006).

86 Nicht zuletzt sieht Schulke (2007, S: 68) in der Eventisierung auch neue Chancen für Public Viewing-Veranstaltungen bei religiösen Ereignissen.
} 
eigentlichen Ereignis wie beim Public Viewing loslösen.

\subsection{Zusammenfassung}

In diesem Kapitel wurde das Ziel verfolgt, das Entstehen von Emotionen durch die Inszenierung von Erlebnissen zu analysieren. Resümierend lässt sich festhalten, dass die veränderten gesellschaftlichen Rahmenbedingungen zum verstärkten Ausleben hedonistischer Bedürfnisse führen. Das Subjekt sucht als Umkehrschluss auf die sozialen Wandlungsprozesse besonders in der freien Zeit nach Befriedigung innenorientierter Wünsche und wählt seine Aktivitäten nach den eigenen Bedürfnissen aus. In emotionalen Erlebnisgemeinschaften, die auch im Rahmen von Public Viewing-Veranstaltungen denkbar sind, wird gesellschaftliche Orientierung gesucht. Soziale gemeinschaftliche Erlebnisse erhalten so einen neuen Stellenwert. Aus diesen Strukturen heraus ergeben sich für Public ViewingAnbieter neue Möglichkeiten, Besucher zu gewinnen und zu binden. Der Erlebniswert eines Freizeitangebots, wie z.B. Public Viewing, rückt weiter in das Zentrum des Interesses. Allerdings sind Erlebnisse nicht per se herstellbar. Sie werden individuell, subjektiv durch Erlebnisarbeit erfahrbar. Dennoch scheint es im Rahmen von großen Ereignissen durch Prozesse der Sozialstrukturierung, denkbar ist hier z.B. die Emergente-Normen-Theorie (s. Kap. 9.3), eine emotionale Intersubjektivität zu geben.

Die Erlebnisökonomie sieht das Erlebnis als Endglied der Differenzierung von Produkten. Gerade für das Public Viewing, welches letztlich eine mediale Rezeption zweiter Ordnung darstellt, scheint die Verstärkung des Erlebnispotentials geeignet. Um der Frage nachzugehen, wie bestmögliche Vorraussetzungen für den Erfolg von Public Viewing zu schaffen sind, wurde auf das ursprünglich für den Tourismus entwickelte Konzept des Erlebnis-Setting zurückgegriffen. Es konnte die Erkenntnis gewonnen werden, dass es Ziel der Verwantwortlichen sein sollte, durch das Settingprogramm die Voraussetzungen für eine anziehend-erregende Atmosphäre zu sorgen, die von den Besuchern als positiv bewertet wird. Inszenierung als Werkzeug des Erlebnis-Setting wird bzgl. ihrer Anwendbarkeit für Public Viewing diskutiert. TV-Vorführungen können anhand der vorgestellten Instrumente als Erlebnis-Setting oder als Event platziert werden. Letzteres stellt eine Extremform der Inszenierung dar, auch weil sie sich 
in der Tendenz vom eigentlichen Ereignis ablöst. Darüber hinaus können Events als Antwort auf die postmoderne Gesellschaft gesehen werden. Insbesondere Sport-Mega-Events wie die Fußball-WM sind in der Lage, weitreichende gesellschaftliche Funktionen zu erfüllen. Die Medien, vor allem das Fernsehen, dienen der erweiterten Kommunikation und nehmen die Rolle von Mulitplikatoren ein. 


\section{Methodik und Forschungsdesign}

Im ersten Teil der Ausarbeitung wurden theoretische Erklärungsansätze für Public Viewing als Forschungsgegenstand diskutiert. In den folgenden Kapiteln wird es um die Erhebung bzw. Bewertung von empirischen Daten gehen, die den theoretischen Erkenntnissen ergänzend hinzugezogen werden. Es erfolgt Forschung zu den Grundlagen des Public Viewing mit Anwendungsbezug. Im Mittelpunkt steht dabei die Gewinnung allgemeiner Erkenntnisse aus der Erhebung von zwei verschiedenen Datensätzen. In diesem Sinne befasst sich Kap. IV zunächst mit dem methodischen Hintergrund und dem Design der Untersuchungen. Zu diesem Zweck erfolgt eine Auseinandersetzung mit methodischen Fragen und Problemen der vorliegenden Befragungen zum Public Viewing (11). Anschließend wird die Anwendung von qualitativen und quantitativen Verfahren im methoden-triangulären Mix diskutiert und Bewertungs- bzw. Qualitätskriterien der methodologischen Triangulation eruiert (11.1). Da für beide methodischen Gebiete jeweils die Befragung als Instrument gewählt wurde, erscheint es sinnvoll, dieser Vorgehensweise besondere Beachtung zu gewähren und auf diese vertieft einzugehen (11.2). Eine Zusammenfassung der Erkenntnisse bildet den Kapitelabschluss (11.3).

Darauf folgend wird das Forschungsdesign dieser methodologischen Triangulation, die eine Momentaufnahme in Form einer punktuellen Erhebung bildet, erläutert (12). Dem zeitlichen Forschungsablauf folgend, wird zunächst das explorative Vorgehen bei den Expertengesprächen vorgestellt (12.1). Zu diesem Zwecke werden der Aufbau des Gesprächsleitfadens eruriert (12.1.1), der Gegenstandsbereich der Expertengespräche abgegrenzt (12.1.2), der Zugang zum Feld erläutert (12.1.3) und das Zeitfenster der Befragung festgelegt (12.1.4). Der gleichen Systematik folgend, schließt sich die Analyse des Designs der Onlinebefragung von Besuchern von Public Viewing-Veranstaltungen an (12.2). Zunächst wird der Aufbau des Onlinefragebogens erörtert (12.2.1), dann der Gegenstandsbereich der Onlineuntersuchung definiert (12.2.2), der Feldzugang dargelegt (12.2.3) sowie das Zeitfenster der empirischen Umfrage abgegrenzt (12.2.4). 


\section{Methodik}

Ziel der praktischen Untersuchung ist es, ein umfassendes Bild des Public Viewing zusammenzufügen. Zu diesem Zweck wird derselbe Gegenstand aus verschiedenen Richtungen auf unterschiedliche Weise beleuchtet, um ein möglichst erschöpfendes Ergebnis zu erreichen. In einem ersten Schritt sind qualitative Interviews mit Public Viewing-Experten geführt worden. Diese Ergebnisse wurden zu einem späteren Zeitpunkt durch eine quantitative Onlinebefragung von Public Viewing-Besuchern ergänzt. Folgerichtig widmet sich der ersten Abschnitt dieses Kapitels der Diskussion um die Anwendung von qualitativen und quantitativen Verfahren im Methodenmix als methodologische Triangulation. Die qualitativen und quantitativen Erhebungsschritte sind zwar nacheinander erfolgt, die Gewichtung der Daten wird aber analog gewertet. Diese Vorgehensweise stellt im Rahmen der Diskussion um geeignete Qualitätskriterien der methodologischen Triangulation eine Maßnahme dar (11.1). Beide Befragungen wurden mit jeweils eigenen Datensätzen durchgeführt und die resultierenden Forschungsergebnisse anschließend aufeinander bezogen. Es kann nicht Aufgabe dieser Ausarbeitung sein, eine grundlegende methodologische Diskussion anzustreben, aber es ist unerlässlich, die angewandten Instrumente präzise darzulegen und zu beschreiben, um das Vorgehen zu rechtfertigen und transparent sowie nachvollziehbar zu gestalten. Da für beide Untersuchungen die Befragung als Instrument verwendet wurde, ist die analytische Auseinandersetzung mit diesem Werkzeug notwendig (11.2). Letztlich erfolgt die Zusammenfassung der erörterten Punkte (11.3). 


\subsection{Triangulation: Qualitative und quantitative Verfahren im Methodenmix}

Wie eingangs erläutert, ist die Postmoderne dadurch gekennzeichnet, dass sich fest gefügte soziale Lebenswelten und -stile auflösen. Es kommt zu einer Differenzierung von Lebensstilen und -arten, die Untersuchungsstrategien erfordern, die zunächst nach akkuraten und weitestgehend vollständigen Beschreibungen der Sichtweisen der Personen fragen, somit also die soziale und subjektive Konstruktion der Welt berücksichtigen (vgl. Flick, v. Kardorff \& Steinke, 2005a, S. 17). Public Viewing ist ein gesellschaftliches Phänomen, welches bislang kaum erforscht ist. Demzufolge ist nur eine explorative empirische Untersuchung möglich. Durch das Erfassen qualitativer Aspekte der Public Viewing-Experten können Wertungen und Beziehungen von den befragten Personen in spezifischen sozialen Situationen erhoben werden. Das Instrument des Leitfaden-Interviews ermöglicht die offene Antwortgestaltung der Spezialisten und gibt dem Interviewer die Chance auf Fragen, Anmerkungen oder interessante Gebiete näher einzugehen. Nur so ist es möglich, detaillierte Befunde über soziale Prozesse zu erheben.

Empirische Sozialforschung hängt von einer Vielzahl an Bedingungen, wie z.B. der Qualität der theoretischen Annahmen, der Angemessenheit der Forschungsmethoden aber auch von materiellen Grenzen, wie Kosten und Zeit ab. Die divergierenden Voraussetzungen, unter denen wissenschaftliche Sozialforschung betrieben wird, machen klar, dass es vom Forschungsziel und den Bedingungen abhängt und nicht von den Gegensätzen zwischen qualitativem und quantitativem Vorgehen, welche Erhebungsverfahren geeignet sind.

Den Oberbegriff der Qualitativen Sozialforschung etwas genauer betrachtend, differenziert diese sich nach Flick et al. (2005a, S. 18) in ihren theoretischen Annahmen, ihrem Gegenstandsverständnis und methodischen Fokus. Kennzeichen qualitativer Forschung sei zum einen der Anspruch, „soziale Wirklichkeit als Ergebnis gemeinsam in sozialer Interaktion hergestellter Bedeutungen und Zusammenhänge zu verstehen" (ebd., S. 20). Und zum anderen werde die soziale Wirklichkeit der konkreten Situation in die jeweils subjektiven Relevanzhorizonte eingeordnet und stelle so die Basis für die Handlungsentwürfe und schließlich das Handeln der Akteure dar (vgl. ebd., S. 20). 
Überträgt man die genannten Annahmen auf die Problembenennung dieser Arbeit, erscheint es logisch, zunächst anhand von qualitativen Gesprächen die wichtigen Relevanzen und Handlungsorientierungen der Experten zu entdecken und herauszufiltern (vgl. auch Hopf, 2005, S. 350). Somit liefern die Interviews mit den Spezialisten Informationen und Hilfestellungen zur Eingrenzung des Gegenstandsbereichs, aber auch für das Verständnis der komplexen Zusammenhänge, was mit quantitativen Methoden nicht möglich gewesen wäre. So lassen sich die theoretischen Grundannahmen qualitativer Forschung wie folgt benennen:

\begin{tabular}{|cl|}
\hline 1. & $\begin{array}{l}\text { Soziale Wirklichkeit als gemeinsame Herstellung und } \\
\text { Zuschreibung von Bedeutungen }\end{array}$ \\
2. & Prozesscharakter und Reflexivităt sozialer Wirklichkeit \\
$3 . \quad \begin{array}{l}\text { <Objektives Lebensbedingungen werden durch subjektive } \\
\text { Bedeutungen fär die Lebenswelt relevant }\end{array}$ \\
2. $\begin{array}{l}\text { Der kommunikative Charakter sozialer Wirklichkeit lăsst die } \\
\text { Rekonstruktion von Konstruktionen sozialer Wirklichkeit } \\
\text { zum Ansatzpunkt der Forschung werden. }\end{array}$ \\
\end{tabular}

Abb. 17: Theoretis che Grundannahmen qualitativer Forschung (nach Flick et al., 2005a, S. 22)

Durch die Herangehensweisen der Qualitativen Sozialforschung ist es folglich möglich, einen offeneren und dem Phänomen 'näheren' Zugang zu erhalten (vgl. ebd., S. 17). In der Sozialforschung haben sich qualitative und quantitative Forschungsweisen zu eigenständigen Bereichen entwickelt. Sie unterscheiden sich laut Flick et al. (2005a, S. 24f.) in zwei ausschlaggebenden Punkten:

1. Bezüglich der Rolle des Forschenden greift der qualitative Forscher in zwingend theoretisch und methodisch untermauerter Weise mit seiner Wahrnehmung in den empirischen Erhebungsprozess ein87, während vom quantitativem Forscher die Unabhängigkeit vom Gegenstand gefordert wird.

2. Die quantitative Forschung ist für ihre vergleichend-statistischen Auswertungen auf ein hohes $M a ß$ an Standardisierung der Datenerhebung angewiesen.

So verdeutlichen Denzin und Lincoln (1994, p. 8),

87 Natürlich ist es möglich, dass der Interviewer den Experten in irgendeiner Form beeinflusst. Ob und wie er dieses tut, ist jedoch schwer kontrollierbar (vgl. Atteslander, 2003, S. 67). 
"Such researchers [qualitative Sozialforscher] emphasize the value-laden nature of inquiry. They seek answers to questions that stress how social experience is created and given meaning. In contrast quantitative studies emphasize the measurement and analysis of causal relationships between variables, not processes. Inquiry is pu rported to be within a value-free framework." ${ }^{88}$

In der vorliegenden Ausarbeitung besteht die empirische Untersuchung aus zwei Teilen, einem qualitativen und einem quantitativen. Das analoge Gewichten der Daten und das Triangulieren der Methoden dienen dazu, die Ergebnisse zu validieren. Denn nach Atteslander (2003, S. 58) würden die „Ziele und Aufgaben explorativer Sozialforschung [verkannt], wenn diese immer nur als Vorstufe, sozusagen als Vorbereitung für 'wissenschaftlichere' Anwendung quantitativer Methoden angesehen" würden.

In der einschlägigen Literatur zum Thema Triangulation ${ }^{89}$ herrscht eine Varianz an Integrationsansätzen von qualitativer und quantitativer Forschungsmethodik. So werden z.B. von Flick (2004, S. 67-84; 2005, S. 313f.) und Mayring (2001, Abs. 22) verschiedene Triangulationsmodelle vorgeschlagen, die die vielfältigen Möglichkeiten des Methodenmixes aufzeigen. Im weiteren Verlauf wird in dieser Arbeit folgende Definition befolgt:

"In der Sozialforschung wird mit dem Begriff < Triangulation> die Betrachtung eines Forschungsgegenstandes von (mindestens) zwei Punkten aus bezeichnet" (Flick, 2005, S. 309).

Diese recht offene Begriffsbenennung kann mit der Beschreibung des Triangulationsmodell von Mayring $(2001$, Abs. 21, 25) ergänzt werden.

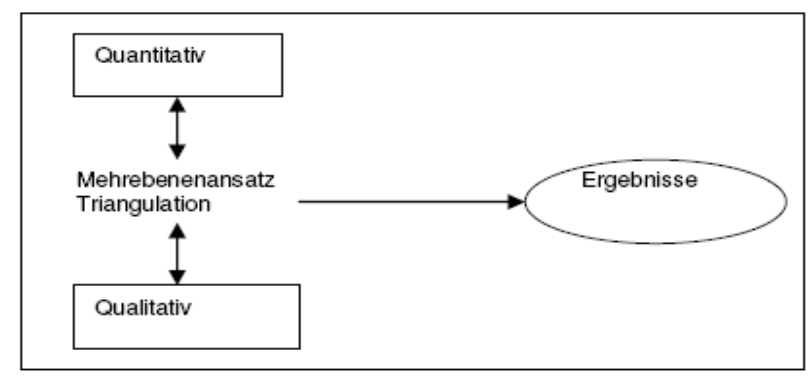

Abb. 18: Triangulationsmodell (nach Mayring, 2001, Abs. 21)

Dieses sei, so Mayring (2001, Abs. 25), „die komplexeste Verschränkung

88 Die Diskussion um die Gegensätze und Gemeinsamkeiten von qualitativer und quantitativer Forschung soll an dieser Stelle nicht vertieft werden, kann aber in Flick 2004, Flick et al. (2005; 2005a), Mayring 2001 [u.a.] nachgelesen werden. Im Bereich Sportwissenschaft bietet z.B. Engels (2004) einen Überblick.

89 Weitere mögliche Triangulationsansätze sind in Flick (2004, S. 13-17; 2005), Mayring (2001, Abs. 9) [u.a.] einzusehen. 
qualitativer und quantitativer Analyseschritte in einem Analyseprozess“. In dieser Weise könnten sich die Untersuchungsergebnisse gegenseitig unterstützen, und die Kreuzung der Resultate ergebe das Endergebnis. Es wird also das Ziel durch „ein schrittweises Erweitern der Erkenntnis durch gegenseitiges Vergleichen unterschiedlicher Herangehensweisen“ verfolgt. Dieses Triangulationsmodell wird von Flick (2005, S. 313) als „Between-Method-Triangulation“ bezeichnet. In diesem Sinne definiert er (2004, S. 12) wie folgt:

\begin{abstract}
„Triangulation beinhaltet die Einnahme unterschiedlicher Perspektiven auf einen untersuchten Gegenstand oder allgemeiner: bei der Beantwortung von Forschungsfragen. Diese Perspektiven können in unterschiedlichen Methoden, die angewandt werden, und/oder unterschiedlichen gewählten theoretischen Zugängen konkretisiert werden, wobei beides wiederum mit einander in Zusammenhang steht bzw. verknüpft werden sollte. Weiterhin bezieht sie sich auf die Kombination unterschiedlicher Datensorten jeweils vor dem Hintergrund der auf die Daten jeweils eingenommenen theoretischen Perspektiven. Diese Perspektiven sollten so weit als möglich gleichberechtigt und gleichermaßen konsequent behandelt und umgesetzt werden. Gleichermaßen sollte durch die Triangulation (etwa verschiedener Methoden oder verschiedener Datensorten) ein prinzipieller Erkenntniszuwachs möglich sein, dass also bspw. Erkenntnisse auf unterschiedlichen Ebenen gewonnen werden, die damit weiter reichen, als es mit einem Zugang möglich wäre."
\end{abstract}

Es sei hier darauf hingewiesen, dass diese Vorgehensweise Irrtümer in der Datenanalyse nicht ausschließt, sondern eher begünstigt. Die mitunter schwierige Vergleichbarkeit von qualitativen und quantitativen Daten ist einer der Punkte, die von Kritikern des Öfteren angesprochen werden. Die im weiteren Verlauf dieser Arbeit zu diskutierenden Untersuchungen beziehen sich zwar auf dasselbe Phänomen, allerdings aber auf z.T. unterschiedliche Aspekte desselben. Somit haben sie nicht exakt denselben Gegenstandsbereich und ergreifen verschiedene Gesichtspunkte. Demnach sind unterschiedliche Ergebnisse schlicht zu erwarten, ohne „dass dies den Schluss auf fehlende Validität erlaubt“ (Kelle \& Erzberger, 2005, S. 303).

Dennoch müssen natürlich für beiderlei Untersuchungen gewisse Qualitätsmerkmale angestrebt werden. Zunächst einmal ist zu beachten, dass sich durch die Divergenz der qualitativen und quantitativen Erkenntnisarten und deren Analyse auch Unterschiede in der Zweckmäßigkeit der Gütekennzeichen ergeben. Die Qualitätskriterien, die in einem Bereich sinnvoll sind, sind nicht ohne weiteres auf den anderen Bereich übertragbar.

In der wissenschaftlichen Fachliteratur (vgl. z.B. Flick, 2004, S. 100; Steinke 1999, 2005) ist man sich uneinig über die Frage, ob es in der qualitativen Forschung 
überhaupt sinnvoll ist, Gütekriterien zu verlangen. In dieser Arbeit ist aber versucht worden, für beide Datensätze ein möglichst weites Maß an Qualitätsmerkmalen zu erreichen. Die weithin anerkannten Kriterien der Objektivität, Validität und Reliabilität werden für beide Untersuchungen eingehalten (s. Kap. 13.1). Wobei mit Objektivität die größtmögliche Ausschaltung des Subjektiven gemeint ist, ein Maß, welches sich im wissenschaftlichen Sinne auf die möglichst sachliche Erhebung und Dokumentation der Erkenntnisse bezieht. Eine Untersuchung ist dann valide, wenn die genutzte Forschungsmethodik auch wirklich das erhebt, was erhoben werden soll. Es stellt sich folglich die Frage, ob die Ergebnisse inhaltlich nachvollziehbar, argumentativ schlüssig und überprüfbar sind. Wohingegen die Reliabiliät die Verlässlichkeit der Daten bzgl. ihrer Ergebniskonstanz bei wiederholtem Erheben unter gleichen Bedingungen meint (vgl. Atteslander, 2003, S. 255).

Ein weiteres Gütekriterium stellt die Repräsentativität der Untersuchung dar. Soll eine Befragung repräsentativ in dem Sinne sein, dass sie reale Einstellungen und Meinungen zu einem Sachverhalt widerspiegelt, lassen sich nach Atteslander (2003, S. 74) drei mögliche Störbereiche identifizieren:

1. Auswahl der Probanden (Stichprobenrepräsentativität),

2. Erhebung von Daten (Erhebungsart),

3. Auswertung der Umfrage und Ergebnisformulierung (d.h. statistischmathematische Analyse und Interpretation).

An dieser Stelle soll der Diskussionsfokus auf der Stichprobenrepräsentativität liegen, während die Wahl der Datenerhebung bereits im Rahmen der Triangulationsdiskussion weiter vorne in diesem Kapitel angesprochen und auf die Art und Weise der Auswertbarkeit der zwei divergierenden Erkenntnisbasen auf Kap. 13 verwiesen wird.

Bevor man eine Untersuchung beginnt, muss man sich die Frage stellen, für wen deren Erkenntnisse gelten sollen. Dementsprechend gilt es, die Hypothesen zu formulieren und die empirischen Befragungen zu gestalten. Ein genereller Nachteil von qualitativer Forschung ist es, dass grundsätzlich keine statistische Repräsentativität möglich ist. Die Befunde der Experteninterviews können folglich nicht für eine gesellschaftliche Grundgesamtheit oder einen definierten Teilbereich dieser gelten, sondern lediglich eine Tendenz abbilden. Ebenso können natürlich nur diejenigen Verantwortlichen befragt werden, die dies auch möchten und damit 
u.U. aus eigenem Interesse handeln.

Aber auch in der hier vorliegenden quantitativen Untersuchung kann keine Repräsentativität gewährleistet werden. So kann es, u.a. begründet durch das Befragungsmedium Internet, keine Zufallsstichprobe geben, die sich auf eine Grundgesamtheit bezieht. Es kann nicht von einer willkürlichen Stichprobe gesprochen werden, da es trotz der stark zunehmenden Nutzung des Internet nach wie vor große Unterschiede bspw. in der altersmäßigen Verteilung gibt. Zugang und Nutzen des Internets sind in den Bevölkerungsteilen verschieden. Couper und Coutts (2004, S. 221f.) sprechen von der 'digitalen Kluft', die sich im Einkommen, in der Bildung, im Geschlecht und im Alter der Nutzer widerspiegelt. Es kann in dem vorliegenden Zusammenhang aber betont werden, dass gerade jüngere Menschen Internetnutzer sind, und gerade diese haben vermehrt Public Viewing-Veranstaltungen besucht (s. Kap. 13), was den Nachteil der digitalen Kluft z.T. aufhebt. Des Weiteren geben Couper und Coutts (2004, S. 219) an, dass sich oftmals gar nicht festgelegen lasse, welche Auswahlgesamtheit für Onlinebefragungen gelte. Auch der ADM (2001, S. 2) hebt hervor, dass es weder eine vollständige aktuelle Liste -ähnlich einem Telefonbuch- aller Internetnutzer gebe, noch Webseiten existieren, deren Besucherstruktur für die der Nutzer insgesamt repräsentativ sei.

Die meisten Fragestellungen, führen Couper und Coutts (2004, S. 219) weiter aus, bezögen sich darüber hinaus auf eine Zielpopulation, die über die der Internetnutzer hinausginge, es sei denn, diese würde explizit auf die zum Erhebungszeitraum aktiven Nutzer eingeschränkt. Ebenso sieht der Arbeitskreis Deutscher Markt- und Sozialforschungsinstitute e.V. (ADM) (2001, S. 2) eine Repräsentativität bei Onlinebefragungen in der Regel nur dann als gewährleistet an, wenn von Internetnutzern insgesamt oder speziellen Internetnutzern bzw. Nutzern bestimmter Websites als Zielpopulation der Untersuchung gesprochen würde. Generalisierbare Aussagen über andere Personengruppen, so führt er weiter aus, seien dagegen nur dann möglich, wenn die Onlinepenetration dieser Population nachweislich sehr weit fortgeschritten sei, ähnlich der der privaten Haushalte mit Telefon, oder wenn die offline für die Untersuchung ausgewählten Personen im Bedarfsfall mit einem Internetzugang ausgestattet würden. Erhebe man den Anspruch, für die Nutzer bestimmter Webseiten zu sprechen, sei die Auswahl der Teilnehmer per Zufallsgenerator zu empfehlen. 
Thielsch (2008, S. 97) bieten eine für diese Arbeit sinnvoll erscheinende 'Zwischenlösung' an, indem er postuliert:

„Für eine möglichst gute Datenqualität in Online-Untersuchungen empfiehlt sich die Nutzung eines aktiv rekrutierten und/oder sehr gut gepflegten Online-Panels ${ }^{90}$. Da dies aus Kostengründen in der Forschung oftmals nicht möglich ist, müssen stattdessen die Methoden der aktiven und passiven Auswahl von Probanden verwendet werden. Um Stichprobeneffekte zu reduzieren, empfiehlt sich bei aktiver und passiver Auswahl die Einladung zur Untersuchung möglichst breit zu streuen und große Stichproben zu erheben."

Durch dieses Vorgehen kann das hier angewandte Verfahren trotzdem nicht als repräsentativ gewertet werden, aber es wurde versucht, sich durch ein weites Verbreiten des Links einer Zufallsstichprobe anzunähern. Die Befragten entscheiden sich bei diesem Verfahren aber natürlich aus eigener Motivation zur Teilnahme (Selbstselektion), was übrigens auch für die Expertengespräche gilt. $\mathrm{Da}$ diese Störvariablen im Rahmen beider Untersuchungen nicht vollends kontrolliert werden können und darüber hinaus auch die Frage nach individueller Relevanz für die einzeInen Befragten (Zentralität) nicht geprüft werden kann, ist in beiden Erhebungen nicht der Anspruch auf Repräsentativität zu erheben.

Über die Fragen der Repräsentativität hinaus, bergen Onlinebefragungen weitere methodische Probleme, wie z.B. die Nichtkontrollierbarkeit von Mehrfachabstimmungen. Dies könnte z.B. durch die Speicherung der IP-Adressen oder die Verwendung von 'Cookies' (vgl. ADM, 2001, S. 5) unterbunden werden. Auf die Sicherung der Adressen ist aber, um die Anonymität der Befragten zu sichern, verzichtet worden. Dies ist zu rechtfertigen, da keiner der Abstimmenden einen persönlichen Vorteil, z.B. finanzielle Anreize, durch das Mehrfachausfüllen erreichen konnte und nicht von einer Verfälschung der Teilnehmer durch "'professionelle' Befragungsteilnehmer" (ebd., S. 2) auszugehen ist. ${ }^{92}$

Wie ersichtlich wurde, kann die folgende Interpretation der erhobenen Daten keine Aussagen über die gesamte Gesellschaft leisten, sondern sie zeigt, auch im

90 Ein Online-Panel besteht „aus einem Pool von registrierten Personen, die sich bereit erklärt haben, an Onlinebefragungen teilzunehmen. In der Regel werden mit den Namen und Adressen verschiedene soziodemographische und andere Merkmale dieser befragungsbereiten Personen gespeichert. Aus dem Pool der Panel-Teilnehmer werden mittels verschiedener Auswahlverfahren -abhängig vom Ziel einer Untersuchung- spezielle Teilgruppen ausgewählt und zumeist per E-Mail zur Teilnahme an einer bestimmten Onlinebefragung gebeten" (ADM, 2001, S. 6).

91 Der ADM (2001, S.2) empfiehlt an dieser Stelle, darüber hinaus auf die regionale Verteilung und internetnutzungsspezifische Streuung der Stichprobe zu achten.

92 Weitere Maßnahmen zur Sicherung der Grundgesamtheitsrepräsentativität sind beim ADM (2001) nachzulesen. Für die vorliegende Ausarbeitung soll die kurze Diskussion der wichtigsten Fehlerquellen genügen. 
Hinblick auf die Hypothesen, lediglich Tendenzen und Trendaussagen auf. Allerdings handelt es sich bei der vorliegenden Untersuchung um eine explorative Studie; und es liegt nicht im Interesse der Autorin, Schlussfolgerungen für alle Bürger der Bundesrepublik Deutschland oder den Mitgliedern einer Population, die einmal an einem Public Viewing teilgenommen haben abzuleiten.93 Es soll vielmehr ein erster Blick auf ein gesellschaftliches Phänomen geworfen werden, welches an vielerlei Stellen weiteren Forschungsbedarf eröffnet.

Zurückkehrend auf die eingangs begonnene Erörterung des methodologischen Triangulationsmodells, könne, so Mayring (2001, Abs. 31) allerdings generell angenommen werden, dass "qualitativ orientierte Forschung durch Hinzuziehen quantitativer Analyseschritte in aller Regel an Verallgemeinerbarkeit der Ergebnisse" gewinne. In dieser Arbeit wird „Triangulation als Ergänzung von Perspektiven, die eine umfassendere Erfassung, Beschreibung und Erklärung eines Gegenstandsbereichs ermöglichen“ (Kelle \& Erzberger, 2005, S. 304; vgl. auch Steinke, 1999, S. 46), angesehen. Denn jedes der kombinierten Verfahren, hebt Flick (2005, S. 314) hervor, müsse für sich als gleichwertig verstanden werden, also nicht „ein Verfahren als das zentrale und die anderen als Vorstufe oder Illustration" bewertet werden. Demnach sind die qualitativen Interviews sowohl als Vorstufe, um sich dem Gegenstand explorativ zu nähern, als auch als gleichgestellte Perspektive mit der qualitativen Onlinebefragung anzusehen. Durch dieses methodische Vorgehen ist es möglich, sowohl die Seite der Experten als auch die Seite der Besucher zu erfassen und somit einen breiteren und tieferen Zugriff auf Public Viewing als Forschungsgegenstand zu erhalten.

Bei der Bewertung der in der methodologischen Triangulation erhobenen Ergebnisse könne nach Flick (2004, S. 99f.) folglich gar nicht „ein Ansatz für die Bewertung der ganzen Studie dominieren, sondern [es müsse] auch dabei der Besonderheit beider Ansätze Rechnung getragen werden“. Bei einer Triangulationsstudie mit qualitativen und quantitativen Methoden stell sich in einem weiteren Schritt zwangsläufig die Frage nach der Verknüpfung der Daten

93 Durch den Abgleich mit den repräsentativen Daten der Befragungen von Behn et. al. (2007) bzw. Pilz (2007) zur wissenschaftlichen Begleitung und Evaluation des Fan- und Besucherbetreuungskonzepts der FIFA WM $2006^{\mathrm{TM}}$ wird allerdings versucht, den dieser Arbeit zugrunde liegenden Untersuchungsergebnissen mehr Tiefgang zu verleihen. 
und ihrer Auswertung. Während die quantitativen Daten mit Statistikprogrammen ${ }^{94}$ ausgewertet werden, ist das bei den qualitativen Aussagen nicht möglich. Somit ist die Auswertung der Ergebnisse, die mit unterschiedlichen Ansätzen erhoben wurden, auch mit verschiedenen Methoden erfolgt (s. auch Kap. IV) (vgl. ebd., S. 95ff.). Als einen Aussagewert zur Qualität von Triangulationsstudien nennt Flick (2004, S. 100) die Begründung des Aufwandes der Verwendung verschiedener Methoden, und ergänzt, dass darüber tatsächlich verschiedene Ebenen und Aspekte des zu umfassenden Phänomens zugänglich bzw. erfasst wurden. Diesem Verlangen kann die vorliegende Arbeit entsprechen, so hat sie Public Viewing von der Ebene der Experten genauso wie aus der Perspektive der Besucher zu erfassen versucht. So stellt nun in dieser Ausarbeitung die Triangulation selber in ihrem Aufbau, in ihrer Methodik, die Absicherung der Daten dar. $^{95}$

Es lässt sich also festhalten, dass das qualitative Erkunden der Meinungen, Erfahrungen und Einschätzungen, also der subjektiven Relevanzhorizonte der Experten, und das Erweitern und Ergänzen dieser Aussagen durch quantitative Daten der Besucher den Forschungsgegenstand im Rahmen der 'BetweenMethod-Triangulation' als dem Erkenntnisgewinn dieser Arbeit zuträgliche Methodik angesehen wird. Die Triangulation im Sinne eines Methodenmixes von qualitativen und quantitativen Daten erscheint als eine geeignete Vorgehensweise, um sich Public Viewing als Forschungsgegenstand explorativ zu nähern.

\subsection{Befragung}

Je nach Forschungsziel und Forschungsvorhaben können also in ein und derselben Untersuchung verschiedene Typen der Befragung, gerade eben auch Mix von qualitativen und quanitativen Verfahren, angewendet werden. In dieser Studie sind sowohl qualitative wie quantitative Befragungen durchgeführt worden. Die Befragung als methodisches Instrument hat, wie jedes andere auch Vor- und Nachteile, die es zu beachten gilt. So soll doch in diesem Kapitel das angewandte

94 In diesem Falle ist die Auswertung der quantitativen Daten mithilfe des Statistikprogramms SPSS erfolgt. Für die grafische Gestaltung der Abbildungen und Tabellen ist bei beiden Erhebungen auf MS-Exel zurückgegriffen worden.

95 Interessante Aspekte zur Triangulations diskussion sind z.B. in Denzin und Lincoln (1994, pp. 811) oder Flick (2004; 2005, S. 310f.) nachzulesen. 
Untersuchungsdesign fundiert begründet werden. Außerdem ist es für die Qualität von methodologischen Triangulationen wichtig, dass die gewählten Werkzeuge, zu dem zu untersuchenden Gegenstand passen (vgl. Kap. 11.1). So ist es nicht richtig, sich nur dem Design des Fragebogens zu widmen, sondern es müssen auch immer die Bedingungen Beachtung finden, unter denen er entwickelt und angewandt worden ist. Aus den genannten Gründen wird an dieser Stelle eine kurze Diskussion der Befragungsthematik erfolgen.

\begin{abstract}
„Befragungen bedeuten Kommunikation zwischen zwei oder mehreren Personen. Durch verbale Stimuli (Fragen) werden verbale Reaktionen (Antworten) hervorgerufen: Dies geschieht in bestimmten Situationen und wird geprägt durch gegenseitige Erwartungen. Die Antworten beziehen sich auf erlebte und erinnerte soziale Ereignisse, stellen Meinungen und Bewertungen dar" (Atteslander, 2003, S. 120).
\end{abstract}

Nun scheint diese Begriffseingrenzung genauso auf jede alltägliche Unterhaltung zwischen Person A und Person B oder/und weiteren Individuen zuzutreffen. Den Unterschied zwischen einer wissenschaftlichen und einer alltäglichen Befragung besteht allein in der theoriegeleiteten Kontrolle der gesamten Befragung: „Wissenschaftlichkeit beruht auf systematischer Zielgerichtetheit und Theorie“ (ebd., S. 123) (vgl. auch Gläser \& Laudel, 2004, S. 108). Im Rahmen dieser Arbeit ist das Vorgehen im qualitativen Bereich z.B. anhand des vor dem Zugang zum Feld erstellten Fragebogenleitfadens gewährleistet worden. Dieser basiert auf einer ersten Sichtung der wissenschaftlichen Literatur und ist auf die Fragestellungen hin ausgerichtet. Durch die sorgfältige und exakte Ausarbeitung des Leitfadens kann die Situation wissenschaftlich kontrolliert ablaufen. Ein Vorteil der Befragung ist z.B. die Ermittlung von persönlichen Erfahrungen, Meinungen und Relevanzhorizonten der beteiligten Personen, also die breite Anwendbarkeit auf Tatbestände, die nicht beobachtbar sind oder zukünfitge Handlungen und Intentionen betreffen. Des Weiteren sind u.a. die Möglichkeit der Themenzentrierung, also die Steuerung des Gesprächleitfadens durch die entsprechende Formulierung und das Erfassen von Selbst- und Fremdbeobachtung zu nennen (vgl. Heinemann, 1998, S. 91).

Im Folgenden wird das Expertengespräch als eine Form der Befragung diskutiert. Jede Befragung, ob dies eine qualitative Expertenbefragung oder eine quantitative Online umfrage ist, stellt eine soziale Situation dar. Von sozialer Situation ist selbst 
dann zu sprechen, wenn jemand für sich allein auf einen schriftlichen Fragebogen Antwort gibt oder wenn er telefonisch befragt wird. Sozial nicht nur, weil die beteiligten Personen untereinander kommunizieren, sondern auch, weil die spezifische Umgebung eine Rolle spielt. Immer wirken die Erwartungen beider Gesprächsteilnehmer, sowie deren Wahrnehmungen und die verbale wie nonverbale Reaktion einer Rolle (vgl. Atteslander, 2003, S. 123; Heinemann, 1998, S. 92ff.). Bei den qualitativen Telefongesprächen nimmt der Interviewer, wie bei jeder persönlichen Befragung, immer auch direkten Einfluss auf den Gesprächsverlauf. Der Gesprächführende stellt also einen schwer einzuschätzenden Einflussfaktor dar. Andererseits kann er auch in gewissem Maße eine Regel- und Kontrollinstanz übernehmen. Bei schriftlichen Befragungen entfallen beide positive und negative Faktoren. Denn normalerweise kann nicht festgestellt werden, wer wann die Fragen, unter welchen Umständen, ob alleine oder mithilfe eines anderen beantwortet hat (vgl. Atteslander, 2003, S. 149; Hermanns, 2005).

Bei den Expertengesprächen erscheint das so genannte 'neutrale Interview' eine geeignete Form der Interviewführung $z u$ sein. Mit dieser Art der Gesprächslenkung wird versucht, die genannten Einflussfaktoren möglichst gering zu halten. Wobei es zu beachten gilt, dass es in einer menschlichen Beziehung, und sei das eine Befragungssituation, keine Neutralität gibt ( $\mathrm{vgl}$. Atteslander, 2003, S. 152). Es kann bspw. nicht verhindert werden, dass sich der Befragte über den Interviewer als Person und die Intention des Gesprächs informiert und somit eine Einstellung zu der Person entwickelt. Folglich ist es nicht möglich, als Interviewer reine Senderfunktion für Stimuli und Empfängerstation für Reaktionen zu sein. Das Ziel einer möglichst uniformen und neutralen Interviewsituation um die Vergleichbarkeit der Daten hoch zu halten, ist generell erstrebenswert, jedoch ist es nicht realitätsnah. Aus diesem Grund wird die bereits 1974 von Maccoby und Maccoby angeführte Form des 'gelockerten neutralen Interviews' in dieser Arbeit vertreten, und die Interviews wurden in dieser Form von der Autorin selbst durchgeführt.

„Der Interviewer (soll sich) bis zu einem gewissen Grad zurückhalten; er sollte einen seriösen Eindruck machen und deutlich werden lassen, dass er das Interview ernst nimmt. Gleichzeitig sollte er keinen zu steifen Eindruck machen: es ist von größter Wichtigkeit für den Interviewer, ein echtes Interesse an dem zu zeigen, was der Befragte sagt" (Maccoby \& Maccoby, 1974, S. 63 zit.n. Atteslander, 2003, S. 152).

So scheint es der Autorin unerlässlich, den Befragten und den von diesem dem 
Interviewer zugestandenen zeitlichen Aufwand zu würdigen. Nur so kann es gelingen, das Interview erfolgreich zu Ende zu führen. So sollte es zwar zum Grundsatz jeder Befragung gehören, die Meinung des Interviewers zum Untersuchungsgegenstand zu verbergen. Doch muss nach den Vorstellungen der Autorin auf den Interviewten bspw. durch unterstützende Bemerkungen eingegangen werden, um die Motivation für die Durchführung des Interviews am Laufen zu halten (vgl. auch Hermanns, 2005). So ist natürlich dem Interviewer immer mehr daran gelegen, Antworten zu erhalten, als dem Befragten daran gelegen ist, solche zu geben. Allerdings ist auch kein Experte gänzlich ohne Interesse, verbraucht das Gespräch doch Arbeitszeit- und kapazität. Natürlich ist hier immer die Position der befragten Person innerhalb des Unternehmens, der Agentur u.a. sowie deren Rechtsform zu beachten, denn von diesen Faktoren hängt selbstverständlich die Möglichkeiten der Zeit- und Kapazitätseinteilung ab. In der vorliegenden Ausarbeitung werden explorative Ziele hinsichtlich der Ergründung des Phänomens Public Viewing verfolgt. Die Experten sind zur Rekonstruktion des „sozialen Prozesses“ (Gläser \& Laudel, 2004, S. 107) Public Viewing befragt worden. Zu der Erkundung neuer wissenschaftlicher Territorien eignen sich Experteninterviews in ihrer wenig strukturierten Interviewsituation (vgl. Atteslander, 2003, S. 153) (vgl. auch Kap. 11.1). Die durchgeführten Interviews sind nach teilstrukturierter Form angelegt (Befragungstyp III), was bedeutet, dass es sich hier um Gespräche handelt, die aufgrund vorbereiteter und vorformulierter Fragen stattfinden, deren Abfolge jedoch relativ offen ist. Diese in der qualitativen Forschung häufig angewandte Form der qualitativen Interviewführung lässt Spielräume in der Frageforumlierung, in der Nachfragestrategie und in der Abfolge der Fragen offen (vgl. Hopf, 2005, S. 351). 


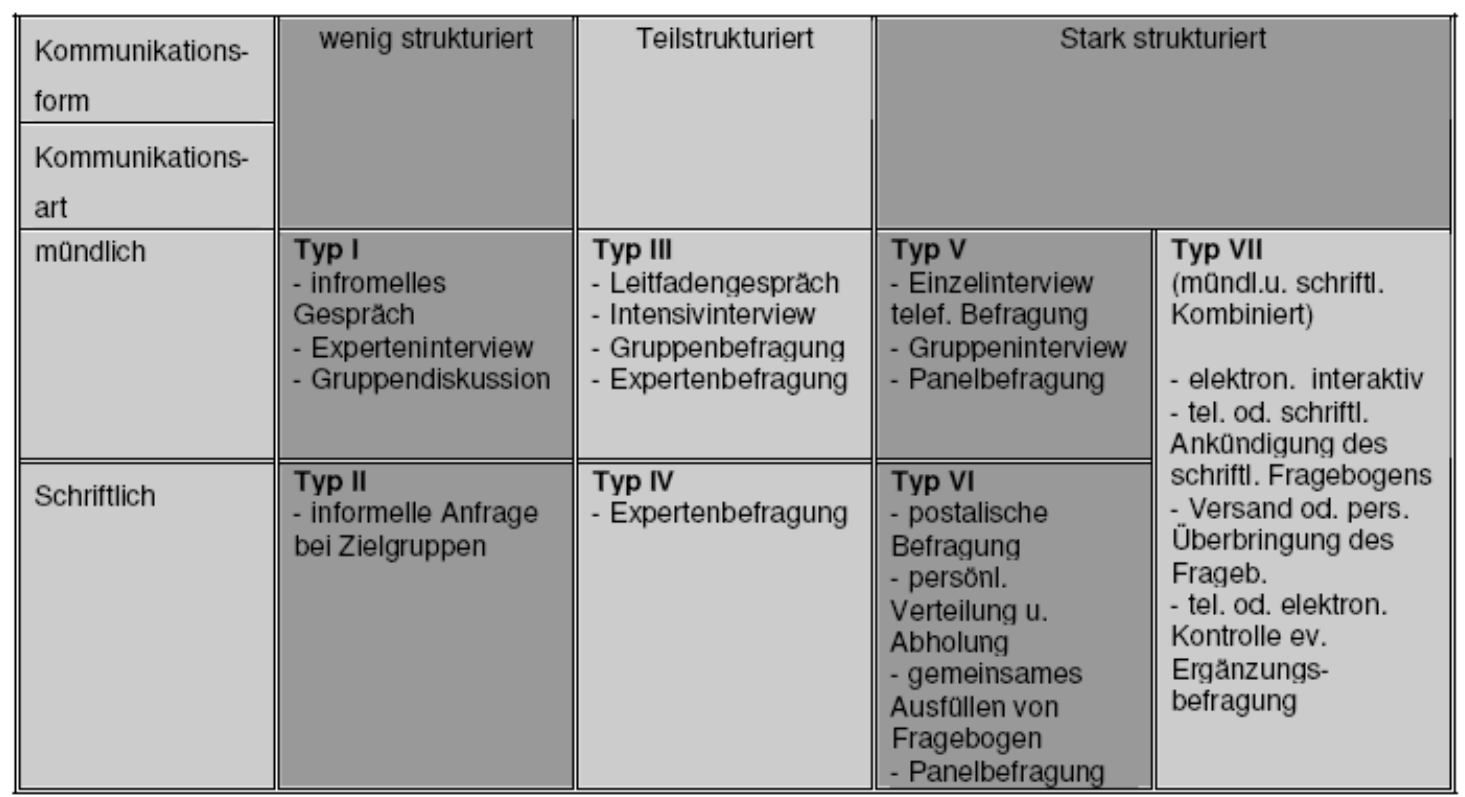

Abb. 19: Typen der Befragung (nach Atteslander, 2003, S. 145)

Es handelt sich folglich um einen teilstrukturierten standardisierten Gesprächsleitfaden. Dadurch besteht die Möglichkeit, sich aus dem Gespräch ergebende Themen aufzunehmen und sie entsprechend weiter zu verfolgen. Obwohl ein späterer Vergleich der Befragungen in ihrer Tendenz erfolgen soll, wurde an dieser Stelle von einer stark strukturierten Befragung abgesehen, da die Einschränkung der Handlungs- und Freiheitsspielräume aller Beteiligten in einem solchen Verfahren relativ groß sind (vgl. ebd., S. 148).

Nachteile gegenüber standardisierten Interviews sind nach Schnell, Hill und Esser (1999, S. 353f.) in den

- $\quad$ höheren Anforderungen an den Interviewer, den

- $\quad$ stärkere Interviewereinflüssen, der

- $\quad$ Abhängigkeit der Datenqualität von der Qualität der Interviewer, im

- $\quad$ höheren Zeitaufwand sowie in der

- $\quad$ geringeren Vergleichbarkeit der Ergebnisse und damit in der

- $\quad$ schwierigere Auswertbarkeit zu sehen.

Dennoch muss eine gewisse Struktur für den hier vorliegenden Zusammenhang, ein Leitfaden für die theoretische Untermauerung vorhanden sein. Dieser gibt dem Gespräch einen Sinn und eine Richtung. Gerade dann, wenn in einem Interview mehrere verschiedene Themen behandelt werden sollen, die durch das Ziel der Befragung und nicht durch die Antworten des Interviewten bestimmt werden. 
Dasselbe gilt, wenn in dem Gespräch u.a. spezifische fassbare Auskünfte erkundigt werden sollen. Schnell et al. (1999, S. 353f.) weisen zudem darauf hin, dass eine höhere Bereitschaft des Befragten zur Mitarbeit anzunehmen sei. Diese drei Intentionen sind in den Telefoninterviews in dieser Arbeit verfolgt worden.

Die Expertengespräche fanden als Telefoninterview statt. Die Vorteile dieses Verfahrens sind eindeutig in der zeitlichen Effizienz, der Praktikabilität und der Kostenersparnis zu sehen. Nachteilig ist neben der erschwerten Kontrolle der Interviewsituation (s. oben) die Unmöglichkeit, sich an die Interviewsituation und den Gegenüber anhand dessen Mimik und Gestik zu gewöhnen. Aus diesem Grund wurde zu Beginn des Gesprächs nochmals kurz auf die Intention der Interviewerin sowie den organisatorischen Ablauf eingegangen. ${ }^{96}$ Darüber hinaus wurden den Experten zu Beginn des Interviews einige Fragen als Aufwärmphase gegeben (s. Anhang B). Dies dient der Gewöhnung an den Gesprächsverlauf und dem Wachrufen der Erinnerungen. Die wichtigsten Fragen wurden aus diesem Grund nicht sogleich am Anfang gestellt. Um die relativ höhere Reaktivität von Telefoninterviews verglichen mit schriftlichen Befragungen zu mindern, schlägt Atteslander (2003, S. 180) vor, den Fragebogen vor dem Gespräch dem Interviewten zuzusenden. Dies ist im Bedarfsfall erfolgt.

Wie verhält es sich nun mit einer Onlinebefragung? Nach dem ADM (2001, S. 1) schließt der Begriff Befragungen ein, bei denen die Teilnehmer

- $\quad$ einen Fragebogen per E-Mail zugesandt bekommen und inn selber zurücksenden,

- $\quad$ den Fragebogen von einem Server herunterladen und inn wieder per E-Mail zurücksenden und

- $\quad$ einen auf einem Server hinterlegten Fragebogen direkt im Internet online ausfüllen.

In der vorliegenden Arbeit wurde letztere Lösung angewandt. Der Onlinefragebogen wurde direkt auf dem Server gespeichert. Die Probanden hatten also keinen zusätzlichen Aufwand durch das Zurücksenden des ausgefültten

96 Pilshofer (2001, S. 12) weist auf die Wichtigkeit des einleitenden Gesprächteils bzw. bei schriftlichen Befragungen auf den Einleitungstext hin. Sie nennt u.a. den Hinweis auf den Wert jedes beantworteten Fragebogens bzw. Interviews und die Bitte um ehrliche Antworten als wichtige Bestandteile. 
Fragebogens per E-Mail.

Im Prinzip gelten für Onlinebefragungen dieselben Regeln und Verhaltensmaßnahmen wie für jede Befragung. Sie hat jedoch unschlagbare Vorteile, wie geringe Erhebungskosten, eine hohe Zeit- und Ortsunabhängigkeit und eine erhebliche erlebte Anonymität der Teilnehmer. Zudem hat der Ausführende einen geringeren Aufwand in der Auswertung, da die Dateien direkt in eine Datenbank auf dem Server gelesen und gespeichert werden (vgl. Thielsch, 2008, S. 100). ${ }^{97}$ Dadurch sind die Daten sofort einsehbar, und bei Bedarf kann sogar ein Zwischenreport angesehen werden. Der größte Vorteil einer schriftlichen Befragung im Internet liegt sicherlich darin, dass mit geringem Personalaufwand und in relativ kurzer Zeit eine verhältnismäßig große Zahl an Befragten erreicht werden kann. Der den genannten Vorteilen gegenüberstehende große Nachteil ist natürlich, dass nur die Teile der Gesamtbevölkerung erfasst werden können, die zum Einen elektronisch erreichbar und zum Anderen im Umgang mit dem Internet geübt sind (vgl. Atteslander, 2003, S. 186) (s. Kap. 11.1). ${ }^{98}$ Es wäre wünschenswert gewesen, die nicht erfassbare Gruppe zur Erreichung eines angemesseren Grades der Repräsentativität unbedingt in die Analyse der Daten einzuschließen. Insgesamt sehen die von Pötschke und Simonson (2001, S. 34) befragten Experten aus Wissenschaft und Wirtschaft dennoch, trotz dieses Makels, in dieser Form der Befragung ein so hohes Potential, dass sie die Vermittlung von Kenntnissen in diesem Verfahren in sozialwissenschaftlichen Studiengängen als notwendig ansehen. Auch Atteslander (2003, S. 190) gibt an, dass elektronisch schriftliche Umfragen zukünftig vermehrt Anwendung finden würden, vorausgesetzt allerdings, dass die Forschungsgegenstände dem Charakter der 'Internet-Population' entsprächen.

Für die vorliegende Onlineuntersuchung wurde ein schriftlich stark strukturiertes Verfahren gewählt, diese ist damit dem Befragungstyp $\mathrm{VI}^{99}$ (s. Abb. 19) zuzurechnen. Im Gegensatz zu den qualitativen Expertengesprächen mit ihrer geringen Strukturierung wird hier eine starke Strukturierung gewählt, um

\footnotetext{
97 Darüber hinaus werden Fehler in der Auswertung durch das automatische Einlesen der Daten vermieden.

98 Eine dezidierte Auseinandersetzung mit den Vor- und Nachteilen der jeweiligen Formen von Befragungen sind bei Atteslander (2003), Heinemann (1998) und Mayring (2002) nachzulesen. Eine weitergehende Diskussion wird an dieser Stelle nicht als zweckmäßig empfunden.

99 Obwohl in der ursprünglichen Grafik von Atteslander (2003, S. 145) die Onlinebefragung in dieser Sektion unerwähnt bleibt.
} 
quantitative Aspekte zu erfassen. Den Befragten wurde die absolut vertrauliche und anonyme Verwendung der Antworten zugesichert; für eventuell aufkommende Fragen war es jedoch jederzeit möglich, der Autorin eine E-Mail zu senden. Natürlich kann nicht ausgeschlossen werden, dass während des Ausfüllens des Fragebogens andere Personen die Antworten des Befragten beeinflussen. Somit kann die völlige Kontrolle der Befragungssituation nicht gewährleistet werden. Um die unvoreingenommene Beantwortung der Fragen aber so weit wie möglich zu gewährleisten, ist so wenig Information wie möglich zur Untersuchung preisgegeben worden (s. Anhang C).

Letztlich kann festgehalten werden, dass die Methodik der Befragung, sowohl in Form eines Leitfadengesprächs als auch als standardisierte Onlinebefragung eine Reihe von z.T. kaum zu exkludierenden und zu beurteilenden möglichen Einflussgrößen besitzt. Es ist jedoch bestmöglich versucht worden, diese Faktoren gering zu halten, bzw. diese nicht zuletzt durch die methodologische Triangulation auszugleichen.

\subsection{Zusammenfassung}

Das Ziel des vorliegenden Kapitels ist es gewesen, die Methodik der durchgeführten Untersuchungen zu rechtfertigen und transparent zu machen. Es konnte aufgezeigt werden, dass sich das leitfadengestütze Experteninterview eignet, einen nahen Zugang zum sozialen Geschehen zu erlangen und möglichst detaillierte Befunde über gesellschaftliche Prozesse -und gerade die in der differenzierten Postmoderne- zu erheben. In einer zweiten Untersuchung wurden quantitative Werte durch die Onlinebefragung von Public Viewing-Besuchern erhoben. Die Kombination und analoge Bewertung der zwei Befragungen im Sinne der Between-Method-Triangulation stellt eine dem Untersuchungsgegenstand adäquate empirische Maßnahme dar. Ebenso ist das methodische Instrument der Befragung, sowohl in Form des per Telefon durchgeführten Leitfadengesprächs als auch das in der Form der Onlinebefragung, als für den vorliegenen Zusammenhang geeignet anzusehen. Die in der vorliegenden Ausarbeitung angewandte Methodik ist demnach dem Erkenntnisgewinn zuträglich und kann folglich in ihrer Gesamtheit als dem Forschungsstand und -vorhaben entsprechend angesehen

werden. 


\section{Forschungsdesign}

Public Viewing lässt sich aufgrund seiner Komplexität auf die unterschiedlichsten Theoriebereiche zurückführen. An der Schnittstelle von Sportwissenschaft, Soziologie, (Sozial-)Psychologie, Medienwissenschaft und Ökonomie sind zwei sich ergänzende Befragungen entworfen worden. Diese entsprechen in ihrer Gesamtheit der Komplexität der Hypothesen H1, H2 und H3. Im Folgenden wird explizit auf den Aufbau, den Gegenstandsbereich, die Herangehensweise und die zeitliche Eingrenzung der beiden Befragungen eingegangen. Das qualitative Design der Experteninterviews stellt den Anfang dar (12.1). Die Public ViewingSpezialisten der Fußball-WM 2006 wurden mit Hilfe eines GesprächleitfadenInterviews befragt. Dieser gliederte sich in mehrere zunächst als explorativ zu sehende Sektionen, die sich mit jeweils einer Facette des Phänomens auseinandersetzen (12.1.1). Trotz des explorativen Vorgehens ist eine Eingrenzung des Gegenstandsbereichs für eine Auswertung der Daten unumgänglich (12.1.2). Zudem ist der gewählte Zugang zu den Experten (12.1.3) und der Zeitraum der Befragung (12.1.4) offen zu legen.

Der zweite Teil des Kapitels besteht aus der Erörterung des quantitativen Aufbaus der Besucherbefragung (12.2). Die Public Viewing-Gäste sind mit einem starkstrukturierten Onlinefragebogen konfrontiert worden (12.2.1). Im nächsten Schritt wird der Gegenstandsbereich der Befragung ab- und eingegrenzt (12.2.2). Darauffolgend gilt es den gewählten Feldzugang zu erörtern (12.2.3) und das Zeitfenster für die Onlinestellung der Untersuchung einzugrenzen (12.2.4). 


\subsection{Design der Experteninterviews}

Im Rahmen dieser Arbeit wurden Expertengespräche per Telefon durchgeführt. Nach Atteslander (2003, S. 155) werden Expertengespräche wie folgt definiert:

„Bei Experteninterviews sprechen wir mit Menschen, die entweder im Umgang mit unseren Probanden Erfahrung haben: z.B. Lehrer, Sozialarbeiter, Sportfunktionäre, oder die über unseren Forschungsgegenstand besondere und umfassende Erfahrung haben."

In der vorliegenden Arbeit sind 21 Public Viewing-Spezialisten der FußballWeltmeisterschaft 2006 befragt worden. Als Experten gelten in diesem Fall die mit der Konzeption, Planung und Durchführung des Public Viewing vertrauten Personen von offiziellen Fan Festen, kommerziellen sowie nicht-kommerziellen Veranstaltungen. Es wird von diesen Personen angenommen, dass sie besondere und umfassende Erfahrungen bzgl. des Forschungsgegenstands besitzen (vgl. auch Gläser \& Laudel, 2004, S. 113).

Die Experteninterviews sind per Telefon durchgeführt und währenddessen durch Tonbandaufzeichnungen konserviert worden. In der Vorbereitungsphase ist zunächst die Festlegung auf die explorativ zu erfragenden Themenkomplexe, die sich vor allem aus einer ersten Literaturrecherche der Autorin gespeist haben, erfolgt. Bei der Realisierung des Fragenkatalogs ist darauf geachtet worden, die Fragen mit möglichst einfachen Worten, kurz und konkret zu formulieren, weil gerade in der telefonischen Befragung die Wahrscheinlichkeit von Missverständnisse erhöht ist (12.1.1). In einem weiteren Schritt ist der Gegenstandsbereich der Untersuchung festgelegt worden. So wurde sich an die von der FIFA und Infront differenzierten Kategorien von Public ViewingVeranstaltungstypen orientiert (12.1.2). Der Erstkontakt mit den Experten ist aktiv über das Internet erfolgt. In einem Anschreiben wurden die Befragten darüber informiert, wer für die Befragung verantwortlich ist, warum die Untersuchung durchgeführt wird und welches Interesse der Befragte selbst an der Beantwortung des Fragenkomplexes haben könnte (12.1.3). Schließlich wird erörtert, in welchem zeitlichen Rahmen die Telefoninterviews durchgeführt worden sind. Einerseits war es wichtig, aufgrund des explorativen Vorgehens der Untersuchung, bei dem Wert auf die noch frischen Erinnerungen und Eindrücke der Geschehnisse gelegt wurde, die Interviews zeitlich nah an der im Sommer 2006 stattfindenden FußballWM zu führen. Andererseits ließen aber auch weniger wissenschaftliche Faktoren, 
wie bspw. die bevorstehende Urlaubszeit es unbedingt wichtig erscheinen, die Gespräche möglichst zeitnah durchzuführen (12.1.4).

\subsubsection{Aufbau des Gesprächleitfadens}

Der Interviewleitfaden gliedert sich in acht Bereiche. Teil A beschäftigt sich mit 'Allgemeine[n] Informationen zu den Räumlichkeiten', Teil B fragt nach dem 'Besucherverhalten und [der] Gästeanzahl', im dritten Teil C geht es um 'finanzielle Aspekte'100 der Veranstaltungen. In den darauf folgenden Bereichen schließen sich Fragen zu 'Motiven, Erwartungen und Erfahrungen' (Teil D), den benutzten 'Technologien' (Teil E), dem 'Sicherheitskonzept' (Teil F) und dem 'Emotionsmanagement' (Teil G) an. Letztlich sind im Teil H noch 'persönliche Angaben' zu machen. ${ }^{101}$

Der Leitfaden gilt in diesem Zusammenhang als Orientierung. So ist der Gedanke verfolgt worden, mit weniger wichtig erscheinenden Fragen zu beginnen, um dem Interviewten die Eingewöhnung in die Situation zu erleichtern (s. Kap. 11.2). Von primärem Interesse muss es aber sein, die Aufmerksamkeit des Experten zu halten, um das Interview vollständig führen zu können. Die Abfolge der vorbereiteten und vorformulierten Fragen ist vorher aber nicht streng festgelegt worden. Es ist in der Tendenz die Möglichkeit verfolgt worden, aus dem Gespräch sich ergebende Themen aufzunehmen und sie von den Antworten der Interviewten ausgehend weiter zu verfolgen. Letztlich ist es nur so möglich gewesen, die für die Aufgabenstellung wichtigen Themenbereiche für den weiteren Forschungsverlauf herauszufiltern und wichtige Impulse für die Handlungsempfehlungen zu erhalten. Um Irrtümern und Missverständnissen weitestgehend vorzubeugen, sind Pre-Interviews durchgeführt worden. So ist die Vollständigkeit und Klarheit der Fragestellungen nochmals überprüft werden und in einigen Bereichen geringfügig modifiziert worden.

Es ist darüber hinaus durch offene Fragestellungen versucht worden, den Gesprächskontakt und das Interesse am Interview fördern. Zudem konnten so am

100 Aus den Experteninterviews ist der Teil C, in dem es um finanzielle Aspekte ging, herausgenommen worden, da er sich zum einen als schwer beantwortbar und zum anderen für die Schwerpunktlegung dieser Ausarbeitung als nebensächlich herausstellte. In den sich auf der Daten-CD befindlichen Transkripten als Tertiärdaten wird Teil $C$ nicht weiter berücksichtig werden. 101 Ein exemplarischer Leitfaden ist im Anhang $(B)$ einzusehen. 
einfachsten die obig angesprochenen Komplexe aussortiert werden, bei denen es entweder Probleme in der Beantwortung gab, oder die sich anderweitig als nicht so ergiebig herausstellten. Weiter ist versucht worden, den Befragten das Gefühl zu geben, Wert auf ihr Urteil zu legen und sie in ihrer Person wie ihren Meinungen und Erfahrungen ernst zu nehmen (vgl. auch Kap. 11). Nach Atteslander (2003, S. 165) ziehen vor allem gut informierte Personen mit eigener Meinung offene Fragen aus diesen Gründen vor. Denn es darf nicht außer Acht gelassen werden, dass sich die Befragten die Zeit des Interviews von ihrer sonstigen Arbeitszeit abzweigen. Das kann für sie Mehrarbeit nach Feierabend oder ein erhöhtes Arbeitspensum für den Rest des Tages bedeuten.

\subsubsection{Gegenstandsbereich}

Im Vorfeld der Untersuchung ist die Einteilung der zu befragenden Experten in Kategorien erfolgt. Mit der Disposition ist das Ziel verfolgt worden, das Feld zu entschlüsseln. Hierbei wurde sich an die Untergliederung der FIFA und des Vermarkters der Public Viewing-Rechte Infront angelehnt. Diese hatten bei den Public Viewing-Veranstaltungen zu den Spielen der Weltmeisterschaft 2006 die TV-Vorführungen wie folgt eingeteilt:

1. Das Public Viewing auf den Fan Festen der FIFA WM $2006^{\mathrm{TM}}$ : Sie werden von den FIFA WM-Städten mit finanzieller Hilfe von fünf offiziellen FIFAPartnern ausgerichtet.

2. Das nicht-kommerzielle Public Viewing: Diese von privater oder öffentlicher Hand organisierten Veranstaltungen müssen zwar bei Infront registriert sein, eine Lizenzgebühr muss aber auch hier nicht entrichtet werden. Allerdings dürfen die Veranstalter keinerlei kommerziellen Nutzen verfolgen, also Eintritt o.ä. verlangen.

3. Das kommerzielle Public Viewing. Hier handelt es sich um Veranstaltungen mit gewerblichem Charakter. Nach Erwerb der Rechte bei Infront darf in diesem Fall Eintritt verlangt werden.

Nach der genannten Differenzierung sind Experten angeschrieben worden. Die zeitlich ersten Interviewpartner jeder der drei Kategorien galten als Pre-Interviews, 
d.h. es wurden an angesprochenen Stellen die Fragen bzw Antworten für die bessere Verständlichkeit leicht modifiziert. Diese Interviews werden mit berücksichtigt, da keine gravierenden Änderungen vorgenommen worden sind und schließlich nur so ein möglichst vollständiges Bild erzielt werden kann.

\subsubsection{Feldzugang}

In einem ersten Schritt wurde über das Internet versucht, Kontakt zu den Experten herzustellen. Dieses war bei den offiziellen Fan Festen z.B. durch die Homepages wesentlich einfacher als bei kleinen kommerziellen und nicht-kommerziellen Anbietern, die z.T. über Suchläufe der gängigen Onlinesuchmaschinen oder auch über den Internetauftritt von regionalen und lokalen Veranstaltungsmagazinen ausfindig gemacht wurden. Der Erstkontakt wurde über eine Ankündigungsemail (s. Anhang A) hergestellt. Natürlich konnten über das Internet nur eingeschränkt die Public Viewing-Veranstaltungen erreicht werden, die online vertreten waren oder über die dort entsprechend berichtet wurde. Dies wurde aber in Kauf genommen, da davon ausgegangen wurde, dass fast alle Veranstaltungen, die Publikum anziehen möchten, online vertreten sein würden. Auf Wunsch wurde, um die vergleichsweise höhere Reaktivität bei Telefoninterviews im Vergleich zu schriftlichen Befragungen weitestgehend einzudämmen ( $\mathrm{vgl}$. A tteslander, 2003, S. 180), vor dem Gespräch ein blanko Leitfaden an den zu Befragenden gesandt. Vor dem Beginn des eigentlichen Interviews wurden nochmals alle wichtigen Informationen zum geplanten Forschungsvorhaben und die Kontaktdaten der Autorin genannt. Zudem wurde den Experten die vollständige Anonymisierung ihrer Daten zugesichert. Die Transkription der erhobenen sozialen Daten ist folglich maskiert erfolgt. Unter Transkription wird pauschal die "graphische Darstellung ausgewählter Verhaltensaspekte von Personen, die an einem Gespräch (z.B. einem Interview oder einer Alltagsunterhaltung) teilnehmen" (Kowall \& O'Conell, 2005, S. 438; s. auch Dittmar, 2004, S. 50) verstanden. In dem vorliegenden Fall ist auf die graphische Darstellung von parasprachlichen Elementen, wie z.B. Sprechpausen oder außersprachlichen Äußerungen verzichtet worden. Diese wurden im vorliegenden Erkenntniszusammenhang als nicht unbedingt notwenig erachtet (vgl. Dittmar, 2004, S. 51f.; Gläser \& Laudel, 2004, S. 188), deshalb ist es vorgezogen worden, die Gespräche in 
Standardorthographie zu verschriftlichen. Die Standardorthographie orientiert sich an den Normen der geschriebenen Sprache, vernachlässigt aber, wie vorher erwähnt, Besonderheiten der gesprochenen Sprache (vgl. Kowall \& O'Conell, 2005, S. 441), indem der Sprachstil geglättet wird, wird das Lesen der Transkripte vereinfacht. Eine derartige Protokolltechnik kann nach Mayring (2002, S. 91) dann angewendet werden, wenn ausschließlich inhaltlich-thematische Aspekte für die spätere Auswertung und Interpretation wie in der vorliegenden Arbeit wichtig sind.

\subsubsection{Zeitfenster}

Ziel der Leitfadengespräche ist es gewesen, die Meinungen, Eindrücke und Einstellungen der Verantwortlichen möglichst zeitnah hinsichtlich der FIFA Fußball-Weltmeisterschaft $2006^{\mathrm{TM}}$ festzuhalten. Die Spiele der WM haben vom 20. Juni bis zum 09. Juli 2006 stattgefunden. Die Interviews erfolgten in den Wochen vom 11. Juli bis 18. August 2006.

Für das Einfangen möglichst unverfälschter Impressionen und zur Vermeidung von Verfälschungen der Expertenmeinungen durch spätere Medienberichte o.ä., ist das Ziel verfolgt worden, die Gespräche so früh wie möglich im Anschluss a n das Weltmeisterschafts-Turnier durchzuführen. Darüberhinaus war es aber nötig, die Verantwortlichen nach der Stressphase während und kurz nach der WM, aber wiederum vor der Urlaubszeit danach zu erreichen. Damit stand für die Befragungen nur ein sehr begrenzter Zeitrahmen zur Verfügung. Vielleicht kann dieses enge Zeitfenster der Grund dafür gewesen sein, dass z.B. nur fünf der elf Verantwortlichen für die Fan Feste zu einem Interview bereit gewesen sind.

\subsection{Design des Onlinefragebogens}

Die quantitative Datenerhebung fand über eine Onlinebefragung im Internet statt, die mithilfe des Freeware-Programms 'oFb' (Onlinefragebogen) erstellt und durchgeführt wurde. ${ }^{102}$ Dieses am Institut für Kommunikationswissenschaften der Ludwig-Maximilians-Universität München entwickelte Programm kann über den Server http://ofb.msd-media.de/ erreicht und bedient werden. Hierzu stellt oFb neben dem Speicherplatz auch die wichtigsten Tools zur Erstellung von

102 Ein blanko Fragebogen ist im Anhang (C) einsehbar. 
Fragebögen zur Verfügung.

Die Befragung im Internet ist in starkem Maße strukturiert erfolgt und wurde sogleich auf dem Server gespeichert. Das Instrument Fragebogen ist in diesem Falle u.a. durch geschlossene Antwortkategorien stark standardisiert worden (12.2.1). Bei der folgenden quantitativen Onlinedatenerhebung sind die Besucher von Public Viewing-Veranstaltungen Gegenstandsbereich (12.2.2). Die Verbreitung des Links zum Fragebogen ist mit einem kurzen informativen Anschreiben per E-Mail und über das Posting in Internetforen erfolgt (12.2.3). Abschließend erfolgt die Erläuterung über den Zeitraum, in dem der Fragebogen online zugänglich war (12.2.4).

\subsubsection{Aufbau des Onlinefragebogens}

Bei der Vorbereitung des Onlinefragebogens hat die Priorität auf dem Fokus gelegen, die durch die qualitative Befragung und die theoretischen Gedanken aufgekommenen Ansätze zur Deutung des Phänomens Public Viewing zu vertiefen. Bei der Konstruktion ist durch die starke Strukturierung der Anzahl und der Reihenfolge der Fragen der Inhalt festgelegt worden. Insgesamt ist der Fragebogen stark standardisiert und auch der Inhalt der Antwortkategorien wurde bis auf das ergänzende Feld für 'Sonstiges' ${ }^{\text {,103 }}$ im Voraus bestimmt. Mit diesem Vorgehen ist der in der methodologischen Triangulation (s. Kap. 11.1) geforderte Mix von qualitativen und quantiativen Instrumenten erfüllt worden. Zudem ist versucht worden, die Triangulation an sich als Gütekriterium insoweit zu erfüllen (s. Kap. 11.1), als dass die Daten sich sowohl ergänzen als auch unterstützen. Nach einem Probedurchlauf ist der Fragebogen noch im Detail modifiziert worden, um Verständnisschwierigkeiten zu minimieren und einen reibungslosen Ablauf zu sichern.

An dieser Stelle soll ein kurzer Überblick über die Gliederung der Untersuchung und die Sektionen des Fragebogens gegeben werden. Dem Fragebogen vorweg geschaltet ist ein Anschreiben, welches kurz über die Autorin, den Hintergrund der Untersuchung und die Verwendung der erhobenen Daten informiert. Je nachdem,

103 Mit dem Feld 'Sonstiges' ist dem Grundsatz dieser explorativen Studie Folge geleistet worden, ein möglichst umfassendes Bild des Forschungsgegenstands zusammenzufügen. Demnach sollte den Besuchern von Public Viewing-Veranstaltungen an ausgewählten Stellen die Möglichkeit gegeben werden eine individuelle Antwort zu geben. 
ob der Teilnehmer den Fragebogen über den aktiven oder passiven Weg der Kontaktaufnahme (s. Kap. 12.2.3) eingesehen hat, sind diese in dem E-MailAnschreiben oder über das Posting im Internet auf die Beschränkung der Teilnehmer auf Personen, die eine Public Viewing-Veranstaltung besucht haben, hingewiesen worden. Selbstverständlich ist den Teilnehmern die anonymisierte Verwendung ihrer Daten zugesichert worden. Die 21 Fragen -plus ein Feld für Anmerkungen- sind in sechs Komplexe eingeteilt worden, von denen der letzte lediglich die soziodemographischen und sozioökonomischen Daten der Befragten erfasst. Der mittlere Abschnitt, welcher die Fragen beinhaltet, unterteilt sich wiederum in fünf thematische Bereiche. Zunächst wird nach dem 'Veranstaltungstyp' gefragt, der besucht wurde. Dann nach ist nach der 'Motivation für den Public Viewing Besuch im Bereich Sport' gefragt worden, während sich der dritte Fragenkatalog mit 'Emotionen' beschäftigt. Im vierten Bereich wird das 'Sportinteresse' und im fünften nach den zukünftigen 'Public Viewing-Aktivitäten' der Personen gefragt. Die Fragen sind so gewählt, dass sie jeweils zu relevanten Aspekten passen. Die Beantwortung der Fragen ist über das Anklicken eines bzw. bei einigen Fragen mehrerer Antwortdimensionen möglich. Durch die geschlossenen Anwortkategorien sollen die Befragten zu möglichst eindeutigen und klaren Äußerungen bewegt werden. Um Artefakte zu vermeiden, ist auf eine Bewertungsskala verzichtet worden. Die Teilnehmer konnten einer Antwort also nur durch Anklicken dieser zustimmen oder durch Nicht-Anklicken diese ablehnen.

\subsubsection{Gegenstandsbereich}

Um die erwünschten Implikationen zu durchdringen, ist es wichtig, nur diejenigen Personen zu befragen, die auch ein Public Viewing besucht haben. Dabei wurde sich im Gegensatz zu den qualitativen Telefongesprächen nicht auf die FußballWeltmeisterschaft 2006 beschränkt. Stattdessen ist die Schere auch auf Veranstaltungen in anderen Sportarten und zu anderen Gegenheiten geöffnet worden.

In dem Fragebogen ist hauptsächlich der Versuch unternommen worden, die Implikationen von Emotionen herauszufiltern. Die Fragen richteten sich also z.B. nach der emotionalen Motivation zum Besuch oder nach der Art der Verbreitung von Gefühlen durch das Publikum. Dabei ist zu beachten, dass die Personen sich 
natürlich nur zum Teil bewusst erinnern und abschätzen könnnen, warum sie eine bestimmte Verhaltensweise gezeigt haben. Viele dieser Prozesse laufen unterbewusst ab. Diese sind wahrscheinlich nicht über das Instrument Befragung abfrag- und erforschbar. Eine die Onlinebefragung unterstützende und ergänzende Beobachtung im Feld wäre sehr wünschenswert gewesen, konnte aber aus Zeit- und Kostengründen leider nicht geleistet werden. An dieser Stelle ist aber ein lohnender und nötiger Ansatz für zukünftige Forschungen zu sehen.

\subsubsection{Feldzugang}

In dieser Onlineuntersuchung ist versucht worden, sich einer Zufallsstichprobe anzunähern. Dazu wurde sowohl auf eine aktive als auch auf eine passive Auswahl an Probanden zurückgegriffen. Unter 'aktiver Auswahl' wird die Einladung von Personen per E-Mail verstanden, in der versucht wird, durch eine inhaltsvolle Textgestaltung, Probanden zur Teilnahme zu bewegen ( $\mathrm{vgl}$. Thielsch, 2008, S. 95). Für die vorliegende Befragung wurde sowohl auf diese Technik zurückgegriffen, als auch nach dem Schneeball-Prinzip verfahren. Das heißt, in der Einladungs-E-Mail sind die Empfänger aufgefordert worden, die E-Mail mit dem Link zum Fragebogen an andere Personen weiterzuleiten. Darüber hinaus wurde der Fragebogen online bei Portalen und thematisch relevanten Foren veröffentlicht. Dieses Posting hat einen Einladungstext mit dem Link auf die Webseite des oFb-Servers auf dem die Befragung hinterlegt gewesen ist, enthalten. Thielsch (2008, S. 96) nennt diese Form der Rekrutierung von Probanden 'passive Auswahl'. Diese Personen stoßen während des Surfens im Internet mehr oder weniger zufällig auf die Aufforderung zur Teilnahme. Folglich wurden alle drei genannten Formen der Probandenrekrutierung kombiniert angewandt. Die Befragten werden dabei nicht kontrolliert als Stichprobe aus einer Grundgesamtheit gewählt, sondern entschließen sich selbst zur Teilnahme (Selbstselektion). ${ }^{104}$

104 Die Risiken und Probleme die sich aus dieser Art der Teilnehmeransprache ergeben sind bereits in Kap. 11.1 diskutiert worden. 


\subsubsection{Zeitfenster}

Der Bogen war vom 07. April bis 30. Juni 2008 online geschaltet. Dabei gilt es zu beachten, dass vom 07. bis zum 29. Juni die Fußball-Europameisterschaft 2008 in Österreich und der Schweiz ausgetragen wurde. Es ist beabsichtigt worden, die Befragung direkt vor bzw. auch während der Europameisterschaft 2008 durchzuführen. Die Fragen verlangen von den Teilnehmern eine deutliche Erinnerung an den Forschungsgegenstand. Es ist versucht worden, die erneute Aufmerksamkeit, die dem Public Viewing im Vorfeld der Europameisterschaft 2008 geschenkt wurde, dafür zu nutzen. So sollte auch die Gefahr der Suggestivwirkung, die bei stark strukturierten und geschlossenen Verfahren, vor allem bei Themen, über die sich der Befragte noch keine eigene Meinung gebildet hat, auftreten kann, minimiert werden (vgl. Atteslander, 2003, S. 164). Natürlich können diese quantitativen Daten nur eine Momentaufnahme für das Jahr 2008 darstellen. 


\section{Analyse der empirischen Daten}

Dieses Kapitel ist der Darstellung und Erörterung der Untersuchungsergebnisse gewidmet. Dabei werden die qualitativen Expertenaussagen der Public ViewingSpezialisten und die quantitativen Werte der Befragung von Public ViewingBesuchern in Bezug auf die Hypothesen deskripitiv dargestellt und diskutiert (13). Zunächst werden die Datensätze der zwei Erhebungen aufgeschlüsselt und nach den Gütekriterien eruriert (13.1). Im Anschluss werden die Hypothesen überprüft (13.2). Da eine direkte Gegenüberstellung der Leitfadengespräche untereinander und mit den quantiativen Daten nicht möglich ist, erfolgt bereits in der Auswahl der zu erörternden Aussagen und Werte eine interpretative Leistung zur Überprüfung der Hypothesen H1 (13.1.1), H2 (13.1.2) und H3 (13.1.3). Im letzten Schritt werden die empirischen Erkenntnisse des Kapitels zusammengefasst, hinsichtlich ihrer Forschungsleistung eruiert und in den Relevanzhorizont der Arbeit gestellt (13.3). 


\section{Diskussion der Untersuchungsergebnisse}

Im folgenden Kapitel erfolgt in einem ersten Schritt die deskriptive Darstellung und Erörterung der allgemeinen Daten der zwei im Rahmen dieser Ausarbeitung durchgeführten empirischen Studien. Aufgrund der Differenzierung in zwei unterschiedliche Erhebungen sind die Aufschlüsselung des Untersuchungsgegenstandes und die Eruierung der Gütekriterien ebenso in zwei Bereiche unterteilt. Der erste Teil extrahiert die qualitativen Experteninterviews und der zweite Abschnitt die Datensätze der Onlinebefragung (13.1). Da es sich bei den geführten Expertengesprächen um qualitative Interviews handelt, können diese nicht zahlenmäßig gewertet und verglichen werden (s. Kap. 11). Eine Gegenüberstellung der persönlichen Erfahrungen der befragten Experten ist daher ohne ein Zusammenwirken mit dem interpretatorischen Anteil kaum möglich. Darüberhinaus werden die Werte beider Untersuchungen nicht im vollen Umfang der erhobenen Daten dargestellt. Stattdessen werden die Werte selektiert und damit gleichzeitig bereits interpretativ bewertet (13.2). Die Diskussion der Hypothesen orientiert sich an den Fragestellungen zu den Entwicklungsfaktoren (H1) (13.2.1) und der Publikumsbildung (H2) (13.2.2) beim Public Viewing. $\mathrm{H} 3$ befasst sich darüber hinaus mit der Steuerbarkeit von positiven Emotionen bei den TV-Vorführungen (13.2.3). Abschließend werden die Erkenntnisse des Kapitels in gebotener Kürze zusammengefasst und in ihrer Bedeutung für den Aussagewert und die Reichweite dieser Ausarbeitung eruiert (13.3). 


\subsection{Allgemeine Daten zu Experteninterviews und Onlinebefragung}

Die im Rahmen der qualitativen Leitfadeninterviews befragte Spezialistengruppe umfasste 21 Public Viewing-Experten. Zu Beginn jedes Interviews hat sich die Autorin nochmals vorgestellt und ihr Anliegen erläutert. Jeder der Befragten ist erneut darauf hingewiesen worden, dass es um eine erste Einschätzung des Phänomens Public Viewing geht und aus diesem Grund besonderer Wert auf die persönliche Meinung gelegt wird. Diesbezüglich kann die Erhebungsmethode des Experteninterviews als valide für die erwünschten Aussagen angenommen werden, denn in einem nur von einem Leitfaden begleiteten Fragegespräch sind die individuellen Vorstellungen, Meinungen und Erfahrungen der Spezialisten gut abfragbar. Es wurde darauf hingewiesen, wie wichtig ehrliche Antworten für fundierte Erkenntnisse in der Arbeit sind. Die Autorin bedankte sich nochmals für die Interviewbereitschaft und erkundigte sich bei den Spezialisten nach Fragen, die vor Beginn des Gesprächs zu klären seinen. Die ungefähre Zeitdauer des Interviews wurde mit 45 Minuten angegeben. ${ }^{105}$

Die Interviews mit den Experten unterteilen sich in fünf Gespräche in der Kategorie Fan Fest, zwölf Interviews in der Kategorie nicht-kommerzielles Public Viewing und vier Fragegespräche in der Kategorie kommerzielles Public Viewing (s. Tab. 6).

Tab. 6: Aufschlüsselung der Expertise der befragten Experten in die Kategorien Fan Fest, nichtko mmerzielles Public Viewing und kommerzie lles Public Viewing (absolute Zahlen) (N=21)

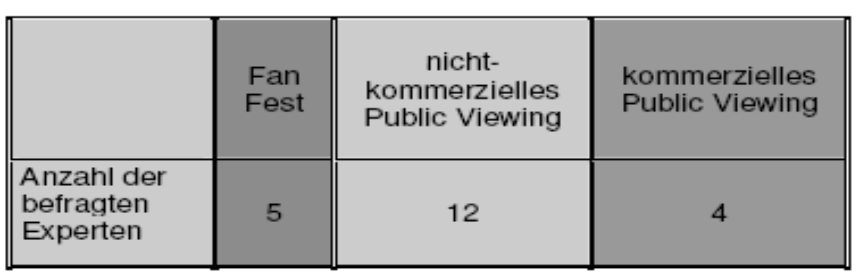

Die Eingrenzung des Untersuchungsgegenstands auf Gespräche dieser drei Kategorien ist sowohl aus den theoretischen Erkenntnissen als auch aus den geführten Interviews heraus entstanden. Es wurde ersichtlich, dass für den in dieser Arbeit diskutierten Forschungsgegenstand die im britischen Raum sehr

\footnotetext{
105 Um eine bessere und ökonomischere Lesbarkeit der Expertengespräche zu ermöglichen, wurde das erläuterte einführende Gespräch bei der Transkription der Interviews im Anhang herausgenommen, da es sich jedes Mal sehr ähnlich wiederholt hat. Ebenso sind die Danksagung und das sich in einigen Fällen anfügende Gespräch am Ende des Interviews aus diesem Grund nicht mit transkribiert.
} 
verbreitete Kultur des gemeinsamen Sehens von Sportveranstaltungen in Pubs bzw. Kneipen mit 100 bis 200 Personen wenig relevant ist. Sie kann vielmehr als eine Zwischendimension angesehen werden, die von den Experten nicht als dem Public Viewing zugehörig erwähnt wird. ${ }^{106}$

Ein Anspruch auf Repräsentativität kann diese explorative Studie nicht erheben, denn die explizite Befragung von Experten schließt eine Verallgemeinerung der Ergebnisse auf die Gesamtgesellschaft von vornherein aus. Der Erkenntnisgewinn, der durch die Spezialisteninterviews gewonnen werden konnte, rechtfertigt jedoch dieses Vorgehen, denn es lassen sich trotzdem gesellschaftliche Trends und Tendenzen ableiten. Bezüglich der Reliabilität der Expertengespräche muss zunächst in die Verlässlichkeit hinsichtlich der Experten und der Verlässlichkeit hinsichtlich der Veranstaltungen unterschieden werden. Um diesem Gütekriterium zu entsprechen, wird an dieser Stelle zunächst der soziodemographische Hintergrund der Experten ${ }^{107}$ erläutert. In Kap. 13.2 werden die Unterschiede und die sich aus diesen gegebenenfalls abzuleitenden Differenzen in den Aussagen der Spezialisten mit in die Diskussion einfließen. Der Personenkreis der Experten unterteilt sich in einen weiblichen und 20 männliche Gesprächspartner.

Die 21 Public Viewing-Spezialisten weisen folgende Altersstruktur auf.

Tab. 7: Aufschlüsselung der Alters struktur der befragten Experten in die Kategorien 20-30 Jahre, 30-40 Jahre, 40-50Jahre und über 50 Jahre (absolute Zahlen) $(\mathrm{N}=21)$

\begin{tabular}{||l|c|c||c|c|}
\hline & $\begin{array}{c}20-29 \\
\text { Jahre }\end{array}$ & $\begin{array}{c}30-39 \\
\text { Jahre }\end{array}$ & $\begin{array}{c}40-49 \\
\text { Jahre }\end{array}$ & $\begin{array}{c}\text { ab 50 } \\
\text { Jahre }\end{array}$ \\
\hline $\begin{array}{l}\text { Alter der } \\
\text { befragten } \\
\text { Experten }\end{array}$ & 3 & 10 & 7 & 1 \\
\hline
\end{tabular}

Die auffällige Konzentration von zehn und sieben Personen bei den 30- bis 39Jährigen und bei den 40 - bis 49-Jährigen lässt sich mit großer Wahrscheinlichkeit auf die berufliche Position zurückführen. Der Gedanke des Expertentums schließt es nahezu aus, dass Berufsanfänger mit geringer Erfahrung und Expertise als Interviewpartner zur Verfügung stehen. In dieser Untersuchung gelten nur die

106 Sicherlich tut sich an dieser Stelle ein interessanter Anknüpfungspunkt für weitere Forschungsarbeiten auf, die z.B. den kulturellen Unterschied des gemeinsamen Sehens von Sportereignissen im Fernsehen zum Gegenstand hat.

107 Auf eine Darstellung der Herkunft der Experten wurde verzichtet, da die Anonymisierung sonst nicht in ausreichendem Maße gewährleistet werden kann. 
Personen als Experten, die ein umfangreiches Wissen bzgl. des Forschungsgegen-standes aufweisen können (s. Kap. 12.1).

Tab. 8: Aufschlüsselung der Positionen der befragten Experten zur WM 2006 in die Kategorien Press es precher, Leiter Stabstelle Presse- u. Öffentlichke itsarbeit und Chefredaktuer; Geschäftsführer, Vorsitzender, Prokurist und Manager; leitender Angestellter, WM-Beauftragter der Stadt und WMProjektleiter der Stadt; Finanzrefe rent und Schatzme is ter; Sozialarbeiter (absolute Zahlen) (N=21)

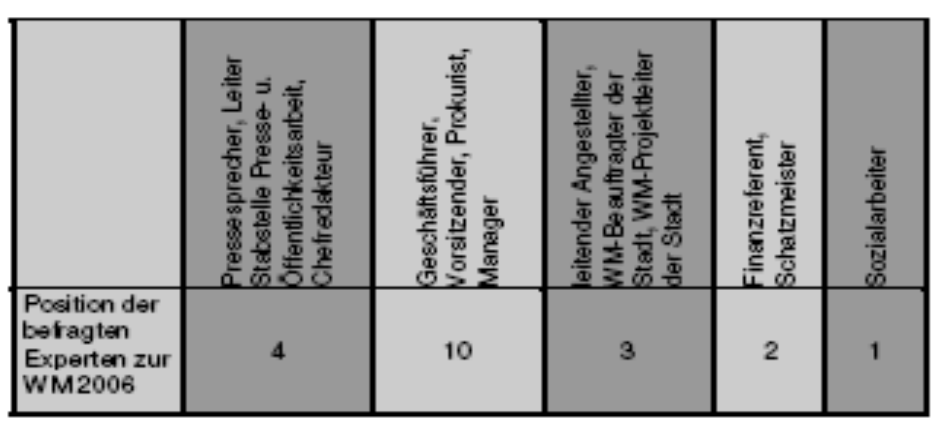

Die auffällige Konzentration der Verteilung bei der zweiten Kategorie der Geschäftsführer, Vorsitzenden, Prokuristen und Manager weist darauf hin, dass mit Personen gesprochen wurde, die einen hervorragenden Überblick über die Veranstaltung haben, da sie in der Verantwortung stehen. Ebenso kann dies für die dritte Kategorie gelten, die leitende Angestellte, WM-Beauftragte der Stadt und WM-Projektleiter der Stadt inkludiert. Auch die Kategorie des Sozialarbeiters könnte in diese Gruppe mithineinzählen, da die Expertin die alleinige Verantwortung für die von ihr durchgeführte Veranstaltung hatte. Die Kategorien Pressesprecher, Leiter Stabstelle Presse- und Öffentlichkeitsarbeit und Chefredakteur sowie die Kategorie vier -Finanzreferent und Schatzmeister- lassen vermuten, dass es sich hier um Personen handelt, die ebenso ein umfangreiches Public Viewing-Wissen haben, jedoch u.U. aus ihrer Position heraus argumentieren. Weiteren Aufschluss über die Zusammensetzung der Spezialisteng ruppe liefert folgende Darstellung des Bildungshi ntergrundes. 
Tab. 9: Aufschlüsselung des Bildungshintergrundes der befragten Experten in die Kategorien Rechts wis sens chaften, Informatik; Wirtschafts geographie, -ingenieurwesen und -wiss ens chaften; Verwaltungs wis senschaften; Politikwis senschaften, Journalis mus, Lehramt und Sozialpädagogik; Zweiter Bildungsweg, Event management und Messe-, Kongress- u. Tagungs wirtschaft (absolute Zahlen) (N=21)

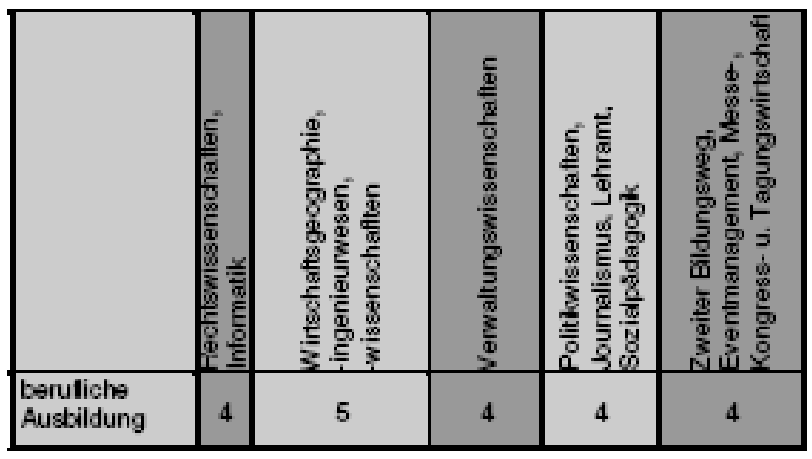

Tab. 9 zeigt, dass der Bildungshintergrund der befragten Experten recht vielschichtig ist. Ein Schwerpunkt kann in der zweiten Kategorie, die 'Wirtschaftsgeographie, -ingenieurwesen und -wissenschaften' zusammenfasst, erkannt werden, weshalb bei der Bewertung der Antworten von einer ökonomischen Ausrichtung auszugehen ist. In dieser Argumentationslinie liegt vermutlich auch die fünfte Kategorie, die den 'zweiten Bildungsweg, Eventmanagement und Messe-, Kongress-, und Tagungswirtschaft' umfasst. Es kann des Weiteren angenommen werden, dass auch die vier Verwaltungswissenschaftler, die vier Personen der Kategorie 'Politikwissenschaften, Journalismus, Lehramt und Sozialpädagogik' sowie auch die vier Experten der Kategorie 'Rechtswissenschaften, Informatik' die finanziellen Aspekte nicht außer Acht lassen können und werden. Jedoch ist es möglich, dass diese Experten auch einem sozialen Auftrag, z.B. für das Allgemeinwohl der Bürger einer Stadt folgen könnten. Deshalb schließt sich an dieser Stelle in Tab. 10 die Aufschlüsselung über die berufliche Anstellung der befragten Experten an. Die Anzahl von elf bei einer Gesellschaft angestellten Experten überwiegt eindeutig vor den sieben Personen, die eine Stadt als Arbeitgeber angeben. Die drei Individuen, die für ihren Verein sprechen, sind in der Minderzahl. Bei der Heranziehung der Expertenaussagen zur Prüfung der Hypothesen müssen die erörterten Hintergründe, um die Reliabilität zu gewährleisten, mit einbezogen werden. 
Tab. 10: Aufschlüsselung der beruflichen Anstellung der befragten Experten in die Kategorien Stadt, Gesellschaft und Verein (absolute Zahlen) $(\mathrm{N}=21)$

\begin{tabular}{||l||c||c||c|}
\hline & Stadt & Gesellschaft & Verein \\
\hline $\begin{array}{l}\text { berufliche } \\
\text { Anstellung } \\
\text { der befragten } \\
\text { Experten }\end{array}$ & 7 & 11 & 3 \\
\hline
\end{tabular}

Für die Analyse von qualitativen Interviews werden in der wissenschaftlichen Literatur mehrere Auswertungstechniken genannt. Die 'Auswertungsstrategie' kann auch eine Zusammenstellung verschiedener Auswertungstechniken sein. Die Auswahl der Auswertungstechnik wird, wie auch die Methodenauswahl von den vorhandenen Ressourcen, wie zeitlichen und personellen Kapazitäten beeinflusst (vgl. z.B. Schmidt, 2005a, S. 447). In der vorliegenden Arbeit ist das Gütekriterium der Objektivität zunächst durch das Aufnehmen der Interviews auf Tonband gewährleistet worden. Im Anschluß wurden die Aufnahmen vorerst mehrfach gehört und anschließend in Standardorthographie transkribiert. Die Vorannahmen, die bereits für die Erstellung des Fragebogens herangezogen wurden, und die forschungsleitenden Fragestellungen sowie die theoretisch diskutierten Hypothesen dienen als Suchraster für Textstellen, die für die Diskussion der Annahmen geeignet sind. Generell ist es wünschenswert, wie es z.B. Mayring (2002, S. 114) vorschlägt, die erhobenen Daten zu kategorisieren, d.h. Fragenkomplexe zu bilden, die für die Diskussion einer Hypothese herangezogen werden. Allerdings erscheint dieses Operationalisieren der qualitativen Daten für leitfadengestütze Interviews nur bedingt möglich. Der Leitfaden dient lediglich als Gedächtnisstütze, da den Experten die Möglichkeit zur Eigenpräsentation gegeben werden soll. Von daher ähneln sich die Interviews nur bedingt, sodass in der vorliegenden Arbeit die thematische Sortierung der Daten ausschließlich hinsichtlich des Begründungszusammenhangs der Hypothesen erfolgt ist. Durch das Selektieren und Zuweisen erfolgt bereits eine Interpretation. So erfolgt das Sichten und Ordnen des Datenmaterials nicht nach einem willkürlichen Muster, sondern wird in einem spezifischen theoretischen Kontext vollzogen (vgl. Atteslander, 2003, S. 355). In diesem Sinne werden im folgenden Kapitel (13.2) Gemeinsamkeiten und Unterschiede der Expertenaussagen herausgearbeitet und mit den Daten der Internetumfrage ergänzt und erweitert. 
Das Erhebungsverfahren der Onlinebefragung ist als valide anzusehen, denn es wurde der Zweck verfolgt, zu spezifischen Punkten ein quantitatives Mehr an kategorisierten Antworten zu erhalten. Insgesamt riefen 595 Personen die Internetbefragung auf. Dadurch, dass der Fragebogen online ausgefüllt werden musste und die Daten direkt in das Statistikprogramm SPSS übertragen und dort ausgewertet wurden, kann von einer objektiven Untersuchung gesprochen werden. Durch dieses Verfahren konnte Z.B. die Drop-Out-Quote (Abb. 20) ermittelt werden.

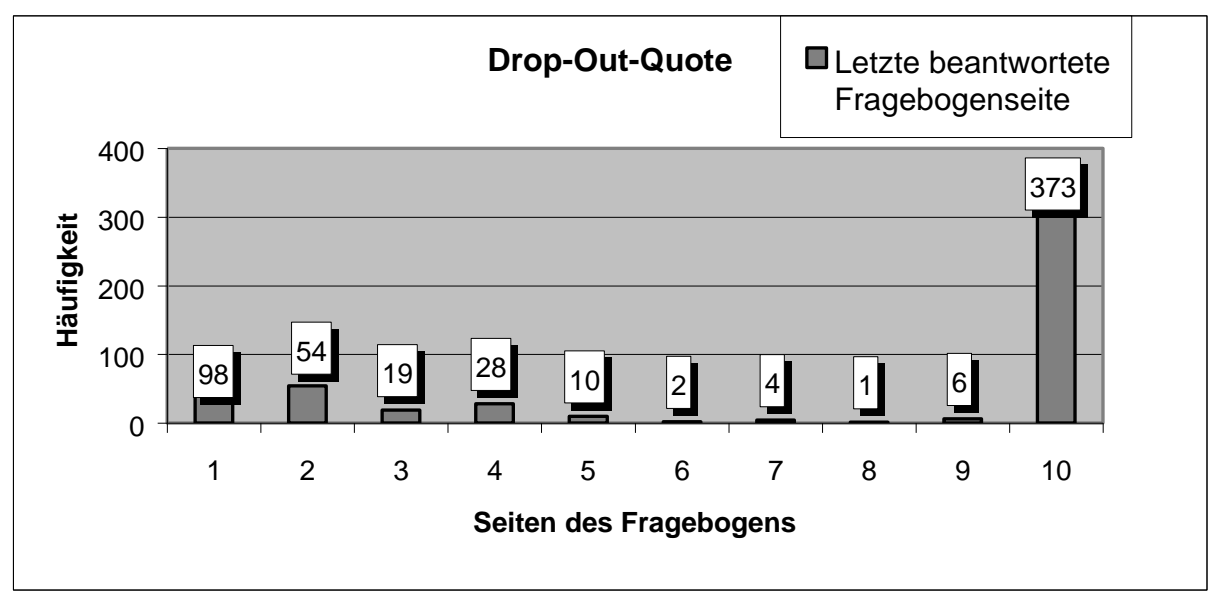

Abb. 20: Drop-Out-Quote der Befragungsteilnehmer aufgeführt nach der letzten beantworteten Seite des Fragebogens $(n=595)^{108}$

98 Teilnehmer verließen die Umfrage nach Aufrufen der Startseite wieder. 130 Befragte brachen inn während der weiteren Bearbeitung ab. Somit bleiben letztlich 373 vollständig bis zur letzten Seite ausgefüllte Fragebögen übrig. Vor Beginn der Untersuchung hat sich die Autorin gegen eine Antwortpflicht entschieden, um die Abbrecherquote möglichst gering zu halten. Aus diesem Grund sind jedoch nicht alle Fragebögen vollständig auswertbar, da, wie obig dargelegt, nicht von allen Teilnehmern alle Fragen beantwortet wurden. Es ist offensichtlich zu einer hohen Abbrecherquote, dem so genannten 'Drop-Out' gekommen, was allerdings bei Onlinebefragungen oftmals der Fall ist. Der hohe Drop-Out stellt nach Thielsch

108 Eine Online durchgeführte Datenerhebung bringt den Nachteil mit sich, dass die Teilnehmer nicht der Kontrolle des Versuchsleiters unterliegen. Die Identität der Befragten kann somit nicht endgültig geklärt werden. Daher wird in anonym erhobenen Online-Stichproben der Stichprobenumfang nicht mit $\mathrm{N}=x$ Personen, sondern nur mit $\mathrm{n}=\mathrm{x}$ Datensätzen angegeben. Zwar wird in den folgenden Untersuchungen von Probanden ges prochen, die Stichprobenumfänge aber jeweils korrekterweise durch das kleine ' $n$ ' gekennzeichnet (vgl. Thielsch, 2008, S. 97). 
(2008, S. 98) nur dann eine Schwierigkeit dar, wenn viele Probanden während der laufenden Befragung ausgestiegen sind, der Abbruch also selektiv erfolgt ist. Bei den Personen, die sich nur die Startseite der Untersuchung ansehen, ist dieses Problem nicht gegeben, denn so kann dieses Sichten der ersten Seite wie das Ansehen eines Experiment-Aushangs in der nicht-virtuellen Welt angesehen werden. Abb. 20 zeigt, dass die relativ hohen Abbrecherquoten von 98 und 54 Personen auf den Seiten eins und zwei zu verzeichnen sind, demzufolge wird die Drop-out-Quote als nicht relevant angesehen.

Es ist versucht worden, sich mit der aktiven und passiven Rekrutierung der Probanden an eine repräsentative Zufallsstichprobe anzunähern (s. Kap. 12.2.3). Ein Großteil (63,76\%) der Befragungsteilnehmer ist zwischen 14 und 29 Jahre alt.

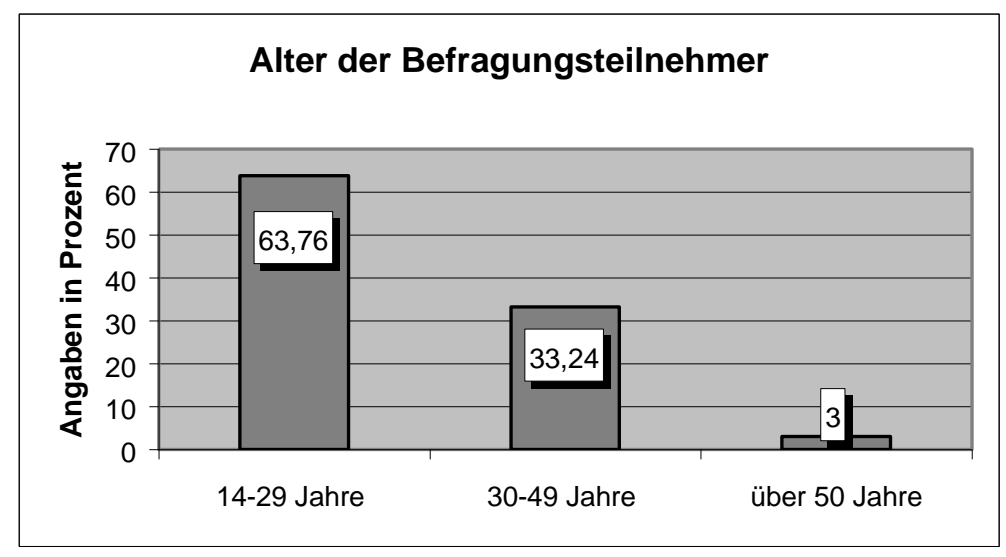

Abb. 21: Altersstruktur der Teilneh mer der Onlinebefragung $(n=367)^{109}$

Dies ist sehr wahrscheinlich zum einen auf den gewählten Feldzugang und zum anderen auf das Umfragemedium Internet zurückzuführen. So ist davon auszugehen, dass jüngere Menschen das Medium häufiger nutzen als ältere. Nach der ARD/ZDF-Onlinestudie (2007) lag die gelegentliche Onlinenutzung im Jahr 2007 bei 14 - bis 19 -Jährigen bei $95,8 \%$ und bei 20 - bis 29 -Jährigen bei $94,3 \%$. Die Nutzung bei 30 bis 39-Jährigen wird noch mit $81,9 \%$ angegeben, während bei 40- bis 49-Jährigen diese nur noch bei 73,8\% liegt. Diese Ergebnisse sind mit der Altersstruktur der Teilnehmer der Onlinebefragung in dieser Arbeit vergleichbar. Für die Altersgruppe 50 bis 59 Jahre gibt die Studie einen Wert von $64,2 \%$ und für die Kategorie $60+$ einen Wert von $25,1 \%$ an, was die geringe Teilnahmequote von nur $3 \%$ von über 50 -Jährigen in der vorliegenden

109 Die Prozentwerte sind immer auf zwei Dezimalstellen nach dem Komma gerunden, um eine bessere Übersicht zu bewahren. 
Untersuchung zumindest z.T. erklärt. Behn et.al. (2007) bzw. Pilz (2007) untersuchten das Fan- und Besucherbetreuungsprogramm während der Fußball WM 2006. Sie befragten u.a. knapp 2.000 Personen aus 60 Nationen am Rande der offiziellen Fan Feste. Diese repräsentativen Daten erfassen nur einen Teil, nämlich die offiziellen Fan Feste, der vorliegenden Dissertation. Allerdings lässt sich durch den Abgleich der Zuschauerdaten mit den dieser Arbeit zugrunde liegenden Angaben, ein Stück weit mehr Tiefe gewinnen. 44,4\% der Befragten 110 gaben an zwischen 21 und 30 Jahre alt zu sein. Nimmt man die Kategorie der bis 20 Jahre alten Personen (16,3\%) hinzu, sowie es in der vorliegenden Arbeit gemacht wurde, kommt man auf einen Wert von 60,7\% 111 . Diese Angabe deckt sich nahezu mit den $63,76 \%$ der 14 bis 29-Jährigen Besucher von Public ViewingVeranstaltungen, die an dieser Onlinebefragung teilgenommen haben. Es kann folglich angenommen werden, dass das Alter der Befragungsteilnehmer sowohl Rückschlüsse auf das Medium Internet zulässt, aber auch das Alter der an Public Viewing Interessierten repräsentiert.

Die Geschlechterverteilung der Befragungsteilnehmer (Abb. 22) betrachtend, fällt auf, dass das Interesse, an der Umfrage teilzunehmen, mit $56,87 \%$ bei den männlichen und $43,13 \%$ bei den weiblichen Probanden recht ausgeglichen ist. Dieses gleichmäßig verteilte Interesse ist so nicht zu erwarten gewesen. Es kann bereits als ein Indikator angesehen werden, dass sich das Public ViewingPublikum bis dato von der gängigen Struktur des Sportpublikums etwa in der Bundesliga unterscheidet (s. Kap. 7.1). Behn et. al. (2007, S. 41 f.) konnten dagegen feststellen, dass an allen Austragungsorten deutlich mehr Männer $(73,2 \%)$ als Frauen $(26,5 \%))^{112}$ an der Befragung teilnahmen. 113

\footnotetext{
110 Als Vergleichswerte werden die Angaben der Besucher aus Deutschland herangezogen da davon ausgegangen wird, dass die Teilnehmer der Onlineumfrage ebenfalls aus Deutschland stammen.

111 Das Durchschnittsalter der Gesamtstichprobe für die Fan Fest-Umfrage liegt nach Behn et.al. (2007, S. 43) bei 30 Jahren. Für die vorliegende Untersuchung liegt es bei 33,33 Jahren, welches einen weiteren Hinweis für die Aussagekraft der Daten liefert.

112 Vier Personen (0,2\%) machten keine Angabe zu ihrem Geschlecht.

113 Hier wurden die Daten der deutschen und ausländischen Fan Fest-Besucher gemeinsam bewertet.
} 


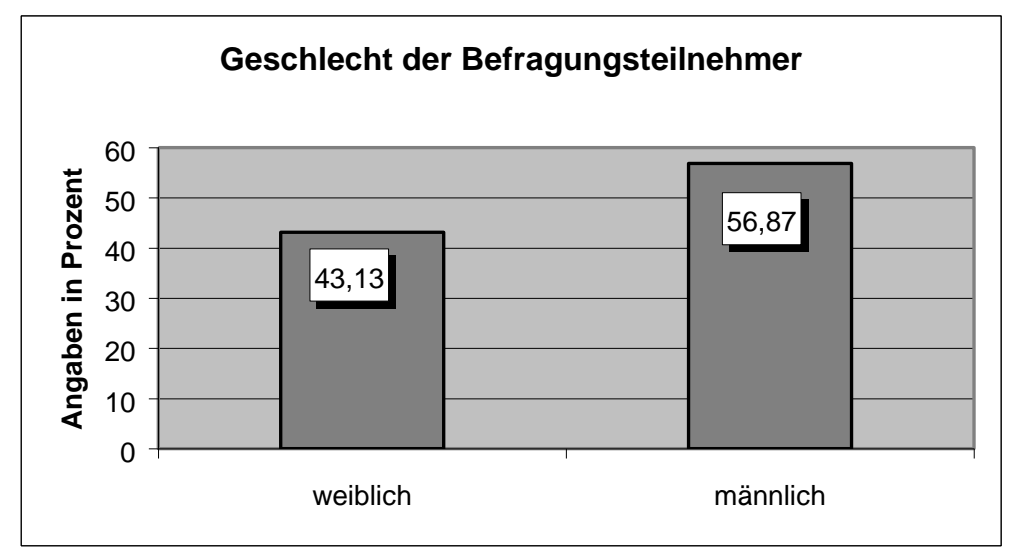

Abb. 22: Geschlechterverteilung der Teilneh mer der On linebefragung $(n=364)$

Die Aufschlüsselung des Bildungsniveaus der Befragungsteilnehmer zeigt eine eindeutige Konzentration von 62,4\% Personen, die ein Studium abgeschlossen haben, wohingegen nur 4,36\% der Probanden die mittlere Reife und $11,17 \%$ der Befragten eine abgeschlossene Berufsausbildung oder Lehre als höchste Qualifizierung angegeben haben.

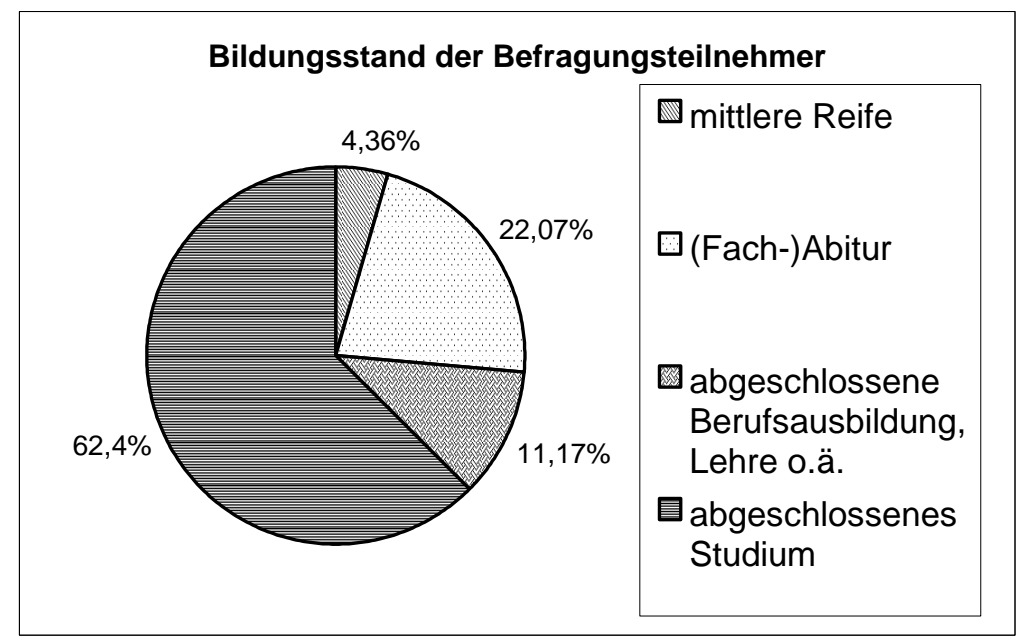

Abb. 23: Bildungsniveau der Teilneh mer der On linebefragung $(n=367)$

Der hohe Prozentsatz der Akademiker könnte sich, ähnlich wie die Altersstruktur, auf die Verfahren der Probandenrekrutierung und das Umfragemedium Internet zurückführen lassen. Eine weitere Möglichkeit wäre aber auch, dass sich das Public Viewing-Publikum tatsächlich aus dieser zunächst hoch erscheindenden Akademikerzahl zusammensetzt. Diese These kann durch die Daten von Behn et al. (2007, S. 43) gestützt werden, denn auch bei dieser Erhebung gaben 22,5\% der Befragten an ein Studium beendet zu haben. Darüber hinaus gaben 19\% an 
im Studium zu sein und weitere 5\% teilten mit ein Aufbaustudium zu besuchen. ${ }^{114}$

Die Herkunft der Teilnehmer nach Bundesländern verteilt sich in der vorliegenden Onlinebefragung wie folgt.

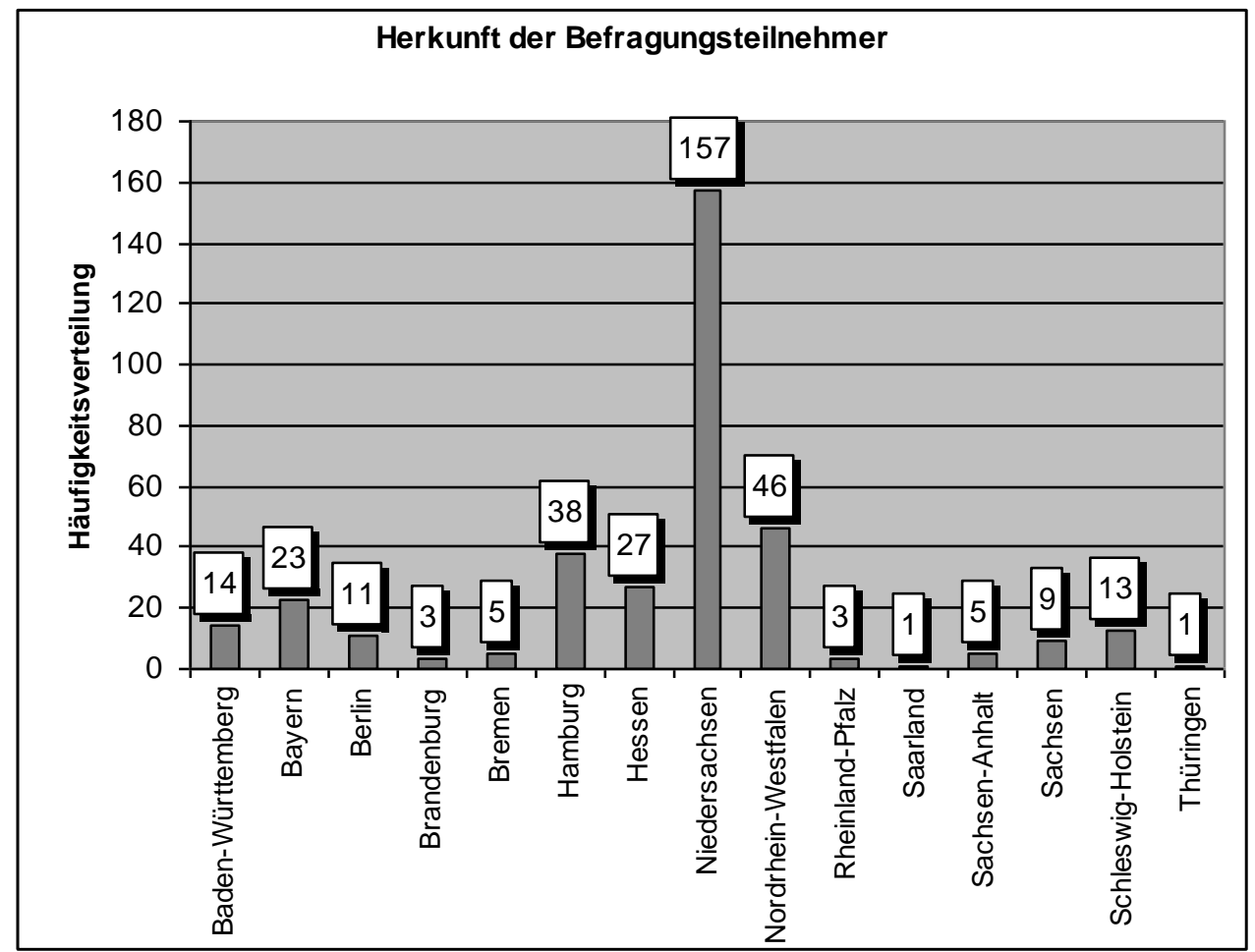

Abb. 24: Herkunft der Teilneh mer der Onlinebefragung nach Bundesländern $(n=356)$

In diesem Fall kann vermutet werden, dass die hohe Anzahl von 157 Personen, die einen Wohnort in Niedersachsen angeben, an dem demographischen Hintergrund der Autorin und der aktiven Probandenauswahl durch das Schneeballverfahren begründet ist. Da aber unter den gleichen Befragungsbedingungen und derselben Art der Probandenrekrutierung die gleiche Teilnehmerverteilung zu erwarten ist, gilt die Reliabilität der Untersuchung als gesichert. Die Repräsentativität dieser Onlineuntersuchung ${ }^{115}$ kann, wie z.B. durch die Rückschlüsse in der Verteilung vor allem bzgl. des Bildungsniveaus und der Herkunft ersichtlich, nicht gewährleistet werden. Der Erkenntnisgewinn der

\footnotetext{
114 Bezüglich des Bildungsstandes der Fan Fes-Besucher wurden die Angaben der ausländischen und der deutschen Befragten zusammen gewertet, wobei bei den ausländischen Gästen immer der adäquate ausländische Abschluss gewählt wurde.

115 Zu der grundsätzlichen Problematik der Repräsentativität von Onlineuntersuchungen vgl. Kap. 11.1.
} 
Untersuchung rechtfertigt aber die Vorgehensweise in vollem Maße. ${ }^{116}$

\section{2 Überprüfung der Hypothesen}

Die eingangs erörterten Fragestellungen (s. Kap. 1) sind bereits in den Theoriekapiteln verdichtet worden. Sie bilden die erste Grundlage für die nun folgende Diskussion der Hypothesen. In diese Basis werden die Erkenntnisse aus den zwei empirischen Studien eingeflochten. Die qualitativen Expertenmeinungen gemeinsam mit den in der quantitativen Onlinebefragung erhobenen Daten und den theoretischen Kapiteln bilden folglich den Hintergrund für die Überprüfung der drei Hypothesen $\mathrm{H} 1, \mathrm{H} 2$ und $\mathrm{H} 3$. Zunächst werden im Rahmen der ersten Hypothese $(\mathrm{H} 1)$ die für die Entwicklung des Public Viewing verantwortlichen Faktoren diskutiert (13.2.1). Danach erfolgt die Diskussion der Publikumsbildung beim Public Viewing im Sinne der zweiten Hypothese (H2) (13.2.2). Auf die dritte Hypothese (H3) Bezug nehmend, wird des Weiteren die Steuerbarkeit positiver Emotionen bei Fernseh-Vorführungen im Sport eruiert (13.2.3).

\footnotetext{
116 Für folgende Untersuchungen wäre es, auch für die Spezifizierung der Hypothesen, wünschenswert, die Grundgesamtheit der befragten Personen näher zu definieren. Da sich dies bei Onlineuntersuchungen grundsätzlich schwierig gestaltet (s.Kap. 11.1) wäre z.B. eine telefonische Befragung mit Zufallsgenerator, bspw. durch ein Sozialforschungsinstitut, denkbar. Hier müsste es in einem ersten Schritt darum gehen, passgenau zu definieren, wie sich die Population der Public Viewing-Besucher zusammensetzt. Zu diesem Zwecke ist ein Herunterbrechen auf die Besucher der einzelnen Kategorien (Fan Feste, kommerzielles und nichtkommerzielles Public Viewing) zu empfehlen.
} 


\subsubsection{Entwicklungsfaktoren des Public Viewing}

Nachdem die theoretische Basis für die Hypothesendiskussion erörtert wurde und die Aufschlüsselung der empirischen Befragungen erfolgt ist, gilt es nun die in Kap. 1 genannten Fragestellungen anhand von Hypothesen zu erurieren. Im Folgenden ist zunächst nach den Faktoren, die zur Entwicklung des Public Viewing geführt haben, zu fragen. Zu diesem Zweck ist Hypothese 1 mit ihren Teilhypothesen $\mathrm{H} 1 \mathrm{a}$ und $\mathrm{H} 1 \mathrm{~b}$ wie folgt formuliert worden:

H1. Public Viewing ist durch zwei sich gegenseitig beeinflussende Entwicklungen entstanden. Soziale Prozesse (Wertewandel, Individualisierungs- und Pluralisierungstendenzen) in der postmodernen Gesellschaft mit den daraus entstehenden Bedürfnissen und Tendenzen sowie der technologische Fortschritt in der Übertragungstechnik, insbesondere der LED-Technik, sind Voraussetzungen für

a. die verstärkte Bedeutung des Sports, insbesondere internationaler Fußballturniere, und

b. im Zusammenspiel mit der Mediatisierung der Gesellschaft dafür, dass immer mehr Zuschauer wichtige Ereignisse des Sports im Fernsehen verfolgen.

Wie in Kap. 6 erörtert wurde, vollzieht sich in der Postmoderne ein tiefgehender gesellschaftlicher Strukturwandel, der die Individualisierung und Pluralisierung von Individuen und ihren Lebensstilen zur Folge hat. Es kann ein schrittweiser Umschwung von traditionellen Normen, Schichten, Klassenzugehörigkeiten und Arbeitswerten hin zu neuen Sinnstrukturen und hedonistischen Freizeitwerten erfolgen. Der Sport als körperbezogenes, ritualisiertes Handlungsfeld scheint als Orientierungspunkt geeignet. Er bietet Möglichkeiten der Identifikation, der Repräsentation sowie der Abgrenzung und kann so ein Ansatzpunkt für die Bildung einer individuellen Sozialstruktur sein. Der Fußballsport spielt darüber hinaus durch seine große Beliebtheit in Deutschland und vielen anderen Staaten weltweit besonders bei internationalen Turnieren für das nationale Zusammengehörigkeitsgefühl eine große Rolle. Daneben weisen der Sport und die Übertragungsmedien eine parallele symbiotisch begründete Entwicklung auf, die die Entstehung des Public Viewing begünstigt hat. 
Um folglich in einem ersten Schritt die Hypothese H1a überprüfen zu können, muss zunächst diskutiert werden, welche Aussagen die Experten bzgl. des Fußballs und bzgl. eventueller weiterer Sportarten, für die eine Public ViewingVeranstaltung denkbar ist, treffen. Danach gilt es den Bedeutungsunterschied für Public Viewing zwischen einem Fußballpiel im Regelbetrieb und einem internationalen Fußballturnier herauszufiltern. Diese Ausführungen werden von den aus der quantiativen Besucherbefragung entnommenen Werten ergänzt. Hier wird nach dem Anlass des Public Viewing-Besuchs, der Motivation für einen weiteren und nach dem Ereignis für diesen zukünftigen Besuch einer FernsehVorführung gefragt.

Dem theoretischen Argumentationsstrang und dem Auftreten des Public Viewing vornehmlich bei großen internationalen Fußballturnieren folgend, werden als erstes die Expertenaussagen hinsichtlich der Bedeutung des Fußballs interpretierend dargestellt. Wie in den theoriegeleiteten Kapiteln bereits verdeutlicht wurde, scheint der Sport und insbesondere der Fußball eine besondere Rolle in der Gesellschaft einzunehmen, die die Entwicklung des Public Viewing gefördert hat. Alle Experten (21 von 21) sehen im Fußballsport einen wichtigen Faktor für die Ausmaße und die Beliebtheit des Public Viewing. Fußball wird als der größte Breitensport in Deutschland, bzw. in der Welt eingeschätzt (vgl. $\mathrm{NN}_{5}$, S. 2, Z. 51f.; $\mathrm{NN}_{10}$, S. 2, Z. 48f.; $\mathrm{NN}_{21}$, S. 2, Z. 54f. [u.v.m.]), der allein in der Lage scheint, eine, die Kapazität in den Sportstätten übersteigende Ticketnachfrage zu generieren.

„Fußball ist im Vergleich mit anderen Sportarten eine der meist gesehenen und auch die publikumswirksamsten, die man sich vorstellen kann. Wir hatten das in der Vergangenheit schon für uns getestet” ( $\mathrm{NN}_{20}$, S. 2, Z. 53ff.).

Nach Meinung der Experten ist diese Verbreitung ein großer Faktor, was die Aussage von $\mathrm{NN}_{18}$ (S. 2, Z. 64): „Basketball würden wir zum Beispiel nicht übertragen", veranschaulicht. Wie bereits theoretisch erläutert wurde, spielt die Beliebtheit und Verbreitung einer Sportart eine ausschlaggebende Rolle. So wird bspw. betont:

„Also, ich glaube, dass Fußball ideal für Public Viewing ist. Ich kann mir, ich denke mir, wenig Sportereignisse vorstellen, auch Mannschaftssportarten, also für unseren Kultur- und Sportkulturkreis, wo das ähnlich gut funktionieren würde. Also ich könnte mir vorstellen, dass Rugby in Australien auch als Public Viewing interessant sein kann, aber in Deutschland ist Fußball da glaube ich schon ideal für g eeignet“ $\left(\mathrm{NN}_{17}, \mathrm{~S}\right.$. 2f., Z. 83-93). 
$\mathrm{NN}_{19}$ (S. 3, Z. 111-114) schlägt für andere Sportarten vor, Public Viewing nur punktuell zu veranstalten:

„[W]enn jetzt z.B. in Mönchengladbach die Hockeyweltmeisterschaft ist und man erwartet da viele Touristen, auch die nichts mit Hockey zu tun haben, dann kann man sagen, man macht so etwas, genau in dem Stil in Mönchengladbach, das ja."

$\operatorname{Er}($ Z. 118f.) führt weiter aus;

„[a]ber dass man sagt, es ist hier Handball-WM, jetzt bauen wir lauter solche Stadien in ganz Deutschland auf. Das haut überhaupt nicht hin (...)."

Ein Experte eines nicht-kommerziellen Public Viewing ( $\mathrm{NN}_{16}$, S. 2, Z. 67f.) stellt die Überlegung an, ob es „zur Olympiade, wenn beim Zehnkampf eine bedeutende Persönlichkeit als Favorit mitwirkt“, möglich wäre, fügt dann aber sogleich hinzu: „Ich denke mir, eine Public Viewing-Veranstaltung wird schon für den Fußballsport beschränkt bleiben, denke ich“ (Z. 69ff.). Es kann folglich nach einhelliger Meinung der Experten angenommen werden, dass Public Viewing, zumindest in der Art und Weise, wie es zur WM 2006 stattgefunden hat, nur im Fußball möglich ist (vgl. $\mathrm{NN}_{1}$, S. 2, Z. 81-85; $\mathrm{NN}_{14}$, S. 2, Z. 71-75). ${ }^{117}$

Eine weitere Teilaussage in $\mathrm{H} 1 \mathrm{a}$ ansprechend, gilt es herauszufinden, ob Public Viewing nur im Rahmen von internationalen Fußballturnieren stattfinden kann, oder ob es auch andere Anlässe geben kann. Einzelne Experten können diesbezüglich bereits auf Erfahrungswerte zurückgreifen. $\mathrm{NN}_{20}$ (S. 2, Z. 53-61) gibt bspw. an, in einzelnen Städten mit Vereinen in der ersten Fußballbundesliga Spiele des Europa-Pokals, insbesondere die Auswärtsspiele, oder auch Spiele des DFB-Pokals gegen attraktive Gegner, gezeigt zu haben. Hier wird also nicht in allen Fällen von internationalen Turnieren gesprochen, aber doch sind die Pokalspiele aus dem Ligaalltag herausstechende Ereignisse. Diese sind offenbar nötig, um ein entsprechendes Publikum zu generieren. Ebenso weist $\mathrm{NN}_{17}$ (S. 2, Z. 83-93) auf die Bedeutung von außeralltäglichen Ereignissen für das Public Viewing hin:

„Fußball geht nicht im Regelbetrieb, es muss ein Fußballereignis sein. Das heißt, entweder ein großes Turnier oder ein Finale, ich sage mal im DFB-Pokal oder so etwas, eine Meisterschaft. Da haben wir das auch schon mal vereinzelt gemacht. Ich kann mir jetzt auch nicht vorstellen, dass man nur eine Leinwand aufstellen muss und irgendeine Sportart auflegen, und dann stellen sich die Leute davor. Also, das ist schon anders zu verstehen. Also, jetzt auch gerade der besondere Erfolg der WM."

117 An dieser Stelle bieten sich interessante Anknüpfungspunkte, z.B. für eine vergleichende Studie anlässlich der Frauen Fußball-WM 2011 in Deutschland. 
Diese Aussagen verdeutlichen den Eindruck, dass Public Viewing nur zu einem besonderen Ereignis möglich ist. Was als Ereignis definiert wird und wie besonders dieses sein muss, das scheint allerdings von der Stadt, von dem Verein und seinen Fans abzuhängen. Als Begründungen werden von den interviewten Spezialisten die besondere Zusammensetzung des Publikums bei international bedeutsamen Fußballturnieren und dessen Vergrößerung durch 'Modefans' genannt (s. Kap. 13.2.2). $\mathrm{NN}_{16}$ (S. 2, Z. 74f.) gibt bspw. an, man könne „[d]efinitiv keine Champions League oder derartige Sportsachen, da da die Fangemeinde zu verschieden ist, [übertragen]. Man kann da keine Schwerpunkte ausmachen“; weiterführend zweifelt er (Z. 75.78): „Also ich bin sehr Köln nah, aber würde bezweifeln, auch wenn Köln mal irgendwann in einem Endspiel stehen sollte, dass die Kölnfans hier zu uns kommen. Die würden in das Stadion gehen“.

$\mathrm{NN}_{3}$ (S. 2f., Z. 80-95), Experte eines Fan Festes, betont auf die Frage nach der Bedeutung der Weltmeisterschaft für die Public Viewing-Veranstaltung:

\begin{abstract}
„Wichtig. Dadurch, dass der Fußball eh eine sehr hohe Medienaufmerksamkeit und auch öffentliche Aufmerksamkeit genießt, ist so eine Weltmeisterschaft ganz anders als eine Tour de France. Hier haben sie mindestens ein gleichwertiges Ereignis. Dies hat aber längst nicht die Wahrnehmung wie die Fußball-Weltmeisterschaft. Daher schätze ich die Bedeutung des Fußballs sehr hoch. Letztlich ist auch gerade der Punkt, dass Deutschland Ausrichter der WM war, ein ganz wichtiger Punkt. Denn Fußball und Deutschland ist der Renner, und Fußball ist immer. Also, wenn man mit Sponsoren über Fußball redet, dann ist es immer anders, als wenn sie sagen Tennis, Golf oder weiß der Kuckuck was. Also, wenn Sie ein Gespräch mit einem Geschäftsführer oder einem Vorstandsvorsitzenden haben, und sie sagen Fußball, und der hat Bock drauf, dann ist das halt so. Die Leute interessieren sich selber dafür. Bei einer anderen Sportart kann es sein, dass sie abgesehen von dem Logo und ein paar Promotionständen nichts von dem Unternehmen spüren. Beim Fußball ist es so, da kommt dann der Vorstands vorsitzende selber und guckt sich die Veranstaltung an und freut sich tierisch über einen Pin. Das ist halt Fußball in Deutschland, die Leute habe da selber Lust drauf. Letztlich hat dieses Fandasein in Deutschland und [unserer Stadt-Name von Autorin anonymisiert] durch die WM noch zugenommen."
\end{abstract}

Zusammenfassend lässt sich also sagen, dass Public Viewing sich nach Meinung der befragten Spezialisten nur für ein besonderes Ereignis im (internationalen) Fußball zu eignen scheint (vgl. NN $N_{14}$, S. 2, Z. 79f.; $\mathrm{NN}_{15}$, S. 2, Z. 71-74; $\mathrm{NN}_{18}$, S. 2 , Z. 68f. [u.v.m.]). Wobei $\mathrm{NN}_{19}$, Experte eines kommerziellen Public Viewing (S. 4, Z. 123-126), zu bedenken gibt:

„Weltmeisterschaft und Europameisterschaft haben einen ähnlichen Stellenwert, nur muss man es ganz genau auf das Land, wo es stattfindet, mit anpassen. Wir könnten bspw. nicht sagen, wir machen das gleiche jetzt wieder, nur weil in Österreich und der Schweiz die Europameisterschaft stattfindet. Das würde in Deutschland nicht hinhauen, nicht in der Größenordnung, so wie wir es gemacht haben."

Es ist also nicht von der Hand zu weisen, dass Public Viewing sich dann 
besonders zu entwickeln scheint, wenn das Ereignis im eigenen Staat stattfindet. $\mathrm{NN}_{4}$ (S. 2, Z. 63ff.) weist darauf hin, dass Public Viewing in Südkorea während der WM 2002 erstmalig in dieser Größenordnung aufgetreten sei und im Rahmen der WM in Deutschland eine Art 'Hype' erreicht hätte. Generell kann das Public Viewing aber, wie bereits im Theorieteil ersichtlich wurde, eine erstaunliche Entwicklungsdynamik aufweisen: „Es ist etwas in Bewegung gekommen, aufgrund der Stimmung, aufgrund des Engagements der Menschen, aber auch das Interesse an solchen Veranstaltungen“ ( $\mathrm{NN}_{20}$, S. 2, Z. 64f.). Dieses Zitat verdeutlicht, dass es hinsichtlich weiterer Entwicklungsprognosen die nächsten großen Fußball(sport)ereignisse bzw-turniere abzuwarten gilt.

In der Onlineerhebung wurde gefragt, zu welchem Anlass eine Public ViewingVeranstaltung besucht worden ist. Es standen sechs feste Kategorien und eine Kategorie 'Sonstiges' für die Beantwortung zur Verfügung. Wie zu erwarten, äußerte der Großteil der Befragten (83,18\%), dass sie mindestens eine Public Viewing-Übertragung während der Fußball-WM 2006 besucht haben.

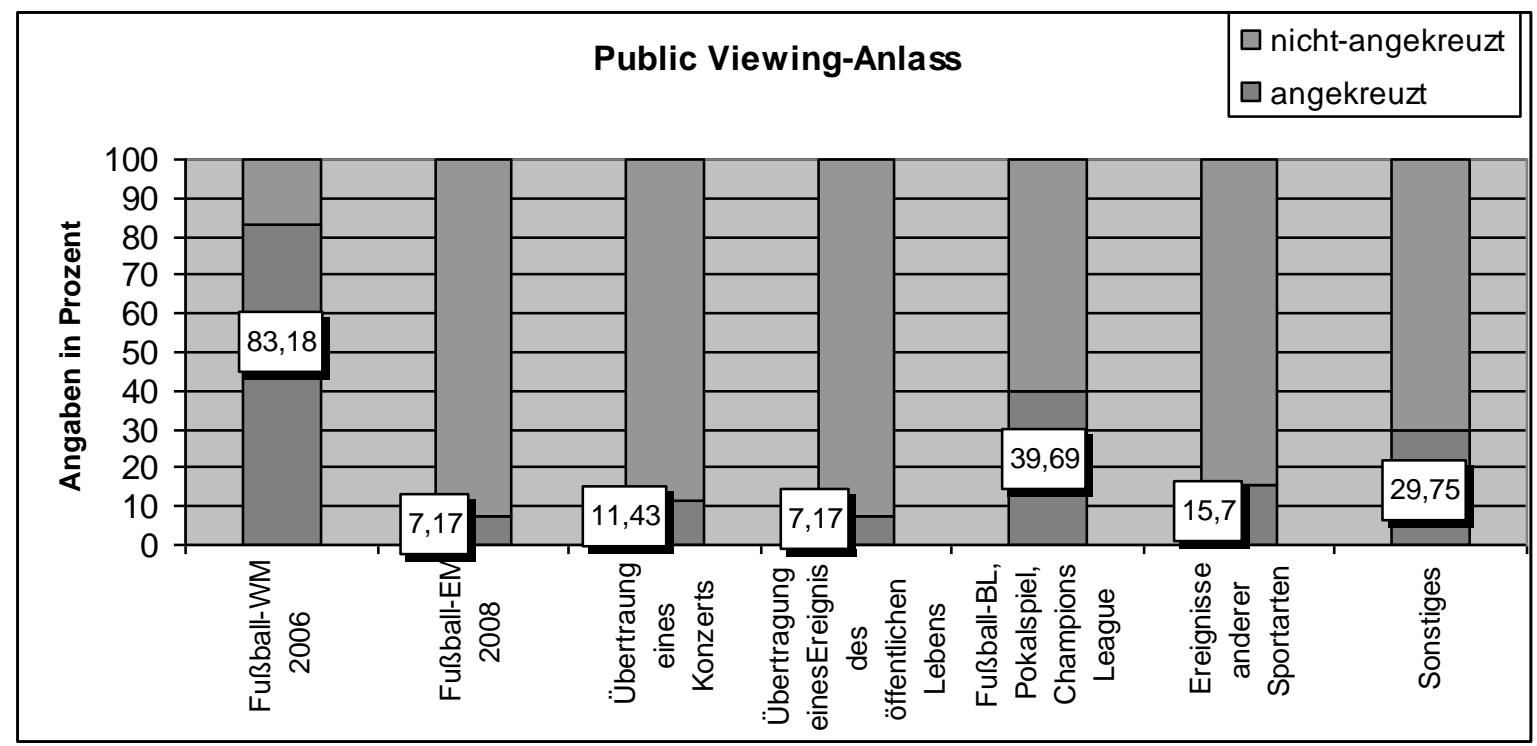

Abb. 25: Anlass, zu demeine Public Viewing-Veranstaltung besucht worden ist $(n=446)$ (Mehrfachnennung möglich) „Im Rahmen welches Ereignisses haben Sie eine Public Viewing-Veranstaltung besucht?“

$11,43 \%$ besuchten eine Konzertübertragung, immerhin 7,17\% ein Ereignis des öffentlichen Lebens, welches auf einer Großleinwand dargestellt wurde, und 15,7\% der Teilnehmer besuchten die TV-Vorführung von Ereignissen anderer Sportarten. 29,75\% trugen eine weitere Antwort ein, hier reichten die Angaben von 
Open-Air-Kino-Veranstaltungen zu Schlager-Grand-Prix-Übertragungen, hin zu den Olympischen Spielen und Fußballspielen unterer Ligen. Aus diesem Ergebnis sticht zunächst die zweite Säule, die den Besuch eines Public Viewings zur Fußball-EM 2008 anzeigt, heraus. Diese Kategorie haben nur 7,17\% der Befragten bestätigt. Diese geringe Prozentzahl könnte auf das Zeitfenster der Befragung zurückgeführt werden. So stellten die Tage der Euro 2008 die letzten Tage der Onlineschaltung des Fragebogens dar. Erfahrungsgemäß füllen aber die meisten Interessierten in den ersten Tagen, nachdem sie auf die Umfrage aufmerksam geworden sind, diese auch aus, womit der niedrige Prozentwert erklärt werden kann. Demnach decken sich die Ergebnisse aus der quantitativen Onlinebefragung mit den Meinungen der Experten. Es werden vornehmlich internationale Fußballereignisse, wobei die Weltmeisterschaft 2006 in Deutschland deutlich heraussticht, als Anlass genommen, eine Public Viewing-Veranstaltung zu besuchen. ${ }^{118}$ Denn es gaben von $n=360$ Personen 247 Personen an, erneut ein Public Viewing besuchen zu wollen.

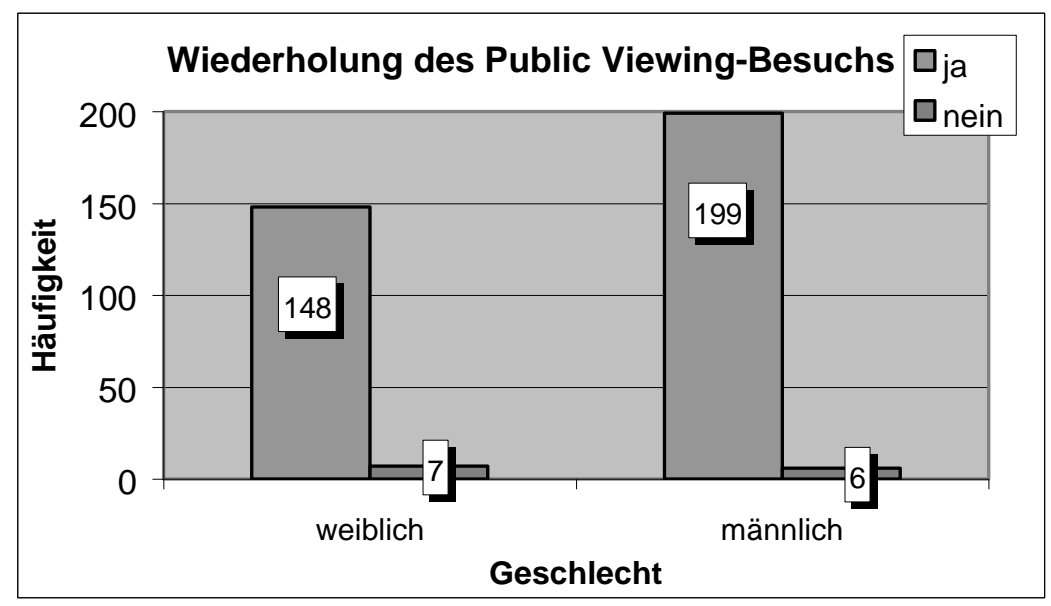

Abb. 26: Vorhaben erneut ein Public Viewing zu besuchen $(n=360)$ „Werden Sie wieder zum Public Viewing gehen?“"

Obwohl die Erhebung eine Momentaufnahme des Meinungsbildes im Mai/Juni 2008 darstellt, lässt sich ein reges Interesse an zukünftigen Public ViewingVeranstaltungen ablesen. Aus Abb. 27 kann entnommen werden, dass sich der Sport, und insbesondere internationale Fußballereignisse, sehr wahrscheinlich

118 In diesem Zusammenhang hätte eine fortführende Befragung zum Public Viewing-Besuch bei der Europameisterschaft 2008 eine weitere Aufschlüsselung des Forschungsgegenstandes mitsichgebracht. Dies konnte im Rahmen der vorliegenden Arbeit nicht geleistet werden, eröffnet aber neue Forschungsperspektiven. 
auch in Zukunft als geeigneter Public Viewing-Hintergrund erweisen werden. Diese Entwicklung hängt auch vom parallel stattfindenden technologischen Fortschritt ab (s. Kap. 8.2). Nach Aussagen der Experten ist aber die LED-Technik von nicht so großer Bedeutung für das Public Viewing, wie zuvor angenommen. Sie gab zwar mit den Impuls die Fan Feste zu veranstalten, doch war es den Zuschauern wichtiger „vernünftig große Bilder“ (NN6, S. 2, Z. 58) (vgl. auch $\mathrm{NN}_{11}$, S. 3, Z. 122; $\mathrm{NN}_{12}$, S. 4, Z. 135, 139, 142; $\mathrm{NN}_{13}$, S. 3, Z. 115f., 120f.) in der bestmöglichen Umsetzung, also auch bspw. mit Abdunklungsverfahren zu sehen (vgl. $\mathrm{NN}_{14}$, S. 5, Z. 191f.).

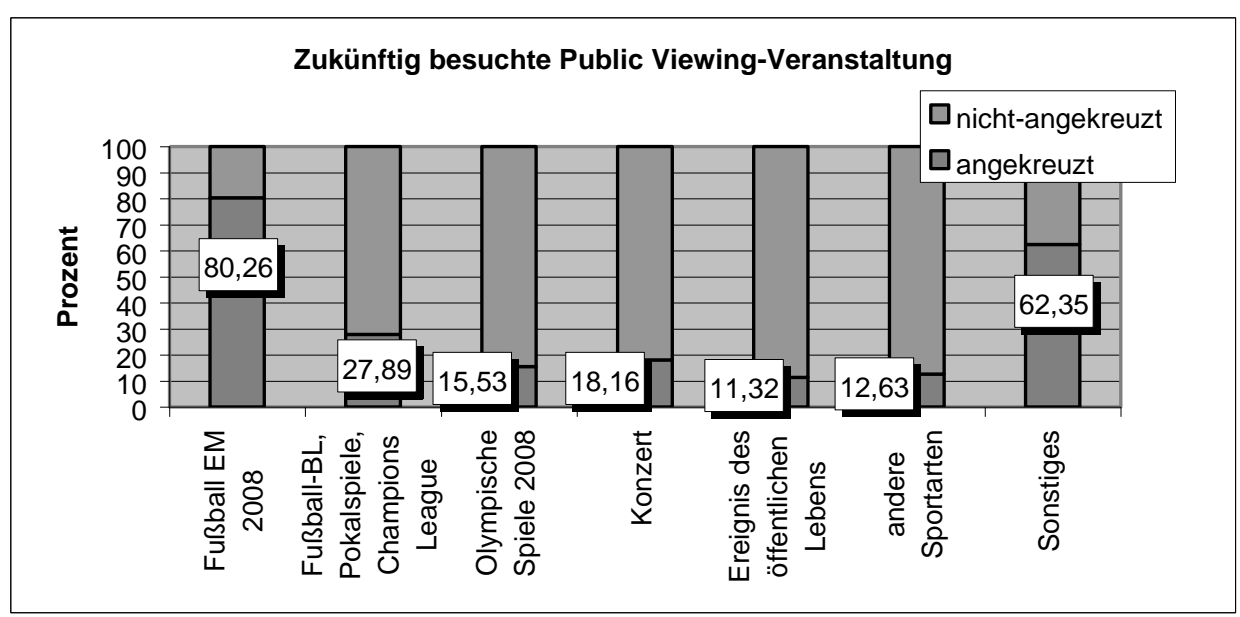

Abb. 27: Ereignis, zu demeine Public Viewing besucht werden soll ( $\mathrm{n}=380)$ (Mehrfachnennung möglich) „Die nächste Public Viewing-Veranstaltung, die ich besuchen werde, wird zu folgendem Ereignis sein:..."

So gaben 80,26\% der Befragten an, bei der Fußball-EM 2008 wieder ein Public Viewing besuchen zu wollen (vgl. Kap. 5.2.1). 27,89\% werden dies bei einem Fußballbundesliga- oder -Pokalspiel sowie in der Champions League tun. Wobei es an dieser Stelle zu beachten gilt, dass es natürlich auch immer auf das Angebot an entsprechenden Veranstaltungen ankommt. Selbstverständlich werden auf dem Markt mehr TV-Vorführungen für Fußballereignisse angeboten, als für andere Sportarten u.ä.. Die Olympischen Spiele 2008 wollten 15,53\% vor dem Großbildschirm verfolgen, 18,2\% die nächste Konzertübertragung, und $11,32 \%$ haben vor, sich in Zukunft ein Ereignis des öffentlichen Lebens anzusehen. $12,63 \%$ der Teilnehmer haben dies bei anderen Sportarten vor, und $62,35 \%$ geben unter 'Sonstiges' spezifische Veranstaltungen, wie ein Spiel der Basketball-Bundesliga oder der Fußball-WM 2010, aber auch Antworten von ,je nach Lust und Angebot“ hin zu „wenn sich zufällig etwas ergibt“ an. Diese Aussagen lassen darauf schließen, dass die Hypothese $\mathrm{H} 1 \mathrm{a}$ zu bestätigen ist. 
Neben der verstärkten Bedeutung des Sports und insbesondere internationaler Fußballturniere wird in $\mathrm{H} 1 \mathrm{~b}$ davon ausgegangen, dass im Zusammenwirken mit der bedeutenderen Rolle der Medien in der Postmoderne eine steigende Zuschauerzahl sich wichtige Ereignisse des Sports im TV ansieht. Wie in Kap. 5.2 deutlich wurde, haben die Fernsehquoten von Fußball-Welt- und Europameisterschaften eine sukzessive Steigerung über die letzten Turniere erhalten. Immer mehr Menschen möchten, in Deutschland wie in vielen anderen Staaten, an diesen internationalen Großereignissen des Fußballsports über das Medium Fernsehen teilhaben. Zur Überprüfung der Hypothese H1b muss folglich zunächst evaluiert werden, inwiefern das Interesse, wichtige Ereignisse des Sports regelmäßig im Fernsehen zu verfolgen, bei denjenigen, die die Onlineumfrage ausgefültt haben, gegeben ist. In einem nächsten Schritt gilt es dann die Korrelation zwischen dem Besuch einer Public Viewing-Veranstaltung und dem Einfluss auf die Meinungsbildung, den die Befragten den Medien zuweisen, zu erörtern. Wie in Kap. 5.3 bereits angedeutet wurde, hatten öffentliche Stellen, aber auch die Medien einen großen Einfluss auf die Entwicklung des Public Viewing während der Fußball-WM 2002 in Südkorea. In einem nöchsten Schritt werden die Expertenaussagen hinsichtlich dieser Wirkungsgröße evaluiert.

Der Aussage, regelmäßig wichtige Partien des Sports im Fernsehen anzusehen, haben $68,42 \%$ der Befragten zugestimmt. 52,11\% der Befragten gab sogar an, sich regelmäßig Spiele der Fußballbundesliga, Pokalspiele und/oder Spiele der Champions League anzuschauen. Diese hohen Werte lassen darauf schließen, dass bei den Teilnehmern der Befragung ohnehin ein Interesse am Public Viewing und somit auch, so ist anzunehmen, am Sport vorhanden ist. So gaben $80 \%$ der Befragten an -bei dieser Frage war eine Mehrfachnennung möglich-, selber regelmäßig Sport zu treiben. 


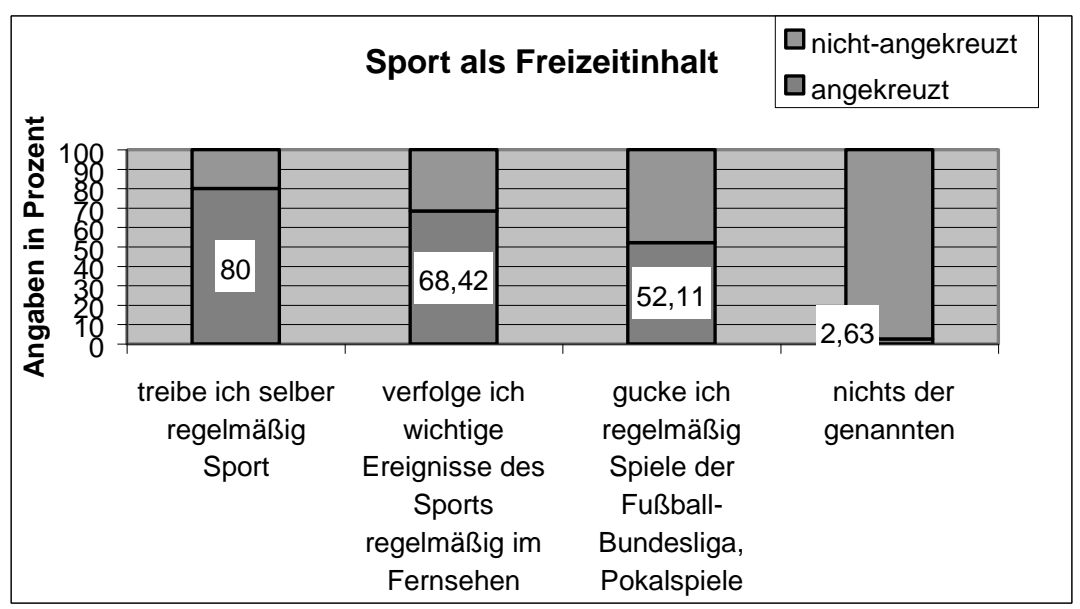

Abb. 28: Sport als Freizeitinhalt (Mehrfachnennung möglich) „In meiner Freizeit...“

In der Onlineerhebung wurde die Frage gestellt, in welchem Rahmen über Public Viewing kommuniziert wurde. Es sollte so eruiert werden, inwiefern die Konfrontation mit dem Thema öffentliche TV-Übertragung der Begegnungen in den Medien bei den Rezipienten auch aufgenommen worden ist; oder ob es vielleicht andere Kommunikationskanäle gab, die einen größeren Einfluss auf das Besucherverhalten gehabt haben. So wurde nach Angabe der Befragungsteilnehmer Public Viewing zu 67,71\% in den Medien thematisiert, was ein nicht unerheblicher Prozentsatz ist.

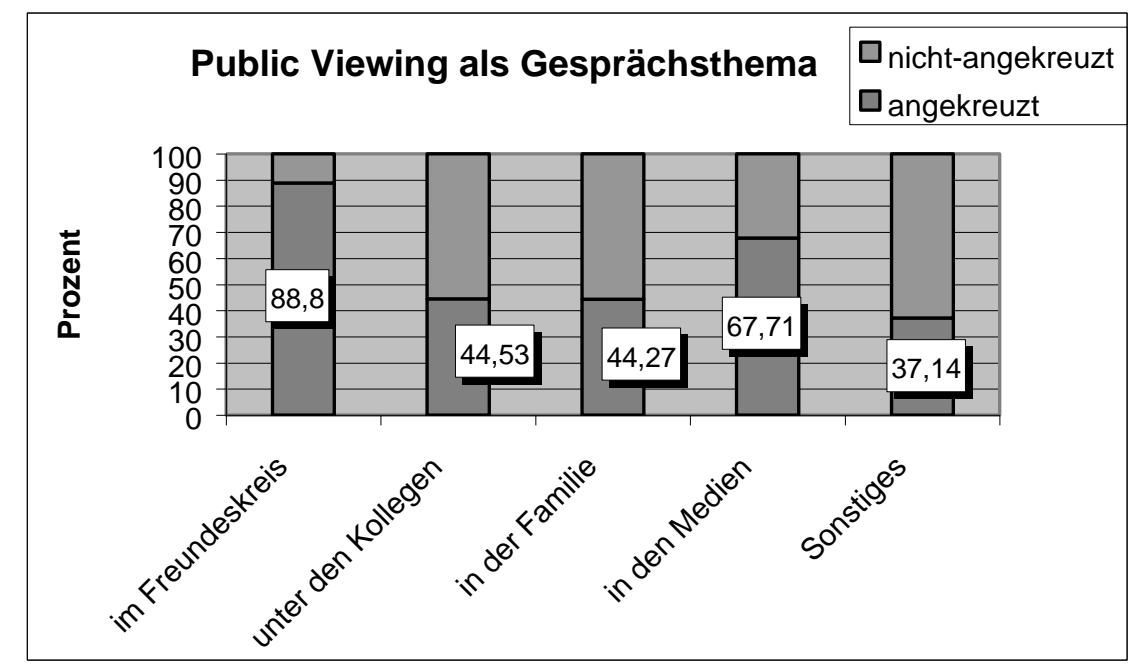

Abb. 29: In welchem Rahmen wurde über Public Viewing kommuniziert $(n=384)$ (Mehrfachnennung möglich) „Außerhalb des Events war Public Viewing Gesprächsthema...“

Erstaunlich sind jedoch die 88,8\%, die die Kommunikation über Public Viewing im Freundeskreis angegeben haben. Dieser Anteil lässt darauf schließen, dass die hohe Intensität der Berichterstattung in den Medien zwar präsent ist, dass aber die soziale Interaktion einen größeren Anteil an der Verbreitung des Themas Public 
Viewing in der Gesellschaft hat. Es haben 44,53\% der Befragten Public Viewing als Gesprächsthema unter den Kollegen angegeben und bei 44,27\% wurde in der Familie über Fernseh-Vorführungen kommuniziert. Diese Zahlen belegen die obig geäußerte Annahme und lässt darauf schließen, dass Public Viewing recht weite Kreise in der Bevölkerung gezogen hat, wie auch unter der Rubrik 'Sonstiges' gemachte Angaben, die von der Behandlung als Unterrichtsthema hinzu „überall“ reichten, bestätigen.

Die Aussagen der Experten lassen erkennen, dass sie einen Zusammenhang zwischen Deutschland als Austragungsstaat und der Präsenz des Themas Public Viewing ausmachen. Es wird darauf hingewiesen, dass aus diesem Grund die Berichterstattung in den Medien einen besonderen Stellenwert eingenommen hat.

\begin{abstract}
„Die Medien haben sehr viel intensiver und umfangreicher über die Weltmeisterschaft gesprochen und die Wichtigkeit der Weltmeisterschaft ganz anders herausgestellt, als wenn die WM in einem anderen Land stattgefunden hätte. Insofern war die Weltmeisterschaft sehr viel bewusster und wurde als Erlebnis natürlich auch sehr viel enger wahrgenommen",
\end{abstract}

berichtet $\mathrm{NN}_{16}$ (S. 7, Z. 267-270). Ein Fan Fest-Experte $\left(\mathrm{NN}_{1}\right.$, S. 3, Z. 122f.) beschreibt, dass die Medien das Thema Public Viewing stark aufgebaut hätten, so dass bereits im Mai und Juni die Vorfreude zu bemerken gewesen wäre. Es wird der Zusammenhang zwischen der hohen Intensität der Berichterstattung und dem großen Public Viewing-Interesse insofern hergestellt, als dass ein jeder auf die Möglichkeit, die Spiele in Form eines Public Viewing zu sehen, deutlich aufmerksam gemacht wurde (vgl. z.B. $\mathrm{NN}_{8}$, S. 5, Z. 203-206; $\mathrm{NN}_{14}$, S. 6, Z. 250). $\mathrm{NN}_{5}$, Fan Fest-Experte (S. 4, Z. 132ff.), berichtete bspw. von der guten Arbeit der Medienpartner, die im Vorfeld und während des Turniers für die Veranstaltung geworben haben.

Die Kommunikation bzgl. des Public Viewing über die Medien scheint also zunächst einen geringeren Stellenwert einzunehmen als zu vermuten war. Viel wichtiger scheint ein anderer Effekt zu sein: Die 'Mund-zu-Mund-Propaganda'. Die Spezialisten berichteten, dass begeisterte Gäste im Verlauf des Turniers immer weitere Kreise gezogen hätten und immer mehr Menschen zu den Veranstaltungen gekommen seien (vgl. z.B. $\mathrm{NN}_{15}$, S. 2, Z. 58ff., Z. 204; $\mathrm{NN}_{16}$, S. 6f., Z. 258f.). Es hat sich offenbar mit der WM in Deutschland eine Dynamik entwickelt (vgl. $\mathrm{NN}_{11}$, S. 5, Z. 189f.; $\mathrm{NN}_{19}$, S. 9. Z. 345), deren Ursachen und 
Zusammenhänge sehr komplex und auch für die Spezialisten nur schwer greifbar sind. Während $\mathrm{NN}_{12}$ (S. 5, Z. 210ff.), Experte eines nicht-kommerziellen Public Viewing angibt, seit 1996 zur Europa- und Weltmeisterschaften im Fußball diese auf Großbildschirme zu übertragen und einen 'Selbstläufereffekt' jedes Mal festgestellt zu haben, wird diese Euphorie von einigen anderen nicht in dem Maße geteilt. Es wird z.B. in Ausrichter- und Nicht-Ausrichterstädte unterschieden (vgl. $\mathrm{NN}_{20}$, S. 5, Z. 187-191); darüber hinaus scheint es sehr vom Einzugsgebiet abzuhängen, ob sich die Public Viewing-Begeisterung durch 'Mund-zu-MundPropaganda' ausgebreitet hat oder nicht. Ein Experte (vgl. NN $N_{10}$, S. 5, Z. 189f.) spricht von gerade mal 15 ausgehängten Plakaten, während ein anderer (vgl. $\mathrm{NN}_{21}$, S. 5, Z. 180-183) deutlich auf die Notwendigkeit von Werbung hinweist. Die Meinungsbildung der Besucher zum Thema Public Viewing ist folglich nur multidimensional erklärbar, auch oder gerade deshalb, weil man sich der besonderen Situation der Fußball-Weltmeisterschaft 2006 in Deutschland bewusst ist. Faktoren wie das sonnige, warme Wetter, das gute Spiel und das gute Abschneiden der deutschen Nationalelf, werden als wichtige Einflüsse wahrgenommen, die die Reproduzierbarkeit des Public Viewing in dieser Form bei nächsten großen Fußballereignissen in Frage stellt (vgl. z.B. NN $N_{17}$, S. 6, Z. $220-$ 234). Es herrscht zwar das gängige Meinungsbild, dass sich etwas wie eine neue "Art von Kultur" ( $\mathrm{NN}_{4}$, S. 5, Z. 193f.) entwickelt hat. $\mathrm{NN}_{1}$ (S. 2, Z. 65f.) spricht von einer neuen „Erscheinung“, die „in Deutschland ungeahnte Höhenflüge erlebt hat". Diese Aussagen verdeutlichen erneut die Multidimensionalität des Public Viewing und stellen die eventuelle Temporiarität der Erscheinung heraus. Das Medium Fernsehen besitzt eine herausragende Stellung und steht in symbiotischer Beziehung mit dem Spitzensport, insbesondere mit dem Fußball. Es konnte festgestellt werden, dass der regelmäßige Sportkonsum im Fernsehen bei den Befragten einen hohen Stellenwert einnimmt und das Public Viewing auch dort als Thema wahrgenommen wird. Noch mehr scheint jedoch die soziale Interaktion in Form der 'Mund-zu-Mund-Propaganda' einigen Public Viewing-Veranstaltungen einen 'Selbstläufer-Effekt' beschert zu haben. Resümierend sind diese Prozesse als inneinandergreifend und sich auf einander beziehend $z u$ verstehen. Die Hypothese H1b kann folglich in dieser Form nur in Ansätzen bestätigt werden. 


\subsubsection{Publikumsbildung beim Public Viewing}

Im vorangehenden Kapitel ist die Frage der Entwicklungsfaktoren des Public Viewing diskutiert worden. Im Folgenden wird die Publikumsbildung der FernsehÜbertragungen evaluiert. In diesem Sinne lautet die Hypothese (H2) mit ihren drei Unterhypothesen $\mathrm{H} 2 \mathrm{a}, \mathrm{H} 2 \mathrm{~b}$ und $\mathrm{H} 2 \mathrm{c}$ :

H2: Die Motive der Zuschauer, eine Public Viewing-Veranstaltung zu besuchen, sind nur multikausal und interdisziplinär erklärbar. Individuelle und soziale Beweggründe greifen in diesem Zusammenhang ineinander. Der Fokus wird auf folgende Aspekte gelegt:

a. Zuschauer besuchen Public Vieuing-Veranstaltungen, weil sie Emotionen in der Gemeinschaft erleben wollen. Internationale Sport-, vor allem Fußballveranstaltungen, bieten ideale Voraussetzungen für die Entstehung eines außergewöhnlichen emotionalen Erlebnisses.

b. Insbesondere der Nationalsport Fußball besitzt in der Gesellschaft repräsentative und gemeinschafsstiftende Funktionen, die identitätsbildend wirken und die verloren gehenden gesellschaftlichen Strukturen ersetzen bzw. diese neu ausrichten. Sie lassen sich vor allem in internationalen sportlichen Großereignissen bekräftigen, indem die einzelnen Zuschauer aktiv interagieren und zu einem Publikum zusammenwachsen.

c. Die Medien, insbesondere das Fernsehen, besitzen eine Kommunikator- und Multiplikatorfunktion, indem sie kollektives emotionales Wissen thematisieren, festigen und aktualisieren. Aus einzelnen Mikro-Ereignissen (Public ViewingVeranstaltungen) disperser Publika entsteht eine imaginierte Gemeinschaft.

Wie in Kap. 7.1 erörtert wurde, ist das Sportpublikum an sich differenziert zu betrachten. Im Falle der hier vorrangig behandelten internationalen Fußballturniere sind vor allem die als 'sport spectator' bzw. 'sport consumer' oder auch 'temporärer Fußballfan' bezeichneten Zuschauer von Bedeutung. Diese eignen sich zu Zeiten großer, wichtiger Turniere ein Expertentum an, um z.B. mittreden und teilhaben zu können. Sie sind aber keine Zuschauer, die bspw. im Bundesligaalltag anzutreffen sind (s. auch Kap. 13.2.1). So heterogen wie die Zusammensetzung des Sportpublikums sind auch die Beweggründe einzelner Zuschauer, der Fernsehübertragung eines sportlichen Großereignisses 
beizuwohnen. Im Theorieteil ist in Anlehnung an Riedl (2006) die Bündelung der Motive in die drei Motivcluster 'Emotionen erleben', 'Identifikation und Gemeinschaft' sowie 'Attraktion und Unterhaltung' erfolgt. Innerhalb dieser drei Kategorien greifen sowohl persönliche Beweggründe der Individuen als auch soziale Motive ineinander (s. Kap. 7.2).

Ein Schwerpunkt scheint der Wunsch, durch das Beiwohnen an einem besonderen Ereignis (s. Kap. 13.2.1) Emotionen gemeinsam mit Anderen erleben zu wollen, zu sein. Emotionen sind letztlich ausschließlich multidimensional fassbar. Sie entstehen aus der subjektiven Interpretation eines jeden Einzelnen auf der Basis von kulturellen und gesellschaftlichen Hintergründen. Gesellschaftliche Regeln und Normen stellen die soziale Basis der emotionalen Deutung dar, aus der sich der Einfluss des geteilten Wissens auf die Emotionsentstehung und -regulierung beim Sportpublikum begründet (vgl. Kap. 9.2). Hier greifen besonders die sozialen Beweggründe, die zeigen, dass das Publikum über das gemeinsame Erlebnis Public Viewing zu einem Personenkollektiv zusammenwachsen kann. Dieses hält sich nach dem Gedanken der sozialen Systemtheorie über Kommunikation in Form von Selbst- und Fremdzuschreibungen zusammen. Das Publikum als kollektive Identität und die Intention der Zuschauer, an einem Großereignis gemeinsam teilhaben zu wollen, reichen beim Public Viewing aus, um kollektives Emotionswissen zu generieren. Es bedarf sowohl einer gemeinsamen emotionalen Wissensbasis als auch eines Konglomerats aus verschiedenen psychologischen und soziologischen Prozessen, die Erzeugung von kollektiven Emotionen zu entwickeln. Besonders bei ungewohnten Ereignissen scheint die Orientierung an anderen Personen in Form von emotionalen 'Ansteckungsmechanismen' ausgeprägt zu sein (vgl. Kap. 9.3). Die Massenmedien, und insbesondere das Fernsehen, können in diesem Prozess eine Multiplikatorfunktion einnehmen. Sie sind in der Lage, aus einzelnen MikroEreignissen disperser Publika, hier in Form von einzelnen Public ViewingVeranstaltungen, eine imaginierte Gemeinschaft, wie z.B. beim 'Wunder von Bern', herzustellen (vgl. Kap. 9.4).

Für die Überprüfung der Hypothese H2a gilt es demnach, die Motive der Gäste aus der Onlinebefragung eine Public Viewing-Veranstaltung zu besuchen, zu eruieren. Die Beweggründe werden ergänzt mit den Angaben der Zuschauer zu der Lokation der TV-Vorführung und der Begleitung, mit der sie diese besucht 
haben. Ergänzt wird die deskriptive Auswertung der quantitativen Daten mit ausgewählten Angaben der Experten hinsichtlich der vermuteten Motivation ihrer Gäste für den Public Viewing-Besuch.

Die Motivlage der Teilnehmer der Onlinebefragung, zum Public Viewing zu gehen, gliedert sich in den drei Kategorien 'Emotionen erleben', 'Identifikation und Gemeinschaft' und 'Attraktion und Unterhaltung' wie folgt auf:

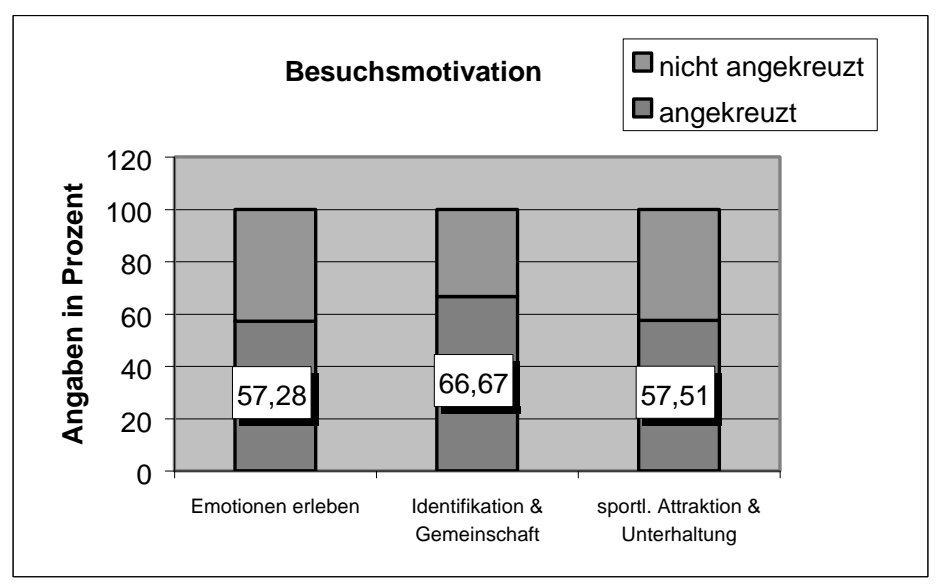

Abb. 30: Besuchsmotivation ( $\mathrm{n}=426)$ (Mehrfachnennung möglich) „Ich habe eine Public ViewingVeranstaltung besucht, weil..."

$57,28 \%$ der Befragten gaben an, zum Public Viewing zu gehen, um Emotionen erleben zu wollen. Unter dieser Kategorie, die sich, wie die anderen beiden auch, aus den Ausführungen in Kap. 9.2 ergibt, wird der Wunsch nach Spannungserleben, Flucht aus dem Alltag, Emotionen ausleben, etwas Besonderes miterleben usw. zusammengefasst. Der höchste Wert mit 66,67\% der Teilnehmer zeigt die Gründe für den Besuch mit dem Bedürfnis nach Identifikation und Gemeinschaft an. Dieses Konglomerat fügt die Motive bzgl. der Identifikation mit einem Sportler bzw. einer Mannschaft und das Genießen der Gemeinschaft zusammen. Wobei sich das letztgenannte Cluster aus den Unterkategorien Zeit mit Freunden/Familie, Stars sehen, Gruppenzugehörigkeit genießen und Gesprächsthema haben, zusammensetzt. Allerdings nimmt mit 57,51\% das Sehen von sportlicher Attraktion und das Unterhalten-Werden, welches sich im Wunsch nach einem guten Spiel/Wettkampf, Spielwitz und einem interessanten Rahmenprogramm ausdrückt, ebenso einen hohen Anteil an. Diese Ergebnisse zeigen, dass offensichtlich das Suchen nach und Erleben von Emotionen einen wichtigen Stellenwert einnimmt. Ebenso ist die Motivation, sich mit dem Sportler 
bzw. der Mannschaft zu identifizieren und die Gemeinschaft zu genießen, ein bedeutsamer Faktor. Aber es scheint auch der Fall zu sein, dass die sportliche Attraktivität und der Unterhaltungswert einer Public Viewing-Veranstaltung stimmen sollten. Letztlich greifen also auch diese Dimensionen ineinander. Denn bspw. besitzt natürlich ein Spiel der deutschen Nationalelf eine hohe Wahrscheinlichkeit, dass ein deutscher Zuschauer sich identifiziert und insbesondere bei der Endrunde der Fußball-Weltmeisterschaft im eigenen Land ein Gemeinschaftsgefühl erfährt. Eine Begegnung bei einem solchen Turnier und mit der favorisierten Mannschaft sorgt aber auf der anderen Seite auch wieder für eine erhöhte Emotionalität und eben über die Emotionen auch für eine größere Attraktion und einen ausgeprägten Unterhaltungswert. ${ }^{119}$

Offenbar ist also das Erleben von Emotionen ein zentrales Motiv für den Public Viewing-Besuch. Öffentliche Orte in Form von 'liminal spaces' scheinen für die spontan-kollektive Emotionsentfaltung des Gros' der Besucher besonders geeignet (vgl. Kap. 8.1).

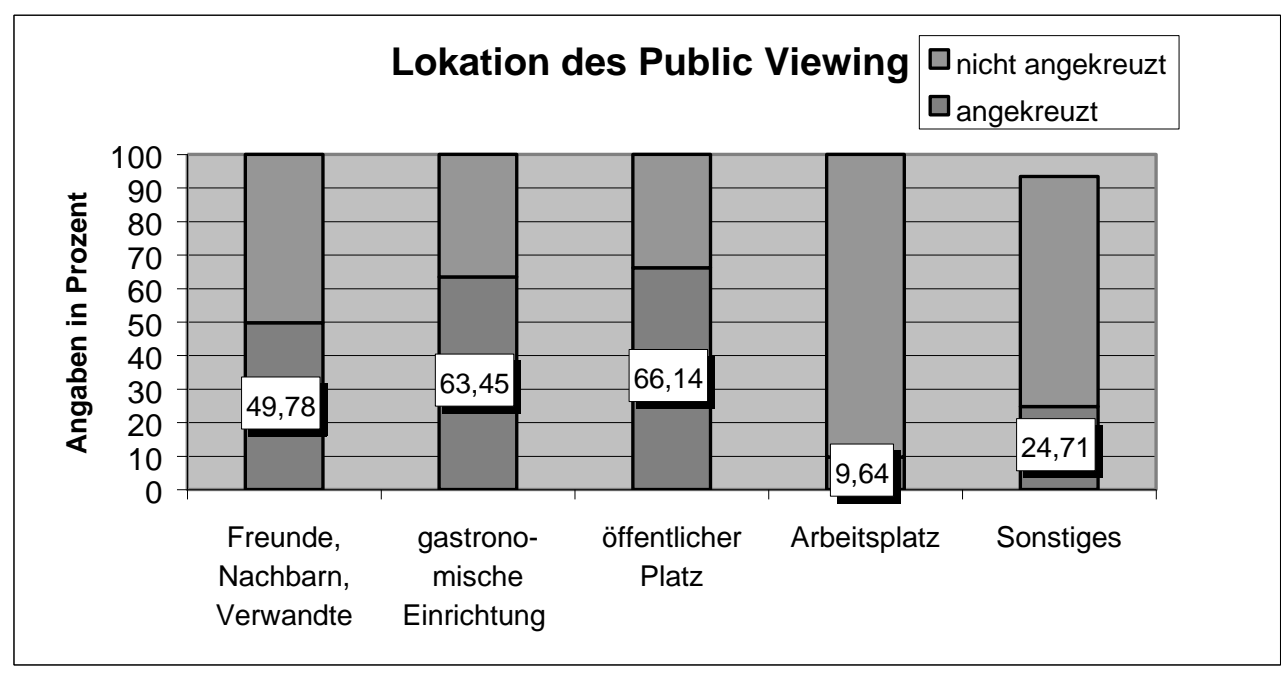

Abb. 31: Lokation der besuchten Public Viewing-Veranstaltung ( $\mathrm{n=446)}$ (Mehrfachnennung möglich) „Wo fand die von Ihnen besuchte Public Viewing-Veranstaltung statt?“

Im Internetfragebogen wurde nach der Lokalität des besuchten Public Viewing gefragt. Die Majorität von $66,14 \%$ der Gäste hat auf einem öffentlichen Platz eine TV-Übertragung gesehen. An zweiter Stelle befinden sich die gastronomischen Einrichtungen, zu denen in der vorliegenden Untersuchung Biergärten, Bars und Cafés gezählt haben, mit 63,45\%. 49,78\% sahen sich das Ereignis bei Freunden,

119 Dass die Spiele der deutschen Nationalelf beim deutschen Fernse hpublikum, unabhängig von der Lokalität, mit Abstand am häufigsten verfolgt wurden, ist in Kap. 5.2.3 bereits herausgestellt worden. 
Nachbarn und Verwandten an. 24,71\% der Teilnehmer nahmen eine Eintragung bei 'Sonstiges' vor. Diese ergänzenden Angaben reichten von Universitätshörsälen über Konzerthallen zu Open-Air-Kinos und Stadien. Nur 9,64\% gaben an, die Spiele in Form eines Public Viewing am Arbeitsplatz verfolgt zu haben. Wobei damit zu rechnen ist, dass es sich um die Übertragungen zur WM 2002 gehandelt hat, da diese aufgrund der Zeitverschiebung vorzugsweise am Vormittag gezeigt worden sind (vgl. Abb. 4). Dass der mit 66,14\% höchste Wert bei den Übertragungen auf einem öffentlichen Platz zu finden ist, zeigt die große Attraktivität, die diese Art von Veranstaltung offenbar ausübt. Dennoch scheint aber das gemeinsame Verfolgen der Spiele außerhalb des eigenen Haushalts in gastronomischen Einrichtungen und auch bei Freunden, Familie und Verwandten weiterhin einen großen Stellenwert einzunehmen. Diese Ergebnisse decken sich mit den in Abb. 4 dargestellten Werten, aus denen ebenfalls zu schlussfolgern ist, dass die TV-Vorführungen im Rahmen von 'öffentliche Veranstaltungen/Großleinwände', so der in dieser Befragung benutzte Kategoriename, an Beliebtheit zunimmt, das Verfolgen der Spiele in gastronomischen Einrichtungen und im privaten Kreis jedoch weiterhin ein wichtiger Faktor bleibt.

Gemein ist den Angaben aber der Wunsch nach dem emotionalen Gemeinschaftserlebnis, für welches international bedeutsame Fußballturniere besonders geeignet scheinen (s. H1). Das 'Gemeinsame-Erleben-Wollen' äußert sich auch in den Prozentangaben bzgl. der Frage nach der Begleitung für den Public Viewing-Besuch.

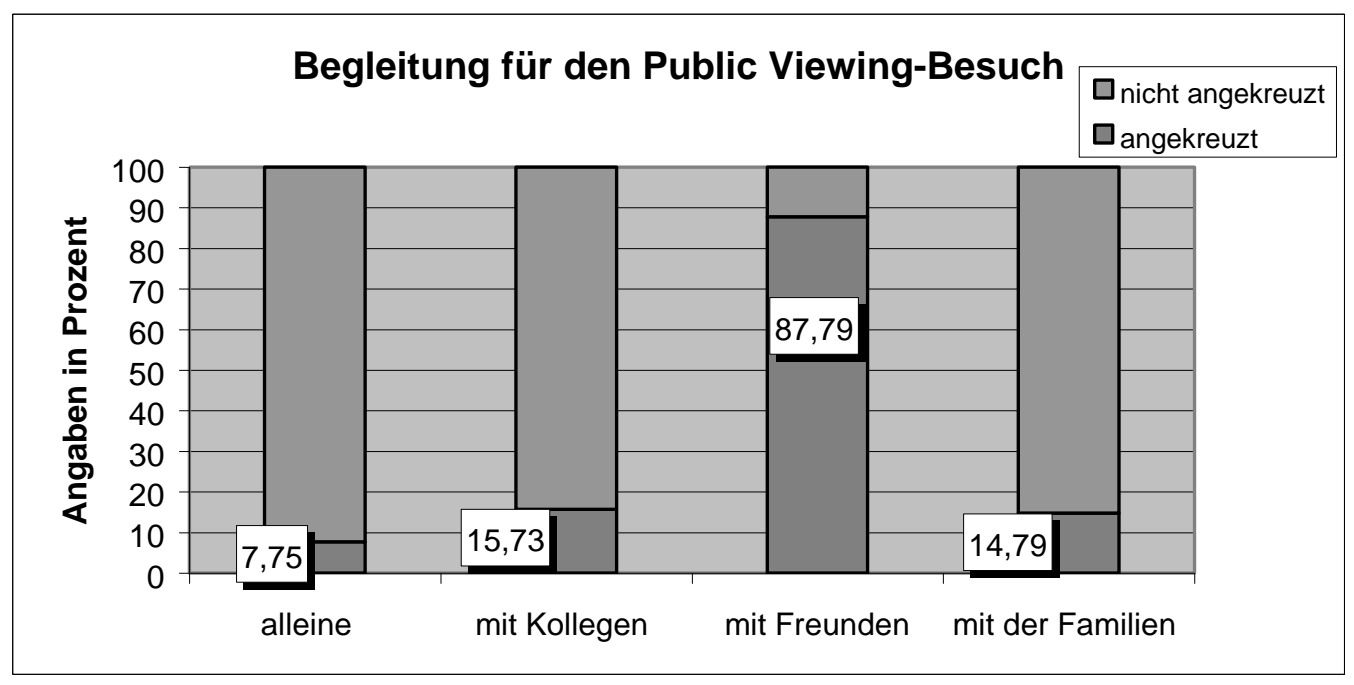

Abb. 32: Begleitung für den Public Vie wing-Besuch (n=426) (Mehrfachnennung möglich) „Ich bin ...“ 
Mit $87,8 \%$ ist der Großteil der Befragten mit Freunden zu der Übertragung auf eine Großleinwand gegangen. $15,73 \%$ haben mit Kollegen eine Public ViewingVeranstaltung besucht und 14,79\% gaben an, mit der Familie gemeinsam der TVÜbertragung beigewohnt zu haben. Wobei hier darauf hingewiesen werden muss, dass es natürlich in den genannten Kategorien auch zu Überschneidungen kommen kann. Dies stellt aber für den Aussagewert der Untersuchung kein Hindernis dar, da herausgestellt werden soll, dass Public Viewing als ein Gemeinschaftserlebnis verstanden wird. 7,75\% der Teilnehmer gingen allerdings alleine zu der Übertragung. Dieser unerwartet hohe Wert der alleine zum Public Viewing Gehenden lässt aber die Vermutung zu, dass die Besucher davon ausgehen, dort Gleichgesinnte zu treffen und letztlich dann in der imaginierten Gemeinschaft dennoch ein kollektives Erleben zu finden.

Public Viewing bei der Fußball-WM 2006 scheint ideale Voraussetzungen für das Entstehen eines außergewöhnlichen, gemeinschaftlichen, emotionalen Erlebnisses geboten zu haben. Zu diesem Schluss kommen 21 von 21 Experten (vgl. $\mathrm{NN}_{14}$, S. 6, Z. 253; $\mathrm{NN}_{15}$, S. 3, Z. $101 ; \mathrm{NN}_{20}$, S. 3, Z. 99 [u.v.m.]). Motive wie gemeinsam Spaß zu haben, Fußball zu gucken und zusammen mitzufiebern (vgl. Z.B. $\mathrm{NN}_{4}$, S. 3, Z. 91; $\mathrm{NN}_{12}$, S. 2, Z. 85) werden oftmals genannt. Ein Experte eines kommerziellen Public Viewing geht davon aus, dass gerade das Bedürnnis nach gemeinsamem emotionalem Erleben auch für eine gute Sicherheitslage gesorgt hat: „Die Besucher haben es sehr als Gemeinschaftserlebnis gesehen und waren nicht übermäßig engagiert, als ihre Mannschaft verloren hatte“( $\left(N_{20}, S .4, Z\right.$. 143ff.). Für $\mathrm{NN}_{13}$ (S. 2, Z. 74f.) schweißt das gemeinsame Erleben und Mitfiebern für eine Sache sogar zusammen. Sie gibt an, das Public Viewing u.a. aus diesem Grund veranstaltet zu haben, wohingegen $\mathrm{NN}_{1}$ (S. 4, Z. 152f.) in dem Bedürfnis nach gemeinschaftlichem Erleben eher eine „Naturellfrage“ sieht, und gibt als Beispiel den Karnevalszug der Rheinländer an. Den in diesem Zusammenhang bereits obig hervorgehobenen Aspekt der besonderen Emotionsentfaltung auf öffentlichen Plätzen beschreibt ein Fan Fest-Spezialist ( $\mathrm{NN}_{2}$, S. 4, Z. 134ff.) folgendermaßen:

„Dann die Stadionatmosphäre, wenn 70.000 jubeln, ist das ein Gäns ehautgefühl. Das kriegen sie nur ab einer kritischen Masse, dass kriegen sie nicht, wenn sie vor dem Ferns eher sitzen oder mit 120 in der Kneipe sitzen."

Es kann also gesagt werden, dass insbesondere auf öffentlichen Plätzen und ab 
einer gewissen Publikumsgröße das emotionale Erleben am wahrscheinlichsten ist. Abschließend ist festzuhalten, dass Zuschauer Public ViewingVeranstaltungen besuchen, weil sie emotionale Gemeinschaftserlebnisse suchen. Diese können in besonderem Maße auf öffentlichen Plätzen und ab einer bestimmten Publikumsgröße erreicht werden. Die Gesamtheit der getätigten Aussagen bestätigt $\mathrm{H} 2 \mathrm{a}$.

Um die Hypothese $\mathrm{H} 2 \mathrm{~b}$ überprüfen zu können, muss im Folgenden durchleuchtet werden, inwiefern die Besucher von Public Viewing-Veranstaltungen neben der subjektiven Interpretation durch das emotionale Gemeinschaftserleben von kollektiven Emotionsregeln und -normen beeinflusst werden. In einem ersten Schritt wird der Frage nachgegangen, inwieweit sich individuelle und soziale Einflüsse aus Sicht der Zuschauer auf ihr Emotionserleben auswirken. Danach gilt es, den Einfluss der Verhaltensunsicherheit bei einem außeralltäg lichen Ereignis sowie die Beeinflussung über Selbst- und Fremdkommunikation hinsichtlich der Emotionsentstehung und -regulierung beim Public Viewing-Publikum zu eruieren. $\mathrm{Zu}$ diesem Zwecke werden verschiedene Einflussebenen von sozialen Emotionsregeln aufgegriffen und erörtert. Im Anschluss wird die Wahrnehmung der Besucher hinsichtlich der Einflussnahme von sozialer Orientierung diskutiert.

Als erstes wurden die Besucher nach den Gründen für ihr positives Emotionserleben befragt. Auf die Aussage "Ich habe positive Emotionen erlebt, weil", standen die Antwortkategorien "die von mir favorisierte Mannschaft ein erfolgreiches Turnier gespielt hat', 'im ganzen Land eine positive Stimmung geherrscht hat', 'ich bereits positive Erfahrungen bei einem vorherigen Besuch gemacht habe'und 'Sonstiges' zur Verfügung. ${ }^{120}$

120 In der Grafik sind diese Kategorien zugunsten der besseren Darstellbarkeit in 'erfolgreiche Mannschaft', 'gute Stimmung im Land', 'positive Erfahrung' und 'Sonstiges' zusammengefasst worden. 


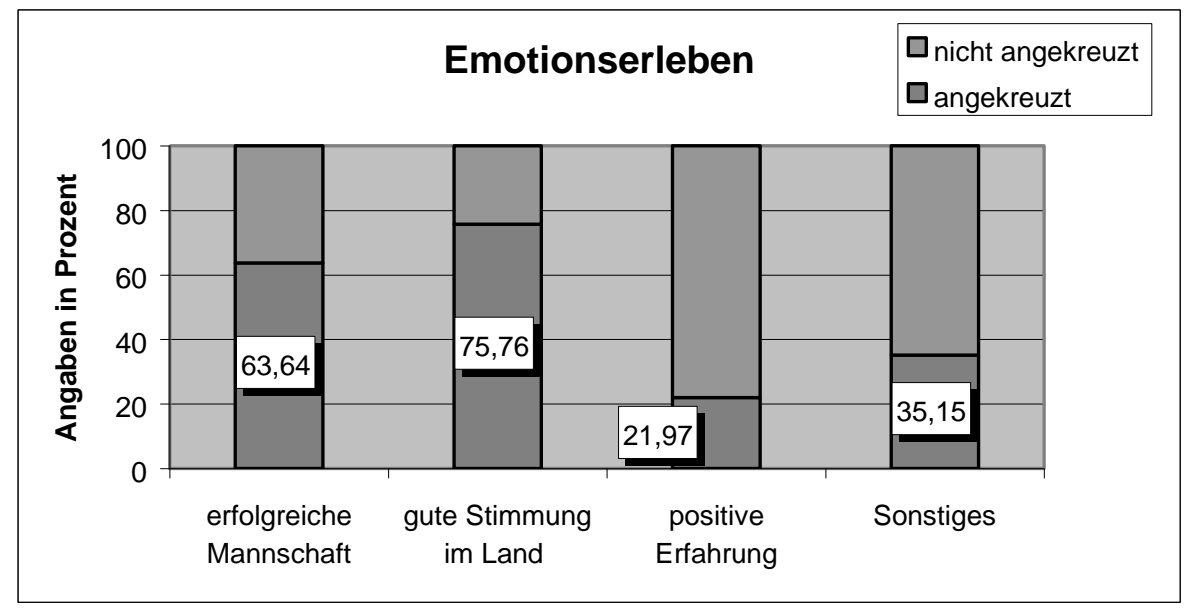

Abb. 33: Emotionserleben (n=396) (Mehrfachnennung möglich) „Ich habe positive Emotionen erlebt, weil..."

Wie in Abb. 33 ersichtlich, machten $75,76 \%$ die im Land ${ }^{121}$ herrschende gute Stimmung für das Erleben von positiven Emotionen beim Public Viewing verantwortlich. Offenbar wirken hier äußere soziale Faktoren auf das individuelle Emotionserleben ein. Darüberhinaus hat das erfolgreiche Spiel der favorisierten Mannschaft und somit ihr gutes Abschneiden im Turnierverlauf für einen Großteil der Besucher $(63,64 \%)$ ebenfalls einen großen Einfluss. Nur 21,97\% gaben dagegen an Emotionen bei einer Fernseh-Vorführung zu empfinden, weil sie bereits positive Erfahrungen gemacht haben. Dieser relativ niedrige Wert kann allerdings auf die bis zu dem Zeitpunkt eher geringen Möglichkeiten ein Public Viewing zu besuchen zurückzuführen sein. Unter der Kategorie 'Sonstiges' (35,15\%) überwiegen die Anmerkungen, die auf das gemeinsame Erleben eines besonderen sportlichen Ereignisses mit Freunden hinweisen. Festzuhalten ist also, dass sich das Emotionserleben des Einzelnen aus individuellen Faktoren, wie bspw. den Erfahrungswerten und sozialen Einflüssen, wie der guten Stimmung im Land, zusammensetzt.

Soziale Wirkungszusammenhänge nehmen immer dann an Gewicht für die Emotionsentstehung und -regulierung $\mathrm{zu}$, wenn bei einer außeralltäglichen Situation, einem hervorstechenden Ereignis oder einer Veranstaltungsart, die noch relativ neu ist, wie dem Public Viewing, eine gewisse Verhaltensunsicherheit -wie etwa 1954- herrscht.

121 Gemeint ist an dieser Stelle der deutsche Staat. Da die Onlineumfrage aber möglichst einfach gestaltet werden sollte, wurde davon ausgegangen, dass eine Umschreibung im alltäglichen Sprachgebrauch angemessener ist. 


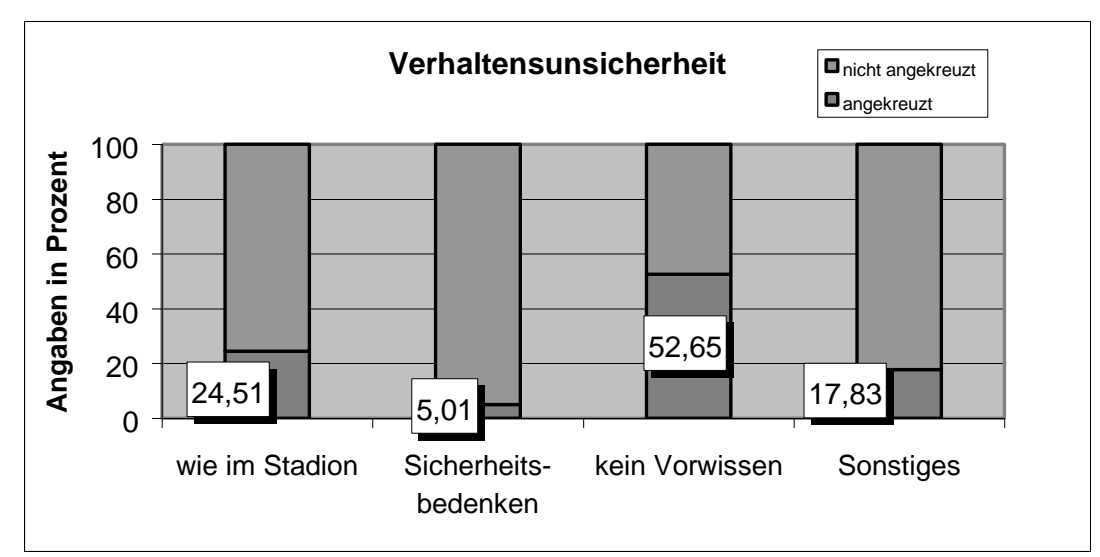

Abb. 34: Verhaltensunsicherheit (n=359) (Mehrfachnennung möglich) „Vor me inem ersten Besuch bei einem Public Viewing..."

Abb. 34 zeigt, dass 52,65\%, also über die Hälfte der Befragten, nicht wusste, was sie bei einem Public Viewing erwarten wird. 24,51\% gaben an, sie dächten, es sei wie im Stadion, und nur 5,01\% äußerten Sicherheitsbedenken. Unter den 17,83\% Nennungen für 'Sonstiges' sind Angaben wie „wusste ich, dass die Stimmung besser sein wird als im kleinen Kreis“, „hatte ich schon Vorstellungen durch Freunde und Medien“, „habe ich mich auf Bier und laue Sommerluft gefreut“ hin zu „hab mir keine Gedanken gemacht“. Obwohl in den Angaben unter 'Sonstiges' keine Tendenz in Richtung einer Aussage auszumachen ist, können diese Meinungsbilder zusammen mit der Gewichtung der gesetzten Kategorien doch einen guten Überblick über die herrschende Verhaltensunsicherheit beim Public Viewing aufzeigen. Die unbekannte Situation vereinfacht die Entstehung neuer emotionaler Gruppen- bzw. Massennormen, an die der Einzelne sich individuell anpasst und durch die ein Zusammengehörigkeitsgefühl unter sich eigentlich fremden Zuschauern entstehen kann.

Die Zuschauer können also über das gemeinsame Erlebnis Public Viewing zu einem Personenkollektiv bzw. Publikum zusammenwachsen. Nach der sozialen Systemtheorie halten sich Systeme über Kommunikation in Form von Selbst- und Fremdzuschreibungen zusammen. Folglich lässt sich daraus ableiten, dass die Entstehung und Regulierung von Emotionen durch Kommunikation beeinflusst werden kann. Neben den verbalen sind vor allem non-verbale Kommunikationsformen als die dominanten Kanäle, durch die Emotionen ihren Ausdruck erhalten, erkennbar (vgl. Kap. 9.2). Die Teilnehmer der Onlinebefragung hatten nach der Einleitung „Während des Public Viewings habe ich..." die 
Antwortkategorien 'geklatscht', 'Spielverlauf angeregt verfolgt (gejubelt, gemeckert etc.)', 'Fangesänge gesungen' und 'bei der LaOla-Welle mitgemacht' zur Verfügung. ${ }^{122}$

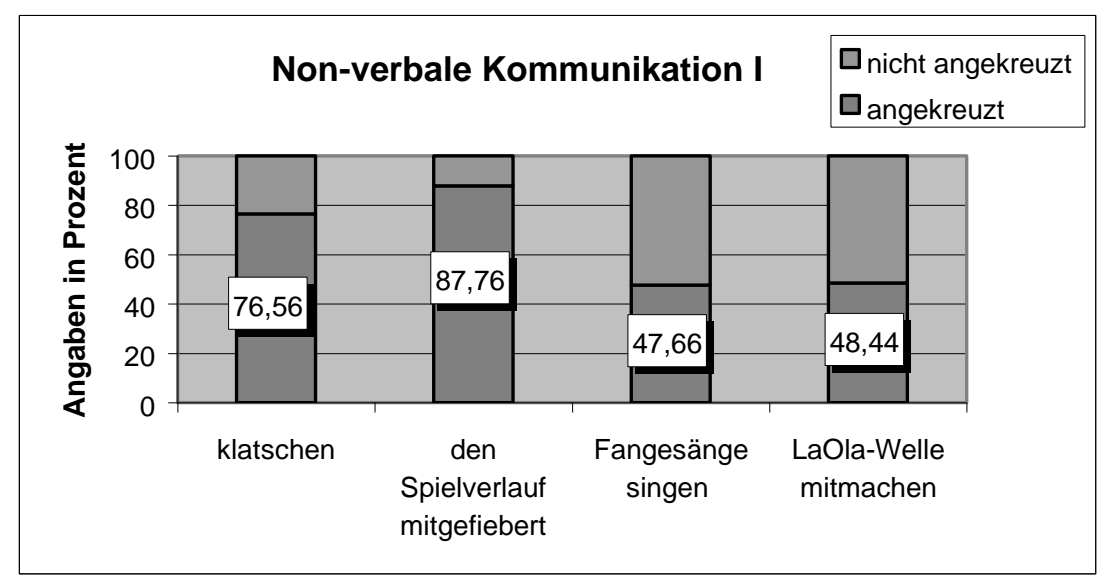

Abb. 35: Non-verbale Kommunikation I ( $\mathrm{n}=384)$ (Mehrfachnennung möglich) „Während des Public Viewing habe ich..."

Abb.35 veranschaulicht, dass das Publikum sich verschiedener Mittel der nonverbalen Kommunikation ${ }^{123}$ für den Ausdruck seiner Emotionen bedient. Den höchsten Wert erzielt das Mitfiebern des Spielverlaufs (87,76\%); das Klatschen (76,56\%) wird als zweithäufigste Aktivität gewählt, und die zwei weiteren Kategorien des Anstimmens von Fangesängen und des Mitmachens der LaOlaWelle rangieren etwa gleich auf mit 47,66\% bzw. 48,44\% dahinter. Der einzelne Zuschauer drückt seine Emotionen anhand dieser Mittel aus und nimmt dadurch eine wichtige Position in der emotionalen Gemeinschaft des Public ViewingPublikums ein. Das Ausdrücken bzw. Ausleben der eigenen Emotionen wird durch die Anpassung an die emotionalen Ausdrücke der anderen Zuschauer, was natürlich als ein Rückkopplungseffekt zu begreifen ist, zu einer gemeinsamen Emotionsnorm.

122 Diese wurden zur besseren graphischen Darstellbarkeit in die Kategorien 'klatschen', 'den Spielverlauf mitfiebern', 'Fangesänge singen' und 'LaOla-Welle mitmachen' eingeteilt.

123 Die Kategorie 'Fangesänge singen' ist eine unspezifische, nicht zielgerichtete verbale Kommunikation und wird aus diesem Grund in Abb. 34 und Abb. 35 unter non-verbaler Kommunikation mitgeführt. 


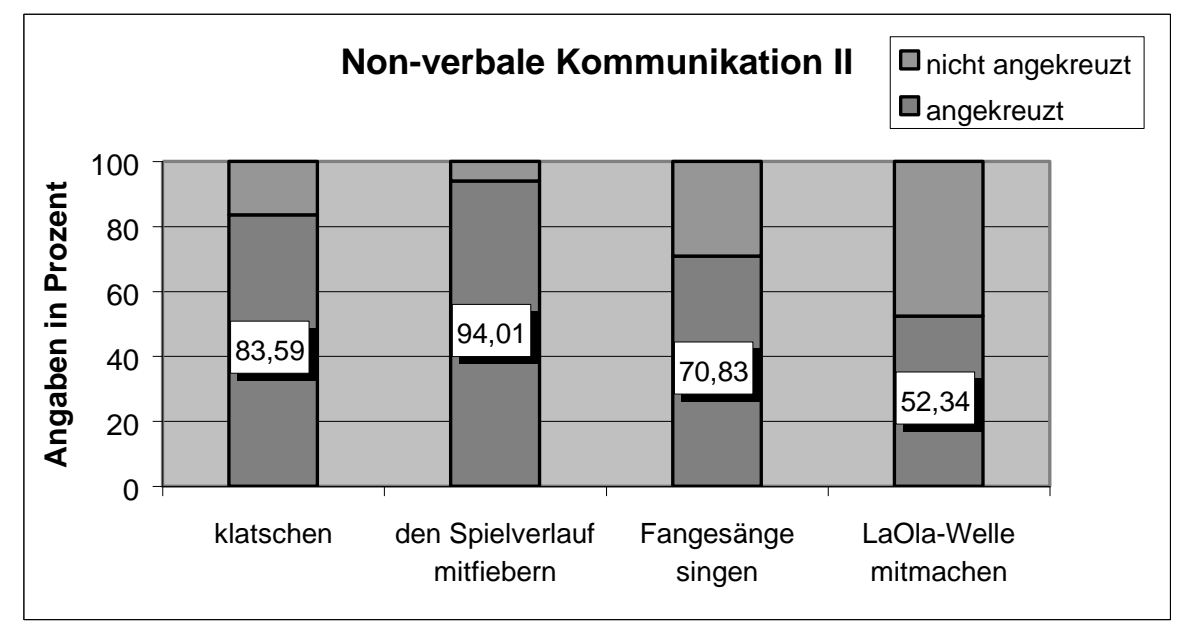

Abb. 36: Non-verbale Kommunikation II (n=384) (Mehrfachnennung möglich) „Welche Handlungen haben die Personen um Sie heru m während der Public Viewing-Veranstaltung gezeigt?““

Abb. 36 verdeutlicht, dass sich die in Abb. 35 genannten Werte zum eigenen Emotionsverhalten und die bei anderen Besuchern beobachteten Handlungen in ihren Prozentwerten ähneln. So gaben 94,01\% der Teilnehmer an, bei anderen das Mitfiebern mit dem Spielverlauf gesehen zu haben, 83,59\% nannten das Klatschen, $70,83 \%$ gaben Fangesänge singen und $52,34 \%$ das LaOla-WelleMitmachen an.

Wie in Kap. 9.2 verdeutlicht wurde, wirken verschiedene Emotionscodes auf verschiedenen Ebenen auf die Entstehung und Regulierung von Emotionen ein. Sie beziehen sich nach Gerhards (1988, S. 199) auf die Qualität, die Intensität und die Dauer der Emotionen. Zunächst wird herausgefiltert, welche Reaktion die befragten Besucher aufgrund von emotionalen Normen als von ihnen erwartet annehmen. Dazu wurde die Anleitung vorgegeben: „Wenn ein Spieler der von mir favorisierten Mannschaft ein Tor geschossen hat, weiß ich, welche emotionale Reaktion angemessen ist,...". Als Antwortkategorien sind 'aus Erfahrung', 'von anderen Besuchern', 'aus den Medien', 'von einem Wortführer/Anführer', 'von den Spielern auf dem Feld', 'vom Veranstalter bzw. Sprecher und dem gezeigten Unterhaltungskonzept ${ }^{124}$ vorgeschlagen, mit denen die Entstehung und Regulierung der Qualität der Emotionen herausgefunden werden soll.

124 Diese aus formulierten Kategoriebezeichnungen gelten für Abb. 37, Abb. 38 und Abb. 39. 


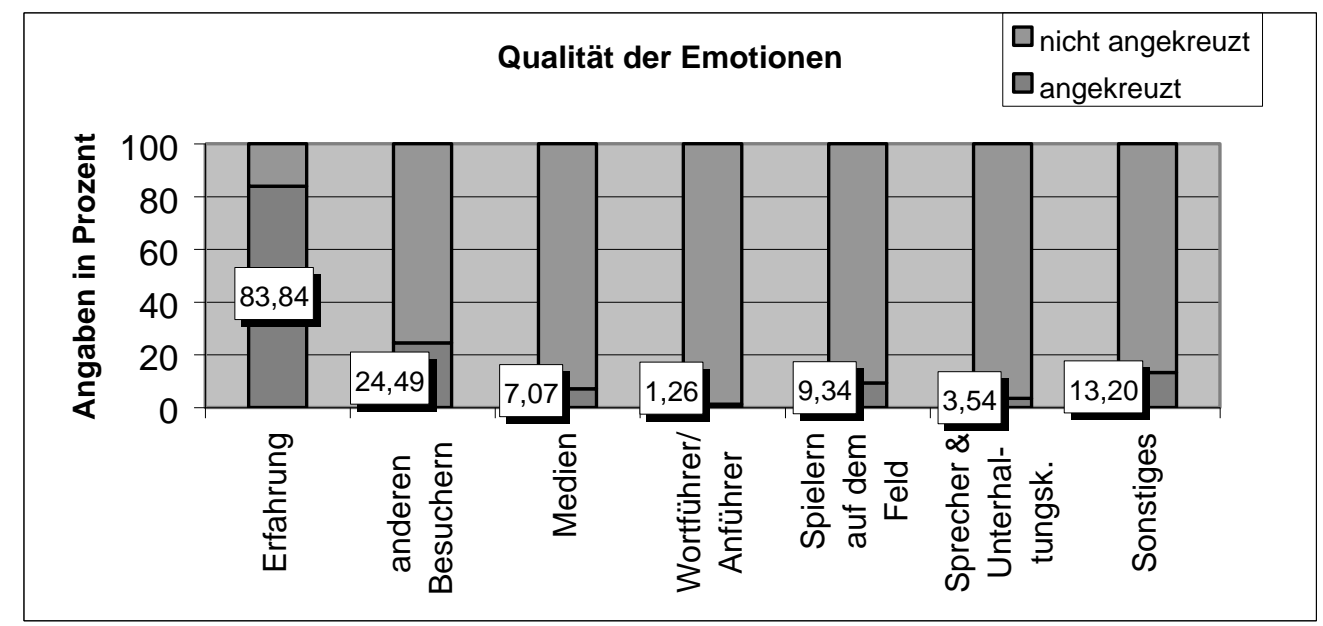

Abb. 37: Qualität der Emotionen (n=396) (Mehrfachnennung möglich) „Wenn die von mir favorisierte Mannschaft ein Tor geschossen hat, weiß ich, welche Emotionen von mir erwartet werden“

Abb 37 zeigt, dass 83,84\% der Besucher die Qualität ihrer Emotionen aus eigenen, individuellen Erfahrungswerten ableiten. Aber auch soziale Strukturen und Kommunikationsprozesse scheinen einen Einfluss auf die gezeigte emotionale Qualität zu haben. So gaben $24,49 \%$ an, ihr Verhalten von dem anderer Besucher abzuleiten. Weniger Teilnehmer beziehen die Informationen über ihre Emotionshandlungen von den Spielern auf dem Feld $(9,34 \%)$ oder aus den Medien (7,07\%). Eine kaum erwähnenswerte Rolle nehmen Wortführer bzw. Anführer $(1,26 \%)$ und 'Veranstalter bzw. Sprecher und das gezeigte Unterhaltungskonzept' $(3,54 \%)^{125}$ ein. $13,2 \%$ nutzen die Möglichkeit, in der Kategorie 'Sonstiges' einen Eintrag zu machen.

Im Anschluß an die Frage nach den sozial erwarteten Emotionshandlungen, wurden die Public Viewing-Besucher, das Foul-Beispiel aus dem Theoriekapitel aufnehmend, gefragt, woher sie wissen, welche Emotionen in welcher Form angemessen sind, wenn bspw. ein Spieler i hrer Mannschaft gefoult wird.

$125 \mathrm{Um}$ eine Zerfaserung des Argumentationsstranges zu vermeiden, wird an dieser Stelle noch nicht auf die Bedeutung von Abb. 37 für die Steuerbarkeit von Emotionen eingegangen. Dieses wird in Kap. 13.2.2 erfolgen. 


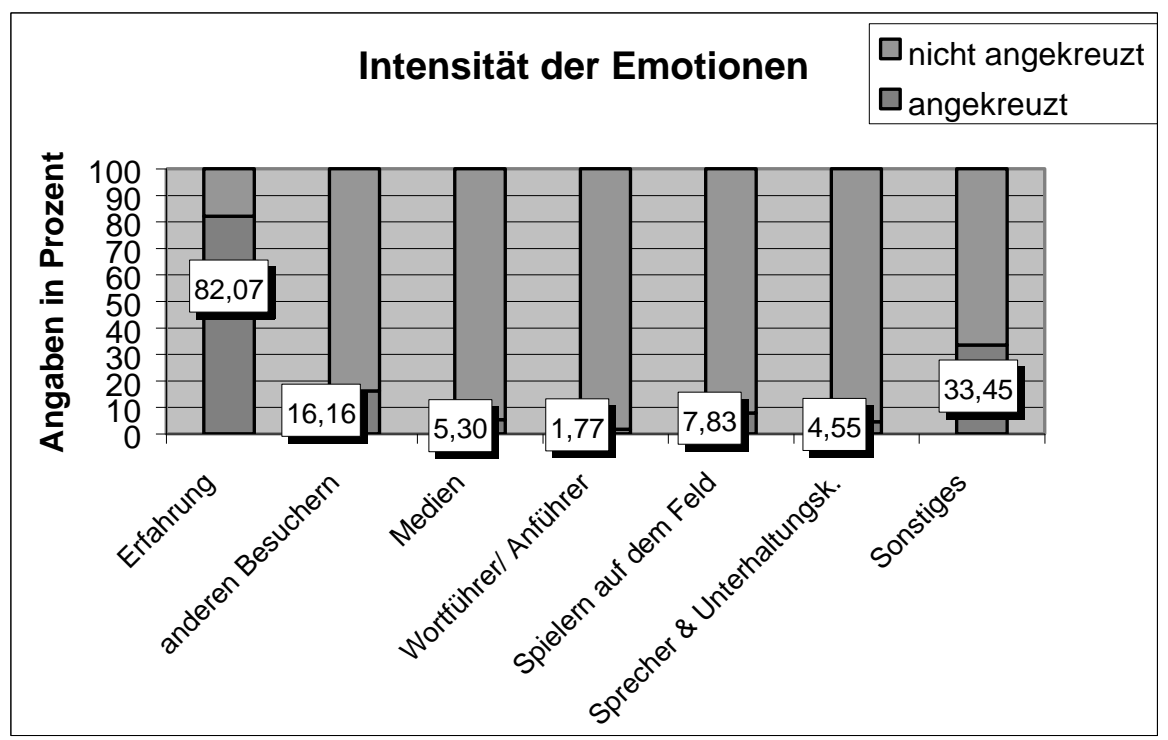

Abb. 38: Intensität der Emotionen (n=396) (Mehrfachnennung möglich) „Wenn ein Spieler der von mir favorisierten Mannschaft gefoult wird, weiß ich welche Reaktion angemessen ist..."

Abb. 38 zeigt, dass auch bzgl. der Intensität der für angemessen erachteten Emotionen die Majorität der Besucher (82,07\%) ihr Emotionsverhalten aus Erfahrungen ableitet. $16.16 \%$ der Teilnehmer greifen in diesem Zusammenhang auf die von anderen Besuchern gezeigten Verhaltensweisen zurück. 5,3\% beziehen Informationen bzgl. des emotionalen Verhaltens aus den Medien, 7,83\% von den Spielern auf dem Feld und 4,55\% vom Sprecher bzw. dem Unterhaltungskonzept der Veranstaltung, wohingegen lediglich $1,77 \%$ der Befragten ihr Verhalten dem eines Wort-bzw. Anführers anpassen. Des Weiteren wurden unter 'Sonstiges' Anmerkungen wie „....wenn alle schreien, schrei ich mit“, „Intuition“ oder „Erziehung“ u.a. gemacht.

Die dritte von Gerhards genannte Ebene von Emotionscodes bezieht sich auf die Dauer der Emotionshandlung. Wie in Abb. 39 ersichtlich, orientieren 78,54\% der Besucher die Dauer des Emotionserlebens nach der Intensität der erlebten Emotionen und 74,75\% nach der Wichtigkeit des Ereignisses. Wie in der Erörterung der $\mathrm{H} 1 \mathrm{a}$ hinsichtlich der Motivcluster bereits erwähnt, sind das Emotionserleben und die Wichtigkeit des Ereignisses, die mit der Attraktion bzw. dem Unterhaltungswert einhergehen, immer in ihrer Wechselwirkung zu betrachten. Desweiteren geben 26,01\% der Teilnehmer an, die Regulation der Emotionen an anderen Public Viewing-Gästen zu orientieren. Ein Wert, der erneut auf Kommunikation als einen Motor für die Entstehung eines Personenkollektivs Publikum schließen lässt. Darüberhinaus stützen die 13,13\% der Teilnehmer, die 
die Dauer der Emotionen an der Berichterstattung in den Medien orientieren, diese Annahme. Die Orientierung an dem Verhalten der Spieler auf dem Feld und an einem Wort- bzw. Anführer nehmen mit 9,34\% bzw. 0,25\% einen geringen Anteil ein. An weiteren Anmerkungen machen die Zuschauer "eigene Bewertung" „individuelle Stimmungslage“ oder „persönliche Bedeutung des Ereignisses für mich“, welche in der Tendenz wieder auf die Mischung der zuvor genannten sozialen Einflüsse mit diesen eher individuellen Werten hindeuten.

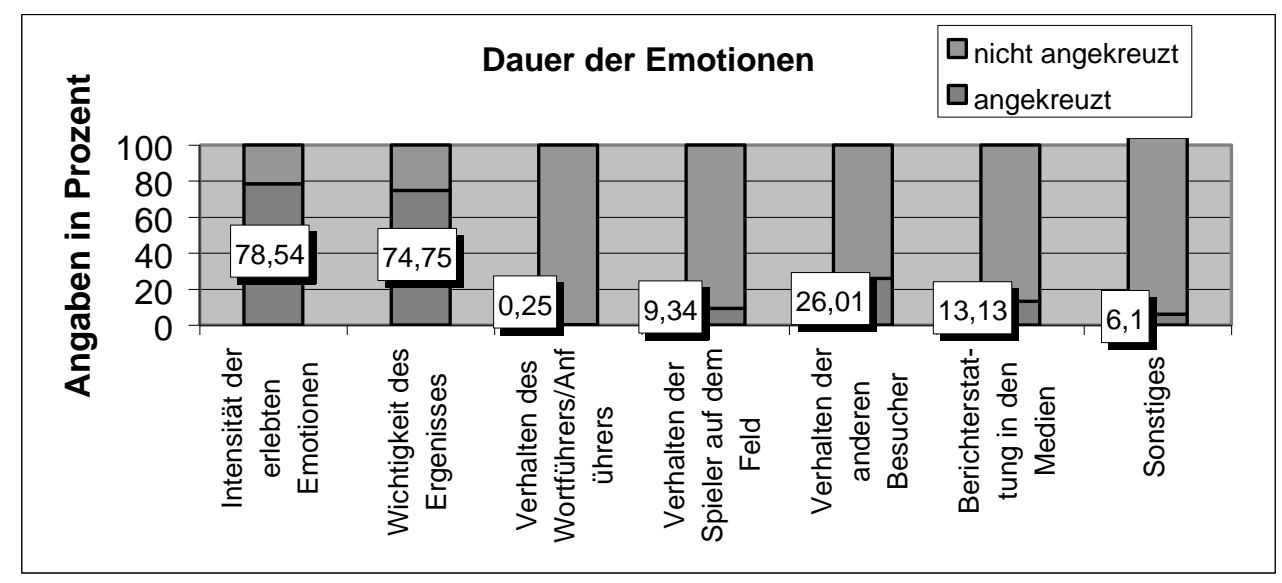

Abb. 39: Dauer der Emotionen (n=396) (Mehrfachnennung möglich), „Die von Ihnen favorisierte Mannschaft hat gewonnen. Wovon machen Sie es abhängig, wie lange Sie sich über den Sieg freuen?“

Emotionsentstehung und -regulation beim Public Viewing ist auf einen Mix zwischen individuellen Erfahrungen und sozialen Emotionscodes bzw. -normen zurückzuführen. Kollektive Emotionen entwickeln sich folglich durch die aktive Interaktion der Zuschauer untereinander (emotionalisierte Kommunikation) und der Kommunikation über Emotionen z.B. mit äußeren Faktoren wie den Medien (s. hierzu auch H2c). Ansteckungsmechanismen, wie bspw. das 'Crowding' bei Massenveranstaltungen, können diese Effekte noch verstärken (s. Kap. 9.3). So gaben 48,91\% an: „Ich hatte das Gefühl, dass sich die Emotionen durch das enge Beisammensein leicht von einem auf den anderen Besucher übertragen“" ${ }^{126}$, und $43,75 \%$ haben ein Wir-Gefühl gespürt. Die Verschmelzung mit anderen Besuchern gaben dagegen nur $1,36 \%$ an, und der Anteil der Teilnehmer, die nichts dergleichen gespürt haben, ist mit 5,98\% ebenfalls eher gering.

126 Auch in Abb. 39 sind aus Gründen der graphischen Darstellbarkeit die Kategoriebezeichnungen nur in einer Kurz form genannt. 


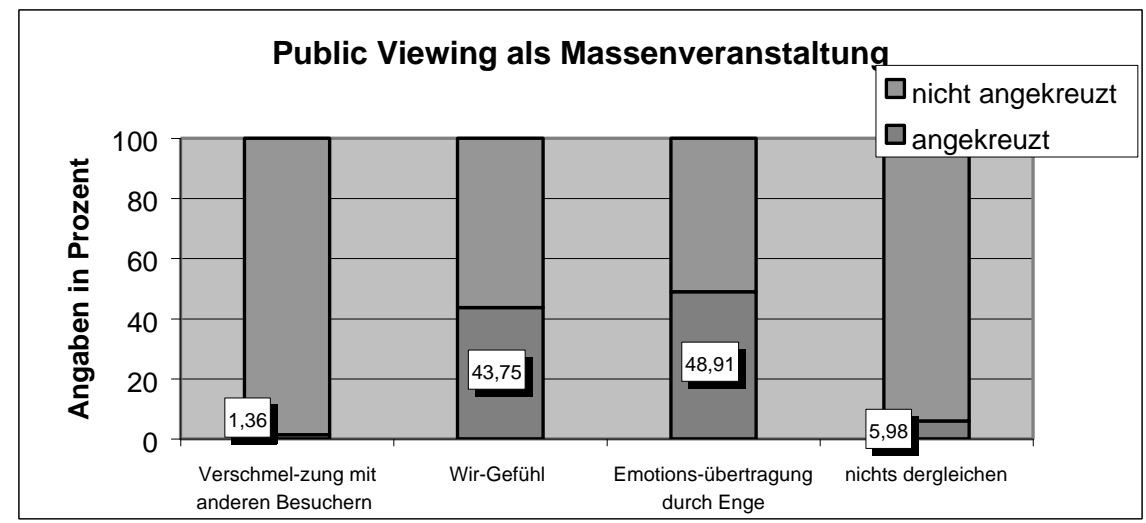

Abb. 40: Public Viewing als Massenveranstaltung ( $\mathrm{n}=368$ ) (Mehrfachnennung möglich) „Public Vie wing ist eine Massenveranstaltung“"

Die Gesamtheit der erörterten Aussagen und Erhebungen bestätigen die Annahmen der $\mathrm{H} 2 \mathrm{~b}$, dass der einzelne Zuschauer seine Emotionsentstehung und -regulation aus individuellen Erfahrungen und sozialen Inhalten zusammensetzt. Die verbale und non-verbale Kommunikation über sich selbst und die Orientierung an äußeren Faktoren, wie anderen Besuchern oder den Spielern auf dem Feld, bedingen die aktive Interaktion der Zuschauer. Der Einfluss von sozialen Emotionsregeln wächst insbesondere in neuen, unbekannten Situation, die das Public Viewing für eine Vielzahl dargestellt hat, und bei Massenveranstaltungen an.

Eine Fortführung der unter $\mathrm{H} 2 \mathrm{~b}$ genannten Bausteine des Publikums als Personenkollektiv findet sich im Konstrukt der 'imagined community'bzw. der imaginierten Gemeinschaft. Dieses geht davon aus, dass dispers verteilte Individuen über die Vorstellung einer Gemeinschaft ohne face-to-faceKommunikation diese empfinden können. Als Mittler dient an dieser Stelle die Kommunikation über die Medien, insbesondere über das Fernsehen. Medienereignisse wie die Fußball-WM besitzen eine große kollektive Emotionalität und sind in der Lage, durch einzelne Mikro-Ereignissen aus dispersen Publika eine imaginierte Gemeinschaft zu erzeugen. Um die Hypothese H2c zu prüfen, gilt es demnach die Meinungsbilder der Experten bezüglich des Einflusses der Massenmedien, und insbesondere des Fernsehens, für die Entstehung einer imaginierten Gemeinschaft zu eruieren.

Ein Beitrag der Medien wird von den Public Viewing-Spezialisten im Wecken der Vorfreude auf das Weltmeisterschaftsturnier gesehen. Diese hätten, u.a., weil die 
WM in Deutschland stattgefunden hat, bereits frühzeitig durch eine intensive Berichterstattung das Interesse und die Begeisterung der Bürger entfacht (vgl. $\mathrm{NN}_{1}$, S. 3, Z. 122f.; $\mathrm{NN}_{8}$, S. 2, Z. 52f.). Diese kann als ein Grund für das ausgeprägte Bedürfnis des Einzelnen angesehen werden, bei diesem Großereignis live dabei zu sein.

\begin{abstract}
„Draußen zu feiern, gemeinsam zu feiern, diese Stimmung, diese WM-Atmos phäre im eigenen Land. Dieses einmalige Erlebnis. Die Großveranstaltung mit aufzusaugen, nicht alleine im Wohnzimmer oder beim Grillen alleine zu erleben" $\left(\mathrm{NN}_{19}\right.$, S. 5, Z. 178ff.) (vgl. auch $\mathrm{NN}_{1}$, S. 2, Z. 64-69).
\end{abstract}

Die Experten haben erkannt, dass Public Viewing-Veranstaltungen den Gästen einen höheren Grad an 'Dabeisein' bieten können als der heimische Fernsehsessel (vgl. $\mathrm{NN}_{15}$, S. 3, Z. 92ff.). Ein Experte eines nicht-kommerziellen Public Viewing ( $\mathrm{NN}_{14}$, S. 4, Z. 137f.) stellt heraus: „Also, dass der Eventcharakter natürlich weit mehr rüber kommt als das jetzt privat, am heimischen Fernseher oder PC“ der Fall ist. Das Gefühl des 'Live-Dabeiseins' scheint sich aber nicht nur über die Berichterstattung und die Kommunikation durch die Medien zu generieren (s. hierzu auch Kap. 13.2.1). Vielmehr ist an der Stelle wiederholt auf einen Wechselwirkungsmechanismus zu verweisen. So wird die positive Stimmung im Ausrichterland Deutschland als ein Ergebnis des gemeinschaftlichen Verfolgens der Spiele in der Öffentlichkeit gesehen (vgl. NN16, S. 6, Z. 239-244). Diese hätten, so Experte $\mathrm{NN}_{2}$ (S. 3f. Z. 132f.), den Gästen die Chance gegeben, „sich selber zu inszenieren, sich selber zu feiern“ und somit das Gefühl gegeben, an dem Großereignis im Kleinen teilzuhaben.

In einigen Fällen konnte dieser Effekt noch durch externe Maßnahmen, wie bspw. dadurch, dass das Public Viewing einen Tag Teil einer übergeordneten regionalen Werbemaßnahme eines Großunternehmens (BASF Roadshow) war, verstärkt worden. So wurde die Chance genutzt, den Gästen das Gefühl zu geben Teil eines großen Ganzen zu sein (vgl. $\mathrm{NN}_{15}$, S. 1, Z. 18f.). Natürlich standen die Fan Feste in erhöhtem Maß in der medialen Öffentlichkeit:

"[l]ch denke schon, dass sie [die Zuschauer] dadurch schon das Gefühl hatten, etwas näher dran zu sein. (...) Aber ich glaube, es ist schon ein Unterschied, die Fans hatten auch durch die Kooperation mit der FIFA das Gefühl näher dran zu sein; wenn Goleo vorbeigeschaut hat, und wir hatten auch Bildmaterial von der FIFA" $\left(\mathrm{NN}_{2}\right.$, S. 4, Z. 152157).

Dennoch scheint aber die Verbindung verschiedener Faktoren stärker an der Erzeugung einer imaginierten Gemeinschaft zu sein, als zunächst vermutet wurde. 
So wurde bspw. zwar auch das erhöhte Medieninteresse an den Public Viewings in Quartierstädten von den Experten bekräftigt (vgl. $\mathrm{NN}_{2}$, S. 166-169; $\mathrm{NN}_{14}, \mathrm{~S}$. 2, Z. 81-85; $\mathrm{NN}_{18}$, S. 3, Z. 123f.), aber es wird auch auf "Rückkopplungseffekte“ $\left(\mathrm{NN}_{14}\right.$, S. 2, Z. 110-114), wie z.B. die Möglichkeit, gleich im Anschluss an die TVÜbertragung in einem Autokorso gemeinsam den Sieg der Mannschaft zu feiern, verwiesen. H2c kann folglich nur in Ansätzen bestätigt werden.

\subsubsection{Steuerbarkeit positiver Emotionen beim Public Viewing}

Nachdem in den letzten beiden Kapiteln geklärt werden konnte, welche Faktoren die Entwicklung des Public Viewing beeinflusst haben und wie sich das Public Viewing-Publikum bildet, soll an dieser Stelle auf die Steuerbarkeit von positiven Emotionen bei den TV-Übertragungen eingegangen werden. Die dritte Hypothese lautet demnach:

H3: Die Wahrscheinlichkeit des Entstehens von positiven Emotionen bei einer Public Viewing-Veranstaltung kann positiv beeinflusst werden.

a. Die Orientierung an hedonistischen Werten führt dazu, dass der Einzelne seine Freizeitaktivitäten zur Erfüllung der eigenen Bedürfnisse auswählt. Dabei stellt das Freizeitangebot mit Erlebnispotential Public Viewing ein geeignetes Ziel dar.

b. Die Steigerung des Erlebnispotentials des Public Viewing kann in Form des Konzepts des Erlebnis-Setting gefördert werden. Die Inszenierung als Instrument des Erlebnis-Setting trägt durch gezielte Maßnahmen dazu bei, alle Sinne zu erreichen und eine positiv bewertete anziehend-erregende emotionale Atmosphäre zu erreichen.

Der individualisierte Einzelne in der Postmoderne ist geneigt, sich über eine innenorientiere Lebenseinstellung soziale Struktur zu schaffen. Denkbar ist dies z.B. in Form von sogenannten Erlebnisgemeinschaften, die sich über das gemeinsame Erleben von emotionalen Ereignissen definieren. Soziale gemeinschaftliche Ereignisse erhalten so einen neuen Stellenwert. Erlebnisse sind jedoch nicht per se herstellbar, sondern sie sind nur in Form von Erlebnisarbeit individuell, subjektiv möglich. Trotzdem scheint es durchaus eine gewisse ErlebnisIntersubjektivität zu geben, z.B. durch soziale Vereinfachungsprozesse wie die 
Strukturierung von Emotionen durch die 'Emergente-Normen-Theorie' (s. Kap. 10.1). Der Erlebniswert eines Freizeitangebots wie Public Viewing rückt folglich in den Vordergrund des Interesses der Besucher und somit auch der Verantwortlichen. Im zweiten Teil der Diskussion der dritten Hypothese wird der Frage nach der Möglichkeit der Steigerung des Erlebnispotentials beim Public Viewing durch gezielte Inszenierungsmaßnahmen zur Erzeugung einer als anziehend-erregend bewerteten Atmosphäre im Rahmen eines Erlebnis-Settings nachgegangen (vgl. $10.3 \& 10.4)$.

Um H3 überprüfen zu können, werden im Folgenden, nach der Erörterung einer Frage an die Teilnehmer der Onlinebefragung bzgl. des Flow-Gefühls (s. Kap. 10.1), vornehmlich die Aussagen der Experten von Public ViewingVeranstaltungen der Fußball-Weltmeisterschaft 2006 hinsichtlich des Erlebnispotentials des Freizeitangebots Public Viewing und der Inszenierungsmaßnahmen diskutiert.

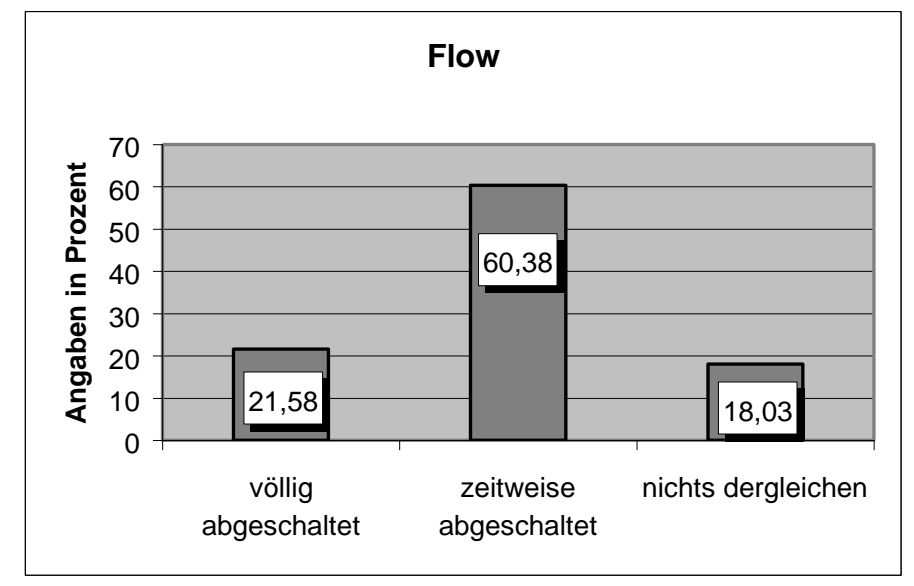

Abb. 41: Flow (n=366) (Mehrfachnennung möglich) „Während des Public Viewing habe ich...“

60,38\% der Besucher gaben an, während des Public Viewing zeitweise völlig abgeschaltet und die Zeit vergessen zu haben sowie in der Situation aufgegangen zu sein. Ein erstaunlich hoher Anteil von 21,58\% nennt sogar das völlige Abschalten während der gemeinschaftlichen TV-Rezeption als gegeben, und nur 18,03\% konnten keinerlei Flow-Gefühl verspüren. Abb. 41 kann demnach zunächst die Möglichkeit des Public Viewing aufzeigen, den Gästen nicht nur positive Emotionen zu bieten, sondern auch Erlebnis zu sein.

Es gilt festzustellen, dass der Großteil (63,76\%) der Teilnehmer der Onlinebefragung zwischen 14- und 29 Jahren alt ist. Von der grundsätzlichen 
Annahme ausgehend, dass nur diejenigen an der Befragung teilgenommen haben, die ein gewisses $M a ß$ an thematischem Interesse haben, abzüglich der durch das Medium bedingten generell jüngeren Benutzer, lässt sich der Schluss auf ein im Durchschnitt junges Public Viewing-Publikum ziehen. Diese Aussage wird von der Majorität der Experten (18 von 21) gestützt. In Interviewauszügen wie: "Wir haben sehr viele junge bis allenfalls 30 -jährige Leute hier gehabt" $\left(\mathrm{NN}_{1}\right.$, S. 2, Z. 70) wird deutlich, dass das Publikum während der WM 2006 hauptsächlich aus jungen Personen bestanden hat (vgl. auch $\mathrm{NN}_{2}$, S. 2, Z. 48f.; $\mathrm{NN}_{3}$, S. 2, Z. 56f.; $N_{7}$, S. 2, Z. 44f.). Offenbar sehen gerade junge Personen im Public Viewing eine Freizeitaktivität, mit der sie ihre Bedürfnisse erfüllen können. Die FernsehÜbertragungen der Begegnungen der Fußball-WM 2006 bieten offenbar vor allem für junge Menschen eine geeignete Grundlage für eine gute Partystimmung (vgl. z.B. $\mathrm{NN}_{3}$, S. 4, Z.142; $\mathrm{NN}_{7}$, S. 2, Z. 79; $\mathrm{NN}_{17}$, S. 4, Z. 151f.). Immer wieder kommt in den Interviews zum Ausdruck, dass die eigentliche Übertragung der Fußballspiele in den Hintergrund rückte.

\begin{abstract}
„Also, es waren sehr viele junge Leute da und vor allem junge Leute, die feiern wollten. Denen ging es eben dann darum, ich sage es mal so: Das FußballAnschauen war Mittel zum Zweck. Aber letztendlich war eine unglaubliche Feierstimmung da, und die haben einfach Spaß daran gehabt. Wir sind ja auch schon lange in dem Geschäft, und das sind auch Erkenntnisse, die wir so noch nicht hatten" $\left(\mathrm{NN}_{1}\right.$, S. 2, Z. 74-79).
\end{abstract}

Das enorme Potential, welches Public Viewing, wenn es als Freizeitangebot mit Erlebniswert gestaltet wird, besitzen kann, hat die Mehrzahl der Experten (17 von 21) bereits im Vorfeld erkannt. Ihre Aussagen lassen darauf schließen, dass hinter dem Gedanken des Public Viewing bei der Fußball-Weltmeisterschaft 2006 die Aufmachung als Freizeitangebot gestanden hat.

\footnotetext{
„Wir wollten einen Kern mit Public Viewing, aber drumherum ein Fan Fest. Da sind wir ja eigentlich die ersten gewesen, mit gastronomischen Einrichtungen, mit Programm, mit Ausstellern, mit einer Kinderwelt etc..",
}

berichtet ein Experte ( $\mathrm{NN}_{1}, \mathrm{~S} .1$, Z. 34ff.). Weiterführend ergänzt er:

\begin{abstract}
„Ja, wir sind nicht auf der grünen Wiese oder einem Stadtplatz, sondern wir haben schon öfter Feste dieser Art gemacht. Dass es ein Motiv in der Mitte gab, hier das Public Viewing, aber dass es auch ein Volksfest drumherum gab."
\end{abstract}

Wie in diesem Zitat deutlich wird, wurde das Übertragen von Spielen der FußballWeltmeisterschaft 2006 zwar als Aufhänger der Gesamtveranstaltung gesehen, aber je nach Professionalisierungsgrad und finanziellem Hintergrund der 
Organisation wurde Wert auf eine Freizeitorientierung gelegt. Als einen weitere $n$ geeigneten Rahmen für Public Viewing-Veranstaltungen zeichnet sich das Familienfest ab. Dass

„....Fußball in der Dimension auch ein Erlebnis für die ganze Familie ist, in dem sich alle wieder finden. Es sollte abwechslungsreich sein, dass man Freude hat, neben dem Fußball, auch immer wieder so auf das Fan Fest zu gehen" $\left(\mathrm{NN}_{5}\right.$, S. 2, Z. $67 \mathrm{ff}$., s. auch $\mathrm{NN}_{4}$, S. 3, Z. 86f., S. 5 Z. 173ff.).

Hinter dem Gedanken des Familienfestes steht auch die Hoffnung, dass so eine friedliche, ruhige Atmosphäre erzielt werden kann (vgl. $\mathrm{NN}_{7}$, S. 3, Z. 86ff.). Man wolle den Gästen einfach eine schöne Zeit, ein paar schöne Stunden bescheren, so zwei der Experten (vgl. NN 6 , S. 4, Z. 158ff.; NN 10 , S. 2, Z. 78f.). Ein Experte eines nicht-kommerziellen Public Viewing $\left(\mathrm{NN}_{8}, \mathrm{~S}\right.$. 3, Z. 88-90) führte diesen Gedanken noch weiter aus:

„....[W]ir wollten einen lokalen Treffpunkt organisieren, wo jetzt nicht nur Leute aus dem Ort, sondern auch aus der näheren Umgebung sich treffen können und gemeinsam Fußball schauen können."

Hier zeigen sich die veränderten Erwartungen der Sportveranstaltungsbesucher. Viele wollen nicht mehr nur den reinen Sport sehen, sondern auch unterhalten werden. Public Viewing bietet diese Möglichkeit in nahezu optimaler Art und Weise. Auf diesem Hintergrund lassen sich natürlich auch Vermarktungsgesichtspunkte verschiedenster Couleur einbringen. Einige der Experten bringen als ein Motiv, Public Viewing zu veranstalten, Imagegründe für die Wertsteigerung der Stadt (vgl. z.B. $\mathrm{NN}_{2}$, S. 3, Z. 114-120; $\mathrm{NN}_{3}$, S. 3, Z. 121127; NN 6 , S. 1f., Z. 38-44) an, die sie mit der stimmungsvollen Gestaltung und dem Aufbau des Erlebnispotentials des Public Viewing erreichen wollen. Die Gesamtheit dieser Aussagen bestätigt die Richtigkeit des ersten Teils der Hypothese (H3a).

Um den zweiten Teil der dritten Hypothese (H3b) diskutieren zu können, werden zum einen Informationen über das Erlebnis-Setting und zum anderen über die genutzten Inszenierungsmaßnahmen innerhalb des Settings benötigt. Darüber hinaus ist es sinnvoll, die Aussagen der Experten nach der Gewichtung der von ihnen angewendeteten Maßnahmen auszuwerten.

Es ist auch hier, wie in $\mathrm{H} 3 \mathrm{a}$ bereits erwähnt, ersichtlich, dass sich viele Experten über die Möglichkeiten, die sich durch ein ansprechendes Setting und 
Inszenierungsmaßnahmen ergeben, bereits bewusst sind. Es lassen sich bzgl. der Setting-Gestaltung zwei hervorstechende Tendenzen ableiten: Die Inszenierung als Stadionersatz und die Inszenierung als interkulturelle Veranstaltung, welche jedoch beide auf die Erzeugung einer anziehend-erregenden Atmosphäre abzielen.

Das Bestreben, sich einer Stadionatmosphäre anzunähern und das Setting dementsprechend zu arrangieren, wird vielfach genannt (vgl. NN $N_{2}$, S. 3, Z. 115; $\mathrm{NN}_{3}$, S. 4, Z. 148 , S. 6 , Z. $258 ; \mathrm{NN}_{4}$, S. 3, Z. $101 ; \mathrm{NN}_{21}$, S. 3, Z. 89 [u.a.]):

„Wir hatten ein Sportprogramm mit Show, mit Tanz, mit Warm-Up, mit Mannschaftsaufstellung, also im Prinzip war es das Konzept. Ich sage mal Stadionsprecher, Stadionatmosphäre, die ganzen Rituale, die Sie auch im Stadion kennen mit Mannschaftsaufstellung, also Vornamen-Zuname-Spielchen, das kennen Sie ja" $\left(\mathrm{NN}_{17}\right.$, S. 3f., Z. 130-134).

Diese beispielhafte Textstelle unterstreicht die Varianz an Inszenierungselementen, mit denen bereits gearbeitet worden ist. Hinsichtlich der stadionähnlichen Gestaltung des Public Viewing wird des weiteren auch auf bauliche Maßnahmen, wie bspw. das Anordnen der Sitz- und Stehplätze im ansteigenden Halbrund genauso gesetzt, wie den Einsatz eines 'Stadionsprechers' mit den im Stadion üblichen Animationsspielen, wie z.B. dem 'Vorname-Zuname-Rufen'. Wobei bspw. die einem Stadion ähnlichen Aufbauten im Rahmen des Erlebnis-Setting als konstante Faktoren zu bezeichnen sind, die musikalische Untermalung aber unter die direkten Wirkfaktoren fallen könnte. Allerdings kann der Musikstil, ähnlich wie die Art des 'Stadionsprechers', auch unter die indirekten Wirkfaktoren fallen, da deren Akzeptanz stark von Motiven und Erwartungen bedingt ist (vgl. Kap. 10.3).

Der zweite Settingansatz ist in der Anreicherung des Public Viewing mit lokalen und regionalen Charakteristika zu sehen. Es wird versucht, den Charakter oder die Besonderheit der eigenen Stadt hervorzuheben. Ein Fan Fest-Experte weist gezielt auf die Inszenierung des Public Viewing im Zusammenhang mit den städtischen Wahrzeichen und dem typischen Flair hin (vgl. NN 2 , S. 4, Z. 139ff.). Bei der Inszenierung des Erlebnis-Setting ist Wert darauf gelegt worden, sich international als guter Gastgeber zu präsentieren. Es wurde der Versuch unternommen auf die ausländischen Mannschaften und ihre Fans einzugehen und einen kleinen Beitrag zur Völkerverständigung zu leisten. Dass dieser Anspruch bei den öffentlichkeitswirksamen offziellen Fan Festen weitaus höher lag als bei 
den z.T. recht kleinen nicht-kommerziellen Public Viewing-Veranstaltungen, liegt auf der Hand. $\mathrm{NN}_{2}$ (S. 3, Z. 124-129) äußert sich dazu folgendermaßen:

"Quantitativ gesehen wollten wir eine Millionen Besucher haben, qualitativ ein hochwertiges Fest der Völker. Wir wollten den Fans die Möglichkeit geben, eins der Kernelemente einer WM, nämlich Völkerverständigung, auszuleben. Deswegen haben wir den [Name eines Setting-Elements] errichtet. Alle 32 Nationen hatten hier die Möglichkeit sich darzustellen. Das waren im Prinzip auch die zwei weiteren Motive."

Wie in Kap. 10.4 theoretisch erläutert, kann das Thema Ausgangspunkt der Inszenierungspyramide sein. An diesem orientieren sich die weiteren Maßnahmen, wie bspw. die Szenerie. Generell lässt sich feststellen, dass der Professionalisierungsgrad auch bei den nicht-kommerziellen Public ViewingVeranstaltungen hoch ist.

„Das Konzept beinhaltet ein ganz normales Veranstaltungskonzept. Dass man sich im Vorfeld Gedanken gemacht hat um Dekoration, um Produkte und Preise, die man dort anbietet. Das ganze Setting wurde konzeptualisiert usw., also vom Würstchenstand: Was für Würstchen grillt man, wie teuer dürfen die sein? Es sollte familienfreundlich sein, d.h. niedrige Preise im gastronomischen Bereich“ ( $\mathrm{NN}_{12}$, S. 3, Z. 90-94).

Wie an diesem Zitat deutlich wird, hat man sich im Vorfeld vielerlei Gedanken gemacht, wie das Public Viewing bestmöglich gestaltet werden kann. Natürlich gilt es auch hier zu bedenken, dass auch die nicht-kommerziellen Veranstalter die Rückprojektion des Public Viewing auf ihre Veranstaltungslokalität oder auf ihre Stadt als Hintergedanken mitbringen. Der Unterschied zu den kommerziellen Veranstaltern oder auch nur zu den TV-Vorführungen mit einem stärkeren finanziellen Hinterhalt, äußert sich z.T. in den Ansprüchen der Experten. Während $\mathrm{NN}_{17}$ (S.3, Z. 123-127) äußert:

„Zu unserem Gesamtkonzept gehörte Public Viewing, dazu [Es wurden Angaben an dieser Stelle herausgenommen, da sie nur schwer anonymisierbar sind]..., das richtige Personal bei Experten und bei der Moderation, ein guter Veranstaltungsort, ein schönes Setting, ein schönes Bühnenbild mit Tribüne."

$\mathrm{NN}_{6}$ (S. 4, Z. 158ff.) weist darauf hin, dass es in dem Sinne nicht um ein „großes Unterhaltungsprogramm mit Stars und Sternchen und Musikgruppen“ ginge, sondern, dass sie „einfach versucht [hätten], thematisch etwas zu gestalten und die Spiele so zu umrahmen“. Aber auch Veranstaltungen mit einem geringeren Budget haben diesen Gedanken getragen und sich ideenreich gezeigt. So gibt $\mathrm{NN}_{11}$ (S. 4, Z. 162-166) an: 


\begin{abstract}
„Also, wir hatten ein passives Sicherheitskonzept. Teil davon war auch, dass wir jeden Tag Livemusik hatten mit Künstlern aus der Region. Die haben sich bereit erklärt, für wenig Geld aufzutreten. Die kamen aus ganz verschiedenen Musikrichtungen wie Boogie-Woogie, Jazz etc.. Das hat sich zu einem Kulturfestival entwickelt. Wir hatten Leute, die nur gekommen sind, um sich in den Biergarten zu setzen und Musik zu hören."
\end{abstract}

Im Rahmen der Interviews zeichneten sich einige Maßnahmen ab, die oftmals genutzt und als gut befunden worden sind. Besonders hervorgehoben wird die Untermalung der Veranstaltung mit affiner Musik ( $v g l . N_{3}$, S. 6, Z. 237; $\mathrm{NN}_{10}$, S. 4, Z.170; $\mathrm{NN}_{12}$ S. 5 , Z. 184 [u.a.]). Ob diese vom Band oder von einer Liveband kam oder ob die Band Fußballmusik oder, wie es z.B. von $\mathrm{NN}_{9}$ (S. 4 Z. 165) oder $\mathrm{NN}_{16}$ (S. 5, Z. 214) benannt wurde, Sambamusik spielt, scheint dabei nicht so wichtig zu sein. Als entscheidend wird nur die Nähe zum Fußball und seinem Publikum unterstrichen.

Einen weiteren wichtigen Inszenierungsbaustein stellt die Moderation dar. So hebt $\mathrm{NN}_{1}$ (S. 5, Z. 213f.) hervor: „Was sicherlich gut war, waren die Moderatoren. Wenn keine Gruppen gespielt hatten, hatten wir einen DJ da. Sie brauchen schon einen 'Zeremonienmeister'" (vgl. auch $\mathrm{NN}_{4}$, S. 4, Z. 170; $\mathrm{NN}_{12}$, S. 5, Z. 134). Darüber hinaus werden Expertentalks (vgl. $\mathrm{NN}_{2}$, S. 6, Z. 228), die Übertragung der Vorund Nachberichterstattung (vgl. $\mathrm{NN}_{15}$, S. 5, Z. 181f.) und Varieté-Künstler (vgl. $\mathrm{NN}_{16}$, S. 5, Z. 211) genannt. Als permanente Unterhaltungsangebote werden Tischfußball (vgl. NN 4 , S. 4, Z. 169), Torwandschießen, das Verlosen von Bällen und Ratespiele (vgl. $\mathrm{NN}_{7}$, S. 5, Z. 213f.), eine fußball-affine Souvenirmesse (vgl. $\mathrm{NN}_{2}$, S. 4, Z. 158f.), Human Table Soccer (vgl. $\mathrm{NN}_{18}$, S. 3, Z. 97) und Speedkick (S. 4, Z. 169) genannt. $\mathrm{NN}_{8}$ (S. 2, Z. 65f.) berichtet von einer Mini-WM, bei der regionale Hobbyteams ein kleines Turnier gespielt haben, und einem Onlinetippspiel. Des Weiteren wurden eine Kletterwand, ein Hochseilgarten, eine Go-Kart-Bahn (vgl. NNg, S. 4, Z. 149ff.) erwähnt. Eine Auswahl an gastronomischen Angeboten ist für die meisten Experten selbstverständlich gewesen. Zum Teil wurden recht umfangreiche Unterhaltungspakete geschnürt. $\mathrm{NN}_{5}$ (S. 3, Z. 121ff.) hebt hervor:

„Das waren Musikgruppen, Filmvorführungen mit Thementagen, z.B. emotionale Fußball-Filme, Comedy, mit allem, was auf Bühnen geht und spezielle Kindertage, Rentnertag und dem Fernsehgarten mit Publikum über 50."

Ebenso weist $\mathrm{NN}_{3}$ (S. 6, Z. 236f.) auf die Nutzung des Geländes für kleinere Feten und Motto-Partys hin. Eine gute Möglichkeit, die Besucher in das Public Viewing 
einzubinden und so die Entstehung von Erlebnissen im Sinne des BesucherInstruments (s. Kap. 10.4) zu fördern, bietet die Möglichkeit, einen Gast eine Spielszene moderieren zu lassen und dieses entsprechen zu honorieren (vgl. $\mathrm{NN}_{20}$, S. 4, Z. 163f.). Allerdings ist zu registrieren, dass die oft kostspieligen Musikgruppen nicht in dem Maße angenommen worden sind, wie es von den Spezialisten erwartet wurde.

„Wir hatten ein Eröffnungskonzert mit [Name der Band], sonst hatten wir ausländische Musikgruppen, die unterhalten sollten. Aber die Leute waren doch sehr auf Fußball fokussiert" ( $\mathrm{NN}_{1}$, S. 5, Z. 201f.).

Diese Erfahrung wird von Fan Fest-Experte $\mathrm{NN}_{3}$ (S. 6, Z. 237ff.) unterstützend erwähnt, indem er angibt, man habe aus der nicht unbedingten Notwendigkeit heraus bewusst auf größere Sachen verzichet.

Es schien mancherortens ein Problem zu sein, die zu erwartende Zielgruppe einzuschätzen und ein affines Unterhaltungsprogramm zusammenzustellen. Dies soll ein Textbeispiel aus dem Interview mit einem Experten für ein nichtkommerzielles Public Viewing verdeutlichen. Auf die Frage der Interviewerin nach der gezielten Gestaltung des Unterhaltungsprogramms auf eine bestimmte Zielgruppe, antwortete $\mathrm{NN}_{16}$ (S. 6, Z. 220-223) folgendermaßen:

„Wir haben schon versucht, unser [Name des Veranstaltungsortes] Publikum zu erreichen, was uns aber nicht so gelungen ist. Wir hatten schon die Fußballfans hier und nicht so das Publikum, was sonst so über das Jahr hier ist, die sich für kulturelle Veranstaltungen aller Art interessieren."

Generell kann gesagt werden, dass dies schlichtweg Erfahrungswerte sind, aus denen man Rückschlüsse für zukünftige Public Viewing-Veranstaltungen ziehen kann. So hebt $\mathrm{NN}_{20}$ (S. 5, Z. 194ff.) auf die Frage nach Verbesserungsmöglichkeiten für das nächste Public Viewing auch hervor:

„...[I]ch würde die Highlights in den Vordergrund stellen. Zum Beispiel das Proben von Fangesängen vor den Spielen. Anderes würde ich weglassen, z.B. ein Gewinnspiel, was an den Gästen vorbeigelaufen ist, weil das Interesse am Nachfolgespiel zu groß war."

Nicht zuletzt sei darauf zu achten, so ein Experte $\left(\mathrm{NN}_{2}\right.$, S. 4, Z. 147f.), dass die Zuschauer „ihr Fandasein ausspielen“ und sich „nicht als Werbeempfänger missbraucht" fühlen würden.

Zu bedenken ist allerdings, dass die Notwendigkeit der Förderung von Emotionen bzw. der Förderung der Stimmung für das Public Viewing kontrovers beschrieben wurde. Auf die diesbezügliche Frage der Interviewerin äußerten sich die 
Spezialisten sehr unterschiedlich. Während $\mathrm{NN}_{3}$ (S. 6, Z. 250-353) als Experte eines Fan Festes es pragmatisch umschreibt:

„Das ist ganz normal, man muss sich ja am Markt orientieren. So als wenn sie Autos verkaufen, es geht um das Geldverdienen. Sie brauchen Autos, dafür gibt es einen Markt. Dieser Markt ist begrenzt. Bei Veranstaltungen gibt es halt einen Markt von Leuten, die kommen raus, feiern und geben Geld aus. Aber dieser Markt ist begrenzt",

sehen vor allem die nicht-kommerziellen Anbieter eher soziale Aspekte: „Das ist schon sehr wichtig, damit die Leute mehr Spaß haben und es noch mehr in Erinnerung behalten“ ( $\mathrm{NN}_{10}$, S. 5, Z. 178f.). Die Mehrzahl der Experten ist davon überzeugt, dass den Besuchern an Unterhaltung gelegen ist. „Die Leute wollen animiert und unterhalten werden", gibt $\mathrm{NN}_{1}$ (S. 5, Z. 205) an, weiter führt er (S. 5, Z. 217-220) aus:

„...[S]ie müssen natürlich schon ein Programm auf der Bühne haben mit Moderation und notfalls Musik vom Band. Sie können nicht einfach sagen, da steht eine Lei nwand und da kommt man hin und wartet, bis es losgeht. Das nicht, da brauchen sie schon auch Animateure."

$\mathrm{NN}_{11}$ (S. 5, Z. 184f.) stellt zusammenfassend fest: „Also, das ist schon wichtig. Die Leute wollen unterhalten werden. Sie wollen Fußballgucken, aber auch unterhalten werden" (vgl. auch NN $N_{4}$, S. 5, Z. 179f.; $\mathrm{NN}_{6}$, S. 4, Z. 169; $\mathrm{NN}_{7}$, S. 5, Z. 213ff.). In diesem Zitat wird der hauptsächliche Punkt der Kontroverse deutlich. $\mathrm{NN}_{12}$ (S. 5, Z. 192ff.) formuliert ähnlich, er führt an, dass es „[b]eim Fußballspiel (...) weitestgehend so emotional [sei], dass man weitestgehend tatsächlich darauf [andere Formen der Unterhaltung-Anmerkung der Autorin] verzichten kann, bis auf die Hintergrundmusik,..... Dieser Zwiespalt zwischen der eigenen, als ausreichend empfundenen Emotionalität des Fußballs ansich und der in einem Nachsatz erfolgten Aussage, dass die musikalische Untermalung aber doch dazugehöre, wird oftmals deutlich. Wobei an dieser Stelle darauf hingewiesen werden muss, dass dies natürlich auch vom geplanten Charakter des Public Viewing abhängig ist. Wenn ein eher ruhiges Beisammensein erreicht werden soll, ist eine stadionähnliche Inszenierung eher weniger angebracht (vgl. z.B. NN $N_{8}$, S. 5, Z. 188f.). Es ist nicht sinnvoll, um jeden Preis eine Partystimmung hervorrufen zu wollen. Die Art, das Ausmaß und die Sinnhaftigkeit von geeigneten emotionsfördernden Maßnahmen sind als sehr veranstaltungsspezifisch anzusehen. Der Grundgedanke der Erlebnisarbeit und der Inszenierung als Instrument des Erlebnis-Setting geht z.B. aus einem Interviewausschnitt mit $\mathrm{NN}_{17}$ 
(S. 5, Z. 204-206) hervor: „Man muss da nicht gezwungen Stimmung erzeugen, aber einfach auch, um das, was da war, dann auch positiv zu verstärken." Ebenso unterstreicht $\mathrm{NN}_{16}$ (S. 6, Z. 227-235) auf die Frage nach der Relevanz der Förderung von Stimmung bzw. Emotionen für das Public Viewing:

\begin{abstract}
„Unabdingbar, das kann man ja, die Frage ist ja etwas gemein. Man kann Stimmung ja so oder so erzeugen. Wir haben es jetzt versucht mit einem hochwertigen Unterhaltungsprogramm, das recht teuer war, und es hat das Publikum nicht so gezogen, wie wir es erwartet haben. Es hat ein anderes Publikum gezogen. Dieses Publikum hätte aber ebenso gut den DJ als völlig ausreichend erachtet. Eine Public Viewing-Veranstaltung ohne einen solchen Rahmen zu machen, kann ich mir nicht vorstellen. Weil, wir wollen immer an die Emotionen appellieren, ganz klar, abgesehen vom Programm wollen wir auch die Zuschauer und dem Ganzen ein rundherum gelungenes Wohlfühlerlebnis bieten. Sie sollen in ein tolles Haus kommen und sich rundherum wohl fühlen und sich erstklassig unterhalten fühlen."
\end{abstract}

Eine Expertin ( $\mathrm{NN}_{13}$, S. 4, Z. 149f) zeigte sich dann auch überrascht: „Ich hätte gedacht, dass es wichtiger ist im Vorhinein, aber das war nachher eigentlich ganz unwichtig“, über die Entwicklung, die das von ihr verantwortete Public Viewing nahm.

Letztlich kann gesagt werden, dass besonders die Fan Feste bereits in großem Maße von den Möglichkeiten der Inszenierung zur Erzeugung einer positivanregenden Atmosphäre im Rahmen des Erlebnis-Setting Gebrauch gemacht haben. Dies ist mit Sicherheit u.a. auf das in großen Teilen wesentlich höhere finanzielle Budget z.B. im Vergleich mit den nicht-kommerziellen Veranstaltungen, zurückzuführen. Hier wurde bereits der Gedanke des Events (s. Kap. 10.5) verfolgt, die Public Viewing-Veranstaltungen insoweit unabhängig von dem eigentlichen Ereignis Fußball-WM zu machen, dass die Besucher auch an spielfreien Tagen gekommen sind. Diese Ergebnisse aus den qualitativen Experteninterviews unterstützen die Aussage der $\mathrm{H} 3 \mathrm{~b}$. 


\subsection{Zusammenfassung}

Zunächst konnte in der Diskussion der Entwicklungsfaktoren des Public Viewing (H1) bestätigt werden, dass Fernseh-Vorführungen in hohem Maße für ein besonderes Ereignis im (internationalen) Fußball prädestiniert sind. An dieser Stelle ist aber auch die erstaunliche Entwicklungsdynamik zu nennen, die es in Zukunft ermöglichen kann Public Viewing in stärkerem Maße neben der Übertragung von internationalen Fußballturnieren bspw. auch auf Spiele des UEFA-Pokals, der Champions League oder andere Sportarten auszuweiten (H1a). Die Medien nehmen in der Kommunikation bzgl. des Public Viewing einen geringeren Stellenwert ein als in $\mathrm{H} 2 \mathrm{a}$ vermutet. Die soziale Interaktion in Form der 'Mund-zu-Mund-Propaganda' konnte als bedeutenderer Faktor herausgefiltert werden.

Die Evaluierung der Publikumsbildung beim Public Viewing (H2) fokussierte die Verschränkung von individuellen und sozialen Beweggründen. Zuschauer besuchen die TV-Übertragungen, weil sie emotionale Gemeinschaftserlebnisse suchen, die nach der Erörterung der quantitativen und qualitativen Daten insbesondere auf öffentlichen Plätzen und ab einer gewissen Publikumsgröße am wahrscheinlichsten sind ( $\mathrm{H} 2 \mathrm{a})$. Die Emotionsentstehung und -regulierung des Publikums erfolgt über die verbale und non-verbale aktive Kommunikation über sich selbst und die Orientierung an äußeren Faktoren, wie anderen Besuchern oder den Spielern auf dem Feld. Bei Massenveranstaltungen wie dem Public Viewing wächst aufgrund der Verhaltensunsicherheit der Einfluss der sozialen Emotionsregeln (H2b). Ähnlich wie bei H1b ist der Einfluss der Medien und des Fernsehens auch für die Publikumsbildung als weniger relevant als angenommen einzuschätzen. Wichtiger erweisen sich Rückkopplungseffekte der Medien, mit der Möglichkeit live an einem wichtigen Großereignis teilzuhaben und sich Selbst darzustellen $(\mathrm{H} 2 \mathrm{c})$.

Die Steuerbarkeit von positiven Emotionen $(\mathrm{H} 3)$ lässt sich zunächst über die Anerkennung des Public Viewing als Freizeitangebot mit Erlebnispotential durch die Besucher bestätigen. Das Bedürfnis des Individuums an einem sportiven Unterhaltungsangebot, das durch stimmungsvolle Gestaltung die Möglichkeit des Emotionserlebens birgt, ist beim Public Viewing gegeben (H3a). Insbesondere die Fan Feste haben bereits vielfach von Inszenierungselementen im Rahmen des 
Erlebnis-Setting Gebrauch gemacht. Sie lassen bereits z.T. das eigentliche Ereignis hinter dem 'Event' Public Viewing verblassen (H3b).

Letztlich lässt sich feststellen, dass die anfangs aufgeworfenen Fragestellungen in der Diskussion der theoretischen Bausteine, der qualitativen Aussagen und quantitativen Daten zur Überprüfung der Hypothesen den Relevanzhorizont des Public Viewing auszuleuchten sowie erste Aussagewerte zu treffen vermochten. Lediglich der mediale Einfluss auf das Phänomen scheint eher in der mehrdimensionalen Verästelung mit anderen sozialen Prozessen, wie bspw. dem Bedürfnis der Selbstdarstellung zu liegen. 


\section{Schlussbemerkung und Ausblick}

In dieser Arbeit wurde das Ziel verfolgt, erste Erklärungsansätze für das Phänomen Public Viewing herauszuarbeiten. Der Forschungszugang erfolgte über interdisziplinäre theoretische Erkenntnisbausteine und über die methodologische Triangulation im empirischen Teil. Breit angelegte explorative Leitfadengespräche mit Public Viewing-Experten der Fußball-Weltmeisterschaft 2006 machten den Anfang. Die qualitativen Aussagen wurden durch eine quantitative Onlinebefragung der Besucher von Public Viewing-Veranstaltungen unterstützt und ergänzt. Diese konzentrierte sich auf ausgewählte Aspekte, die sich aus den Experteninterviews und den theoretischen Ansätzen herauskristallisierten. Auf diesem Weg ist eine erste explorative systematisch-analytische Annäherung an das Thema Public Viewing über einen multidimensionalen Blickwinkel erfolgt. Die vorliegende Arbeit kann aufgrund des geringen Vorwissens über die Erscheinung als Anfang eines wissenschaftlichen Diskurses gewertet werden. Während der Auseinandersetzung mit dem Forschungsgegenstand haben sich darüber hinaus eine Reihe interessanter Fragen, aber auch Ideen, Einsichten und Schlussfolgerungen ergeben, die weitestgehend bereits mit in die Arbeit eingeflossen sind. Der Erkenntnisgewinn liegt in den, dem Forschungsgegenstand adäquat erscheinenden mehrdimensionalen Verbindungen, vielschichtigen theoretischen Ergebnissen und Ableitungen sowie den triangulären empirischen Werten und in der Überprüfung der Hypothesen. Um die Komplexität des Forschungsgegenstandes überschaubar zu gestalten, wurde Wert darauf gelegt, am Ende jedes größeren Kapitels eine Zusammenfassung der jeweiligen Erkenntnisse zu verfassen und diese in den Gesamthorizont der Arbeit einzuordnen. Es kann folglich gesagt werden, dass der Forschungsstand zum Thema Public Viewing deutlich erweitert wurde. Diese Arbeit leistet einen wichtigen ersten Schritt für die Erkundung des Phänomens und eröffnet vielerlei Möglichkeiten für weitere Forschungsansätze.

Folgende Fragestellungen sind nach einer ersten Sichtung der Literatur formuliert worden:

1. Welche Faktoren führen zur Entwicklung des Public Viewing?

2. Warum besuchen Zuschauer Public Viewing-Veranstaltungen?

3. Ist das Entstehen von positiven Emotionen bei einer Public Viewing-Veran- 
staltung steuerbar?

Diese sind auf der Grundlage der qualitativen Expertengespräche und der theoretischen Bausteine zu Hypothesen verfeinert worden. Zur Erweiterung und Absicherung der Meinungsbilder wurden im Anschluss Public Viewing-Besucher befragt. Diese gesammelten Erkenntnisse bilden die Basis der Diskussion von $\mathrm{H} 1$, $\mathrm{H} 2$ und $\mathrm{H} 3$. In $\mathrm{H} 1$ werden zunächst die Entwicklungsfaktoren des Public Viewing diskutiert:

H1. Public Viewing ist durch zwei sich gegenseitig beeinflussende Entwicklungen entstanden. Soziale Prozesse (Wertewandel, Individualisierungs- und Pluralisierungstendenzen) in der postmodernen Gesellschaft mit den daraus entstehenden Bedürfnissen und Tendenzen sowie der technologische Fortschritt in der Übertragungstechnik, insbesondere der LED-Technik, sind Voraussetzungen für

a. die verstärkte Bedeutung des Sports, insbesondere internationaler Fußballturniere, und

b. im Zusammenspiel mit der Mediatisierung der Gesellschaft dafür, dass immer mehr Zuschauer wichtige Ereignisse des Sports im Fernsehen verfolgen.

Wie in den Erörterungen zur H1 gezeigt werden konnte, ist die Entwicklung des Public Viewing durch gesellschaftliche Modernisierungsprozesse wie den möglichen Wandel zu hedonistischen Werten und Individualisierungstendenzen gefördert worden. Das sich aus diesen Entwicklungen ergebende Bedürfnis des Individuums nach neuen sozialen Strukturen kann offenbar insbesondere durch das 'Dabeisein' bei internationalen Fußballturnieren befriedigt werden. Nach Meinung der Experten zeigt sich an dieser Stelle eine große Differenz zu anderen Sportarten, aber auch zum Fußball im Regelbetrieb. Die Möglichkeiten des Public Viewing bspw. für einen normalen Bundesligaspieltag werden durch die nicht in ausreichendem Maße vorhandene Nachfrage nach Tickets und der Heterogenität des Publikums in Frage gestellt (H1a). Ebenso gab die Mehrzahl der Besucher an, die nächste TV-Vorführung im Rahmen eines internationalen Fußballturniers, nämlich zur EM 2008 besuchen zu wollen, was die in Kap. 5.2.1 nachzulesenden Zuschauerzahlen bestätigen können. Der Einfluss der technologischen Entwicklungen ist zwiespältig zu betrachten. Einerseits haben die LEDVideowände, die die Übertragung auch bei starkem Tageslicht ermöglichen, zur 
Entscheidung für die Fan Feste beigetragen, andererseits werden sie generell aber als für das Public Viewing nicht unbedingt notwendig erachtet $(\mathrm{H} 2 \mathrm{a})$.

Die Publikumsbildung des Public Viewing bildet den Untersuchungsgegenstand der zweiten Hypothese:

H2: Die Motive der Zuschauer, eine Public Viewing-Veranstaltung zu besuchen, sind nur multikausal und interdisziplinär erklärbar. Individuelle und soziale Beweggründe greifen in diesem Zusammenhang ineinander. Der Fokus wird auf folgende Aspekte gelegt:

a. Zuschauer besuchen Public Viewing-Veranstaltungen, weil sie Emotionen in der Gemeinschaft erleben wollen. Internationale Sport-, vor allem Fußballveranstaltungen, bieten ideale Voraussetzungen für die Entstehung eines außergewöhnlichen emotionalen Erlebnisses.

b. Insbesondere der Nationalsport Fußball besitzt in der Gesellschaft repräsentative und gemeinschafsstiftende Funktionen, die identitätsbildend wirken und die verloren gehenden gesellschaftlichen Strukturen ersetzen bzw. diese neu ausrichten. Sie lassen sich vor allem in internationalen sportlichen Großereignissen bekräftigen, indem die einzelnen Zuschauer aktiv interagieren und zu einem Publikum zusammenwachsen.

c. Die Medien insbesondere das Fernsehen, besitzen eine Kommunikator- und Multiplikatorfunktion, indem sie kollektives emotionales Wissen thematisieren, festigen und aktualisieren. Aus einzelnen Mikro-Ereignissen (Public ViewingVeranstaltungen) disperser Publika entsteht eine imaginierte Gemeinschaft.

In der Diskussion der $\mathrm{H} 2$ konnte bestätigt werden, dass das Herausfiltern von allgemeingültigen Besuchsmotiven schwierig ist. Vielmehr ist es immer ein Motivbündel, welches das Individuum zum Besuch eines Public Viewing bewegt. Die Ambivalenz des Einzelnen zwischen Individualisierungstendenzen und der Suche nach Gemeinschaftserlebnissen im Rahmen großer, weltweiter Sportveranstaltungen stellt offenbar ein Paradoxon der Postmoderne dar. Das gemeinschaftliche Emotionserleben ist, so konnte herausgestellt werden, auf einem öffentlichen Platz und ab einer bestimmten Masse am intensivsten (H2a). Die Emotionsentstehung und -regulierung erfolgt über Kommunikation und Interaktion vor allem mit anderen Public Viewing-Besuchern. Bei einer Veranstaltung, die in dieser Massenhaftigkeit ein Novum darstellt, greift der 
Einfluss sozialer Emotionsregeln in verstärktem Maße und ermöglicht das Zusammenwachsen einzelner Zuschauer zu einem Publikum (H2b). Durch das Wissen, verbreitet über soziale Interaktion und Selbstdarstellung und weniger als zunächst angenommen durch die Medien, über das Vorhandensein vieler anderer Public Viewing-Veranstaltungen kann der Gedanke einer imaginierten Gemeinschaft enstehen (H2c).

Letztlich ist die Steuerbarkeit von positiven Emotionen beim Public Viewing vornehmlich anhand der Expertenaussagen eruiert worden:

H3: Die Wahrscheinlichkeit des Entstehens von positiven Emotionen bei einer Public Viewing-Veranstaltung kann positiv beeinflusst werden.

a. Die Orientierung an hedonistischen Werten führt dazu, dass der Einzelne seine Freizeitaktivitäten zur Erfüllung der eigenen Bedürfnisse auswählt. Dabei stellt das Freizeitangebot mit Erlebnispotential Public Viewing ein geeignetes Ziel dar.

b. Die Steigerung des Erlebnispotentials des Public Viewing kann in Form des Konzepts des Erlebnis-Setting gefördert werden. Die Inszenierung als Instrument des Erlebnis-Setting trägt durch gezielte Maßnahmen dazu bei, alle Sinne zu erreichen und eine positiv bewertete anziehend-erregende emotionale Atmosphäre zu erreichen.

Es konnte aufgezeigt werden, dass Public Viewing offenbar im Rahmen internationaler Fußballturniere ein Freizeitangebot mit Erlebnispotential darstellt. Vornehmlich für junge Besucher zwischen 19 und 29 Jahren, aber auch für Familien sind die TV-Übertragungen attraktiv (H3a). Die Mehrzahl der Public Viewing-Experten hat die Möglichkeit gesehen, die Entstehung von positiven Emotionen durch Inszenierung zu steuern, um das Publikum zu generieren und zu halten. Insbesondere die Fan Feste haben hier bereits auf ein z.T. ausgereiftes Repertoire des Erlebnis-Setting zurückgegriffen. Es hat sich jedoch gezeigt, dass sich keine konkreten Anleitungen und allgemeingültigen Erfolgsmaßnahmen herausstellen lassen, da sich die inneren und äußeren Umstände und die Art der Veranstaltungen zu multivariant darstellen. Lediglich die musikalische Untermalung sowie die Moderation des Public Viewing werden in der Tendenz hervorgehoben $(\mathrm{H} 3 b)$.

Natürlich konnte durch den selektiven Forschungszugang nur ein Teilbereich des 
komplexen Gegenstands Public Viewing näher erläutert werden. Dennoch sind die Erkenntnisse der vorliegenden Arbeit dazu in der Lage, wesentlich zum Verständnis des Phänomens beizutragen und praktische Handlungsanregungen aufzuzeigen. In diesem Sinne vermag diese Dissertation in dem anvisierten Bereich der drei Fragestellungen und den sich aus diesen ergebenden Hypothesen die sportwissenschaftliche Forschung um wichtige Aspekte zu erweitern. Wie einleitend beschrieben, ist das Hauptaugenmerk dieser Ausarbeitung auf die Frage nach den Ursachen bzw. mehreren möglichen Aspekten für die Entstehung der neuen Dynamik, die das gemeinsame Sehen vor allem im Laufe der Fußball-Weltmeisterschaft 2006 in Deutschland erlangt hat, gelegt worden. Darüber hinaus wurde nach den Beweggründen der Gäste, eine Public Viewing-Veranstaltung zu besuchen, gefragt und die Möglichkeit, positive Emotionen bewusst zu steuern, eruiert.

Die bislang nur in wenigen Arbeiten vorhandene sportwissenschaftliche Forschung zum Thema Public Viewing kann in einzelnen Bereichen um wichtige Ideen und Erklärungsansätze bereichert werden. Diese Abhandlung beschreibt bspw. erstmals dezidiert Aspekte des individuellen und gemeinschaftlichen Emotionserlebens sowie die Erlebnissuche von Public Viewing-Besuchern. Zudem schließt sie mit den zu diesem Thema erstmalig erfolgten empirischen Befragungen, sowohl aus der Perspektive der Experten von Public ViewingVeranstaltungen zur Fußball-WM 2006 als auch der Besucher von TVVorführungen nicht nur eine Forschungslücke. Vielmehr eröffnet sie geradezu einen neuen wissenschaftlichen Diskurs zu einem hoch aktuellen, gesellschaftlich weit reichenden Thema. Folglich fält es schwer, die Bedeutung dieser Dissertation für den Stand der Forschung nur in den einzelnen Teilbereichen zu gewichten. Die interdisziplinäre Verzweigung der Erklärungsansätze ermöglicht es, wichtige vorhandene Konstrukte und Ideen zu einem schlüssigen Gesamtbild zusammenzufügen. Beispielhaft sei hier die Verquickung medienwissenschaftlicher Konstrukte $\mathrm{zu}$ Medienereignissen mit den soziologischen Theorieelementen zum kollektiven Emotionserleben und den massenpsychologischen Ansätzen bzgl. geteilter gesellschaftlicher Normen zu nennen. Gleichzeitig impliziert Gesamtbild in diesem Zusammenhang ein schlüssiges Ganzes für die vorliegende Arbeit. Das bedeutet aber nicht, dass diese geradezu nach weiteren wissenschaftlichen Forschungsleistungen an vielen 
Ecken und Enden sucht. Es besteht also die unbedingte Notwendigkeit von weiteren sportwissenschaftlichen und interdisziplinären Arbeiten, um die gewonnenen Erkenntnisse zu festigen, zu vertiefen und zu erweitern.

Geht man in die Thematik hinein, wird an erster Stelle deutlich, dass sich die gesellschaftliche Stellung des Sports in der Postmoderne zu wandeln scheint. In den klassischen Sport, bestehend aus dem sportlichen Wettstreit mit Siegern und Verlierern, werden zunehmend andere kulturelle Elemente wie Musik, Mode oder Video integriert, was auf der einen Seite von außen beabsichtigt geschieht, auf der anderen Seite sich aber wie von selbst entwickelt hat. Hier bildet sich ein neues Feld der sport- und sozialwissenschaftlichen Forschung, ein Bereich, welcher auf dem Weg zur Erkundung (sport-)gesellschaftlicher Erscheinungen nach gemeinsamen integrativen Erkenntnissen sucht. Denn dieses ist notwendig, um die Begründungszusammenhänge zu hinterfragen, die Public Viewing im Jahr 2006 in Deutschland in diese neue Dimension hinsichtlich medialer Präsenz, organisatorischer Professionalität, technologischer Qualität und der Größe des Publikumszulaufs katapuliert haben. Auffallend erscheint die sozialstrukturelle Bedeutung die der Sport dem Individuum der Postmoderne liefern kann. Vor allem der Fußball erweist sich wieder einmal als Volkssport und scheint in Deutschland nichts von seiner Bedeutung seit dem Wunder von Bern eingebüßt zu haben. Ein Novum birgt diese Arbeit, weil sie sich erstmals nicht mit dem Geschehen im Stadion oder mit den Sehgewohnheiten in den heimischen Wohnzimmern auseinandersetzt. Sie greift in das und hier kann getrost wieder auf das Ereignis von 1954 Bezug genommen werden ein, was dazwischen liegt und doch so viel mehr Bedeutung hat als zunächst vermutet werden mag. Die damaligen Fernsehstuben und Versammlungen vor Schaufenstern wurden abgelöst durch Großbildleinwände und Videoscreens auf öffentlichen Plätzen, großen Hallen oder Kinos, aber immer wird durch Public Viewing das sportliche Großereignis in den öffentlichen Raum gebracht. Dieser öffentliche Raum vermag offenbar in einem Rückkopplungseffekt, etwa durch die Möglichkeit der Selbstdarstellung in Form von Autokorsos, die gesellschaftliche und mediale Präsenz und Relevanz des Ereignisses zu verstärken. Der Sport kehrt aus den isolierten Arenen und außerhalb gelegenen Multisportkomplexen zurück in das soziale Leben, in die Mitte der Gesellschaft. Eine neue Kultur des gemeinsamen Sporterlebens des 
Einzelnen zwischen dem gleichzeitig konträr angesiedelten, aber auch sich ergänzenden Bedürfnis nach Individualität und Gemeinschaft und geteiltem emotionalen Erleben bahnt sich an. Letztlich kann dies in der offensichtlichen Intensität und der erlebten sowie dokumentierten gesellschaftlichen Verbreitung nur durch die Neuordnung der Grenzen zwischen Raum und Zeit durch das Bildermedium Fernsehen geschehen. Es öffnet sich eine neue Perspektive auf sportliche Großveranstaltungen, die im Sinne der Medienereignisse immer mehr einen Charakter der Eigenständigkeit erlangen. Public Viewing wurde anlässlich der Fußball-WM 2006 erstmals offiziell von den verantwortlichen Akteuren geplant, aber die Strahlkraft der Fan Feste reichte hinaus auf die vielen privaten und öffentlichen TV-Vorführungen. So waren diese vielen Vorführungen mit den tausenden Besuchern nahezu unabhängig vom eigentlichen Ereignis; intermedial vernetzt durch die Bilder und das Wissen um die Fans vor Ort, die Zuschauer zu Hause und die dispersen Publika auf den einzeInen Public ViewingVeranstaltungen. An dieser Stelle vermag die vorliegende Dissertation eine konkrete Verbindung zwischen den vor allem in den Medienwissenschaften diskutierten Ansätzen und einem sportlichen Großereignis herzustellen. Es scheint unumgänglich, sich in zukünftigen Forschungsarbeiten mit dieser neuen Verknüpfung von Sport, Medium und Veranstaltung dezidiert auseinanderzusetzen.

Die vielfach diskutierten Beweggründe von Sportzuschauern, sowohl als Livepublikum als auch vor dem heimischen Fernseher, können -insbesondere hinsichtlich der Suche des postmodernen Individuums nach gemeinschaftlichen emotionalen Erlebnissen- vertieft werden. Durch die Verknüpfung von mikro- und makrosoziologischen Theorien mit sozial- und gruppen- bzw. massenpsychologischen Modellen sowie emotionspsychologischen Konstrukten ist im Speziellen ein anwendungsbezogener Blick hinter die gesellschaftliche Bedeutung von Emotionen am Beispiel des Sport- bzw. Public Viewing-Publikums gelungen. Gerade im Hinblick auf die durchschnittlich junge Zuschauerschaft bei sportlichen TV-Vorführungen scheint hier ein Bedürfnis zu liegen, was der näheren Betrachtung bedarf. Dies birgt sicherlich darüber hinaus interessante Aspekte über die Möglichkeiten der Inszenierungsinstrumente des Erlebnis-Setting hinsichtlich ihrer Anwendung beim Public Viewing. So drückt sich hier doch die Sehnsucht des postmodernen Menschen nach Teilhabe an etwas Besonderem, nach 
emotionalem Erleben und nach einer an hedonistischen Zielen orientierten Freizeitgestaltung aus. Es wurde versucht, anhand der Verbindung von sportwissenschaftlichen mit touristischen und ökonomischen Ideen einen Diskurs für die erlebnisorientierte Angebotsgestaltung zu eröffnen. Mit einem erweiterten Blickwinkel auf die Möglichkeiten des Public Viewing als -eigenständiges- Event konnte zunächst ein Kreis um die Möglichkeiten der Inszenierung von emotionalen Erlebnissen mit Raum für individuelle Erlebnisarbeit gezogen werden. Es ist aber unbedingt wünschenswert, auch im Hinblick auf bevorstehende Ereignisse, diese Ansätze zu vertiefen.

Letztlich birgt diese Arbeit auch die Erkenntnis, dass sich ein weites Untersuchungsfeld aufgetan hat, welches -nicht nur im Rahmen der Sportwissenschaften- weiterer Studien bedarf. Denkbar wären z.B. Analysen in anderen Ländern und von anderen Sportarten oder Veranstaltungen. ${ }^{127}$ Des Weiteren wäre es wünschenswert, wenn in weiteren Studien z.B. anhand von Beobachtungen im Feld die methodischen Herausforderungen angegangen würden. Eine weitere Forschungsmöglichkeit wäre es, eine Vergleichsgruppe zu der nicht-repräsentativen Internetpopulation mit den Besuchern einzelner Veranstaltungen vor Ort zu befragen oder durch die Nutzung eines Online-Panels eine weitergehende Annäherung an eine Zufallsstichprobe zu erreichen. Zum Abschluss lässt sich sagen, dass diese Arbeit von der Annnahme ausgehend, dass Public Viewing auch in Zukunft bei großen internationalen Sportveranstaltungen ein wichtiges Thema sein wird, einen grundlegenden Beitrag für die sportwissenschaftliche Forschung auf diesem Gebiet leisten konnte.

\footnotetext{
127 Als aktueller Ansatzpunkt ist z.B. das nahezu völlig ausgebliebene Public Viewing während der Olympischen Spiele in Peking 2008 zu nennen. In China hat offenbar die Warnung der Regierung vor terroristischen Attentaten auf Menschenansammlungen vor Videowänden genügt, um den Einzelnen vor dem Fernseher zu Hause zu halten. So blieben die Plätze vor den Leinwänden meist leer. Ebenso ist abzuwarten, wie es sich mit der Sicherheitsproblematik u.a. bei der FußballWeltmeisterschaft in Südafrika 2010 verhält. Hier wird sich wohl der u.a. von Pilz (2007, S. Gleichfalls muss die Zukunft zeigen, ob Public Viewing in Deutschland auch zur Frauen-FußballWeltmeisterschaft 2011 eine ähnliche Popularität erfahren wird. Denn die Stadien, in denen die Spiele stattfinden werden, sind im Verhältnis zur Kartennachfrage -vergleicht man die Faktoren mit den Rahmenbedingungen zur Weltmeisterschaft der Männer 2006- wesentlich größer. Das heißt, es können anteilsmäßig mehr Zuschauer direkt in die Stadien gehen, womit weniger Personen für Public Viewing in Betracht kämen. Es sei denn, Public Viewing etabliert sich bis dahin zu einer eigenständigen Veranstaltungsform, wie es in der Tendenz bereits in dieser Arbeit angeklungen ist.
} 


\section{Literatur und Quellen}

\section{Literaturverzeichnis}

Abercrombie, N. \& Longhurst, B. (1998). Audiences. A Sociological Theory of Performance and Imagination. London, Thousand Oaks, New Delhi: Sage Publications.

Alfes, H. (1995). Literatur und Gefühl. Emotionale Aspekte literarischen Schreibens und Lesens. Opladen: Westdeutscher Verlag.

Anderson, B. (1988). Die Erfindung der Nation. Zur Karriere eines erfolgreichen Konzepts. Frankfurt a.M., New York: Campus Verlag.

Arpagaus, R. (2008). Fragen im Zusammenhang mit den Uefa-Lizenzen für das Public Viewing bei der Fußball-Europameisterschaft 2008. In sic!, 2, Zürich: Schulthess, 87-100.

Atteslander, P. (2003). Methoden der empirischen Sozialforschung. (10. neu bearbeitete und erweiterte Aufl). Berlin, New York: Walter de Gruyter.

Bachleitner, R. (2004). „Erlebnis“ kritisch betrachtet. In H. Kagelmann, R. Bachleitner \& M. Rieder (Hrsg.). ErlebnisWelten. Zum Erlebnisboom in der Postmoderne (S. 16-20). München, Wien: Profil Verlag.

Barbalet, J. (2002). Introduction: why emotions are crucial. In J. Barbalet (Ed.). Emotions and Sociology (pp. 1-9). Malden, Oxford, Vicotria: Blackwell Publishing.

Bartsch, A. \& Hübner, S. (2004). Emotionale Kommunikation-ein integratives Modell. Dissertation, Universität Halle a.d.S.

Bausenwein, Ch. (1995). Geheimnis Fußball: Auf den Spuren eines Phänomens. Göttingen: Verlag Die Werkstatt.

Beck, U. (1986). Risikogesellschaft. Auf dem Weg in eine andere Moderne. Frankfurt a.M.: Suhrkamp Verlag.

Beck, U. (1997). Kinder der Freiheit: Wider das Lamento über den Werteverfall. In U. Beck (Hrsg.). Kinder der Freiheit (S. 9-33). (3. Aufl.). Frankfurt a.M.: Suhrkamp Verlag.

Becker, Ch. (2000). Freizeit und Tourismus in Deutschland-eine Einführung. In Ch. Becker \& H. Job (Hrsg.). Nationalatlas Bundesrepublik Deutschland. (Bd.10, Freizeit und Tourismus, S. 12-21.). Heidelberg, Berlin: Spektrum Verlag.

Beckmann, U. (1993). Bandenwerbung wirksam gestalten. Wirkungen von Techniken der Konkreten Poesie auf Handballzuschauer. (Beiträge und Quellen zu Sport und Gesellschaft, Bd. 6), Berlin: Verlagsgesellschaft Tischler GmbH. 
Behn, S., Kuhlmann, D., Pilz, G.A. \& Wölki, F. (2007). „Die Welt zu Gast bei Freunden." Evaluation des Fan- und Besucherbetreuungsprogramms zur FIFA WM 2006TM in Deutschland- Abschlußbericht für den Deutschen Fußball-Bund und die Daniel Nivel Stiftung. Hannover/Berlin.

Bendelow, G. \& Williams, S. (Eds.). Emotions in Social Life. Critical Themes and Contemporary Issues. London [u.a.]: Routledge.

Bernstein, A. \& Blain, N. (2003). Sport and Media: The Emergence of a Major Research Field. In A. Bernstein \& N. Blain (Eds.). Sport, Media, Culture. Global and Local Dimensions (pp. 1-29). London, Portland: Frank Cass.

Bertling, Ch. \& Eggers, E. (2004). Fußballer im Bild der Medien-Anmerkungen zur medialen Visualisierung. In T. Schierl (Hrsg.). Die Visualisierung des Sports in den Medien. (Sportkommunikation, Bd. 2, S. 187-213). Köln: Harlem Verlag.

Bette, K.-H. (1993). Sport und Individualisierung. In Spectrum der Sportwissenschaft, 1.34-55.

Bette, K.-H. \& Schimank, U. (1995). Doping im Hochleistungsport. Anpassung durch Abweichung. Frankfurt a.M: Suhrkamp.

Bette, K.-H. \& Schimank, U. (2000). Sportevents. Eine Verschränkung von „erster“ und „zweiter Moderne“. In W. Gebhardt, R. Hitzler \& F. Liebl (Hrsg.). Events. Soziologie des Außergewöhnlichen. (Erlebniswelten, Bd. 2, S. 307324). Opladen: Leske+Budrich.

Blain, N. (2003). Beyond 'Media Culture': Sport as Dispersed Symbolic Activity. In A. Bernstein \& N. Blain (Eds.). Sport, Media, Culture. Global and Local Dimensions (pp. 227-254). London, Portland: Frank Cass.

Bösch, F. \& Borutta, M. (Hrsg.) (2006). Die Massen bewegen. Medien und Emotionen in der Moderne. Frankfurt, New York: Campus Verlag.

Bösch, F. \& Borutta, M. (2006a). Medien und Emotionen in der Moderne. Historische Perspektiven. In F. Bösch \& M. Borutta (Hrsg.). Die Massen bewegen. Medien und Emotionen in der Moderne (S. 13-41). Frankfurt, New York: Campus Verlag.

Bösch, F. \& Borutta, M. (2006b). Vorwort. In F. Bösch \& M. Borutta (Hrsg.). Die Massen bewegen. Medien und Emotionen in der Moderne (S. 9f.). Frankfurt, New York: Campus Verlag.

Breitmeier, F. (2004). Ein Wunder, wie es im Drehbuch steht: Die WM 1954-ein deutscher Erinnerungsfilm. In W. Pyta (Hrsg.) Der lange Weg zur Bundesliga. Zum Siegeszug des Fußballs in Deutschland (S. 127-150). Münster: LIT Verlag.

Brint, S. (2001). Gemeinschaft Revisited: A Critique and Reconstruction of the Community Concept. Sociological Theory, 19(1). 1-23.

Brown, J. (2000). What is a Psychoanalytic Sociology of Emotion? Psychoanalytic Studies, 2 (1). 35-49.

Brown, J. \& Richards, B. (2000). Introduction to the Psychoanalytic Sociology of Emotion. Psychoanalytic Studies, 2 (1). 31-33. 
Brunner-Sperdin, A. (2006). Das Phänomen des „Flow“-Erlebens, Emotionen und Kundenzufriedenheit. In K. Weiermair \& A. Brunner-Sperdin (Hrsg.). Erlebnisinszenierung im Tourismus. Erfolgreich mit emotionalen Produkten und Dienstleistungen (S. 23-34). Berlin: Erich Schmidt Verlag.

Bryant, J., Zillmann, D. \& Raney, A. (2000). Violence and the Enjoyment of Media Sports. In L. Wenner (Ed.). MediaSport (pp. 252-265). New York, London: Routledge.

Buhr, E. (2005). "Ich liebe euch": Robbie Williams singt zu 8000 in Berlin und zu Millionen im Rest der Welt. Frankfurter Rundschau, 256, 11. Oktober 2005. 29.

Chi-dong, L. (2002). Millions unite in Street-side Cheering. In Korean Information Service (Ed.), Korean Experience with the World Cup (pp. 26-31). Seoul: Moojin.

Ciompi, L. (1997). Die emotionalen Grundlagen des Denkens. Entwurf einer fraktalen Affektlogik. Göttingen: Vandenhoeck \& Ruprecht.

Cordsen, O. \& Deilmann, C. (2005). Auf dem Platz, im Stadion und vor dem Bildschirm: Sport und Emotionen. In S. Schmidt (Hrsg.). Medien und Emotionen (Medien: Forschung und Wissenschaft, S. 308-349). Münster: LIT Verlag.

Couldry, N. (2005). The extended audience: scanning the horizon. In M. Gillespie (Ed.). Media Audiences (pp. 183-222). Berkshire, New York: Open University Press.

Couper, M. \& Coutts, E. (2004). Online-Befragung. Probleme und Chancen verschiedener Arten von Online-Erhebungen. In A. Diekmann (Hrsg.), Methoden der Sozialfoschung (Kölner Zeitschrift für Soziologie und Sozialpsychologie, S. 217-243). Wiesbaden: VS Verlag für Sozialwissenschaften.

Crossley, N. (1998). Emotion and communicative action. Habermas, linguistic philosophy and existentialism. In G. Bendelow \& S. Williams (Eds.). Emotions in Social Life. Critical Themes and Contemporary Issues (pp. 1638). London [u.a.]: Routledge.

Croteau, D. \& Hoynes, W. (2003). Media Society. Industries, Images and Audiences (3rd ed.). Thousand Oaks, London, New Delhi: Pine Forge Press.

Cybulska, M. (2007). Public Viewing. Das mediale Ereignis im öffentlichen Raum. Marburg: Tectum Verlag.

Csikszentmihalyi, M. (2003). Flow. Das Geheimnis des Glücks (11. Aufl.). Stuttgart: Klett Verlag.

Dayan, D. \& Katz, E. (1994). Media Events. The Live Broadcasting of History. Cambridge, London: Harvard University Press.

Dayan, D. \& Katz, E. (2001). Medienereignisse. In R. Adelmann, J. Hesse, J. Keilbach, M. Stauff \& M. Thiele (Hrsg.). Grundlagentexte zur Fernsehwissenschaft. Thoerie - Geschichte - Analyse (S. 413-453). Konstanz: UVK Verlagsgesellschaft mbH. 
Denzin, N. \& Lincoln, Y. (1994). Introduction: Entering the Field of Qualitative Research. In N. Denzin \& Y. Lincoln (Eds.). Handbook of Qualitative Research (pp. 1-17). London, Thousand Oaks, New Delhi: Sage Publications.

Diederichsen, D. (1999). „We were half a million strong..... Massenereignisse, Festivals und Paraden in Pop- und Gegenkulturen. In A. Klein \& F. Nullmeier. Masse-Macht-Emotionen: zu einer politischen Soziologie der Emotionen (S. 330-344). Opladen, Wiesbaden: Westdt. Verlag.

Digel, H. (1995). Sportentwicklung in Deutschland - Chancen und Risiken gesellschaftlicher Modernisierung. In J. Rode \& H. Philipp (Hrsg.). Sport in Schule, Verein und Betrieb (11. Sportwissenschaftlicher Hochschultag der dvs vom 22.-24.9.1993 in Potsdam. Schriften der Deutschen Vereinigung für Sportwissenschaft; 64, S. 13-42) Sankt Augustin: Academia-Verl..

Digel, H. \& Burk, V. (1999). Zur Entwicklung des Fernsehsports in Deutschland. Sportwissenschaft, 29 (1), 22-41.

Digel, H. \& Burk, V. (2001). Sport und Medien. Entwicklungstendenzen und Probleme einer lukrativen Beziehung. In G. Roters, W. Klinger \& M. Gerhards. Sport und Sportrezeption (Schriftenreihe Forum Medienrezeption, Bd. 5, S. 15-31) Baden-Baden: Nomos Verlagsgesellschaft.

Dittmar, N. (2004). Transkription. Ein Leitfaden mit Aufgaben für Studenten, Forscher und Laien (Qualitative Sozialforschung, Bd. 10., 2.Aufl.). Wiesbaden: VS Verlag für Sozialwissenschaften.

Doelfs, G. (2005). Studie: Berlin bevorzugtes Ziel für WM-Besucher. Berliner Morgenpost, 226, 19. August 2005, 14.

Döhle, M. \& Vowe, G. (2006). Der Sport auf der „Mediatisierungstreppe“? Ein Modell zur Analyse medienbedingter Veränderungen des Sports. In Merz: Medien+Erziehung. Zeitschrift für Medienpädagogik, 30 (6),18-28.

Döhle, M., Klimmt, Ch. \& Schramm, H. (2006). König Fußball: Rezeptionsmotive und Medienmenüs an einem Bundesliga-Spieltag. Merz: Medien+Erziehung. Zeitschrift für Medienpädagogik, 30 (6), 51-62.

Dreyer, A. (2002). Vermarktung von Destinationen mit Events. In Th. Bieger \& Ch. Laesser (Hrsg.). Jahrbuch 2001/2002 (S. 345-371). St. Gallen: Schweizerische Tourismuswirtschaft.

Eisenberg, Ch., Lanfranchi, P., Mason, T. und Wahl, A. (2004). FIFA 1904-2004: 100 Jahre Weltfussball. Göttingen: Verlag Die Werkstatt.

Engels, U. (2004). Studienabbruch als biographische Arbeit. Dissertation, GeorgAugust-Universität Göttingen: Göttingen.

Feldenkirchen, M. (2006). Die Macht des Leders. Der Ball ist rund, und die Welt guckt zu: Der Sport und das Geld und der große Moment. In Das Parlament, 19, 1.

Flam, H. (1999). Soziologie der Emotionen heute. In A. Klein \& F. Nullmeier (Hrsg.). Masse-Macht-Emotionen: zu einer politischen Soziologie der Emotionen (S. 179-199). Opladen, Wiesbaden: Westdt. Verlag. 
Flam, H. (2002). Soziologie der Emotionen: Eine Einführung. Konstanz: UVK Verlagsgesellschaft $\mathrm{mbH}$.

Flick, U. (2004). Triangulation. Eine Einführung. (Qualitative Sozialforschung, Bd. 12). Wiesbaden: VS Verlag für Sozialwissenschaften.

Flick, U. (2005). Triangulation in der qualitativen Forschung. In U. Flick, E. v. Kardorff \& I. Steinke (Hrsg.). Qualitative Sozialforschung. Ein Handbuch (S. 309-318). (4. Aufl.). Reinbek bei Hamburg: Rowohlt Taschenbuch Verlag.

Flick, U., v. Kardorff, E. \& Steinke, I. (2005). Was ist qualitative Sozialfo rschung? Einleitung und Überblick. In U. Flick, E. v. Kardorff \& I. Steinke (Hrsg.). Qualitative Sozialforschung. Ein Handbuch (S. 13-29). (4. Aufl.). Reinbek bei Hamburg: Rowohlt Taschenbuch Verlag.

Freyer, W. \& Groß, S. (Hrsg.) (2002). Tourismus und Sport-Events. Dresden: Forschungsinstitut für Tourismus (FIT).

Freyer, W. \& Groß, S. (2002a). Events im Sport und ihr Bezug zum Tourismus. In W. Freyer \& S. Groß (Hrsg.). Tourismus und Sport-Events (S. 1-6). Dresden: Forschungsinstitut für Tourismus (FIT).

Frick, B. (1997). Kollektivproblematik und externe Effekte im professionellen Team-Sport: "Spannungsgrad" und Zuschauerentwicklung im bezahlten Fußball. In H.-D. Horch, J. Heydel \& A. Sierau (Hrsg.). Professionalisierung im Sportmanagement: Beiträge des 1. Kölner Sportökonomie-Kongresses 1997. (Edition Sportökonomie und Sportmanagement, Bd.1, S. 144-160.). Aachen: Meyer \& Meyer.

Friedrichs, J. (1998). Die Individualisierungs-These. Opladen: Liske+Budrich.

Früh, W. (1991). Medienwirkungen: Das dynamisch-transaktionale Modell. (Theorie und empirische Forschung). Opladen: Westdeutscher Verlag.

Führer, K. \& Ross, C (2006). Mass Media, Culture and Society in TwentiethCentury Germany: An Introduction. In K. Führer \& C. Ross (Eds.). Media, Culture and Society in Twentieth-Century Germany (pp. 1-22). Houndmill, New York: Palgrace Macmillian.

Gabler, H. (1998). Zuschauen im Sport-Sportzuschauer. In B. Strauß (Hrsg.), Zuschauer (S. 113-138). Göttingen, Bern, Toronto [u.a.]: Hogrefe, Verl. f. Psychologie.

Gärtner, M. \& Pommerehne, W. (1978). Der Fußballzuschauer - ein homo oeconomicus?: Eine theoretische und empirische Analyse. Jahrbuch für Sozialwissenschaft, 29, 88-107.

Gebauer, G. (2002). Sport in der Gesellschaft des Spektakels. (Sport, Spiele, Kämpfe, Bd. 5). Sankt Augustin: Academia Verlag.

Gebhardt, W. (2000). Feste, Feiern und Events. Zur Soziologie des Außergewöhnlichen. In W. Gebhardt, R. Hitzler \& F. Liebl (Hrsg.). Events. Soziologie des Außergewöhnlichen. (Erlebniswelten, Bd. 2, S. 17-31). Opladen: Leske+Budrich.

Gebhardt, W., Hitzler, R. \& Liebl, F. (Hrsg.). (2000). Events. Soziologie des Außergewöhnlichen. (Erlebniswelten, Bd. 2). Opladen: Leske+Budrich. 
Gebhardt, W., Hitzler, R. \& Pfadenhauer, M. (2000). Einleitung. In W. Gebhardt, R. Hitzler \& F. Liebl (Hrsg.). Events. Soziologie des Außergewöhnlichen. (Erlebniswelten, Bd. 2, S. 9-16.). Opladen: Leske+Budrich.

Geese, S., Zeughardt, C. \& Gerhard, H. (2006). Die Fußball-Weltmeisterschaft 2006 im Fernsehen. Daten zur Rezeption und Bewertung. Media Perspektiven, 9, 454-464.

Geese, S. \& Gerhard, H. (2008). Die Fußball-Europameisterschaft 2008 im Fernsehen. Daten zur Rezeption und Bewertung. Media Perspektiven, 9, 442-449.

Gelis, M. (2005). König Fußball huldigen. Frankfurter Rundschau, 142, 21. Juni $2005,41$.

Gerhard, H. (2006). Die Fußball-WM als Fernsehevent. Analyse der Zuschauerakzeptanz bei Fußball-Weltmeisterschaften 1954 bis 2006. Media Perspektiven, 9, 465-475.

Gerhard, H. (2006a). Fußball im Fernsehen. Wie die Tiefe des Raumes die Höhe der Einschaltquoten bestimmt. In Ch. Holtz-Bacha (Hrsg.). FußballFernsehen-Politik (S. 44-70). Wiesbaden: VS Verlag für Sozialwissenschaften.

Gerhards, J. (1988). Soziologie der Emotionen: Fragestellungen, Systematik und Perspektiven. Weinheim, München: Juventa-Verlag.

Gläser, J. \& Laudel, G. (2004). Experteninterviews und qualitative Inhaltsanalyse als Instrumente rekonstruierender Untersuchungen. (1. Aufl.). Wiesbaden: VS Verlag für Sozialwissenschaften.

Gleich, U. (1998). Sport, Medien und Publikum-eine wenig erforschte Allianz. Media Perspektiven, 3, 144-148.

Gleich, U. (2000). Merkmale und Funktionen der Sportberichterstattung. Sport und Medien - ein Forschungsüberblick. Media Perspektiven,11. 511-516.

Gleich, U. (2001). Sportberichterstattung in den Medien: Merkmale und Funktionen. Ein zusammenfassender Forschungsüberblick. In G. Roters, W. Klinger \& M. Gerhards. Sport und Sportrezeption. (Schriftenreihe Forum Medienrezeption, Bd. 5, S. 167-182). Baden-Baden: Nomos Verlagsgesellschaft.

Godbey, G. (1993). Time, Work, and Leisure: Trends That Will Shape the Hospitality Industry. Journal of Hospitality \& Tourism Research, 17, 49-58.

Godbey, G. (2003). Leisure in Your Life. An Exploration (6th ed.). State College, PA: Venture Publishing, Inc..

Goes, N. \& Wiedenfels, M. (2006). Public Viewing: Lizenzen ohne Kraft. Horizont Sport Business. Monthly, 1, $6 \mathrm{f}$. .

Goffman, E. (1969). Wir alle spielen Theater. Die Selbstdarstellung im Alltag. München: R. Piper \& Co. Verlag.

Göring, A. (2006). Risikosport - Interdisziplinäre Annäherung, empirische Befunde und Anwendungsbezüge. Dissertation, Georg-August-Universität Göttingen: Göttingen. 
Goy, M. (2005). Ein neues Stadion für Hamburg. Die Welt, 281, 01. Dezember $2005,35$.

Grötsch, K. (2006). Aha-Ein Erlebnis! Über Erlebnisinszenierung und Emotionmanagement. In K. Weiermair \& A. Brunner-Sperdin (Hrsg.). Erlebnisinszenierung im Tourismus. Erfolgreich mit emotionalen Produkten und Dienstleistungen (S. 49-80). Berlin: Erich Schmidt Verlag.

Grünberg, R. (2005). Das Woodstock des Fußballs. Hamburger Abendblatt, 3, 05. Januar 2005, 23.

Güldenpfennig, S. (1996). Sport: Autonomie und Krise. (Soziologie der Texte und Kontexte des Sports). Sankt Augustin: Academia-Verlag.

Gundlach, A. (2007). Gelungene Geschichten - Grundzüge der Eventdramaturgie. In O. Nickel (Hrsg.). Eventmarketing. Grundlagen und Erfolgsbeispiele (S. 81-96). München: Verlag Franz Vahlen.

Günther, A. (2006). 20 Jahre Erlebnisgesellschaft-und mehr Fragen als Antworten. Zwischenbilanz oder Abgesang auf die Erlebniswelt-Diskussion. In P. Reuber \& P. Schnell (Hrsg.). Postmoderne Freizeitstile und Freizeiträume. Neue Angebote im Tourismus. (Schriften zu Tourismus und Freizeit, Bd. 5, S. 47-61). Berlin: Erich Schmidt Verlag.

Guttmann, A. (1986). Sports Spectators. New York: Columbia University Press.

Hackforth, J. (2001). Auf dem Weg in die Sportgesellschaft? In G. Roters, W. Klinger \& M. Gerhards. Sport und Sportrezeption. (Schriftenreihe Forum Medienrezeption, Bd. 5, S. 33-40). Baden-Baden: Nomos Verlagsgesellschaft.

Hafkemeyer, L. (2003). Die mediale Vemarktung des Sports: Strategien und Institutionen. Wiesbaden: Dt. Universitäts-Verlag.

Hagenah, J. (2004). Einfluss von Persönlichkeit auf das Erleben von Sport in den Medien. In H. Schramm (Hrsg.). Die Rezeption des Sports in den Medien. (Sportkommunikation, Bd. 3, S. 75-96). Köln: Halem Verlag.

Halbwachs, M (1985). Das Gedächtnis und seine sozialen Bedingungen. Frankfurt a.M.: Suhrkamp.

Hamburger Abendblatt (2005a). WM-2006 die größte Fußball-Party aller Zeiten. Hamburger Abendblatt, 81, 08. April 2005, 28.

Hamburger Abendblatt (2005b). Noch ein Jahr bis zum Anpfiff. Hamburger Abendblatt, 132, 09. Juni 2005, 28.

Hanauer, F. (2005). Hamburgs Pläne für die WM 2006. Welt am Sonntag, 47, 20. November 2005, 6.

Harney, K. \& Jütting, D. (2007). Massenhaftes Zuschauen, FIFA-WM und Projekt Klinsmann. Beobachtungen zur FIFA-Weltmeisterschaft 2006. In D. Jütting (Hrsg.). Die Welt ist wieder heimgekehrt. Studien zur Evaluation der FIFAWM 2006. (Edition Global-lokale Sportkultur, Bd. 23, S. 11-24). Münster, New York. München [u.a]: Waxmann Verlag. 
Haut, J. (2006). Excitement and Identification. Explanations of Sports Spectating from Figurational Perspective. SportZeiten. Sport in Geschichte, Kultur und Gesellschaft, 6 (1). 145-160.

Hediger, V. (2006). Gefühlte Distanz. Zur Modellierung von Emotion in der Filmund Medientheorie. In F. Bösch \& M. Borutta (Hrsg.). Die Massen bewegen. Medien und Emotionen in der Moderne (S. 42-62). Frankfurt, New York: Campus Verlag.

Heinemann, K. (1998). Einführung in die Methoden und Techniken empirischer Forschung im Sport (Sport und Sportunterricht, Bd. 15). Schorndorf: Hofmann Verlag.

Heinemann, K. (2007). Einführung in die Soziologie des Sports (Sport und Sportunterricht., Bd. 1., 5. überarbeitete und aktualisierte Aufl.). Schorndorf: Hofmann Verlag.

Henecka, H. (2000). Grundkurs Soziologie. UTB für Wissenschaft. Opladen: Leske Verlag+Budrich $\mathrm{GmbH}$.

Hermanns, H. (2005). Interviewen als Tätigkeit. In U. Flick, E. v. Kardorff \& I. Steinke (Hrsg.). Qualitative Sozialforschung. Ein Handbuch (S. 360-368). (4. Aufl.). Reinbek bei Hamburg: Rowohlt Taschenbuch Verlag.

Hickethier, K. \& Bleicher, J. (1998). Die Inszenierung der Information im Fernsehen. In H. Willems \& M. Jurga (Hrsg.). Inszenierungsgesellschaft. Ein einführendes Handbuch (S. 369-383). Opladen, Wiesbaden: Westdeutscher Verlag.

Hochschild, A. (1990). Das gekaufte Herz. Zur Kommerzialisierung der Gefühle. New York, Frankfurt a.M.: Campus Verlag.

Hochschild, A. (1998). The sociology of emotion as a way of seeing. In G. Bendelow \& S. Williams (Eds.). Emotions in Social Life. Critical Themes and Contemporary Issues (pp. 3-15). London [u.a.]: Routledge.

Hopf, Ch. (2005). Qualitative Interviews-ein Überblick. In U. Flick, E. v. Kardorff \& I. Steinke (Hrsg.). Qualitative Sozialforschung. Ein Handbuch (S. 349-360). (4. Aufl.). Reinbek bei Hamburg: Rowohlt Taschenbuch Verlag.

Horak, R. (1995). Vom Erlebnis zur Wahrnehmung: Der Wiener Fußball und seine Zuschauer 1945-1990. Wien: Verlag Turia+Kant.

Horky, Th. (2006). Das schnelle Ende der Ehe von RTL und Beachvolleyball-Zur Inszenierung von Sport im Fernsehen. Merz: Medien+Erziehung. Zeitschrift für Medienpädagogik, 30 (6), 29-40.

Horn, M. \& Zemann, Ch. (2002). Erfassung und Bewertung sozioökonomischer Wirkungen von Sportgroßveranstaltungen. In W. Freyer \& S. Groß (Hrsg.). Tourismus und Sport-Events (S. 131-152). Dresden: FIT.

Horne, J. \& Manzenreiter, W. (2004). Accouting for Mega-Events. Forecast and Actual Impacts of the 2002 Football World Cup Finals on the Host Countries of Japan/Korea. International Review for the Sociology of Sport, 39 (2). 187203. 
Horne, J. \& Manzenreiter, W. (2006). An introduchtion to the sociology of sports mega-events. In J. Horne \& W. Manzenreiter (Eds.). Sports Mega-Events. Social Scientific Analyses of a Global Phenomenon. (The Sociological Review, pp. 1-24). Malden, Oxford, Vicotria: Blackwell Publishing.

Horx, M. (2007). Anleitung zum Zukunftsoptimismus. Warum die Welt nicht schlechter wird. München, Zürich: Piper Verlag.

Hüttl, T. (2005). Die hohe Kunst des Fußballs. taz, die tageszeitung, 235. 30. August 2005, 23

Inden-Lohmar, T. (2007). Typische Probleme bei der Planung und Durchführung von Events. In O. Nickel (Hrsg.). Eventmarketing. Grundlagen und Erfolgsbeispiele (S. 3-36). München: Verlag Franz Vahlen.

Inglehart, R. (1977). The Silent Revolution. Changing Values and Political Styles Among Western Publics. Princeton, New Jersey: Princeton University Press.

Jütting, D. (Hrsg.) (2007). Die Welt ist wieder heimgekehrt. Studien zur Evaluation der FIFA-WM 2006. (Edition Global-lokale Sportkultur, Bd. 23). Münster, New York. München [u.a]: Waxmann Verlag.

Kagelmann, H., Bachleitner, R. \& Rieder, M. (Hrsg.) (2004). ErlebnisWelten. Zum Erlebnisboom in der Postmoderne. München, Wien: Profil Verlag.

Kaiser, T. (2005). Sponsoren fiebern der Fußball-WM entgegen. Welt am Sonntag, 159,12 . Juni 2005, 28

Kelle, U. \& Erzberger, Ch. (2005). Qualitative und quantitative Methoden: kein Gegensatz. In U. Flick, E. v. Kardorff \& I. Steinke (Hrsg.). Qualitative Sozialforschung. Ein Handbuch (S. 299-309). (4. Aufl.). Reinbek bei Hamburg: Rowohlt Taschenbuch Verlag.

Keilbach, J. \& Stauff, M. (2006). Technical Innovation, Social Participation, Societal Self-Reflection: Televised Sport in (West)German Society. In K. Führer \& C. Ross (Eds.). Media, Culture and Society in Twentieth-Century Germany (pp. 165-180). Houndmill, New York: Palgrace Macmillian.

Kellermann, G. (2007). Deutschland als Ausrichter der Fußball-WM 2006-kritische Rückblicke. In D. Jütting (Hrsg.). Die Welt ist wieder heimgekehrt. Studien zur Evaluation der FIFA-WM 2006. (Edition Global-lokale Sportkultur, Bd. 23, S. 147-172). Münster, New York. München [u.a]: Waxmann Verlag.

Kemper, Th. (1978). A social interactional theory of emotions. New York, Chichester, Brisbane [u.a.]: Wiley \& Sons.

Kemper, Th. (2002). Predicting emotions in groups: some lessons from Sepember 11. In J. Barbalet (Ed.). Emotions and Sociology (pp. 53-68). Malden, Oxford, Vicotria: Blackwell Publishing.

Kemper, Th. (2007). Power and Status and the Power-Status Theory of Emotions. In J. Stets \& J. Turner, (Eds.). Handbook of the Sociology of Emotions (pp. 87-113). New York: Springer Science+Business Media.

Keupp, H. (1995). Solidarisch und doch frei. Für eine kommunitäre Individualität. Psychologie heute, 7, 50-55. 
Klages, H. (1984). Wertorientierung im Wandel. Rückblick, Gegenwartsanalyse, Prognosen. Frankfurt, New York: Campus Verlag.

Klein, A. \& Nullmeier, F. (1999). Masse-Macht-Emotionen: zu einer politischen Soziologie der Emotionen. Opladen, Wiesbaden: Westdt. Verlag.

Klein, A., Nullmeier, F. \& v. Wersch, O. (1999). Einleitung. In A. Klein \& F. Nullmeier. Masse-Macht-Emotionen: zu einer politischen Soziologie der Emotionen (S. 9-26). Opladen, Wiesbaden: Westdt. Verlag.

Knoblauch, H. (2000). Das strategische Ritual der kollektiven Einsamkeit. Zur Begrifflichkeit und Theorie des Events. In W. Gebhardt, R. Hitzler \& F. Liebl (Hrsg.). Events. Soziologie des Außergewöhnlichen. (Erlebniswelten, Bd. 2, S. 33-50). Opladen: Leske+Budrich.

Köck, C. (2005). Die Konstruktion der Erlebnisgesellschaft. In K. Wöhler (Hrsg.). Erlebniswelten. Herstellung und Nutzung touristischer Welten. (Beiträge zu Wissenschaft und Praxis, Bd. 5, S. 3-16). Münster: LIT Verlag.

König, T. (2002). Fankultur: Eine soziologische Studie am Beispiel des Fußballfans. (Studien zur Sportsoziologie, 11). Münster: LIT Verlag.

Kowal, S. \& O'Conell, D. (2005). Zur Transkription von Gesprächen. In U. Flick, E. v. Kardorff \& I. Steinke (Hrsg.). Qualitative Sozialforschung. Ein Handbuch (S. 437-447). (4. Aufl.). Reinbek bei Hamburg: Rowohlt Taschenbuch Verlag.

Kruck, P. \& Stuke, F. (2003). WM-Monitor 2002. In Th. Horky (Hrsg.). Die Fußballweltmeisterschaft als Kommunikationsthema. (Sport \& Kommunikation, Bd. 1, S. 35-54). Norderstedt: Books on Demand GmbH.

Krüger, A. (1990). The Ritual in Modern Sport: A Sociobiological Approach. In A. Krüger \& J. M. Carter (Eds.). Ritual and Record. Ritual and Records: Sports Record and Quantification in Pre-Modern Societies (pp. 135-152). Westport, Conn. [u.a.]: Greenwood.

Krüger, A. (1993). Cui Bono? Die Rolle des Sports in den Massenmedien. In A. Krüger \& S. Scharenberg (Hrsg.). Wie die Medien den Sport aufbereiten. Ausgewählte Aspekte der Sportpublizistik. (Beiträge und Quellen zu Sport und Gesellschaft, Bd. 5, S. 24-63). Berlin: Verlagsgesellschaft Tischler $\mathrm{GmbH}$.

Krüger, A. (1999). Geschichte des modernen Massensports. In Museum für Völkerkunde (Hrsg.). Das Gemeinsame Haus Europa. Handbuch zur europäischen Kulturgeschichte (S. 1222-1228). München: Deutscher Taschenbuch Verlag.

Kurscheidt, M. (2002). Tourismuswirtschaft und Sport-Mega-Events: das Beispiel der Fußball-WM 2006. In W. Freyer \& S. Groß (Hrsg.). Tourismus und Sport-Events (S. 35-70). Dresden: FIT.

Le Bon, G. (1982). Psychologie der Massen (Kröners Taschenausgabe, Bd. 99, 15. Aufl.). Stuttgart: Kröner Verlag. (Original veröffentlicht 1911).

Levine, E. (2008). Distinguishing television: the changing meanings of television liveness. Media, Culture \& Society, 30 (3). London [u.a.]: SAGE Publications. 393-409. 
Livingstone, S. (2005). Media audiences, interpreters and users. In Gillespie, M. (Ed.). Media Audiences (pp. 10-50). Berkshire, New York: Open University Press.

Loosen, W. (2001). „Das wird alles von den Medien hochsterilisiert“. Themenkarrieren und Konjunkturkurven der Sportberichterstattung. In G. Roters, W. Klinger \& M. Gerhards. Sport und Sportrezeption. (Schriftenreihe Forum Medienrezeption, Bd. 5, S. 133-147). Baden-Baden: Nomos Verlagsgesellschaft.

Loosen, W. (2004). Sport als Berichtungsgegenstand der Medien. In H. Schramm (Hrsg.). Die Rezeption des Sports in den Medien (Sportkommunikation, Bd. 3, S. 10-27). Köln: Halem Verlag.

Lucerna, C. (1997). Vermarktung von Sportereignissen: eine sozialpsychologische Perspektive. Wiesbaden: Dt. Univ.-Verlag.

Lyon, M. (1998). The limitations of cultural constructionism in the study of emotions. In G. Bendelow \& S. Williams (Eds.). Emotions in Social Life. Critical Themes and Contemporary Issues (pp. 39-59). London [u.a.]: Routledge.

Maennig, W. (2007). Fußball-WM 2006 - eine ökonomische Nachschau. In Olympisches Feuer. Zeitschrift des Deutschen Olympischen Sportbundes und der Deutschen Olympischen Gesellschaft, 3, 34-37.

Maier, U, (1999). Sinn und Gefühl in der Moderne. Zu Robert Musils Gefühlstheorie und einer Soziologie der Emotionen. Aachen: Shaker Verlag.

Manzenreiter, W. \& Horne, J. (2007). Playing the Post-Fordist Game in/to the Far East: The Footballisation of China, Japan and South Korea. Soccer \& Society, 8 (4), 561-577.

Marsh, P. \& Morris, D. (1989). Die Horde Mensch. Individuum und Gruppenverhalten. München: Heyne Verlag.

Mayring, Ph. (2002). Einführung in die qualitative Sozialforschung: Eine Einleitung zu qualitativem Denken (5. überarbeitete und neu ausgestattete Aufl.). Weinheim, Basel: Beltz.

McCarthy, A. (2001). Ambient Television. Visual Culture and Public Space. Durham, London: Duke University Press.

McLuhan, M. (2001). Das Medium ist die Botschaft=The medium ist the message. Dresden: Philo Fine Arts Verlag der Kunst.

Mehrabian, A. \& Russell, J. (1974). An Approach to Environmental Psychology. Cambridge, London: The MIT Press.

Meyer, W.-U., Schützwohl, A. \& Reisenzein, R. (1993). Einführung in die Emotionspsychologie. (Bd. 1). Bern, Göttingen, Toronto [u.a.]: Verlag Huber.

Meyer, W.-U., Schützwohl, A. \& Reisenzein, R. (1997). Einführung in die Emotionspsychologie. (Bd. 2). Bern, Göttingen, Toronto [u.a.]: Verlag Huber. 
Meyer-Odewald, J. \& Grünberg, R. (2005). An 31 Tagen: WM-Party auf St. Pauli. Hamburger Abendblatt, 279, 29. November 2005, 11.

Messing, M. \& Lames, M. (1996). Empirische Untersuchungen zur Sozialfigur des Sportzuschauers (Mainzer Studien zur Sportwissenschaft, Bd. 17). Niedernhausen: Schors-Verlag.

Müller, H. \& Scheurer, R. (2004). Tourismus-Destination als Erlebniswelt. Ein Leitfaden zur Angebots-Inszenierung. FIF Universität Bern.

Mummendey, H. (1995). Psychologie der Selbstdarstellung. (2. überarbeitete und erweiterte Aufl.). Göttingen, Bern, Toronto [u.a.]: Hogrefe, Verl. f. Psychologie.

Murray, B. (1996). The world's game. A history of soccer. Urbana: University of Illionois Press.

Muth, C. (1999). Auf der Suche nach latenten Sinnstrukturen in der Persönlichkeit Lady Dianas und ihr Einfluß auf die Entstehung einer modernen Massengemeinschaft. Gleichzeitig der Versuch einer Anwendung des Enneagramms. In S. Berghahn \& S. Koch-Baumgarten (Hrsg.). Mythos Diana-von der Princess of Wales zur Queen of Hearts (S. 133-148). Gießen: Psychosozial-Verlag.

Neal, D. (1993). A Further Examination of Anonymity, Contagion, and Deindividuation in Crowd and Collective Behavior. Sociological Focus, 26 (2). 93-107.

Neue Zürcher Zeitung (2001). Die andere Sicht. Neue Zürcher Zeitung, 129, 07. Juni 2001, 57.

Nickel, O. (2007) (Hrsg.). Eventmarketing. Grundlagen und Erfolgsbeispiele. München: Verlag Franz Vahlen.

Ohmann, S., Jones, I. \& Wilkes, K. (2006). The Perceived Social Impact of the 2006 Football World Cup on Munich Residents. Journal of Sport \& Tourism, $11(2), 129-152$.

Olsson, T. \& Viscovi, D. (2006). Who Whatches What and why?-The Media Sport Audience. In Merz: Medien+Erziehung. Zeitschrift für Medienpädagogik, 30 (6), 41-50.

Opaschowski, H. (1996). Die Zukunft des Sports. Zwischen Inszenierung und Vermarktung. Hamburg: BAT-Freizeit-Forschungsinstitut der BritishAmerican-Tobacco.

Opaschowski, H. (2000). Kathedralen des 21. Jahrhunderts. Erlebniswelten im Zeitalter der Eventkultur. (1. Aufl.). Hamburg: Germa Press.

Opaschowski, H. (2000a). Zukunftstrends im nächsten Jahrzehnt: Folgen und Folgerungen. In G. Trosien \& M. Dinkel (Hrsg.). Ökonomische Dimensionen von Sport-Events: Faktoren-Folgerungen-Fallbeispiele (S. 155-175). Butzbach-Griedel: AFRA-Verlag.

Opaschowski, H. (2001). Show, Sponsoren und Spektakel - Breitensport und Zuschauersport. In G. Roters, W. Klinger \& M. Gerhards. Sport und Sportrezeption. (Schriftenreihe Forum Medienrezeption, Bd. 5, S. 77-96). Baden-Baden: Nomos Verlagsgesellschaft. 
Opaschowski, H. (2006). Deutschland 2020. Wie wir morgen leben - Prognose der Wissenschaft. Wiesbaden: VS Verlag für Sozialwissenschaften.

Opaschowski, H. (2008). Deutschland 2030. Wie wir in Zukunft leben. Gütersloh: Gütersloher Verlagshaus.

Oswald, R. (2006). Emotionale Volksgemeinschaften. Das Wunder von BernF 1954 als Rundfunkereignis in Ungarn und Deutschland. In F. Bösch \& M. Borutta (Hrsg.). Die Massen bewegen. Medien und Emotionen in der Moderne (S. 369-386). Frankfurt, New York: Campus Verlag.

Paperlein, J. (2005). Städte beklagen Unsicherheit. Die WM und ihre Vermarktung (Teil 12). Horizont Sport Business, 50, 09. Dezember 2005, 28.

Peterson, G. (2007). Cultural Theory and Emotions. In J. Stets \& J. Turner, (Eds.). Handbook of the Sociology of Emotions (pp. 114-134). New York: Springer Science+Business Media.

Pfaff, S. (2002). Freizeitdienstleistung „Sport“-Besuchervermarktung von Sportgroßveranstaltungen. In W. Freyer \& S. Groß (Hrsg.) Tourismus und Sport-Events (S. 153-188). Dresden: Forschungsinstitut für Tourismus (FIT).

Pfaff, S. (2002a). Erlebnismarketing für die Besucher von Sportveranstaltungen. Erlebnisstrategien und -instrumente am Beispiel der Fußballbundesliga. Dissertation, Georg-August-Universität Göttingen.

Pfaff, S. (2007). Trend zum Event? Chancen und Risiken von Eventisierungstendenzen im universitären Wettkampfsport. In I. Behrens \& A. Göring. Abschied von Humboldt? Reformprozesse an deutschen Hochschulen (Hochschulsport: Bildung und Wissenschaft, S. 57-66). Universitätsverlag Göttingen.

Pilz, G. (2002). Fußball ist unser Leben!? Leerformel oder gesellschaftspolitische Herausforderung. In Württembergischer Fußballverband e.V. (Hrsg.). Der Fußball...ein Beitrag zu einer Gesellschaftskultur der Zukunft (S. 59-77). Sindelfingen: Röhm GmbH Druckerei und Verlag.

Pilz, G. (2007). Evaluation und wissenschaftliche Begleitung des Fan- und Besucherbetreuungskonzeptes der FIFA WM 2006 ${ }^{\mathrm{TM}}$. In D. Jütting (Hrsg.). Die Welt ist vieder heimgekehrt. Studien zur Evaluation der FIFA-WM 2006. (Edition Global-lokale Sportkultur, Bd. 23, S. 73-98). Münster, New York. München [u.a]: Waxmann Verlag.

Pine, J. \& Gilmore, J. (1999). The Experience Economy: Work Is a Theatre and Every Business a Stage. Boston: Harvard Business School Press.

Pötschke, M. \& Simonson, J. (2001). Soziologie und Marktforschung: Ausbildung am Arbeitsmarkt vorbei? Planung \& Analyse, 2001 (6). 32-38.

Pyta, W. (Hrsg.) (2004). Der lange Weg zur Bundesliga. Zum Siegeszug des Fußballs in Deutschland. Münster: LIT Verlag.

Pyta, W. (2004a). Einleitung: Der Beitrag des Fußballsports zur kulturellen Identitätsstiftung in Deutschland. In W. Pyta (Hrsg.) Der lange Weg zur Bundesliga. Zum Siegeszug des Fußballs in Deutschland (S. 1-30). Münster: LIT Verlag. 
Pritchard, M. \& Funk, D. (2006). Symbiosis \& Substitution in Spectactor Sport. Journal of Sportmanagement, 20, 299-321.

Rahmann, B., Weber, W., Groening, Y., Kurscheidt, M., Napp, H.-G- und Pauli, M. (Hrsg.) (1998). Sozio-ökonomische Analyse der Fußball-Weltmeisterschaft 2006 in Deutschland: Gesellschaftliche Wirkungen, Kosten-Nutzen-Analyse und Finanzierungsmodelle einer Sportgroßveranstaltung (Wissenschaftliche Berichte und Materialien des Bundes-instituts für Sportwissenschaft, Bd. 4). Köln: Sport und Buch Strauss.

Raney, A. (2004). Motives for Using Sport in the Media: Motivational Aspects of Sport Reception Processes. In H. Schramm. (Hrsg.). Die Rezeption des Sports in den Medien (Sportkommunikation, Bd. 3, S. 49-74). Köln: Harlem Verlag.

Redhead, S. (2007). Those Absent From the Stadium are Always Right: Accelerated Culture, Sport Media, and Theory at the Speed of Light. Journal of Sport \& Social Issues, 3 (31). 226-241.

Reicher, S. (2001). The Psychology of Crowd Dynamics. In M. Hogg \& R. Tindale (Eds.) Blackwell Handbock of Social Psychology: Group Processes (pp. 182-208). Malden, Oxford, Vicotria: Blackwell Publishing.

Reng, R. (1997). Die Welt steht still für die Herz-Dame. Süddeutsche Zeitung, 256, 08. September 1997. 3.

Reuter, H. (2008). Abgespecktes Sommermärchen. Werben \& Verkaufen (W \& V), 23, $26 f .$.

Riedl, L. (2006). Spitzensport und Publikum. Überlegungen zu einer Theorie der Publikumsbildung. Schorndorf: Hoffmann-Verlag.

Riedl, L. (2008). „Und dann jubelte das ganze Stadion!“. Zur Entstehung und Steuerung kollektiver Emotionen im Spitzensport. Sport und Gesellschaft Sport and Society 5 (3), 221-250.

Rittner, V. \& Breuer, Ch. (2000). Soziale Bedeutung und Gemeinwohlorientierung des Sports. (Wissenschaftliche Berichte und Materialien des Bundesinsituts für Sportwissenschaft, Bd. 13), Köln: Sport und Buch Strauss.

Ritzer, G. (1997). Postmodern Social Theory. New York: ReGrowth.

Rivenburgh, N. (2003). The Olympic Games: Twenty-First Centura Challenges as a Global Media Event. In A. Bernstein \& N. Blain (Eds.). Sport, Media, Culture. Global and Local Dimensions (pp. 31-50). London, Portland: Frank Cass.

Röger, K. (2006). Die Devise heißt: Dabei sein ist alles. „Public Viewing“ ist angesagt: Um die Spiele zu sehen, gehen die Fans in die Szenebar. In Das Parlament 19 (56). 11.

Roche, M. (2000). Mega-events and Modernity. Olympics and expos in the grouth of global culture. London, New York: Routledge.

Romeiß-Stracke, F. (2006). Ist optimale Inszenierung möglich? In K. Weiermair \& A. Brunner-Sperdin (Hrsg.). Erlebnisinszenierung im Tourismus. Erfolgreich mit emotionalen Produkten und Dienstleistungen (S. 35-46). Berlin: Erich Schmidt Verlag. 
Roost, F. \& Schöning, B. (2006). Public Screens und Public Screening. Zur Kommerzialisierung der öffentlichen Kontrolle. In Neue Gesellschaft für Bildende Kunst (Hrsg.). HEIMspiel 2006. Standort, Sport, Spektakel. NG BK Berlin, 36-39.

Roth, R. (1999). Bewegung statt Masse. Der Massediskurs aus Sicht der Bewegungsforschung. In A. Klein \& F. Nullmeier. Masse-Macht-Emotionen: zu einer politischen Soziologie der Emotionen (S. 241-259). Opladen, Wiesbaden: Westdt. Verlag.

Rühle, A. (2003). Sportprofile deutscher Fernsehsender 2002. Das Sportang ebot im deutschen Free-TV. Media Perspektiven, 5, 216-230.

Sawyer, R. (2005). Social Emergence. Societies as complex Systems. Cambridge: University Press.

Schauerte, T. \& Schwier, J. (2004). Die Telegenisierung von Sportereignissen Anpassung von Sportarten und ihrem Regelwerk an mediale Bedingungen. In T. Schierl (Hrsg.) Die Visualisierung des Sports in den Medien. (Sportkommunikation, Bd. 2, S. 164-186). Köln: Harlem.

Scherer, K. (2001). Emotion. In W. Stroebe, K. Jonas \& M. Hewstone (Hrsg.) (2001). Sozialpsychologie. Eine Einführung (S. 165-214). (4. überarbeitete und erweiterte Aufl.). Berlin, Heidelberg, New York: Springer-Verlag.

Scherer, H. (2004). Die Darstellung von Emotionen in der Sportberichterstattung. In T. Schierl (Hrsg.). Die Visualisierung des Sports in den Medien. (Sportkommunikation, Bd. 2, S. 214-236). Köln: Harlem.

Scheurer, R. (2003). Erlebnis-Setting. Touristische Angebotsgestaltung in der Erlebnisökonomie. (Berner Studien zu Freizeit und Tourismus, Heft 43). Hünibach: Jost Druck AG.

Schiffer, J. (2004). Fußball als Kulturgut: geschichtliche, soziologische, ökonomische, rechtliche, politische und philosophische Aspekte. Eine kommentierte Bibliografie (Teil 1), Köln: Sport und Buch Strauß.

Schimmel, K., Harrington, C. \& Bielby, D. (2007). Keep Your Fans to Yourself: The Disjuncture between Sport Studies' and Pop Culture Studies' Perspectives on Fandom. Sport in Society, 10 (4), 580-600.

Schlicht, W. \& Strauß, B. (2003). Sozialpsychologie des Sports. Eine Einführung. (Sozialpsychologie des Sports, Bd. 2.). Göttingen, Bern, Toronto [u.a.]: Hogrefe, Verl. f. Psychologie.

Schmidt, W. (2002). Das Mannschaftsspiel Fußball im Wandel der Zeit: Chancen und Risiken der individuellen und sozialen Entwicklung. In Württembergischer Fußballverband e.V. (Hrsg.). Der Fußball...ein Beitrag zu einer Gesellschaftskultur der Zukunft (S. 14-33). Sindelfingen: Röhm $\mathrm{GmbH}$ Druckerei und Verlag.

Schmidt, S. (Hrsg.) (2005). Medien und Emotionen: Zum Management von Bezugnahmen. In S. Schmidt (Hrsg.). Medien und Emotionen. (Medien: Forschung und Wissenschaft, S. 11-39). Münster: LIT Verlag.

Schmidt, Ch. (2005a). Analyse von Leitfadeninterviews. In U. Flick, E. v. Kardorff \& I. Steinke (Hrsg.). Qualitative Sozialforschung. Ein Handbuch (S. 447456). (4. Aufl.). Reinbek bei Hamburg: Rowohlt Taschenbuch Verlag. 
Schnell, R., Hill, P. \& Esser, E. (1999). Methoden der empirischen Sozialforschung. München, Wien: Oldenbourg Verlag.

Schober, R. (1995). Kreative Wege zum besseren Angebot. Eine Planungshilfe für Tourismus-Praktiker. München: ADAC.

Schramm, H., Döhle, M. \& Klimmt, C. (2004). Das Erleben von Fußball im Fernsehen. In H. Schramm (Hrsg.) Die Rezeption des Sports in den Medien. (Sportkommunikation, Bd. 3, S. 121-142). Köln: Harlem Verlag.

Schulke, H.-J. (2006). Perspektiven und Werthaltigkeit des Public Viewing: Zu den Dimensionen einer neuen Qualität des Zuschauerve rhaltens beim Fußball. In M.-P. Büch (Hrsg). Zur Ökonomie der Rechte bei Sportveranstaltungen (S. 17-30). (1. Aufl.). Köln: Sportverlag Strauß.

Schulke, H.-J. (2006a). Ein Weltereignis als Gemeinschaftserlebnis. Olympisches Feuer, 3, 18-21.

Schulke, H.-J. (2007). Fan und Flaneur: Public Viewing bei der FIFAWeltmeisterschaft 2006-Organisatorische Erfahrungen, soziologische Begründungen und politische Steuerung bei einem neuen Kulturgut. In D. Jütting (Hrsg.). Die Welt ist wieder heimgekehrt. Studien zur Evaluation der FIFA-WM 2006. (Edition Global-lokale Sportkultur, Bd. 23, S. 25-72). Münster, New York, München [u.a]: Waxmann Verlag.

Schulze, G. (1997). Die Erlebnis-Gesellschaft: Kultursoziologie der Gegenwart. (7. Aufl., Studienausgabe); Frankfurt a.M., New York: Campus Verlag.

Schulze, G. (2000). Vier Faktoren für wirkungsvolle Events. In G. Trosien \& M. Dinkel (Hrsg.). Ökonomische Dimensionen von Sport-Events: FaktorenFolgerungen-Fallbeispiele (S. 19-31). Butzbach-Griedel: AFRA-Verlag.

Schulze, G. (2007). Die Zukunft der Erlebnisgesellschaft. In O. Nickel. Eventmarketing. Grundlagen und Erfolgsbeispiele (S. 309-323). (2. vollständig überarbeitete Aufl.), München: Verlag Franz Vahlen.

Schwark, J. (2007). Der Weltmeister zu Gast in Meiderich - Ökonomische Effekte der Fußball-WM 2006 für die Stadt Duisburg. In D. Jütting (Hrsg.). Die Welt ist wieder heimgekehrt. Studien zur Evaluation der FIFA-WM 2006. (Edition Global-lokale Sportkultur, Bd. 23, S. 99-114). Münster, New York. München [u.a]: Waxmann Verlag.

Schwier, J. (Hrsg.) (2002). Mediensport: Ein einführendes Handbuch. Baltmannsweiler: Schneider Verlag Hohengehren.

Schwier, J. \& Schauerte, Th. (2006). "Mittendrin statt nur dabei"-Inszenierungsformen des Fernsehsports. In Merz: Medien+Erziehung. Zeitschrift für Medienpädagogik 30 (6), 9-17.

Seel. M. (2001). Inszenieren als Erscheinenlassen. Thesen über die Reichweite eines Begriffs. In J. Früchtl. \& J. Zimmermann (Hrsg.), Ästhetik der Inszenierung. Dimensionen eines künstlerischen, kulturellen und gesellschaftlichen Phänomens (S. 48-62). Frankfurt a.M.: Suhrkamp.

Shilling, Ch. (2002). The two traditions in the sociology of emotions. In J. Barbalet (Ed.). Emotions and Sociology (pp. 10-32). Malden, Oxford, Vicotria: Blackwell Publishing. 
Siegel, G. (2002). Double Vision: Large-Screen Video Display and Live Sports Spectacle. Television NewMedia,3, 49-73.

Sloan, L. R. (1989). The Motives of Sport Fans. In J. H. Goldstein (Ed.), Sports, Games and Play (pp. 175-240). Hillsdale/N.J. [u.a.]: Erlbaum.

Soon-Hee, W. (2004). Football, fashion and fandom. Sociological reflections on the 2002 World Cup and collective memories in Korea. In W. Manzenreiter \& J. Horne (Eds.). Football goes east. Business, culture and the people's game in China, Japan and South Korea (pp. 148-164). London: Routledge.

Soo-min, S. (2002). Ears of Many, World Cup Echoes On. In Korean Information Service (Ed.). Korean Experience with the World Cup (pp. 32-35). Seoul: Moojin.

Soon-Hee, W. (2004). Football, fashion and fandom. Sociological reflections on the 2002 World Cup and collective memories in Korea. In W. Manzenreiter \& J. Horne (Eds.). Football goes east. Business, culture and the people's game in China, Japan and South Korea (pp. 148-164). London: Routledge.

Spellerberg, A. (1996). Soziale Differenzierung durch Lebensstile: eine empirische Untersuchung zur Lebensqualität in West- und Ostdeutschland. Berlin: Ed Sigma.

Städele, K. (2005). Fußball ante portas. Media und Marketing, 5, 12. Mai 2005, 31.

Stets, J. \& Turner, J. (Eds.) (2007). Handbook of the Sociology of Emotions. New York: Springer Science+Business Media.

Steinke, I. (1999). Kriterien qualitativer Forschung. Ansätze zur Bewertung qualitativ-empirischer Sozialforschung. Weinheim und München: Juventa Verlag.

Steinke, I. (2005). Gütekriterien qualitativer Forschung. In U. Flick, E. v. Kardorff \& I. Steinke (Hrsg.). Qualitative Sozialforschung. Ein Handbuch (S. 319-331). (4. Aufl.). Reinbek bei Hamburg: Rowohlt Taschenbuch Verlag.

Stiehler, H.-J. \& Friedrich, J. (2003). Fußball am Morgen: Zuschauerprofile der Berichterstattung zur Fußball-Weltmeisterschaft 2002 im deutschen Fernsehen. In Th. Horky (Hrsg.). Die Fußballweltmeisterschaft als Kommunikationsthema. (Sport \& Kommunikation, Bd. 1, S. 55-80). Norderstedt: Books on Demand GmbH.

Stollenwerk, H.J. (1996). Sport-Zuschauer-Medien. (Edition Sport \& Freizeit; Bd. 4). Aachen: Meyer \& Meyer Verlag.

Strauß, B. \& Jürgensen, S. (1998). Facetten des Zuschauers. In B. Strauß (Hrsg.). Zuschauer (S. 7-26). Göttingen, Bern, Toronto [u.a.]: Hogrefe, Verl. f. Psychologie.

Stroebe, W., Jonas, K. \& Hewstone, M. (Hrsg.) (2001). Sozialpsychologie. Eine Einführung. (4. überarbeitete und erweiterte Aufl.). Berlin, Heidelberg, New York: Springer-Verlag.

Süddeutsche Zeitung Magazin (Hrsg.) (2006). Was ist Public Viewing? Süddetusche Zeitung, 12, 26. 
Sydnor, S. (2000). Sport, Celebrity and Liminality. In N. Dyck (Ed.). Games, Sports and Cultures (pp. 221-240). Oxford, New York: Berg.

Tedeschi, J., Madi, N. \& Lyakhovitzky, D. (1998). Die Selbstdarstellung von Zuschauern. In B. Strauß (Hrsg.), Zuschauer (S. 93-110). Göttingen, Bern, Toronto [u.a.]: Hogrefe, Verl. f. Psychologie.

Tester, K. (1998). 'Bored and blasé'. Television, the emotions and Georg Simmel. In G. Bendelow \& S. Williams (Eds.). Emotions in Social Life. Critical Themes and Contemporary Issues (pp. 83-96). London [u.a.]: Routledge.

Thielsch, M. (2008). Einführung in die Methodik von Online-Untersuchungen. In M. Thielsch (Hrsg.). Ästhetik von Websites. Wahrnehmung von Ästhetik und deren Beziehung zu Inhalt, Usability und Persönlichkeitsmerkmalen (S. 95101). Münster: MV Wissenschaft.

Tritt, K. (1992). Emotionen und ihre soziale Konstruktion: Vorarbeiten zu einem wissenssoziologischen, handlungstheoretischen Zugang zu Emotionen. (Europäische Hochschulschriften, Reihe 22, Soziologie, Bd. 226). Frankfurt a.M. [u.a.]: Lang.

Trosien, G. \& Dinkel, M.(Hrsg.).(2000). Ökonomische Dimensionen von SportEvents: Faktoren-Folgerungen-Fallbeispiele. Butzbach-Griedel: ALFRAVerlag.

Trosien, G. \& Dinkel, M. (2000a). Einführung. In G. Trosien \& M. Dinkel (Hrsg.). Okonomische Dimensionen von Sport-Events: Faktoren-FolgerungenFallbeispiele (S. 9-18). Butzbach-Griedel: ALFRA-Verlag.

Turner, J. \& Stets, J. (2007). Sociological Theories of Human Emotions. In J. Stets \& J. Turner (Eds.). Handbook of the Sociology of Emotions (S. 25-52). New York: Springer Science+Business Media.

Vester, H.-G. (1991). Emotionen, Gesellschaft und Kultur: Grundzüge einer soziologischen Theorie der Emotionen. Opladen: Westdt.-Verlag.

Vester, H.-G. (1993). Soziologie der Postmoderne. München: Quintessenz.

Vester, M., v. Oertzen, P., Geiling, H., Hermann, Th. \& Müller, D. (1993). Soziale Milieus im gesellschaftlichen Strukturwandel. Zwischen Integration und Ausgrenzung. Köln: Bund-Verlag.

Wann, D. \& Branscombe, N. (1993). Sport Fans: Measuring Degree of Identification with Their Team. International Journal of Sport Psychology, 24, 1-17.

Wann, D. (1995). Preliminary Validation of the Sport Motivation Scale. Journal of Sport and Social Issues, 19 (4), 377-396.

Wann, D., Melnick, M., Russell, G. \& Pease, D. (2001). Sport Fans. The psychological and social impact of spectators. New York, London: Routledge.

Weber, R. \& Gehrau, V. (2004). Sportrezeption auf Mikroebene. Eine empirische Analyse der Rezeption von Fußballspielen im Fernsehen. In H. Schramm. (Hrsg.). Die Rezeption des Sports in den Medien. (Sportkommunikation, Bd. 3, S. 143-166). Köln: Harlem Verlag. 
Weiermair, K. \& Brunner-Sperdin, A. (Hrsg.) (2006). Erlebnisinszenierung im Tourismus. Erfolgreich mit emotionalen Produkten und Dienstleistungen. Berlin: Erich Schmidt Verlag.

Weiermair, K. (2006). Von der Dienstleistung zum Erlebnis. In K. Weiermair \& A. Brunner-Sperdin (Hrsg.). Erlebnisinszenierung im Tourismus. Erfolgreich mit emotionalen Produkten und Dienstleistungen (S. 13-22). Berlin: Erich Schmidt Verlag.

Weinberg, P. \& Nickel, O. (2007). Grundlagen für die Erlebniswirkung von Marketingevents. In O. Nickel (Hrsg.). Eventmarketing. Grundlagen und Erfolgsbeispiele (S. 37-50). München: Verlag Franz Vahlen.

Weinhold, K.-P. (2002). Ethik und Leistung-Grundpfeiler einer humanen Sport und Spielkultur. In Württembergischer Fußballverband e.V. (Hrsg.). Der Fußball...ein Beitrag zu einer Gesellschaftskultur der Zukunft (S. 34-44). Sindelfingen: Röhm GmbH Druckerei und Verlag.

Wenner, L. \& Gantz, W. (2000). Watching Sports on Television: Audience Experience, Gender, Fanship, and Marriage. In L. Wenner (Ed.). MediaSport (pp. 233-251). New York, London: Routledge.

Whannel, G. (2000). Reading the Sports Media Audience. In L. Wenner (Ed.). MediaSport (pp. 221-232). New York, London: Routledge.

Whannel, G. (2005). Pregnant with anticipation: The pre-history of television sport and the politics of recycling and preservation. International Journal of Cultural Studies, 8 (4), 405-426.

Wiswede, G. (2000). Einführung in die Wirtschaftspsychologie. (3. überarbeitete und erweiterte Aufl.). München, Basel: E. Reinhardt Verlag.

Williams, S. \& Bendelow, G. (1998). Introduction: emotions in social life. Mapping the sociological terrain. In G. Bendelow \& S. Williams (Eds.). Emotions in Social Life. Critical Themes and Contemporary Issues (pp. xv-Xxx). London [u.a.]: Routledge.

Wöhler, K. (2004). Was soll die Diagnose: Überall Erlebnis? In H. Kagelmann, R. Bachleitner \& M. Rieder (Hrsg.). ErlebnisWelten. Zum Erlebnisboom in der Postmoderne (S. 220-226). München, Wien: Profil Verlag.

Woldt, R. (2006). HDTV: Erfolg im zweiten Anlauf? Das hochauflösende Fernsehen hat noch einige Hürden zu überwinden. Media Perspektiven, 4, 235-242.

Xu, X. (2006). Modernizing China in the Olympic spotlight: China's national identiy and the 2008 Beijing Olympiad. In J. Horne \& W. Manzenreiter (Eds.). Sports Mega-Events. Social Scientific Analyses of a Global Phenomenon. (The Sociological Review, pp. 91-107). Malden, Oxford, Vicotria: Blackwell Publishing.

Zanger, C. (2007). Eventmarketing als Kommunikationsinstrument. Entwicklungsstand in Wissenschaft und Praxis. In O. Nickel (Hrsg.). Eventmarketing. Grundlagen und Erfolgsbeispiele (S. 3-36). München: Verlag Franz Vahlen.

Zillmann, D. \& Bryant, J. (1998). Fernsehen. In B. Strauß (Hrsg.), Zuschauer (S. 175-212). Göttingen, Bern, Toronto [u.a.]: Hogrefe, Verl. f. Psychologie. 
Zubayr, C. \& Gerhard, H. (2002). Fußball-WM 2002: Ein Fernsehhighlight aus Sicht der Zuschauer. Nutzung und Bewertung der WM-Berichterstattung im Fernsehen. Media Perspektiven, 7, 308-313.

Zubayr, C. \& Gerhard, H. (2004). Zur Nachfrage nach Sportangeboten in den Medien. In H. Schramm (Hrsg.). Die Rezeption des Sports in den Medien (S. 28-48). Köln: Halem Verlag.

Zubayr, C. \& Gerhard, H. (2004a). Die Fußball-Europameisterschaft im Fernsehen. Nutzung und Bewertung der Live-Berichterstattung bei ARD und ZDF. Media Perspektiven, 9, 421-425.

Zubayr, C. \& Gerhard, H. (2005). Tendenzen im Fernsehverhalten. Fernsehgewohnheiten und Fernsehreichweiten im Jahr 2004. Media Perspektiven, 3, 94-104.

Zubayr, C. \& Gerhard, H. (2007). Tendenzen im Fernsehverhalten. Fernsehgewohnheiten und Fernsehreichweiten im Jahr 2006. Media Perspektiven, 4, 187-199.

Zubayr, C. \& Gerhard, H. (2008). Tendenzen im Fernsehverhalten. Fernsehgewohnheiten und Fernsehreichweiten im Jahr 2007. Media Perspektiven, 3, 106-119. 


\section{Internetquellen}

Aimiller, K. \& Kretschmar, H. (1995). Motive des Sportzuschauers. Umfeldoptimierung durch motivationale Programmselektion (MPS). Unterföhring: DSF. Zugriff am 20. September 2007 unter http://www.dsf.de/media/pdf/ unternehmen/medien/research/MotivedesSportzuschauers.pdf

Arbeitskreis Deutscher Markt- und Sozialforschungsinstitute e.V. (ADM) (2001, Mai). Standards zur Qualitätsicherung für Onlinebefragungen. Zugriff am 10. Mai 2008 unter http://www.adm-ev.de/pdf/Onlinestandards_D.PDF

ARD/ZDF-Onlinestudie (2007). Entwicklung der Onlinenutzung in Deutschland 1997 bis 2007. Zugriff am 06. Januar 2009 unter http://ard-zdfonlinestudie.de/index.php?id=20

Aschemann-Pilshofer, B. (2001, Januar). Wie erstelle ich einen Fragebogen? Ein Leitfaden für die Praxis. (Wissenschaftsladen Graz, 2. Auf.). Zugriff am 12. Dezember 2008 unter http://www.aschemann.at/Downloads/ Fragebogen.pdf

Brehm, W., Sygusch, R., Ballmert, J. \& Reuter, A. (2007). Public Viewing bei der FIFA-WM 2006. Ein sportökonomischer Blick auf das Sommermärchen 2006. Spectrum, 2, 7ff.

Brinker, H. (2004, 19. Oktober). Die Wiege des Fußballs. Zugriff am 06. Dezember 2005 unter http://www.fifa.com/de/regulations/magazine/index/ $0,1569,103477,00$. html?articleid $=103477$

DOSB (2008, 15. Oktober). Bestandserhebung 2008. Zugriff am 28. Dezember 2008 unter http://www.dosb.de/fileadmin/fm-dosb/downloads/ bestandserhebung/2008_Bestandserhebung.pdf

FIFA (Hrsg.) (o.J.a). World Cup \& Television. Info Plus. Zugriff am 06. Dezember 2005 unter http:fifaworldcup.yahoo.com/releases/IP-401-DTV.Pdf

FIFA (Hrsg.) (o.J.b). FIFA Fußball - Weltmeisterschaft 2002 ${ }^{\text {TM }}$ : 41.000 Stunden TV-Berichterstattung in 213 Ländern. Zugriff am 07. Dezember 2005 unter http://www.fifa.com/de/marketing/newmedia/index/0,3509,10,00.html

FIFA (Hrsg.) (o.J.C). Photo gallery. WC2002--KOREA-CROWDS 128639. Zugriff am 21. Dezember 2005 unter http://2002.fifaworldcup.yahoo.com/02/ en/ 020622/1/ d9v.html

FIFA (Hrsg.) (o.J.d). Geschichte der Fussball-Weltmeisterschaft. Zugriff am 07. Dezember 2005 unter http://www.fifa.com/de/history/0,1283,5,00.html

FIFA (Hrsg.) (o.J.e). Über 2000 Jahre Fussball. Aus der bunten Geschichte eines faszinierenden Spiels. Zugriff am 10. Dezember 2005 unter http://www.fifa .com/de/history/history/0,1283,1,00.html

FIFA (Hrsg.) (2005, $14 . \quad$ Juni). FIFA präsentiert erstes StadionUnterhaltungsprogramm beim FIFA Konföderationen-Pokal 2005. Zugriff am 07.Dezember 2005 unter http://fifaworldcup.yahoo.com/06/de/050614/1/ 2392_pf.html 
FIFA Marketing AG Media Services (Hrsg.) (o.J.). FIFA World Cup tм ratings success-results confirm event as a "24/7" viewing experience. Zugriff am 28. September 2005 unter http://2002.fifaworldcup.yahoo.com/02/en/ 020624/2/17xw.html

Habbel, F.-R. (2005). Fussball WM 2006тм Deutschland - eine Fußballarena. Genehmigungsprozesse, Motivation und Zielsetzungen für öffentliche Vorführungen - Public Viewing Events. In TelematicsPRO e.V. (Hrsg.) Public Viewing Events 2006. Gezielte Konzepte schaffen positive Gemeinschafts-erlebnisse. Handbuch für Planung und Durchführung von kommerziellen und nicht-kommerziellen öffentlichen Vorführungen von Spielen der FIFA WM 2006тм (S. 6-13). Berlin. Zugriff am 14. Dezember 2005 unter http://www.public-viewing.de/Download/Handbuch.pdf

Horizont Sportbusiness (2006, 14. Juli). Publikumsmagnet Kino-Fußball. Zugriff am 07. August 2006 unter http://www.sportbusiness.horizont.net/ topstories/pages/show.prl?params=keyword\%3DPublic\%20Viewing\%26all \%3D\%26type\%3D2\%26laufzeit\%3DO\&id=6436\&currPage=1

Infront Sports \& Media AG (Hrsg.) (o.J.). Making History - The 2006 FIFA World Cup $^{T M}$. Zugriff am 12. Dezember 2008 unter http://www.infrontsports.com/ uploades/media/Making History-2006FIFAWorldCup_01.pdf

Infront Sports \& Media AG (Hrsg.) (2002, 05. Dezember). 2002 FIFA World CuptM Final most viewed match in history. Viewers the world over demonstrated their willingness to trade sleep for soccer and change their viewing habits and daily routine. Zugriff am 06. Dezember 2005 unter http://www.infrontsport.com/news/news_detail.asp?rubrik ID=\& ID=92\&suchb egriffe $=$ public $\% 20$ viewing $\&$ curpage $=1$

Infront Sports \& Media AG (2006, 17. Juli). Record television audiences in Europe for the final matches of the 2006 FIFA World Cup ${ }^{T M}$. News Detail. Zugriff am 22. Oktober 2007 unter http://www.infrontsports.com/presscenter/ newsdetail/article/91/record-telev.html

Infront Sports \& Media AG (2006a, 27. Juni). 2006 FIFA World Cup ${ }^{T M}$ viening figures in Germany up to 51 percent on average over 2002. News Detail. $\begin{array}{llll}\text { Zugriff am } 22 . & \text { Oktoaber } 2007 & \end{array}$ http://www.infrontsports.com/presscenter/ newsdetail/article/177/2006-fifawo-7.html

Infront Sports \& Media AG (2006b, 5. Juli). SOS Children's Villages receive a 700 000 Euro donation from Infront, FIFA's television partner. Zugriff am 22. Oktober 2007 unter http://www.infrontsports.com/presscenter/newsdetail/ article/177/sos-children.html

Infront Sports \& Media AG (Hrsg.) (o.J.a). Richtlinien für kommerzielle öffentliche Vorführungen von Spielen der FIFA WM 2006. Zugriff am 09. Dezember 2005 unter http://www.sportministerkonferenz.de/dateien/

Richtlinien\%20kommerzielle\%20Vorfuehrungen.pdf

Infront Sports \& Media AG (Hrsg.) (o.J.b). Richtlinien für nicht-kommerzielle öffentliche Vorführungen von Spielen der FIFA WM 2006. Zugriff am 09. Dezember 2005 unter http://www.sportministerkonferenz.de/dateien/ Richtlinien\%20nicht\%20kommerzielle\%20Vorfuehrungen.pdf 
Infront Sports \& Media AG (o.J.c). 2002 FIFA World Cupтм - making history. Zug riff am 30. Dezember 2005 unter http://www.infrontsport.com/news/

news_detail.asp?rubrik ID=\&ID=84\&suchbegriffe=public\%viewing \&curpag $\mathrm{e}=1 \&$ saison $=2005 \% 2 \mathrm{~F}=\& \&$ soieltag $=7 \&$ gruppe $=7523$

Klebs, F. (2005, 18. August). Fußball-WM als Marketing-Plattform-Studie ermittelt Chancen und Risiken rund um den WM-Kick. Informationsdienst Wissenschaft. Zugriff am 22. August 2005 unter http://idwonline.de/pages/de/ news124673

Kurscheidt, M. (2004). Erfassung und Bewertung der wirtschaftlichen Effekte der Fußball-WM 2006TM (Unabhängiges wissenschaftliches Gutachten). Zugriff am 01. September 2005 unter http://www.dbresearch.de/PROD/

DBR_INTERNET_DE-PROD/PROD0000000000073277.pdf

Mayring, Ph. (2001, Februar). Kombination und Integration qualitativer und quantitativer Analyse [31 Absätze]. Forum Qualitative Sozialforschung/Forum: Qualitative Social Research [Online-Journal], 2 (1). Zugriff am 14. August 2008 unter http://qualitative-research.net/fqs/fqs. htm

Mediaedge: CIA (Hrsg.) (2002, August). Werbung und Fernsehverhalten zur Fußball-WM. Düsseldorf. Zugriff am 25. September 2007 unter http://www.wuv.de/studien/archiv/082002/586/1883.html

Meyers Lexikonverlag (2007, 27. Februar). Public Viewing. Zugriff am 08. Oktober 2007 unter

http://lexikon.meyers.de/index .php?title=Public_Viewing\&oldid=133243

Pank, B. (o.J.). 2002 Football World Cup recorded and broadcast in HD. Bob Pank joins with Franz Beckenbauer to ask 'why don't we have this in Europe?'. Zugriff am 06. Dezember 2005 unter http://www.sonybiz.net/b2b/ sony-business-it/ 46085-sony-biz-italia-2002-football-world-cuprecordedand-broadcast-hd-hdcam-eventi.html

Rheinland-Pfalz WM-Portal (Hrsg.) (o.J.). Public Viewing. Zugriff am 28. September 2005 unter http://www.wm2006.rlp.de/Deutsch/ broker?uCon=b033038a-75d9-1501-6e24b9cf5a06189a\&uTem =9b24069b1d59-4201-6e24-b9cf5a06189a

Rötzer, F. (1997, 23. Dezember). Die globale Massage oder das Geheimnis der Lady Di. Telepolis. Heise Zeitschriften Verlag. Zugriff am 14. August 2008 unter http://www.heise.de/tp/r4/artikel/2/2238/1.html

Rötzer, F. (2006, 08. Juni). Mediale Massage. In Telepolis. Heise Zeitschriften Verlag. Zufriff am 27. September 2007 unter http://www.heise.de/tp/r4/artikel/22/22842/l.html

Schulke, H.-J. (2006b, 28. Dezember). Fan und Flaneur: Public Viewing bei der FIFA-Weltmeisterschaft 2006. Organisatorische Erfahrungen, soziologische Begründungen und politische Steuerung zu einem neuen Kulturgut. (Diskussionsentwurf). Zugriff am 27. Oktober 2007 unter http://www.spowi.uni-bremen.de/personen/ personaldaten-sport/schulke/hans-juergen-schulke.html

Stadt Frankfurt am Main (o.J.). Die 'schwimmende' Videowand. Zugriff am 26. November 2008 unter http://www. frankfurt.de/sixcms/ detail.php?id=1103109\&template=bildanzeigen 
Tagesspiegel (2001, 27. November). Kinowelt vor der Pleite. Zugriff am 22. Oktober 2005 unter http://archiv.tagesspiegel.de/archiv/26.11.2001/ ak-wi-un-4412501.html

UEFA (2008). Public Viewing - Allgemeine Bedingungen. Allgemeine Bedingungen der UEFA für die öffentliche Vorführung von Spielen der UEFA-Fussball-Europameisterschafts-Endrunde 2008. Zugriff am 25. September 2008 unter http://de.uefa.com/newsfiles/533215.pdf

Zils, O. (2005, 03. November). Im Stadion Fernsehen schauen. Das PublicViewing-Konzept Frankfurts sticht aus dem Angebot der WM-Städte heraus. Horizont Sport Business, 4. 


\section{Quellenverzeichnis}

Leitfadeninterviews mit Public Viewing-Experten der Fußball-Weltmeisterschaft 2006 (anonymisierte Transkripte in Daten-CD im Einband)

\section{Fan Feste}

$\mathrm{NN}_{1}$ : Experte für ein Fan Fest der Fußball-Weltmeisterschaft 2006, 12. Juli 2006

$\mathrm{NN}_{2}$ : Experte für ein Fan Fest der Fußball-Weltmeisterschaft 2006, 19. Juli 2006

$\mathrm{NN}_{3}$ : Experte für ein Fan Fest der Fußball-Weltmeisterschaft 2006, 21. Juli 2006

$\mathrm{NN}_{4}$ : Experte für ein Fan Fest der Fußball-Weltmeisterschaft 2006, 01. August, 2006

$\mathrm{NN}_{5}$ : Experte für ein Fan Fest der Fußball-Weltmeisterschaft 2006, 14. August 2006

\section{Nicht-kommerzielles Public Viewing}

$\mathrm{NN}_{6}$ : Experte für ein nicht-kommerzielles Public Viewing der Fußball-Weltmeisterschaft 2006, 11. Juli 2006

$\mathrm{NN}_{7}$ : Experte für ein nicht-kommerzielles Public Viewing der Fußball-Weltmeisterschaft 2006, 17. Juli 2006

$\mathrm{NN}_{8}$ : Experte für ein nicht-kommerzielles Public Viewing der Fußball-Weltmeisterschaft 2006, 17. Juli 2006

$\mathrm{NN}_{9}$ : Experte für ein nicht-kommerzielles Public Viewing der Fußball-Weltmeisterschaft 2006, 19. Juli 2006

$\mathrm{NN}_{10}$ : Experte für ein nicht-kommerzielles Public Viewing der Fußball-Weltmeisterschaft 2006, 21. Juli 2006

$\mathrm{NN}_{11}$ : Experte für ein nicht-kommerzielles Public Viewing der Fußball-Weltmeisterschaft 2006, 21. Juli 2006

$\mathrm{NN}_{12}$ : Experte für ein nicht-kommerzielles Public Viewing der Fußball-Weltmeisterschaft 2006, 26. Juli 2006

$\mathrm{NN}_{13}$ : Experte für ein nicht-kommerzielles Public Viewing der Fußball-Weltmeisterschaft 2006, 26. Juli 2006

$\mathrm{NN}_{14}$ : Experte für ein nicht-kommerzielles Public Viewing der Fußball-Weltmeisterschaft 2006, 09. August 2006

$\mathrm{NN}_{15}$ : Experte für ein nicht-kommerzielles Public Viewing der Fußball-Weltmeisterschaft 2006, 15. August 2006

$\mathrm{NN}_{16}$ : Experte für ein nicht-kommerzielles Public Viewing der Fußball-Weltmeisterschaft 2006, 18. August 2006 
$\mathrm{NN}_{17}$ : Experte für ein nicht-kommerzielles Public Viewing der Fußball-Weltmeisterschaft 2006, 18. August 2006

\section{Kommerzielles Public Viewing}

$\mathrm{NN}_{18}$ : Experte für ein kommerzielles Public Viewing der Fußball-Weltmeisterschaft 2006, 19. Juli 2006

$\mathrm{NN}_{19}$ : Experte für ein kommerzielles Public Viewing der Fußball-Weltmeisterschaft 2006, 24. Juli 2006

$\mathrm{NN}_{20}$ : Experte für ein kommerzielles Public Viewing der Fußball-Weltmeisterschaft 2006, 24. Juli 2006

$\mathrm{NN}_{21}$ : Experte für ein kommerzielles Public Viewing der Fußball-Weltmeisterschaft 2006, 09. August 2006 


\section{Anhang}

A. Anschreiben an die Public Viewing-Experten der FIFA Fußball-WM $2006^{\mathrm{TM}}$

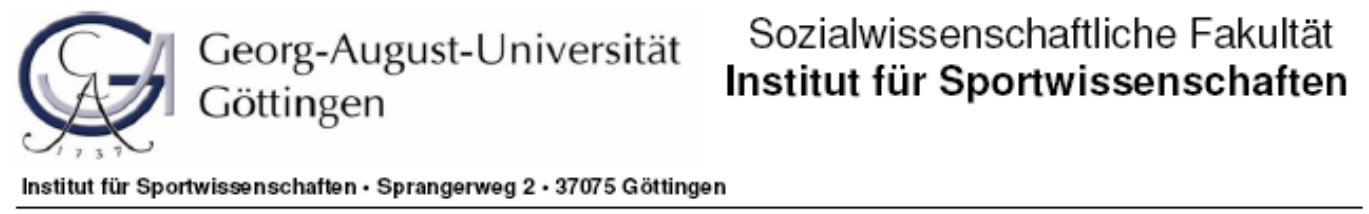

Prof. Dr. Arnd Kräger

Leiter Abteilung Gesellschaft und Training akruege1@gwdg.de

Tel. $+49(0) 551 / 395651$ Fax $+49(0) 551 / 395641$

Tel. $+49(0) 551 / 395653$ ifs@sportuni-goettingen.de

www.sport.uni-goettingen.de

Gottingen, Mai 2006

Sehr geehrte Damen und Herren,

als Doktorandin des Instituts für Sportwissenschaften der Georg-August-Universität Göttingen bei Prof. Dr. Arnd Kruger beschäftige ich mich in meiner Promotion mit dem Thema „Public Viewing bei der FIFA Fußball-WM $2006^{\mathrm{TM}}$ “.

Um die Dimensionen dieses neuartigen Phänomens erfassen zu können, möchte ich die Veranstalter von TV-Vorfuhrungen der Weltmeisterschaftsspiele befragen. Nach Ende des Turniers am 09. Juli 2006 würde ich Ihnen gern schriftlich oder telefonisch Fragen stellen, bei denen es vor allem um Ihre Motive, Erwartungen und Zielvorstellungen sowie um Ihre Erfahrungen geht.

Ihre Ergebnisse würden Sie mir sehr helfen, meine Arbeit möglichst praxisnah zu gestalten. Ich bitte Sie daher vorab um ihr Einverstăndnis.

Für eine kurze Rückmeldung per E-Mail wäre ich Ihnen sehr dankbar. Bitte lassen Sie mich dabei auch wissen, ob Ihnen ein telefonischer oder ein schriftlicher Kontakt lieber wăre.

Die Ergebnisse werden so anonymisiert, dass die Einzelergebnisse nicht erkennbar sind - es sei denn, dass Sie ausdrucklich die Zustimmung fur das Zitieren Ihrer persönlichen Informationen geben.

Mit freundlichen Grußen,

Britta Ufer

(i.A. Arnd Kruger) 
B. Gesprächsleitfaden für die qualitativen Interviews mit den Public Viewing-Experten der FIFA Fußball-WM $2006^{\text {TM }}$

\section{Leitfaden Fan Fest}

Datum:

Uhrzeit:

Gespräch mit:

Aufnahme? Ja/nein

Teil A. Informationen zu den Räumlichkeiten

Wo genau fand ihre Public Viewing-Feier statt?

Warum (evtl. repräsentative Gründe/Wahrzeichen/vorhandene Anschlüsse)?

Liegt Platz/Halle/o.ä. zentral oder außerhalb des Stadtzentrums?

Bieten Sie Ihren Gästen Sitz- und Stehplätze an?

Wie waren diese anteilig verteilt?

Gab es eine VIP-Tribüne?

Wie waren diese Plätze zur Leinwand angeordnet?

Teil B. Besucherverhalten und Gästeanzahl

Welche Besuchergruppe (Alter, Zielgruppe) hat ihre Veranstaltung angezogen?

- Wie war Ihrer Meinung nach der Anteil von Frauen und Männern?

War das Public Viewing während der gesamten Zeit (bezogen auf einen Tag, Wochenende, Uhrzeit) gleich stark besucht?

Wie hoch schätzen Sie die Bedeutung des Fußballsports in Deutschland und der Welt für den Erfolg Ihrer Public-Viewing-Veranstaltung ein?

Und wie hoch die Bedeutung, dass es sich um die Weltmeisterschaft handelte?

\section{Teil C. Finanzielle Aspekte}

Ist es richtig, dass fünf der Offiziellen Partner der FIFA Ihr Public Viewing unterstützt haben?

Haben Nationale Förderer der FIFA WM 2006 Ihre Public Viewing unterstützt?

Wenn ja, welche Nationalen Förderer der FIFA WM 2006 haben mit welchen Mitteln ihre Public Viewing-Veranstaltung unterstützt? 
Haben Unternehmen, welche nicht Offizielle Partner der FIFA oder Nationale Förderer der FIFA WM 2006 sind, Sie als so genannte „Unterstützer“ mitfinanziert?

- Wenn ja, aus welchen Branchen kamen diese „Unterstützer“ und in welcher Form wurden Sie unterstützt?

In welchem Maß haben Sie die Kriterien der FIFA Marketingrichtlinien (dass keines dieser Unternehmen aus den Branchen der FIFA Sponsoren stammen darf) in der Finanzierung Ihres Events belastet?

- Was verursacht aus Ihrer Sicht die größten Kosten bei der Durchführung des Public Viewing?

- $\quad$ Sehen Sie eine Möglichkeit, diese Kosten zu verringern?

- Wenn ja, welche?

- $\quad$ Die Infront Sports \& Media AG hat auf die Lizenzgebühr für die Fan Feste verzichtet. Wenn dies nicht geschehen wäre, hätten Sie das Public Viewing trotzdem durchgeführt?

- Wenn ja, warum ?

- $\quad$ Wenn nein, warum nicht!

- $\quad$ Konnten Sie die Refinanzierung Ihres Public Viewing sicherstellen?

- Wenn nein, warum führen Sie eine solche Veranstaltung durch, obwohl es sich für Sie (Stadt, Gemeinde, Kommune) nicht finanziell rechnet?

- $\quad$ Inwiefern war das Erwirtschaften eines finanziellen Gewinns für Sie wichtig?

\section{Teil D. Motive, Erwartungen und Erfahrungen}

Was waren Ihre Motive, Public Viewing zu veranstalten?

Was haben Sie sich von der Durchführung der Public Viewing-Veranstaltung versprochen (Zielvorstellung)?

- Was haben sich Ihrer Meinung nach Ihre Gäste vom Besuch der Public Viewing-Veranstaltung versprochen?

Hatten Sie ein Konzept für die Veranstaltung (Inhalt)?

Warum haben Ihre Gäste gerade das Fan Fest besucht und keine andere Public-Viewing-Veranstaltung o.ä.?

- $\quad$ Übt der Besuch eines offiziellen Fan Festes einen besonderen Reiz auf die Fans aus (warum)?

Macht Ihre Stadt, als Ausrichterstadt, einen besonderen Reiz für die Fans aus?

- $\quad$ Sind Ihre Gäste, Ihrer Meinung nach mit dem Besuch des Events zufrieden 
gewesen?

- Wie zufrieden waren Sie als Veranstalter mit dem Public Viewing-Fest?

Wie ausschlaggebend für den Erfolg des Fan Festes war das Erreichen des Halbfinales der deutschen Mannschaft?

Mussten Sie nach dem Ausscheiden der deutschen Mannschaft im Halbfinale einen deutlichen Besucherrückgang für die letzten Spieltage verzeichnen?

\section{Teil E. Technologien}

Für die Bildprojektion haben Sie LED-Leinwände genutzt. Warum haben Sie diese Technologie gewählt?

- Wie wichtig ist Ihnen die Bildqualität während der Übertragungen?

Wie wichtig ist Ihnen generell die Tonqualität während der Übertragungen?

Es gibt drei neuartige Technologien (LED-Videowände, Dolby-Surround, HDTV), die die Entwicklung des Public Viewing gefördert haben.

Ist Ihrer Meinung nach eine dieser Technologien wichtig für den Erfolg von Public Viewing-Veranstaltungen?

- Wenn ja, welche der Technologien ist es?

\section{Teil F. Sicherheitskonzept}

Haben Sie an den Eingängen Personenkontrollen durchführen lassen (permanent, Einzäunung)?

- Wie wichtig für einen reibungslosen Veranstaltungsablauf sind Ihrer Meinung nach Personenkontrollen an den Eingängen?

- Haben Sie Videokameras zur Beobachtung des Geländes eingesetzt?

- Wie wichtig für einen reibungslosen Veranstaltungsablauf ist Ihrer Meinung nach der Einsatz von Videokameras?

- Was meinen Sie als Veranstalter, haben sich Ihre Gäste sicher gefühlt?

- Kam es zu gewalttätigen Ausschreitungen?

\section{Teil G. Emotionsmanagement}

Wenn Sie ein Veranstaltungskonzept hatten, haben Sie es im Laufe der Weltmeisterschaft verändert?

- Wenn ja, was haben Sie verändert (warum)? 
Haben Sie neben der Übertragung der Fußballspiele Ihren Gästen Unterhaltung geboten?

- Wenn ja, was haben Sie neben der Übertragung der Fußballspiele an Unterhaltung für die Gäste geboten?

- $\quad$ Wenn ja, warum haben Sie dieses Unterhaltungsprogramm angeboten?

- $\quad$ Wenn ja, wollten Sie mit ihrem Unterhaltungsprogramm auf eine bestimmte Zielgruppe abzielen (welche)?

- $\quad$ Für wie wichtig halten Sie die Förderung von Emotionen/ bzw. die Förderung der Stimmung für den Erfolg Ihres Public Viewing?

Für wie wichtig halten Sie die positive Stimmung im Land für den Erfolg Ihres Public Viewing?

- $\quad$ Meinen Sie, dass viele Gäste Ihr Public Viewing besucht haben, weil die WM in Deutschland stattgefunden hat?

- In wiefern sind Sie der Meinung, dass sich Public Viewing zu einer Art „Selbstläufer“ entwickelt hat? Also keine Werbung für die Veranstaltung mehr nötig war, sondern die Gäste u.a. gekommen sind, weil sie Angst hatten etwas zu verpassen?

- Würden Sie eine derartige Veranstaltung wiederholen?

- Wenn ja, was würden Sie anders machen?

\section{Teil H: Persönliche Angaben}

- Frau oder Mann?

- Alter?

- Berufsausbildung/Berufsabschluss?

- Position in Ihrer Firma/lhrem Betrieb/lhrem Verein etc.?

DANKSAGUNG

Haben Sie noch Fragen oder Anregungen?

Eine Bitte? 


\section{Onlinefragebogen für die Besucher von Public Viewing-Veranstaltungen}

Fragebogen

Seite 1 von 1

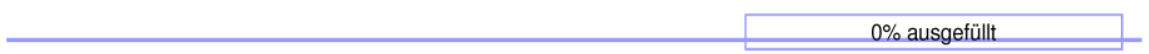

\section{Liebe(r) Public Viewing-Interessierte(r),}

vielen Dank für Ihre Bereitschaft, an dieser Befragung für meine Dissertation am Institut für Sportwissenschaften der Georg-August-Universität Göttingen teilzunehmen.

Die Beantwortung der Fragen dauert ca. 10 Minuten. Die Daten werden selbstverständlich anonym erhoben und natürlich auch anonym in der Dissertationsschrift verwendet.

Es findet selbstverständlich keine Erfassung der IP-Nummer statt, deswegen bitte ich Sie um eine wahrheitsgemäße Beantwortung der Fragen.

Für Ihre Mithilfe bedanke ich mich herzlichst im Voraus!

Ich würde mich freuen, wenn Sie den entsprechenden Link an interessierte Personen aus ihrem Freundes- und Bekanntenkreis weiterleiten würden.

Für die bessere Lesbarkeit wurde auf die Anführung der weiblichen Form verzichtet. Die männliche Ansprache bezieht sich hier auf beide Geschlechter.

Britta Ufer

Britta Ufer, Institut für Sportwissenschaften, Georg-August-Universität Göttingen - 2008

http://ofb.msd-media.de/pv/?set=PV\&admin_access=true \&PHPSESSID=9a1020326c ... 27.07.2008 


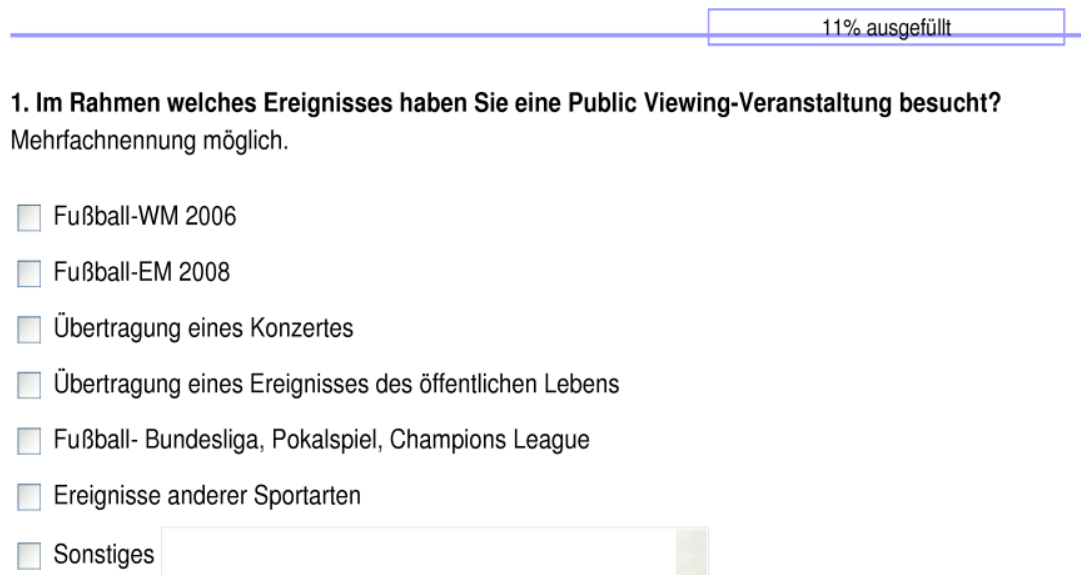

Britta Ufer, Institut für Sportwissenschaften, Georg-August-Universität Göttingen - 2008 
3. Ich habe eine Public Viewing-Veranstaltung besucht,weil ich ... Mehrfachnennung möglich.

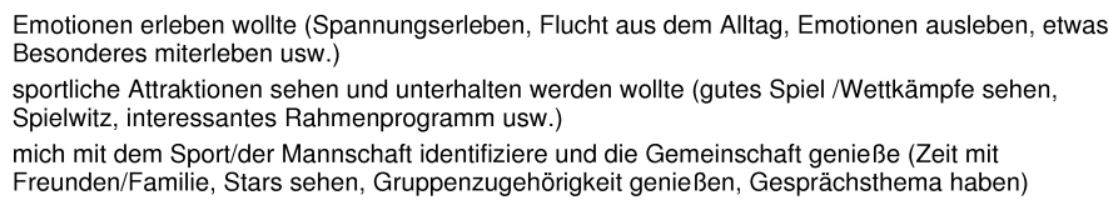

4. Ich bin...

Mehrfachnennung möglich.

alleine zum Public Viewing gegangen.

mit Kollegen zum Public Viewing gegangen.

mit Freunden zum Public Viewing gegangen.

mit der Familie zum Public Viewing gegangen.

Britta Ufer, Institut für Sportwissenschaften, Georg-August-Universität Göttingen - 2008 


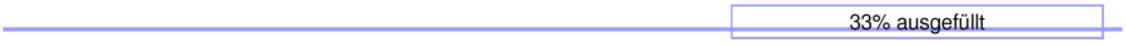

5. Ich habe positive Emotionen erlebt, weil...

Mehrfachnennung möglich.

die von mir favorisierte Mannschaft ein gutes Turnier gespielt hat.

im ganzen Land eine gute Stimmung geherrscht hat.

$\square$ ich bereits positive Erfahrungen bei einem vorherigen Besuch gemacht habe

Sonstiges

6. Wenn ein Spieler der von mir favorisierte Mannschaft gefoult wird, weiß ich, welche emotionale Reaktion angemessen ist,...

Mehrfachnennung möglich.

aus Erfahrung

von anderen Besuchern

aus den Medien

von einem Wortführer/Anführe

von den Spielern auf dem Feld

vom Veranstalter bzw. Sprecher und dem gezeigten Unterhaltungskonzept

Sonstiges

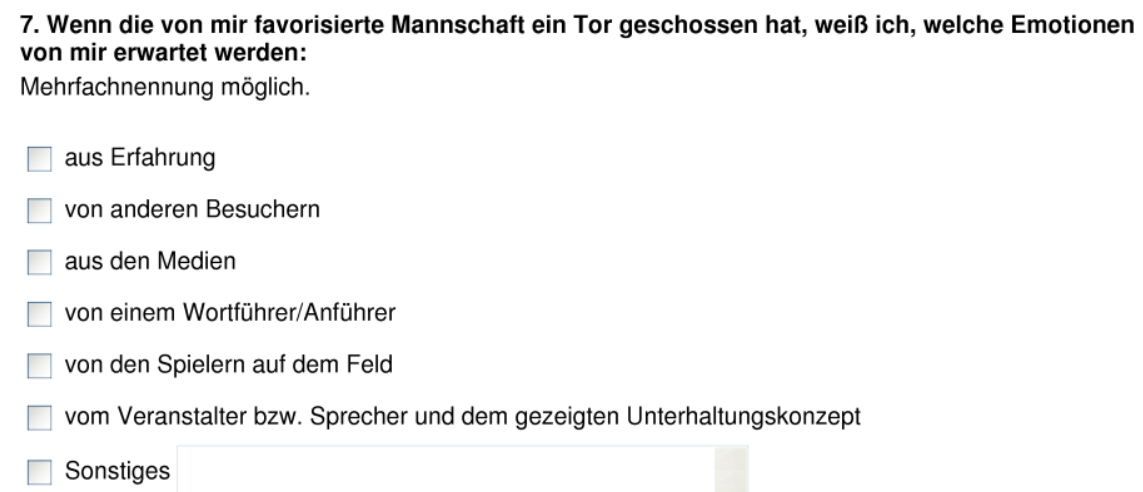

8. Die von Ihnen favorisierte Mannschaft hat gewonnen. Wovon machen Sie es abhängig, wie lange Sie sich über den Sieg freuen?

Mehrfachnennung möglich. 
9. Vor meinem ersten Besuch bei einem Public Viewing...

Bitte Zutreffendes ankreuzen.

habe ich gedacht, daß es wie im Stadion sein wird.

war ich unsicher, was die Sicherheit angeht.

wußte ich garnicht, was mich erwartet.

Sonstiges

10. Public Viewing ist eine Massenveranstaltung.

Bitte kreuzen Sie Zutreffendes an.

Ich hatte das Gefühl, mit den anderen Besuchern zu verschmelzen.

Ich habe ein Wir-Gefühl gespürt

Ich hatte das Gefühl, dass sich die Emotionen durch das enge Beisammensein leicht von einem auf den anderen Besucher übertragen.

Ich habe nichts dergleichen gespürt.

11. Während des Public Viewing... Bitte kreuzen Sie Zutreffendes an.

habe ich völlig abgeschaltet, die Zeit vergessen und bin in der Situation aufgegangen

habe ich zeitweise völlig abgeschaltet, die Zeit vergessen und bin in der Situation aufgegangen.

habe ich nichts dergleichen gespürt.

Britta Ufer, Institut für Sportwissenschaften, Georg-August-Universität Göttingen - 2008 


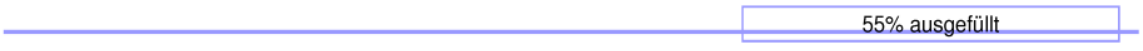

12. Außerhalb des Events war Public Viewing Gesprächsthema... Mehrfachnennung möglich.

im Freundeskreis.

unter den Kollegen.

in der Familie.

in den Medien.

Sonstiges

13. Welche Handlungen haben die Personen um Sie herum während der Public ViewingVeranstaltung gezeigt?

Mehrfachnennung möglich.

Sie haben geklatscht.

Sie haben mit dem Spielverlauf gefiebert (jubeln, meckern etc.)

Sie haben Fangesänge gesungen.

Sie haben bei der LaOla-Welle mitgemacht.

14. Während des Public Viewings habe ich..

Mehrfachnennung möglich.

geklatscht

Spielverlauf angeregt verfolgt (gejubelt, gemeckert etc.).

Fangesänge gesungen.

bei der LaOla-Welle mitgemacht.

Britta Ufer, Institut für Sportwissenschaften, Georg-August-Universität Göttingen - 2008 


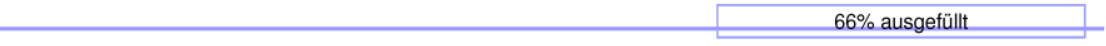

15. In meiner Freizeit..

treibe ich selber regelmäßig Sport.

verfolge ich wichtige Ereignisse des Sports regelmäßig im Fernsehen.

gucke ich regelmäßig die Spiele der Fußball-Bundesliga, Pokalspiele und/oder Spiele der

Championsleague.

gehe ich regelmäßig auf Konzerte.

engagiere ich mich in der Gesellschaft und verfolge aktuelle Ereignisse.

nichts der genannten.

16. Werden Sie wieder zum Public Viewing gehen?

ja

nein

17. Wenn ja, wird die nächste Public Viewing-Veranstaltung die ich besuche, zu folgendem Ereignis sein:

Wenn nein, bitte überspringen Sie diese Frage.

Mehrfachnennung möglich

Fußball EM 2008

Fußball- Bundesliga, Pokalspiele, Champions League

Olympische Spiele 2008

Konzert

Ereignis des öffentlichen Lebens

andere Sportarten

Sonstiges

Britta Ufer, Institut für Sportwissenschaften, Georg-August-Universität Göttingen - 2008 


\begin{tabular}{cc}
\hline \\
\hline
\end{tabular}
18. Ich bin...
weiblichen Geschlechts.
männlichen Geschlechts.
19. Ich bin...
14-29 Jahre alt.
30-49 Jahre alt.
über 50 Jahre alt.

\section{In welchem Bundesland leben Sie?}

[Bitte auswählen]

21. Welches ist ihr höchster Bildungsabschluss?
mittlere Reife
(Fach-)Abitur
abgeschlossene Berufsausbildung, Lehre o.ä.
abgeschlossenes Studium

Britta Ufer, Institut für Sportwissenschaften, Georg-August-Universität Göttingen - 2008 
22. Falls Ihnen noch etwas einfällt, was Sie gerne zum Thema Public Viewing erzählen würden, ist hier Platz dafür.

Anmerkung

Britta Ufer, Institut für Sportwissenschaften, Georg-August-Universität Göttingen - 2008 


\section{Herzlichen Dank für Ihre Teilnahme!}

Britta Ufer, Institut für Sportwissenschaften, Georg-August-Universität Göttingen - 2008 


\section{Interviewtranskripte}




\section{E. Wissenschaftlicher Bildungsgang}

\section{Wissenschaftlicher Bildungsgang}

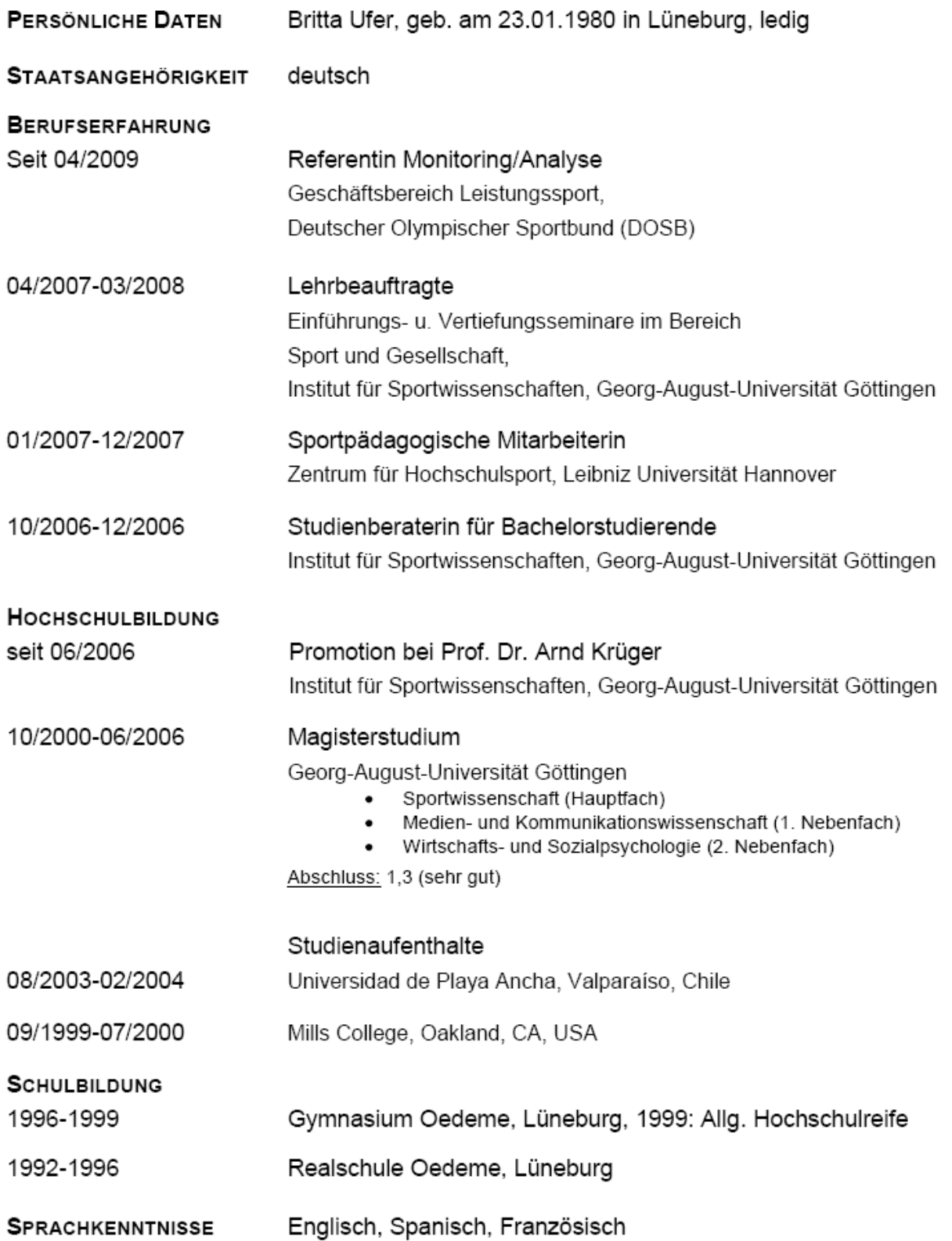


Ich versichere, dass ich die eingereichte Dissertation „Emotionen und Erlebnisse beim Public Viewing. Explorativ interdisziplinäre Analyse eines gesellschaftlichen Phänomens“, selbstständig und ohne unerlaubte Hilfsmittel verfasst habe. Anderer als der von mir angegebenen Hilfs mittel und Schriften habe ich mich nicht bedient. Alle wörtlich oder sinngemäß den Schriften anderer Autoren entnommenen Stellen habe ich kenntlich gemacht.

Reppenstedt, 13. April 2009 